Kazuwa Nakao - Nagahiro Minato Shinji Uemoto Editors
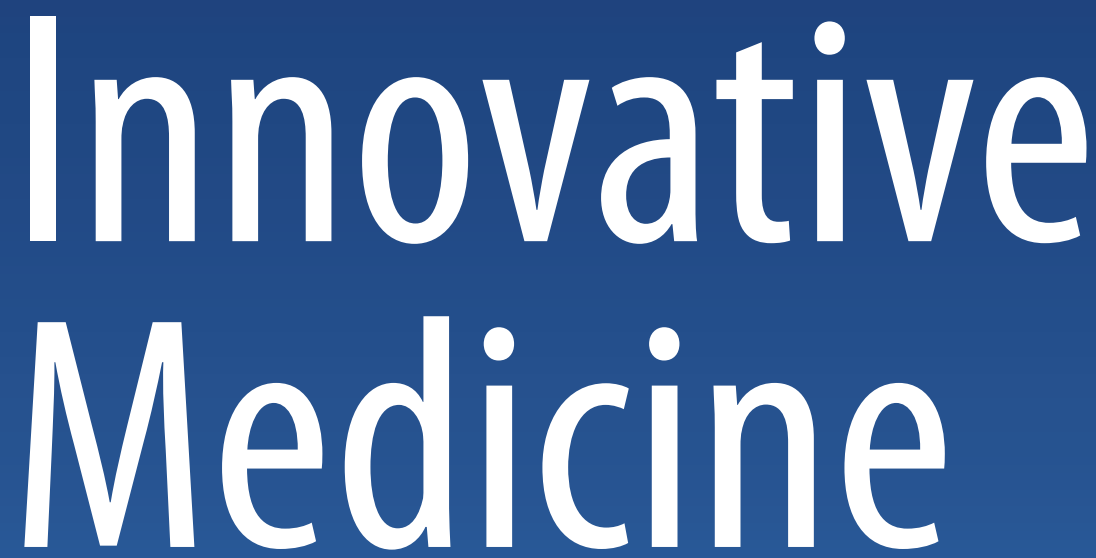

Basic Research and Development 
Innovative Medicine 

Kazuwa Nakao - Nagahiro Minato

Shinji Uemoto

Editors

\section{Innovative Medicine}

Basic Research and Development

照 Springer Open 


\author{
Editors \\ Kazuwa Nakao, M.D., Ph.D., Professor \\ Medical Innovation Center \\ Kyoto University Graduate School \\ of Medicine \\ Kyoto, Japan \\ Shinji Uemoto, M.D., Ph.D., Professor \\ Division of HBP Surgery and \\ Transplantation, Department of Surgery \\ Kyoto University Graduate School \\ of Medicine \\ Kyoto, Japan

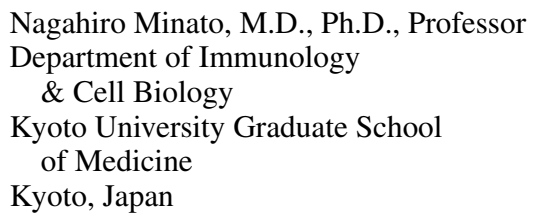

ISBN 978-4-431-55650-3 ISBN 978-4-431-55651-0 (eBook)

DOI 10.1007/978-4-431-55651-0

Library of Congress Control Number: 2015951664

Springer Tokyo Heidelberg New York Dordrecht London

(C) The Editor(s) (if applicable) and the Author(s) 2015. This book is published with open access at SpringerLink.com.

Open Access This book is distributed under the terms of the Creative Commons Attribution Noncommercial License, which permits any noncommercial use, distribution, and reproduction in any medium, provided the original author(s) and source are credited.

All commercial rights are reserved by the Publisher, whether the whole or part of the material is concerned, specifically the rights of translation, reprinting, reuse of illustrations, recitation, broadcasting, reproduction on microfilms or in any other physical way, and transmission or information storage and retrieval, electronic adaptation, computer software, or by similar or dissimilar methodology now known or hereafter developed.

The use of general descriptive names, registered names, trademarks, service marks, etc. in this publication does not imply, even in the absence of a specific statement, that such names are exempt from the relevant protective laws and regulations and therefore free for general use.

The publisher, the authors and the editors are safe to assume that the advice and information in this book are believed to be true and accurate at the date of publication. Neither the publisher nor the authors or the editors give a warranty, express or implied, with respect to the material contained herein or for any errors or omissions that may have been made.

Printed on acid-free paper

Springer Japan KK is part of Springer Science+Business Media (www.springer.com) 


\section{Preface}

Despite rapid and remarkable advances in basic medical science, discoveries in basic science can successfully be translated into clinical applications only after a time-consuming process and, unfortunately, only in extremely rare cases. The process has often been likened to a seemingly endless trip through a long and dark tunnel. The research field of translational science has thus been craved for, to unite basic and clinical sciences and make innovative medical technology a reality.

There are multiple vital steps in the creation of innovative medical technology: development and analysis of optimal animal models of human diseases, interpretation of data from genome science and epidemiology to address human disease and pathology, and establishment of "proof of concept" that plays a pivotal role in transitional to preclinical stages of translational science. Besides drug research and development, great expectations have been harbored for progress in diagnostic technology, new surgical procedures, and new clinical devices and equipment. Original research targets may well be rare diseases. More importantly, one can hope and try to expand the scope of the research into common diseases with the aid of "clinical wisdom."

In 2012, the Uehara Memorial Foundation launched the Innovative Medicine: Basic Research and Development project with the intention of making a contribution to the promotion and acceleration of medical research in Japan. Twenty outstanding Japanese researchers were selected to be part of the project team consisting of basic and clinician scientists, aiming at the goal of innovative medicine.

In the international symposium, held in Tokyo, 15-17 June 2014, fresh new findings of the project team and cutting-edge research developments were presented by leading basic and clinician scientists from around the globe who were invited speakers at the symposium, similarly aiming at the realization of innovative medicine.

Core themes were:

1. Basic research for innovative medicine

2. Translational research for innovative medicine

3. New technology for innovative medicine 
We sincerely hope that the symposium has sparked an upsurge of basic medical science, translational research, and the realization of innovation in its true sense in medical science and practice.

We are very grateful for the speakers and participants and are pleased to be able to publish the proceedings of this exciting symposium.

Kyoto, Japan

Kazuwa Nakao 


\section{Contents}

Part I Basic Research for Innovative Medicine

Diverting Glycolysis to Combat Oxidative Stress

Edouard Mullarky and Lewis C. Cantley

Metabolic Regulation by Nuclear Receptors

Sihao Liu, Michael Downes, and Ronald M. Evans

Fighting Fire with Fire in Cancer

Thorsten Berger, Mary E. Saunders, and Tak W. Mak

Linear Polyubiquitination: A Crucial Regulator of NF-кB Activation

Kazuhiro Iwai

VCP, a Major ATPase in the Cells, as a Novel Drug Target

for Currently Incurable Disorders

Akira Kakizuka

Roles of E-cadherin in Hepatocarcinogenesis

Shin Maeda and Hayato Nakagawa

The Hippo Signaling Pathway: A Candidate New Drug Target

for Malignant Tumors.

Miki Nishio, Hiroki Goto, Miki Suzuki, Aya Fujimoto, Koshi Mimori, and Akira Suzuki

Inhibitory Immunoreceptors on Mast Cells in Allergy and Inflammation

Akira Shibuya, Chigusa Nakahashi-Oda, and Satoko Tahara-Hanaoka 
Doxycycline-Inducible Autoimmune Blistering Skin Disease Model 109 Wataru Nishie and Hiroshi Shimizu

T Cell Senescence and Autoimmunity

Nagahiro Minato

Part II Translational Research for Innovative Medicine

IL-6: A New Era for the Treatment of Autoimmune

Inflammatory Diseases

Tadamitsu Kishimoto, Sujin Kang, and Toshio Tanaka

Pathogenesis of Non-alcoholic Steatohepatitis and Its Potential

Therapeutic Strategies

Yoshihiro Ogawa, Takayoshi Suganami, Michiko Itoh, and Miyako Tanaka

Multifaceted Translational Approach to Major Mental Illness

Akira Sawa

Translational Research of Leptin in Lipodystrophy

and Its Related Diseases

Ken Ebihara and Kazuwa Nakao

Translational Research of the Activation of the C-Type

Natriuretic Peptide (CNP)-Guanylyl Cyclase-B

Pathway for Skeletal Dysplasia

Akihiro Yasoda and Kazuwa Nakao

Clarity and Challenges in Tissue Fibrosis

Scott L. Friedman

TRP Channels: Their Function and Potentiality as Drug Targets

Motohiro Nishida, Koichiro Kuwahara, Daisuke Kozai,

Reiko Sakaguchi, and Yasuo Mori

Autophagic Cell Death and Cancer Chemotherapeutics.

Shigeomi Shimizu

Adrenomedullin as a Potential Therapeutic Agent for Refractory Ulcerative Colitis

Kazuo Kitamura, Shinya Ashizuka, Haruhiko Inatsu, and Toshihiro Kita

RNA Activation

X.Y. Zhao, J. Voutila, Nagy A. Habib, and Vikash Reebye 


\section{Part III New Technology for Innovative Medicine}

Proceedings of the Uehara Memorial Foundation. Innovative

Medicine: Basic Research and Development. Cardiac

Reprogramming for Heart Repair

Masaki Ieda

Development of a New In Vivo Optical Probe for Biological

Diagnosis and Therapy

Michitaka Ozaki, Takeaki Ozawa, and Yuma Yamada

Introduction of Mesenchymal Stem Cells for Liver Surgery

(Hepatectomy and Transplantation)

Shinji Uemoto, Yasuhiro Fujimoto, Takumi Teratani, Hiroyuki Kanazawa,

Junji Iwasaki, Zhao Xiangdong, Yuki Masano, Shintaro Yagi, Koichiro Hata, and Eiji Kobayashi

Synaptic and Axonal Plasticity Induction in the Human

Cerebral Cortex

Yoshikazu Ugawa

TIM-3 Is a Novel Therapeutic Target for Eradicating Acute

Myelogenous Leukemia Stem Cells

Koichi Akashi

TGF- $\beta$ LAP Degradation Products, a Novel Biomarker

and Promising Therapeutic Target for Liver Fibrogenesis

Mitsuko Hara, Tomokazu Matsuura, and Soichi Kojima

Cell-Based Regenerative Therapy for Liver Disease

Kenichi Horisawa and Atsushi Suzuki 
Part I

Basic Research for Innovative Medicine 


\title{
Diverting Glycolysis to Combat Oxidative Stress
}

\author{
Edouard Mullarky and Lewis C. Cantley
}

\begin{abstract}
Reactive oxygen species (ROS) are an intricate part of normal cellular physiology. In excess, however, ROS can damage all three major classes of macromolecules and compromise cell viability. We briefly discuss the physiology of ROS but focus on the mechanisms cells use to preserve redox homeostasis upon oxidative stress, with particular emphasis on glycolysis. ROS inhibits multiple glycolytic enzymes, including glyceraldehyde 3-phosphate dehydrogenase, pyruvate kinase M2, and phosphofructokinase-1. Consistently, glycolytic inhibition promotes flux into the oxidative arm of the pentose phosphate pathway to generate NADPH. NADPH is critically important, as it provides the reducing power that fuels the protein-based antioxidant systems and recycles oxidized glutathione. The unique ability of pyruvate kinase M2 inhibition to promote serine synthesis in the context of oxidative stress is also discussed.
\end{abstract}

Keywords Oxidative stress $\bullet$ Glycolysis $\bullet$ Pentose phosphate pathway $\bullet$ PKM2 • $\mathrm{GAPDH} \bullet \mathrm{ROS} \bullet \mathrm{NADPH}$

\section{Chemical Definition and Sources of ROS}

Reactive oxygen species (ROS) is a vague moniker used to describe a variety of oxygen-containing, chemically reactive small molecules, such as superoxide $\left(\bullet \mathrm{O}_{2}^{-}\right)$, the hydroxyl radical $(\mathrm{HO} \bullet)$, and hydrogen peroxide $\left(\mathrm{H}_{2} \mathrm{O}_{2}\right)$, that cause oxidative stress. ROS can be generated from exogenous sources like ionizing radiation or

\footnotetext{
E. Mullarky

Department of Medicine, Weill Cornell Medical College, New York, NY 10065, USA

Biological and Biomedical Sciences Graduate Program,

Harvard Medical School, Boston, MA 02115, USA

e-mail: edouardm2010@gmail.com

L.C. Cantley $(\varangle)$

Department of Medicine, Weill Cornell Medical College, New York, NY 10065, USA

e-mail: lcantley@med.cornell.edu
} 
redox-cycling xenobiotics $[1,2]$. Endogenously, ROS are an obligate by-product of aerobic metabolism. Typically, molecular oxygen is reduced by single- or two-electron mechanisms, yielding superoxide or hydrogen peroxide, respectively. Mitochondria are the predominant source of ROS owing to the electron transport chain (ETC), but peroxisomes and the endoplasmic reticulum contribute. During normal respiration, 1-2\% of molecular oxygen is converted to superoxide owing to electron leak at Complexes I and III [1, 3, 4]. Perturbations in mitochondrial metabolism such as changes in oxygen tension and the actions of mitochondrial uncoupling proteins can modulate superoxide production $[5,6]$. In addition, enzymes including the NADPH oxidases, which are particularly important in phagocytic cells, xanthine oxidases, uncoupled nitric oxide synthases, and cytochrome P-450s actively produce ROS [7]. Redox-active metal ions, such as iron, can generate the highly reactive hydroxyl radical from hydrogen peroxide via the Fenton reaction [8]. While diverse reactive oxygen species are commonly grouped together under the term ROS, it is important to remember that their chemistry, and hence biology, differ substantially. For instance, hydroxyl radicals react with near diffusion-limited rate constants with almost any organic molecule. The more limited reactivity of hydrogen peroxide enables it to diffuse across membranes and oxidize thiols specifically, thus making it a more suitable ROS second messenger [9, 10]. In general, reactivity comes at the expense of specificity.

\section{Physiology of ROS}

In excess, ROS can lead to widespread oxidative damage of all three macromolecular classes - lipids, protein, nucleic acids - and ultimately to cell death via apoptotic or necrotic pathways [11]. For instance, the hydroxyl radical and a protonated form of superoxide can initiate dangerous autocatalytic lipid peroxidation [11-13]. ROS are mutagenic and may therefore promote tumorigenesis [8]. Hydroxyl radicalinduced 8-oxoguanine lesions promote genomic G-to-T and C-to-A substitutions due to mismatched base pairing [14]. The hydroxyl radicals produced via ionizing radiation or Fenton reactions are such strong oxidants that they can abstract hydrogen atoms from a polypeptide backbone to generate a carbon radical [8, 15]. In addition, ROS-mediated proline oxidation can result in the cleavage of a protein peptide backbone. Amino acid side chains, such as those of methionine and cysteine and the aromatic groups of phenylalanine, tryptophan, tyrosine, and histidine, are also vulnerable to attack. Protein carbonylation is commonly used as a marker for oxidative stress. Oxidative protein modification can result in protein-protein cross-links. For example, the amino group of a lysine residue can attack a carbonyl of another protein. Importantly, some of the protein oxidative modifications, particularly protein cross-links, are resistant to proteasomal degradation and can inhibit the activity of the proteasome towards other proteins [16]. 
In moderate amounts, however, ROS are intricately linked with "normal" cellular physiology. In nonphagocytic cells, stimulating tyrosine kinase receptors via epidermal growth factor (EGF), platelet-derived growth factor (PDGF), and vascular endothelial growth factor (VEGF) induces a transient increase in cellular ROS [8, 17-19]. The signaling can be attenuated by antioxidant treatment. Nature has exploited the redox sensitivity of cysteine thiol groups to develop biochemical switches poised to functionally respond to changes in cellular ROS [20, 21]. Several of these thiol switches respond to growth factor stimulation-induced ROS. Specifically, ROS reversibly inhibits catalytic cysteine residues of the lipid phosphatase PTEN (phosphatase and tensin homolog) by disulfide bond formation and protein tyrosine phosphatases (PTPs) by cyclic sulfonamide formation. Thus, ROS-mediated phosphatase inhibition serves to enhance phosphatidylinositol-3 kinase (PI3K) and tyrosine kinase proliferative and survival signaling [20, 22, 23]. Most cytosolic protein thiol groups have a pKa greater than the physiological $\mathrm{pH}$ and are thus protonated and insensitive to the more mild forms of ROS such as hydrogen peroxide. However, the thiol switch local environment significantly reduces the cysteine side chain $\mathrm{pKa}$ such that the more nucleophilic thiolate anion predominates $[9,20]$. Thus, the thiolate anion is sensitized to changes in cellular ROS and ready to respond. In addition, thiols can react with electrophilic species via a Michael addition mechanism to form a covalent adduct potentially triggering the thiol switch [9].

ROS can both activate and repress transcription factors via thiol switch-based mechanisms. Rather than inhibiting enzymatic activity, as with the phosphatases discussed above, thiol oxidation induces conformational changes to regulate transcription factor subcellular localization. In Saccharomyces cerevisiae, for example, the AP-1-like transcription factor Yap1p responds to oxidative stress via $\mathrm{H}_{2} \mathrm{O}_{2}$ induced inter- and intramolecular disulfide exchanges that result in a conformational change in Yap1p. Conformational remodeling masks the nuclear export signal promoting nuclear stabilization and antioxidant gene expression. The Yap1p thiol switch thus permits a yeast cell to regulate an antioxidant gene program that responds to ROS directly [20]. Similarly, mammalian cells utilize a thiol redox switch to induce an antioxidant gene expression program in response to oxidative and xenobiotic stresses. Under "normal" conditions, Keap1 (Kelch-like ECHassociated protein 1) negatively regulates NRF2 (nuclear factor erythroid 2-related factor 2) by acting as an adapter for a CUL3 E3 ligase that targets NRF2 for ubiquitination and proteasomal degradation [24]. Keap1 contains multiple cysteine residues that are targeted by oxidants, including ROS and exogenous or endogenous electrophiles, to disrupt NRF2 repression [25-28]. Thus stabilized, NRF2 can induce expression of approximately 200 genes to promote both antioxidant and xenobiotic responses. Important NRF2 targets include glutathione (GSH) synthesis genes, such as the catalytic (GCLC) and modifier (GCLM) subunits of the ratelimiting step in GSH synthesis, and glutathione reductase (GSR). 


\section{Biochemical Mechanisms that Preserve Redox Homeostasis}

In addition to transcriptional responses like that of NRF2, cells employ a number of strategies to maintain redox homeostasis. The cytosol is maintained at a negative reducing potential of approximately $-250 \mathrm{mV}$ using the abundant (1-10 $\mathrm{mM}$ ) tripeptide glutathione (GSH) and its oxidized form (GSSG) as a redox couple buffer [20]. High-catalytic-activity enzymes rapidly scavenge ROS as they are produced. Cytoplasmic and mitochondrial isoforms of superoxide dismutase (SOD) enhance 10,000-fold the spontaneous dismutation of superoxide to hydrogen peroxide [29]. Peroxisomal catalase (CAT) and glutathione peroxidases (GPx) can further degrade hydrogen peroxide to water and molecular oxygen [10]. Were ROS to evade direct enzymatic scavenging and oxidize protein thiols, the parallel thioredoxin (Trx) and glutaredoxin (Grx) systems reduce the damage. Trx and Grx are small proteins $(9-16 \mathrm{kD})$, which share a dicysteine active site motif (CxxC) in a Trx fold [30]. The Trx mechanism involves a Trx-to-target protein-mixed disulfide that is subsequently nucleophilically attacked, by the remaining active site cysteine, to form an intramolecular Trx disulfide fully reducing the target protein. Grx prefers to attack S-glutathionylated target proteins forming a mixed Grx-glutathione disulfide that is resolved by a second GSH molecule releasing reduced Grx and GSSG. Both systems are ultimately dependent on cellular NADPH-reducing equivalents to regenerate them: Trx reductase (TrxR) and glutathione reductase (GSR) use NADPH to reduce oxidized Trx and GSSG, respectively (Fig. 1) [30]. In addition, glutathione peroxidases such GPx4 use GSH to reduce lipid and cholesterol peroxides [4, 31]. NRF2 activation induces the expression of multiple metabolic enzymes that directly generate NADPH, including glucose-6-phosphate dehydrogenase (G6PD), 6-phosphogluconate dehydrogenase (PGD), isocitrate dehydrogenase (IDH1), and malic enzyme (ME1), while downregulating genes for fatty acid synthesis that consume NADPH $[32,33]$. This allows NRF2 to stimulate the production of NADPH, the fundamental source of cellular reducing power. While catalase does not require NADPH for its enzymatic activity, it has an allosteric site for NADPH that maintains catalase in its active conformation [34]. ROS can activate mitogen-activated kinase (MAPK) signaling cascades that respond to cellular stress. Under normal conditions, ASK1 (apoptosis signaling-regulated kinase) is bound to Trx and inhibited. Trx binding requires the Trx dicysteine motif to be reduced. Following oxidation, ASK1 is released and free to oligomerize and autophosphorylate. Thus activated, ASK1 induces MAPK cascades that activate the p38 and JNK stress kinases to promote apoptosis [35]. Interestingly, the $\alpha$-arrestin family member Trx-interacting protein (TXNIP) seems to integrate glucose availability and ROS. As its name indicates, TXNIP forms intermolecular disulfides with Trx, inhibiting it and promoting oxidative stress [36]. TXNIP furthermore regulates the glucose transporter Glut1 by suppressing Glut1 mRNA and promoting its internalization via clathrin-coated pits. AMP-activated protein kinase (AMPK) - the cellular energy sensor - is activated under low-energy conditions to suppress ATP consumption and 


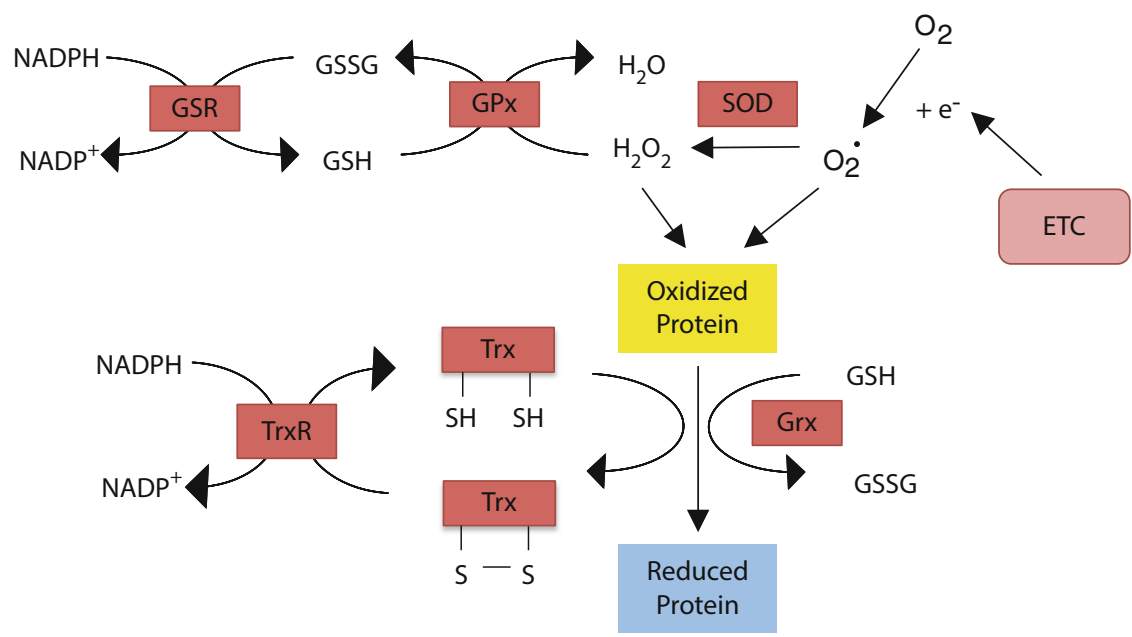

Fig. 1 Antioxidant systems that preserve redox homeostasis. Electron $\left(\mathrm{e}^{-}\right)$leak from the electron transport chain (ETC) produces superoxide $\left(\cdot \mathrm{O}_{2}^{-}\right)$. Superoxide dismutase (SOD) converts superoxide to hydrogen peroxide. Glutathione peroxidases (GPx) reduce peroxides, such as hydrogen peroxide $\left(\mathrm{H}_{2} \mathrm{O}_{2}\right)$, oxidizing glutathione (GSH) to GSSG. Reactive oxygen species (ROS) can oxidize proteins. The parallel thioredoxin (Trx) and glutaredoxin (Grx) systems can reduce proteins by oxidizing their dicysteine motif or GSH, respectively. Trx reductase (TrxR) and glutathione reductase (GSR) consume NADPH to restore Trx and GSH

increase ATP production. As such, AMPK phosphorylates TXNIP, thereby promoting its degradation via the proteasome to stabilize Glut1 mRNA and maintain Glut1 transporters at the plasma membrane [37].

\section{Metabolic Adaptations to ROS}

Metabolism is profoundly affected by oxidative stress. In excess, oxidation can provoke metabolic failure, compromising cell viability by inactivating enzymes of glycolysis, the Krebs cycle, and the ETC [11,38]. For example, oxygen-labile ironsulfur clusters, such as those of aconitase or ETC complexes, are often targeted $[4,39]$. However, metabolism has also evolved to respond to such stresses in an adaptive manner. Frequently, the mechanism revolves around thiol-based switches that allow the cell to rewire metabolism in a way that promotes an antioxidant response independent of transcriptional or signaling pathways. As such, metabolism is one of the faster responders; metabolic rewiring is evident within minutes of oxidative stress [40]. We will explore how cells tune glycolytic metabolism to cope with oxidative damage. Much of the antioxidant systems ineluctably rest on the NADPH to $\mathrm{NADP}^{+}$ratio. Thus, a recurring theme will be how glycolytic flux is diverted into NADPH-generating processes. 


\section{The Pentose Phosphate Pathway and NADPH Production}

After glucose is imported into the cell via GLUT transporters, it is phosphorylated by hexokinase (HK) at the 6 position to generate glucose-6-phosphate (G6P). Glucose phosphorylation has the dual benefits of trapping glucose within the cell and providing a trans-membrane concentration gradient to draw more glucose in. G6P lies at the nexus of glycolysis, glycogen synthesis - via conversion to glucose1-phosphate-and the oxidative arm of the pentose phosphate pathway (ox-PPP). The predominant fate of G6P is a function of cell type and metabolic demand. The ox-PPP is traditionally considered the predominant producer of cellular NADPH and is thus critical for antioxidant defense [41]. Conceptually, the ox-PPP is distinct from the reversible non-oxidative phase of the PPP, which does not produce NADPH (Fig. 2) [42]. G6PD catalyzes the first committed and rate-limiting step of the ox-PPP, generating one unit of NADPH and 6-phosphoglucolactone [34]. The unstable lactone ring is opened by phosphogluconolactonase to yield 6-phosphogluconate, which is subsequently decarboxylated by PGD to give an additional unit of NADPH

Glycolysis

Oxidative PPP

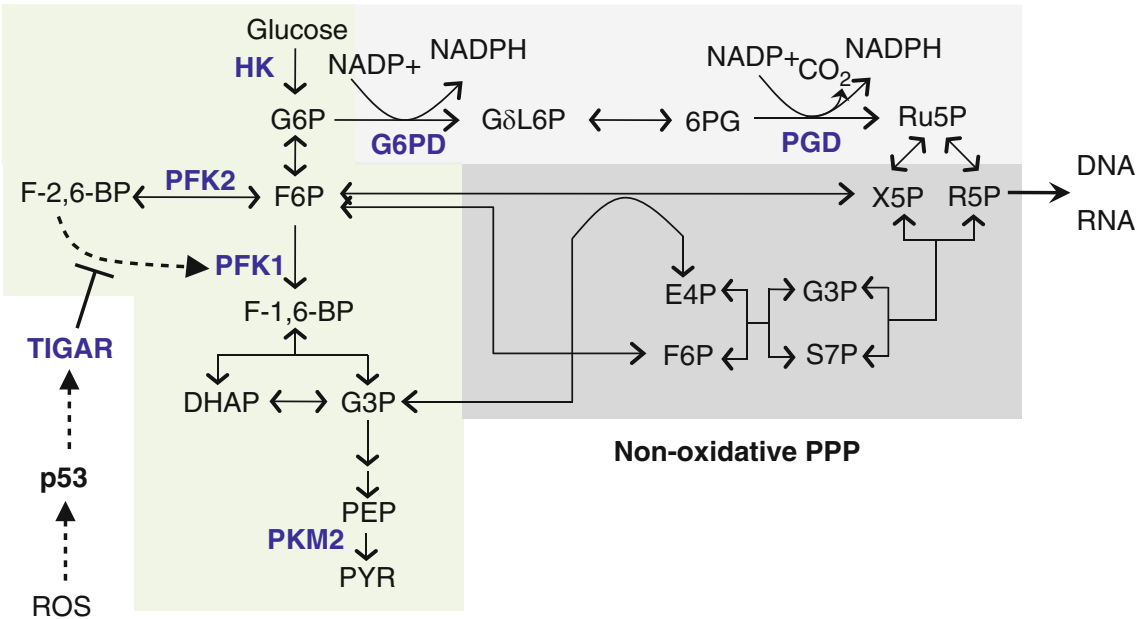

Fig. 2 Glycolysis and the pentose phosphate pathway (PPP). The PPP is composed of two distinct arms, the oxidative branch (light blue) and the non-oxidative branch (gray). While both arms produce ribose-5-phosphate, a precursor for nucleotide synthesis, only the oxidative branch concomitantly produces NADPH. Glycolytic flux enters the oxidative branch via glucose-6-phosphate dehydrogenase (G6PD). Fructose-2,6-bisphosphate (F-2,6-BP) activates phosphofructokinase-1 (PFK1) to promote glycolysis (light green). In response to reactive oxygen species (ROS) and UV stress, p53 activates TIGAR (TP53-induced glycolysis and apoptosis regulator). TIGAR degrades F-2,6-BP, thereby inhibiting PFK1. This allows glycolytic flux to be diverted into the oxidative arm and enhances NADPH production to fuel the cellular antioxidant systems. Metabolic enzymes are shown in dark blue 
and ribulose-5-phosphate [34, 43]. The net yield per unit of G6P is therefore two NADPH and ribulose-5-phosphate. Ribulose-5-phosphate is an immediate precursor for the ribose-5-phosphate used in the synthesis of nucleotide sugar moieties. The G6P carbon may be recycled back into glycolysis as the non-oxidative arm PPP enzyme transketolase produces the glycolytic intermediates glyceraldehyde-3-phosphate (G3P) and fructose-6-phosphate (F6P) (Fig. 2) [34, 43]. Post-translationally, G6PD is regulated by phosphorylation, protein-protein interaction, and translocation to the plasma membrane upon growth factor stimulation [34, 44-47]. Importantly, G6PD is allosterically activated by the $\mathrm{NADP}^{+}$to NADPH ratio [34, 48, 49]. Thus, as antioxidant enzymes, including those of the Grx and Trx systems, consume NADPH to reduce ROS-induced damage, $\mathrm{NADP}^{+}$levels increase, stimulating the activity of the ox-PPP to produce more NADPH and maintain cellular reducing power.

The importance of ox-PPP in protecting against oxidant stress is clearly evident from X-linked G6PD deficiency, the most common human enzyme defect in the world. Erythrocytes are sensitive to oxidative stress and are highly dependent on ox-PPP to maintain NADPH and reduced GSH. Thus, one well-documented and potentially lethal clinical manifestation of G6PD deficiency is acute hemolytic anemia following ingestion of oxidative stress-inducing agents. Such agents include the antimalarial primaquine, sulfonamides, and fava beans. Other patients suffer from chronic anemia $[50,51]$. In agreement with the human pathology, in vitro experiments in a variety of cell types show that G6PD inhibition or genetic knockout increases sensitivity to oxidizing agents, including exogenous and endogenous $\mathrm{H}_{2} \mathrm{O}_{2}$ [52, 53]. G6PD knockout increases the apoptotic response of CHO cells exposed to ionizing radiation consistent with the role of ROS in apoptosis [54]. Conversely, G6PD overexpression increases resistance to exogenous $\mathrm{H}_{2} \mathrm{O}_{2}[52,53]$. The combination of human and in vitro data argues that the diversion of glycolytic flux into the ox-PPP pathway plays a vital role in antioxidant defense at both a cellular and organismal level.

Different cell types likely rely on different metabolic pathways to generate their basal level of NADPH. Mutant KRas-driven pancreatic ductal adenocarcinoma cells (PDAC) use glutamine-derived malate to generate basal NADPH, via malic enzyme (ME1), and keep ROS in check. In PDAC, G6PD knockdown does not affect NADPH levels, suggesting that it is not necessary for redox balance [56]. PDAC rely on the non-oxidative PPP branch to promote ribose biogenesis for nucleic acid production, hence decoupling it from NADPH synthesis [57]. In contrast, HEK293T cells are not dependent on ME1 but instead use the ox-PPP and folate cycle to generate basal NADPH and maintain reduced GSH pools [58]. Whether ox-PPP activation from a more inhibited state due to high NADPH levels under "normal" cellular conditions is the predominant NADPH stress response pathway, as some have suggested, needs further investigation [52, 54, 59]. For example, HEK293T cells derive a majority of their NADPH from the ox-PPP with the folate cycle producing a substantial amount [58]. Knockdown of the folate cycle enzymes methylenetetrahydrofolate dehydrogenase 1 (MTHFD1) and MTHFD2 sensitizes HEK293T cells to acute hydrogen peroxide and diamide stress, indicating that the folate cycle also plays a role in dealing with oxidative stress presumably through its substantial 
NADPH contribution. Whether folate cycle NADPH production is directly activated by ROS stress, like the ox-PPP, remains to be determined. The fact that NRF2 has evolved to regulate the expression of the NADPH-generating enzymes IDH1 and ME1, in addition to G6PD and PGD, suggests that it is beneficial to activate NADPH production not only via the induction of the ox-PPP [28]. In the context of ME1 knockdown PDAC cells, why the increases in $\mathrm{NADP}^{+}$and ROS do not trigger increased ox-PPP pathway flux directly through G6PD or indirectly via NRF2 is not clear and is surprising given that other cell types are known to do so [53].

\section{Phosphofructokinase-1 Inhibition}

Once glucose is trapped within the cell as G6P, it undergoes a reversible isomerization reaction to fructose-6-phosphate (F-6-P) catalyzed by phosphoglucose isomerase (PGI). Phosphofructokinase-1 (PFK1) subsequently phosphorylates F-6-P at the 1 position, yielding fructose-1,6-bisphosphate (F-1,6-BP). Importantly, the PFK1 step is both rate limiting and the first committed step of glycolysis; above PFK1, glycolytic intermediates can enter into glycogen synthesis, the ox-PPP, or the hexosamine pathway $[60,61]$. PFK1 functions as the gatekeeper of glycolysis and is therefore highly regulated. ATP and citrate are allosteric inhibitors, while AMP and fructose-2,6-bisphosphate (F-2,6-BP) are activators [60, 61]. The exact PFK1 kinetic parameters are determined by the specific subunit composition [62]. Releasing ATP-based PFK1 inhibition is important to stimulate glucose metabolism in proliferating cells [63]. This is in part achieved by F-2,6-BP-induced PFK1 activation. F-2,6-BP is produced by phosphofructokinase-2 (PFK2) phosphorylating F-6-P at the 2 position (Fig. 2). PFK2 is a bifunctional enzyme containing a kinase domain and bisphosphatase (BPase) domain at the $\mathrm{N}$ and $\mathrm{C}$-termini, respectively $[64,65]$. Thus, the cellular F-2,6-BP concentration depends on the rates of the two opposing activities. The kinase and BPase activities are regulated transcriptionally and post-translationally via, for example, hormonal stimulation [64, 65]. Conceptually, the F-2,6-BP shunt not only provides a PFK1 feed-forward mechanism to accelerate glycolysis when intracellular F-6-P accumulates but also helps decouple glycolytic flux from the cellular ATP charge. Unsurprisingly, PFK1 and PFK2 are deregulated in cancer $[64,66]$.

TIGAR (TP53-induced glycolysis and apoptosis regulator) was identified as a p53 target gene induced by ionizing radiation [67, 68]. TIGAR has a single BPase activity that degrades F-2,6-BP to F-6-P [64, 65]. By decreasing F-2,6-BP levels, TIGAR inhibits glycolytic flux downstream of PFK1. PFK1 inhibition allows the G6P and F6P pools to accumulate as their consumption is greatly diminished. The increased G6P can flow into the ox-PPP to generate NADPH. Consistent with this, TIGAR knockdown, or inhibition of upstream positive regulators, leads to increased ROS and a decrease in NADPH and reduced GSH [68-71]. The intestinal crypts of TIGAR knockout mice subjected to whole body irradiation are acutely more apoptotic and have a greater difficulty in regenerating themselves compared with those 
of wild-type animals [72]. The apoptotic response is suggestive of a failure in dealing with ROS; left unchecked, ROS can trigger apoptosis. Use of an in vitro three-dimensional crypt culture model showed that the TIGAR knockout crypts also have a proliferation defect. The defect can be rescued by exogenous antioxidants or nucleosides. Interestingly, nucleoside addition was found to help sustain a favorable GSH to GSSG ratio [72]. Overall, these mechanisms can be understood in that PFK1 inhibition allows for a buildup of G6P that pushes into the ox-PPP in which a rising $\mathrm{NADP}^{+}$to NADPH ratio is furthermore activating G6PD. The NADPH thus produced provides reducing power to deal with the oxidative stress. The antioxidant effect of TIGAR under hypoxia is partially independent of its BPase activity and instead depends on TIGAR translocating to the mitochondria and associating with mitochondrial hexokinase-2 [73].

\section{Glyceraldehyde 3-Phosphate Dehydrogenase Inhibition}

The redirection of glycolytic flux through the ox-PPP to combat oxidative stress is also achieved by targeting glycolytic enzymes downstream of PFK1. Frequently, the process involves ROS directly oxidizing thiol switches within these enzymes. Subsequent to the PFK1 step, aldolase cleaves F-1,6-BP into two three-carbon molecules: dihydroxyacetone phosphate (DHAP) and G3P. G3P is the substrate of glyceraldehyde 3-phosphate dehydrogenase (GAPDH). GAPDH catalyzes the reversible oxidative phosphorylation of G3P to 1,3-bisphosphoglycerate (1,3-BPG) using $\mathrm{NAD}^{+}$and inorganic phosphate. 1,3-BPG is a strong product inhibitor of GAPDH [74]. Mechanistically, GAPDH employs a conserved active site cysteine (Cys152 in humans) for a nucleophilic attack on the aldehyde moiety of G3P forming a thiohemiacetal that rearranges to an acyl-enzyme intermediate with a hydride transfer to $\mathrm{NAD}^{+}$. The acyl-enzyme intermediate is resolved by an inorganic phosphate attack [74]. The same active site cysteine involved in catalysis functions as a thiol switch, as discussed below. Interestingly, GAPDH has other enzymatic activities including S-nitrolase, ADP-ribosylase, kinase, and peroxidase [74].

The GAPDH reaction is not at equilibrium and is therefore a potential regulatory point of glycolysis [75]. In mammalian cells, GAPDH is inhibited within minutes of exposure to oxidants predominantly via direct enzyme inactivation and loss of the $\mathrm{NAD}^{+}$cofactor presumably through PARP activation $[40,76]$. The GAPDH active site cysteine is highly sensitive to inhibitory oxidative modifications of ROS and reactive nitrogen oxide species (RNS). With $\mathrm{H}_{2} \mathrm{O}_{2}$, the modifications include, in order of increasing oxidation, sulfenic, sulfinic, and sulfonic acid. Additionally, the active site cysteine can oxidize by forming an intramolecular disulfide with a proximal cysteine [20, 40, 77-80].

Beyond direct ROS thiol oxidation, GAPDH is rapidly S-thiolated following both endogenous (e.g., monocyte respiratory bursts) and exogenous oxidative stress. $\mathrm{S}$-thiolation is a posttranslational modification in which proteins form mixed disulfides with low molecular weight thiols. In human cells, the majority of adducts are 
formed using GSH, but free cysteine also contributes. GAPDH S-thiolation is inhibitory. Activity can be restored by dithioerythritol (DTE) treatment or if the oxidative insult or stimulus is removed, indicating that the inhibition is reversible [81, 82]. $S$. cerevisiae knockout strains defective in GSH biosynthesis cannot recover GAPDH enzymatic activity, suggesting that GSH is necessary to protect against irreversible thiol hyperoxidation [83]. The process seems to be regulated, because S-thiolation is specific to the Tdh3 isoform of GAPDH in S. cerevisiae, but not the Tdh2 isoform, despite high sequence homology (96\% identity). Tdh3 recovers activity within a 2-h period, but not Tdh2. Interestingly, the isozymes are required to deal with different types of exogenous oxidative stress-lethal dose versus a continuous low-level challenge [84]. ATP levels plummet following ROS stress as both mitochondrial and glycolytic ATP synthesis is inhibited [40, 76]. Protecting GAPDH from irreversible oxidation via S-thiolation may allow a cell to quickly resume glycolysis and hence ATP production after the stress wanes. Without a sufficiently rapid recovery of ATP synthesis, cell death may ensue. Oxidative stress can also induce GAPDH aggregation via intermolecular disulfide bonds dependent on the active site cysteine. Such aggregates are found in brain extracts from Alzheimer's disease $(\mathrm{AD})$ patients and may participate in the proapoptotic functions of GAPDH $[20,85,86]$. Importantly, GAPDH inhibition helps divert glycolytic flux into the ox-PPP pathway by allowing metabolites to accumulate upstream of the point of inhibition consistent with the observed induction of PPP enzymes following $\mathrm{H}_{2} \mathrm{O}_{2}$ treatment (Fig. 3) [83, 87]. Triose phosphate isomerase (TPI) immediately precedes GAPDH in glycolysis. Both Caenorhabditis elegans and S. cerevisiae mutants with reduced TPI activity are resistant to oxidative stress. Using a combination of genetic knockouts of PPP enzymes and metabolomic studies, it was shown that low-TPI-activity mutants or ROS inhibition of GAPDH rerouted flux through the PPP $[88,89]$. Thus, GAPDH is an important target of ROS that mediates cellular antioxidant response.

\section{Pyruvate Kinase M2 Inhibition}

Pyruvate kinase (PK) catalyzes the final reaction of glycolysis transferring the phosphate moiety of phosphoenolpyruvate (PEP) to ADP, thus generating pyruvate and ATP. Mammals have four PK isoforms. The liver (PKL) and erythrocyte (PKR) isoforms are produced from the PKLR gene. The PKM1 and PKM2 isoforms derive from alternate splicing of exons 9 and 10 of the $P K M$ gene, respectively [90-93]. PKM1 is predominantly expressed in adult differentiated tissues with a high ATP demand, such as the brain, heart, and muscle. PKM2 is expressed over the course of development, in cancers, and in tissues such as the spleen and lungs [94, 95]. PKM2 differs from PKM1 in that it has a lower intrinsic enzymatic activity and has unique regulatory properties. PKM2 allosteric activators include AMP, the de novo purine synthesis intermediate SAICAR (succinylaminoimidazolecarboxamide ribose-5-phosphate), the glycolytic intermediate F-1,6-BP, and the amino acid serine [95-98]. Cellular PKM2 is in a dynamic equilibrium between a less active 


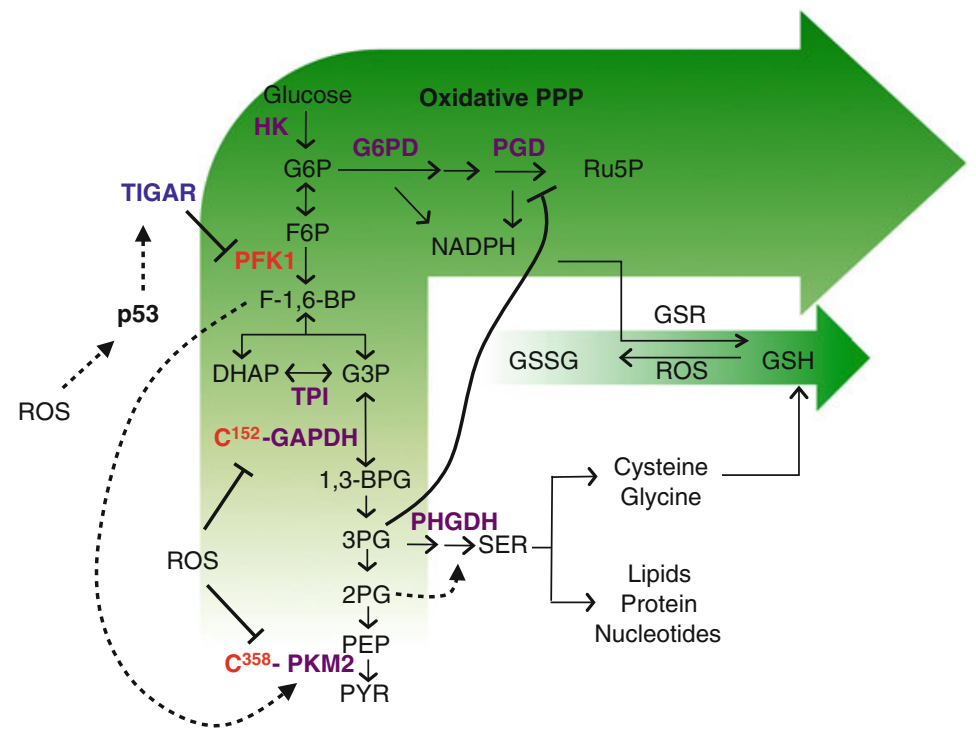

Fig. 3 Reactive oxygen species (ROS)-mediated inhibition of glycolysis reroutes flux into the oxidative arm of the pentose phosphate pathway. ROS inactivates glyceraldehyde 3-phosphate dehydrogenase (GAPDH) and the pyruvate kinase isoform PKM2 by directly targeting cysteine residues. Alternatively, ROS and UV stress can trigger p53-dependent TIGAR (TP53-induced glycolysis and apoptosis regulator) activation that inhibits phosphofructokinase-1 (PFK1). Glycolytic inhibition promotes flux into the oxidative pentose phosphate pathway to produce NADPH and fuel cellular antioxidant systems (graded green arrow). For example, NADPH is consumed by glutathione reductase (GSR) to recycle oxidized glutathione (GSSG). PKM2 inhibition is unique in that it allows for a diversion of flux into the serine synthesis pathway. Serine not only contributes to the synthesis of macromolecules but is also a precursor for glutathione (GSH). Serine synthesis is activated by a buildup of 2-phosphoglycerate (2PG), which prevents 3-phosphoglycerate (3PG)induced inhibition of the oxidative pentose phosphate arm. Enzymes are shown in purple. ROS targets are shown in red

monomeric form and a more active tetrameric form. Mechanistically, F-1,6-BP allosterically activates PKM2 by stabilizing the tetramer. Conversely, as F-1,6-BP levels drop, the monomeric form prevails, inhibiting PKM2 activity. Thus, F-1,6-BP provides a regulatory loop to coordinate PKM2 activity based on the product of the critical PFK1 step and glucose availability [99-101]. Phosphotyrosine protein binding, tyrosine phosphorylation (Y105), and lysine acetylation (K433) prevent F-1,6-BP binding, thereby inhibiting PKM2 activity [102-104]. Surprisingly, multiple non-glycolytic functions unique to PKM2 have been proposed, including protein kinase and transcriptional coactivator activities. The role of PKM2 in cancer is under intensive study, in part because it has been argued that PKM2 is critical for the metabolic rewiring needed to support cancer cell proliferation, and also because of its novel non-glycolytic activities [96, 102, 105-111]. In studying the glycolytic function of PKM2 in cancer cells, it has become clear that PKM2 contains a thiol switch that is targeted by ROS [112]. 
Across diverse organisms ranging from Escherichia coli to humans, PK activity is inhibited by oxidative stresses [86,112-114]. One of the earlier observations was that $E$. coli $\mathrm{PK}$ stored cold for prolonged periods of time without a reducing agent lost activity. Activity was unresponsive to the conventional activators AMP and F-1,6-BP, but could be recovered by incubating the inactive species with the reducing agents beta-mercaptoethanol or dithiothreitol (DTT) [113]. Whether the inhibition was an in vitro artifact or physiologically relevant was unclear. Prompted by the link between oxidative stress and Alzheimers disease (AD), proteomic studies to identify oxidatively modified proteins in the hippocampi of patients suffering from mild cognitive impairment, a condition that commonly progresses to $\mathrm{AD}$, revealed that PKM2 was significantly more carboxylated in those patients than in controls [86]. Interestingly, in $S$. cerevisiae, low PK activity activates respiration. Despite increased oxidative phosphorylation, increased ROS production is suppressed, hinting at some antioxidant function of low PK activity [115]. PEP functions as a competitive inhibitor of human and yeast TPI. Crystallographic studies indicate that the PKM2 substrate PEP binds directly in the TPI catalytic pocket $[115,116]$. Thus, low PK activity enables PEP to accumulate, to form a negative feedback loop that reduces GAPDH substrate availability by preventing the interconversion of DHAP and G3P. TPI inhibition redirects flux into the PPP pathway and protects yeast from a variety of oxidative stresses explaining how the increased respiration resulting from low PK activity does not promote ROS [115]. Previous work had shown that TPI loss-of-function mutants in S. cerevisiae and C. elegans are similarly resistant to exogenous oxidative stresses in a manner genetically dependent on PPP enzymes [88, 89].

Studying PKM2 in the context of cancer cell metabolism not only elucidated the mechanism whereby ROS inactivates PKM2, but also identified the functional significance of PMK2 inhibition [112]. In human cancer cells, several types of oxidative stresses, including $\mathrm{H}_{2} \mathrm{O}_{2}$, diamide, and hypoxia, inactivate PKM2. DTT restores PKM2 activity to levels commensurate with those of untreated cells. Neither PKM1 nor heteromers of PKM1 and PKM2 are inhibited by oxidation. Oxidation was shown to directly target Cys358 of PMK2 and decrease the levels of the active tetramer thereby explaining the reduced PKM2 activity. Mutating Cys358 to serine abrogates oxidative stress-induced PKM2 dissociation thus preserving the enzymatic activity under stress. Adding small molecule activators that bind to the PKM2 subunit interface and stabilize the tetrameric form similarly prevent ROS-induced dissociation and loss of PKM2 activity [101, 112]. Functionally, PKM2 inhibition allows cells to increase G6P levels and ox-PPP pathway flux to generate more NADPH and hence preserve reduced GSH and prevent intracellular ROS accumulation (Fig. 3). The ROS inducible PKM2 inhibition not only translates into greater survival when cells are exposed to acute oxidative stress, or chronic ROS stress induced by hypoxia, but also increases the tumorigenic potential of cells in xenografts. Both activator-treated and PKM2 ${ }^{\mathrm{C} 358 \mathrm{~S}}$ mutant cells are defective in their antioxidant response indicating how critical tetramer dissociation is to protect against oxidative stress [112]. ROS-mediated PKM2 inhibition also suggests a mechanism whereby PEP levels can accumulate and inhibit TPI, as in the yeast study described above. 
PEP inhibition of recombinant human TPI in biochemical assays has been demonstrated $[115,116]$. Whether TPI inhibition is necessary for the protective effects of PKM2 inhibition in human cells remains unknown. PKM2 has been reported to interact with the HIF $1 \alpha$ and HIF $2 \alpha$ transcription factors to promote expression of glycolytic genes (e.g., SLC2A1, LDHA, PDK1) and VEGFA. Thus, PKM2 may also promote ROS detoxification by alleviating tumor hypoxia $[117,118]$.

\section{De Novo Serine Synthesis}

While PKM2 inhibition allows cells to fend off ROS by activating the ox-PPP, it may also help cells deal with more chronic oxidative stress by enabling a buildup of the glycolytic intermediate 3-phosphoglycerate (3PG). 3PG can be diverted into the phosphoserine pathway for de novo serine synthesis [119-121]. Alternatively, serine can be imported from the extracellular space by a variety of transporters, including the commonly expressed ASC system (ASCT1 and ASCT2), that mediate the symport of serine, alanine, or cysteine with sodium [122, 123]. Serine plays a vital role in the antioxidant defense system because it is a precursor for the synthesis of GSH (Fig. 3). The phosphoserine synthesis pathway consists of three sequential reactions: first, 3-phosphoglycerate dehydrogenase (PHGDH) oxidizes 3PG using $\mathrm{NAD}^{+}$to give 3-phosphohydroxypyruvate (3-PHP); second, the PLP-dependent phosphoserine aminotransferase (PSAT1) transaminates 3-phosphohydroxypyruvate to phosphoserine (PSER) utilizing glutamate as the nitrogen donor; finally, phosphoserine phosphatase (PSPH) hydrolyzes the PSER phosphate group to release serine [119-121]. PHGDH, which catalyzes the first committed step of the pathway, was found to be focally amplified in human tumors, particularly those of the breast and melanoma. Cancer cell lines harboring the amplification, and some nonamplified lines overexpressing PHGDH, are uniquely sensitive to knockdown of any enzyme in the pathway [124, 125]. Although some have speculated, the mechanism by which the phosphoserine pathway promotes tumorigenesis and why extracellular serine is unable to compensate remain to be determined [121]. Interestingly, 3PG is a competitive inhibitor of PGD. Thus, an extensive buildup of 3PG can inhibit ox-PPP NADPH production. 3PG levels are kept sufficiently low via a feedback loop that activates 3PG diversion into the phosphoserine pathway. In glycolysis, 3PG is converted to 2-phosphoglycerate (2PG) by phosphoglycerate mutase 1 (PGAM1). 2PG activates PHGDH to deplete excess $3 \mathrm{PG}$, thereby promoting the synthesis of serine and preventing ox-PPP inhibition [126].

Oxidative stress is known to damage all three principal classes of macromolecules -lipids, nucleic acids, and protein [11]. Macromolecules that cannot be repaired by the cellular antioxidant systems can be replaced by newly synthesized molecules. Serine is an important precursor for de novo macromolecule synthesis. Serine is directly incorporated into proteins and the head groups of certain abundant lipids such as sphingosine and phosphatidylserine [127, 128]. Serine hydroxymethyltransferases (SHMTs) convert serine to glycine in a retro-aldol cleavage reaction 
concomitantly charging the folate pool with a methylene group. In fact, the SHMT reaction is a major source of one-carbon units for the folate cycle. Glycine and the folate cycle donate carbon for the synthesis of purine and pyrimidines [129]. Thus, by contributing to protein, nucleic acid, and lipid synthesis, serine can help cells recover from oxidative damage to macromolecules.

The importance of serine in dealing with oxidative stress is further highlighted by its contribution to GSH synthesis. GSH is an enzymatically synthesized tripeptide composed of glutamate, cysteine and glycine. Cysteine and glycine can both be produced from serine or imported from the extracellular space. Serine combines with homocysteine in the transsulfuration pathway to yield cystathionine, which is subsequently hydrolyzed to cysteine and homoserine [42]. Glycine is formed from serine via SHMTs as described above. Thus, up to two moles of serine can be consumed per mole of GSH produced. In certain cell types, a large fraction of cytosolic NADPH, comparable to that produced via the PPP, is produced from the oxidation of folate cycle one-carbon units derived from serine via the SHMT reaction [58]. Hence, the conversion of serine to glycine may have the twin benefits of fuelling GSH synthesis and providing the NADPH-reducing power to maintain GSH in its reduced form via glutathione reductase. Alternatively, the NADPH could fuel fatty acid synthesis to aid recovery from lipid oxidation damage [130]. There is significant heterogeneity in the propensity of different cell types to synthesize serine de novo suggesting that the anabolic functions of serine following oxidative stress may similarly diverge across cell types [124, 125].

\section{Conclusion}

We have seen that ROS can inhibit glycolysis at multiple nodes. A recurring theme is that the inhibition of glycolysis allows cells to divert flux into the ox-PPP pathway to promote NADPH synthesis and protect against oxidative stress. However, there are also differences depending on the exact point of inhibition. Inhibition at the PKM2 step allows cells to promote flux into the serine synthesis pathway, while PFK1 and GAPDH inhibition does not. Furthermore, both GAPDH and PKM2 inhibition can promote dihydroxyacetone phosphate accumulation, which is an important precursor for the glycerol-3-phosphate shuttle and the synthesis of glycerol needed for triglycerides $[88,115]$. As of yet, we only have a limited understanding of what determines which glycolytic node is targeted by ROS and what the advantages are for each. For example, both GAPDH and PKM2 are inhibited by hydrogen peroxide, but is the order of inactivation simply determined by the relative order of the redox potentials of their respective cysteines or are other mechanisms involved $[81,112]$ ? Presumably, GAPDH inhibition overrides PKM2 inhibition, as it is upstream of the latter. One could imagine a hierarchical model where PKM2 responds first to oxidative stress, then GAPDH, and finally PFK1. Given the importance of ROS in tumor development and anticancer therapies, a better understanding of how central metabolism and ROS intertwine could uncover interesting biology and suggest mechanisms to enhance current therapies [1]. 
Acknowledgments We would like to thank Gina M. DeNicola, Jared L. Johnson, and Costas A. Lyssiotis for helpful discussions and comments on the manuscript. This work was supported by National Institutes of Health grants to L.C.C.: R01 GM041890, P01 CA117969, P01 CA120964.

Open Access This chapter is distributed under the terms of the Creative Commons Attribution Noncommercial License, which permits any noncommercial use, distribution, and reproduction in any medium, provided the original author(s) and source are credited.

\section{References}

1. Gorrini C, Harris IS, Mak TW (2013) Modulation of oxidative stress as an anticancer strategy. Nat Rev Drug Discov 12:931-947. doi:10.1038/nrd4002

2. Yoshida T, Goto S, Kawakatsu M et al (2012) Mitochondrial dysfunction, a probable cause of persistent oxidative stress after exposure to ionizing radiation. Free Radic Res 46:147-153. doi:10.3109/10715762.2011.645207

3. Starkov AA (2008) The role of mitochondria in reactive oxygen species metabolism and signaling. Ann N Y Acad Sci 1147:37-52. doi:10.1196/annals.1427.015

4. Andreyev AY, Kushnareva YE, Starkov AA (2005) Mitochondrial metabolism of reactive oxygen species. Biochemistry (Mosc) 70:200-214

5. Boveris A, Chance B (1973) The mitochondrial generation of hydrogen peroxide. General properties and effect of hyperbaric oxygen. Biochem J 134:707-716

6. Nègre-Salvayre A, Hirtz C, Carrera G et al (1997) A role for uncoupling protein-2 as a regulator of mitochondrial hydrogen peroxide generation. FASEB J 11:809-815. doi:10.1096/fj.1530-6860

7. Handy DE, Loscalzo J (2012) Redox regulation of mitochondrial function. Antioxid Redox Signal 16:1323-1367. doi:10.1089/ars.2011.4123

8. Valko M, Rhodes CJ, Moncol J et al (2006) Free radicals, metals and antioxidants in oxidative stress-induced cancer. Chem Biol Interact 160:1-40. doi:10.1016/j.cbi.2005.12.009

9. Wall SB, Oh J-Y, Diers AR, Landar A (2012) Oxidative modification of proteins: an emerging mechanism of cell signaling. Front Physiol 3:1-9. doi:10.3389/fphys.2012.00369

10. Forman HJ, Maiorino M, Ursini F (2010) Signaling functions of reactive oxygen species. Biochemistry 49:835-842. doi:10.1021/bi9020378

11. Avery SV (2011) Molecular targets of oxidative stress. Biochem J 434:201-210. doi:10.1042/BJ20101695

12. Wang G, Hong Y, Johnson MK, Maier RJ (2006) Lipid peroxidation as a source of oxidative damage in Helicobacter pylori: protective roles of peroxiredoxins. Biochim Biophys Acta 1760:1596-1603. doi:10.1016/j.bbagen.2006.05.005

13. Bansal AK, Bilaspuri GS (2009) Antioxidant effect of vitamin E on motility, viability and lipid peroxidation of cattle spermatozoa under oxidative stress. Anim Sci Paper Rep 27:5-14

14. Jang S, Imlay JA (2007) Micromolar intracellular hydrogen peroxide disrupts metabolism by damaging iron-sulfur enzymes. J Biol Chem 282:929-937. doi:10.1074/jbc.M607646200

15. Stadtman ER (1992) Protein oxidation and aging. Science 257:1220-1224. doi:10.1126/ science. 1355616

16. Stadtman ER, Levine RL (2003) Free radical-mediated oxidation of free amino acids and amino acid residues in proteins. Amino Acids 25:207-218. doi:10.1007/s00726-003-0011-2

17. Neufeld G, Cohen T, Gengrinovitch S, Poltorak Z (1999) Vascular endothelial growth factor (VEGF) and its receptors. FASEB J 13:9-22. doi:10.1096/fj.1530-6860

18. Bae YS, Kang SW, Seo MS et al (1997) Epidermal growth factor (EGF)-induced generation of hydrogen peroxide. Role in EGF receptor-mediated tyrosine phosphorylation. J Biol Chem 272:217-221. doi:10.1074/jbc.272.1.217 
19. Catarzi S, Degl'Innocenti D, Iantomasi $\mathrm{T}$ et al (2002) The role of $\mathrm{H} 2 \mathrm{O} 2$ in the plateletderived growth factor-induced transcription of the gamma-glutamylcysteine synthetase heavy subunit. Cell Mol Life Sci 59:1388-1394

20. Brandes N, Schmitt S, Jakob U (2009) Thiol-based redox switches in eukaryotic proteins. Antioxid Redox Signal 11:997-1014. doi:10.1089/ARS.2008.2285

21. Groitl B, Jakob U (2014) Thiol-based redox switches. Biochim Biophys Acta 1844:1335-1343. doi:10.1016/j.bbapap.2014.03.007

22. Xu D, Rovira II, Finkel T (2002) Oxidants painting the cysteine chapel: redox regulation of PTPs. Dev Cell 2:251-252

23. Leslie NR, Bennett D, Lindsay YE et al (2003) Redox regulation of PI 3-kinase signalling via inactivation of PTEN. EMBO J 22:5501-5510. doi:10.1093/emboj/cdg513

24. Kobayashi A, Kang M-I, Okawa $\mathrm{H}$ et al (2004) Oxidative stress sensor Keap1 functions as an adaptor for Cul3-based E3 ligase to regulate proteasomal degradation of Nrf2. Mol Cell Biol 24:7130-7139. doi:10.1128/MCB.24.16.7130-7139.2004

25. Dinkova-Kostova AT, Holtzclaw WD, Cole RN et al (2002) Direct evidence that sulfhydryl groups of Keap1 are the sensors regulating induction of phase 2 enzymes that protect against carcinogens and oxidants. Proc Natl Acad Sci 99:11908-11913. doi:10.1073/pnas. 172398899

26. Zhang DD, Hannink M (2003) Distinct cysteine residues in Keap1 are required for Keap1dependent ubiquitination of $\mathrm{Nrf} 2$ and for stabilization of $\mathrm{Nrf} 2$ by chemopreventive agents and oxidative stress. Mol Cell Biol 23:8137-8151. doi:10.1128/MCB.23.22.8137-8151.2003

27. Adam J, Hatipoglu E, O'Flaherty L et al (2011) Renal cyst formation in Fh1-deficient mice is independent of the Hif/Phd pathway: roles for fumarate in KEAP1 succination and Nrf2 signaling. Cancer Cell 20:524-537. doi:10.1016/j.ccr.2011.09.006

28. Hayes JD, Dinkova-Kostova AT (2014) The Nrf2 regulatory network provides an interface between redox and intermediary metabolism. TIBS 39:199-218. doi:10.1016/j.tibs.2014.02.002

29. Forman HJ, Fridovich I (1973) Superoxide dismutase: a comparison of rate constants. Arch Biochem Biophys 158:396-400

30. Holmgren A, Johansson C, Berndt C et al (2005) Thiol redox control via thioredoxin and glutaredoxin systems. Biochem Soc Trans 33:1375-1377. doi:10.1042/BST20051375

31. Thomas JP, Maiorino M, Ursini F, Girotti AW (1990) Protective action of phospholipid hydroperoxide glutathione peroxidase against membrane-damaging lipid peroxidation. In situ reduction of phospholipid and cholesterol hydroperoxides. J Biol Chem 265:454-461

32. Wu KC, Cui JY, Klaassen CD (2011) Beneficial role of Nrf2 in regulating NADPH generation and consumption. Toxicol Sci 123:590-600. doi:10.1093/toxsci/kfr183

33. Mitsuishi Y, Taguchi K, Kawatani Y et al (2012) Nrf2 redirects glucose and glutamine into anabolic pathways in metabolic reprogramming. Cancer Cell 22:66-79. doi:10.1016/j. ccr.2012.05.016

34. Stanton RC (2012) Glucose-6-phosphate dehydrogenase, NADPH, and cell survival. IUBMB Life 64:362-369. doi:10.1002/iub.1017

35. McCubrey JA, Lahair MM, Franklin RA (2006) Reactive oxygen species-induced activation of the MAP kinase signaling pathways. Antioxid Redox Signal 8:1775-1789. doi:10.1089/ ars.2006.8.1775

36. Yoshihara E, Masaki S, Matsuo Y et al (2014) Thioredoxin/Txnip: redoxisome, as a redox switch for the pathogenesis of diseases. Front Immunol 4:514. doi:10.3389/fimmu.2013.00514

37. Wu N, Zheng B, Shaywitz A et al (2013) AMPK-dependent degradation of TXNIP upon energy stress leads to enhanced glucose uptake via GLUT1. Mol Cell 49:1167-1175. doi:10.1016/j.molcel.2013.01.035

38. Cecarini V, Gee J, Fioretti E et al (2007) Protein oxidation and cellular homeostasis: emphasis on metabolism. Biochim Biophys Acta 1773:93-104. doi:10.1016/j.bbamcr.2006.08.039

39. Gardner PR (2002) Aconitase: sensitive target and measure of superoxide. Meth Enzymol 349:9-23

40. Cochrane CG (1991) Cellular injury by oxidants. Am J Med 91:23S-30S 
41. Voet D, Voet JG, Pratt CW (2008) Fundamentals of biochemistry. Hoboken, New Jersey, USA

42. Salway JG (2004) Metabolism at a glance. Blackwell, Malden

43. Riganti C, Gazzano E, Polimeni M et al (2012) The pentose phosphate pathway: an antioxidant defense and a crossroad in tumor cell fate. Free Radic Biol Med 53:421-436. doi:10.1016/j.freeradbiomed.2012.05.006

44. Cosentino C, Grieco D, Costanzo V (2011) ATM activates the pentose phosphate pathway promoting anti-oxidant defence and DNA repair. EMBO J 30:546-555. doi:10.1038/ emboj.2010.330

45. Jiang P, Du W, Wang X et al (2011) p53 regulates biosynthesis through direct inactivation of glucose-6-phosphate dehydrogenase. Nat Cell Biol 13:310-316. doi:10.1038/ncb2172

46. Tian WN, Pignatare JN, Stanton RC (1994) Signal transduction proteins that associate with the platelet-derived growth factor (PDGF) receptor mediate the PDGF-induced release of glucose-6-phosphate dehydrogenase from permeabilized cells. J Biol Chem 269:14798-14805

47. Pan S, World CJ, Kovacs CJ, Berk BC (2009) Glucose 6-phosphate dehydrogenase is regulated through c-Src-mediated tyrosine phosphorylation in endothelial cells. Arterioscler Thromb Vasc Biol 29:895-901. doi:10.1161/ATVBAHA.109.184812

48. Holten D, Procsal D, Chang HL (1976) Regulation of pentose phosphate pathway dehydrogenases by NADP+/NADPH ratios. Biochem Biophys Res Commun 68:436-441

49. Kotaka M, Gover S, Vandeputte-Rutten L et al (2005) Structural studies of glucose-6phosphate and NADP+ binding to human glucose-6-phosphate dehydrogenase. Acta Crystallogr D Biol Crystallogr 61:495-504. doi:10.1107/S0907444905002350

50. Cappellini MD, Fiorelli G (2008) Glucose-6-phosphate dehydrogenase deficiency. Lancet 371:64-74. doi:10.1016/S0140-6736(08)60073-2

51. Luzzatto L, Seneca E (2014) G6PD deficiency: a classic example of pharmacogenetics with on-going clinical implications. Br J Haematol 164:469-480. doi:10.1111/bjh.12665

52. Pandolfi PP, Sonati F, Rivi R et al (1995) Targeted disruption of the housekeeping gene encoding glucose 6-phosphate dehydrogenase (G6PD): G6PD is dispensable for pentose synthesis but essential for defense against oxidative stress. EMBO J 14:5209-5215

53. Tian WN, Braunstein LD, Apse K et al (1999) Importance of glucose-6-phosphate dehydrogenase activity in cell death. Am J Physiol 276:C1121-C1131

54. Tuttle S, Stamato T, Perez ML, Biaglow J (2000) Glucose-6-phosphate dehydrogenase and the oxidative pentose phosphate cycle protect cells against apoptosis induced by low doses of ionizing radiation. Radiat Res 153:781-787. doi:10.1667/0033-7587(2000)153\%5B0781:GP DATO\%5D2.0.CO;2

55. Tian WN, Braunstein LD, Pang J et al (1998) Importance of glucose-6-phosphate dehydrogenase activity for cell growth. J Biol Chem 273:10609-10617. doi:10.1074/jbc.273.17.10609

56. Son J, Lyssiotis CA, Ying $\mathrm{H}$ et al (2014) Glutamine supports pancreatic cancer growth through a KRAS-regulated metabolic pathway. Nature 496:101-105. doi:10.1038/ nature 12040

57. Ying H, Kimmelman AC, Lyssiotis CA et al (2012) Oncogenic Kras maintains pancreatic tumors through regulation of anabolic glucose metabolism. Cell 149:656-670. doi:10.1016/j. cell.2012.01.058

58. Fan J, Ye J, Kamphorst JJ et al (2014) Quantitative flux analysis reveals folate-dependent NADPH production. Nature 510:298-302. doi:10.1038/nature13236

59. Tuttle SW, Varnes ME, Mitchell JB, Biaglow JE (1992) Sensitivity to chemical oxidants and radiation in $\mathrm{CHO}$ cell lines deficient in oxidative pentose cycle activity. Int $\mathrm{J}$ Radiat Oncol Biol Phys 22:671-675

60. Yalcin A, Telang S, Clem B, Chesney J (2009) Regulation of glucose metabolism by 6-phosphofructo-2-kinase/fructose-2,6-bisphosphatases in cancer. Exp Mol Pathol 86:174-179. doi:10.1016/j.yexmp.2009.01.003 
61. Jenkins CM, Yang J, Sims HF, Gross RW (2011) Reversible high affinity inhibition of phosphofructokinase-1 by acyl-CoA: a mechanism integrating glycolytic flux with lipid metabolism. J Biol Chem 286:11937-11950. doi:10.1074/jbc.M110.203661

62. Dunaway GA, Kasten TP, Sebo T, Trapp R (1988) Analysis of the phosphofructokinase subunits and isoenzymes in human tissues. Biochem $\mathrm{J}$ 251:677-683

63. Lunt SY, Vander Heiden MG (2011) Aerobic glycolysis: meeting the metabolic requirements of cell proliferation. Annu Rev Cell Dev Biol 27:441-464. doi:10.1146/annurevcellbio-092910-154237

64. Mor I, Cheung EC, Vousden KH (2011) Control of glycolysis through regulation of PFK1: old friends and recent additions. Cold Spring Harb Symp Quant Biol 76:211-216. doi:10.1101/sqb.2011.76.010868

65. Lee P, Vousden KH, Cheung EC (2014) TIGAR, TIGAR, burning bright. Cancer Metab 2:1. doi:10.1186/2049-3002-2-1

66. Yi W, Clark PM, Mason DE et al (2012) Phosphofructokinase 1 glycosylation regulates cell growth and metabolism. Science 337:975-980. doi:10.1126/science.1222278

67. Jen K-Y, Cheung VG (2005) Identification of novel p53 target genes in ionizing radiation response. Cancer Res 65:7666-7673. doi:10.1158/0008-5472.CAN-05-1039

68. Bensaad K, Tsuruta A, Selak MA et al (2006) TIGAR, a p53-inducible regulator of glycolysis and apoptosis. Cell 126:107-120. doi:10.1016/j.cell.2006.05.036

69. Peña-Rico MA, Calvo-Vidal MN, Villalonga-Planells R et al (2011) TP53 induced glycolysis and apoptosis regulator (TIGAR) knockdown results in radiosensitization of glioma cells. Radiother Oncol 101:132-139. doi:10.1016/j.radonc.2011.07.002

70. Lui VWY, Wong EYL, Ho K et al (2011) Inhibition of c-Met downregulates TIGAR expression and reduces NADPH production leading to cell death. Oncogene 30:1127-1134. doi:10.1038/onc. 2010.490

71. Wanka C, Steinbach JP, Rieger J (2012) Tp53-induced glycolysis and apoptosis regulator (TIGAR) protects glioma cells from starvation-induced cell death by up-regulating respiration and improving cellular redox homeostasis. J Biol Chem 287:33436-33446. doi:10.1074/ jbc.M112.384578

72. Cheung EC, Athineos D, Lee $P$ et al (2013) TIGAR is required for efficient intestinal regeneration and tumorigenesis. Dev Cell 25:463-477. doi:10.1016/j.devcel.2013.05.001

73. Cheung EC, Ludwig RL, Vousden KH (2012) Mitochondrial localization of TIGAR under hypoxia stimulates HK2 and lowers ROS and cell death. Proc Natl Acad Sci U S A 109:20491-20496. doi:10.1073/pnas.1206530109

74. Seidler NW (2012) GAPDH: biological properties and diversity. Adv Exp Med Biol 985:21-29

75. Williamson JR (1965) Glycolytic control mechanisms. I. Inhibition of glycolysis by acetate and pyruvate in the isolated, perfused rat heart. J Biol Chem 240:2308-2321

76. Hyslop PA, Hinshaw DB, Halsey WA et al (1988) Mechanisms of oxidant-mediated cell injury. The glycolytic and mitochondrial pathways of ADP phosphorylation are major intracellular targets inactivated by hydrogen peroxide. J Biol Chem 263:1665-1675

77. Leichert LI, Gehrke F, Gudiseva HV et al (2008) Quantifying changes in the thiol redox proteome upon oxidative stress in vivo. Proc Natl Acad Sci U S A 105:8197-8202. doi:10.1073/pnas.0707723105

78. Maller C, Schröder E, Eaton P (2011) Glyceraldehyde 3-phosphate dehydrogenase is unlikely to mediate hydrogen peroxide signaling: studies with a novel anti-dimedone sulfenic acid antibody. Antioxid Redox Signal 14:49-60. doi:10.1089/ars.2010.3149

79. Jeong J, Jung Y, Na S et al (2011) Novel oxidative modifications in redox-active cysteine residues. Mol Cell Proteomics 10(3):M110.000513. doi:10.1074/mcp.M110.000513

80. Schmalhausen EV, Pleten AP, Muronetz VI (2003) Ascorbate-induced oxidation of glyceraldehyde-3-phosphate dehydrogenase. Biochem Biophys Res Commun 308:492-496. doi:10.1016/S0006-291X(03)01421-9

81. Schuppe-Koistinen I, Moldéus P, Bergman T, Cotgreave IA (1994) S-thiolation of human endothelial cell glyceraldehyde-3-phosphate dehydrogenase after hydrogen peroxide treatment. Eur J Biochem 221:1033-1037 
82. Ravichandran V, Seres T, Moriguchi T (1994) S-thiolation of glyceraldehyde-3-phosphate dehydrogenase induced by the phagocytosis-associated respiratory burst in blood monocytes. J Biol Chem 269:25010-25015

83. Shenton D, Grant CM (2003) Protein S-thiolation targets glycolysis and protein synthesis in response to oxidative stress in the yeast Saccharomyces cerevisiae. Biochem J 374:513-519. doi:10.1042/BJ20030414

84. Grant CM, Quinn KA, Dawes IW (1999) Differential protein S-thiolation of glyceraldehyde3-phosphate dehydrogenase isoenzymes influences sensitivity to oxidative stress. Mol Cell Biol 19:2650-2656

85. Nakajima H, Amano W, Kubo T et al (2009) Glyceraldehyde-3-phosphate dehydrogenase aggregate formation participates in oxidative stress-induced cell death. J Biol Chem 284:34331-34341. doi:10.1074/jbc.M109.027698

86. Butterfield DA, Hardas SS, Lange MLB (2010) Oxidatively modified glyceraldehyde-3phosphate dehydrogenase (GAPDH) and Alzheimer's disease: many pathways to neurodegeneration. J Alzheimers Dis 20:369-393. doi:10.3233/JAD-2010-1375

87. Godon C, Lagniel G, Lee J et al (1998) The H2O2 stimulon in Saccharomyces cerevisiae. J Biol Chem 273:22480-22489

88. Ralser M, Wamelink MM, Kowald A et al (2007) Dynamic rerouting of the carbohydrate flux is key to counteracting oxidative stress. J Biol 6:10. doi:10.1186/jbiol61

89. Ralser M, Heeren G, Breitenbach M et al (2006) Triose phosphate isomerase deficiency is caused by altered dimerization - not catalytic inactivity - of the mutant enzymes. PLoS One 1:e30. doi:10.1371/journal.pone.0000030

90. Clower CV, Chatterjee D, Wang Z et al (2010) The alternative splicing repressors hnRNPA1/ A2 and PTB influence pyruvate kinase isoform expression and cell metabolism. Proc Natl Acad Sci U S A 107:1894-1899. doi:10.1073/pnas.0914845107

91. Noguchi T, Yamada K, Inoue H et al (1987) The L- and R-type isozymes of rat pyruvate kinase are produced from a single gene by use of different promoters. J Biol Chem 262:14366-14371

92. Noguchi T, Inoue H, Tanaka T (1986) The M1- and M2-type isozymes of rat pyruvate kinase are produced from the same gene by alternative RNA splicing. J Biol Chem 261:13807-13812

93. David CJ, Chen M, Assanah M et al (2010) HnRNP proteins controlled by c-Myc deregulate pyruvate kinase mRNA splicing in cancer. Nature 463:364-368. doi:10.1038/nature08697

94. Imamura K, Tanaka T (1972) Multimolecular forms of pyruvate kinase from rat and other mammalian tissues. I. Electrophoretic studies. J Biochem 71:1043-1051

95. Mazurek S (2011) Pyruvate kinase type M2: a key regulator of the metabolic budget system in tumor cells. Int J Biochem Cell Biol 43:969-980. doi:10.1016/j.biocel.2010.02.005

96. Keller KE, Doctor ZM, Dwyer ZW, Lee Y-S (2014) SAICAR induces protein kinase activity of PKM2 that is necessary for sustained proliferative signaling of cancer cells. Mol Cell 53:700-709. doi:10.1016/j.molcel.2014.02.015

97. Keller KE, Tan IS, Lee Y-S (2012) SAICAR stimulates pyruvate kinase isoform M2 and promotes cancer cell survival in glucose-limited conditions. Science 338:1069-1072. doi:10.1126/science.1224409

98. Chaneton B, Hillmann P, Zheng L et al (2013) Serine is a natural ligand and allosteric activator of pyruvate kinase M2. Nature 491:458-462. doi:10.1038/nature11540

99. Ashizawa K, Willingham MC, Liang CM, Cheng SY (1991) In vivo regulation of monomertetramer conversion of pyruvate kinase subtype M2 by glucose is mediated via fructose 1,6-bisphosphate. J Biol Chem 266:16842-16846

100. Ikeda Y, Noguchi T (1998) Allosteric regulation of pyruvate kinase M2 isozyme involves a cysteine residue in the intersubunit contact. J Biol Chem 273:12227-12233

101. Anastasiou D, Yu Y, Israelsen WJ et al (2012) Pyruvate kinase M2 activators promote tetramer formation and suppress tumorigenesis. Nat Chem Biol 8:839-847. doi:10.1038/ nchembio. 1060

102. Christofk HR, Vander Heiden MG, Wu N et al (2008) Pyruvate kinase M2 is a phosphotyrosinebinding protein. Nature 452:181-186. doi:10.1038/nature06667 
103. Lv L, Xu Y-P, Zhao D et al (2013) Mitogenic and oncogenic stimulation of K433 acetylation promotes PKM2 protein kinase activity and nuclear localization. Mol Cell 52:340-352. doi:10.1016/j.molcel.2013.09.004

104. Hitosugi T, Kang S, Vander Heiden MG et al (2009) Tyrosine phosphorylation inhibits PKM2 to promote the Warburg effect and tumor growth. Sci Signal 2:ra73. doi:10.1126/ scisignal.2000431

105. Gao J, Aksoy BA, Dogrusoz U et al (2013) Integrative analysis of complex cancer genomics and clinical profiles using the cBioPortal. Sci Signal 6:pl1-pl1. doi:10.1126/ scisignal.2004088

106. Gao X, Wang H, Yang JJ et al (2012) Pyruvate kinase M2 regulates gene transcription by acting as a protein kinase. Mol Cell 45:598-609. doi:10.1016/j.molcel.2012.01.001

107. Israelsen WJ, Dayton TL, Davidson SM et al (2013) PKM2 isoform-specific deletion reveals a differential requirement for pyruvate kinase in tumor cells. Cell 155:397-409. doi:10.1016/j. cell.2013.09.025

108. Yang W, Xia Y, Hawke D et al (2012) PKM2 phosphorylates histone H3 and promotes gene transcription and tumorigenesis. Cell 150:685-696. doi:10.1016/j.cell.2012.07.018

109. Christofk HR, Vander Heiden MG, Harris MH et al (2008) The M2 splice isoform of pyruvate kinase is important for cancer metabolism and tumour growth. Nature 452:230-233. doi:10.1038/nature06734

110. McKnight SL (2014) Please keep me 2uned to PKM2. Mol Cell 53:683-684. doi:10.1016/j. molcel.2014.02.022

111. Yang W, Xia Y, Ji H et al (2011) Nuclear PKM2 regulates $\beta$-catenin transactivation upon EGFR activation. Nature 480:118-122. doi:10.1038/nature10598

112. Anastasiou D, Poulogiannis G, Asara JM et al (2011) Inhibition of pyruvate kinase M2 by reactive oxygen species contributes to cellular antioxidant responses. Science 334:1278-1283. doi:10.1126/science.1211485

113. Maeba P, Sanwal BD (1968) The regulation of pyruvate kinase of Escherichia coli by fructose diphosphate and adenylic acid. J Biol Chem 243:448-450

114. McDonagh B, Ogueta S, Lasarte G, Padilla CA (2009) Shotgun redox proteomics identifies specifically modified cysteines in key metabolic enzymes under oxidative stress in Saccharomyces cerevisiae. J Proteomics 72:677-689. doi:10.1016/j.jprot.2009.01.023

115. Grüning N-M, Rinnerthaler M, Bluemlein K et al (2011) Pyruvate kinase triggers a metabolic feedback loop that controls redox metabolism in respiring cells. Cell Metab 14:415-427. doi:10.1016/j.cmet.2011.06.017

116. Grüning N-M, Du D, Keller MA et al (2014) Inhibition of triosephosphate isomerase by phosphoenolpyruvate in the feedback-regulation of glycolysis. Open Biol 4:130232. doi:10.1098/rsob.130232

117. Luo W, Hu H, Chang R et al (2011) Pyruvate kinase M2 is a PHD3-stimulated coactivator for hypoxia-inducible factor 1. Cell 145:732-744. doi:10.1016/j.cell.2011.03.054

118. Luo W, Semenza GL (2011) Pyruvate kinase M2 regulates glucose metabolism by functioning as a coactivator for hypoxia-inducible factor 1 in cancer cells. Oncotarget 2:551-556

119. Snell K, Natsumeda Y, Eble JN et al (1988) Enzymic imbalance in serine metabolism in human colon carcinoma and rat sarcoma. Br J Cancer 57:87-90

120. Snell K (1986) The duality of pathways for serine biosynthesis is a fallacy. TIBS 11(6):241-243

121. Mullarky E, Mattaini KR, Vander Heiden MG et al (2011) PHGDH amplification and altered glucose metabolism in human melanoma. Pigment Cell Melanoma Res 24:1112-1115. doi:10.1111/j.1755-148X.2011.00919.x

122. Barker GA, Ellory JC (1990) The identification of neutral amino acid transport systems. Exp Physiol 75:3-26

123. Palacín M, Estévez R, Bertran J, Zorzano A (1998) Molecular biology of mammalian plasma membrane amino acid transporters. Physiol Rev 78:969-1054 
124. Possemato R, Marks KM, Shaul YD et al (2011) Functional genomics reveal that the serine synthesis pathway is essential in breast cancer. Nature 476:346-350. doi:10.1038/nature 10350

125. Locasale JW, Grassian AR, Melman T et al (2011) Phosphoglycerate dehydrogenase diverts glycolytic flux and contributes to oncogenesis. Nat Genet 43:869-874. doi:10.1038/ng.890

126. Hitosugi T, Zhou L, Elf S et al (2012) Phosphoglycerate mutase 1 coordinates glycolysis and biosynthesis to promote tumor growth. Cancer Cell 22:585-600. doi:10.1016/j. ccr.2012.09.020

127. Futerman AH, Riezman H (2005) The ins and outs of sphingolipid synthesis. Trends Cell Biol 15:312-318. doi:10.1016/j.tcb.2005.04.006

128. Kuge O, Hasegawa K, Saito K, Nishijima M (1998) Control of phosphatidylserine biosynthesis through phosphatidylserine-mediated inhibition of phosphatidylserine synthase I in Chinese hamster ovary cells. Proc Natl Acad Sci 95:4199-4203

129. de Koning TJ, Snell K, Duran M et al (2003) L-serine in disease and development. Biochem J 371:653-661. doi:10.1042/BJ20021785

130. Vander Heiden MG, Cantley LC, Thompson CB (2009) Understanding the Warburg effect: the metabolic requirements of cell proliferation. Science 324:1029-1033. doi:10.1126/ science. 1160809 


\title{
Metabolic Regulation by Nuclear Receptors
}

\author{
Sihao Liu, Michael Downes, and Ronald M. Evans
}

\begin{abstract}
Nuclear receptors (NRs) are a large family of ligand-dependent transcriptional regulators that control development, reproduction, metabolism, and inflammation. Cognate ligands include fatty acids, bile acids, steroids, vitamins A and D, and thyroid hormone, which enable NRs to sense lipophilic nutrients levels and control their respective flux and metabolism. While major metabolic outputs of some individual receptors are well studied, an integrated understanding of their contributions to systemic metabolic homeostasis is just starting to be revealed. In this chapter, we summarize recent advances in NR signaling in metabolism, with a focus on the emerging paradigm that positions NRs as hubs that translate environmental signals of a particular physiological state into daily metabolic rhythms. As NRs are a proven class of therapeutic targets, these novel findings provide insight into therapeutics for the metabolic syndrome.
\end{abstract}

Keywords Nuclear receptors • Energy homeostasis • Metabolite signaling • Microbiome $\bullet$ FGFs $\bullet$ Metabolic diseases

\section{Introduction}

The human body maintains relatively stable levels of key energy substrates through metabolic flexibility [1]. After a meal, elevated blood glucose triggers insulin release to facilitate glucose uptake in the liver and muscles while simultaneously suppressing free fatty acid release from adipose tissue. Between meals, a complex

\footnotetext{
S. Liu $\bullet$ M. Downes

Gene Expression Laboratory, Salk Institute for Biological Studies, 10010 North Torrey Pines Road, La Jolla, CA 92037, USA

R.M. Evans $(\varangle)$

Gene Expression Laboratory, Salk Institute for Biological Studies, 10010 North Torrey Pines Road, La Jolla, CA 92037, USA
}

Howard Hughes Medical Institute, Salk Institute for Biological Studies, 10010 North Torrey Pines Road, La Jolla, CA 92037, USA

e-mail: evans@salk.edu 
counter-regulatory hormonal network maintains a steady blood glucose level via hepatic glycogen breakdown and gluconeogenesis while concurrently decreasing circulating insulin levels to allow adipose tissue lipolysis to release free fatty acids as the major source of energy. Hence, metabolic flexibility can also be described as the ability to switch between carbohydrates and lipids as the predominant source of energy [1].

While metabolic flexibility undoubtedly provided a survival advantage during our early hunter-gatherer times, in combination with excess nutrients it is now driving the human body to a crisis point. Obesity is now a worldwide epidemic and is often accompanied by pathologies such as type 2 diabetes, hepatosteatosis, and cardiovascular diseases. The excessive accumulation of lipids in tissues brought about by modern day excess nutrient uptake, a condition termed as lipotoxicity [2], often results in insulin resistance and hyperglycemia, and leads to metabolic inflexibility of the body.

At the cellular level, metabolic flexibility utilizes energy sensors, among which are nuclear receptors (NRs), that either activate or inhibit specific metabolic pathways. NRs in humans are comprised of a family of 48 ligand-dependent transcription factors with conserved molecular architecture that includes a DNAbinding domain and a ligand-binding domain [3]. Ligands for NRs consist of a diverse array of small lipophilic molecules [3], which, upon binding to their cognate $\mathrm{NR}$, recruit enzymatic cofactors to modulate their target gene expression and, thereby, physiological output. A small subset of NRs have been identified as major players in the maintenance of metabolic flexibility via their ability to sense key lipophilic dietary nutrients and regulate a complex network of genes to control the metabolic flux of these nutrients.

Intensive research over the past two decades has established the fundamental mode of action of a subset of individual receptors (Table 1), including the peroxisome proliferator-activated receptors (PPARs), farnesoid X receptor (FXR), and liver X receptors (LXRs). We will summarize the recent progress in elucidating how these receptors coordinate metabolic homeostasis in response to dietary intake and environmental stressors.

\section{NR Endogenous Ligands Are Dietary Sensors}

The generation and degradation of prototypical endogenous NR ligands through enzymatic modifications, such as those catalyzed by cytochrome P450 (CYP) enzymes [3, 9], allows for integration with NR-independent regulatory pathways. For example, regulation of FOXO1 activity by hepatic insulin signaling determines the expression level of CYP7A1, the rate-limiting P450 enzyme in the synthesis of bile acids, the endogenous ligands for FXR [10].

In the case of the PPARs, each binds fatty acids with relatively low affinity and regulates metabolic homeostasis through canonical direct repeat 1 (DR1) elements within promoters and enhancers of target genes. Specificity is achieved at the level 
Table 1 Nutrient-sensing nuclear receptors

\begin{tabular}{|c|c|c|c|}
\hline & Ligand & Expression & Function \\
\hline PPAR $\alpha$ & $\begin{array}{l}\text { Short-chain fatty } \\
\text { acids }\end{array}$ & Liver & $\begin{array}{l}\text { PPAR } \alpha \text { was first identified as the } \\
\text { target of the lipid-lowering drug } \\
\text { phenofibrate. PPAR } \alpha \text { is a master } \\
\text { regulator of the adaptive fasting } \\
\text { response in the liver. In the fasted } \\
\text { state, elevated circulating free fatty } \\
\text { acids activate hepatic PPAR } \alpha \text {, } \\
\text { whose targets include rate-limiting } \\
\text { enzymes in fatty acid oxidation and } \\
\text { ketogenesis [4]. PPAR } \alpha \text { knockout } \\
\text { mice die upon prolonged fasting } \\
\text { with excessive hepatic fatty acid } \\
\text { accumulation }\end{array}$ \\
\hline $\operatorname{PPAR} \gamma$ & $\begin{array}{l}\text { 15-deoxy-Delta- } \\
12,14 \text {-prostaglandin } \\
\mathrm{J} 2 \text { and } \\
\text { thiazolidinedione } \\
\text { antidiabetic drugs }\end{array}$ & Adipose tissue & $\begin{array}{l}\text { PPAR } \gamma \text { is the master regulator of } \\
\text { adipogenesis and lipogenesis, and is } \\
\text { required for both white and brown } \\
\text { adipose tissue formation. } \\
\text { Postprandial increase in fatty acids } \\
\text { activates adipose PPAR } \gamma \text { to facilitate } \\
\text { fat storage and production of } \\
\text { adipokines (leptin, adiponectin, } \\
\text { resistin, etc.), which are important } \\
\text { regulators of whole-body insulin } \\
\text { sensitivity }[4,5]\end{array}$ \\
\hline PPAR $\delta$ & $\begin{array}{l}\text { Long-chain fatty } \\
\text { acids }\end{array}$ & $\begin{array}{l}\text { Ubiquitously } \\
\text { expressed }\end{array}$ & $\begin{array}{l}\text { PPAR } \delta \text { activation enhances fatty } \\
\text { acid oxidation and mitochondrial } \\
\text { respiration. PPAR } \delta \text { activation in } \\
\text { white adipose tissue promotes its } \\
\text { conversion to brown fat, whereas in } \\
\text { the muscle, it increases oxidative } \\
\text { type I fiber formation. In the liver, } \\
\text { PPAR } \delta \text { controls fatty acid synthesis } \\
\text { through direct activation of the } \\
\text { rate-limiting enzyme Acaca }[4,6]\end{array}$ \\
\hline FXR & Bile acids & $\begin{array}{l}\text { Liver, kidney, and } \\
\text { intestine }\end{array}$ & $\begin{array}{l}\text { FXR regulates bile acid homeostasis } \\
\text { by promoting bile acid reabsorption } \\
\text { in the gut and suppressing de novo } \\
\text { bile acid synthesis in the liver }[7,8]\end{array}$ \\
\hline LXR & Oxysterols & $\begin{array}{l}\text { LXR } \alpha \text { : liver, } \\
\text { adipose tissue, and } \\
\text { macrophages; } \\
\text { LXR } \beta \text { : } \\
\text { ubiquitously } \\
\text { expressed }\end{array}$ & $\begin{array}{l}\text { LXRs promote cholesterol efflux in } \\
\text { the liver via } 3 \text { mechanisms: } \\
\text { (1) suppression of cholesterol uptake } \\
\text { from LDL recycling; (2) production } \\
\text { of VLDL for export; (3) conversion } \\
\text { of cholesterol to bile acids for } \\
\text { excretion. LXRs exert an opposing } \\
\text { effect to FXR on bile acid } \\
\text { metabolism [8] }\end{array}$ \\
\hline
\end{tabular}

Acaca acetyl-coA carboxylase $1, F X R$ farnesoid $X$ receptor, $L D L$ low-density lipoprotein, $L X R$ liver $\mathrm{X}$ receptor, $P P A R$ peroxisome proliferator-activated receptor, $V L D L$ very low-density lipoprotein 
of the ligand, enabling the body to enact different physiological responses using common elements within the genome. Long-chain fatty acids preferentially activate PPAR $\delta$ to stimulate fatty acid oxidation in skeletal muscle. In contrast, PPAR $\alpha$ activation by short-chain fatty acids switches on a feed-forward mechanism in the liver to promote fuel oxidation, thereby preventing the intracellular accumulation of fatty acids and consequent lipotoxicity. Thus, the coordinated actions of these PPARs protect against lipotoxicity through the regulation of different metabolic target tissues in response to the uptake of fatty acids.

\section{Gut and Gut Microbiota}

There is growing evidence that the modern lifestyle, most notably the high-fat/highsugar "Western" diet, has altered the genetic composition and metabolic activity of our resident microorganisms. The microbiome communicates with NRs through its ability to modify dietary nutrients to generate specific NR ligands. An early example is oleoylethanolamide (OEA), a high-affinity PPAR $\alpha$ ligand generated in enterocytes from oleic acid [11]. OEA-induced activation of intestinal PPAR $\alpha$ enhances fat absorption through induction of the target gene, CD36, and promotes satiety through a secondary signaling cascade in the brain [12].

More recently, an enhanced bile acid flux was observed throughout the intestinal tract of germ-free mice [13], suggestive of dysregulated FXR activity. While it was known that intestinal microbiota metabolize primary bile acids to increase their hydrophobicity and chemical diversity $[13,14]$, it was not known to what extent the microbiota influence FXR activity. The serum bile acid composition of the germfree mice revealed elevated levels of tauro-conjugated alpha- and beta-muricholic acids (T $\alpha / \beta \mathrm{MCA})$, known FXR antagonists $[13,15]$, which result in a loss of feedback inhibition of bile acid synthesis. Importantly, this study demonstrates that endogenous FXR antagonists are default products of the canonical bile acid synthesis pathway, raising interesting questions about the co-evolution of crucial NR-dependent metabolic pathways and the intestinal microbiome.

An added biological complexity to this system is that reciprocally, bile acids can influence the composition of the intestinal microbiome. A diet high in saturated fatty acids increases tauro-conjugated cholic acid concentrations to promote the growth of sulfate-consuming bacteria, increasing the susceptibility of mice to colitis [16]. This dynamic relationship between bile acids and microbiota is still not well understood, making it difficult to predict the metabolic outcome when deregulation occurs. For example, while FXR null mice are more susceptible to metabolic disorders [17-19], intestinal-specific FXR knockout mice are protected [15]. Clinically, the importance of this pathway was demonstrated in a recent study, which showed that the metabolic benefits of vertical sleeve gastrectomy were mediated by the gut microbiota and FXR signaling in the intestine [20]. 


\section{De Novo Lipogenesis}

In contrast to the relatively well-understood metabolic actions of dietary lipids, the effects of the largely monounsaturated fatty acids generated in the liver through de novo lipogenesis are now being explored [21]. Utilizing genetic mouse models to disrupt hepatic de novo lipogenesis, researchers have demonstrated a broad spectrum of metabolic changes on fat-free diets [22, 23]. Loss of fatty acid synthase (FAS) - a rate-limiting enzyme in de novo lipogenesis - in the liver leads to the development of a fatty liver upon fasting, similar to a PPAR $\alpha$ knockout phenotype $[24,25]$. Importantly, this fatty liver phenotype can be rescued with a PPAR $\alpha$ synthetic ligand, implicating de novo fatty acid synthesis in the generation of endogenous PPAR $\alpha$ ligands. Furthermore, the authors identified a phosphocholine (16:0/18:1-GPC) as an endogenous ligand for PPAR $\alpha$ [24]. In adipose tissue, lipogenesis has been implicated in producing PPAR $\gamma$ endogenous ligands [26], such as 1-O-octadecenyl-2-palmitoyl-3-glycerophosphocholine (18:1e/16:0-GPC) [27].

Direct biochemical approaches have identified endogenous ligands for PPAR $\alpha$ and PPAR $\gamma$. How these ligands function in normal physiology when both dietary and de novo synthesized lipids are present is not clear [28]. Although not directly evaluated under diet-induced or genetic obesity, $\mathrm{PC}(16: 0 / 18: 1)$ is one of two serum phosphocholine species that were observed to have aberrant diurnal rhythmic concentrations [29]. It should be cautioned that "ligands" identified through blocking of an endogenous pathway can be misleading, illustrated by the fact that these phospholipids are weak activators of their respective targets in cell-based reporter systems [24, 27]. However, weak pharmacological activation of PPAR $\alpha$ or PPAR $\gamma$ is beneficial for some metabolic disorders, while, conversely, strong NR activators can carry significant side effects (e.g. rosiglitazone). It was recently shown that a weak PPAR $\gamma$ ligand could achieve similar therapeutic responses to rosiglitazone with limited side effects [30, 31]. Exploring the therapeutic values of these endogenous ligands will be an exciting next step.

\section{Integrative Physiology-Nuclear Receptor-Mediated Crosstalk}

Metabolic flexibility results from cohesive actions of major metabolic organs in response to nutrient flux. It is a collective consequence of localized activation of cellular nutrient-sensing pathways and systemic hormonal actions. Whereas hormones can be released from specialized endocrine organs that directly sense nutrients, they can also be produced from metabolic organs to amplify or propagate local nutrient cues. One example of the latter is the adipose PPAR $\gamma$-mediated production of adipokines such as leptin and adiponectin [5]. In the postprandial setting, 

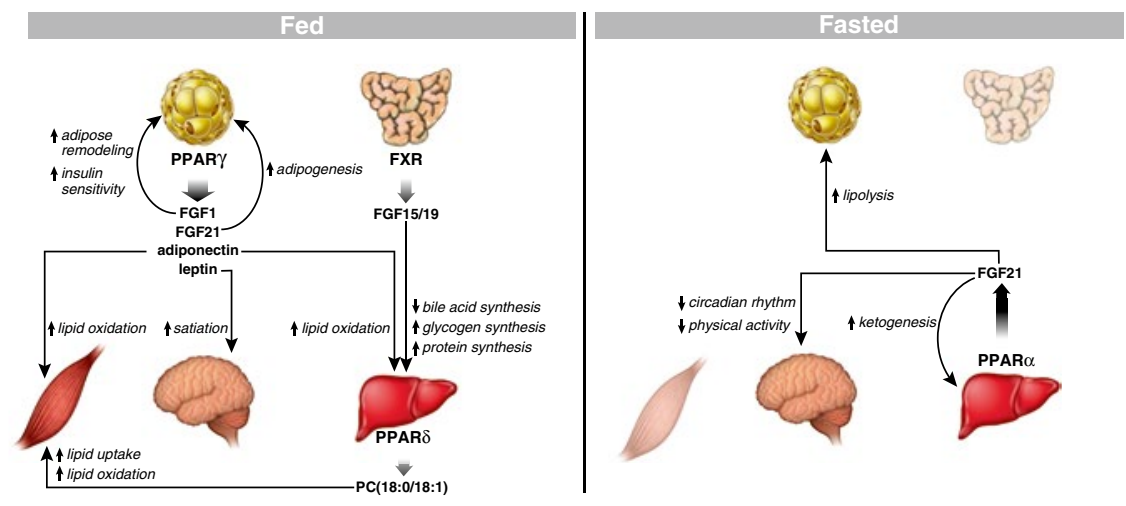

Fig. 1 Integrative physiology through nuclear receptor $(N R)$-mediated crosstalk. Nutrient-sensing NRs amplify or propagate nutrient signals through local and long-range hormone actions. In the fed state, intestinal farnesoid $\mathrm{X}$ receptor $(F X R)$ activation resulting from bile acid release produces fibroblast growth factor $(F G F) 15 / 19$. FGF15/19 limits hepatic bile acid synthesis and promotes glycogen and protein synthesis to store nutrients. Postprandial carbohydrate and lipid influx activate a hepatic peroxisome proliferator-activated receptor $(P P A R) \delta$-controlled lipid synthesis program. The phosphocholine $\mathrm{PC}(18: 0 / 18: 1)$ is secreted as a long-range hormone to promote muscle fat utilization. Feeding activates adipose tissue PPAR $\gamma$, which, in turn, controls the release of multiple adipokines. Locally, FGF21 amplifies PPAR $\gamma$ signaling and promotes further adipogenesis. FGF1 is another paracrine signal that is essential for healthy adipose tissue remodeling and for maintaining whole-body insulin sensitivity. Systemically, adiponectin enhances lipid oxidation in muscle and liver, whereas leptin increases satiation by acting on the central nervous system. During fasting, hepatic PPAR $\alpha$ activation secretes FGF21, whose pleiotropic effects, including elevated adipose tissue lipolysis and hepatic ketogenesis, are important for adaptive fasting response. In addition, FGF21 interferes with the circadian clock in the brain and modulates physical activities

PPAR $\gamma$ induces leptin production to enhance satiation in the central nervous system (CNS), as well as the release of adiponectin to promote fatty acid oxidation in the liver. The dual regulation of central and peripheral responses creates the needed coordination to maintain metabolic balance.

Given the critical roles of the aforementioned NRs in cellular nutrient sensing, it is perhaps not surprising that some of these NRs have been shown to produce hormonelike molecules (Fig. 1). To date, two classes of secreted molecules have been prominently linked with these NRs: the fibroblast growth factors (FGFs) and lipids.

\section{Fibroblast Growth Factors}

Screening for genes activated by nutrient-sensing NRs identified several FGFs as highly induced [32, 33] (Table 2). There are 22 FGFs (FGF1-FGF23) in the human genome (FGF15 is the mouse ortholog of human FGF19 [34]) that are classified as classic or endocrine FGFs. Most classic FGFs are secreted, heparan sulfate-binding 
Table 2 Nutrient-regulated fibroblast growth factors (FGFs)

\begin{tabular}{l|l|l}
\hline Nuclear receptor & FGF & Physiological state \\
\hline FXR & FGF15/19 & Fed \\
\hline PPAR $\alpha$ & FGF21 & Fasted \\
\hline PPAR $\gamma$ & FGF21 & Fed \\
\hline PPAR $\gamma$ & FGF1 & Fed \\
\hline
\end{tabular}

FXR farnesoid $\mathrm{X}$ receptor, $P P A R$ peroxisome proliferatoractivated receptor

proteins that interact locally with fibroblast growth factor receptors (FGFRs) as autocrine/paracrine factors to modulate cell growth, angiogenesis and wound healing [34]. In contrast, the endocrine FGFs (FGF15/19, FGF21, and FGF23) bind heparan sulfate poorly, thereby escaping the extracellular matrix to circulate in the blood. Furthermore, the endocrine FGFs require $\alpha$ - and $\beta$-klotho co-receptors for signal transduction.

After consuming a meal, the liver takes up 25-35\% of dietary carbohydrates [35]. This effect cannot be replicated by either systemic hyperglycemia or hyperinsulinemia, suggesting that crosstalk between the gastrointestinal tract and liver is critical for hepatic glucose utilization. More importantly, feeding-induced hepatic glycogen synthesis remains functional in a genetic model that lacks insulin signaling in the liver $[36,37]$, raising the possibility of a parallel pathway to control postprandial hepatic metabolic reprogramming. Postprandial bile acid release and subsequent reabsorption are integral responses to feeding. The reabsorption of bile acids in the intestine leads to the activation of intestinal FXR and the subsequent secretion of FGF15/19, a direct target of FXR. Originally found to suppress hepatic de novo synthesis of bile acids [32], FGF15/19 has recently been found to promote hepatic glycogen synthesis and protein synthesis by activating the Ras-extracellular signal-related kinase (ERK) pathway, a parallel pathway to the insulin-activated PI3K-AKT pathway [38]. These findings implicate FGF15/19 in the coordination of intestinal feeding responses with hepatic metabolic changes.

In the fasted state, the coordinated activation of hepatic fatty acid oxidation and white adipose tissue lipolysis constitutes a major axis of energy substrate switch from carbohydrate to lipids. FGF21, a hepatic PPAR $\alpha$ target gene, was found to play important roles in liver-adipose crosstalk during fasting [33, 39]. As serum levels of FGF21 correlate closely with hepatic FGF21 expression [40], the liver is thought to be the major source of circulating FGF21. FGF21 promotes free fatty acid release from white adipose tissue by activating several lipases, thereby providing substrates for hepatic fatty acid oxidation and ketogenesis. In addition, FGF21 enhances hepatic ketogenesis through increasing the protein levels of rate-limiting enzymes in ketogenesis.

However, the adaptive fasting response, especially after prolonged fasting, requires the coordinated actions of all major organs to enhance survival. Recently, FGF21 was found to suppress physical activity, alter circadian behavior, and reduce female fertility $[41,42]$ - actions known to be involved in the physiological response to prolonged fasting. Moreover, these adaptive responses were attributed to FGF21 activity in the suprachiasmatic nucleus $(\mathrm{SCN})$ region of the brain. 
Interestingly, pharmacological administration of FGF21 elicits pleiotropic effects beyond the changes seen in the adaptive fasting response [43, 44], suggesting additional roles of FGF21 in other physiological settings. Indeed, FGF21 was found to be a target of PPAR $\gamma$ in adipose tissue, where its paracrine actions potentiate PPAR $\gamma$ activation in the postprandial state [40]. Notably, the effects of FGF21 on glucose homeostasis and hepatic lipid metabolism were shown to be dependent on adiponectin, a PPAR $\gamma$-induced adipokine [44].

In addition to liver and white adipose tissue, FGF21 is produced in brown fat [45] and skeletal muscle [46] in response to specific stimuli, suggesting that FGF21 coordinates the tissue-specific responses of the major metabolic organs.

The expressions of FGF15/19 and FGF21 are exquisitely dynamic during fasting and feeding cycles [32, 33, 40, 47]. The nutrient-responsive nature of FGF21 and FGF15 expression opens a new chapter for their respective roles in metabolism. In contrast, the prototypic FGF1 and FGF2 [34] are ubiquitously expressed [48], with established roles in wound healing and brain development [34]. Whereas FGF2 knockout mice have mild brain development and wound healing defects, FGF1 knockout mice are phenotypically normal [49]. In search of additional FGFs that may act as nutrient-controlled hormones, FGF1 was found to be highly induced in white adipose tissue upon feeding of a high-fat diet [50]. Conversely, FGF1 knockout (FGF1KO) mice developed marked adipose tissue fibrosis, impaired glucose homeostasis, and insulin insensitivity when fed a high-fat diet. Furthermore, upon withdrawal of the high-fat diet, FGF1KO mice developed pronounced fat necrosis. Given that adipose tissue expansion accompanied by increased vascularization is required to maintain insulin sensitivity under conditions of nutrient surplus [51], the poor white adipose tissue vascularization observed in FGF1 knockout mice may be responsible for their severe insulin-resistant phenotype on a high-fat diet. Interestingly, FGF1 is shown to be directly regulated by PPAR $\gamma$, and PPAR $\gamma$ and its target genes are increased in FGF1 knockout mice [50]. Therefore, FGF1 appears to be an essential output for PPAR $\gamma$-mediated insulin sensitization. Distinct from FGF21-mediated amplification of PPAR $\gamma$-mediated adipogenesis and adipokine production [40], FGF1 does not promote adipogenesis in 3 T3-L1 cells [52]. These properties raise the intriguing possibility of recombinant FGF1 (rFGF1) as an antidiabetic drug that may minimize the side effects associated with the thiazolidinedione class of PPAR $\gamma$ agonists. Indeed, rFGF1 injection markedly improves insulin sensitivity in genetic and diet-induced obesity models [53]. These effects are insulin dependent, suggesting a role as an insulin sensitizer, and require adipose FGFR1 expression, as rFGF1 fails to lower blood glucose in FGFR1 adiposespecific knockout mice. Interestingly, $\mathrm{rFGF} 1$ treatment does not alter adipose tissue vasculature, indicating differences between pharmacological administration and genetic studies. Importantly, an N-terminally truncated FGF1 analog with markedly reduced mitogenic activity was similarly able to normalize blood glucose levels in 
diabetic mice [53]. The ability to dissociate the mitogenic and glucose-lowering activities of FGF1 suggests that these different effects may be receptor specific. Nevertheless, these results demonstrate that FGF1 is a critical mediator of nutrient responsiveness in adipose tissue and has significant therapeutic potential.

\section{Lipids}

De novo lipogenesis has emerged as a novel pathway that produces lipid signaling molecules, including NR ligands. It was found that activation of PPAR $\delta$ in the liver enhanced hepatic de novo lipogenesis but paradoxically reduced serum lipid concentrations $[6,29]$. The reduction of serum lipids was also correlated with increased fatty acid uptake and oxidation in the skeletal muscle. Through unbiased metabolomics profiling, phosphocholine $\mathrm{PC}(18: 0 / 18: 1)$ was identified as a signaling molecule that facilitates postprandial crosstalk between the liver and muscle to promote fatty acid utilization. Furthermore, the effects of PC(18:0/18:1) are mediated by another NR, PPAR $\alpha$, in the muscle, underlying a new model of tissue crosstalk through NRs.

\section{Concluding Remarks}

In 1995, 10 years after the cloning of the first NR, the glucocorticoid receptor, the challenge of the field was proposed: "...to elucidate the contributions of these individual systems to the integrated and complex processes associated with the multihormonal nature of metabolic regulation..." [54] This review summarizes our current understandings toward this challenge. An immediate implication of our expanded knowledge of endogenous ligands and downstream hormonal signals is to explore their potential as novel therapeutics. Indeed, several compounds are in clinical trials for metabolic syndrome. In addition, these recent advances have highlighted the role of nutrient-sensing NRs in coordinating metabolic homeostasis. Through receptor activation, environmental cues are transduced as local metabolic outputs and long-range hormonal actions (Fig. 2). In a reductionist's perspective, these receptors may be important on-off switches for specific physiological states [55]. While wholesale, prolonged activation of any of these receptors may not be desirable, the use of high-affinity synthetic ligands offers a window to tease out beneficial downstream targets, especially in light of the identification of several hormone-like molecules that are good therapeutic candidates. With readily available synthetic ligands and mouse genetic models, it is possible that these receptors will become platforms for drug discovery. 

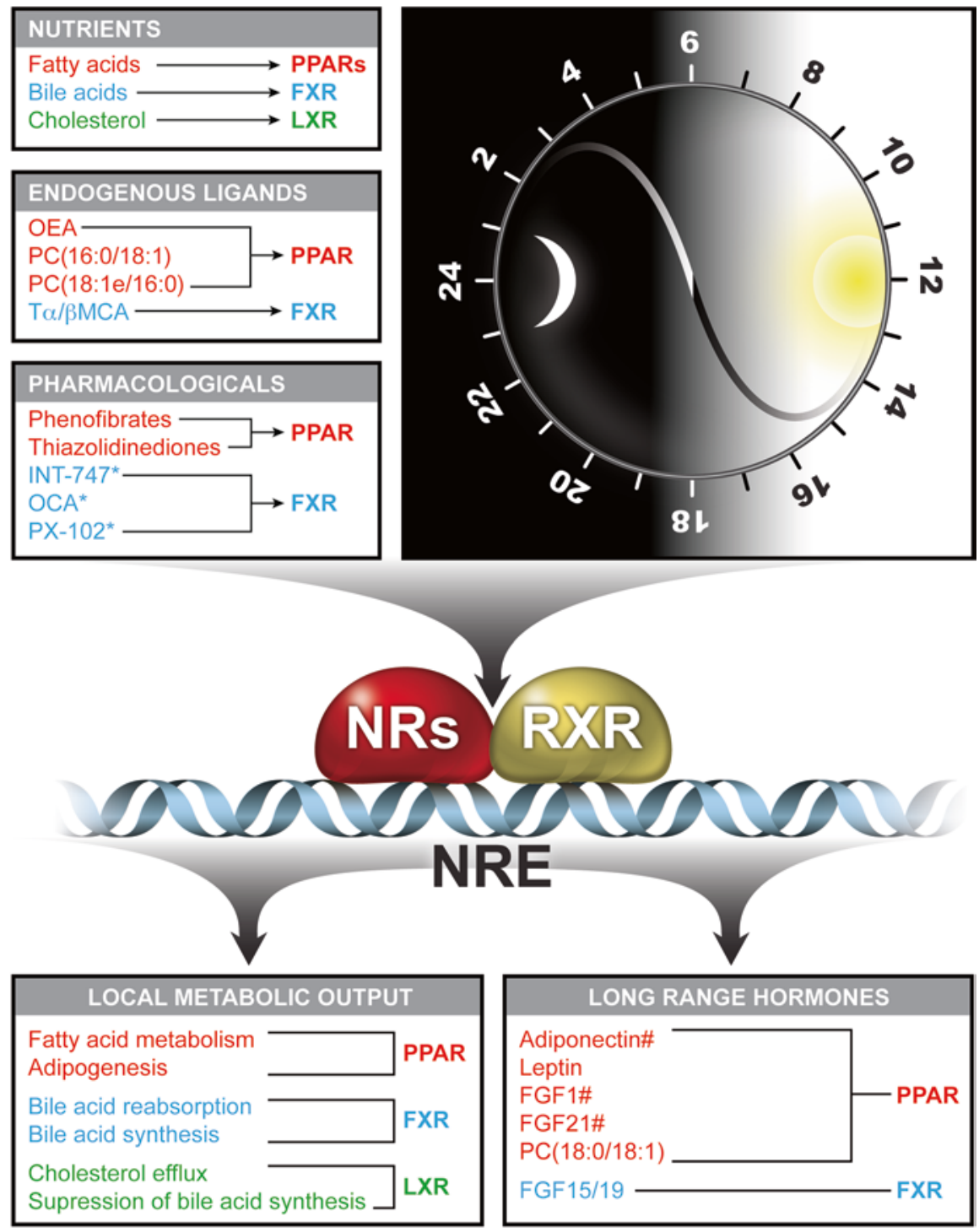

*currently under clinical trial \#pharmacological target

Fig. 2 Nuclear receptors $(N R s)$ as hubs to transduce environmental signals into daily metabolic rhythms. Originally identified as sensors for lipophilic nutrients to control their respective ligand metabolism, emerging evidence points to an integrative nature of the regulation and action of the peroxisome proliferator-activated receptor $(P P A R)$, farnesoid $\mathrm{X}$ receptor $(F X R)$, and liver $\mathrm{X}$ receptor $(L X R)$ families of NRs. In addition to nutrients, their activities are fine-tuned by endogenous ligands and the circadian clock. Activation of these NRs not only controls key cellular metabolic programs but also produces hormones that further connect metabolic tissues for a cohesive response to nutrient flux. Given the central roles of NRs in nutrient signaling, several pharmacological agents have been developed or are in development for metabolic diseases. Additionally, the NR-controlled hormones represent yet another emerging venue for therapeutics 
Open Access This chapter is distributed under the terms of the Creative Commons Attribution Noncommercial License, which permits any noncommercial use, distribution, and reproduction in any medium, provided the original author(s) and source are credited.

\section{References}

1. Muoio DM, Newgard CB (2006) Obesity-related derangements in metabolic regulation. Annu Rev Biochem 75:367-401

2. Samuel VT, Shulman GI (2012) Mechanisms for insulin resistance: common threads and missing links. Cell 148(5):852-871

3. Evans RM, Mangelsdorf DJ (2014) Nuclear receptors, RXR, and the big bang. Cell 157(1):255-266

4. Evans RM, Barish GD, Wang YX (2004) PPARs and the complex journey to obesity. Nat Med 10(4):355-361

5. Ahmadian $\mathrm{M}$ et al (2013) PPARgamma signaling and metabolism: the good, the bad and the future. Nat Med 19(5):557-566

6. Liu S et al (2011) Role of peroxisome proliferator-activated receptor $\{$ delta $\} /\{$ beta $\}$ in hepatic metabolic regulation. J Biol Chem 286(2):1237-1247

7. de Aguiar Vallim TQ, Tarling EJ, Edwards PA (2013) Pleiotropic roles of bile acids in metabolism. Cell Metab 17(5):657-669

8. Calkin AC, Tontonoz P (2012) Transcriptional integration of metabolism by the nuclear sterolactivated receptors LXR and FXR. Nat Rev Mol Cell Biol 13(4):213-224

9. Chawla A et al (2001) Nuclear receptors and lipid physiology: opening the X-files. Science 294(5548): 1866-1870

10. Haeusler RA et al (2012) Impaired generation of 12-hydroxylated bile acids links hepatic insulin signaling with dyslipidemia. Cell Metab 15(1):65-74

11. Fu J et al (2003) Oleylethanolamide regulates feeding and body weight through activation of the nuclear receptor PPAR-alpha. Nature 425(6953):90-93

12. Gaetani $\mathrm{S}$ et al (2010) The fat-induced satiety factor oleoylethanolamide suppresses feeding through central release of oxytocin. J Neurosci 30(24):8096-8101

13. Sayin SI et al (2013) Gut microbiota regulates bile acid metabolism by reducing the levels of tauro-beta-muricholic acid, a naturally occurring FXR antagonist. Cell Metab 17(2):225-235

14. Swann JR et al (2011) Systemic gut microbial modulation of bile acid metabolism in host tissue compartments. Proc Natl Acad Sci U S A 108(Suppl 1):4523-4530

15. Li F et al (2013) Microbiome remodelling leads to inhibition of intestinal farnesoid X receptor signalling and decreased obesity. Nat Commun 4:2384

16. Devkota $S$ et al (2012) Dietary-fat-induced taurocholic acid promotes pathobiont expansion and colitis in Il10-/- mice. Nature 487(7405):104-108

17. Cariou $B$ et al (2006) The farnesoid $X$ receptor modulates adiposity and peripheral insulin sensitivity in mice. J Biol Chem 281(16):11039-11049

18. Ma K et al (2006) Farnesoid X receptor is essential for normal glucose homeostasis. J Clin Invest 116(4):1102-1109

19. Zhang Y et al (2006) Activation of the nuclear receptor FXR improves hyperglycemia and hyperlipidemia in diabetic mice. Proc Natl Acad Sci U S A 103(4):1006-1011

20. Ryan KK et al (2014) FXR is a molecular target for the effects of vertical sleeve gastrectomy. Nature 509(7499):183-188

21. Fu S et al (2011) Aberrant lipid metabolism disrupts calcium homeostasis causing liver endoplasmic reticulum stress in obesity. Nature 473(7348):528-531

22. Liu S, Alexander RK, Lee CH (2014) Lipid metabolites as metabolic messengers in interorgan communication. Trends Endocrinol Metab 25(7):356-363 
23. Lodhi IJ, Wei X, Semenkovich CF (2011) Lipoexpediency: de novo lipogenesis as a metabolic signal transmitter. Trends Endocrinol Metab 22(1):1-8

24. Chakravarthy MV et al (2009) Identification of a physiologically relevant endogenous ligand for PPARalpha in liver. Cell 138(3):476-488

25. Chakravarthy MV et al (2005) "New" hepatic fat activates PPARalpha to maintain glucose, lipid, and cholesterol homeostasis. Cell Metab 1(5):309-322

26. Kim JB, Spiegelman BM (1996) ADD1/SREBP1 promotes adipocyte differentiation and gene expression linked to fatty acid metabolism. Genes Dev 10(9):1096-1107

27. Lodhi IJ et al (2012) Inhibiting adipose tissue lipogenesis reprograms thermogenesis and PPARgamma activation to decrease diet-induced obesity. Cell Metab 16(2):189-201

28. Kim YG, Lou AC, Saghatelian A (2011) A metabolomics strategy for detecting protein-metabolite interactions to identify natural nuclear receptor ligands. Mol Biosyst 7(4):1046-1049

29. Liu $\mathrm{S}$ et al (2013) A diurnal serum lipid integrates hepatic lipogenesis and peripheral fatty acid use. Nature 502(7472):550-554

30. Choi JH et al (2010) Anti-diabetic drugs inhibit obesity-linked phosphorylation of PPARgamma by Cdk5. Nature 466(7305):451-456

31. Choi JH et al (2011) Antidiabetic actions of a non-agonist PPARgamma ligand blocking Cdk5mediated phosphorylation. Nature 477(7365):477-481

32. Inagaki $\mathrm{T}$ et al (2005) Fibroblast growth factor 15 functions as an enterohepatic signal to regulate bile acid homeostasis. Cell Metab 2(4):217-225

33. Inagaki $T$ et al (2007) Endocrine regulation of the fasting response by PPARalpha-mediated induction of fibroblast growth factor 21. Cell Metab 5(6):415-425

34. Beenken A, Mohammadi M (2009) The FGF family: biology, pathophysiology and therapy. Nat Rev Drug Discov 8(3):235-253

35. Moore MC et al (2012) Regulation of hepatic glucose uptake and storage in vivo. Adv Nutr 3(3):286-294

36. Dong XC et al (2008) Inactivation of hepatic Foxo1 by insulin signaling is required for adaptive nutrient homeostasis and endocrine growth regulation. Cell Metab 8(1):65-76

37. Lu M et al (2012) Insulin regulates liver metabolism in vivo in the absence of hepatic Akt and Foxo1. Nat Med 18(3):388-395

38. Kir S et al (2011) FGF19 as a postprandial, insulin-independent activator of hepatic protein and glycogen synthesis. Science 331(6024):1621-1624

39. Badman MK et al (2007) Hepatic fibroblast growth factor 21 is regulated by PPARalpha and is a key mediator of hepatic lipid metabolism in ketotic states. Cell Metab 5(6):426-437

40. Dutchak PA et al (2012) Fibroblast growth factor-21 regulates PPARgamma activity and the antidiabetic actions of thiazolidinediones. Cell 148(3):556-567

41. Bookout AL et al (2013) FGF21 regulates metabolism and circadian behavior by acting on the nervous system. Nat Med 19(9):1147-1152

42. Owen BM et al (2013) FGF21 contributes to neuroendocrine control of female reproduction. Nat Med 19(9):1153-1156

43. Xu J et al (2009) Fibroblast growth factor 21 reverses hepatic steatosis, increases energy expenditure, and improves insulin sensitivity in diet-induced obese mice. Diabetes 58(1):250-259

44. Lin Z et al (2013) Adiponectin mediates the metabolic effects of FGF21 on glucose homeostasis and insulin sensitivity in mice. Cell Metab 17(5):779-789

45. Hondares E et al (2011) Thermogenic activation induces FGF21 expression and release in brown adipose tissue. J Biol Chem 286(15):12983-12990

46. Kim KH et al (2013) Autophagy deficiency leads to protection from obesity and insulin resistance by inducing Fgf21 as a mitokine. Nat Med 19(1):83-92

47. Stroeve JH et al (2010) Intestinal FXR-mediated FGF15 production contributes to diurnal control of hepatic bile acid synthesis in mice. Lab Invest 90(10):1457-1467

48. Fon Tacer $\mathrm{K}$ et al (2010) Research resource: comprehensive expression atlas of the fibroblast growth factor system in adult mouse. Mol Endocrinol 24(10):2050-2064 
49. Miller DL et al (2000) Compensation by fibroblast growth factor 1 (FGF1) does not account for the mild phenotypic defects observed in FGF2 null mice. Mol Cell Biol 20(6):2260-2268

50. Jonker JW et al (2012) A PPARgamma-FGF1 axis is required for adaptive adipose remodelling and metabolic homeostasis. Nature 485(7398):391-394

51. Sun K, Kusminski CM, Scherer PE (2011) Adipose tissue remodeling and obesity. J Clin Invest 121(6):2094-2101

52. Hutley L et al (2004) Fibroblast growth factor 1: a key regulator of human adipogenesis. Diabetes 53(12):3097-3106

53. Suh JM et al (2014) Endocrinization of FGF1 produces a neomorphic and potent insulin sensitizer. Nature 513(7518):436-439

54. Mangelsdorf DJ, Evans RM (1995) The RXR heterodimers and orphan receptors. Cell 83(6):841-850

55. Fan W et al (2013) Road to exercise mimetics: targeting nuclear receptors in skeletal muscle. J Mol Endocrinol 51(3):T87-T100 


\title{
Fighting Fire with Fire in Cancer
}

\author{
Thorsten Berger, Mary E. Saunders, and Tak W. Mak
}

\begin{abstract}
Cancer will not be cured until we understand and target the unique alterations that distinguish tumor cells from normal cells. This chapter briefly describes four new approaches to anticancer therapy based on boosting the immune system's response to tumor cells, countering the metabolic adaptations that allow tumor cells to thrive under conditions that kill normal cells, manipulating the increased oxidative stress associated with the tumor environment, and exploiting the aneuploidy characteristic of many advanced tumor cells. The long-term goal is to devise biomarkers and novel therapeutic agents able to more effectively fight aggressive cancers.
\end{abstract}

Keywords Warburg effect $\bullet$ Metabolism $\bullet$ CTLA-4 $\bullet$ IL-7 $\bullet$ CPT1C $・$ IDH $\bullet$ BRCA

\section{Introduction}

Why has it been so difficult to cure cancer? The answer lies in our inability to define all of the elements that contribute to the transformation and survival of tumor cells, and challenges in dissecting the body's responses to these malignant growths. As far back as 1924, Otto Warburg proposed that "The cause of cancer is the replacement of the respiration of oxygen in normal body cells by a fermentation of sugar," a concept now widely known as the "Warburg effect" $[1,2]$. Because this change would not alter the "antigenic face" of a cancer cell, the immune system would not be able to recognize these aberrant cells and remove them, necessitating externally imposed therapies. Oncologists of the day therefore focused on eliminating all fastreplicating cells, normal or cancerous, by radiation or chemotherapy, a trend that remained firmly in place into the 1990s (Table 1).

T. Berger • M.E. Saunders $\bullet$ T.W. Mak $(\bowtie)$

The Campbell Family Institute for Breast Cancer Research and Ontario

Cancer Institute, University Health Network, 620 University Avenue, Suite 706,

Toronto, ON M5G 2M9, Canada

e-mail: tmak@uhnres.utoronto.ca 
Table 1 Chemotherapeutic agents approved

Table 2 Targeted agents approved

\begin{tabular}{l|l}
\hline Year approved & Agent \\
\hline 1942 & Nitrogen mustard \\
\hline 1948 & 6-Mercaptopurine \\
\hline 1958 & Methotrexate \\
\hline 1959 & Cyclophosphamide \\
\hline 1975 & 5-Fluorouracil \\
\hline 1978 & Cisplatin \\
\hline 1992 & Paclitaxel \\
\hline 1996 & Gemcitabine \\
\hline 1996 & Topotecan \\
\hline 2004 & Pemetrexed
\end{tabular}

\begin{tabular}{l|l|l}
\hline $\begin{array}{l}\text { Year of } \\
\text { approval }\end{array}$ & Target & Drug name \\
\hline 1998 & Her-2 & Herceptin \\
\hline 2001 & Bcr-abl & Imatinib \\
\hline 2003 & EGFR & Gefitinib \\
\hline 2003 & Proteasome & Bortezomib \\
\hline 2004 & VEGF & Bevacizumab \\
\hline 2006 & HDAC & Vorinostat \\
\hline 2007 & mTOR & Temsirolimus \\
\hline 2011 & CTLA-4 & Ipilimumab \\
\hline 2011 & ALK & Crizotinib \\
\hline 2011 & B-Raf & Vemurafenib \\
\hline 2012 & Hedgehog & Vismodegib \\
\hline 2013 & Btk & Ibrutinib \\
\hline
\end{tabular}

In a talk at a 1966 Nobel Laureates' meeting, Warburg reiterated his belief that cancer cells ultimately arise when a switch from normal respiration to fermentation is caused by (for example) damage to the enzymatic machinery required for respiration (see http://www.mediatheque.lindau-nobel.org/videos/31517/on-the-primarycauses-and-on-the-secondary-causes-of-cancer-german-presentation-1966/ laureate-warburg). However, in the 1970s, Warburg's hypothesis was again sidelined as scientists became convinced that the underlying cause of cancer was aberrant function of either oncogenes or tumor suppressor genes. After the discovery of Src in 1976 [3], a multitude of other oncogenes were identified, including EGFR, Her2, Abl, Raf, Alk, Btk, PI3'K, Tor, and many others. This focus on a genetic origin for cancer initiation was then reflected in the development of anticancer therapeutics. Since 1998, numerous therapeutic agents targeting specific oncogenes or other relevant molecules or structures have been approved (Table 2).

Although anti-oncogene agents have proven undeniably helpful, it has unfortunately become clear that the cancer cell genome is too varied and oncogenes are too numerous for these strategies to be able to eradicate all tumors. As Robert Weinberg 
said, "There are more paths to developing tumors than there are stars in the sky." Pathways now known to be deregulated in cancer include the Hedgehog, Wnt/ Notch, TGF $\beta$ R, FAS, and VEGFR pathways, as well as those involving receptor tyrosine kinases $[4,5]$. Concomitantly, the development of new chemotherapeutics has slowed down significantly, with no new agent being approved in the last 10 years. Researchers have instead returned to Warburg's hypothesis and are exploring ways of disrupting tumor cell metabolism and mitosis, as well as seeking means of boosting anticancer immune responses. The long-term goal is to develop novel anticancer therapeutics that differ from existing classes of agents and so may be more effective. In the following sections, we will describe our work on four novel avenues that show promise as future anticancer strategies: (1) boosting the immune system; (2) targeting cancer cell metabolism; (3) targeting reactive oxygen species; and (4) exploiting cancer cell aneuploidy.

\section{Boosting the Immune System}

The objective of immunotherapy is to strengthen the body's ability to recognize and attack tumor cells - that is, fight the fire of cancer with the fire of an aggressive immune response. Activation of the $\mathrm{T}$ lymphocytes that underpin any adaptive immune response requires two stimuli: (1) binding of the T cell receptor to a complex of antigenic peptide plus MHC (pMHC), which delivers an antigen-specific signal; and (2) binding of CD28, a receptor expressed on the T cell surface, to CD80 or CD86 ligands expressed on the surface of antigen-presenting cells (APCs), which delivers a co-stimulatory signal. In 1995, our group reported that $\mathrm{T}$ cell activation is negatively regulated by cytotoxic T lymphocyte antigen-4 (CTLA-4) in a manner vital for the control of lymphocyte homeostasis [6]. Subsequent work demonstrated that CTLA-4 exerts its inhibitory activity by binding to CD80 or CD86, thereby preventing the binding of $\mathrm{CD} 28$ to these surface proteins and blocking the costimulation needed for optimal T cell activation [7]. Several other negative regulators of $\mathrm{T}$ cell activation, including PD1, have since been discovered $[8,9]$.

There are multiple reasons why the immune system is often unable to completely eradicate a cancer without help from targeted therapeutics. First, there are very few truly tumor-specific antigens, since healthy and malignant tissues are identical in most of their components. Second, the negative homeostatic regulation imposed by CTLA-4 and PD1 decreases the activation of antitumor T cells. Third, like chronic viral infections, cancers often induce $\mathrm{T}$ cell exhaustion, in which hyperactivated $\mathrm{T}$ cell clones display specific profiles of transcription factor and inhibitory receptor expression that eventually suppress the antitumor response [10]. Fourth, the general lack of co-stimulation characteristic of malignant tissue prevents the activation of antitumor $\mathrm{T}$ cells [11]. Fifth, cancers impede the homing of naïve $\mathrm{T}$ lymphocytes into tumor-draining lymph nodes, decreasing the probability of the rare interaction between T cells and APCs displaying the relevant pMHC complex [12]. This plethora of difficulties indicates that strengthening the antitumor response in vivo will 
require a battery of complementary approaches. Accordingly, immunotherapies used to treat cancer patients have been devised that are antibody based, cytokine based, or cell based. In the following sections, we discuss some exciting results based on the first two approaches.

\section{Targeting CTLA-4}

Because CTLA-4 is a negative regulator of $\mathrm{T}$ cell activation, agents have been designed to block CTLA-4 and thereby hopefully sustain antitumor T cell responses. In a phase 3 study of a human anti-CTLA-4 antibody (ipilimumab), a cohort of melanoma patients treated with this agent gained a significant survival advantage [13]. Suppression of the inhibitory signals generated by CTLA-4 apparently allowed prolonged activation of antitumor T cells. The success of this trial has led to renewed efforts to determine why antitumor $\mathrm{CD}^{+} \mathrm{T}$ cells do not routinely attack and kill cancer cells on their own, and how their activities can be boosted.

\section{IL-7 Treatment}

Several groups have attempted to strengthen antitumor $\mathrm{T}$ cell responses in vivo by vaccination with tumor antigens, but clinical trials of this approach have not shown the expected efficacy [14]. In searching for ways to improve this result, our group discovered that vaccine-induced immune responses to tumors could be augmented and sustained by providing exogenous IL-7 [15]. In this study, we employed the RIP-TAG2 transgenic mouse model, in which mice expressing the SV40 large T antigen (TAG) under the control of the rat insulin promoter (RIP) develop pancreatic $\beta$-islet cell tumors [16]. We crossed these mice with transgenic mice expressing the lymphocytic choriomeningitis virus (LCMV) glycoprotein (GP) under the control of RIP [17] to produce RIP(GP $x$ TAG2) mice. Tumors arising in these mice were not cleared by the immune system even though they expressed the foreign GP antigen [18]. Even after LCMV infection, which mimics administration of a live antitumor vaccine, only a limited increase in overall mouse survival occurred and no sustained antitumor response was observed [18]. However, if IL-7 treatment of LCMV-vaccinated tumor-bearing RIP(GP x TAG2) mice was initiated at 8 days after LCMV infection-a point that coincides with the peak of the CD8 ${ }^{+} \mathrm{T}$ cell response - the virus was eliminated, and mouse survival was prolonged by over 100 days $[11,15]$.

Subsequent work focused on determining how IL-7 can overcome immune inhibitory networks during a chronic viral infection, which mimics the continuous production of a tumor antigen by a cancer [19]. Although IL-7 treatment had already shown significant therapeutic promise [20-22], and had been successfully used in several nonhuman primate SIV infection models [23-25], the efficacy of IL-7 in 
promoting viral clearance had not been fully explored [19]. We administered IL-7 to mice infected with LCMV clone 13, which establishes a chronic infection and generates massive viral antigen levels [26]. In part by enhancing thymic output, IL-7 treatment of LCMV-13-infected mice increased both the magnitude of the immune response and the size of the entire naive $\mathrm{T}$ cell pool, including $\mathrm{T}$ cell clones directed against non-LCMV epitopes [19]. LCMV-13-specific CD8 ${ }^{+}$T cells showed enhanced degranulation kinetics and cytokine production upon IL-7 exposure, resulting in PD-1 downregulation on effector $\mathrm{T}$ cells and efficient viral clearance [19]. IL-7 treatment also induced a cytokine milieu favoring leukocyte activation and production of the cytoprotective cytokine IL-22 [19]. At the molecular level, IL-7 led to a reduction in Socs3 expression in T cells that was FoxO dependent [19]. These model system data indicate that a combination of IL-7 and a cancer vaccine may result in an antitumor response capable of benefitting cancer patients.

\section{Targeting Cancer Cell Metabolism}

"Oncogene addiction" is a concept devised to explain the observation that the inhibition of crucial oncogenes, or the reconstitution of previously lost or repressed tumor suppressor genes, can have a broad antitumorigenic effect. However, in addition to their genetic and epigenetic alterations, cells undergoing transformation implement specific metabolic adaptations that are induced by their altered microenvironment. These adaptations lead to upregulation of the stress response and other pathways that are not inherently tumorigenic but allow developing tumor cells to survive under conditions that would kill normal cells [27]. This "metabolic addiction" of pre-cancerous and ultimately cancerous cells provides new opportunities for specific therapeutic intervention, since normal cells, which have not had to endure the same constant internal and external stress, should be unaffected by agents targeting cancer cell metabolic adaptations.

\section{Targeting Carnitine Palmitoyltransferase-1C}

Our group has discovered that carnitine palmitoyltransferase-1C (CPT1C), a brainspecific metabolic enzyme, may be involved in tumor cell metabolic adaptation to heightened environmental stress [28]. Expression of CPT1C, but not the ubiquitous CPT1A or heart-specific CPT1B, correlated inversely with mTOR pathway activation in tumor cells, indicating that CPT1C may act in a pathway parallel to mTORenhanced glycolysis $[28,29]$. CPT1C contributes to rapamycin resistance in murine primary tumors and is overexpressed in human non-small-cell lung carcinomas (NSCLC) [28]. CPT1C overexpression in a human cancer cell line led to increased fatty acid oxidation and ATP production, and resistance to glucose deprivation or hypoxia [28]. Importantly, siRNA-mediated depletion of CPT1C reduced tumor 
growth in an in vivo xenograft mouse model [28], and delayed tumor development and increased mouse survival in a neurofibromatosis type I tumor model [30-32]. Subsequent studies have established that CPT1C is a p53 target gene, and that CPT1C expression is induced by metabolic stress factors such as hypoxia and glucose deprivation in a p53- and AMPK-dependent manner [32]. These results indicate that p53 initially protects cells from metabolic stress via induction of CPT1C but that excessive CPT1C expression can promote carcinogenesis [32]. Because CPT1C expression is normally restricted to the brain, and most drugs cannot penetrate the blood-brain barrier, CPT1C may be an ideal candidate for specific smallmolecule inhibition as a treatment for hypoxic and otherwise treatment-resistant cancers $[29,32]$.

\section{Targeting Mutated Isocitrate Dehydrogenases}

Isocitrate dehydrogenase (IDH)- 1 and IDH2 are metabolic enzymes that govern the important NADP/NADPH ratio in the cytoplasm and mitochondria, respectively. Oncogenic mutations to these enzymes have recently garnered much interest since their discovery during cancer genome sequencing projects [33-35]. The normal function of IDH $1 / 2$ is to convert isocitrate to $\alpha$-ketoglutarate $(\alpha \mathrm{KG})$ while reducing NADP to NADPH and liberating $\mathrm{CO}_{2}$ [36]. To date, mutations of IDH1 altering a single arginine residue (R132) in the enzymatic active site have been found at high frequency in glioblastoma multiforme (GBM) [34], acute myeloid leukemia (AML) [33], cholangiocarcinoma [37, 38], and chondrosarcoma [39]. IDH1 R132 mutations occur less frequently in other types of cancers such as melanoma, NSCLC, and prostate and colon cancers [40]. IDH2 mutations, predominantly R172K and R140Q [36, 41], have been identified in cholangiocarcinoma [37, 38], myelodysplastic syndrome (MDS) and myeloproliferative disorder (MPD) [42-44], AML [33], chondrosarcoma [39], angioimmunoblastic T cell lymphoma (AITL) [45], and D2HG aciduria [46].

In 2009, scientists at Agios Pharmaceuticals used a metabolite profiling strategy to make the breakthrough discovery that the tumorigenic effect of IDH1/2 mutations is not due to a loss of function of these proteins. Instead, the mutant IDH enzymes acquire a neomorphic activity in which the normal product $\alpha \mathrm{KG}$ is converted to 2-hydroxyglutarate (D2HG) in a reaction that consumes, rather than produces, NADPH [36, 47].

To examine the pathophysiological consequences of IDH mutations in the most relevant in vivo system possible, our group generated a conditional knock-in mouse model using the lox-stop-lox (LSL) system. In the absence of Cre recombinase (Cre), neither the LSL IDH1 R132 mutant allele nor the wild type IDH allele is expressed, but when Cre is present, a stop codon is excised and the mutant IDH1 protein is expressed from the endogenous locus [48]. Initial characterization of various mouse strains revealed that IDH1 knockout mice were viable and fertile but that expression of the mutant IDH1 enzyme and its consequent D2HG production were 
embryonic lethal [36]. Mutant IDH1 enzyme expressed solely in the myeloid compartment (LysM promoter) resulted in splenomegaly, decreased bone marrow cellularity, and extramedullary hematopoiesis by age 6 months [48]. LysM IDH1 knock-in LSK cells showed an increase in highly methylated CpG sites and histone hypermethylation [48], consistent with the DNA methylation changes observed in human IDH1- or IDH2-mutant gliomas [49] and AML [50].

The available data indicate that mutant IDH enzymes exert their tumor-promoting function through their novel enzymatic activity, which generates massive quantities of D2HG. Mechanistic studies of D2HG have focused on its competitive inhibition of 2-OG-dependent dioxygenases (2OGD), which use $\alpha \mathrm{KG}$ as a cosubstrate [36]. In mammalian cells, there are more than 60 2OGD involved in collagen biosynthesis, fatty acid metabolism, DNA repair, RNA and chromatin modifications, and hypoxia detection [51]. The general enzymatic reaction performed by 2OGD converts $\alpha K G$ to succinate and $\mathrm{CO}_{2}$, and requires oxygen, ascorbate, and iron as cofactors [51]. D2HG has been shown in vitro to competitively inhibit 2OGD [52], and the high concentration of D2HG measured in cells and tissues of IDH-mutant tumors makes it very likely that D2HG impairs the activity of this class of enzymes in vivo as well [36]. Additional potential targets of D2HG inhibition include the TET proteins involved in DNA methylation, the JumonjiC domain-containing histone demethylases, the prolyl hydroxylases (PHD) and lysyl hydroxylases (LHD) required for collagen folding and maturation, and the PHDs that regulate hypoxia-inducible factor (HIF) signaling [36].

In early 2014, an oral, potent, reversible, and selective inhibitor (AG-221) of the mutated IDH2 protein underwent evaluation in a clinical trial of patients with advanced IDH2-mutant hematologic malignancies. Encouragingly, AG-221 treatment reduced D2HG levels and demonstrated a dose-dependent survival benefit [53]. Pursuit of a similar compound to combat IDH1-mutant cancers is ongoing.

\section{Targeting Reactive Oxygen Species}

An important cellular stress factor increased in cancer cells is the level of reactive oxygen species (ROS). ROS regulation is critical for normal cellular functions and survival, and the accelerated growth of tumor cells generates increased ROS. Cancers therefore need to adjust signaling pathways linked to ROS regulation to cope with their enhanced ROS. Elevated ROS are generated by hypoxia, defective metabolism, endoplasmic reticulum (ER) stress, and oncogene activity [54]. Conversely, ROS are eliminated routinely via NADPH, glutathione, and dietary antioxidants, and under stress conditions through the activation of transcription factors such as NRF2 and the activity of tumor suppressors such as BRCA1, p53, PTEN, and ATM [54]. During carcinogenic progression from normal tissue to neoplastic transformation to carcinoma in situ and finally to invasive carcinoma, cellular ROS levels progressively increase because of metabolic aberrations acquired following transformation. Unlike normal cells, cancer cells can cope with this inexorable rise 
in ROS by upregulating the above antioxidant pathways and transiently lowering ROS levels. Targeted therapeutics that interfere with this upregulation may therefore result in ROS increasing to the point where the apoptotic death of the cancer cell is induced. Alternatively, agents that increase ROS production beyond the capacity of the upregulated antioxidant mechanisms to cope may kill the tumor cell while sparing normal cells in which these pathways are not activated.

We recently explored whether altered ROS regulation could explain the tissue specificity of BRCA1-related cancers, which occur almost exclusively in the breast and ovary. We found that BRCA1 deficiency enhanced ROS levels in breast cancer cells and that Nrf2-driven antioxidant pathways were defective [55]. Further analysis revealed that BRCA1 directly interacts with Nrf2 and that this interaction affects Keap1-mediated Nrf2 ubiquitination, stability, and activation [55]. Interestingly, estrogen treatment partially restored Nrf2 levels and enhanced tumor growth in the absence of BRCA1 [55, 56]. We hypothesize that loss of BRCA1 in heterozygous carriers of somatic BRCA1 mutations has different effects depending on the tissue. In tissues without an estrogen-rich environment, BRCA1 deficiency impairs Nrf2 antioxidant signaling, leading to an accumulation of ROS in the BRCA1-deficient cells that kills them. However, in the breast and ovary, estrogen activates Nrf2 via a mechanism that depends on PI3K-AKT and protects BRCA1-deficient cells from ROS-induced death. If a BRCA1-deficient breast or ovarian cell also loses PTEN function, the PI3K-AKT pathway may be further stimulated and may reinforce estrogen-mediated Nrf2 signaling. Mitogenic and antioxidant pathways acting downstream of AKT, coupled with the genomic instability caused by a lack of BRCA1-mediated DNA repair, might then eventually drive the complete malignant transformation of the BRCA1-deficient cells [56]. Exploitation of the altered ROS regulation in these cells may serve as the basis for an effective therapy in the future.

\section{Exploiting Cancer Cell Aneuploidy}

A long-term goal of our laboratory is to develop novel anticancer therapeutics that differ from existing classes of agents. We have recently taken advantage of an alteration shared by many advanced cancer cells but not found in normal cells: aneuploidy.

By using a systematic approach that combines RNAi screening with gene expression analysis in human breast cancers and cell lines and focusing on cancer cell aneuploidy, we have identified polo-like kinase-4 (PLK4), an enzyme critical for aneuploidy maintenance, as a promising therapeutic target [57]. A drug discovery program culminated in the isolation of CFI-400945, a potent and selective smallmolecule PLK4 inhibitor [57]. In vitro treatment of human cancer cells with CFI400945 results in effects similar to those of siRNA-mediated PLK4 kinase inhibition, including mitotic defects, centriole duplication, and cell death [57]. In in vivo mouse models based on human ovarian or breast cancer xenografts, tumor growth was significantly inhibited by CFI-400945 in a manner influenced by the PTEN status of 
the tumor [57]. PTEN-deficient xenografts showed a greater response to CFI400945 than xenografts expressing wild type PTEN, making PTEN status a potential predictive biomarker for therapy with this first-in-class agent [57].

\section{Conclusion}

This chapter has briefly outlined four innovative anticancer approaches under investigation in our laboratory. Our belief is that by concentrating on unique aspects of tumor biology, we can identify strategies and targets that are applicable to a broad range of cancers and less likely to induce damaging side-effects in normal tissues. By fighting the fire of malignancy with the fire of creative thinking, we hope to indeed conquer cancer in our lifetime.

Open Access This chapter is distributed under the terms of the Creative Commons Attribution Noncommercial License, which permits any noncommercial use, distribution, and reproduction in any medium, provided the original author(s) and source are credited.

\section{References}

1. Koppenol WH, Bounds PL, Dang CV (2011) Otto Warburg's contributions to current concepts of cancer metabolism. Nat Rev Cancer 11(5):325-337

2. Warburg O, Posener K, Negelein E (1924) Über den Stoffwechsel der Tumoren. Biochem Z 152:309-344

3. Stehelin D et al (1976) DNA related to the transforming gene(s) of avian sarcoma viruses is present in normal avian DNA. Nature 260(5547):170-173

4. Hanahan D, Weinberg RA (2000) The hallmarks of cancer. Cell 100(1):57-70

5. Hanahan D, Weinberg RA (2011) Hallmarks of cancer: the next generation. Cell 144(5):646-674

6. Waterhouse $\mathrm{P}$ et al (1995) Lymphoproliferative disorders with early lethality in mice deficient in Ctla-4. Science 270(5238):985-988

7. Egen JG, Kuhns MS, Allison JP (2002) CTLA-4: new insights into its biological function and use in tumor immunotherapy. Nat Immunol 3(7):611-618

8. Pentcheva-Hoang T, Corse E, Allison JP (2009) Negative regulators of T-cell activation: potential targets for therapeutic intervention in cancer, autoimmune disease, and persistent infections. Immunol Rev 229(1):67-87

9. Pardoll DM (2012) The blockade of immune checkpoints in cancer immunotherapy. Nat Rev Cancer 12(4):252-264

10. Wherry EJ (2011) T cell exhaustion. Nat Immunol 12(6):492-499

11. Sadelain M, Riviere I, Brentjens R (2003) Targeting tumours with genetically enhanced T lymphocytes. Nat Rev Cancer 3(1):35-45

12. Carriere V et al (2005) Cancer cells regulate lymphocyte recruitment and leukocyteendothelium interactions in the tumor-draining lymph node. Cancer Res 65(24):11639-11648

13. Hodi FS et al (2010) Improved survival with ipilimumab in patients with metastatic melanoma. N Engl J Med 363(8):711-723 
14. Prendergast GC, Jaffee EM (2007) Cancer immunologists and cancer biologists: why we didn't talk then but need to now. Cancer Res 67(8):3500-3504

15. Pellegrini $M$ et al (2009) Adjuvant IL-7 antagonizes multiple cellular and molecular inhibitory networks to enhance immunotherapies. Nat Med 15(5):528-536

16. Hanahan D (1985) Heritable formation of pancreatic beta-cell tumours in transgenic mice expressing recombinant insulin/simian virus 40 oncogenes. Nature 315(6015):115-122

17. Ohashi PS et al (1991) Ablation of "tolerance" and induction of diabetes by virus infection in viral antigen transgenic mice. Cell 65(2):305-317

18. Speiser DE et al (1997) Self antigens expressed by solid tumors do not efficiently stimulate naive or activated T cells: implications for immunotherapy. J Exp Med 186(5):645-653

19. Pellegrini $M$ et al (2011) IL-7 engages multiple mechanisms to overcome chronic viral infection and limit organ pathology. Cell 144(4):601-613

20. Levy Y et al (2009) Enhanced T cell recovery in HIV-1-infected adults through IL-7 treatment. J Clin Invest 119(4):997-1007

21. Sereti I et al (2009) IL-7 administration drives T cell-cycle entry and expansion in HIV-1 infection. Blood 113(25):6304-6314

22. Sportes $\mathrm{C}$ et al (2008) Administration of rhIL-7 in humans increases in vivo TCR repertoire diversity by preferential expansion of naive T cell subsets. J Exp Med 205(7):1701-1714

23. Beq $S$ et al (2006) IL-7 induces immunological improvement in SIV-infected rhesus macaques under antiviral therapy. J Immunol 176(2):914-922

24. Fry TJ et al (2003) IL-7 therapy dramatically alters peripheral T-cell homeostasis in normal and SIV-infected nonhuman primates. Blood 101(6):2294-2299

25. Nugeyre MT et al (2003) IL-7 stimulates T cell renewal without increasing viral replication in simian immunodeficiency virus-infected macaques. J Immunol 171(8):4447-4453

26. Wherry EJ et al (2003) Viral persistence alters CD8 T-cell immunodominance and tissue distribution and results in distinct stages of functional impairment. J Virol 77(8):4911-4927

27. Galluzzi L et al (2013) Metabolic targets for cancer therapy. Nat Rev Drug Discov 12(11):829-846

28. Zaugg K et al (2011) Carnitine palmitoyltransferase 1C promotes cell survival and tumor growth under conditions of metabolic stress. Genes Dev 25(10):1041-1051

29. Reilly PT, Mak TW (2012) Molecular pathways: tumor cells Co-opt the brain-specific metabolism gene CPT1C to promote survival. Clin Cancer Res 18(21):5850-5855

30. Reilly KM et al (2000) Nf1; Trp53 mutant mice develop glioblastoma with evidence of strainspecific effects. Nat Genet 26(1):109-113

31. Vogel KS et al (1999) Mouse tumor model for neurofibromatosis type 1. Science 286(5447):2176-2179

32. Sanchez-Macedo N et al (2013) Depletion of the novel p53-target gene carnitine palmitoyltransferase $1 \mathrm{C}$ delays tumor growth in the neurofibromatosis type I tumor model. Cell Death Differ 20(4):659-668

33. Mardis ER et al (2009) Recurring mutations found by sequencing an acute myeloid leukemia genome. N Engl J Med 361(11):1058-1066

34. Parsons DW et al (2008) An integrated genomic analysis of human glioblastoma multiforme. Science 321(5897): 1807-1812

35. Yan H et al (2009) IDH1 and IDH2 mutations in gliomas. N Engl J Med 360(8):765-773

36. Cairns RA, Mak TW (2013) Oncogenic isocitrate dehydrogenase mutations: mechanisms, models, and clinical opportunities. Cancer Discov 3(7):730-741

37. Wang P et al (2013) Mutations in isocitrate dehydrogenase 1 and 2 occur frequently in intrahepatic cholangiocarcinomas and share hypermethylation targets with glioblastomas. Oncogene 32(25):3091-3100

38. Borger DR et al (2012) Frequent mutation of isocitrate dehydrogenase (IDH) 1 and IDH2 in cholangiocarcinoma identified through broad-based tumor genotyping. Oncologist 17(1):72-79 
39. Amary MF et al (2011) IDH1 and IDH2 mutations are frequent events in central chondrosarcoma and central and periosteal chondromas but not in other mesenchymal tumours. J Pathol 224(3):334-343

40. Kang MR et al (2009) Mutational analysis of IDH1 codon 132 in glioblastomas and other common cancers. Int J Cancer 125(2):353-355

41. Ward PS et al (2010) The common feature of leukemia-associated IDH1 and IDH2 mutations is a neomorphic enzyme activity converting alpha-ketoglutarate to 2-hydroxyglutarate. Cancer Cell 17(3):225-234

42. Shih AH et al (2012) The role of mutations in epigenetic regulators in myeloid malignancies. Nat Rev Cancer 12(9):599-612

43. Rakheja D et al (2012) IDH mutations in acute myeloid leukemia. Hum Pathol 43(10):1541-1551

44. Patnaik MM et al (2012) Differential prognostic effect of IDH1 versus IDH2 mutations in myelodysplastic syndromes: a Mayo Clinic study of 277 patients. Leukemia 26(1):101-105

45. Cairns RA et al (2012) IDH2 mutations are frequent in angioimmunoblastic T-cell lymphoma. Blood 119(8):1901-1903

46. Kranendijk M et al (2010) IDH2 mutations in patients with D-2-hydroxyglutaric aciduria. Science 330(6002):336

47. Dang L et al (2009) Cancer-associated IDH1 mutations produce 2-hydroxyglutarate. Nature 462(7274):739-744

48. Sasaki M et al (2012) IDH1(R132H) mutation increases murine haematopoietic progenitors and alters epigenetics. Nature 488(7413):656-659

49. Noushmehr $\mathrm{H}$ et al (2010) Identification of a $\mathrm{CpG}$ island methylator phenotype that defines a distinct subgroup of glioma. Cancer Cell 17(5):510-522

50. Figueroa ME et al (2010) Leukemic IDH1 and IDH2 mutations result in a hypermethylation phenotype, disrupt TET2 function, and impair hematopoietic differentiation. Cancer Cell 18(6):553-567

51. Rose NR et al (2011) Inhibition of 2-oxoglutarate dependent oxygenases. Chem Soc Rev 40(8):4364-4397

52. Chowdhury R et al (2011) The oncometabolite 2-hydroxyglutarate inhibits histone lysine demethylases. EMBO Rep 12(5):463-469

53. Stein E et al (2014) Clinical safety and activity in a phase I trial of AG-221, a first in class, potent inhibitor of the IDH2-mutant protein, in patients with IDH2 mutant positive advanced hematologic malignancies. In: Proceedings of the 105th annual meeting of the American Association for Cancer Research, San Diego, 5-9 April

54. Gorrini C, Harris IS, Mak TW (2013) Modulation of oxidative stress as an anticancer strategy. Nat Rev Drug Discov 12(12):931-947

55. Gorrini $\mathrm{C}$ et al (2013) BRCA1 interacts with Nrf2 to regulate antioxidant signaling and cell survival. J Exp Med 210(8):1529-1544

56. Gorrini $\mathrm{C}$ et al (2014) Estrogen controls the survival of BRCA1-deficient cells via a PI3KNRF2-regulated pathway. Proc Natl Acad Sci U S A 111(12):4472-4477

57. Mason JM et al (2014) Functional characterization of CFI-400945, a polo-like kinase 4 inhibitor, as a potential anticancer agent. Cancer Cell 26(2):163-176 


\title{
Linear Polyubiquitination: A Crucial Regulator of NF-кB Activation
}

\author{
Kazuhiro Iwai
}

\begin{abstract}
NF- $\kappa \mathrm{B}$ is a transcription factor known to be involved in pleomorphic biological phenomena such as inflammation and immune responses. Abnormal activation of NF- $\mathrm{KB}$ has been reported in many pathological conditions, including malignant tumors. Therefore, the NF- $\mathrm{KB}$ activation pathway has been extensively studied and involvement of the ubiquitin conjugation system in the NF- $\kappa \mathrm{B}$ activation pathways has been revealed. Although the ubiquitin conjugation system was discovered as a part of a protein degradation pathway, non-degradable roles of the ubiquitin system have been revealed recently. Several types of polyubiquitin chains exist in cells and the type of chain seems to determine how ubiquitinated proteins are regulated. We have identified that a new type of polyubiquitin chain, the linear polyubiquitin chain, plays a crucial role in regulating the $\mathrm{NF}-\kappa \mathrm{B}$ activation pathway in non-degradable manner. In this chapter, the discovery, roles in NF- $\kappa \mathrm{B}$ activation, and involvement in the pathogenesis of cancers of linear ubiquitination will be discussed.
\end{abstract}

Keywords NF- $\kappa$ B $\bullet$ Ubiquitin $\bullet$ LUBAC $\bullet$ Linear ubiquitin chain $\bullet$ cpdm $\bullet$ B cell lymphoma

\section{The Ubiquitin Conjugation System}

The ubiquitin system, which is one of the most extensively studied post-translational protein modification systems, has been identified as part of an energy-dependent degradation system [1]. Ubiquitin is a protein-based modifier composed of 76 amino acids. Through the function of three enzymes called the ubiquitin-activating enzyme (E1), the ubiquitin conjugating enzyme (E2) and the ubiquitin ligase (E3), ubiquitin is conjugated to the substrate proteins that are recognized by E3s. Ubiquitin is added onto ubiquitin pre-conjugated to the substrates to generate polyubiquitin chains - polymer of ubiquitin. The proteasome recognizes the polyubiquitin chains

K. Iwai $(\bowtie)$

Department of Molecular and Cellular Physiology, Graduate School of Medicine,

Kyoto University, Yoshida-konoe-cho, Sakyo-ku, Kyoto 606-8501, Japan

e-mail: kiwai@mcp.med.kyoto-u.ac.jp

K. Nakao et al. (eds.), Innovative Medicine, DOI 10.1007/978-4-431-55651-0_4 
and destines ubiquitinated proteins for degradation (Fig. 1) [2]. The physiological significance of the ubiquitin proteolytic system mainly arises from its timely and selective recognition of the substrate proteins [2]. However, timely and selective protein modification is a desirable feature not only for degradation but also for other modes of protein regulation. As expected, these non-degradation functions of the ubiquitin system are now widely recognized [3]. Among the post-translational modification systems, the ubiquitin system has the unique feature that the system regulates the function of proteins by conjugating polyubiquitin chains in most cases. There are several types of polyubiquitin chains in cells, and the type of polyubiquitin chain is thought to determine the mode of regulation of the conjugated proteins [3]. Polyubiquitin chains have been believed to be exclusively generated via linkage between the carboxyl group of a ubiquitin monomer and $\varepsilon$-amino group of one of seven Lys residues within ubiquitin. For example, ubiquitin chains generated via Lys(K)48 of ubiquitin (K48 chains) function as a degradation signal and chains generated via K63 (K63 chains) are involved in DNA repair or signal transduction without a functioning degradation signal (Fig. 1) [3]. We have identified a new type of polyubiquitin chain, the linear polyubiquitin chain, in which the carboxyl group of a ubiquitin monomer forms a peptide bond with an N-terminal Met residue of another ubiquitin molecule (Fig. 1) [4]. We have also identified the ubiquitin ligase
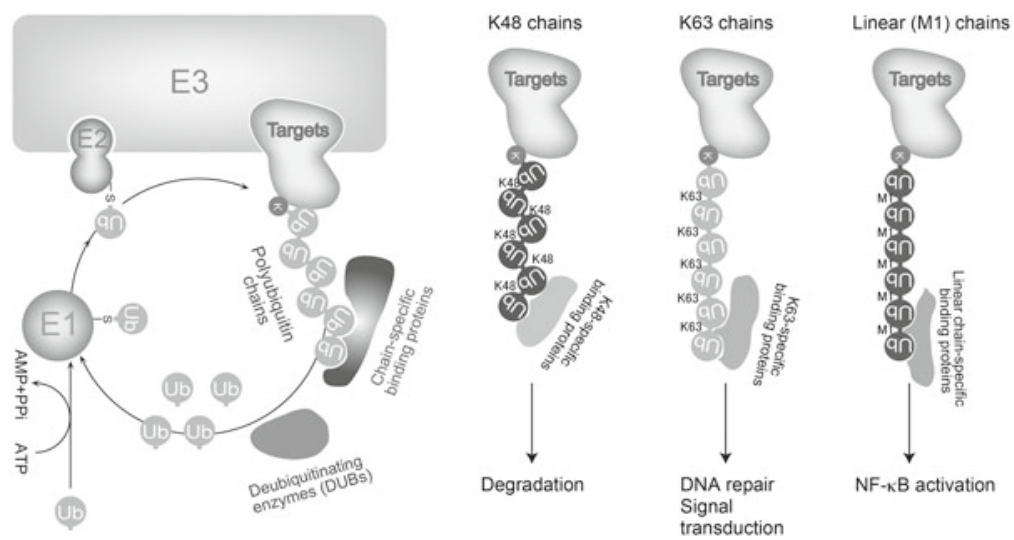

Fig. 1 The ubiquitin conjugation system. Through the function of three enzymes, called ubiquitin activating enzymes (E1), ubiquitin conjugating enzymes (E2), and ubiquitin ligases (E3), ubiquitin is conjugated to target proteins that are specifically recognized by E3s. Once an ubiquitin is conjugated to the target protein, additional ubiquitins are conjugated successively onto the terminal ubiquitin already conjugated to the target protein to form polyubiquitin chains. The polyubiquitin chains have been velieved to be generated by iso-peptide bond formation between the C-terminal carboxyl group of one ubiquitin and an $\varepsilon$-amino group of one of seven Lys residues in another ubiquitin. In the case of the ubiquitin proteolytic pathway, polyubiquitin chains are conjugated via K48. K63 conjugated chains are involved in signaling or DNA repair without the conjugated protein being subject to degradation. However, the linear ubiquitin chain, as reviewed here, is unique because it is generated by peptide bond formation between the C-terminus of one ubiquitin and the $\alpha$-amino group of Met1 residues in another ubiquitin. Conjugated ubiquitins are cleaved by deubiquitinating enzymes (DUBs). The function of conjugated proteins is modulated via specific recognition of conjugated polyubiquitin chains 
complex that specifically generates linear polyubiquitin chains and named it as the linear ubiquitin chain assembly complex (LUBAC) [4]. Further analysis has revealed that linear polyubiquitination is involved in NF- $\mathrm{KB}$ activation [5].

\section{The Canonical NF-кB Activation Pathways}

$\mathrm{NF}-\kappa \mathrm{B}$ is a transcription factor that is involved in a broad array of biological phenomena including cell survival, inflammation, and innate and acquired immune responses [6]. Since aberrant activation of NF- $\kappa \mathrm{B}$ is reported in numerous pathological conditions including autoimmune diseases and neoplasms, the NF- $\mathrm{KB}$ activation pathway has been extensively studied. NF- $\mathrm{BB}$ activation pathways are basically subdivided into two distinct pathways, known as the canonical and alternative pathways [6]. The canonical NF- $\kappa \mathrm{B}$ activation pathway is discussed here since LUBAC-mediated linear polyubiquitination is known to be involved in the pathway [7]. NF- $\mathrm{\kappa B}$ is inactive in resting cells as it resides in the cytoplasm bound

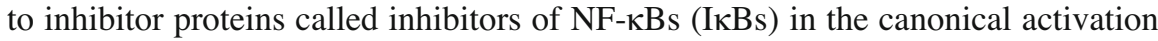
pathway (Fig. 2). The IкB kinase (IKK) complex, which comprises IKK1, IKK2, and NF- $\kappa \mathrm{B}$ essential modulator (NEMO), is activated upon stimulation with various agents such as Toll-like receptor (TLR) ligands or inflammatory cytokines including

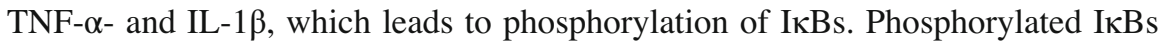
are recognized by the $\mathrm{SCF}^{\beta \mathrm{TrCP}}$ ubiquitin ligase and conjugated with $\mathrm{K} 48$ chains, which leads to phosphorylated IкBs to degradation by the proteasome. Then, liberalized NF- $\kappa \mathrm{B}$ translocates to the nucleus to induce the transcription of target genes (Fig. 2) [6]. Although the precise mechanism leading to the activation of the IKK complex has not been completely solved, K63 polyubiquitin chains have been shown to be involved in the IKK activation in the canonical pathway [8]. Since roles of K63 chains in NF- $\mathrm{KB}$ activation is not the main topic of in this article [8], the current hypothesis of the roles of K63 chains in the TNF- $\alpha$ - and IL- $1 \beta$-induced canonical NF- $\kappa \mathrm{B}$ activation pathway will only be summarized here. Upon binding TNF- $\alpha$ in addition to adaptor molecules, ubiquitin ligases including TRAF2 and cIAPs are recruited to the TNF receptor 1 (TNFR1) and K63 chains conjugated mainly onto RIP1. In the case of IL-1 $\beta$ signaling, TRAF6 E3 recruited to the IL-1 receptor (IL-1R) complex generates K63 chains on TRAF6 itself and on IRAK1. K63 chains, generated in the activated receptor complex, recruit the TAK1-TAB1TAB2/3 complex and the IKK complex via K63-selective binding of TAB2/3 or NEMO, respectively. TAK1 then phosphorylates specific Ser residues of IKK2, which leads to the phosphorylation and degradation of IкBs [8]. However, the involvement of K63-linked chains in NF- $\mathrm{B}$ activation has been challenged. In cells isolated from $\mathrm{KO}$ mice of Ubc13, a crucial component of an Ubc13-Uev1a E2 complex to generate K63 chains specifically, NF- $\kappa \mathrm{B}$ activation mediated by TNF- $\alpha$ is not overtly affected, although TNF- $\alpha$-mediated activation of MAPKs by these stimuli is severely impaired [9]. Moreover, K63 chains are dispensable for TNF- $\alpha-$, but not for IL-1 $\beta$-, induced canonical NF-KB activation [10]. Thus, although K63 


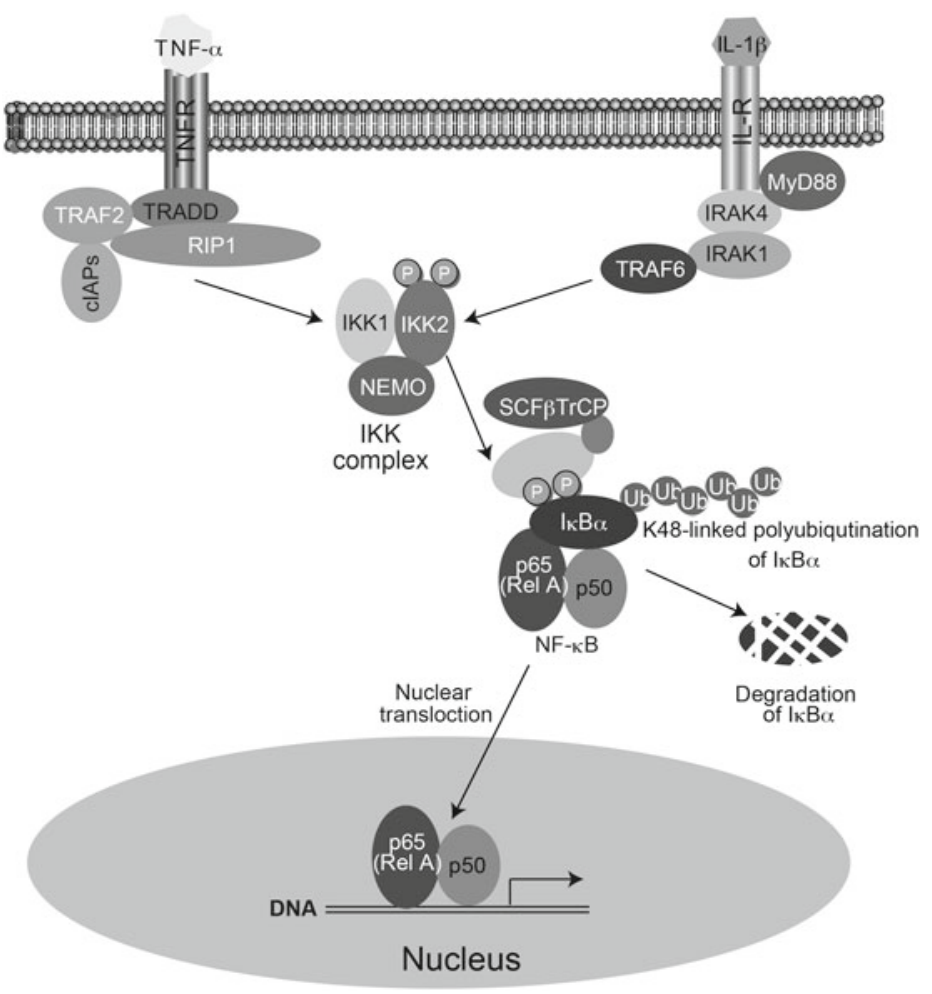

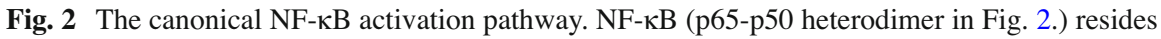
within the cytoplasm of resting cells in a form bound to the inhibitor protein, I $\mathrm{B} B \alpha$. Upon binding to TNF- $\alpha$, TRADD and RIP1 are recruited to the TNF receptor 1 (TNFR1). TRADD then recruits TRAF2 and cIAPs. In the case of IL- $1 \beta$ signaling, MyD88 is recruited to the IL-1 receptor (IL-1R), which recruits IRAK1 and IRAK4 to the receptor. IRAK1 binds to TRAF6. The ubiquitin ligase activities of TRAF6 and cIAPs are involved in the signaling cascade leading to canonical IKK activation, namely IKK2 phosphorylation. Phosphorylated I $\kappa \mathrm{B} \alpha$ is then conjugated with K48linked polyubiquitin chains by the $\mathrm{SCF}^{\beta T r C P}$ ubiquitin ligase and degraded by the proteasome. Liberated NF- $\mathrm{KB}$ translocates to the nucleus and induces the expression of target genes

chains play crucial roles in signaling, they might be dispensable for the canonical $\mathrm{NF}-\kappa \mathrm{B}$ activation, at least on some occasions including TNF- $\alpha$ stimulation.

\section{The Linear Ubiquitin Chains and Their Roles in NF-кB Activation}

One of the important features of the ubiquitin conjugation system is the diversity of the polyubiquitin chains [11]. Ubiquitin chains have been believed to be generated by conjugating $\mathrm{C}$-terminal carboxyl groups of ubiquitin to $\varepsilon$-amino groups of one of seven Lys residues of another ubiquitin. Although polyubiquitin chains are 
HOIL-1L

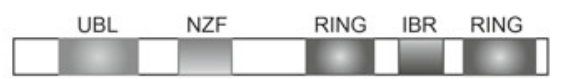

HOIP

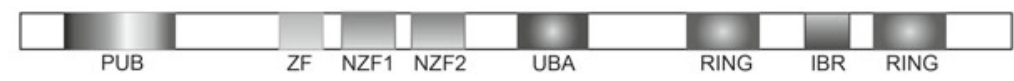

SHARPIN

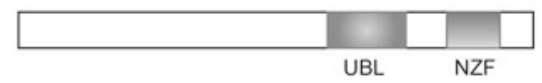

Fig. 3 Schematic representation of LUBAC, which is composed of HOIL-1L, HOIP, and SHARPIN. The zinc finger and RING-IBR-RING domains of HOIP are the substrate-binding site and $\mathrm{E} 3$ active site, respectively. IBR in-between RING, $N Z F$ Npl4-type zinc finger, $P U B$ peptide:Nglycanase/UBA- or UBX-containing proteins, $R I N G$ really interesting new gene, $U B A$ ubiquitinassociated domains, $U B L$ ubiquitin-like, $Z F$ zinc finger

generated by the repetitive functions of three enzymes, E1, E2, and E3, molecular mechanisms underlying polyubiquitin chain generation has not been completely solved [11]. During the dissection of the mechanism underscoring polyubiquitin generation, we have identified a new type of ubiquitin chain called the linear ubiquitin chain, in which the $\mathrm{C}$-terminal carboxyl group of ubiquitin is conjugated to the $\alpha$-amino group of the N-terminus of another ubiquitin [11]. Here, how the linear chains were discovered is discussed. We have identified the E3 complex composed of two RING-IBR-RING proteins, HOIL-1L and HOIP, and realized that HOIL-1L and HOIP possess multiple ubiquitin binding sites in addition to E2 binding sites (Fig. 3) [4, 12]. We thus hypothesized that the ubiquitin ligase complex recognizes ubiquitin as a substrate and conjugates ubiquitin onto it to generate polyubiquitin chains. Further analyses revealed that the HOIL-1L-HOIP complex indeed generates linear polyubiquitin chains exclusively [4].

During the course of seeking the physiological function, we found that siRNAmediated suppression of HOIP attenuated TNF- $\alpha$-mediated NF- $\kappa$ B activation [5]. In primary hepatocytes isolated from mice lacking HOIL- $1 \mathrm{~L}, \mathrm{TNF}-\alpha$-induced nuclear localization of $\mathrm{p} 65$, a subunit of $\mathrm{NF}-\kappa \mathrm{B}$, is heavily impaired. Moreover, TNF- $\alpha$-induced IKK activation and expression of target genes of NF- $\kappa \mathrm{B}$ are severely attenuated in embryonic fibroblasts (MEFs) from the HOIL-1L KO mice [5].

However, although deletion of molecules essential for NF- $\kappa \mathrm{B}$ activation, such as NEMO or IKK2, is embryonic lethal in mice, HOIL-1L KO is viable. HOIP, which is a catalytic subunit of the complex, was heavily decreased but not completely absent in HOIL-1L KO cells, which suggested that HOIP may have another binding partner besides HOIL-1L, and we identified SHARPIN as a binding partner of HOIP. The C-terminus half of SHARPIN exhibits significant homology with the N-terminal half of HOIL-1L, which is essential for binding to HOIP (Fig. 3) [13]. Further analyses revealed that SHARPIN forms the tertiary complex with HOIL-1L and HOIP [13]. The complex composed of HOIL-1L, HOIP, and SHARPIN conjugates to linear polyubiquitin chains, and we designated the complex composed of HOIL-1L, HOIP, and SHARPIN as LUBAC (Fig. 3) [13]. 
Pathophysiological roles of linear polyubiquitin chains were revealed by the analyses of spontaneous mutant mice called chronic proliferative dermatitis in mice (cpdm) [13-15]. Loss of SHARPIN is causative in cpdm mice that exhibit chronic inflammation including chronic dermatitis and immunodeficiency. In cells isolated from cpdm mice, the amount of the other components of LUBAC, HOIL-1L and HOIP, was reduced drastically by the lack of SHARPIN, thereby attenuating canonical NF- $\kappa \mathrm{B}$ activation induced by several stimuli including TNF- $\alpha$ and CD40 [13-15]. Recently, loss-of-function mutation of HOIL-1L provoked a fatal human inherited disorder characterized by chronic autoinflammation, invasive bacterial infections, and muscular amylopectinosis [16]. Loss of HOIL-1L or SHARPIN destabilizes the other two components of LUBAC and thereby suppresses signalinduced NF- $\mathrm{KB}$ activation [5, 13-15]. Thus, loss of regulatory subunits of LUBAC suppresses signal-induced canonical NF- $\kappa \mathrm{B}$ activation and provokes diseases characterized by autoinflammatory and immunodeficiency phenotypes, at least in part [7].

\section{Mechanism Underlying Linear Ubiquitin Chain-Mediated NF-кB Activation}

The ubiquitin conjugation system regulates a wide variety of physiological phenomena by conjugating polyubiquitin chains in a timely and selective manner [3]. The E3 enzymes must recognize target proteins prior to their ubiquitination [3]. In the case of LUBAC-mediated canonical NF- $\kappa \mathrm{B}$ activation, NEMO, an integral subunit of the IKK complex, is the substrate of LUBAC, and linear polyubiquitination of NEMO is shown to be involved in the activation of IKK that leads to canonical NF-кB activation [6]. Recent analyses revealed the molecular mechanism of linear polyubiquitination of NEMO-mediated IKK activation [17]. Upon stimulation with agents such as TNF- $\alpha$, LUBAC recognizes NEMO using the Npl4-type zinc finger 1 (NZF1) domain of HOIP (Fig. 4) and conjugates linear ubiquitin chains onto the proteins. Conjugation of di-ubiquitin (dimer) of linear linkage to NEMO appears enough to activate the IKK complex [17]. Activation of the IKK complex is mediated by the phosphorylation of IKK2 [6]. In general, phosphorylation of kinases is mediated either by trans-autophosphorylation or by upstream kinases [18]. The crystal structural analyses of human IKK2 revealed that homotypic interaction of the IKK2 kinase domain is crucial for IKK2 activation [19]. Moreover, NEMO possesses the ubiquitin binding activity that prefers linear di-ubiquitin, and NEMO's ubiquitin binding activity is critical for IKK activation [20]. We thus showed that linear di-ubiquitin conjugated to NEMO is recognized by another NEMO in trans, which triggers dimerization of the IKK complex and subsequent trans-autophosphorylation of IKK2 (Fig. 4) [17]. 
Fig. 4 The proposed role of linear chains in canonical $\mathrm{NF}-\kappa \mathrm{B}$ activation. NEMO, an integral component of the IKK complex, is recognized by the NZF1 of HOIP upon stimulation and is linearly ubiquitinated by

LUBAC. Linear di-ubiquitin is sufficient to activate IKK. Linear di-ubiquitin conjugated to NEMO is recognized by NEMO in another IKK complex in trans, triggering dimerization and trans-autophosphorylation of IKK2. Activated IKK2 phosphorylates $\mathrm{I} \kappa \mathrm{B} \alpha$, activating the canonical $\mathrm{NF}-\kappa \mathrm{B}$ pathway

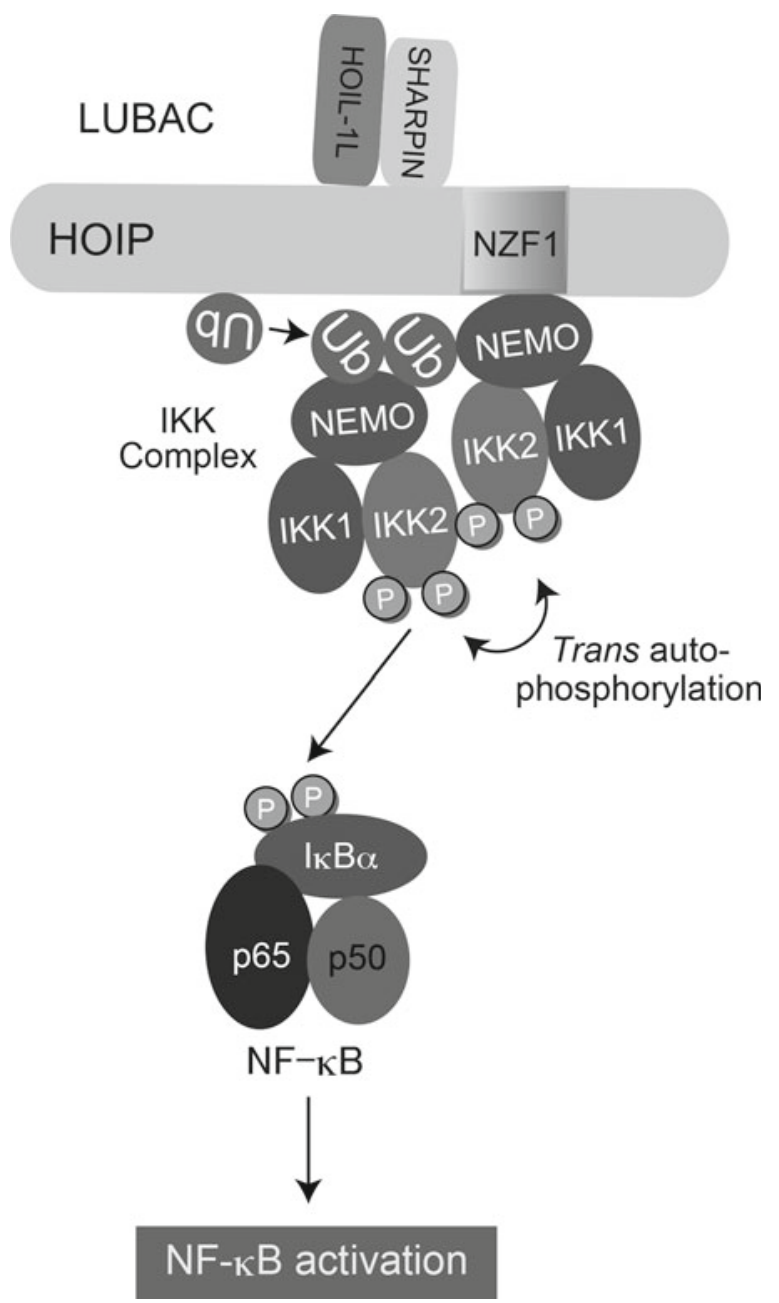

\section{Perspectives}

The involvement of LUBAC-mediated linear polyubiquitination in disease has been shown recently. Aberrant NF- $\kappa \mathrm{B}$ activation plays an essential role in the pathogenesis of activated B cell-like diffuse large B cell lymphomas (ABC DLBCL) [21]. Recent analyses showed that LUBAC is essential for the growth of ABC DLBCL cells through suppression of NF-KB activity. Moreover, two rare SNPs in the HOIP gene are enriched in ABC DLBCL [21]. We have also observed that attenuation of LUBAC suppressed lung metastases of osteosarcomas in mice [22]. Moreover, HOIP attenuates apoptosis induced by platinum-based genotoxins, including cisplatin and carboplatin, that are widely used as anti-cancer drugs [23]. Thus, suppression of LUBAC activity might be a suitable target to control malignant tumors. We have 
identified a new type of ubiquitin modification, linear ubiquitination, during our analyses to address the basic question of how the ubiquitin system generates polyubiquitin chains. We hope our unexpected finding will lead to the development of new therapeutic agents to control cancer.

Open Access This chapter is distributed under the terms of the Creative Commons Attribution Noncommercial License, which permits any noncommercial use, distribution, and reproduction in any medium, provided the original author(s) and source are credited.

\section{References}

1. Ciechanover A, Hod Y, Hershko A (1978) A heat-stable polypeptide component of an ATP-dependent proteolytic system from reticulocytes. Biochem Biophys Res Commun 81(4):1100-1105. doi:0006-291X(78)91249-4 [pii]

2. Hershko A, Ciechanover A (1998) The ubiquitin system. Annu Rev Biochem 67:425-479. doi:10.1146/annurev.biochem.67.1.425

3. Deshaies RJ, Joazeiro CA (2009) RING domain E3 ubiquitin ligases. Annu Rev Biochem 78:399-434. doi:10.1146/annurev.biochem.78.101807.093809

4. Kirisako T, Kamei K, Murata S, Kato M, Fukumoto H, Kanie M, Sano S, Tokunaga F, Tanaka K, Iwai K (2006) A ubiquitin ligase complex assembles linear polyubiquitin chains. EMBO J 25(20):4877-4887. 7601360 [pii] doi:10.1038/sj.emboj.7601360

5. Tokunaga F, Sakata S, Saeki Y, Satomi Y, Kirisako T, Kamei K, Nakagawa T, Kato M, Murata S, Yamaoka S, Yamamoto M, Akira S, Takao T, Tanaka K, Iwai K (2009) Involvement of linear polyubiquitylation of NEMO in NF-אB activation. Nat Cell Biol 11(2):123-132. doi:ncb1821 [pii] 10.1038/ncb1821

6. Hayden MS, Ghosh S (2008) Shared principles in NF-kB signaling. Cell 132(3):344-362. doi:S0092-8674(08)00120-7 [pii] 10.1016/j.cell.2008.01.020

7. Iwai K (2012) Diverse ubiquitin signaling in NF- $\kappa B$ activation. Trends Cell Biol 22(7):355-364. doi:10.1016/j.tcb.2012.04.001

8. Chiu YH, Zhao M, Chen ZJ (2009) Ubiquitin in NF-кB signaling. Chem Rev 109(4):1549-1560. doi:10.1021/cr800554j

9. Yamamoto M, Okamoto T, Takeda K, Sato S, Sanjo H, Uematsu S, Saitoh T, Yamamoto N, Sakurai H, Ishii KJ, Yamaoka S, Kawai T, Matsuura Y, Takeuchi O, Akira S (2006) Key function for the Ubc13 E2 ubiquitin-conjugating enzyme in immune receptor signaling. Nat Immunol 7(9):962-970. doi:. doi:ni1367 [pii] 10.1038/ni1367

10. Xu M, Skaug B, Zeng W, Chen ZJ (2009) A ubiquitin replacement strategy in human cells reveals distinct mechanisms of IKK activation by TNF $\alpha$ and IL-1 $\beta$. Mol Cell 36(2):302-314

11. Hochstrasser M (2006) Lingering mysteries of ubiquitin-chain assembly. Cell 124(1):27-34. doi:S0092-8674(05)01466-2 [pii] 10.1016/j.cell.2005.12.025

12. Yamanaka K, Ishikawa H, Megumi Y, Tokunaga F, Kanie M, Rouault TA, Morishima I, Minato $\mathrm{N}$, Ishimori K, Iwai K (2003) Identification of the ubiquitin-protein ligase that recognizes oxidized IRP2. Nat Cell Biol 5(4):336-340. doi:10.1038/ncb952 ncb952 [pii] 10.1038/ncb952

13. Tokunaga F, Nakagawa T, Nakahara M, Saeki Y, Taniguchi M, Sakata S, Tanaka K, Nakano H, Iwai K (2011) SHARPIN is a component of the NF-kB-activating linear ubiquitin chain assembly complex. Nature 471(7340):633-636. doi:nature09815 [pii] 10.1038/nature09815

14. Gerlach B, Cordier SM, Schmukle AC, Emmerich CH, Rieser E, Haas TL, Webb AI, Rickard JA, Anderton H, Wong WW, Nachbur U, Gangoda L, Warnken U, Purcell AW, Silke J, Walczak H (2011) Linear ubiquitination prevents inflammation and regulates immune signalling. Nature 471(7340):591-596. doi:nature09816 [pii] 10.1038/nature09816 
15. Ikeda F, Deribe YL, Skanland SS, Stieglitz B, Grabbe C, Franz-Wachtel M, van Wijk SJ, Goswami P, Nagy V, Terzic J, Tokunaga F, Androulidaki A, Nakagawa T, Pasparakis M, Iwai K, Sundberg JP, Schaefer L, Rittinger K, Macek B, Dikic I (2011) SHARPIN forms a linear ubiquitin ligase complex regulating NF-кB activity and apoptosis. Nature 471(7340):637-641. doi:nature09814 [pii] 10.1038/nature09814

16. Boisson B, Laplantine E, Prando C, Giliani S, Israelsson E, Xu Z, Abhyankar A, Israel L, Trevejo-Nunez G, Bogunovic D, Cepika AM, Macduff D, Chrabieh M, Hubeau M, Bajolle F, Debre M, Mazzolari E, Vairo D, Agou F, Virgin HW, Bossuyt X, Rambaud C, Facchetti F, Bonnet D, Quartier P, Fournet JC, Pascual V, Chaussabel D, Notarangelo LD, Puel A, Israel A, Casanova JL, Picard C (2012) Immunodeficiency, autoinflammation and amylopectinosis in humans with inherited HOIL-1 and LUBAC deficiency. Nat Immunol 13(12):1178-1186. doi:10.1038/ni.2457

17. Fujita H, Rahighi S, Akita M, Kato R, Sasaki Y, Wakatsuki S, Iwai K (2014) Mechanism underlying IкB kinase activation mediated by the linear ubiquitin chain assembly complex. Mol Cell Biol 34(7):1322-1335. doi:10.1128/MCB.01538-13

18. Hunter T, Lindberg RA, Middlemas DS, Tracy S, van der Geer P (1992) Receptor protein tyrosine kinases and phosphatases. Cold Spring Harb Symp Quant Biol 57:25-41

19. Polley S, Huang DB, Hauenstein AV, Fusco AJ, Zhong X, Vu D, Schrofelbauer B, Kim Y, Hoffmann A, Verma IM, Ghosh G, Huxford T (2013) A structural basis for IkB kinase 2 activation via oligomerization-dependent trans auto-phosphorylation. PLoS Biol 11(6):e1001581. doi:10.1371/journal.pbio.1001581

20. Rahighi S, Ikeda F, Kawasaki M, Akutsu M, Suzuki N, Kato R, Kensche T, Uejima T, Bloor S, Komander D, Randow F, Wakatsuki S, Dikic I (2009) Specific recognition of linear ubiquitin chains by NEMO is important for NF-KB activation. Cell 136(6):1098-1109. doi:S00928674(09)00264-5 [pii] 10.1016/j.cell.2009.03.007

21. Yang Y, Schmitz R, Mitala J, Whiting A, Xiao W, Ceribelli M, Wright GW, Zhao H, Xu W, Rosenwald A, Ott G, Gascoyne RD, Connors JM, Rimsza LM, Campo E, Jaffe ES, Delabie J, Smeland EB, Braziel RM, Tubbs RR, Cook JR, Weisenburger DD, Chan WC, Wiestner A, Kruhlak MJ, Iwai K, Bernal F, Staudt LM (2014) Essential role of the linear ubiquitin chain assembly complex in lymphoma revealed by rare germline polymorphisms. Cancer Discov 4(4):480-493. doi:10.1158/2159-8290.CD-13-0915

22. Tomonaga M, Hashimoto N, Tokunaga F, Onishi M, Myoui A, Yoshikawa H, Iwai K (2012) Activation of nuclear factor-kappa B by linear ubiquitin chain assembly complex contributes to lung metastasis of osteosarcoma cells. Int J Oncol 40(2):409-417. doi:10.3892/ijo.2011.1209

23. Mackay C, Carroll E, Ibrahim AF, Garg A, Inman GJ, Hay RT, Alpi AF (2014) E3 ubiquitin ligase HOIP attenuates apoptotic cell death induced by cisplatin. Cancer Res 74(8):2246-2257. doi:10.1158/0008-5472.CAN-13-2131 


\title{
VCP, a Major ATPase in the Cells, as a Novel Drug Target for Currently Incurable Disorders
}

\author{
Akira Kakizuka
}

Abstract Neuroprotection would be a novel therapeutic strategy for the prevention or retardation of clinical manifestations of currently incurable eye diseases as well as neurodegenerative diseases. A decrease in cellular ATP levels may contribute to the pathologies of these diseases; therefore, stabilization of ATP levels may retard the disease progression. We created novel small compounds (Kyoto University Substances, KUSs) to inhibit the ATPase activity of VCP (valosin-containing protein), the most abundant soluble ATPase in the cell. KUSs did not apparently impair the reported cellular VCP functions. Nevertheless, they significantly suppressed the VCP-dependent decrease of cellular ATP levels. Moreover, KUSs as well as exogenous ATP or ATP-producing compounds suppressed endoplasmic reticulum (ER) stress, and indeed protected various types of cultured cells from cell deathinducing insults. We then examined the efficacies of KUSs in rd10, a mouse model of retinitis pigmentosa. KUSs not only prevented photoreceptor cell death but also preserved visual function. These results reveal an unexpected, crucial role of ATP consumption by VCP for the determination of cell fate in the pathological context, and point to a promising new neuroprotective strategy for currently incurable eye and neurodegenerative diseases.

Keywords VCP $\bullet$ ATP regulation $\bullet$ Incurable diseases $\bullet$ Retinitis pigmentosa $\bullet$ ER stress $\bullet$ Cell death $\bullet$ Drug discovery

\section{Background}

Despite recent advances in medical care, there remain many incurable disorders, e.g. neurodegenerative diseases, incurable eye diseases, etc. In these disorders, the major pathology is early cell death in the affected organs, which precedes the death of the individual, often affecting the quality of life over extended periods.

\footnotetext{
A. Kakizuka, M.D., Ph.D. ( $\square)$

Laboratory of Functional Biology, Kyoto University Graduate School of Biostudies, Kyoto 606-8501, Japan

e-mail: kakizuka@lif.kyoto-u.ac.jp 
To date, no drug has been successful at inhibiting or delaying such early cell death in vivo. In the 1990s, with much optimism, caspase inhibitors were developed and were expected to be such miracle drugs, but they were not able to prevent cell death in vivo. Indeed, later research revealed that caspases determine how cells die but are not able to affect the commitment to cell death.

Retinitis pigmentosa, in which a gradual degeneration and loss of photoreceptors occur along with aging, causes severe visual deterioration, and more than 1.5 million patients worldwide are estimated to be suffering from this eye disorder. There is no effective treatment that can prevent or delay the symptoms of retinitis pigmentosa. At least 60 genes have been implicated in retinitis pigmentosa, and therefore the cellular etiology - that is, the constellation of cellular mishaps that culminate in cell death - is the subject of ongoing debate. Involvement of endoplasmic reticulum (ER) stress has been proposed as a pathological mechanism in retinitis pigmentosa $[1,2]$.

\section{VCP in Neurodegenerative Disorders}

Using polyglutamine disease models, we have long been searching for common molecular bases in neurodegenerative diseases, and have found valosin-containing protein (VCP), an AAA (ATPases Associated with diverse cellular Activities)-type ATPase with ubiquitous expression, as a major player causing neurodegeneration. VCP is a phylogenetically well-conserved protein, and the respective amino acid sequences are identical among mouse, rat, and human, and $84 \%$ identical between human and Drosophila [3]. To search for modifier genes that are involved in the pathogenesis of polyglutamine diseases, we first created Drosophila models of polyglutamine diseases, and then performed genetic analyses. In 2002, our mutant screening revealed that Ter94 loss-of-function alleles mitigated eye degeneration that was induced by the expression of genes with expanded polyglutamine tracts [3]. Consistent with these results, overexpression of wild-type Ter94 exacerbated the polyglutamine-induced eye degeneration [3]. It is notable that the mammalian Ter94 ortholog is $V C P$. Thus, these results implicated VCP in the pathogenesis of human neurodegenerative diseases [4]. In 2004 and 2010, VCP mutations were identified that are causative for IBMPFD (inclusion body myopathy associated with Paget disease of bone and frontotemporal dementia) [5], a human hereditary disease with dementia, and for rare cases of familial amyotrophic lateral sclerosis (ALS) [6], respectively. In our evaluation, all tested pathogenic VCPs showed significant elevations of the ATPase activities, as compared with wild-type VCP [7], and thus we proposed the possibility that the constitutive elevation of its ATPase activity is a pathogenic mechanism.

These lines of evidence suggested that specific inhibitors of the ATPase activity of VCP could protect neuronal cells. In addition to its ATPase activity, however, $\mathrm{VCP}$ has been proposed to act in many important cellular processes [8-10], e.g., proteasome-mediated protein degradation, endoplasmic reticulum-associated degradation (ERAD), cell cycle control, membrane fusion, maintenance of the 
Golgi apparatus, protein trafficking, autophagy, genomic DNA surveillance, etc. Indeed, VCP knockdown and overexpression of dominant-negative forms of VCP induced cell death in essentially all cultured cells $[11,12]$. DBeQ, a recently reported VCP inhibitor (with an in vitro IC50 of $1 \mu \mathrm{M}$ ) [13], also induced cell death. Among the diverse cellular functions of VCP, some would require ATP hydrolysis and others would not. Thus, we believed it would be possible to find or develop small compounds that can inhibit or reduce the ATPase activity of VCP without inducing cellular toxicity.

\section{Development of Novel Inhibitors (KUSs) of VCP ATPase Activity That Do Not Block VCP Cellular Functions}

In our screening for novel VCP ATPase inhibitors, we found that a naphthalene derivative can inhibit the ATPase activity of VCP without showing apparent toxicity in cultured cells. Via the modification of its chemical structure, we created about 200 new compounds and named them Kyoto University Substances (KUSs). Some of them clearly inhibited the ATPase activity of recombinant VCP in vitro with $\mathrm{IC}_{50}$ values ranging from approximately $100 \mathrm{nM}$ to $1 \mu \mathrm{M}$ (Fig. 1). Importantly, the KUSs did not appear to inhibit the ATPase activity of N-ethylmaleimide-sensitive fusion protein (NSF), the protein most closely related to VCP.

As reported, DBeQ induced accumulation of ubiquitinated proteins, ER stress, autophagy, and eventually cell death. In contrast, KUSs (e.g. KUS31, 69, 94, 121, and 187) did not induce any of these phenotypes. These results clearly indicated that ATPase inhibition by KUS31, 69, 94, 121, and 187 (referred to as "KUSs" hereafter) did not interfere with reported cellular VCP functions (referred to as "VCP functions" hereafter). These results indicate that VCP functions do not necessarily require its ATPase activity.
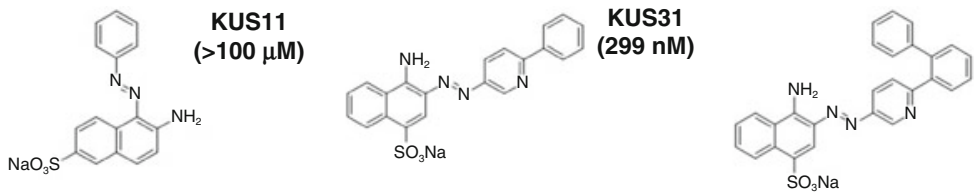

KUS69

$(116 \mathrm{nM})$
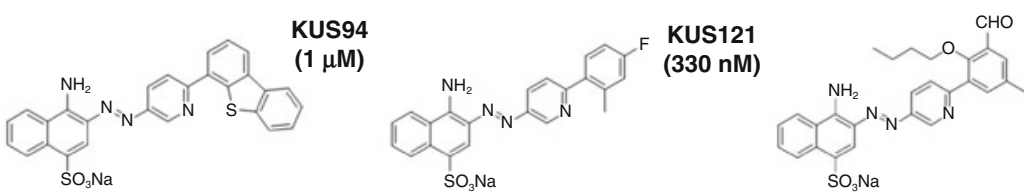

KUS187

(186 nM)

Fig. 1 Structures and $\mathrm{IC}_{50}$ values of Kyoto University Substances (KUSs), novel valosincontaining protein (VCP) modulators (Modified from Ikeda et al. [22]). Structures and $\mathrm{IC}_{50}$ values of KUS11, KUS31, KUS69, KUS94, KUS121, and KUS187 are shown. Note that KUS11 did not inhibit the ATPase activity of recombinant VCP, and it did not share a common structure with the other KUSs 


\section{KUSs Protected Cells Under ER Stress-Inducing Conditions}

Over and above their lack of toxicity, KUSs were found to protect cells from several cell death-inducing insults. For example, HeLa cells died within several days when cultured under low-glucose conditions ( $0.2 \mathrm{~g} / \mathrm{l}$ of glucose) (Fig. 2a). However, the addition of KUSs prevented cell death under these conditions (Fig. 2a, b). Similar protective effects were also observed when HeLa cells were cultured with tunicamycin (Tm) (Fig. 2c), or when HEK293 cells were cultured under serum-free conditions (Fig. 2d). These data indicated that inhibition of VCP ATPase activity by KUSs was beneficial for cells against cell death-inducing insults.

Tunicamycin treatment and glucose starvation cause ER stress and eventually lead to cell death. C/EBP-homologous protein (CHOP) is upregulated during ER stress, and is proposed to mediate the ER stress-induced cell death [14]. Indeed, the induction of CHOP in tunicamycin-treated HeLa cells was suppressed by the addition of KUSs. The induction of $78 \mathrm{kDa}$ glucose-regulated protein (Grp78), another ER stress marker [15], was also suppressed by KUSs in the tunicamycin-treated cells.
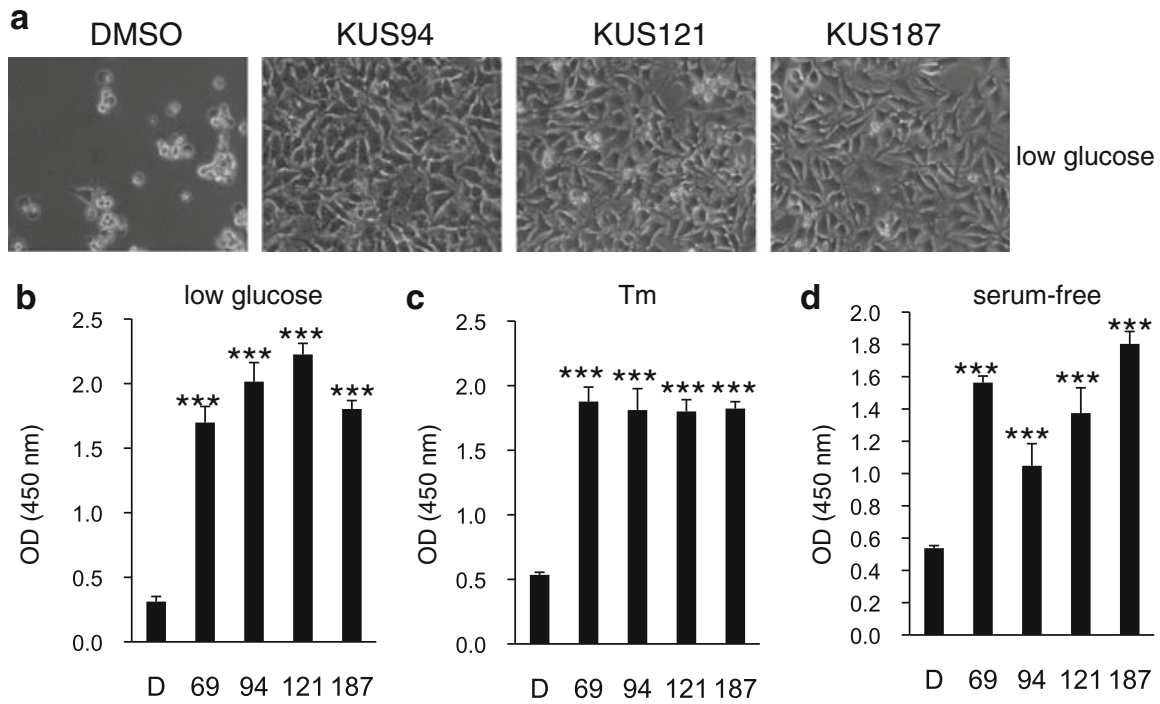

Fig. 2 Prevention of cell death and endoplasmic reticulum (ER) stress by Kyoto University Substances (KUSs) (Modified from Ikeda et al. [22]). (a) Photographs of HeLa cells, cultured with DMSO (DMSO) or KUSs (KUS94, KUS121, and KUS187, $20 \mu \mathrm{M}$ each) for $41 \mathrm{~h}$ in low-glucose $(0.2 \mathrm{~g} / \mathrm{l})$ medium. Scale bar, $100 \mu \mathrm{m}$. (b, c) WST (water-soluble tetrazolium salt) values reflecting relative live cell numbers are shown as optical density (OD) at $450 \mathrm{~nm}$. Error bars indicate standard deviations. (b) WST values of HeLa cells, cultured in low glucose (0.2 g/l) with DMSO (D) or KUSs (50 $\mu \mathrm{M}$ for KUS121; $20 \mu \mathrm{M}$ for KUS69, KUS94, and KUS187; $n=3$ ) for $41 \mathrm{~h}$. (c) Cell viability, indicated by WST values of HeLa cells, cultured with tunicamycin $(\mathrm{Tm})(0.2 \mu \mathrm{g} / \mathrm{ml})$ for $41 \mathrm{~h}$ with DMSO (D) or KUSs $(20 \mu \mathrm{M}$ each; $n=3)$. (d) WST values of HEK293 cells, cultured under serum-free conditions for $65 \mathrm{~h}$ with DMSO (D) or KUSs $(20 \mu \mathrm{M}$ each; $n=3)$. *** $P<0.001$ vs. DMSO control (Dunnett's test) 
These data indicate that KUSs have the ability to suppress ER stress and to promote cell survival in certain conditions.

We next examined the effect of KUSs on cellular ATP levels. After a $20 \mathrm{~h}$ incubation in low-glucose medium $(0.25 \mathrm{~g} / \mathrm{l})$, glucose levels in the medium approached zero, and ATP levels in the cells (control cells) also significantly decreased. In contrast, ATP levels in cells cultured in low-glucose medium and KUSs remained significantly higher than those in the control cells.

\section{KUSs or Exogenous ATP Prevented ER Stress in Cultured Cells}

ER stress has been believed to be induced by the accumulation of misfolded proteins, or protein aggregates, in the ER [16-18]. We recently identified laminin $\gamma 1$ as an aggregation-prone protein in the ER. We therefore examined the change of laminin $\gamma 1$ states in ER stress by tunicamycin treatments. Expression of laminin $\gamma 1$ was diffusely observed throughout the ER in normal cells (Control in Fig. 3a). In tunicamycin-treated cells, laminin $\gamma 1$ formed clear aggregates (DMSO in Fig. 3a). However, $50 \mu \mathrm{M}$ KUSs (KUS69, 94, 121, and 187) or $1 \mathrm{mM}$ ATP treatments prevented its aggregation (Fig. 3a). The KUSs or ATP treatment similarly prevented decreases of ATP levels in tunicamycin-treated cells (Fig. 3b). By contrast, KUS11, which could not inhibit VCP ATPase, was unable to prevent the tunicamycinelicited drop in cellular ATP concentration (Figs. 1 and 3b). The addition of $0.1 \mathrm{mM}$ ATP or 3 to $10 \mathrm{mM}$ methylpyruvate (weakly membrane-permeable pyruvate, which is converted to ATP in mitochondria) was also ineffective in preventing the aggregation of laminin $\gamma 1$ in tunicamycin-treated cells (Fig. 3a), but could nevertheless suppress the induction of ER stress, namely CHOP induction (Fig. 3c). These results indicated that the ER is more sensitive to decreases in ATP levels than to the presence of aggregates.

\section{KUSs Mitigated Pathologies of rd10, a Mouse Model of Retinitis Pigmentosa}

We have long been seeking a new strategy to protect retinal neuronal cells. In retinitis pigmentosa, an involvement of ER stress has been proposed [1, 2]. We wondered whether the protective effects of KUSs in cell culture would translate in vivo to the prevention of degeneration of photoreceptor cells in $\mathrm{rd} 10$ mice, a representative mouse model of retinitis pigmentosa [19]. Rd10 mice carry a mutation in a gene encoding the rod cyclic guanosine monophosphate (cGMP) phosphodiesterase beta subunit (PDE6B) [19]. Similar mutations are found in patients with retinitis pigmentosa. 
a
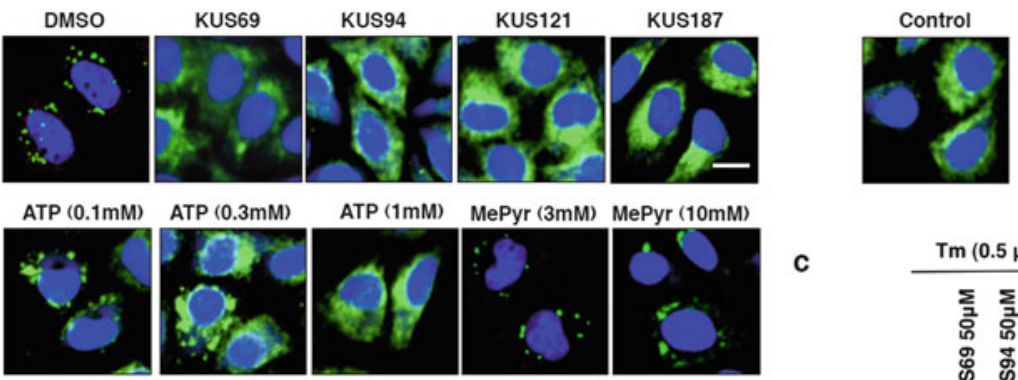

ATP (1mM)

MePyr (3mM) MePyr (10mM)
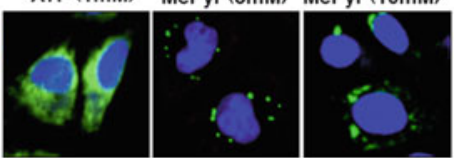

c

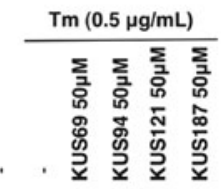

b
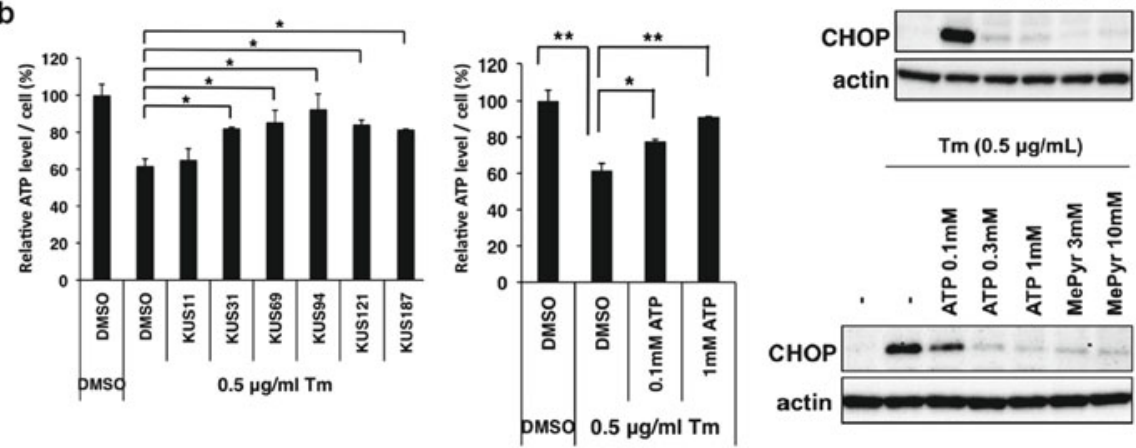

$\operatorname{Tm}(0.5 \mu \mathrm{g} / \mathrm{mL})$

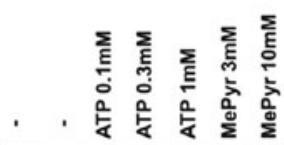

CHOP

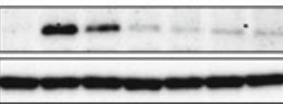

Fig. 3 Kyoto University Substances (KUSs) and ATP each prevented a decrease of ATP levels and ameliorated endoplasmic reticulum (ER) stress in tunicamycin (Tm)-treated cells (Modified from Ikeda et al. [22]). (a) Immunocytochemical analyses of HeLa cells by an anti-laminin $\gamma 1$ antibody. HeLa cells were treated with $0.5 \mu \mathrm{g} / \mathrm{ml}$ of Tm for $5 \mathrm{~h}$ in the presence of KUSs $(50 \mu \mathrm{M})$, ATP $(0.1$, 0.3 , and $1 \mathrm{mM}$ ), methylpyruvate (MePyr) $(3$ and $10 \mathrm{mM})$, or vehicle alone (DMSO). Then, cells were fixed and subjected to immunocytochemical analyses. Normally growing HeLa cells were also analyzed (Control). Scale bar, $10 \mu \mathrm{m}$. (b) Measurements of the relative amounts of ATP per cell. HeLa cells were treated with Tm $(0.5 \mu \mathrm{g} / \mathrm{ml})$ for $24 \mathrm{~h}$ in the presence of KUSs $(50 \mu \mathrm{M})$ or ATP $(0.1$ and $1 \mathrm{mM})$, or vehicle alone (DMSO), and were harvested. Then, ATP amounts from $1.5 \times 10^{5}$ cells were measured. $* P<0.05, * * P<0.01$. Error bars indicate standard deviations. (c) Western blot analyses of C/EBP-homologous protein (CHOP). HeLa cells were treated with $0.5 \mu \mathrm{g} / \mathrm{ml}$ of Tm for $5 \mathrm{~h}$ in the presence of KUSs $(50 \mu \mathrm{M})$, ATP $(0.1,0.3$, and $1 \mathrm{mM}), \operatorname{MePyr}(3$ and $10 \mathrm{mM})$, or vehicle alone (-). Then, cells were harvested and subjected to western blot analyses. Actin served as a loading control

Starting 7 days after birth, KUS121 or KUS187 was administered daily (50 mg/kg) by intraperitoneal injection. Spectral-domain optical coherence tomography (SD-OCT) examination showed that at age 25 days, the thinning of the outer nuclear layer (ONL) was clearly observed in the control rd10 mice (Fig. 4a). The ONL and the junction line between the inner segment and outer segment (arrow heads in Fig. 4a), which is generally considered to be positively associated with visual function $[20,21]$, were clearly detected in the KUS-treated rd10 mice but not in control rd10 mice. A very small electroretinogram response was observed in control rd10 mice, but an almost normal electroretinogram response was observed in most of the KUS-treated rd10 mice (Fig. 4b). At age 29 days, the photoreceptor layer was barely 


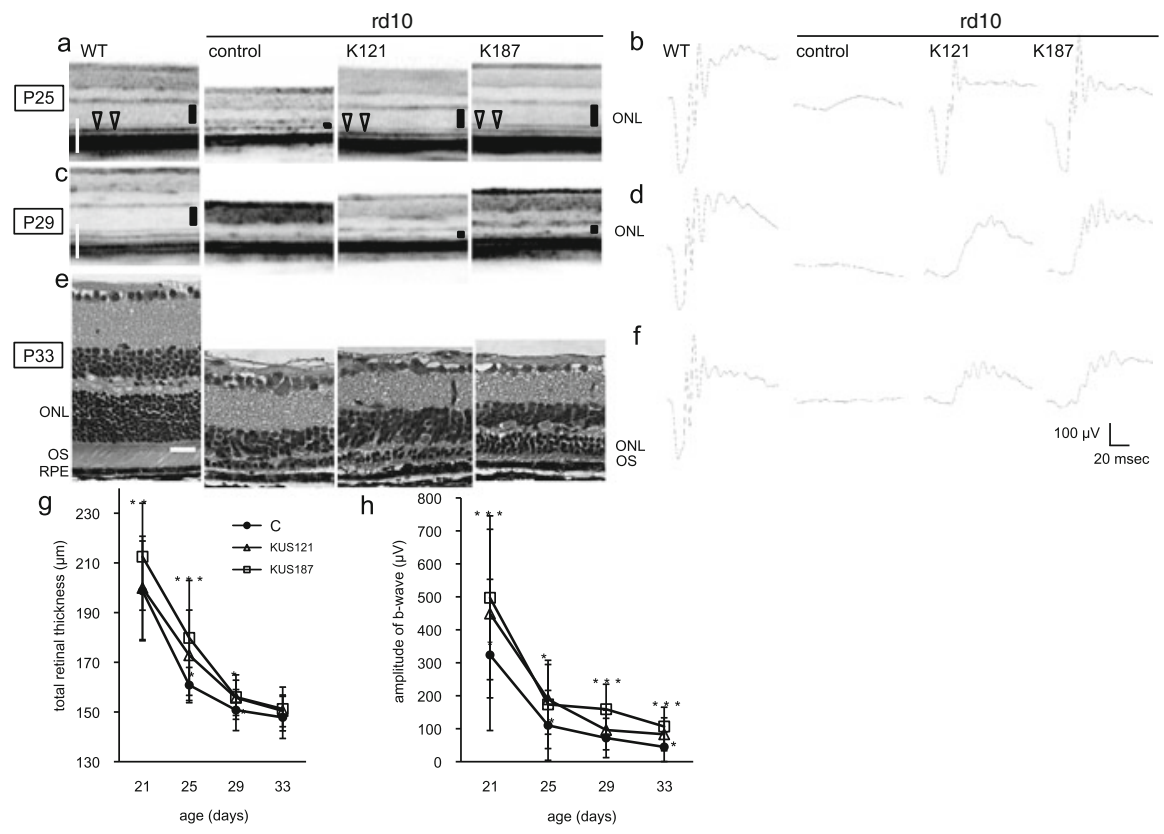

Fig. 4 In vivo efficacies of Kyoto University Substances (KUSs) in the rd10 mouse model of retinitis pigmentosa (Modified from Ikeda et al. [22]). (a, c) Representative live sectional images (vertical sections) by spectral-domain optical coherence tomography (SD-OCT) of retinas in 25-day-old (a) and 29-day-old (c) normal C57BL/6 mice (WT) and rd10 mice, administered KUS $121(n=17)$, KUS187 $(n=21)$, or saline $(n=18)$ as a control. Vertical bars in the images indicate the thickness of the outer nuclear layer (ONL). Note that the ONL was barely detectable in salinetreated control rd10 mice. (b, d, f) Electroretinograms of 25-day-old (b), 29-day-old (d), and 33-day-old (f) normal C57BL/6 mice (WT) and rd10 mice, administered KUSs or saline. (e) HE-stained retinas of 33-day-old normal C57BL/6 mice (WT) and rd10 mice, administered KUSs or saline. Scale bars (shown by white color), $100 \mu \mathrm{m}$ in (a) and (c); $20 \mu \mathrm{m}$ in (e). (g, h) Timedependent changes of total retinal thickness $(\mathbf{g})$ and b-wave amplitude in dark-adapted electroretinograms (h) in rd10 mice administered KUSs or saline (C). $* P<0.05$, $* * P<0.01$, *** $P<0.005$ vs. saline (Dunnett's test). Error bars indicate standard deviations. $O S$ outer segment, RPE retinal pigment epithelium

detected in SD-OCT images, and electroretinogram records were almost flat in control rd10 mice. In the age-matched KUS-treated rd10 mice, the ONL, although thin, and an electroretinogram response were still observed (Fig. 4c, d).

By histological examination, at age 33 days, the ONL in the control rd10 mice consisted of only 1-2 rows of cells, but there remained 5-6 rows of cells in the ONL in the KUS-treated rd10 mice (Fig. 4e). In KUS-treated mice but not in control rd10 mice, the outer segment of the photoreceptors was observed (Fig. 4e). The electroretinogram was non-recordable in the control rd10 mice, but small b-wave and oscillatory potentials were observed in the KUS-treated rd10 mice (Fig. 4f). Timedependent changes in total retinal thickness measured on SD-OCT images (Fig. 4g) and in b-wave amplitudes of dark-adapted electroretinograms (Fig. 4h) showed that 
KUS treatments had the potential to prevent or delay the disease progression. When examined by electron microscopy, the outer segment of the control rd10 mouse retina was mostly disarranged at the age of 21 days, whereas that of the KUS-treated rd10 mouse retina was regularly arranged [22].

\section{Discussion}

We showed that KUSs, new compounds developed as ATPase inhibitors of VCP, have novel functions as "VCP modulators" or "ATP regulators" without apparent inhibition of cellular VCP functions. These new "ATP regulators" have strong neuroprotective effects in vivo on retinal photoreceptor cells. The efficacies were apparently correlated with their abilities to suppress ER stress. From the evidence that KUSs could prevent the decrease in the cellular ATP level in response to several cell death-inducing insults and thereby prevent cell death in pathological conditions, and the evidence that the prevention of early cell death could in turn prevent or delay the deterioration of the affected organs, we posit that a reduction of ATP levels is a common feature in the affected organs of individuals with incurable disorders involving early cell death. Because many proteins require ATP, a reduction of ATP levels would contribute to a functional decline in affected cells or organs in the early stages of the disease. Reducing ATP consumption by way of KUSs and/or enhancing ATP generation by yet-unknown compounds would be a novel strategy to retard these processes and thus to prevent or retard the progression of clinical manifestations [22].

Open Access This chapter is distributed under the terms of the Creative Commons Attribution Noncommercial License, which permits any noncommercial use, distribution, and reproduction in any medium, provided the original author(s) and source are credited.

\section{References}

1. Lin JH, Lavail MM (2010) Misfolded proteins and retinal dystrophies. Adv Exp Med Biol 664:115-121

2. Jiang H, Xiong S, Xia X (2014) Retinitis pigmentosa-associated rhodopsin mutant T17M induces endoplasmic reticulum (ER) stress and sensitizes cells to ER stress-induced cell death. Mol Med Rep 9:1737-1742

3. Higashiyama $\mathrm{H}$ et al (2002) Identification of ter 94 , Drosophila VCP, as a modulator of polyglutamine-induced neurodegeneration. Cell Death Differ 9:264-273

4. Kakizuka A (2008) Roles of VCP in human neurodegenerative disorders. Biochem Soc Trans 36:105-108

5. Watts GD et al (2004) Inclusion body myopathy associated with Paget disease of bone and frontotemporal dementia is caused by mutant valosin-containing protein. Nat Genet 36:377-381

6. Johnson JO et al (2010) Exome sequencing reveals VCP mutations as a cause of familial ALS. Neuron 68:857-864 
7. Manno A, Noguchi M, Fukushi J, Motohashi Y, Kakizuka A (2010) Enhanced ATPase activities as a primary defect of mutant valosin-containing proteins that cause inclusion body myopathy associated with Paget disease of bone and frontotemporal dementia. Genes Cells 15:911-922

8. Stolz A, Hilt W, Buchberger A, Wolf DH (2011) Cdc48: a power machine in protein degradation. Trends Biochem Sci 36:515-523

9. Meyer H, Bug M, Bremer S (2012) Emerging functions of the VCP/p97 AAA-ATPase in the ubiquitin system. Nat Cell Biol 14:117-123

10. Wolf DH, Stolz A (2012) The Cdc48 machine in endoplasmic reticulum associated protein degradation. Biochim Biophys Acta 1823:117-124

11. Hirabayashi $\mathrm{M}$ et al (2001) $\mathrm{VCP} / \mathrm{p} 97$ in abnormal protein aggregates, cytoplasmic vacuoles, and cell death, phenotypes relevant to neurodegeneration. Cell Death Differ 8:977-984

12. Kobayashi T, Tanaka K, Inoue K, Kakizuka A (2002) Functional ATPase activity of p97/valosin-containing protein (VCP) is required for the quality control of endoplasmic reticulum in neuronally differentiated mammalian PC12 cells. J Biol Chem 277:47358-47365

13. Chou TF et al (2011) Reversible inhibitor of $\mathrm{p} 97$, DBeQ, impairs both ubiquitin-dependent and autophagic protein clearance pathways. Proc Natl Acad Sci U S A 108:4834-4839

14. Zinszner $\mathrm{H}$ et al (1998) CHOP is implicated in programmed cell death in response to impaired function of the endoplasmic reticulum. Genes Dev 12:982-995

15. Kim R, Emi M, Tanabe K, Murakami S (2006) Role of the unfolded protein response in cell death. Apoptosis 11:5-13

16. Naidoo N (2009) ER and aging-Protein folding and the ER stress response. Ageing Res Rev 8:150-159

17. Gardner BM, Pincus D, Gotthardt K, Gallagher CM, Walter P (2013) Endoplasmic reticulum stress sensing in the unfolded protein response. Cold Spring Harb Perspect Biol 5:a013169

18. Gorman AM, Healy SJM, Jager R, Samali A (2012) Stress management at the ER: regulators of ER stress-induced apoptosis. Pharmacol Ther 134:306-316

19. Chang B et al (2002) Retinal degeneration mutants in the mouse. Vision Res 42:517-525

20. Ojima Y et al (2010) Restoration of outer segments of foveal photoreceptors after resolution of central serous chorioretinopathy. Jpn J Ophthalmol 54:55-60

21. Oishi A et al (2010) The significance of external limiting membrane status for visual acuity in age-related macular degeneration. Am J Ophthalmol 150:27-32

22. Ikeda $\mathrm{HO}$ et al (2014) Novel VCP modulators mitigate major pathologies of rd10, a mouse model of retinitis pigmentosa. Sci Rep 4:5970 


\title{
Roles of E-cadherin in Hepatocarcinogenesis
}

\author{
Shin Maeda and Hayato Nakagawa
}

\begin{abstract}
Loss of E-cadherin function has been reported to be associated with progression and poor prognosis of liver cancer. However, the precise role of E-cadherin in liver cancer development has not been elucidated. Thus, we generated liver-specific E-cadherin $(C d h 1)$ knockout mice $\left(C d h 1^{\Delta L i v}\right)$ by crossing $C d h 1^{f l o x f f o x}$ mice with albumin-Cre transgenic mice. Interestingly, $C d h 1^{\Delta L i v}$ mice developed spontaneous inflammation in the portal areas, and then developed periductal onion skin-like fibrosis, which resembled primary sclerosing cholangitis. Microarray analysis showed that expression of stem cell markers such as CD44 and Sox9, and inflammatory cytokines such as IL-6 and TNF- $\alpha$, are increased in Cdh1 ${ }^{\text {LLiv }}$ liver compared with $C d h 1^{f l o x f f l o x}$ liver. To investigate the role of E-cadherin in the liver tumorigenesis, we crossed $C d h 1^{\Delta L i v}$ mice with lox-stop-lox Kras ${ }^{G 12 D}$ mice $\left(K r a s+C d h 1^{\Delta L i v}\right) . K r a s+C d h 1^{\Delta L i v}$ mice developed liver tumors at age 28 weeks $(8 / 8,100 \%)$, whereas Kras $+C d h l^{f l o x /+}$ mice did not develop any tumors. Histologically, these tumors were hepatocellular carcinomas with a small proportion of ductal lesions and strongly positive for progenitor cell markers such as CD44 and Sox9. Interestingly, epithelial to mesenchymal transition (EMT) was found in the tumors of $\mathrm{Kras}+C d h 1^{\Delta L i v}$ mice. We also found that diethylnitrosamine-induced tumorigenesis was significantly accelerated in $C d h 1^{\Delta L i v}$ mice. In summary, loss of E-cadherin in the liver leads to sclerosing cholangitis and promotes tumorigenesis. Its tumor-promoting function seemed to be caused by gain of stem cell properties as well as induction of EMT.
\end{abstract}

Keywords Liver cancer $\bullet$ E-cadherin $\bullet$ Stem cell marker $\bullet$ Inflammation $\bullet$ Epithelialmesenchymal transition $($ EMT) $\bullet$ Knockout mouse

\footnotetext{
S. Maeda $(\bowtie)$

Department of Gastroenterology, Yokohama City University,

3-9 Fukuura, Kanazawa-ku, Yokohama 236-0004, Japan

e-mail:smaeda@med.yokohama-cu.ac.jp

H. Nakagawa

Department of Gastroenterology, University of Tokyo,

7-3-1 Hongo, Bunkyo-ku, Tokyo 113-8655, Japan

(C) The Author(s) 2015

K. Nakao et al. (eds.), Innovative Medicine, DOI 10.1007/978-4-431-55651-0_6
} 


\section{Introduction}

Hepatocellular carcinoma (HCC) is the third most common cause of cancer deaths worldwide; therefore, improving the prognosis has become an important issue [1]. Major HCC risk factors include infection with hepatitis B (HBV) or C viruses (HCV), alcohol and non-alcoholic steatohepatitis (NASH). The prognosis of patients with HCC has improved recently because of progress in early diagnosis and treatment, but patients with advanced $\mathrm{HCC}$ are still incurable. Thus, understanding the carcinogenic mechanisms and exploring new therapeutic targets for HCC has become an important issue.

Hanahan et al. showed that all cancers share common hallmarks such as proliferation or anti-cell death that govern the transformation of normal cells to cancer cells [2]. Among them, invasion and metastasis play important roles in tumor promotion and progression, and are associated with prognosis of the patients. Dysregulation of E-cadherin has been reported to contribute to cancer progression [3]. It is reported that decreased expression of E-cadherin is associated with malignant progression in various kinds of cancer, such as gastric cancer or skin cancer [4]. In liver cancers, HCC or cholangiocarcinoma (CCC), E-cadherin expression is decreased by $20-60 \%$ and is associated with invasiveness or poor prognosis $[5,6]$. These findings suggest that E-cadherin may play an important role in tumor suppression in the liver. However, so far, the precise roles of E-cadherin in liver carcinogenesis remain unclear, especially in vivo; thus, we examined the role of E-cadherin in liver tumorigenesis by using liver-specific E-cadherin knockout mice.

\section{Results}

\section{Physiological Function of E-cadherin in the Liver}

To determine the role of E-cadherin in the liver, we generated liver-specific E-cadherin knockout mice by crossing $C d h 1^{F / F}$ and albumin-Cre transgenic mice (both from Jackson Laboratories). In $C d h 1^{F / F}$ control mice, E-cadherin was expressed on the membrane of hepatocytes and interlobular biliary epithelial cells. In contrast, in $C d h 1^{\Delta L}$ mice, the expression was completely deleted in both hepatocytes and biliary epithelial cells (Fig. 1a). Histologically, $C d h 1^{\Delta L}$ liver was shown to be almost normal at 1 month; however, at 2 month of age, $C d h 1^{\Delta L}$ mice spontaneously developed inflammation around the portal area, and at 8 month of age, periductal onion skin-like fibrosis, which resembled human primary sclerosing cholangitis, was observed in $C d h 1^{\Delta L}$ mice (Fig. 1b).

According to the periductal inflammation, we hypothesized that the bile transport system might be impaired by the E-cadherin deletion from the biliary epithelial cells. To prove the hypothesis, fluorescent-labeled bile acid was injected into $C d h 1^{F / F}$ and $C d h 1^{\Delta L}$ mice. After $15 \mathrm{~min}$, in control $C d h 1^{F / F}$ mice, we could see a 
a $\mathrm{CDH} 1 \mathrm{~F} / \mathrm{F}$

$\mathrm{CDH} 1 \Delta \mathrm{L}$
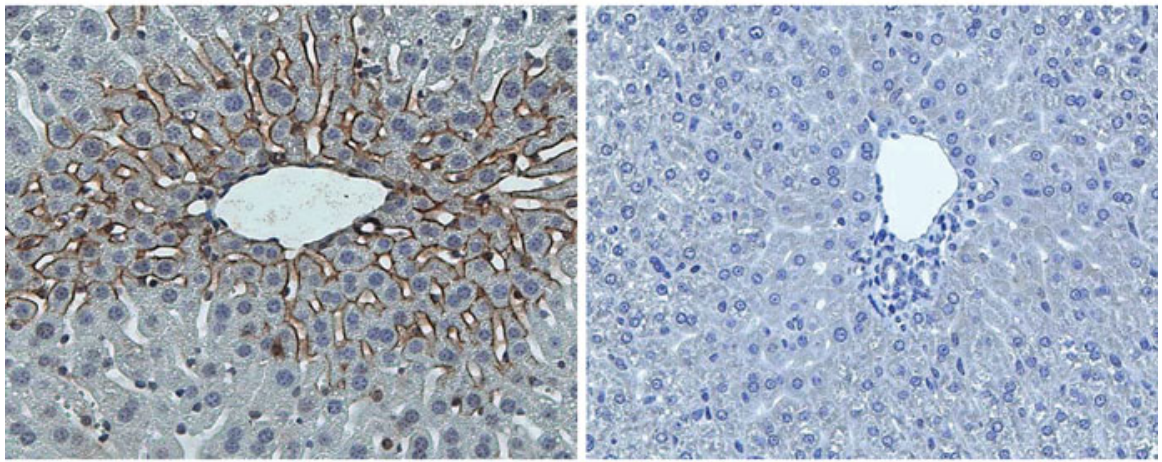

b

$\mathrm{CDH} 1 \Delta \mathrm{L}$

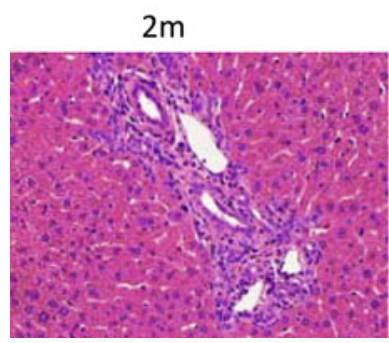

$5 \mathrm{~m}$

$8 \mathrm{~m}$
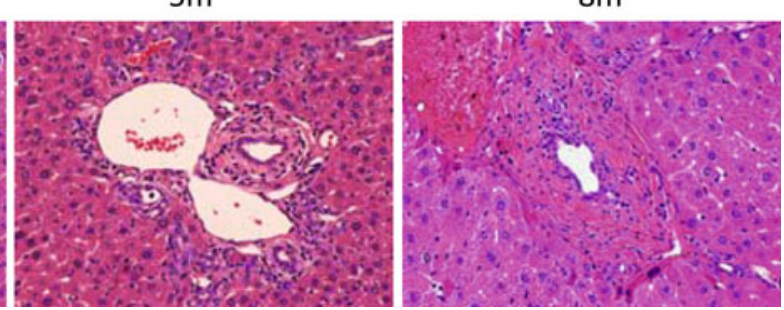

Fig. 1 (a) Analysis of E-cadherin expression by immunohistochemistry of liver sections $(\times 200)$ obtained from 1-month-old $C D H 1^{F / F}$ and $C D H 1^{\Delta L}$ mice. (b) H\&E staining of 2-, 5-, and 8-monthold $C D H 1^{\Delta L}$ mice $(\times 200)$

clear canalicular pattern and bile acid was smoothly transported into the bile duct. In contrast, in $C d h l^{\Delta L}$ mice, the canalicular staining pattern was very fuzzy and bile acid did not reach the bile duct lumen. These observations suggest that the bile canalicular network may be functionally impaired in $C d h 1^{\Delta L}$ mice, and this leads to liver injury and subsequent inflammation.

\section{Progenitor Cell Proliferation in CDH1 ${ }^{L}$ Mice}

To characterize the phenotype of $C D H 1^{\Delta L}$ mice, microarray analysis was performed using whole-liver samples obtained from $C D H 1^{F / F}$ and $C D H 1^{\Delta L}$ mice. The expression of several hepatic progenitor cell markers, such as Sox9, CD44, or Epcam, was upregulated in $C D H 1^{\Delta L}$ mice compared with $C D H 1^{F / F}$ mice. The results were confirmed by immunohistochemical analysis in $C D H 1^{F / F}$ and $C D H 1^{\Delta L}$ mice. We also found a lot of ductal cells expressing these progenitor cell markers in the periportal area. These results suggest that ductal cells with progenitor potential are proliferating in the portal area of $C D H 1^{\Delta L}$ mice. 


\section{Loss of E-cadherin Accelerates Oncogene-Addicted Liver Carcinogenesis}

Because Ras signaling is frequently active in human HCC, we crossed $C D H 1^{\Delta L}$ mice with active Kras conditional knockin $\left(L S L-\operatorname{Kras}^{G 12 D}\right)$ mice $\left(\mathrm{Kras}_{\mathrm{CDH}} \mathrm{Cl}^{\Delta L}\right)$. All male $\mathrm{Kras} / \mathrm{CDH} 1^{\Delta L}$ mice developed multiple liver tumors at 8 months of age $(n=10)$, whereas only 4 of 10 male albumin-Cre/LSL-Kras ${ }^{G 12 D} / C D H 1$ wild-type mice (Kras/CDH1 ${ }^{+/+}$) developed a few visible tumors (Fig. 2a). Most of the tumors arising in $\mathrm{Kras} / \mathrm{CDH}^{\Delta L}$ mice were AFP-positive HCC and ranged from a well to a poorly differentiated type. On the other hand, tumors in the $\mathrm{Kras}_{\mathrm{CDH}} \mathrm{CD}^{+/+}$mice were mostly AFP-negative dysplastic nodules or well-differentiated HCC. These results suggest that loss of E-cadherin accelerates Ras-addicted liver cancer development.

We assessed activation of extracellular signal-regulated kinase (ERK), which is a major downstream transducer of Ras, in non-tumor tissue. Strong ERK phosphorylation was observed in $\mathrm{Kras} / \mathrm{CDHI}^{\mathrm{\Delta L}}$ livers compared with that in Kras/ $\mathrm{CDH1}^{+/+}$livers. We thought that the increased ERK activation was one of the mechanism for the tumor acceleration in $C D H 1^{\Delta L}$ mice.

Epithelial to mesenchymal transition (EMT) is considered a key process for tumor invasiveness, and loss of E-cadherin expression is a hallmark of EMT [7]. Interestingly, in some tumors in $\mathrm{Kras} / \mathrm{CDH} 1^{\Delta L}$ mice, $\mathrm{HCC}$ cells gradually transformed into fibroblast-like cells, and these cells were positive for the mesenchymal marker vimentin, indicating that spontaneous EMT occurred in the tumors of these mice. EMT was shown to be associated with a gain of stem cell properties [7]. Indeed, evident expression of two stem cell markers, CD44 and Sox9, was positive in tumor cells undergoing EMT.

a

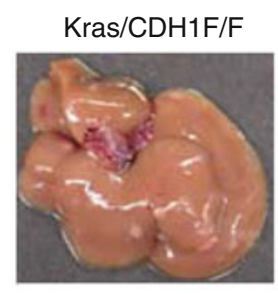

\section{$\mathrm{Kras} / \mathrm{CDH} 1 \Delta \mathrm{L}$}

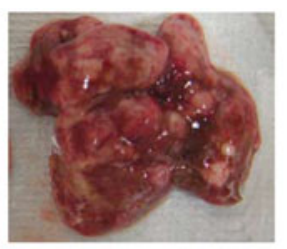

b

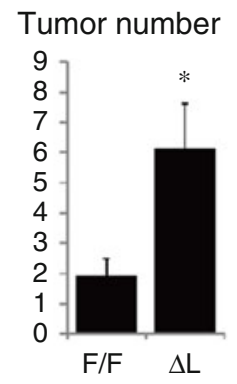

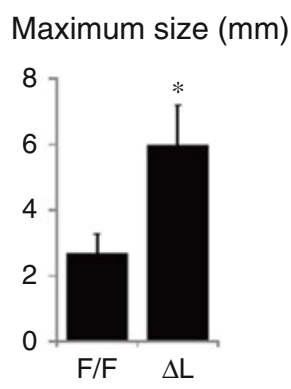

Fig. 2 (a) Representative images of the liver from 8-month-old $\mathrm{Kras} / \mathrm{CDH1} 1^{+/+}$and $\mathrm{Kras} / \mathrm{CDH} 1^{\Delta L}$ mice. (b) Bar graphs of tumor number and tumor size in each mouse after 8 months of diethylnitrosamine (DEN) injections are shown. Data are expressed as means \pm SEMs $(n=10$ per group, $* p<0.05)$ 
In female $\mathrm{Kras} / \mathrm{CDH} 1^{\Delta L}$ mice, only two of eight mice developed tumors by 12 months of age. This indicates gender disparity in this model of liver cancer susceptibility, as was shown in other mouse HCC models [8].

\section{Loss of E-cadherin Promotes Chemical-Induced HCC}

To further examine the role of E-cadherin in hepatocarcinogenesis, we used diethylnitrosamine (DEN) to induce a hepatocyte-derived HCC [9]. $\mathrm{CDH} 1^{F / F}$ mice and $C D H 1^{\Delta L}$ mice were injected with $25 \mathrm{mg} / \mathrm{kg}$ DEN on postnatal day 14 [10]. After 8 months, $C D H 1^{\Delta L}$ mice showed a significantly increased number and size of liver tumors compared with $C D H 1^{F / F}$ mice. In addition, $C D H 1^{\Delta L}$ mice developed histologically more advanced tumors (Fig. 2b). As in $\mathrm{Kras} / \mathrm{CDH} 1^{\Delta L}$ mice, strong ERK phosphorylation was observed in tumors of DEN-treated $C D H 1^{\Delta L}$ mice. Some tumors in $C D H 1^{\Delta L}$ mice strongly expressed CD44 and vimentin, whereas very few tumors in $C D H 1^{F / F}$ mice expressed these markers. These results confirmed that loss of E-cadherin enhances activation of ERK and expression of stem cell and EMT markers in a chemically induced HCC model.

\section{Relationship Between E-cadherin Loss and Mesenchymal and Stem Cell Markers in Human HCC}

To investigate whether E-cadherin loss correlates with mesenchymal and stem cell markers in human HCC, we examined the expression of E-cadherin, CD44, and vimentin in human HCC cell lines. Significant inverse correlations were observed, particularly between E-cadherin and CD44. Among these cell lines, we chose three that expressed E-cadherin, Hep3B, HuH7, and PLC/PRF/5, and we examined the effect of E-cadherin knockdown with siRNA. All three cell lines exhibited elevated expression of mesenchymal markers such as $\mathrm{N}$-cadherin and vimentin, and showed an elongated mesenchymal-like appearance. In addition, invasion capacity was significantly increased by E-cadherin knockdown, suggesting that loss of E-cadherin can be a causal factor of EMT and invasive phenotype of HCC.

\section{Discussion}

Our current data strongly suggest that E-cadherin is a tumor suppressor in the liver. Although various kinds of epithelial tumors showed decreased E-cadherin expression, there have been few reports of direct connections between E-cadherin loss and tumor progression, especially in vivo [11,12]. In this study, when combined with 
Ras activation or chemical carcinogen administration, $C D H 1^{\Delta L}$ display markedly accelerated carcinogenesis and an invasive phenotype. Although it has been unclear whether loss of E-cadherin is a consequence or a cause of EMT, we have demonstrated its causal role in vivo and in vitro. Recent reports have established a direct link between EMT and a gain of stem/progenitor cell properties [7], which is supported by our mouse models since tumor cells undergoing EMT clearly expressed stem cell markers. The expression of stem cell markers such as CD44 and Sox 9 has been reported to be associated with a poor prognosis in patients with HCC [13].

The cellular source of liver cancers still remains unclear. Recent studies suggested that mature hepatocytes could translate into not only HCC but also CCC [14]. We speculated that tumors in $\mathrm{Kras} / \mathrm{CDH} 1^{\Delta L}$ mice originated from two different cell types on the basis of the pathological findings and distribution of the tumors - proliferating duct cells including progenitor cells induced by loss of E-cadherin, and mature hepatocytes transformed by Ras activation. However, to reach a firm conclusion, further analyses such as cell lineage tracing are needed, and we consider this an important future issue.

In summary, loss of E-cadherin in the liver causes impairment of the intrahepatic biliary network and subsequent inflammatory reactions. In mature hepatocytes, loss of E-cadherin leads to EMT induction, upregulation of stem cell markers, and ERK activation, which eventually results in enhanced carcinogenesis and an invasive phenotype [15]. Thus, E-cadherin plays critical roles in maintaining homeostasis and suppressing carcinogenesis in the liver.

Open Access This chapter is distributed under the terms of the Creative Commons Attribution Noncommercial License, which permits any noncommercial use, distribution, and reproduction in any medium, provided the original author(s) and source are credited.

\section{References}

1. El-Serag HB (2011) Hepatocellular carcinoma. N Engl J Med 365:1118-1127

2. Hanahan D, Weinberg RA (2000) The hallmarks of cancer. Cell 100:57-70

3. Paredes J, Figueiredo J, Albergaria A et al (2012) Epithelial E- and P-cadherins: role and clinical significance in cancer. Biochim Biophys Acta 1826:297-311

4. Berx G, van Roy F (2009) Involvement of members of the cadherin superfamily in cancer. Cold Spring Harb Perspect Biol 1:a003129

5. Lee S, Kim WH, Jung HY, Yang MH, Kang GH (2002) Aberrant CpG island methylation of multiple genes in intrahepatic cholangiocarcinoma. Am J Pathol 161:1015-1022

6. Matsumura T, Makino R, Mitamura K (2001) Frequent down-regulation of E-cadherin by genetic and epigenetic changes in the malignant progression of hepatocellular carcinomas. Clin Cancer Res 7:594-599

7. Mani SA, Guo W, Liao MJ et al (2008) The epithelial-mesenchymal transition generates cells with properties of stem cells. Cell 133:704-715

8. Naugler WE, Sakurai T, Kim S et al (2007) Gender disparity in liver cancer due to sex differences in MyD88-dependent IL-6 production. Science 317:121-124

9. Bralet MP, Pichard V, Ferry N (2002) Demonstration of direct lineage between hepatocytes and hepatocellular carcinoma in diethylnitrosamine-treated rats. Hepatology 36:623-630 
10. Maeda S, Kamata H, Luo JL, Leffert H, Karin M (2005) IKKbeta couples hepatocyte death to cytokine-driven compensatory proliferation that promotes chemical hepatocarcinogenesis. Cell 121:977-990

11. Derksen PW, Liu X, Saridin F et al (2006) Somatic inactivation of E-cadherin and p53 in mice leads to metastatic lobular mammary carcinoma through induction of anoikis resistance and angiogenesis. Cancer Cell 10:437-449

12. Shimada S, Mimata A, Sekine M et al (2012) Synergistic tumour suppressor activity of E-cadherin and p53 in a conditional mouse model for metastatic diffuse-type gastric cancer. Gut 61:344-353

13. Yang XR, Xu Y, Yu B et al (2010) High expression levels of putative hepatic stem/progenitor cell biomarkers related to tumour angiogenesis and poor prognosis of hepatocellular carcinoma. Gut 59:953-962

14. Sekiya S, Suzuki A (2012) Intrahepatic cholangiocarcinoma can arise from Notch-mediated conversion of hepatocytes. J Clin Invest 122:3914-3918

15. Nakagawa H, Hikiba Y, Hirata Y et al (2014) Loss of liver E-cadherin induces sclerosing cholangitis and promotes carcinogenesis. Proc Natl Acad Sci U S A 111:1090-1095 


\title{
The Hippo Signaling Pathway: A Candidate New Drug Target for Malignant Tumors
}

\author{
Miki Nishio, Hiroki Goto, Miki Suzuki, Aya Fujimoto, Koshi Mimori, \\ and Akira Suzuki
}

\begin{abstract}
The Hippo pathway has the unique capacity to sense tissue architecture and the external forces that shape it, and dysregulation of this pathway leads to tumorigenesis. The study of mice bearing systemic or tissue-specific mutations of Hippo elements has driven huge progress in understanding this pathway's role in normal physiology and disease. Here, we summarize how disruption of Hippo signaling relates to cancer, and we highlight the importance of this pathway as a new drug target for malignant tumors.
\end{abstract}

Keywords Hippo $\bullet$ Cancer $\bullet$ Function $\bullet$ Mouse model

\section{Introduction}

The Hippo signaling pathway was first identified as a regulator of organ size in Drosophila [1]. In mammals, canonical Hippo signaling is mediated by mammalian sterile 20-like (MST) kinases, large tumor suppressor (LATS) kinases, the adaptor proteins Salvador homolog 1 (SAV1) and Mps one binder kinase activator protein (MOB1), and the downstream transcription cofactors Yes-associated protein 1 (YAP1) and its paralog, transcriptional coactivator with PDZ-binding motif (TAZ).

In a cell exposed to cell-cell contact, mechanical force, or a stress stimulus, MST phosphorylates LATS in a reaction facilitated by SAV1 and MOB1. LATS in turn phosphorylates YAP1 (S127)/TAZ (S89), which then binds to 14-3-3 protein. This binding prevents phosphorylated YAP1/TAZ from accessing the nucleus and

The authors declare that no conflicts of interest exist.

M. Nishio • H. Goto • M. Suzuki • A. Fujimoto • A. Suzuki, M.D., Ph.D. ( $₫)$

Division Cancer Genetics, Medical Institute of Bioregulation, Kyushu University,

3-1-1, Higashiku, Fukuoka 812-8582, Japan

e-mail: suzuki@bioreg.kyushu-u.ac.jp

K. Mimori

Department of Surgery, Kyushu University Beppu Hospital, Beppu 874-0838, Japan

K. Nakao et al. (eds.), Innovative Medicine, DOI 10.1007/978-4-431-55651-0_7 


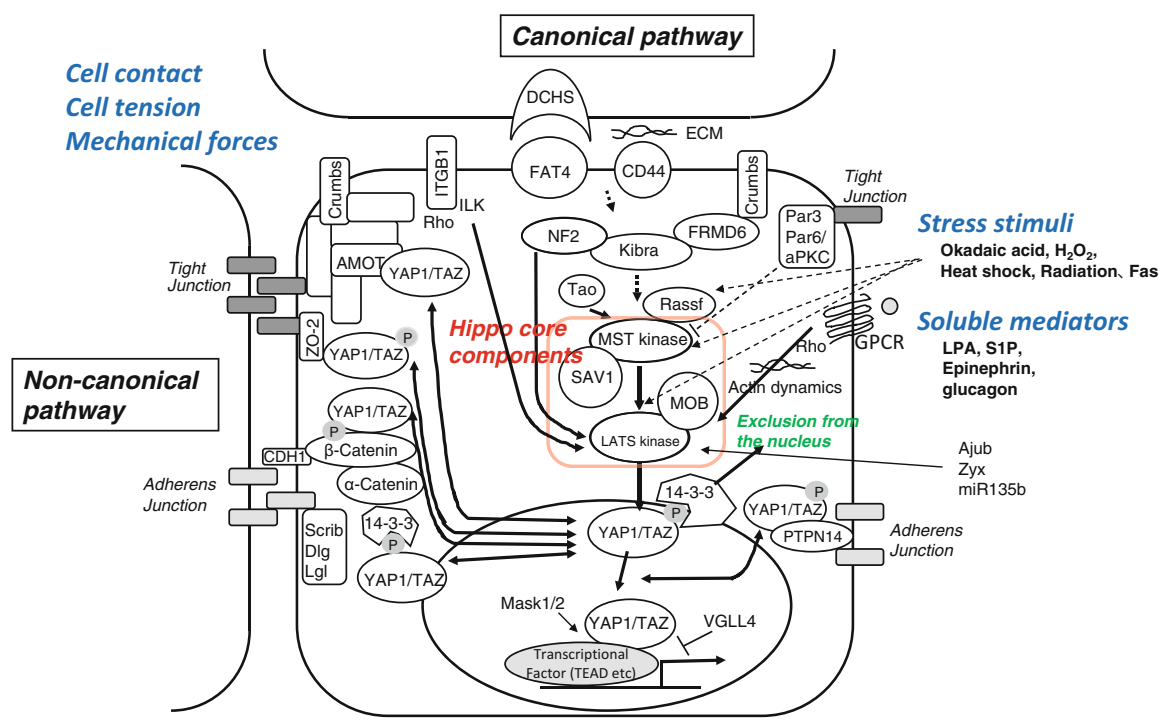

Fig. 1 Mammalian canonical and non-canonical Hippo signaling pathways. The core components of the mammalian Hippo pathway are the MST and LATS kinases, and the SAV1 and MOB adaptor proteins. The Hippo pathway is activated in response to increased cell density, cell tension, mechanical forces, stress stimuli, or G-protein-coupled receptor (GPCR) antagonists, and inhibits cell proliferation and other processes potentially contributing to tumorigenesis. Candidate sensory components upstream of the Hippo core include FAT4, DCHS1, ITGB1, CD44, NF2, FRMD6, KIBRA, TAO, and RASSF. Upon Hippo core activation, SAV1 binding to MST allows this kinase to phosphorylate MOB1. Phospho-MOB1 binding to LATS both enhances LATS catalytic activity and allows it to be phosphorylated by MST. Phospho-LATS then phosphorylates and inactivates YAP (or its paralog, TAZ), promoting their cytoplasmic retention through binding to 14-3-3 protein. LATS-phosphorylated YAP1 (or TAZ) is also degraded so transcription factors (such as the TEADs) promoting cell survival are not activated. In contrast, under conditions of low cell density or minimal stress, YAP dissociates from 14-3-3, translocates into the nucleus, and activates transcription factors that induce the expression of pro-survival genes. Solid lines indicate known direct interactions; dashed lines indicate unknown mechanisms. The non-canonical Hippo pathway operates in the tight and adherens junction complexes and involves AMOT in the Crumb complex in the tight junction, ZO-2 in the tight junction, and $\alpha$-catenin, $\beta$-catenin and PTPN14 in the adherence junction. Each of these molecules can bind to phosphorylated YAP1 to control its localization and activity

activating the transcription of a broad range of growth factors and anti-apoptotic genes (Fig. 1). Additional control is exerted by the binding of phosphorylated YAP1(S397)/TAZ(S311) to the E3 ligase complex SCF ${ }^{\beta-\operatorname{TrCP}}$, which facilitates YAP1/ TAZ ubiquitination and proteasomal degradation [2-5] (Fig. 2). Thus, the promotion of cell proliferation by YAP1/TAZ is negatively regulated by Hippo signaling.

The upstream sensors activating Hippo signaling in mammals are not clearly understood. With respect to extracellular triggers, E-cadherin (CDH1), CD44, $\beta 1$-integrin (ITGB1)-Rho-GTPase, $\beta 1$-integrin-ILK-MYPT1-PP1, G-proteincoupled receptors (GPCRs), and protease-activated receptors (F2Rs) Rho-GTPasesROCK have all been linked to control of NF2, LATS, or YAP1/TAZ activation, as illustrated in Figs. 1 and 2 [2, 6-15], but the precise sequence of events remains 


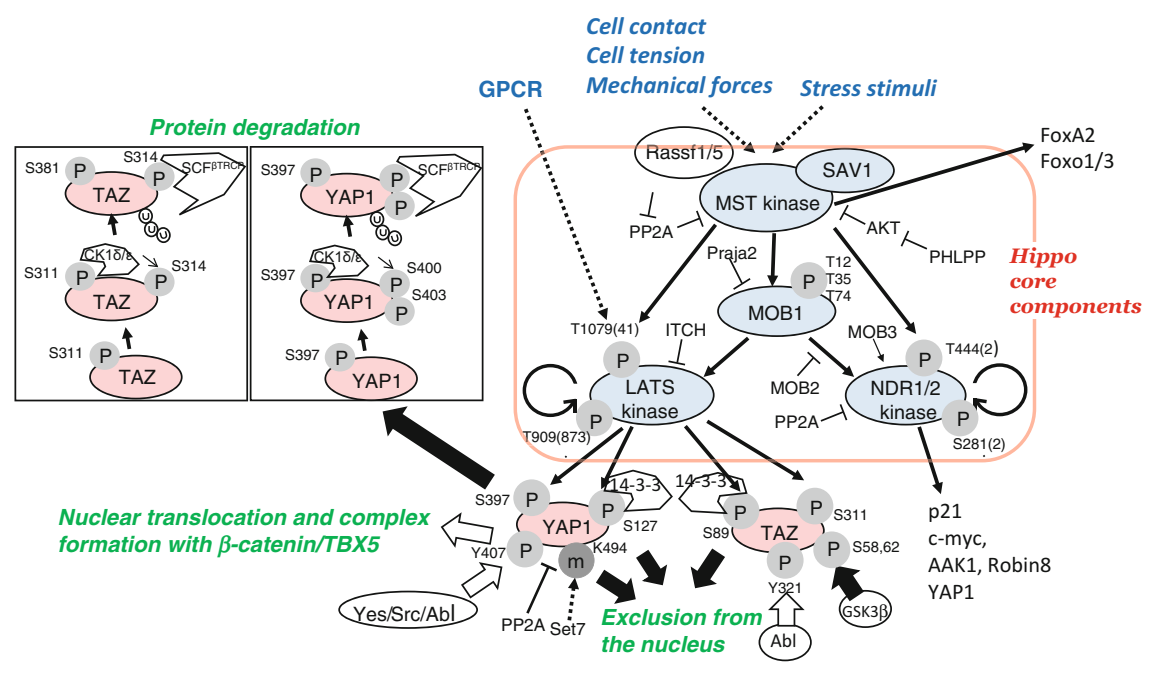

Fig. 2 Mechanisms of YAP1/TAZ inactivation. Activation of the Hippo core, as described in Fig. 1, leads to LATS-mediated phosphorylation of YAP1 (S127) and TAZ (S89) and the cytoplasmic retention of these proteins via 14-3-3 binding. Alternatively, LATS-mediated phosphorylation of YAP1 (S397)/TAZ (S311) triggers the binding of the E3 ligase complex SCF $\beta$-TRCP, which facilitates YAP1/TAZ ubiquitination and proteasomal degradation. Phosphorylation of YAP1 (Y407)/ TAZ (Y321) by SRC family kinases (YES1, SRC, ABL) excludes YAP1/TAZ from the nucleus, whereas methylation of YAP1(K494) by SET7 promotes its nuclear localization. Phosphorylation of TAZ (Ser58 and Ser62) by glycogen synthase kinase $3 \beta$ (GSK3 $\beta$ ) recruits $\beta$-TrCP to target TAZ for degradation. RASSF1A activates MST1 by preventing PP2A-mediated dephosphorylation of MST1/2 and YAP1. NDR1/2 are alternative MST substrates

obscure. The roles of intracellular triggers are even less well defined but include KIBRA binding to NF2 and FRMD6 and the activities of TAOK1, PP2A, and RASSFs [16-23].

Establishment of epithelial apical-basal polarity requires the Crumbs, Par, and Scribble complexes [24, 25]. In addition to the canonical Hippo signaling pathway, a non-canonical Hippo pathway exists that involves AMOT in the Crumb complex in the tight junction, $\mathrm{ZO}-2$ in the tight junction, and $\alpha$-catenin, $\beta$-catenin, and PTPN14 in the adherence junction [26-32]. These molecules all bind to phosphorylated YAP1 to control its localization and activity. The Scribble and Par3 polarity complexes also influence Hippo signaling.

The localization and activity of YAP1/TAZ are also regulated by several other non-Hippo molecular events. For example, the phosphorylation of YAP1 (Y407)/ TAZ (Y321) by SRC family kinases (YES1, SRC, ABL) [33] induces nuclear localization of YAP1, while methylation of YAP1 (K494) by SET7 excludes YAP1 from the nucleus [34]. Like Hippo-mediated phosphorylation, phosphorylation of TAZ (Ser58 and Ser62) by glycogen synthase kinase $3 \beta$ (GSK3 $\beta$ ) recruits $\beta$-TrCP, driving TAZ degradation [35].

The main transcription factors regulated by YAP1/TAZ are the TEADs, but, as illustrated in Fig. 3, many other transcription factors are affected by 


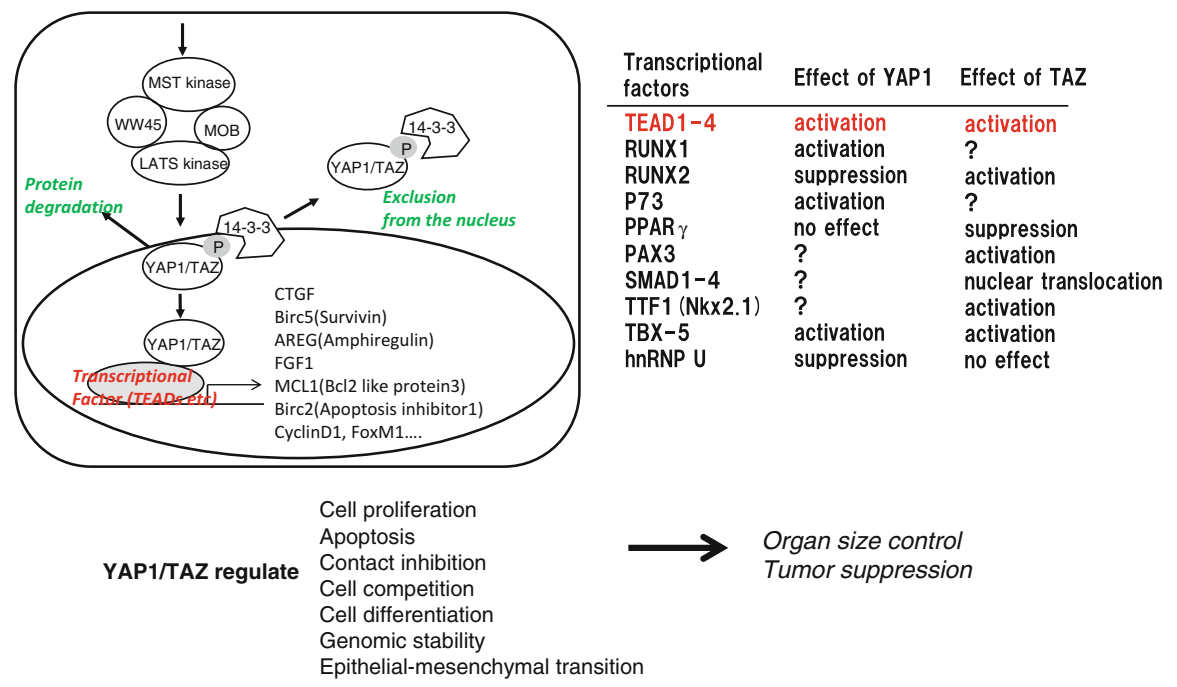

Fig. 3 Transcription factors and downstream target genes activated by Hippo signaling. Left: the main transcription factors regulated by YAP1/TAZ are the TEADs. Following interaction with nuclear YAP1/TAZ, TEADs enhance the transcription of anti-apoptotic genes such as CTGF and BIRC5 (survivin). Right: list of other transcription factors controlled either positively or negatively by YAP1 or TAZ. Bottom: cellular processes regulated by YAP1/TAZ

YAP1/TAZ. Accordingly, the Hippo pathway regulates cell proliferation, organ size, and tumor suppression. As shown in Fig. 4, the Hippo pathway also engages in crosstalk with several morphogenetic signaling pathways whose disruption is linked to morphogenesis and carcinogenesis [36]. While YAP1 and TAZ share most structural features, TAZ lacks two sequences present in YAP1: a $\operatorname{Pxx} \varphi \mathrm{P}$ motif and an SH3-binding motif. The effect of these differences on specific transcriptional activation patterns is under investigation.

Identifying the roles of mammalian Hippo signaling elements has been a challenge because multiple homologs exist for almost all components. Each isoform's function has required separate clarification via the use of specific knockout (KO) mice. Strikingly, mutants lacking NF2, MST1/2, or LATS1, as well as YAP1 transgenic animals, all develop some type of tumor. In human cancers, inactivation of the Hippo pathway due to either genetic (NF2, SAV1, MOB1A, LATS2, or YAP1) or epigenetic changes has been documented [37-40]. Indeed, for a variety of tumors, loss of Hippo signaling can predict patient sensitivity to chemotherapy as well as disease-free survival and overall survival [41]. This function of Hippo components as potent tumor suppressors makes them attractive targets for new anti-cancer therapies. To date, certain porphyrin derivatives, such as verteporfin (VP) and protoporphyrin IX (PPIX), have been reported to inhibit YAP1-TEAD association

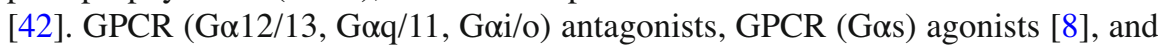
Src family antagonists (dasatinib) [33] have also been proposed as candidate Hippo modulators for tumor therapy. 


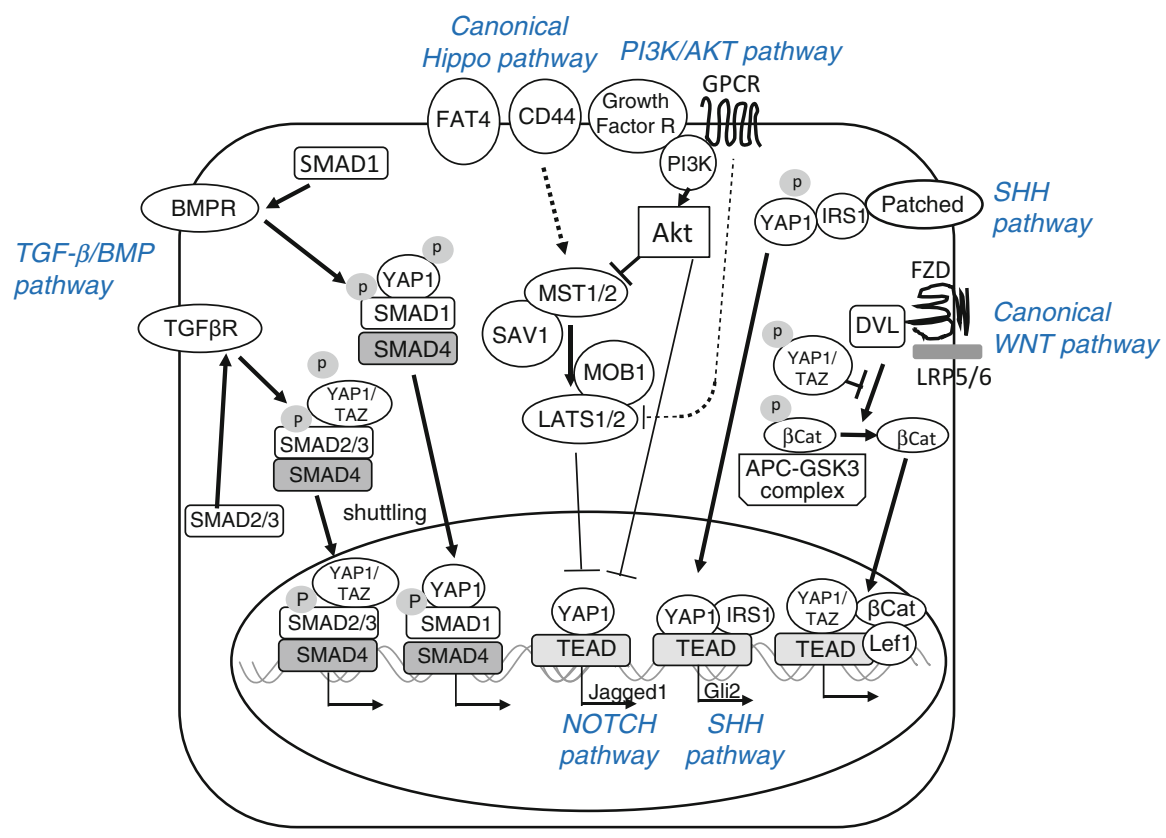

Fig. 4 Crosstalk between the Hippo pathway and other signaling pathways. The Hippo pathway is influenced by and interacts with components of the indicated morphogenetic pathways whose dysregulation is related to tumorigenesis

In this chapter, we describe gene-targeted mouse models that have been useful in characterizing the physiological and tumor-suppressive properties of the Hippo pathway in various tissues, and relate these models to human cancers.

\section{Loss of Hippo Signaling in the Liver}

YAP1 transgenic mice show hepatomegaly due to an increase in liver cell numbers rather than cell size. This hyperplasia was completely reversible in the short term but led to irreversible liver tumorigenesis in the long term [37]. YAP1 hyperactivation caused adult hepatocytes to dedifferentiate into liver progenitor cells that exhibited enhanced self-renewal and engraftment capacities as well as increased Notch2 transcription promoting the cholangiocyte lineage [43]. In contrast, mice deficient for YAP1 specifically showed loss of hepatocytes and cholangiocytes, and resisted the induction of hepatocarcinoma (HCC) [38, 42, 44].

In human HCC, amplification of the YAP1 gene is uncommon ( 5-10\%) [37], but loss of YAP1 (Ser127) phosphorylation and elevated nuclear YAP1 are frequently observed [38, 39]. Increased YAP1 is now considered an independent prognostic marker for HCC [41]. Among human liver tumor subtypes, YAP1 elevation is most 
frequent in combined hepatocellular cholangiocarcinomas (cHC-CC; 67\%). YAP1 is also higher in more HCCs exhibiting "stemness" (EpCAM ${ }^{+}$and keratin 19+; $56 \%$ ) than in HCCs lacking "stemness" (17\%). In addition, impaired phosphorylation of MOB1 occurs in $81 \%$ of human HCCs [40].

Complete knockout of both MST1 and MST2 (DKO) in mice results in embryonic lethality, whereas retention of a single functional copy of either MST1 or MST2 is sufficient for normal embryogenesis. These mutants show enhanced proliferation of liver stem cells and progenitor cells (such as oval cells) that give rise to both hepatocytes and cholangiocytes. Eventually these mutants develop liver tumors due to loss of function of the single MST1 or MST2 allele. These tumors display characteristics of both $\mathrm{HCC}$ and $\mathrm{CC}$, and feature the expansion and transformation of a mixed liver progenitor cell population [45]. Liver-specific MST1/2 DKO mice develop similar hepatomegaly and $\mathrm{HCC} / \mathrm{CC}$ tumors by 5-6 months after birth [46].

It is still unclear whether LATS function is essential for tumor suppression in the liver. One study showed that loss of MST1/2 decreased MOB1 and YAP1 phosphorylation but had no effect on LATS1/2 phosphorylation [45]. However, another study demonstrated reduced levels of phosphorylated YAP1 and LATS1/2 but unexpectedly decreased TAZ protein levels in MST1/2 DKO liver and tumors [40]. These data suggest that LATS1/2 and TAZ may be unlikely to play a major role in this liver phenotype.

Liver-specific deletion of Sav1 in mice enhances oval cell proliferation such that the mutants eventually develop HCC/CC liver tumors. However, levels of phosphoYAP1 and phospho-LATS1/2 are normal in SAV1 KO livers, suggesting that Sav1 promotes oval cell expansion and tumorigenesis in this model but acts independently of LATS1/2 and YAP1 [46, 47].

Liver-specific deletion of NF2 in mice also results in hepatocyte and cholangiocyte proliferation, leading to HCC/CC $[42,48,49]$. In one study, YAP1 and LATS1/2 phosphorylation were reduced in NF2-deficient liver and nuclear YAP1 was increased. Deletion of one copy of YAP1 was sufficient to reverse tumorigenesis in this model, indicating that NF2-mediated tumor suppression is achieved by inactivating YAP1 [48]. However, another group reported that liver-specific deletion of NF2 led to dramatic progenitor cell expansion without altering YAP1 localization or phosphorylation. EGFR inhibition blocked tumorigenesis triggered by NF2 deletion in this model, arguing against a role for YAP1 [49].

\section{Loss of Hippo Signaling in the Pancreas}

Pancreas-specific MST1/2 DKO mice (using PDX1-Cre Tg) exhibit a small pancreas in which the exocrine compartment shows increased cell proliferation but also extensive disorganization and pancreatitis-like autodigestion [50]. Pancreatic MST1/2 deficiency also induces postnatal de-differentiation of acinar cells into duct-like cells and thus reduces the total number of acinar cells [51]. However, neither 
these nor any other pancreas-specific Hippo mutants examined to date develop tumors. Interestingly, doxycycline-dependent YAP1 activation in the mouse pancreas expands its size and increases the number of acinar cells, leading to ductal metaplasia [44]. Thus, the phenotype observed in pancreas-specific MST DKO mice may be YAP1 independent.

In humans, pancreatic ductal adenocarcinoma (PDAC) frequently carries an oncogenic Kras mutation. In an inducible KrasG12D-driven mouse PDAC model, the mutants can develop malignancies in which KrasG12D and downstream MAPK activation are lost, but DNA amplification and/or mRNA overexpression of YAP1 are acquired $[52,53]$. Excessive YAP1 may thus be the major driver of invasive and recurrent PDAC.

\section{Loss of Hippo Signaling in the Intestine}

The role of Hippo signaling in the intestine is complex. YAP1 is overexpressed and localized in the nucleus in most human colon cancers, but silenced in a subset of highly aggressive and undifferentiated human colorectal carcinomas [54]. In mice, reports differ regarding Hippo element functions in intestinal stem cell (ISC) expansion and intestinal regeneration. In one study, loss of YAP1 in intestinal epithelial cells (Villin-Cre) had little effect on intestinal development but severely impaired DSS-induced intestinal regeneration. In the same study, doxycycline-dependent YAP1 activation expanded multipotent undifferentiated dysplastic progenitor cells in the intestine in a manner partly dependent on Notch activation [44]. However, another study found that intestinal YAP1 deficiency (Villin-Cre) resulted in WNT hypersensitivity leading to hyperplasia, ISC expansion, and formation of ectopic crypts and microadenomas. This report also showed that transgenic YAP1 expression reduced WNT target gene expression and induced rapid loss of intestinal crypts. In this latter case, the authors speculated that cytoplasmic YAP1 can bind to DVL and dampen WNT signaling [54]. The discrepancies between these reports require resolution because both increased nuclear YAP1 and decreased cytoplasmic YAP1 are associated with an increased risk of colon cancer in humans.

Intestine-specific Sav1-deficient mice show accelerated crypt regeneration and DSS-induced colonic polyp formation dependent on nuclear YAP1 but independent of $\beta$-catenin [55]. In contrast, intestine-specific MST1/2-deficient mice (Villin-Cre) display YAP1-dependent activation of Notch and $\beta$-catenin, as well as ISC expansion associated with colonic adenoma formation [56]. Recent work has shown that phosphorylation of YAP1(Y407) by the SRC family tyrosine kinase YES1 allows YAP1 to enter the nucleus and form a complex with the transcription factor TBX5 and $\beta$-catenin. This complex then promotes the transcription of anti-apoptotic genes such as BCL2L1 and BIRC5 [33]. Thus, a SRC-YAP1- $\beta$ catenin-TBX5 complex may be essential to the transformation and survival of $\beta$-catenin-driven cancers. 


\section{Loss of Hippo Signaling in the Lung}

Lung-specific MST1/2 DKO mice (TTF1(NKX2.1)-Cre) exhibit perinatal mortality due to respiratory failure [57]. The lungs appear immature, with reduced type I pneumocytes and increased immature type II pneumocytes lacking microvilli, lamellar bodies, and surfactant protein expression. These features are reminiscent of human infant respiratory distress syndrome (IRDS). MST normally phosphorylates and stabilizes the transcription factor FOXA2, which regulates alveolar epithelial cell maturation and surfactant protein expression. The IRDS phenotype of MST1/2 DKO mice is independent of YAP1 hyperactivation but dependent on FOXA2 [57].

Whether MST1/2 deficiency in the adult mouse lung would trigger lung tumorigenesis remains unknown. In Sca ${ }^{+}$murine lung tumor-propagating cells (TPC), TAZ is overexpressed by more than twofold and NF2 is downregulated by more than 15-fold compared with non-TPC [58]. Knockdown of YAP1 or TAZ in lung cancer cell lines decreases their cellular migration capacity and ability to metastasize. Conversely, expression of doxycycline-inducible constitutively active YAP1 in Kras Tg mice results in lung tumor progression in vivo [58].

TAZ KO mice display alveolarization defects similar to those seen in human emphysema patients [59-62]. However, although TAZ activates TTF1, a transcription factor essential for lung formation, TAZ KO lungs show a normal pattern of TTF1 target gene expression [63]. Mutant mice heterozygous for TAZ deficiency are resistant to bleomycin-induced lung fibrosis, suggesting that TAZ regulates fibrotic responses in this tissue [61].

Humans exposed to asbestos often develop malignant mesothelioma (MM). Mutation of NF2 occurs in $50 \%$ of human MM and YAP1 activation is frequent [64]. However, no statistically significant MM development has been reported to date in mice bearing mutations of Hippo elements.

\section{Loss of Hippo Signaling in the Skin}

YAP1 plays a key role in murine skin morphogenesis. Normal basal epidermal progenitors with nuclear YAP1 localization are driven to proliferate, whereas those with cytoplasmic YAP1 localization differentiate into hair follicles. YAP1 overexpression in mouse skin causes expansion of epidermal stem cells and progenitors, resulting in epidermal thickening, hyperkeratosis, hair follicle evagination, and the development of squamous cell-like carcinomas in skin grafts [65, 66]. Conversely, YAP1 deficiency in mouse epidermis, or disruption of YAP1-TEAD interaction, leads to epidermal hypoplasia and decreased keratinocyte proliferation due to loss of epidermal stem/progenitor cells.

At least one wild-type (WT) MOB1A or MOB1B allele is essential for murine embryogenesis, and any loss of this remaining allele results in trichilemmal carcinomas of the skin [67]. YAP1 activation and MOB1A/1B inactivation are also frequently observed in human trichilemmal carcinomas. Newborn MOB1A/1B DKO mice 
show hyperplastic keratinocyte progenitors and defective keratinocyte terminal differentiation, and rapidly die of malnutrition. MOB1A/1B-deficient keratinocytes exhibit hyperproliferation, apoptotic resistance, impaired contact inhibition, enhanced progenitor self-renewal, and increased centrosomes. LATS1/2 and YAP1 activities are altered in these cells [67].

SAV1-deficient mice exhibit a thick epidermal skin layer and succumb to early embryonic lethality [68]. SAV1-deficient primary keratinocytes show hyperproliferation, progenitor expansion, decreased apoptosis, and inhibition of terminal differentiation.

Surprisingly, keratinocyte-specific MST1/2 DKO mice are healthy and show normal YAP1 phosphorylation and activation. YAP1 is therefore likely phosphorylated by an alternative mechanism in keratinocytes [22], perhaps by NDR1/2 kinases or GPCR signaling [8].

As noted above, cell adhesion and junction complex proteins can also regulate YAP1. Keratinocyte-specific loss of $\alpha$-catenin results in keratinocyte hyperplasia and squamous cell carcinomagenesis similar to that observed in YAP1 transgenic mice [22]. In normal keratinocytes, $\alpha$-catenin interacts directly with YAP1 and restricts it to the cytoplasm [39]. YAP1 localization in the skin is also influenced by $\beta$-catenin, PTPN14, AMOT, and ZO-2 [69, 70].

\section{Loss of Hippo Signaling in T Lymphocytes}

In humans, inactivating MST1 mutations occur in families with T cell immunodeficiency or autoimmune disorders [71, 72]. In MST1 KO mice, naïve T cells show impaired CCL19/21-induced LFA-1 clustering and reduced cell migration [73-75]. In addition, MST1-deficient T cells exhibit defective FOXO1-IL-7R-BCL2 signaling, FAS upregulation, and a FOXO1/3-dependent decrease in SOD2 and catalase. As a result, these mutant $\mathrm{T}$ cells possess increased ROS and undergo apoptosis [76], a mechanism that may also underlie the observed immunodeficiency in humans with MST1 mutations. MOB1 phosphorylation is decreased in MST1 KO T cells but LATS $1 / 2$ and YAP1 phosphorylation are essentially normal, suggesting that LATS1/2 and YAP1 are not downstream effectors of MST1 and MOB1 in naïve T cells.

Despite their T cell deficiency, aged MST1 KO mice develop autoimmune disease because MST1 normally enhances FOXP3 expression and thus sustains regulatory $\mathrm{T}$ cell development through stabilizing FOXO1/3 [75]. These mutants are also susceptible to chemical induction of T-ALL and undergo accelerated spontaneous lymphomagenesis in a p53-deficient background [77].

The chemokines CCL19/21 normally induce the binding of DOCK8 to phosphorylated $\mathrm{MOB} 1$, promoting actin polarization and thymocyte migration from the wild-type thymus. MST1/2 DKO thymocytes show a loss of DOCK8 binding to MOB1 [78]. As a result, T cell-specific MST1/2 DKO thymocytes develop normally but undergo apoptosis before they can exit the thymus, leading to an absence of mature $\mathrm{T}$ cells in the circulation and lymphoid tissues [78]. 


\section{Loss of Hippo Signaling in Salivary Glands}

TAZ phosphorylation increases during salivary submandibular gland (SMG) development in mice [79]. In human Sjogren's syndrome (SS) patients, TAZ is localized in the nucleus rather than in the junctional regions of the SMGs, causing an abnormal accumulation of TAZ downstream targets such as extracellular matrix components, fibronectin, and CTGF [79]. LATS2 deficiency also results in defective SMG morphogenesis and a reduction in junction-localized TAZ in mice. Lastly, $5 \%$ of MOB1 partially deficient mice develop salivary gland adenomas and carcinomas [67].

\section{Loss of Hippo Signaling in Neurons}

In humans, mutations of DCHS1 or FAT4 cause Van Maldergem syndrome, which is characterized by a periventricular neuronal heterotopia (the mislocalization of cortical neuron progenitors) accompanied by auditory and renal deficits as well as craniofacial, skeletal, and limb malformations [80]. In mice, reduction of DCHS1 or FAT4 in embryonic neuroepithelium increases neuronal progenitors but reduces their differentiation. These cells are then mislocalized in the neocortex in a YAP1dependent manner [80], producing a phenotype similar to human Van Maldergem syndrome.

Inactivating mutations of NF2 in humans are associated with sporadic schwannomas, meningiomas, and ependymomas [81]. Heterozygous NF2 mutant mice do not develop neuronal tumors, but homozygous loss of NF2 specifically in Schwann cells results in their hyperplasia and eventually schwannomas [81]. Additional loss of one p53 allele results in the appearance of malignant peripheral nerve sheath tumors [82]. Complete loss of NF2 in mice is embryonic lethal. Telencephalonspecific NF2 KO embryos show neural progenitor expansion associated with increased nuclear YAP1/TAZ [83]. YAP1 or TEAD overexpression promotes cell cycle progression and inhibits neuronal differentiation by inducing the expression of downstream targets such as cyclin D1 and Pax3 [84, 85].

In normal brain, YAP1 is a downstream effector of the SHH pathway and increases neural stem cell proliferation [86, 87]. YAP1 and SHH signaling engage in crosstalk, since GLI2, which participates in SHH signaling, also acts downstream of YAP1 to control neuronal differentiation [88]. YAP1 is often upregulated in both human and mouse SHH-dependent or PATCHED1-mutated medulloblastomas [86, 87].

\section{Loss of Hippo Signaling in Bone}

Several Hippo elements have been implicated in controlling tumor suppression in bone. Many human osteosarcoma patients show high levels of nuclear YAP1 in their tumor cells [89], and aged mice bearing heterozygous mutations of NF2 develop osteosarcomas [90]. Some SAV1 heterozygous mouse mutants also develop osteosarcomas [68], as do some MOB1A/1B partially deficient mice [67]. 


\section{Concluding Remarks}

This chapter has summarized evidence showing that heterozygous inactivation of Hippo components leads to tumorigenesis in a wide range of tissues. For more detail on the in vivo functions of the Hippo pathway, please refer to several excellent recent reviews [36, 91, 92]. In general, loss of Hippo signaling or abnormal activation of YAP1/TAZ leads to impaired cell contact inhibition and eventually malignant transformation. Hippo components also control the differentiation/ de-differentiation of many stem/progenitor cells. Thus, proper Hippo signaling not only protects against tumor formation but also regulates a vast array of physiological and pathological phenomena.

The studies we have cited demonstrate a strong correlation between findings in human diseases and the phenotypes of Hippo mutant mouse strains. These animals may therefore be of great value for studying the mechanisms underlying various pathological conditions and for determining the efficacy of new therapeutic strategies. Manipulation of Hippo signaling could spur stem cell expansion for regenerative medicine or halt tumor progression to benefit cancer patients. As noted above, porphyrin derivatives [42], GPCR agonists and antagonists [8], and Src family antagonists [33] have already been proposed as candidate Hippo regulators for cancer therapy. Future work will no doubt uncover other Hippo-based approaches worth exploring for their anticancer potential.

Acknowledgments We gratefully acknowledge the support of the Uehara Memorial Foundation and the Ministry of Education, Culture, Sports, Science and Technology, Japan (MEXT).

Open Access This chapter is distributed under the terms of the Creative Commons Attribution Noncommercial License, which permits any noncommercial use, distribution, and reproduction in any medium, provided the original author(s) and source are credited.

\section{References}

1. Harvey K, Tapon N (2007) The Salvador-Warts-Hippo pathway - an emerging tumoursuppressor network. Nat Rev Cancer 7:182-191

2. Dupont S, Morsut L, Aragona M, Enzo E, Giulitti S, Cordenonsi M, Zanconato F, Le Digabel J, Forcato M, Bicciato S, Elvassore N, Piccolo S (2011) Role of YAP/TAZ in mechanotransduction. Nature 474:179-183

3. Liu CY, Zha ZY, Zhou X, Zhang H, Huang W, Zhao D, Li T, Chan SW, Lim CJ, Hong W, Zhao S, Xiong Y, Lei QY, Guan KL (2010) The hippo tumor pathway promotes TAZ degradation by phosphorylating a phosphodegron and recruiting the SCF-TrCP E3 ligase. J Biol Chem 285:37159-37169

4. Zhao B, Li L, Tumaneng K, Wang CY, Guan KL (2010) A coordinated phosphorylation by Lats and CK1 regulates YAP stability through SCF(beta-TRCP). Genes Dev 24:72-85

5. Zhao B, Li L, Lei Q, Guan KL (2010) The Hippo-YAP pathway in organ size control and tumorigenesis: an updated version. Genes Dev 24:862-874

6. Sainio M, Zhao F, Heiska L, Turunen O, den Bakker M, Zwarthoff E, Lutchman M, Rouleau GA, Jaaskelainen J, Vaheri A, Carpen O (1997) Neurofibromatosis 2 tumor suppressor protein colocalizes with ezrin and CD44 and associates with actin-containing cytoskeleton. J Cell Sci 110(Pt 18):2249-2260 
7. Morrison H, Sherman LS, Legg J, Banine F, Isacke C, Haipek CA, Gutmann DH, Ponta H, Herrlich P (2001) The NF2 tumor suppressor gene product, merlin, mediates contact inhibition of growth through interactions with CD44. Genes Dev 15:968-980

8. Yu FX, Zhao B, Panupinthu N, Jewell JL, Lian I, Wang LH, Zhao J, Yuan H, Tumaneng K, Li H, Fu XD, Mills GB, Guan KL (2012) Regulation of the Hippo-YAP pathway by G-proteincoupled receptor signaling. Cell 150:780-791

9. Mo JS, Yu FX, Gong R, Brown JH, Guan KL (2012) Regulation of the Hippo-YAP pathway by protease-activated receptors (PARs). Genes Dev 26:2138-2143

10. Kim M, Kim M, Lee S, Kuninaka S, Saya H, Lee H, Lee S, Lim DS (2013) cAMP/PKA signalling reinforces the LATS-YAP pathway to fully suppress YAP in response to actin cytoskeletal changes. EMBO J 32:1543-1555

11. Sansores-Garcia L, Bossuyt W, Wada K, Yonemura S, Tao C, Sasaki H, Halder G (2011) Modulating F-actin organization induces organ growth by affecting the Hippo pathway. EMBO J 30:2325-2335

12. Wada K, Itoga K, Okano T, Yonemura S, Sasaki H (2011) Hippo pathway regulation by cell morphology and stress fibers. Development 138:3907-3914

13. Aragona M, Panciera T, Manfrin A, Giulitti S, Michielin F, Elvassore N, Dupont S, Piccolo S (2013) A mechanical checkpoint controls multicellular growth through YAP/TAZ regulation by actin-processing factors. Cell 154:1047-1059

14. Tang Y, Rowe RG, Botvinick EL, Kurup A, Putnam AJ, Seiki M, Weaver VM, Keller ET, Goldstein S, Dai J, Begun D, Saunders T, Weiss SJ (2013) MT1-MMP-dependent control of skeletal stem cell commitment via a beta1-integrin/YAP/TAZ signaling axis. Dev Cell 25:402-416

15. Serrano I, McDonald PC, Lock F, Muller WJ, Dedhar S (2013) Inactivation of the Hippo tumour suppressor pathway by integrin-linked kinase. Nat Commun 4:2976

16. Xiao L, Chen Y, Ji M, Volle DJ, Lewis RE, Tsai MY, Dong J (2011) KIBRA protein phosphorylation is regulated by mitotic kinase aurora and protein phosphatase 1. J Biol Chem 286:36304-36315

17. Yu J, Zheng Y, Dong J, Klusza S, Deng WM, Pan D (2010) Kibra functions as a tumor suppressor protein that regulates Hippo signaling in conjunction with Merlin and expanded. Dev Cell 18:288-299

18. Moleirinho S, Chang N, Sims AH, Tilston-Lunel AM, Angus L, Steele A, Boswell V, Barnett SC, Ormandy C, Faratian D, Gunn-Moore FJ, Reynolds PA (2013) KIBRA exhibits MST-independent functional regulation of the Hippo signaling pathway in mammals. Oncogene 32:1821-1830

19. Poon CL, Lin JI, Zhang X, Harvey KF (2011) The sterile 20-like kinase Tao-1 controls tissue growth by regulating the Salvador-Warts-Hippo pathway. Dev Cell 21:896-906

20. Boggiano JC, Vanderzalm PJ, Fehon RG (2011) Tao-1 phosphorylates Hippo/MST kinases to regulate the Hippo-Salvador-Warts tumor suppressor pathway. Dev Cell 21:888-895

21. Guo C, Zhang X, Pfeifer GP (2011) The tumor suppressor RASSF1A prevents dephosphorylation of the mammalian STE20-like kinases MST1 and MST2. J Biol Chem 286:6253-6261

22. Schlegelmilch K, Mohseni M, Kirak O, Pruszak J, Rodriguez JR, Zhou D, Kreger BT, Vasioukhin V, Avruch J, Brummelkamp TR, Camargo FD (2011) Yap1 acts downstream of alpha-catenin to control epidermal proliferation. Cell 144:782-795

23. Oh HJ, Lee KK, Song SJ, Jin MS, Song MS, Lee JH, Im CR, Lee JO, Yonehara S, Lim DS (2006) Role of the tumor suppressor RASSF1A in Mst1-mediated apoptosis. Cancer Res 66:2562-2569

24. Bryant DM, Mostov KE (2008) From cells to organs: building polarized tissue. Nat Rev Mol Cell Biol 9:887-901

25. Shin K, Fogg VC, Margolis B (2006) Tight junctions and cell polarity. Annu Rev Cell Dev Biol 22:207-235

26. Varelas X, Samavarchi-Tehrani P, Narimatsu M, Weiss A, Cockburn K, Larsen BG, Rossant J, Wrana JL (2010) The Crumbs complex couples cell density sensing to Hippo-dependent control of the TGF-beta-SMAD pathway. Dev Cell 19:831-844 
27. Chen CL, Gajewski KM, Hamaratoglu F, Bossuyt W, Sansores-Garcia L, Tao C, Halder G (2010) The apical-basal cell polarity determinant Crumbs regulates Hippo signaling in Drosophila. Proc Natl Acad Sci U S A 107:15810-15815

28. Robinson BS, Huang J, Hong Y, Moberg KH (2010) Crumbs regulates Salvador/Warts/Hippo signaling in Drosophila via the FERM-domain protein Expanded. Curr Biol 20:582-590

29. Grzeschik NA, Parsons LM, Allott ML, Harvey KF, Richardson HE (2010) Lgl, aPKC, and Crumbs regulate the Salvador/Warts/Hippo pathway through two distinct mechanisms. Curr Biol 20:573-581

30. Ling C, Zheng Y, Yin F, Yu J, Huang J, Hong Y, Wu S, Pan D (2010) The apical transmembrane protein Crumbs functions as a tumor suppressor that regulates Hippo signaling by binding to Expanded. Proc Natl Acad Sci U S A 107:10532-10537

31. Martin-Belmonte F, Perez-Moreno M (2012) Epithelial cell polarity, stem cells and cancer. Nat Rev Cancer 12:23-38

32. Menendez J, Perez-Garijo A, Calleja M, Morata G (2010) A tumor-suppressing mechanism in Drosophila involving cell competition and the Hippo pathway. Proc Natl Acad Sci U S A 107:14651-14656

33. Rosenbluh J, Nijhawan D, Cox AG, Li X, Neal JT, Schafer EJ, Zack TI, Wang X, Tsherniak A, Schinzel AC, Shao DD, Schumacher SE, Weir BA, Vazquez F, Cowley GS, Root DE, Mesirov JP, Beroukhim R, Kuo CJ, Goessling W, Hahn WC (2012) Beta-Catenin-driven cancers require a YAP1 transcriptional complex for survival and tumorigenesis. Cell 151:1457-1473

34. Oudhoff MJ, Freeman SA, Couzens AL, Antignano F, Kuznetsova E, Min PH, Northrop JP, Lehnertz B, Barsyte-Lovejoy D, Vedadi M, Arrowsmith CH, Nishina H, Gold MR, Rossi FM, Gingras AC, Zaph C (2013) Control of the hippo pathway by Set7-dependent methylation of Yap. Dev Cell 26:188-194

35. Huang W, Lv X, Liu C, Zha Z, Zhang H, Jiang Y, Xiong Y, Lei QY, Guan KL (2012) The $\mathrm{N}$-terminal phosphodegron targets TAZ/WWTR1 protein for SCFbeta-TrCP-dependent degradation in response to phosphatidylinositol 3-kinase inhibition. J Biol Chem 287:26245-26253

36. Nishio M, Otsubo K, Maehama T, Mimori K, Suzuki A (2013) Capturing the mammalian Hippo: elucidating its role in cancer. Cancer Sci 104:1271-1277

37. Zender L, Spector MS, Xue W, Flemming P, Cordon-Cardo C, Silke J, Fan ST, Luk JM, Wigler M, Hannon GJ, Mu D, Lucito R, Powers S, Lowe SW (2006) Identification and validation of oncogenes in liver cancer using an integrative oncogenomic approach. Cell 125:1253-1267

38. Dong J, Feldmann G, Huang J, Wu S, Zhang N, Comerford SA, Gayyed MF, Anders RA, Maitra A, Pan D (2007) Elucidation of a universal size-control mechanism in Drosophila and mammals. Cell 130:1120-1133

39. Zhao B, Wei X, Li W, Udan RS, Yang Q, Kim J, Xie J, Ikenoue T, Yu J, Li L, Zheng P, Ye K, Chinnaiyan A, Halder G, Lai ZC, Guan KL (2007) Inactivation of YAP oncoprotein by the Hippo pathway is involved in cell contact inhibition and tissue growth control. Genes Dev 21:2747-2761

40. Zhou D, Conrad C, Xia F, Park JS, Payer B, Yin Y, Lauwers GY, Thasler W, Lee JT, Avruch J, Bardeesy N (2009) Mst1 and Mst2 maintain hepatocyte quiescence and suppress hepatocellular carcinoma development through inactivation of the Yap1 oncogene. Cancer Cell $16: 425-438$

41. Xu MZ, Yao TJ, Lee NP, Ng IO, Chan YT, Zender L, Lowe SW, Poon RT, Luk JM (2009) Yesassociated protein is an independent prognostic marker in hepatocellular carcinoma. Cancer 115:4576-4585

42. Liu-Chittenden Y, Huang B, Shim JS, Chen Q, Lee SJ, Anders RA, Liu JO, Pan D (2012) Genetic and pharmacological disruption of the TEAD-YAP complex suppresses the oncogenic activity of YAP. Genes Dev 26:1300-1305

43. Yimlamai D, Christodoulou C, Galli GG, Yanger K, Pepe-Mooney B, Gurung B, Shrestha K, Cahan P, Stanger BZ, Camargo FD (2014) Hippo pathway activity influences liver cell fate. Cell 157:1324-1338 
44. Camargo FD, Gokhale S, Johnnidis JB, Fu D, Bell GW, Jaenisch R, Brummelkamp TR (2007) YAP1 increases organ size and expands undifferentiated progenitor cells. Curr Biol 17:2054-2060

45. Song H, Mak KK, Topol L, Yun K, Hu J, Garrett L, Chen Y, Park O, Chang J, Simpson RM, Wang CY, Gao B, Jiang J, Yang Y (2010) Mammalian Mst1 and Mst2 kinases play essential roles in organ size control and tumor suppression. Proc Natl Acad Sci U S A 107:1431-1436

46. Lu L, Li Y, Kim SM, Bossuyt W, Liu P, Qiu Q, Wang Y, Halder G, Finegold MJ, Lee JS, Johnson RL (2010) Hippo signaling is a potent in vivo growth and tumor suppressor pathway in the mammalian liver. Proc Natl Acad Sci U S A 107:1437-1442

47. Lee KP, Lee JH, Kim TS, Kim TH, Park HD, Byun JS, Kim MC, Jeong WI, Calvisi DF, Kim JM, Lim DS (2010) The Hippo-Salvador pathway restrains hepatic oval cell proliferation, liver size, and liver tumorigenesis. Proc Natl Acad Sci U S A 107:8248-8253

48. Zhang N, Bai H, David KK, Dong J, Zheng Y, Cai J, Giovannini M, Liu P, Anders RA, Pan D (2010) The Merlin/NF2 tumor suppressor functions through the YAP oncoprotein to regulate tissue homeostasis in mammals. Dev Cell 19:27-38

49. Benhamouche S, Curto M, Saotome I, Gladden AB, Liu CH, Giovannini M, McClatchey AI (2010) Nf2/Merlin controls progenitor homeostasis and tumorigenesis in the liver. Genes Dev 24:1718-1730

50. George NM, Day CE, Boerner BP, Johnson RL, Sarvetnick NE (2012) Hippo signaling regulates pancreas development through inactivation of Yap. Mol Cell Biol 32:5116-5128

51. Gao T, Zhou D, Yang C, Singh T, Penzo-Mendez A, Maddipati R, Tzatsos A, Bardeesy N, Avruch J, Stanger BZ (2013) Hippo signaling regulates differentiation and maintenance in the exocrine pancreas. Gastroenterology 144:1543-1553, 1553 e1541

52. Kapoor A, Yao W, Ying H, Hua S, Liewen A, Wang Q, Zhong Y, Wu CJ, Sadanandam A, Hu B, Chang Q, Chu GC, Al-Khalil R, Jiang S, Xia H, Fletcher-Sananikone E, Lim C, Horwitz GI, Viale A, Pettazzoni P, Sanchez N, Wang H, Protopopov A, Zhang J, Heffernan T, Johnson RL, Chin L, Wang YA, Draetta G, DePinho RA (2014) Yap1 activation enables bypass of oncogenic kras addiction in pancreatic cancer. Cell 158:185-197

53. Zhang W, Nandakumar N, Shi Y, Manzano M, Smith A, Graham G, Gupta S, Vietsch EE, Laughlin SZ, Wadhwa M, Chetram M, Joshi M, Wang F, Kallakury B, Toretsky J, Wellstein A, Yi C (2014) Downstream of mutant KRAS, the transcription regulator YAP is essential for neoplastic progression to pancreatic ductal adenocarcinoma. Sci Signal 7:ra42

54. Barry ER, Morikawa T, Butler BL, Shrestha K, de la Rosa R, Yan KS, Fuchs CS, Magness ST, Smits R, Ogino S, Kuo CJ, Camargo FD (2013) Restriction of intestinal stem cell expansion and the regenerative response by YAP. Nature 493:106-110

55. Cai J, Zhang N, Zheng Y, de Wilde RF, Maitra A, Pan D (2010) The Hippo signaling pathway restricts the oncogenic potential of an intestinal regeneration program. Genes Dev 24:2383-2388

56. Zhou D, Zhang Y, Wu H, Barry E, Yin Y, Lawrence E, Dawson D, Willis JE, Markowitz SD, Camargo FD, Avruch J (2011) Mst1 and Mst2 protein kinases restrain intestinal stem cell proliferation and colonic tumorigenesis by inhibition of Yes-associated protein (Yap) overabundance. Proc Natl Acad Sci U S A 108:E1312-E1320

57. Chung C, Kim T, Kim M, Kim M, Song H, Kim TS, Seo E, Lee SH, Kim H, Kim SK, Yoo G, Lee DH, Hwang DS, Kinashi T, Kim JM, Lim DS (2013) Hippo-Foxa2 signaling pathway plays a role in peripheral lung maturation and surfactant homeostasis. Proc Natl Acad Sci U S A 110:7732-7737

58. Lau AN, Curtis SJ, Fillmore CM, Rowbotham SP, Mohseni M, Wagner DE, Beede AM, Montoro DT, Sinkevicius KW, Walton ZE, Barrios J, Weiss DJ, Camargo FD, Wong KK, Kim CF (2014) Tumor-propagating cells and Yap/Taz activity contribute to lung tumor progression and metastasis. EMBO J 33:468-481

59. Hossain Z, Ali SM, Ko HL, Xu J, Ng CP, Guo K, Qi Z, Ponniah S, Hong W, Hunziker W (2007) Glomerulocystic kidney disease in mice with a targeted inactivation of Wwtr1. Proc Natl Acad Sci U S A 104:1631-1636 
60. Makita R, Uchijima Y, Nishiyama K, Amano T, Chen Q, Takeuchi T, Mitani A, Nagase T, Yatomi Y, Aburatani H, Nakagawa O, Small EV, Cobo-Stark P, Igarashi P, Murakami M, Tominaga J, Sato T, Asano T, Kurihara Y, Kurihara H (2008) Multiple renal cysts, urinary concentration defects, and pulmonary emphysematous changes in mice lacking TAZ. Am J Physiol Renal Physiol 294:F542-F553

61. Mitani A, Nagase T, Fukuchi K, Aburatani H, Makita R, Kurihara H (2009) Transcriptional coactivator with PDZ-binding motif is essential for normal alveolarization in mice. Am J Respir Crit Care Med 180:326-338

62. Tian Y, Kolb R, Hong JH, Carroll J, Li D, You J, Bronson R, Yaffe MB, Zhou J, Benjamin T (2007) TAZ promotes PC2 degradation through a SCFbeta-Trcp E3 ligase complex. Mol Cell Biol 27:6383-6395

63. Park KS, Whitsett JA, Di Palma T, Hong JH, Yaffe MB, Zannini M (2004) TAZ interacts with TTF-1 and regulates expression of surfactant protein-C. J Biol Chem 279:17384-17390

64. Sekido Y (2013) Molecular pathogenesis of malignant mesothelioma. Carcinogenesis 34:1413-1419

65. Silvis MR, Kreger BT, Lien WH, Klezovitch O, Rudakova GM, Camargo FD, Lantz DM, Seykora JT, Vasioukhin V (2011) Alpha-catenin is a tumor suppressor that controls cell accumulation by regulating the localization and activity of the transcriptional coactivator Yap1. Sci Signal 4:ra33

66. Zhang H, Pasolli HA, Fuchs E (2011) Yes-associated protein (YAP) transcriptional coactivator functions in balancing growth and differentiation in skin. Proc Natl Acad Sci U S A 108:2270-2275

67. Nishio M, Hamada K, Kawahara K, Sasaki M, Noguchi F, Chiba S, Mizuno K, Suzuki SO, Dong Y, Tokuda M, Morikawa T, Hikasa H, Eggenschwiler J, Yabuta N, Nojima H, Nakagawa K, Hata Y, Nishina H, Mimori K, Mori M, Sasaki T, Mak TW, Nakano T, Itami S, Suzuki A (2012) Cancer susceptibility and embryonic lethality in Mob1a/1b double-mutant mice. J Clin Invest 122:4505-4518

68. Lee JH, Kim TS, Yang TH, Koo BK, Oh SP, Lee KP, Oh HJ, Lee SH, Kong YY, Kim JM, Lim DS (2008) A crucial role of WW45 in developing epithelial tissues in the mouse. EMBO J 27:1231-1242

69. Zhao B, Li L, Lu Q, Wang LH, Liu CY, Lei Q, Guan KL (2011) Angiomotin is a novel Hippo pathway component that inhibits YAP oncoprotein. Genes Dev 25:51-63

70. Hirate Y, Hirahara S, Inoue K, Suzuki A, Alarcon VB, Akimoto K, Hirai T, Hara T, Adachi M, Chida K, Ohno S, Marikawa Y, Nakao K, Shimono A, Sasaki H (2013) Polarity-dependent distribution of angiomotin localizes Hippo signaling in preimplantation embryos. Curr Biol 23:1181-1194

71. Abdollahpour H, Appaswamy G, Kotlarz D, Diestelhorst J, Beier R, Schaffer AA, Gertz EM, Schambach A, Kreipe HH, Pfeifer D, Engelhardt KR, Rezaei N, Grimbacher B, Lohrmann S, Sherkat R, Klein C (2012) The phenotype of human STK4 deficiency. Blood 119:3450-3457

72. Nehme NT, Pachlopnik Schmid J, Debeurme F, Andre-Schmutz I, Lim A, Nitschke P, RieuxLaucat F, Lutz P, Picard C, Mahlaoui N, Fischer A, de Saint BG (2012) MST1 mutations in autosomal recessive primary immunodeficiency characterized by defective naive T-cell survival. Blood 119:3458-3468

73. Katagiri K, Imamura M, Kinashi T (2006) Spatiotemporal regulation of the kinase Mst1 by binding protein RAPL is critical for lymphocyte polarity and adhesion. Nat Immunol 7:919-928

74. Dong Y, Du X, Ye J, Han M, Xu T, Zhuang Y, Tao W (2009) A cell-intrinsic role for Mst1 in regulating thymocyte egress. J Immunol 183:3865-3872

75. Du X, Shi H, Li J, Dong Y, Liang J, Ye J, Kong S, Zhang S, Zhong T, Yuan Z, Xu T, Zhuang Y, Zheng B, Geng JG, Tao W (2014) Mst1/Mst2 regulate development and function of regulatory $\mathrm{T}$ cells through modulation of Foxo1/Foxo3 stability in autoimmune disease. J Immunol 192:1525-1535 
76. Choi J, Oh S, Lee D, Oh HJ, Park JY, Lee SB, Lim DS (2009) Mst1-FoxO signaling protects Naive T lymphocytes from cellular oxidative stress in mice. PLoS One 4:e8011

77. Kim TS, Lee DH, Kim SK, Shin SY, Seo EJ, Lim DS (2012) Mammalian sterile 20-like kinase 1 suppresses lymphoma development by promoting faithful chromosome segregation. Cancer Res 72:5386-5395

78. Mou F, Praskova M, Xia F, Van Buren D, Hock H, Avruch J, Zhou D (2012) The Mst1 and Mst2 kinases control activation of rho family GTPases and thymic egress of mature thymocytes. J Exp Med 209:741-759

79. Enger TB, Samad-Zadeh A, Bouchie MP, Skarstein K, Galtung HK, Mera T, Walker J, Menko AS, Varelas X, Faustman DL, Jensen JL, Kukuruzinska MA (2013) The Hippo signaling pathway is required for salivary gland development and its dysregulation is associated with Sjogren's syndrome. Lab Investig J Tech Method Pathol 93:1203-1218

80. Cappello S, Gray MJ, Badouel C, Lange S, Einsiedler M, Srour M, Chitayat D, Hamdan FF, Jenkins ZA, Morgan T, Preitner N, Uster T, Thomas J, Shannon P, Morrison V, Di Donato N, Van Maldergem L, Neuhann T, Newbury-Ecob R, Swinkells M, Terhal P, Wilson LC, Zwijnenburg PJ, Sutherland-Smith AJ, Black MA, Markie D, Michaud JL, Simpson MA, Mansour S, McNeill H, Gotz M, Robertson SP (2013) Mutations in genes encoding the cadherin receptor-ligand pair DCHS1 and FAT4 disrupt cerebral cortical development. Nat Genet 45:1300-1308

81. Giovannini M, Robanus-Maandag E, van der Valk M, Niwa-Kawakita M, Abramowski V, Goutebroze L, Woodruff JM, Berns A, Thomas G (2000) Conditional biallelic Nf2 mutation in the mouse promotes manifestations of human neurofibromatosis type 2. Genes Dev 14:1617-1630

82. Robanus-Maandag E, Giovannini M, van der Valk M, Niwa-Kawakita M, Abramowski V, Antonescu C, Thomas G, Berns A (2004) Synergy of Nf2 and p53 mutations in development of malignant tumours of neural crest origin. Oncogene 23:6541-6547

83. Lavado A, He Y, Pare J, Neale G, Olson EN, Giovannini M, Cao X (2013) Tumor suppressor Nf2 limits expansion of the neural progenitor pool by inhibiting Yap/Taz transcriptional coactivators. Development 140:3323-3334

84. Cao X, Pfaff SL, Gage FH (2008) YAP regulates neural progenitor cell number via the TEA domain transcription factor. Genes Dev 22:3320-3334

85. Milewski RC, Chi NC, Li J, Brown C, Lu MM, Epstein JA (2004) Identification of minimal enhancer elements sufficient for Pax 3 expression in neural crest and implication of Tead2 as a regulator of Pax3. Development 131:829-837

86. Fernandez LA, Northcott PA, Dalton J, Fraga C, Ellison D, Angers S, Taylor MD, Kenney AM (2009) YAP1 is amplified and up-regulated in hedgehog-associated medulloblastomas and mediates Sonic hedgehog-driven neural precursor proliferation. Genes Dev 23:2729-2741

87. Kagey JD, Brown JA, Moberg KH (2012) Regulation of Yorkie activity in Drosophila imaginal discs by the Hedgehog receptor gene patched. Mech Dev 129:339-349

88. Lin YT, Ding JY, Li MY, Yeh TS, Wang TW, Yu JY (2012) YAP regulates neuronal differentiation through Sonic hedgehog signaling pathway. Exp Cell Res 318:1877-1888

89. Chan LH, Wang W, Yeung W, Deng Y, Yuan P, Mak KK (2014) Hedgehog signaling induces osteosarcoma development through Yap1 and H19 overexpression. Oncogene 33(40):4857-4866

90. McClatchey AI, Saotome I, Mercer K, Crowley D, Gusella JF, Bronson RT, Jacks T (1998) Mice heterozygous for a mutation at the Nf2 tumor suppressor locus develop a range of highly metastatic tumors. Genes Dev 12:1121-1133

91. Varelas X (2014) The Hippo pathway effectors TAZ and YAP in development, homeostasis and disease. Development 141:1614-1626

92. Mo JS, Park HW, Guan KL (2014) The Hippo signaling pathway in stem cell biology and cancer. EMBO Rep 15:642-656 


\title{
Inhibitory Immunoreceptors on Mast Cells in Allergy and Inflammation
}

\author{
Akira Shibuya, Chigusa Nakahashi-Oda, and Satoko Tahara-Hanaoka
}

\begin{abstract}
Activation of immune cells is regulated by positive and negative signals triggered by activating and inhibitory cell surface immunoreceptors, respectively. Inhibitory receptors are characterized by the immunoreceptor tyrosine-based inhibition motif (ITIM) in their cytoplasmic domains and play an important role in immune regulation by both lymphoid and myeloid cells. Mast cells express the high-affinity receptor for IgE (FceRI) and toll-like receptors (TLR) on the cell surface, and play a central role in allergic and non-allergic inflammations. We identified novel inhibitory immunoglobulin-like receptors, Allergin-1 and CD300a, which are expressed on mast cells. Allergin-1 inhibits mast cell degranulation via suppression of FceRI-mediated signaling. Allergin-1-deficient mice showed significantly exacerbated IgE-associated type 1 immediate hypersensitivity reactions. On the other hand, CD300a recognizes phosphatidylserine exposed on the plasma membrane of apoptotic cells and inhibits production of chemoattractants from mast cells in response to LPS stimulation. CD300a-deficient mice showed significantly prolonged survival after cecum ligation and puncture (CLP). Together, our results suggest that Allergin-1 and CD300a may be candidates as molecular targets for the treatment of mast cell-dependent inflammatory diseases.
\end{abstract}

Keywords Mast cell $\bullet$ Inhibitory immunoreceptor $\bullet$ Allergin-1 $\bullet$ CD300a $\bullet$ Allergy

- Sepsis • Immunoreceptor tyrosine-based inhibitory motif (ITIM)

\footnotetext{
A. Shibuya $(\bowtie) \cdot S$. Tahara-Hanaoka

Department of Immunology, Faculty of Medicine, University of Tsukuba, Tsukuba, Ibaraki 305-8575, Japan

Department of Immunology, Life Science Center of Tsukuba Advanced Research Alliance (TARA), University of Tsukuba, Tsukuba, Ibaraki 305-8575, Japan

Department of Immunology, Japan Science and Technology Agency, Core Research for Evolutional Science and Technology (CREST), University of Tsukuba,

Tsukuba, Ibaraki 305-8575, Japan

e-mail: ashibuya@md.tsukuba.ac.jp

C. Nakahashi-Oda

Department of Immunology, Faculty of Medicine, University of Tsukuba,

Tsukuba, Ibaraki 305-8575, Japan

Department of Immunology, Japan Science and Technology Agency, Core Research for Evolutional Science and Technology (CREST), University of Tsukuba,

Tsukuba, Ibaraki 305-8575, Japan

K. Nakao et al. (eds.), Innovative Medicine, DOI 10.1007/978-4-431-55651-0_8
} 


\section{Introduction}

Activation of immune cells is regulated by positive and negative signals triggered by activating and inhibitory cell surface immunoreceptors, respectively. These [1] immunoreceptors play important roles in regulation of immune responses $[2,3]$. Inhibitory receptors are characterized by the immunoreceptor tyrosine-based inhibition motif (ITIM) in their cytoplasmic domains. The prototype 6-amino acid sequence for ITIM is (I/V/L/S)-X-Y-X-X-(L/V) (x denotes any amino acid), whose tyrosine is phosphorylated upon ligand binding, providing a docking site for the recruitment of Src homology 2 (SH2)-containing cytoplasmic phosphatases $[4,5]$ and shutting down activation signals by dephosphorylating intracellular substrates at the earliest steps of the activation response. The ITIM-bearing cell surface immunoreceptors, including certain NK receptors, Fc receptors (Fc $\gamma \mathrm{RIIb}$ ), and others, play a central role in mediating negative signals in both lymphoid and myeloid cells [6].

Mast cells express the high-affinity receptor for IgE (FceRI) on the cell surface, and play a central role in IgE-associated allergic responses [7, 8]. Crosslinking of FceRI-bound IgE with multivalent antigen initiates the activation of mast cells by promoting the aggregation of FceRI. This process results in the degranulation of mast cells, with the concomitant secretion of chemical mediators, such as histamine, tryptase, carboxypeptidase A, and proteoglycans, which are stored in the cytoplasmic granules, the de novo synthesis of pro-inflammatory lipid mediators, such as prostaglandins and leukotrienes, and platelet-activating factor (PAF) in the early phase (5-30 min after exposure to antigen), and the synthesis and secretion of cytokines and chemokines in the late phase (2-6 h after exposure to antigen) [9].

FceRI-mediated mast cell activation is modulated by several cell surface inhibitory receptors [10], including Fc $\gamma$ RIIB [4], paired Ig-like receptor (PIR)-B [11, 12], gp49B1 [13], mast-cell function-associated antigen (MAFA) [14, 15], and signal regulatory protein (SIRP)- $\alpha$ [16]. The cytoplasmic portion of these inhibitory receptors commonly contains the ITIM. When these inhibitory receptors are co-ligated with FceRI, the tyrosine residue in the ITIM is phosphorylated and recruits src homology 2 (SH2) domain-containing protein tyrosine phosphatase (SHP)-1, SHP-2, and/or SH2 domain-containing inositol 5-phosphatase (SHIP), thereby blocking the early step in the activation signal mediated by FceRI. The regulatory mechanisms of FceRI-mediated mast cell activation have not been completely elucidated, and a molecular target that controls allergic and inflammatory responses has not been identified.

We identified Allergin-1 and CD300a, which are novel inhibitory immunoglobulinlike receptors expressed on mast cells. In this chapter, we describe the molecular and functional characteristics of these receptors and discuss the possibility of these molecules as molecular targets for the therapy of allergy and inflammation. 


\section{Allergin-1}

\section{Identification of Allergin-1}

By using the signal sequence trap method [17], we identified a cDNA (MILRI) encoding an Ig-like receptor, designated Allergin-1, which consists of a 19-amino acid (aa) leader sequence, a 208-aa extracellular region composed of two Ig-like domains, a 21-aa transmembrane domain, and a 95-aa cytoplasmic domain (Fig. 1) [18]. We also identified two cDNAs encoding Allergin-1 isoforms, which lacked the first or second Ig-like domain in the extracellular portion and were designated Allergin-1 short form 1 (Allergin-1S1) and Allergin-1 short form 2 (Allergin-1S2), respectively (Fig. 1). The extracellular portions of mouse Allergin-1 and human Allergin-1L, and those of human Allergin-1S1 and Allergin-1S2, contained six and three potential N-linked glycosylation sites, respectively. Genomic DNA database analyses demonstrated that MILRI consists of ten exons. The genes encoding Allergin-1 (MILR1 and milr1) are located on chromosome 17q23.3 in humans and chromosome 11E1 in mice, near CD300 (or Cd300) family genes that encode

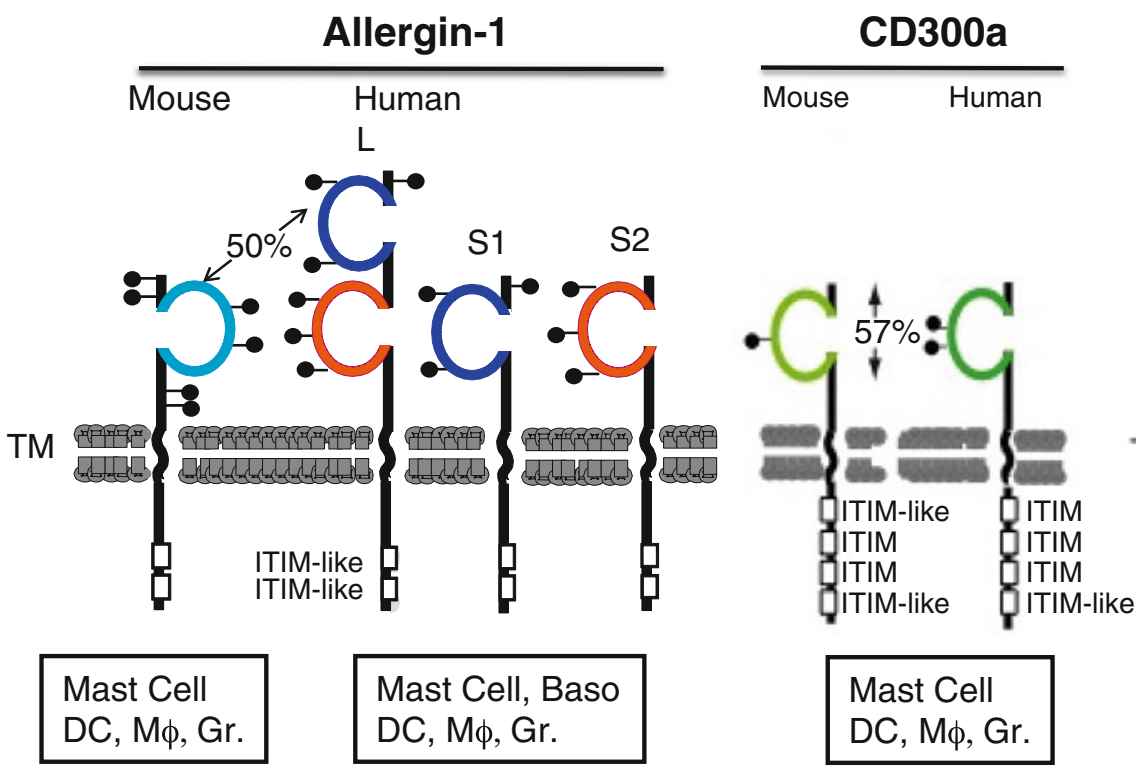

Fig. 1 Schematic diagram of mouse and human Allergin-1 and CD300a proteins. The transmembrane domain (TM) and the potential $\mathrm{N}$-linked glycosylation sites are indicated. The amino acid (aa) sequence of the first Ig-like domain (blue) in human Allergin-1-L is $50 \%$ identical to the Ig-like domain in mouse Allergin-1. The aa sequences of the first (blue) and second (red lined) Ig-like domain in human Allergin-1-L are identical to those of Allergin-1-S1 and Allergin-1-S2. The amino acid sequence of the Ig-like domain in human CD300a is $57 \%$ identical to the Ig-like domain in mouse CD300a. DC (dendritc cell), MФ (macrophage), Gr (neutrophil) 
Ig-like receptors mediating positive or negative signals in myeloid cells [19, 20]. MILRI and milr1 were also close to the gene, which encodes PECAM-1 (CD31 or Cd31), an ITIM-bearing Ig-like receptor expressed on myeloid cells and platelets.

\section{Expression of Allergin-1}

Flow cytometry analyses of spleen cells demonstrated that Allergin-1 is expressed on dendritic cells, macrophages and neutrophils, but not on $\mathrm{T}, \mathrm{B}$, or natural killer (NK) cells in mice. However, Allergin-1 was most strongly expressed on mast cells in the peritoneal cavity. In contrast, Allergin-1 was not detected on basophils in the bone marrow. In agreement with mouse Allergin-1, human Allergin-1L and/or Allergin-1S1 were also expressed in myeloid cell lineages, including monocytes, neutrophils, and dendritic cells, in the peripheral blood. It was noted that, unlike mouse Allergin-1, human Allergin-1L and/or Allergin-1S1 were considerably expressed on peripheral blood basophiles [18].

Anti-human Allergin-1 monoclonal antibodies (mAbs) EX32 and EX33 recognize an epitope of the first and the second Ig-like domain of Allergin-1L, respectively. By using these $\mathrm{mAbs}$ simultaneously together with $\mathrm{mAbs}$ against the lineage markers (CD3, CD19, CD56, CD11b, and CD11c), c-Kit and FceRI $\alpha$, we developed a multi-color flow cytometric method to characterize the human primary mast cells in the bronchial alveolar lavage fluid (BALF). Mast cells were defined as $\mathrm{PI}^{-} \mathrm{CD} 45^{+} \mathrm{Lin}^{-} \mathrm{c}-\mathrm{Kit}^{+} \mathrm{Fc} \varepsilon \mathrm{RI} \alpha^{+} \mathrm{cells}$, which comprised $0.153 \% \pm 0.041 \%(n=28)$ of the total cell population in BALF. We found diverse expression profiles of Allergin-1 isoform on BAL mast cells among donors; however, Allergin-1S1 was dominantly expressed on BAL-MC, compared with Allergin-1L1 or Allergin-1S2 (Fig. 2) [21].

\section{Function of Allergin-1}

\section{In Vitro Analyses of Allergin-1}

Allergin-1 contains immunoreceptor tyrosine-based inhibitory motif (ITIM)-like sequences in the cytoplasmic portion, suggesting that Allergin-1 is tyrosine phosphorylated and recruits the SH2-containing phosphatases, such as SHP-1, SHP-2, or SHIP. Because Allergin-1 is strongly expressed on mast cells, we examined whether mouse Allergin-1 mediates an inhibitory signal against FceRI-mediated degranulation, by using a rat basophil leukemia cell line, RBL-2H3. Co-ligation of Allergin-1 with FceRI significantly decreased degranulation, as determined by $\beta$-hexosaminidase release from the transfectants, when compared with FceRI stimulation alone, suggesting that mouse Allergin-1 inhibits IgE-mediated degranulation of mast cells (Fig. 3).

Because an insufficient number of human primary mast cells can be obtained to conduct the ELISA assay for chemical mediators such as histamine or $\beta$-hexosaminidase released from mast cells of BALF samples, we performed an 

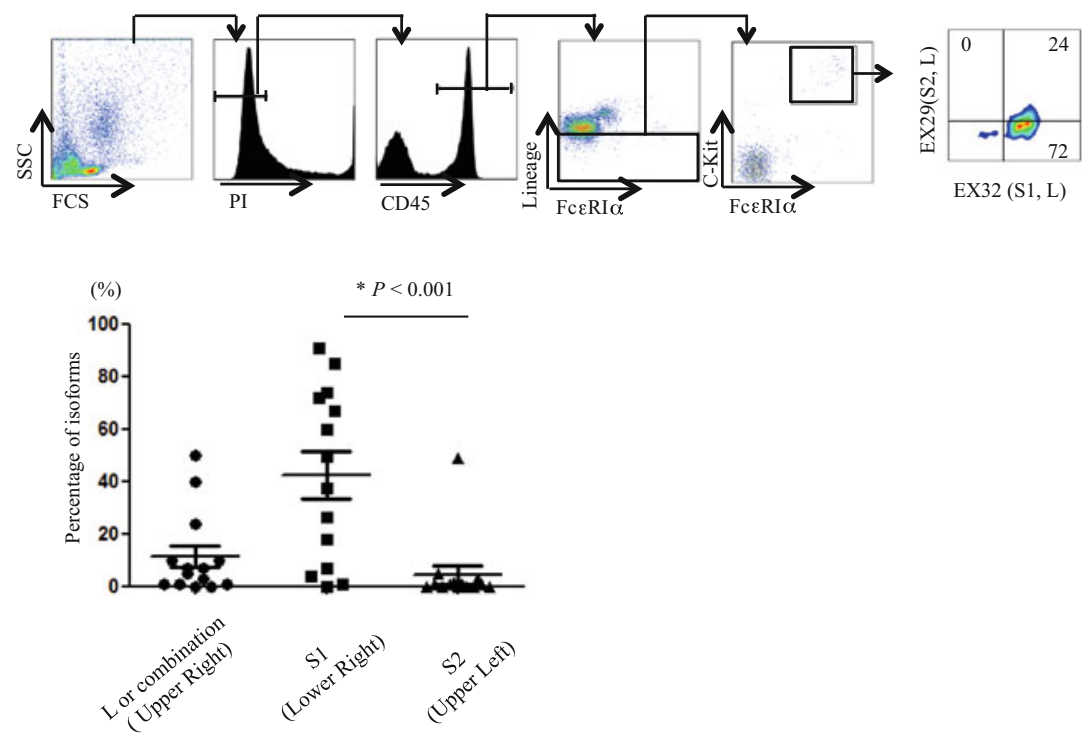

Fig. 2 Expression of Allergin-1 isoforms on human mast cells in bronchial alveolar lavage fluid (BALF). Cells obtained from the BALF of patients with pulmonary diseases $(n=14)$ were stained with PI, anti-CD45, lineage mAb cocktail containing anti-CD3, anti-CD19, anti-CD56, anti$\mathrm{CD} 11 \mathrm{~b}$, and anti-CD11c mAbs, anti-FceRI $\alpha$, and anti-c-Kit mAbs together with isotype control, and EX32 and/or EX29 mAb, and the BAL-MC were analyzed by using flow cytometry. Representative results are shown in the upper panel. "Combinations" indicates mast cells in the upper right quadrant that express either $\mathrm{L}+\mathrm{S} 1, \mathrm{~L}+\mathrm{S} 2, \mathrm{~L}+\mathrm{S} 1+\mathrm{S} 2$, or $\mathrm{S} 1+\mathrm{S} 2$

activation assay by utilizing a multicolor flow cytometry to detect cell surface CD107a as a marker of mast cell degranulation. We detected mast cells derived from the culture of cord blood and peripheral blood stem cells that turned out to be positive for CD107a expression on the cell surface when the mast cells were stimulated with anti-TNP IgE followed by TNP-conjugated control mAb. However, CD $107 \mathrm{a}^{+}$cells were significantly decreased when FceRI $\alpha$ was colligated with Allergin-1 with anti-TNP IgE and TNP-conjugated anti-Allergin-1 mAb [21]. We then examined the inhibitory function of Allergin-1 on mast cells in BALF. BALF cells were also stimulated and then stained with anti-CD107a and mAbs for the identification of BAL mast cells, as described above, and analyzed by using multi-color flow cytometry. We detected a subpopulation of BAL mast cells that was positive for CD107a when the BALF cells were stimulated with anti-TNP IgE followed by TNP-conjugated control mAb. However, the population of CD107a ${ }^{+}$ mast cells was significantly decreased when BAL mast cells were stimulated via anti-TNP IgE and TNP-conjugated anti-Allergin-1 mAb [21]. Since BAL mast cells also preferentially express Allergin-1S1 rather than Allergin-1L or Allergin-1S2, these results indicated that Allergin-1S1 inhibits the IgE-mediated activation of BAL mast cells. 


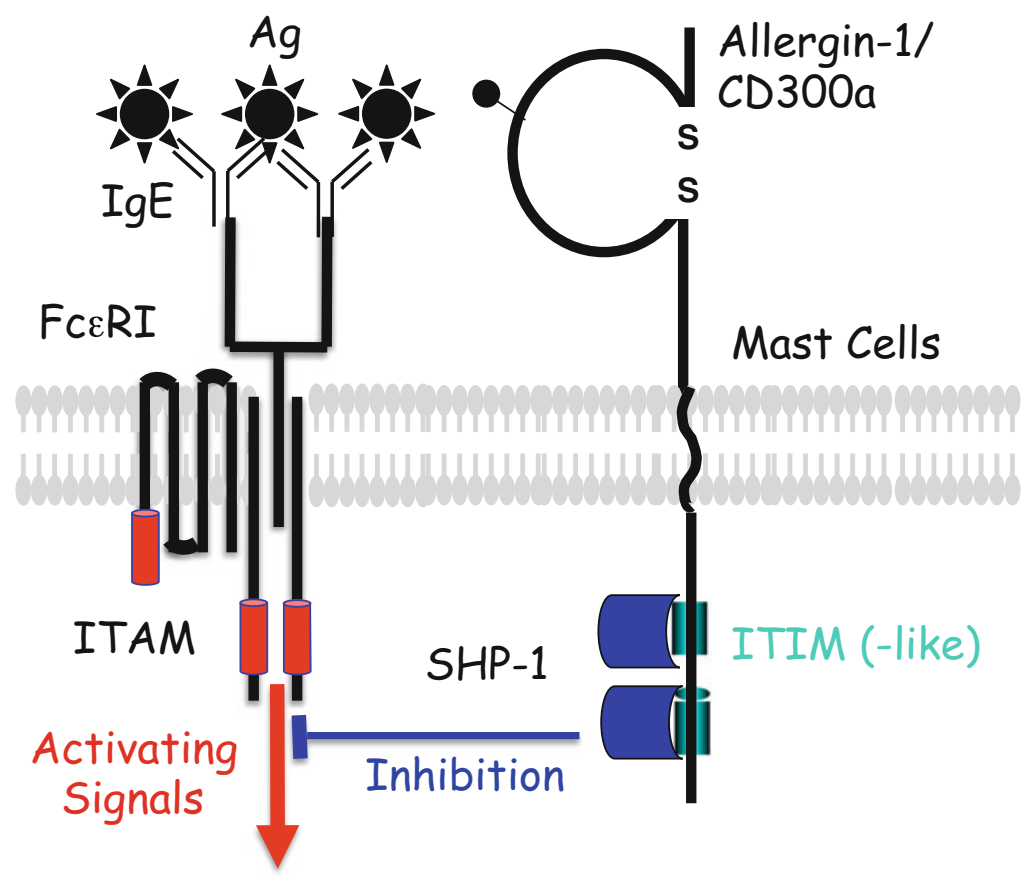

Fig. 3 Schematic model of Allergin-1 and CD300a-mediated inhibition of FceRI signaling in mast cells. Allergin-1 and CD300a recruit tyrosine phosphatases such as SHP-1 and SHP-2 to the ITIM in the cytoplasmic region. The activated tyrosine phosphatases then dephosphorylate tyrosine phosphorylated FceRI $\gamma$-mediated signaling molecules and shut down FceRI-mediated mast cell activation

\section{In Vivo Analyses of Allergin-1}

Since Allergin-1 inhibited FceRI-mediated degranulation of mast cells in vitro, we next examined whether Allergin-1 was involved in passive systemic and cutaneous anaphylaxises (PSA), an IgE-mediated type 1 immediate hypersensitivity reaction. WT and Allergin-1-deficient mice were passively sensitized with anti-TNP IgE $\mathrm{mAb}$ and then intravenously injected with ovalbumin (OVA) or TNP-conjugated OVA (TNP-OVA). WT mice challenged with TNP-OVA showed a progressive decrease in rectal temperature to $4{ }^{\circ} \mathrm{C}$ below the basal temperature by 18 min after injection (Fig. 4). Allergin-1-deficient mice challenged with TNP-OVA showed significantly lower rectal temperatures than the corresponding WT mice (Fig. 4) [18].

WT and Allergin-1-deficient mice were also passively sensitized by intravenous injection of anti-dinitrophenol (DNP) $\operatorname{IgE} \mathrm{mAb}$ and then challenged with epicutaneous application of dinitrofluorobenzene (DNFB) in acetone/olive oil in the left ear and acetone/olive oil alone in the right ear. The ear swelling in Allergin-1-deficient mice was significantly greater than the ear swelling in WT mice during the entire $50 \mathrm{~h}$ 


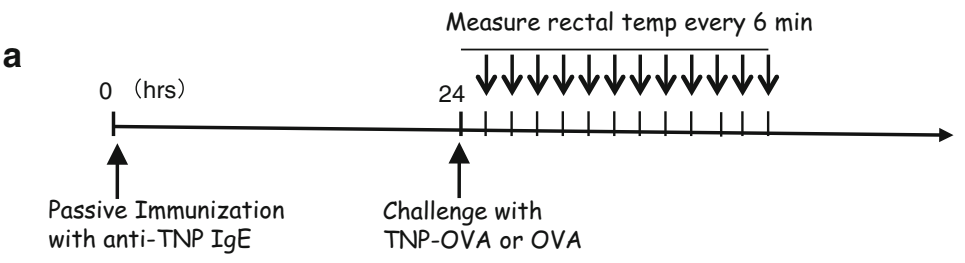

b

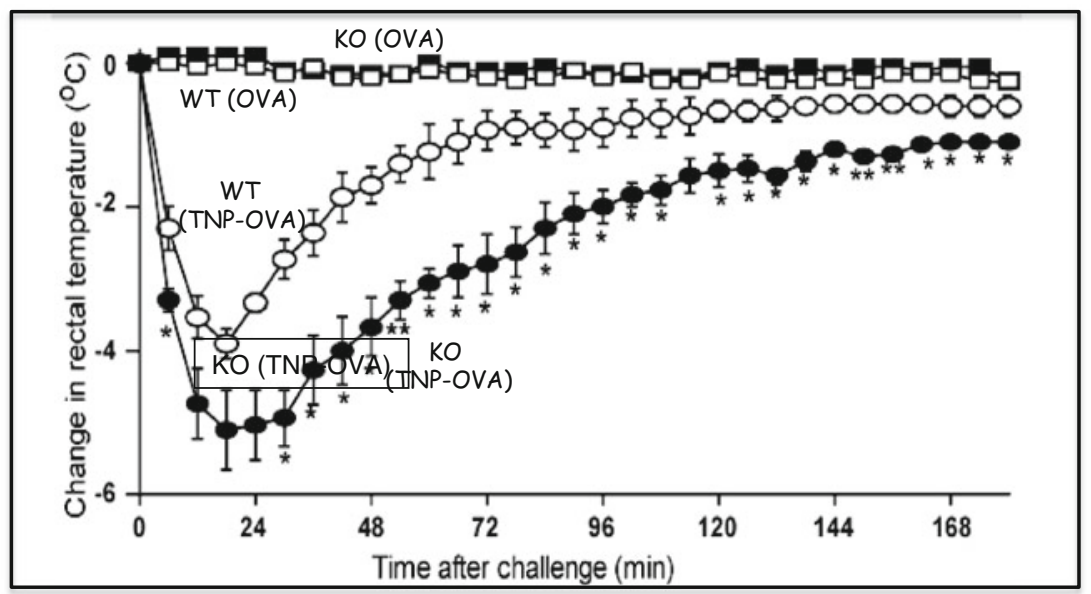

Fig. 4 Enhanced systemic anaphylaxis in Allergin-1-deficient mice. (a) Allergin-1 ${ }^{+/+}$mice (WT) and Allergin-1 $1^{-/}(\mathrm{KO})$ mice were intravenously injected with $20 \mu \mathrm{g}$ of anti-TNP mouse IgE mAb. (b) After $24 \mathrm{~h}$, mice were challenged with $1 \mathrm{mg}$ of OVA or $\mathrm{TNP}_{6}$-conjugated OVA, and rectal temperatures were measured every $6 \mathrm{~min}$. The data are the mean $\pm \operatorname{SEM}(n=3) . * P<0.05$, $* * P<0.01, * * * P<0.005$ for the comparison of WT versus KO mice challenged with TNP-OVA

observation period after the antigen challenge [18]. Taken together, these results suggest that Allergin-1 negatively regulates IgE-mediated mast cell activation in vivo and suppresses the type 1 immediate hypersensitivity reactions.

\section{CD300a}

\section{Identification and Expression of CD300a}

To identify novel genes involved in immune responses by myeloid cells, we performed representative differential analysis (RDA), which is a PCR-based subtractive hybridization, using day 14 fetal livers from PU.1 $1^{-/-}$mice lacking myeloid cells and control littermates, followed by screening of a macrophage cDNA library. We cloned a cDNA encoding a type-1 transmembrane protein with one Ig-like domain in the extracellular portion, indicating that the protein, designated myeloidassociated immunoglobulin-like receptor (MAIR)-I (CD300a), is a member of the 
Ig superfamily (Fig. 1). The cytoplasmic region contains the consensus sequence for ITIMs, and the ITIM-like sequences. The CD300a gene is located in the proximal region of the E2 band of mouse chromosome 11 and consists of six exons, as determined by fluorescence in situ hybridization (FISH) and a genomic DNA sequence database. Biochemical analyses demonstrated that the molecular weight of CD300a is $~ 50 \mathrm{KDa}$ protein when analyzed under both reducing and non-reducing conditions [22].

CD300a is expressed on the majority of myeloid cells, including macrophages, dendritic cells, granulocytes, and bone-marrow-derived cultured mast cells, and a subset of B cells, but neither on T nor on NK cells.

\section{Identification of a Ligand for CD300a}

To identify the ligand for CD300a, we generated a chimeric fusion protein of the extracellular portion of CD300a with the Fc portion of human IgG (CD300a-Fc) (Fig. 5a). Although the CD300a-Fc did not stain any viable cells tested, we found that CD300a-Fc bound apoptotic cells (Fig. 5b). A neutralizing monoclonal antibody (mAb)

a

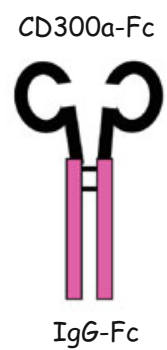

b

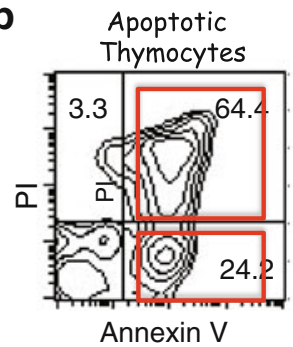

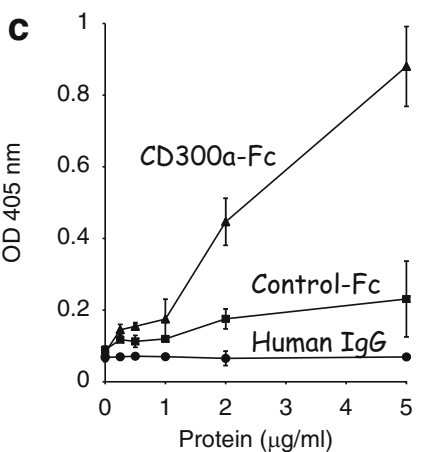

Annexin $\mathrm{V}^{-}, \mathrm{Pl}^{-} \quad$ Annexin $\mathrm{V}^{+}, \mathrm{PI}^{-} \quad$ Annexin $\mathrm{V}^{+}, \mathrm{PI}^{+}$

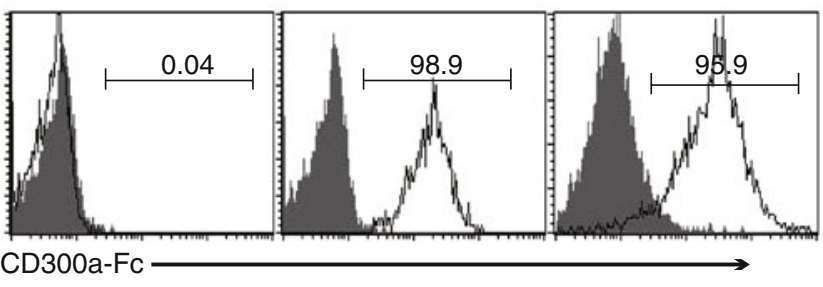

Fig. 5 CD300a-Fc binds phosphatidylserine on apoptotic cells. (a, b) Dexamethasone-treated mouse thymocytes were stained with APC-conjugated annexin $\mathrm{V}$ and mouse $\mathrm{CD} 300 \mathrm{a}-\mathrm{Fc}$ followed by an FITC-conjugated antibody against human IgG and PI in the presence of $\mathrm{CaCl}_{2}$ and analyzed by flow cytometry. (c) Microtiter plates coated with PS were incubated with increasing concentrations of CD300a-Fc, control protein-Fc, or human IgG, and PS binding was analysed by ELISA. Error bars show SDs 
against mouse CD300a (TX41) specifically inhibited the CD300a-Fc binding to apoptotic cells. Solid-phase ELISA confirmed that CD300a directly bound PS in a CD300a dose-dependent manner (Fig. 5c). These results suggested that CD300a is a PS receptor [23].

\section{Function of CD300a}

\section{In Vitro Analyses of CD300a}

Several receptors for PS are expressed on phagocytes and are involved in clearing apoptotic cells in physiological and pathological settings [24-29]. However, we found that CD300a does not mediate phagocytosis of apoptotic cells by macrophages [23].

CD300a was tyrosine phosphorylated and co-immunoprecipitated with SHP-1, SHP-2, and SHIP after stimulation with pervanadate in the RBL-2H3 transfectant upon stimulation with pervanadate. We demonstrated that cross-linking CD300a and FceRI with anti-CD300a mAb and IgE antibody, followed by co-ligation with a common secondary antibody, induced inhibition of IgE-mediated degranulation from RBL-2H3 transfectant expressing CD300a and BM-derived cultured mast cells [30].

To address whether apoptotic cells affect mast cell activation via CD300a, we generated bone marrow (BM)-derived mast cells (BMMCs) from CD300a-deficient or WT mice. WT or CD300a-deficient BMMCs were cocultured with apoptotic cells in the presence of LPS. Although we did not detect any cytokines or chemokines in the culture supernatants in the absence of stimuli, stimulation with LPS induced both WT and CD300a-deficient BMMCs to produce TNF- $\alpha$, IL-13, and MCP-1; however, CD300a-deficient BMMCs produced them at significantly higher concentrations, suggesting that CD300a-PS interaction inhibits these cytokines and chemokines production from BMMCs [23].

\section{In Vivo Analyses of CD300a}

TNF- $\alpha$, IL-13, and MCP-1 produced by mast cells are chemoattractants for neutrophils and play an important role in bacterial clearance in a CLP peritonitis model in mice [31-34]. Therefore, we hypothesized that CD300a affects immune regulation by mast cells at the site of peritonitis, where large numbers of cells undergo apoptosis in the peritoneal cavity [35]. In fact, we observed that more than $10 \%$ of cells in the peritoneal cavity $4 \mathrm{~h}$ after CLP were apoptotic cells, as determined by staining with annexin V. To test the hypothesis, we subjected WT and CD300a-deficient mice to CLP and observed that $C d 300 \mathrm{a}^{-/-}$mice survived significantly longer than did WT mice after CLP [23]. 


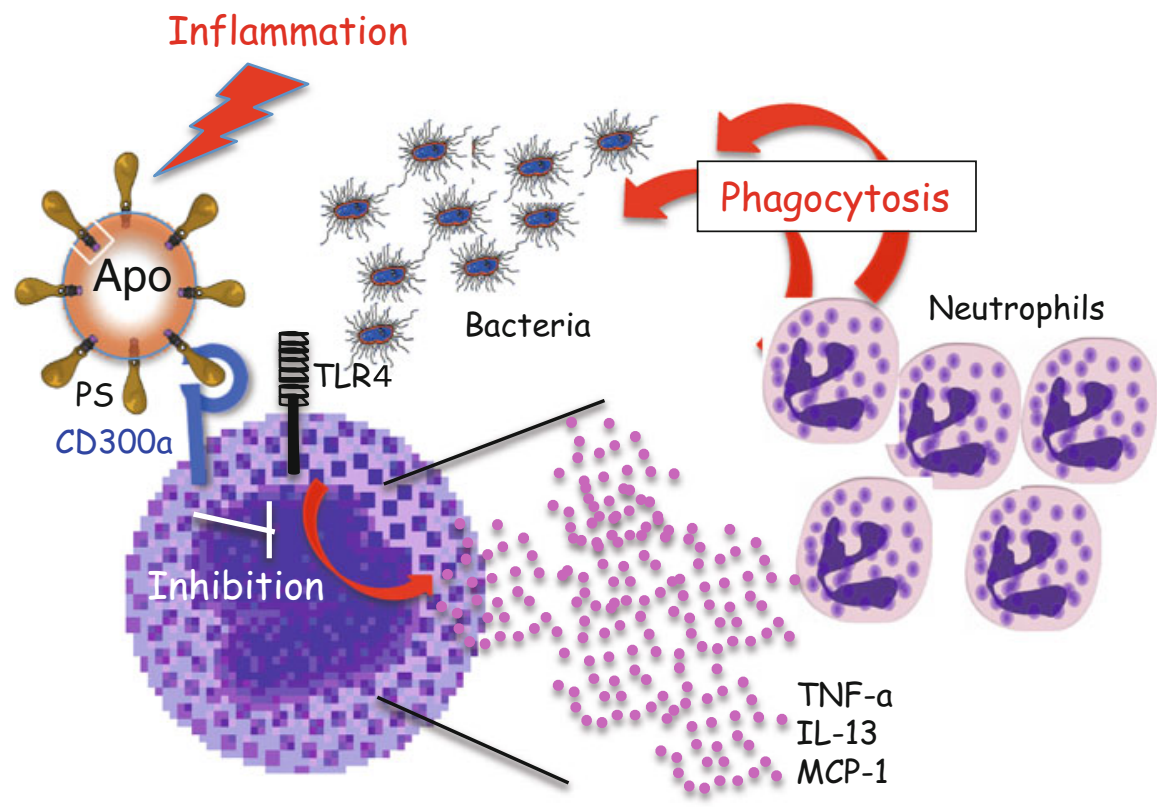

Fig. 6 CD300a inhibits the TLR4-mediated signal for production of chemoattractants for neutrophils. Upon binding to phosphatidylserine (PS) on apoptotic cells, CD300a mediates an inhibitory signal, resulting in the suppression of the TLR4-mediated signal for production of chemoattractants, including TNF- $\alpha$, IL-13, and MCP-1, for neutrophils, which are involved in phagocytosis of Gram-negative bacteria

In the early phase of CLP peritonitis model, mast cells play an important role in recruitment of neutrophils into the peritoneal cavity by secretion of chemoattractants for neutrophils such as TNF- $\alpha$ and prolong the survival $[32,36]$. We demonstrated that CD300a-deficient BMMCs produced a greater amount of chemoattractants for neutrophils than did WT BMMCs in the peritoneal cavity after CLP by transfer experiments into $\mathrm{Kit}^{\mathrm{W}-\mathrm{sh} / \mathrm{W}-\mathrm{sh}}$ mice, which are deficient in mast cells, before CLP [23]. Our results suggested that $\mathrm{CD} 300 \mathrm{a}$ on mast cells was primarily responsible for the phenotype of prolonged survival of CD300a-deficient mice after CLP (Fig. 6).

\section{Blockade of CD300a-PS Interaction Prolonged Survival of Mice After CLP}

Because CD300a-deficient mice survived longer after CLP, we examined whether an antibody against mouse CD300a (TX41) could have a prophylactic effect on CLP-induced sepsis. TX41 does not deplete myeloid cells, including mast cells. Intraperitoneal injection of mice with TX41 $1 \mathrm{~h}$ before and $18 \mathrm{~h}$ after CLP significantly increased neutrophil numbers in the peritoneal cavity, improved bacterial clearance $4 \mathrm{~h}$ after CLP [23], and prolonged survival compared with treatment with 

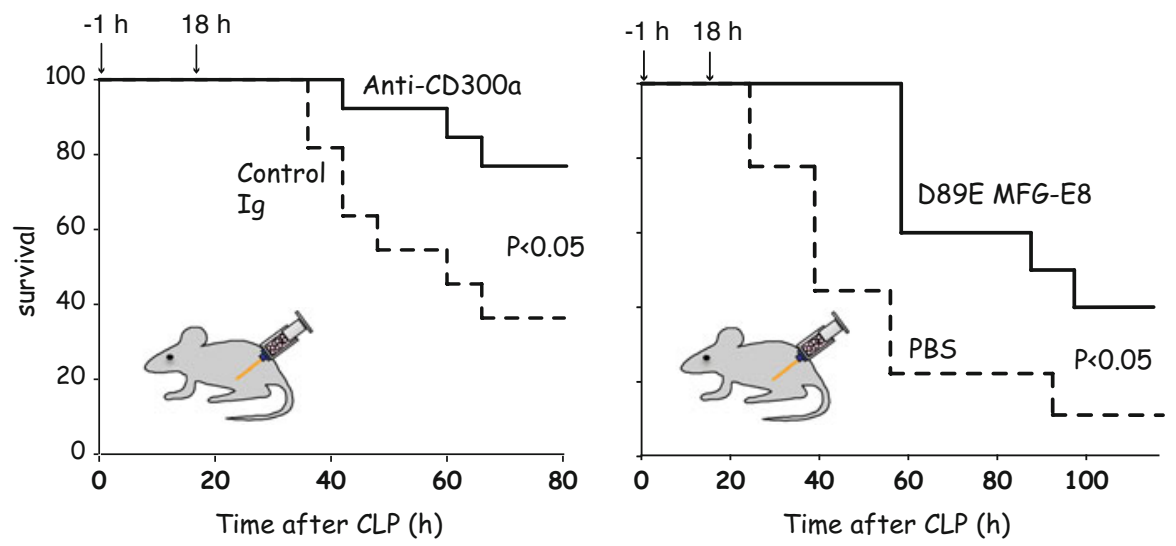

Fig. 7 The blockade of the CD300a-phosphatidylserine interaction prolongs survival of mice after CLP. Mice were injected intraperitoneally with control antibody, anti-CD300a monoclonal antibody (TX41), D89E MFG-E8, or PBS $1 \mathrm{~h}$ before and $18 \mathrm{~h}$ after CLP, and the survival rates are shown

a control antibody (Fig. 7). Similarly, intraperitoneal injection with mutated milk fat globule EGF factor 8 at residue 89 (D89E MFG-E8), which is able to block the interaction between PS and CD300a, also prolonged survival of WT mice compared with treatment with PBS. Moreover, D89E MFG-E8 improved bacterial clearance of WT mice [23]. These results provided the formal evidence that PS on apoptotic cells affected the bacterial clearance. Thus, blocking the interaction between PS and its receptor CD300a is potentially a useful therapy for prophylaxis against peritonitis-induced sepsis.

\section{Conclusion}

Mast cells express pathogen-associated molecular patterns (PAMPs) as well as FceRI, which mediate activation signal in mast cells, resulting in various chemokines' and cytokines' secretion and degranulation from mast cells. Thereby, they are involved in a variety of allergic and non-allergic inflammatory diseases. Here, we have described molecular and functional characteristic of Allergin-1 and CD300a, which are expressed on mast cells and inhibit activating signals mediated by FceRI and TLR-4 in vitro and in vivo. Thus, these inhibitory immunoreceptors play an important role in regulation of mast cell-dependent inflammatory responses. In fact, we showed that anti-CD300a neutralizing antibody prolonged survival in a sepsis mouse model. Further studies on regulation of mast cell activation by using these inhibitory immunoreceptor as targets for the therapy for allergy and inflammation might be promising. 
Open Access This chapter is distributed under the terms of the Creative Commons Attribution Noncommercial License, which permits any noncommercial use, distribution, and reproduction in any medium, provided the original author(s) and source are credited.

\section{References}

1. Bachelet I et al (2005) The inhibitory receptor IRp60 (CD300a) is expressed and functional on human mast cells. J Immunol 175(12):7989-7995

2. Ravetch JV, Lanier LL (2000) Immune inhibitory receptors. Science 290(5489):84-89

3. Lanier LL (2001) Face off-the interplay between activating and inhibitory immune receptors. Curr Opin Immunol 13(3):326-331

4. Malbec $\mathrm{O}$ et al (1998) $\mathrm{Fc}$ epsilon receptor I-associated lyn-dependent phosphorylation of $\mathrm{Fc}$ gamma receptor IIB during negative regulation of mast cell activation. J Immunol 160(4): 1647-1658

5. Smith KG (1998) Decisions to remove asymptomatic third molars require rational criteria. Br Dent J 184(11):546

6. Daeron $\mathrm{M}$ et al (2008) Immunoreceptor tyrosine-based inhibition motifs: a quest in the past and future. Immunol Rev 224:11-43

7. Galli SJ (2000) Mast cells and basophils. Curr Opin Hematol 7(1):32-39

8. Schwartz LB (1994) Mast cells: function and contents. Curr Opin Immunol 6(1):91-97

9. Kawakami T, Galli SJ (2002) Regulation of mast-cell and basophil function and survival by IgE. Nat Rev Immunol 2(10):773-786

10. Li L, Yao Z (2004) Mast cell and immune inhibitory receptors. Cell Mol Immunol 1(6):408-415

11. Maeda A et al (1998) Requirement of SH2-containing protein tyrosine phosphatases SHP-1 and SHP-2 for paired immunoglobulin-like receptor B (PIR-B)-mediated inhibitory signal. J Exp Med 187(8):1355-1360

12. Yamashita Y, Ono M, Takai T (1998) Inhibitory and stimulatory functions of paired Ig-like receptor (PIR) family in RBL-2H3 cells. J Immunol 161(8):4042-4047

13. Katz HR et al (1996) Mouse mast cell gp49B1 contains two immunoreceptor tyrosine-based inhibition motifs and suppresses mast cell activation when coligated with the high-affinity $\mathrm{Fc}$ receptor for IgE. Proc Natl Acad Sci U S A 93(20):10809-10814

14. Jurgens L et al (1996) Proximity relationships between the type I receptor for Fc epsilon (Fc epsilon RI) and the mast cell function-associated antigen (MAFA) studied by donor photobleaching fluorescence resonance energy transfer microscopy. Eur J Immunol 26(1):84-91

15. Xu R et al (2001) SH2 domain-containing inositol polyphosphate 5'-phosphatase is the main mediator of the inhibitory action of the mast cell function-associated antigen. J Immunol 167(11):6394-6402

16. Lienard $\mathrm{H}$ et al (1999) Signal regulatory proteins negatively regulate immunoreceptordependent cell activation. J Biol Chem 274(45):32493-32499

17. Jacobs KA et al (1997) A genetic selection for isolating cDNAs encoding secreted proteins. Gene 198(1-2):289-296

18. Hitomi K et al (2010) An immunoglobulin-like receptor, Allergin-1, inhibits immunoglobulin E-mediated immediate hypersensitivity reactions. Nat Immunol 11(7):601-607

19. Nakahashi-Oda $C$ et al (2012) Apoptotic cells suppress mast cell inflammatory responses via the CD300a immunoreceptor. J Exp Med 209(8):1493-1503

20. Clark GJ et al (2009) The CD300 family of molecules are evolutionarily significant regulators of leukocyte functions. Trends Immunol 30(5):209-217

21. Nagai $\mathrm{K}$ et al (2013) Expression and function of Allergin-1 on human primary mast cells. PLoS One 8(10):e76160 
22. Yotsumoto K et al (2003) Paired activating and inhibitory immunoglobulin-like receptors, MAIR-I and MAIR-II, regulate mast cell and macrophage activation. J Exp Med 198(2):223-233

23. Nakahashi-Oda C et al (2012) Identification of phosphatidylserine as a ligand for the CD300a immunoreceptor. Biochem Biophys Res Commun 417(1):646-650

24. Miyanishi M et al (2007) Identification of Tim4 as a phosphatidylserine receptor. Nature 450(7168):435-439

25. Hanayama R et al (2002) Identification of a factor that links apoptotic cells to phagocytes. Nature 417(6885):182-187

26. Kobayashi N et al (2007) TIM-1 and TIM-4 glycoproteins bind phosphatidylserine and mediate uptake of apoptotic cells. Immunity 27(6):927-940

27. Park D et al (2007) BAI1 is an engulfment receptor for apoptotic cells upstream of the ELMO/ Dock180/Rac module. Nature 450(7168):430-434

28. Park SY et al (2008) Rapid cell corpse clearance by stabilin-2, a membrane phosphatidylserine receptor. Cell Death Differ 15(1):192-201

29. Scott RS et al (2001) Phagocytosis and clearance of apoptotic cells is mediated by MER. Nature 411(6834):207-211

30. Okoshi Y et al (2005) Requirement of the tyrosines at residues 258 and 270 of MAIR-I in inhibitory effect on degranulation from basophilic leukemia RBL-2H3. Int Immunol 17(1):65-72

31. Marshall JS (2004) Mast-cell responses to pathogens. Nat Rev Immunol 4(10):787-799

32. Echtenacher B, Mannel DN, Hultner L (1996) Critical protective role of mast cells in a model of acute septic peritonitis. Nature 381(6577):75-77

33. Matsukawa A et al (1999) Endogenous monocyte chemoattractant protein-1 (MCP-1) protects mice in a model of acute septic peritonitis: cross-talk between MCP-1 and leukotriene B4. J Immunol 163(11):6148-6154

34. Baumhofer JM et al (1998) Gene transfer with IL-4 and IL-13 improves survival in lethal endotoxemia in the mouse and ameliorates peritoneal macrophages immune competence. Eur J Immunol 28(2):610-615

35. Hotchkiss RS, Nicholson DW (2006) Apoptosis and caspases regulate death and inflammation in sepsis. Nat Rev Immunol 6(11):813-822

36. Malaviya R et al (1996) Mast cell modulation of neutrophil influx and bacterial clearance at sites of infection through TNF-alpha. Nature 381(6577):77-80 


\title{
Doxycycline-Inducible Autoimmune Blistering Skin Disease Model
}

\author{
Wataru Nishie and Hiroshi Shimizu
}

\begin{abstract}
Bullous pemphigoid (BP) is the most common autoimmune blistering disorder. BP autoantibodies target two hemidesmosomal components, collagen XVII (COL17) and BP230, with autoimmunity to COL17 being mainly involved in the development of the disease. BP most commonly affects the elderly, and systemic corticosteroids are widely used to treat blisters on the entire body. Therefore, severe cases are sometimes fatal. Toward developing innovative, disease-specific therapies with fewer adverse effects, faithful disease models are essential. However, it has been challenging to reproduce BP in animals, because of inter-species differences in the amino acid sequences of pathological epitopes on human and mouse COL17. Human IgG autoantibodies from BP patients are unable to bind with mouse COL17; thus, the passive transfer of BP-IgG into mice fails to induce blistering disease. To overcome inter-species differences, we have generated genetically modified mice that express human but not mouse COL17 in skin. We call these "COL17-humanized mice". Using these mice, we have produced different BP models to elucidate the BP pathomechanism and to produce novel BP therapies. Currently, another novel model is under development. Here, we introduce the BP models that we have developed by using a novel technique called "humanization of autoantigen".
\end{abstract}

Keywords Autoimmunity $\bullet$ Collagen XVII • BP180 • Blistering disease $\bullet$ Skin $\bullet$ Humanized mouse

W. Nishie, M.D., Ph.D. • H. Shimizu, M.D., Ph.D. $(\bowtie)$

Department of Dermatology, Hokkaido University Graduate School of Medicine,

N15W7, Kita-ku, Sapporo 003-0835, Japan

e-mail: shimziu@med.hokudai.ac.jp

K. Nakao et al. (eds.), Innovative Medicine, DOI 10.1007/978-4-431-55651-0_9 


\section{Bullous Pemphigoid (BP), The Most Common Autoimmune Blistering Disease}

\section{Clinical Features of BP}

Bullous pemphigoid (BP) is the most common autoimmune blistering skin disorder, and the elderly are the most prone to it $[1,2]$. In typical cases of BP, urticarial erythemas with tense blisters on the trunk and extremities associated with pruritus are observed (Fig. 1a). BP usually develops around the seventh to eighth decades of life in both sexes. Individuals younger than age 50 rarely develop BP. Previous retrospective studies in European countries have shown a prevalence of around 6.6:1,000,000 per year, but recent analyses have shown an increased prevalence of 21.7 66:1,000,000 [3]. This increase is probably due to population aging and to development of diagnostic tools such as ELISA assays that can easily detect circulating autoantibodies (autoAbs).

\section{Pathogenesis of BP}

Direct immunofluorescence (IF) studies of the perilesional skin shows linear in vivo deposition of IgG and C3 at the dermal-epidermal junction (DEJ) (Fig. 1b), and circulating IgG autoAbs binding the DEJ of the skin can be found in sera from BP
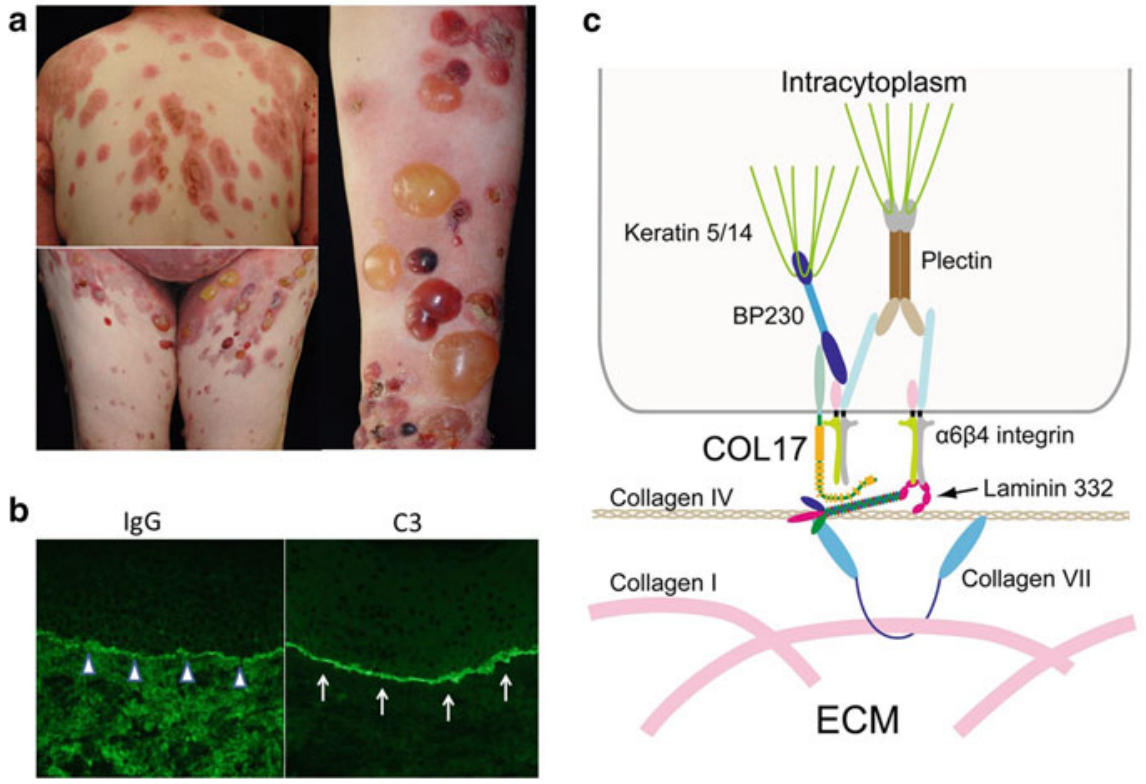

Fig. 1 Clinical and immunopathological manifestations of BP. (a) Urticarial erythema with tense blisters. (b) DIF studies of the lesional skin show linear in vivo deposition of IgG (arrowheads) and C3 (arrows) at the DEJ. (c) COL17 and hemidesmosomal molecules at the DEJ (partially modified from Nishie et al. [4]) 
patients by indirect IF. The autoAbs in BP patients target two hemidesmosomal components: transmembrane collagen XVII (COL17, also called BP180 or BPAG2) and the plakin family protein BP230 (BPAG1) (Fig. 1c). Of these, COL17 is thought to be the major autoantigen (autoAg) [4]. The IgG autoAbs against the COL17 in the $\mathrm{DEJ}$ of the skin are considered to trigger the inflammatory process, resulting in the disruption of dermal-epidermal anchoring [2, 3]. It remains uncertain as to whether anti-BP230 autoantibodies in BP patients are pathogenic for blister formation or whether they are just by-products of epitope-spreading associated with disease extension.

\section{COL17 as a Main AutoAg for BP}

COL17 is a type-II-oriented 1,497-amino-acid transmembrane collagen whose amino (N) terminus is in the cytoplasm and whose carboxyl (C) terminus is in the extracellular matrix (ECM) [5, 6]. As described in Fig. 1c, the extracellular domain of COL17 interacts with laminin 332 and collagen IV in the ECM, and the farthest part of the C-terminal region of COL17 has a flexible tail in vivo [7]. The extracellular domain of COL17 is constitutively cleaved (shed) from the cell surface by ADAM 9/10/17 [8, 9], a phenomenon that occurs within a juxtamembranous extracellular non-collagenous NC16A domain of 77 amino acids. Interestingly, the majority of IgG autoAbs from $\mathrm{BP}$ patients react with this NC16A domain, and epitopes cluster tightly in the $\mathrm{N}$-terminal 45 -amino-acid stretch of this region (Fig. 2a) [10, 11]. These findings indicate that autoAbs to the NC16A domain are pathogenic for blister formation in BP. However, human IgG autoantibodies from BP patients are unable to bind with mouse COL17; thus, the passive transfer of BP-IgG into mice fails to induce blistering disease [12]. This is attributed to the significant differences between human COL17 and mouse COL17 in amino acid sequences of the NC16A domain (Fig. 2a).

\section{BP Models Produced by "Humanization of Autoantigen"}

\section{Generation of COL17-Humanized Mice}

The pathological relevance of Abs to COL17 has been studied by neonatal mouse models that have been produced by injecting rabbit Abs targeting the NC14A domain of mouse COL17 (corresponding to NC16A domain of human COL17). When rabbit Abs targeting mouse COL17 were passively transferred into wild-type neonatal mice, the rabbit Abs bound with the DEJ of the mice skin, after which complement activation, mast cell degradation, neutrophil recruitment and, finally, epidermal detachment by mild friction occurred [12]. Using the experimental BP model mice induced by rabbit IgG against mouse COL17, complement activation followed by mast cell degradation and neutrophil infiltration have been suggested to be involved in the blister formation [13-15]. However, the pathogenic relevance of human autoAbs to COL17 in BP patients has never been proved. In 2007, our group 
a
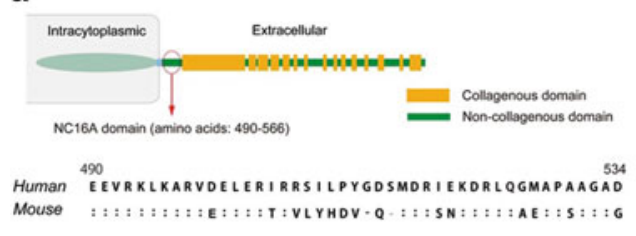

b

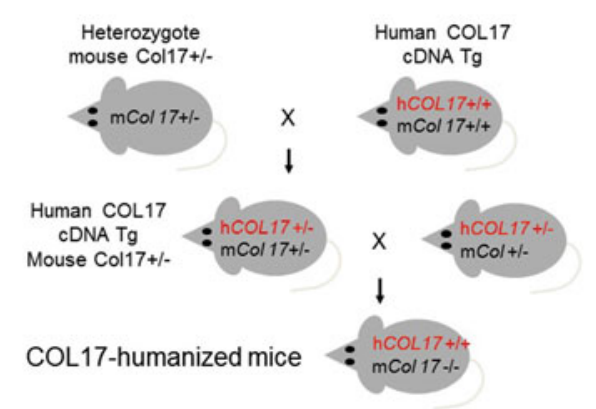

c

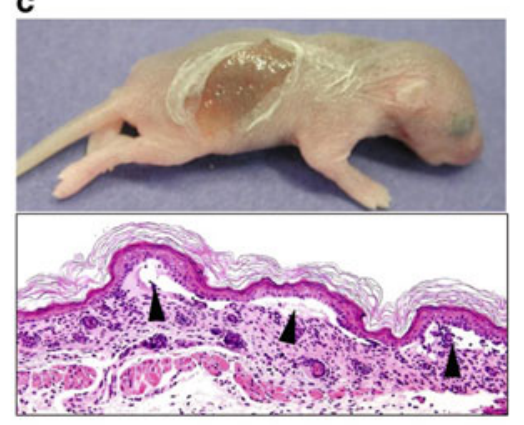

d

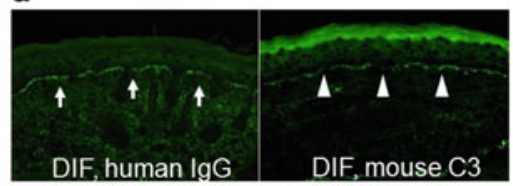

Fig. 2 Generation of the COL17-humanized mice and a BP model. (a) The most pathogenic of the epitopes cluster within the juxtamembranous NC16A domain of COL17. Significant differences in their amino acid sequences exist between the human and the mouse NC16A domains of COL17. (b) Generation of COL17-humanized mice by repeated crossing of heterozygous mouse Coll7knockout $\left(\mathrm{mCol17^{+/ }}\right)$ and human $\mathrm{COL17^{+/+ }} \mathrm{Tg}$ mice. (c) Skin detachment of the neonatal COL17humanized mice is inducible by mild friction $48 \mathrm{~h}$ after the injection of BP-IgG. (d) Histologically, subepidermal blister formation (arrowheads) associated with linear deposition of human IgG (arrows) and mouse C3 (white arrowheads) at the DEJ are observed

succeeded in confirming the pathogenicity of BP autoAbs by using the unique technique of "humanization of autoantigen" [4]. First, we generated murine COL17knockout $\left(\mathrm{mCol17^{-/ } )}\right.$ mice that developed blisters and erosions on the skin, symptoms that reproduce a human disease called non-Herlitz epidermolysis bullosa, which is caused by null mutations in the COL17Al gene [16]. Then, we crossed human $\mathrm{COL} 17^{+/+}\left(h \mathrm{COL} 17^{+/+}\right)$transgenic $(\mathrm{Tg})$ mice [17] with heterozygous $\mathrm{mCol17^{+/- }}$ mice. Mice that carried both the heterozygous null mutation of $\mathrm{mCol17}$ and the transgene of human $\mathrm{COL} 17\left(\mathrm{mCol17}^{+/-}, \mathrm{hCOL17^{+/- }}\right)$ were bred to generate COL17-humanized $\left(\mathrm{hCOL17^{+/+ }}, \mathrm{mColl1}^{-/-}\right)$mice (Fig. 2b). COL17-humanized mice lack mouse COL17 but express human COL17. They showed no apparent clinical phenotype and were able to deliver COL17-humanized mice pups by mating with COL17-humanized mice parents. Neonatal COL17-humanized mice that were passively transferred with either total IgG or IgG affinity-purified against the NC16A domain of COL17 from BP patients developed diffuse erythema and showed epidermal separation by gentle skin friction at $48 \mathrm{~h}$ after transfer (Fig. 2c) [4]. Lesional skin specimens demonstrated dermal-epidermal separation and inflammatory cell infiltration of neutrophils and lymphocytes. DIF analysis revealed linear deposition of human IgG and mouse $\mathrm{C} 3$ at the DEJ, which simulates the human BP phenotype (Fig. 2d). This passive-transfer neonatal mouse model was the first to directly show the pathogenicity of anti-COL17 NC16A IgG autoantibodies in BP patients. 
This COL17-humanized BP mouse model is quite valuable not only for elucidating the pathomechanisms of BP but also for innovating disease-specific therapies. We executed a therapeutic trial using human COL17 NC16A recombinant peptides. After the transfer of total IgG from BP patients, COL17-humanized mice were treated with a recombinant peptide composed of the NC16A domain. The treated mice showed markedly reduced blister formation [4]. In addition, we have developed a novel therapeutic strategy of treating BP by using the recombinant Fab fraction of IgG monoclonal antibodies to COL17 to block the complement activation that would otherwise be induced by pathogenic autoantibodies [18]. We used phage display to generate recombinant Fab fragments of $\mathrm{IgG}$ monoclonal antibodies against the human COL17 NC16A domain from antibody repertoires of BP patients. Two recombinant Fab fragments showed marked ability to inhibit the binding of BP autoantibodies and to inhibit subsequent complement activation in vitro. In the in vivo experiments using the COL17-humanized BP mouse model, these recombinant Fab fragments protected mice against BP-autoantibody-induced blistering disease [18]. Thus, the use of engineered Fab Abs to block pathogenic epitopes seems promising, and it may lead to disease-specific treatments for BP.

\section{Neonatal BP Model Induced by Maternally Transferred Abs to Human COL17}

Using the COL17-humanized mouse, we developed another neonatal BP mouse model, which was induced by maternally transferred antibodies. When heterozygous mouse COL17-deficient $\left(\mathrm{mColl}^{+/-}\right)$female mice are crossed with COL17humanized $\left(\mathrm{hCOL} 17^{+/+}, \mathrm{mCol1} 7^{-/-}\right)$male mice, it is expected that $50 \%$ of the pups will show the genotype of human $\mathrm{COL} 17^{+/-}$, mouse Col17 $7^{-1-}$. We immunized mouse $\mathrm{COL} 17^{+/-}$female mice with human COL17 and mated them with COL17-humanized $\left(\mathrm{hCOL} 17^{+/+}, \mathrm{mCol17^{-/- }}\right.$ ) male mice. As expected, the circulating anti-human COL17 $\mathrm{IgG}$ antibodies that were produced by the immunized mother were transferred into the neonates through not only the placenta but also the milk, and half of the neonates whose skin expressed human but not murine COL17 $\left(\mathrm{hCOL} 17^{+/-}, \mathrm{mCol17}^{-/-}\right)$ developed blisters associated with the histological and immunological features that are seen in BP patients [19]. This novel experimental system has some advantages: (1) the model does not require the technically difficult injection procedure; (2) the pathogenic IgG persists for longer than in conventional passive transfer models; and (3) the immune reaction totally depends on the murine immune system, even though the antigen is human COL17.

\section{Active BP Model}

To develop an active BP mouse model, we generated immunodeficient Rag$2^{-/-} /$COL17-humanized mice by crossing COL17-humanized mice with $R a g-2^{-/-}$ mice. As a next step, we isolated splenocytes from the wild-type mice immunized 
a

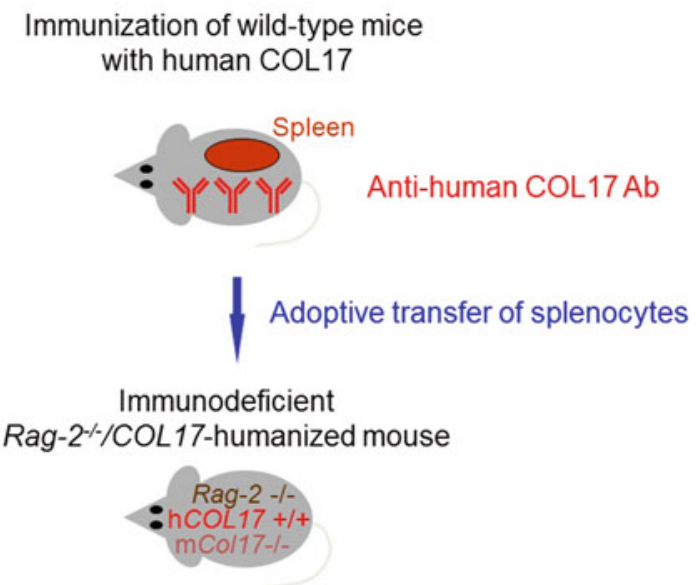

b

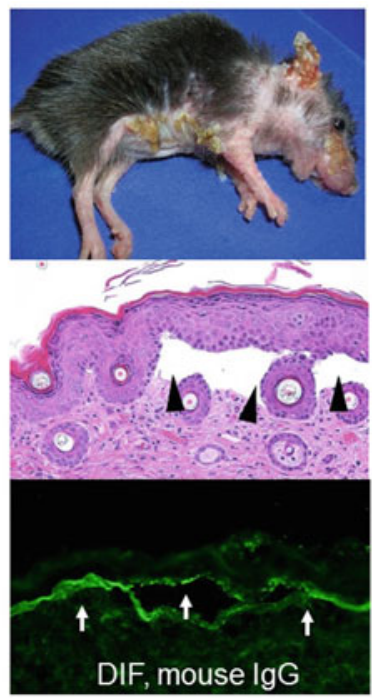

Fig. 3 Generation of an active BP model. (a) agg-2 $^{-/-}$COL17-humanized mice into which had been transferred splenocytes obtained from wild-type mice immunized by human COL17 protein. (b) The treatment induces the continuous production of anti-human COL17 IgG and blister formation corresponding to BP. Histologically, subepidermal blister formation (arrowheads) associated with linear deposition of mouse IgG (arrows) at the DEJ are observed

with human COL17 and adoptively transferred them into the $R a g-2^{-/-} /$COL17humanized mice. Because these Rag-2-/-/COL17-humanized mice had no mature T or B cells, they did not reject the transferred splenocytes. The transferred splenocytes induced continuous production of anti-human COL17 IgG, and blistering disease occurred (Fig. 3) [20]. In that study, CD4+ ${ }^{+}$cells were proven to be crucial for the development of the BP phenotype, because the depletion of $\mathrm{CD}^{+} \mathrm{T}$ cells from immunized splenocytes and the administration of cyclosporin A, a suppressant of T-cell function that works by inhibiting the production of IL-2 from the activated T cells, each significantly suppressed the development of pathogenic IgG and diminished the disease severity.

\section{Doxycycline-Inducible BP Model}

We have produced different BP models using the COL17-humanized mice as described. In these COL17-humanized mice, the innate expression of human COL17 eliminated all abnormal phenotypes observed in the Coll7-knockout (mColl ${ }^{-/-}$) mice [4]. If the expression of human COL17 is induced after birth in the Coll7knockout (mCol17 $\left.{ }^{-/}\right)$mice, what kinds of phenotypes will appear? Blistering diseases observed in adult Coll7-knockout $\left(\mathrm{mColl}^{-/-}\right)$mice may be rescued by 
neo-expression of human $\mathrm{COL} 17$ in the skin, but an immune reaction to human COL17 may occur. However, neo-expression of COL17 in adult Col17-knockout (mCol17 ${ }^{-/}$) mice may induce Abs to human COL17, leading to blistering disease similar to BP (Fig. 4a). We focused on using the Tet system that we commonly use to regulate expression of the gene of interest. By introducing transgenes including a reverse tetracycline-controlled transactivator (rtTA) and a pTRE vector with human COL17 cDNA, the administration of doxycycline induces the neo-expression of human COL17. In contrast, by incorporating a tetracycline-controlled transactivator (tTA) and a pTRE vector with human COL17 cDNA, the stable expression of human COL17 is expected to be suppressed by the administration of doxycycline. By in vitro studies using HEK 293 cells stably expressing pTRE-human COL17 and rtTA or tTA, we have found that the expression of human COL17 can be tightly regulated. In addition, the Tet-on system using rtTA showed less leaking expression of human COL17 (Fig. 4b). On the basis of these results, we started to prepare two

a
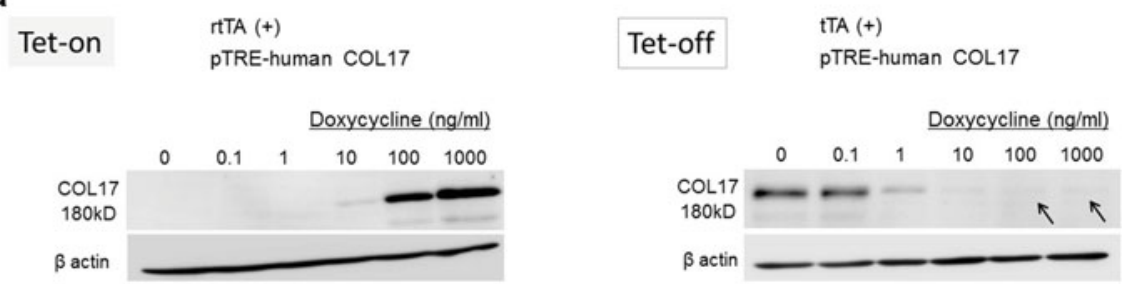

b

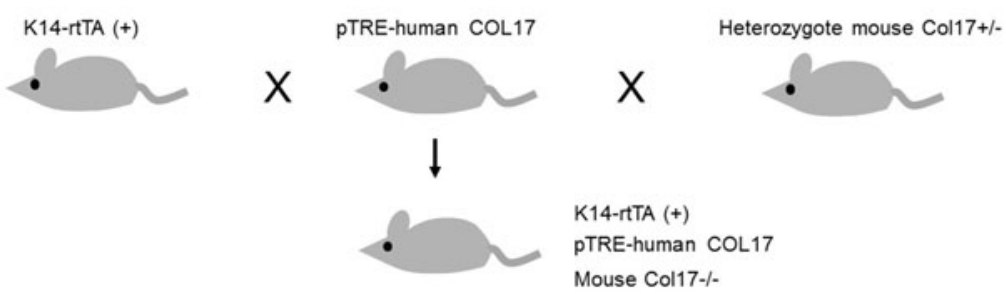

Oral doxycycline

Neo-expression of human COL17

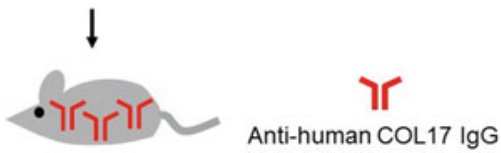

Fig. 4 Strategy for the generation of a doxycycline-inducible BP model. (a) In vitro studies using HEK 293 cells stably expressing pTRE-human COL17 and rtTA or tTA show that the expression of human COL17 can be tightly regulated. A Tet-on system using rtTA results in less leaking expression of human COL17 compared with a Tet-off system (arrows). (b) Oral administration of doxycycline to pTRE-COL17, K14-rtTA, mouse COL17-null mice is expected to induce neo-expression of human COL17 in basal epidermal keratinocytes, which would induce autoimmunity to human COL17 
different Tg mice expressing rtTA under keratin 14 (K14) promoter and pTREhuman COL17 cDNA. In the near future, we will obtain a novel BP model in which blistering disease can be initiated with only oral administration of doxycycline (Fig. 4a).

\section{Perspectives}

Recent studies using animal models have made considerable progress toward our understanding of the pathogenesis of BP as well as toward producing novel therapies. To further advance our knowledge of BP pathomechanisms and to develop novel therapeutic strategies for BP, the establishment of an active, stable disease model is anticipated.

Open Access This chapter is distributed under the terms of the Creative Commons Attribution Noncommercial License, which permits any noncommercial use, distribution, and reproduction in any medium, provided the original author(s) and source are credited.

\section{References}

1. Korman N (1987) Bullous pemphigoid. J Am Acad Dermatol 16(5 Pt 1):907-924

2. Nishie W (2014) Update on the pathogenesis of bullous pemphigoid: an autoantibodymediated blistering disease targeting collagen XVII. J Dermatol Sci 73(3):179-186. doi:10.1016/j.jdermsci.2013.12.001 S0923-1811(13)00386-1 [pii]

3. Schmidt E, Zillikens D (2013) Pemphigoid diseases. Lancet 381(9863):320-332. doi:10.1016/ S0140-6736(12)61140-4 S0140-6736(12)61140-4 [pii]

4. Nishie W, Sawamura D, Goto M, Ito K, Shibaki A, McMillan JR, Sakai K, Nakamura H, Olasz E, Yancey KB, Akiyama M, Shimizu H (2007) Humanization of autoantigen. Nat Med 13(3):378-383. doi:nm1496 [pii] 10.1038/nm1496

5. Diaz LA, Ratrie H 3rd, Saunders WS, Futamura S, Squiquera HL, Anhalt GJ, Giudice GJ (1990) Isolation of a human epidermal cDNA corresponding to the $180-\mathrm{kD}$ autoantigen recognized by bullous pemphigoid and herpes gestationis sera. Immunolocalization of this protein to the hemidesmosome. J Clin Invest 86(4):1088-1094. doi:10.1172/JCI114812

6. Giudice GJ, Squiquera HL, Elias PM, Diaz LA (1991) Identification of two collagen domains within the bullous pemphigoid autoantigen, BP180. J Clin Invest 87(2):734-738. doi:10.1172/ JCI115054

7. Nonaka S, Ishiko A, Masunaga T, Akiyama M, Owaribe K, Shimizu H, Nishikawa T (2000) The extracellular domain of BPAG2 has a loop structure in the carboxy terminal flexible tail in vivo. J Invest Dermatol 115(5):889-892. doi:jid136 [pii] 10.1046/j.1523-1747.2000.00136.x

8. Hirako Y, Usukura J, Uematsu J, Hashimoto T, Kitajima Y, Owaribe K (1998) Cleavage of BP180, a 180-kDa bullous pemphigoid antigen, yields a 120-kDa collagenous extracellular polypeptide. J Biol Chem 273(16):9711-9717

9. Franzke CW, Tasanen K, Schacke H, Zhou Z, Tryggvason K, Mauch C, Zigrino P, Sunnarborg S, Lee DC, Fahrenholz F, Bruckner-Tuderman L (2002) Transmembrane collagen XVII, an epithelial adhesion protein, is shed from the cell surface by ADAMs. EMBO J 21(19):5026-5035 
10. Zillikens D, Rose PA, Balding SD, Liu Z, Olague-Marchan M, Diaz LA, Giudice GJ (1997) Tight clustering of extracellular BP180 epitopes recognized by bullous pemphigoid autoantibodies. J Invest Dermatol 109(4):573-579. doi:S0022202X97890574 [pii]

11. Kobayashi M, Amagai M, Kuroda-Kinoshita K, Hashimoto T, Shirakata Y, Hashimoto K, Nishikawa T (2002) BP180 ELISA using bacterial recombinant NC16a protein as a diagnostic and monitoring tool for bullous pemphigoid. J Dermatol Sci 30(3):224-232. doi:S0923181102001093 [pii]

12. Liu Z, Diaz LA, Troy JL, Taylor AF, Emery DJ, Fairley JA, Giudice GJ (1993) A passive transfer model of the organ-specific autoimmune disease, bullous pemphigoid, using antibodies generated against the hemidesmosomal antigen, BP180. J Clin Invest 92(5):2480-2488. doi:10.1172/JCI116856

13. Liu Z, Giudice GJ, Swartz SJ, Fairley JA, Till GO, Troy JL, Diaz LA (1995) The role of complement in experimental bullous pemphigoid. J Clin Invest 95(4):1539-1544. doi:10.1172/JCI117826

14. Liu Z, Giudice GJ, Zhou X, Swartz SJ, Troy JL, Fairley JA, Till GO, Diaz LA (1997) A major role for neutrophils in experimental bullous pemphigoid. J Clin Invest 100(5):1256-1263. doi:10.1172/JCI119639

15. Liu Z, Shapiro SD, Zhou X, Twining SS, Senior RM, Giudice GJ, Fairley JA, Diaz LA (2000) A critical role for neutrophil elastase in experimental bullous pemphigoid. J Clin Invest 105(1):113-123. doi:10.1172/JCI3693

16. Gatalica B, Pulkkinen L, Li K, Kuokkanen K, Ryynanen M, McGrath JA, Uitto J (1997) Cloning of the human type XVII collagen gene (COL17A1), and detection of novel mutations in generalized atrophic benign epidermolysis bullosa. Am J Hum Genet 60(2):352-365

17. Olasz EB, Roh J, Yee CL, Arita K, Akiyama M, Shimizu H, Vogel JC, Yancey KB (2007) Human bullous pemphigoid antigen 2 transgenic skin elicits specific IgG in wild-type mice. $\mathrm{J}$ Invest Dermatol 127(12):2807-2817. doi:5700970 [pii] 10.1038/sj.jid.5700970

18. Wang G, Ujiie H, Shibaki A, Nishie W, Tateishi Y, Kikuchi K, Li Q, McMillan JR, Morioka H, Sawamura D, Nakamura H, Shimizu H (2010) Blockade of autoantibody-initiated tissue damage by using recombinant fab antibody fragments against pathogenic autoantigen. Am J Pathol 176(2):914-925. doi:10.2353/ajpath.2010.090744 S0002-9440(10)60403-8 [pii]

19. Nishie W, Sawamura D, Natsuga K, Shinkuma S, Goto M, Shibaki A, Ujiie H, Olasz E, Yancey KB, Shimizu H (2009) A novel humanized neonatal autoimmune blistering skin disease model induced by maternally transferred antibodies. J Immunol 183(6):4088-4093. doi:10.4049/jimmunol.0800389 jimmunol.0800389 [pii]

20. Ujiie H, Shibaki A, Nishie W, Sawamura D, Wang G, Tateishi Y, Li Q, Moriuchi R, Qiao H, Nakamura H, Akiyama M, Shimizu H (2010) A novel active mouse model for bullous pemphigoid targeting humanized pathogenic antigen. J Immunol 184(4):2166-2174. doi:10.4049/jimmunol.0903101 jimmunol.0903101 [pii] 


\title{
T Cell Senescence and Autoimmunity
}

\author{
Nagahiro Minato
}

\begin{abstract}
Immune aging results in a decreased competence of adaptive immunity with an increased risk for autoimmunity. However, the mechanistic links between the immune aging and autoimmunity remain elusive. We reported that a PD- $1^{+}$ memory phenotype (MP) $\mathrm{CD}^{+} \mathrm{T}$ cell population is increased as normal mice age, termed senescence-associated (SA-) T cells. The SA-T cells show characteristic signs and features of cellular senescence and emerge as follicular T cells in spontaneous germinal centers (GCs) that occur in aged mice. Spontaneous development of GCs is a hallmark of systemic autoimmune diseases, and we found that the development of SA-T cells is robustly and prematurely accelerated in bona fide lupus-prone mice in association with spontaneous, auto-reactive GCs. A fraction of the SA-T cells defined by CD153 expression is activated by autologous GC B cells to produce a plethora of inflammatory factors in a TCR-dependent manner and contributes to the expansion of the $\mathrm{GC}$ reactions, although the remaining part of them is rendered TCR anergic in situ. The results uncover that accelerated T cell senescence underlies the development of autoimmunity in systemic lupus erythematosus.
\end{abstract}

Keywords Immune aging $\bullet$ Systemic lupus erythematosus $\bullet$ Cell senescence $\bullet$ Senescence-associated T cells $\bullet$ Follicular T cells $\bullet$ Germinal centers $\bullet$ Homeostatic proliferation $\bullet$ Autoantibodies

\section{Introduction: Immune Aging and Senescence- Associated T Cells}

Immune aging (immunosenescence) is characterized by the reduced competence of acquired immunity, leading to increased susceptibility to infection as well as decreased vaccination efficiency. Recent accumulating evidence indicates that immunosenescence underlies an increased proinflammatory trait with age,

\footnotetext{
N. Minato, M.D., Ph.D. ( $ه)$

Professor, Department of Immunology \& Cell Biology,

Kyoto University Graduate School of Medicine, Kyoto, Japan

e-mail: minato@imm.med.kyoto-u.ac.jp 
including various chronic inflammatory and metabolic disorders, such as atherosclerosis and diabetes mellitus, as well as an increased risk for autoimmunity [1]. Cellular senescence is characterized by irreversible arrest of proliferation, grossly altered gene expression, and relative resistance to apoptosis [2]. Notably, senescent cells are often metabolically active and may become foci of host reactions in tissues by secreting various inflammatory factors [3]. The features and consequences of cellular senescence in $\mathrm{T}$ cells in the immune system, however, remain elusive.

One of the most prominent changes occurring in the immune organs with age is an early involution of the thymus. The thymus is a central immune organ to support $\mathrm{T}$ cell development and establish $\mathrm{T}$ cell self-tolerance. The $\mathrm{T}$ cell generation in the thymus is most active at the late embryonic through neonatal stages, but the activity sharply declines after the juvenile stage, eventually replaced almost entirely by fat tissues at later stages of life (Fig. 1a). Although the mechanism of early thymic involution remains elusive, it is suggested that the rapid decrease of thymic epithelial stem cell (TECSC) activity after birth nay play a crucial role in it [4]. In concordance

a
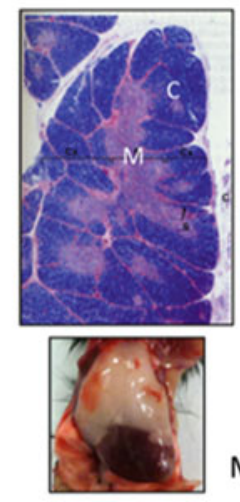

Mouse
Human

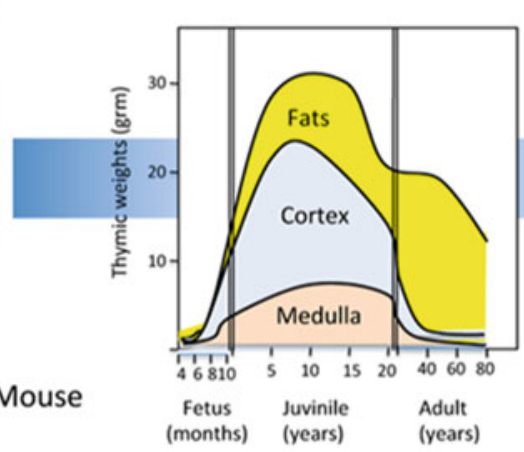

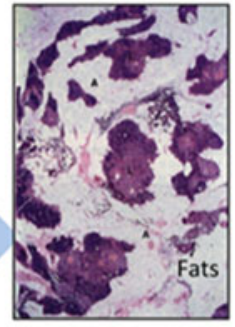

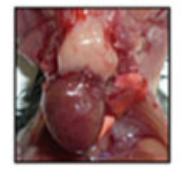

b

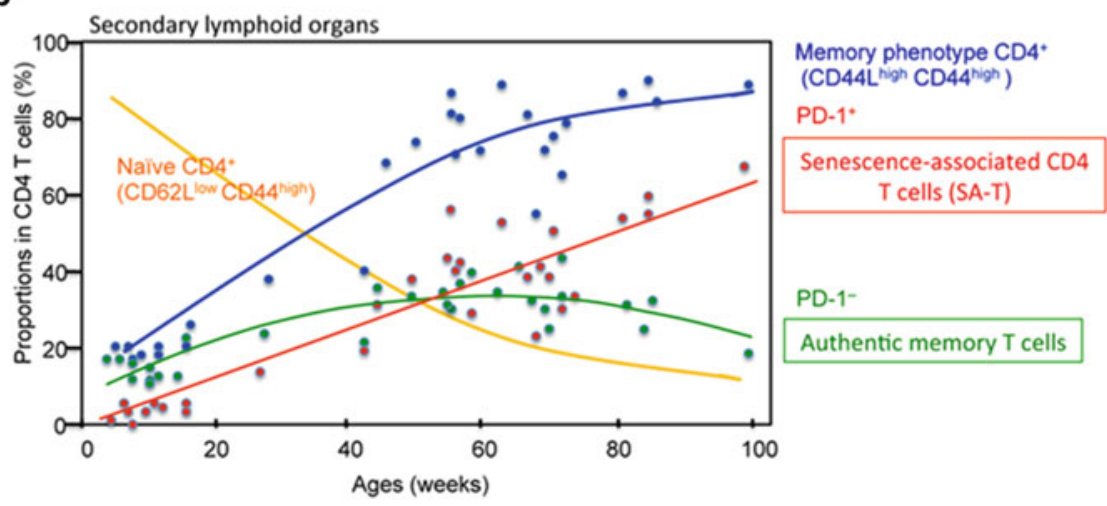

Fig. 1 (a) The thymus shows early involution with age. (b) The proportions of SA-T cells in peripheral CD4+ T cell pool increase with age. 
with the decrease of neo-T cell genesis, the peripheral naïve $\mathrm{T}$ cells are gradually reduced with age. Although the peripheral $\mathrm{T}$ cell pool is well maintained in aged individuals, the population shows a steady increase in the proportions of memory phenotype (MP) T cells [5]. It is suggested that these MP T cells include those that have homeostatically expanded in response to the decreasing drift of the $\mathrm{T}$ cell numbers, in addition to the authentic memory $\mathrm{T}$ cells for specific environmental antigens [5]. We reported that a unique $\mathrm{PD}-1^{+} \mathrm{MP} \mathrm{CD}^{+} \mathrm{T}$ cell population is increased with age [6], now termed senescence-associated (SA-) T cells (Fig. 1b). The SA-T cells showed compromised capacity of clonal proliferation and production of typical T cell-specific cytokines via TCR-stimulation; however, the SA-T cells secrete abundant proinflammatory cytokines such as osteopontin (OPN) [6].

\section{SA-T Cells Represent an Endogenously Arising Follicular T Cell Population with Age}

We found that normal aged mice often developed GCs spontaneously in the secondary lymphoid tissues, and the SA-T cells were detected mostly in the GCs, representing follicular T cells (Fig. 2). In agreement with the follicular localization, the majority of SA-T cells expressed ICOS and CD200, similarly to the typical follicular helper $\mathrm{T}$ cells induced by immunization with exogenous antigens such as sheep red blood cells (SRBC-TFH). However, a significant portion of the SA-T cells additionally expressed CD153 (CD30L), which was rarely expressed in SRBCTFH cells. Functionally, CD153+ SA-T cells showed compromised proliferation but produced large amounts of OPN via TCR-stimulation with much less IL-4 and
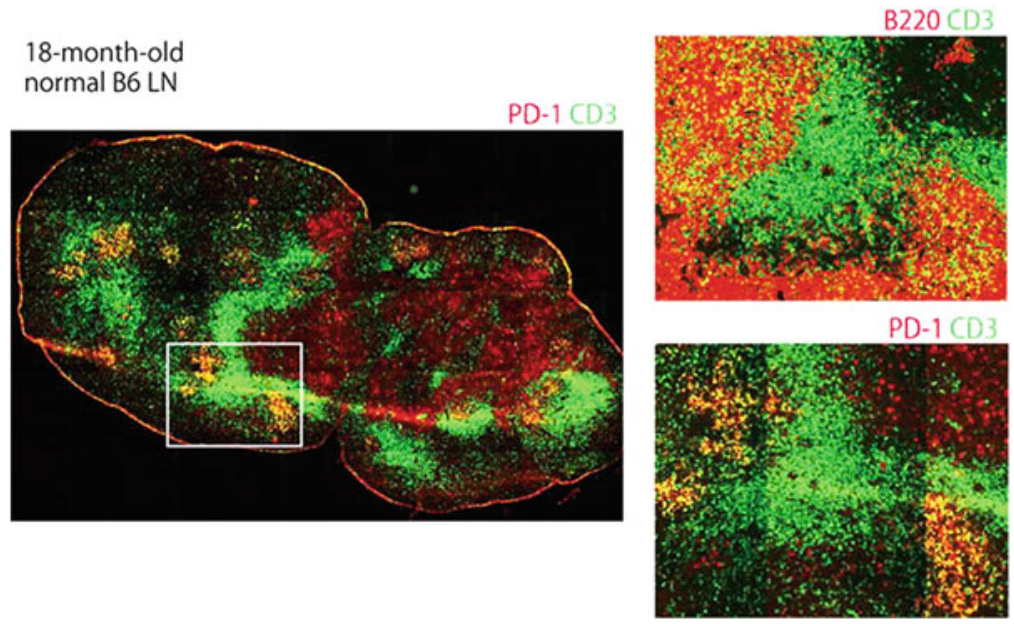

Fig. 2 Spontaneous development of germinal centers (GCs) in the secondary lymphoid tissues of normal aged nice, containing abundant PD- $1^{+}$follicular $\mathrm{T}$ cells 

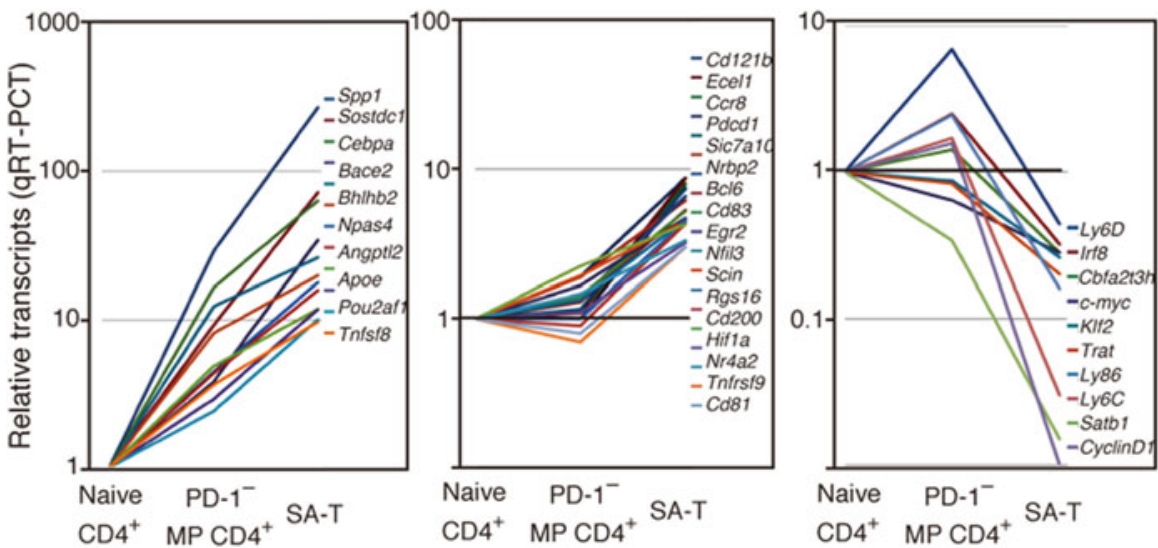

Fig. 3 SA-T cells show a unique transcriptome compared with naïve and $\mathrm{PD}-1^{-} \mathrm{MP} C D 4^{+} \mathrm{T}$ cells

IFN- $\gamma$, whereas the rest of the $\mathrm{CD} 153^{-}$population did not proliferate or produce any of these cytokines at all, suggesting that these cells were TCR unresponsive. The SA-T cells showed a unique transcriptome (Fig. 3) different from those of PD-1 MP $\mathrm{CD}^{+} \mathrm{T}$ cells and SRBC-TFH cells, but the CD153+ and CD153- fractions of the SA-T cells had very similar transcriptome profiles to each other, despite the distinct TCR responsiveness. The results suggest that SA-T cells represent a unique type of follicular $\mathrm{CD}^{+} \mathrm{T}$ cell population, a fraction of which is rendered TCR anergic in situ. Although $\mathrm{CD}_{153^{+}}$and $\mathrm{CD} 153^{-} \mathrm{SA}-\mathrm{T}$ cells became detectable only in normal aged mice, the development was remarkably accelerated in the adult-thymectomized mice or $\gamma$-ray irradiated with transplanted naïve T cells. The results strongly suggest that the SA-T cells are generated in the process of homeostatic $\mathrm{T}$ cell proliferation, either acutely or chronically depending on the extent and tempo of the decrease in a peripheral T cell pool.

\section{SA-T Cells Increase Robustly in Bone Fide Lupus-Prone Mice}

Spontaneous GC reactions are a hallmark of systemic autoimmune diseases, including lupus [7,8], and the findings above prompted us to examine them in female BWF1 mice, a bona fide mouse model of human SLE. Female BWF1 mice robustly developed GCs with a remarkable increase of follicular PD- $1^{+} \mathrm{MP} \mathrm{CD} 4^{+} \mathrm{T}$ cells beginning from as early as 6 months of age (Fig. $4 \mathrm{a}$ ); this was not the case in male BWF1 mice or parental NZW mice. A significant portion of them expressed CD153 in addition to other follicular T cell markers such as CXCR5, ICOS, and Bcl-6 (Fig. 4b). Transcriptome analysis confirmed that both CD153+ and CD153cell fractions showed highly coincidental profiles with the corresponding SA-T 
a

6-month-old female BWF1
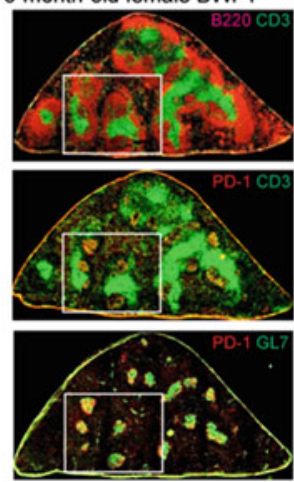

b

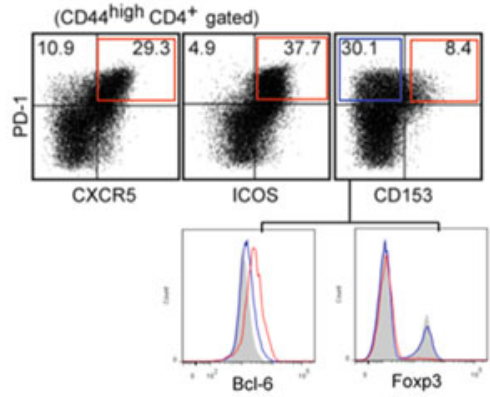

Fig. 4 Lupus-prone female BWF1 mice robustly and spontaneously develop GCs containing abundant SA-T cells much earlier than normal mice

cells in normal aged mice, rather than SRBC-TFH cells. Moreover, the TCRstimulated $\mathrm{CD}_{153}{ }^{+}$cell fraction produced abundant OPN in addition to other inflammatory chemokines $(\mathrm{Ccl} 1, \mathrm{Ccl} 3, \mathrm{Ccl} 4)$ with minimal production of $\mathrm{T}$ cellspecific cytokines, whereas the $\mathrm{CD}_{153^{-}}$fraction produced no cytokines at all, including OPN. Overall, the follicular T cells in female BWF1 mice showed features essentially identical to those of the SA-T cells in normal aged mice, except for more robust and earlier development.

\section{SA-T Cells Show Typical Signs and Features of Cellular Senescence}

Transcriptome profiles of the SA-T cells of both aged B6 and female BWF1 mice revealed the up-regulation of a numbers of genes coding for potentially inflammatory factors including Sppl (Fig. 5a). The feature was reminiscent of a so-called senescence-associated secretory phenotype (SASP) [3], and indeed the expression of $C e b p b$, shown to be involved in the regulation of SASP, was also increased. In addition, the SA-T cells revealed increased expression of Cdknla (Cipl) and $C d k n 2 b$ (Ink4b) (Fig. 5b), which are representative senescence-associated biomarkers $[9,10]$, remarkably increased nuclear heterochromatin foci, and higher SA- $\beta$ Gal activity than PD-1- $1^{-} D 4^{+} \mathrm{T}$ cells (Fig. 5c). Also, the SA-T cells were highly stable in culture and survived for long terms without proliferation. All of these results are consistent with the notion that the SA-T cells represent the T cells in cellular senescence. 

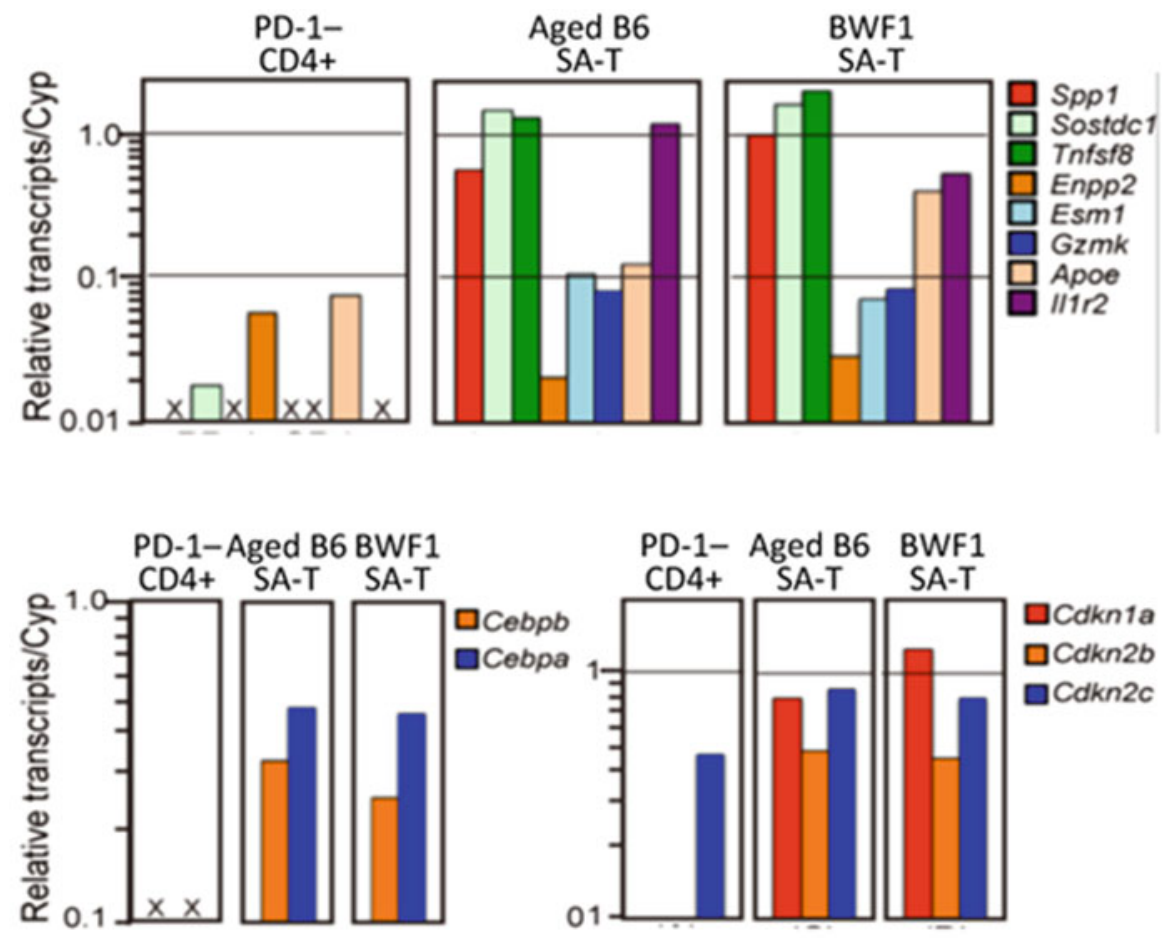

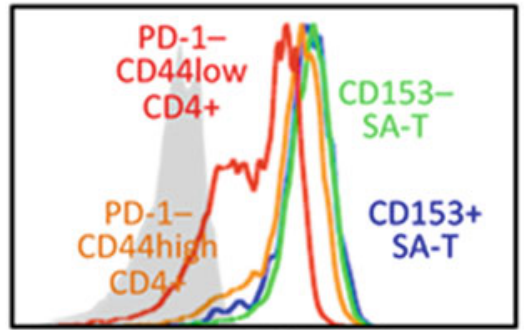

SA- $\beta-$ Gal $(\log 10)$

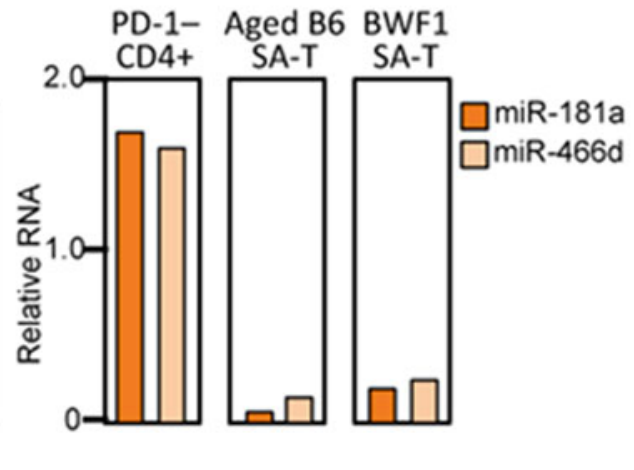

Fig. 5 SA-T cells reveal the signs and features of cellular senescence, including a senescenceassociated secretory phenotype (SASP), senescence biomarkers (Cdkn1a, Cdkn2b), increased SA- $\beta$-gal activity, and decreased aging-associated microRNAs 


\section{SA-T Cells Are Activated in Response to Autologous GC B Cells to Produce OPN that Protects Against B Cell Apoptosis in GCs}

The Sppl transcripts were sharply increased in the spleens of female BWF1 mice from around 5 months of age, in concordance with the overt GC reactions. Analysis with laser-capture microdissection (LMD) revealed that the Sppl was confined to the $\mathrm{PNA}^{+} \mathrm{GC}$ regions, whereas the GCs in SRBC-immunized B6 mice hardly expressed Spp1. When SA-T cells from female BWF1 mice were co-cultured with $\gamma$-ray-irradiated autologous B cells, the CD153+, but hardly TCR-anergic CD153fraction produced significant amounts of OPN. The response was induced by $\mathrm{CD} 95^{+}$ GC B cells more efficiently than $\mathrm{CD}^{-} \mathrm{B}$ B cells and was inhibited significantly in the presence of anti-MHC II or soluble anti-CD3 antibody, suggesting that the SA-T cells recognized self-antigens presented by GC B cells via TCR. Curiously, however, the response was unaffected in the presence of anti-PD-L2 antibody, albeit that the GC-B cells expressed PD-L2, suggesting that the OPN production by CD153+ SA-T cells is mediated by a PD-1-resistant, alternative TCR-signaling pathway (Fig. 6). On the other hand, the OPN production in response to GC-B cells was significantly enhanced by CD30-Ig agonistic to CD153 (Fig. 6). The primary B cells from female BWF1 mice showed significant cell death from stimulation with anti- $\mu$ antibody. However, OPN prevented B cell death and accordingly enhanced the secretion of anti-dsDNA antibody in vitro. Upon analysis using a WEHI231 B1 cell line, it was indicated that OPN inhibited the BCR-induced apoptosis by inducing the expression of Bcl2ala, which is known to be crucial for B cell survival (Fig. 6). The results suggest that the SA-T cells specifically recognize GC-B cells and locally produce OPN, which in turn protects the GC B cells from antigen-induced apoptosis.

\section{Involvement of SA-T Cells in the Expansion of GCs and Autoimmunity in Lupus}

To directly investigate the role of SA-T cells in GC reaction, we isolated PD-1- MP $\mathrm{CD}^{+}$and SA-T cells from female BWF1 mice with overt lupus disease and transferred them into pre-disease young BWF1 mice. We found that the CD153+ SA-T cells caused a significant increase in GC sizes in the recipients, whereas the PD-1- MP CD4 ${ }^{+}$cells barely affected the GC reactions. Moreover, a partial depletion of CD153+ SA-T cells in female BWF1 mice at 4 months of age by treatment with a rat anti-CD153 antibody $(500 \mu \mathrm{g} / \mathrm{head})$ twice a week for 4 weeks resulted in significant reduction of GC sizes as well as BC-B cells; none of these mice developed significant anti-dsDNA antibody, whereas $20 \%$ (3 out of 15) of control mice showed a burst of anti-dsDNA antibody. These results suggest that CD153+ SA-T cells are directly involved in the growth of spontaneous GCs and autoantibody production in female BWF1 mice. 


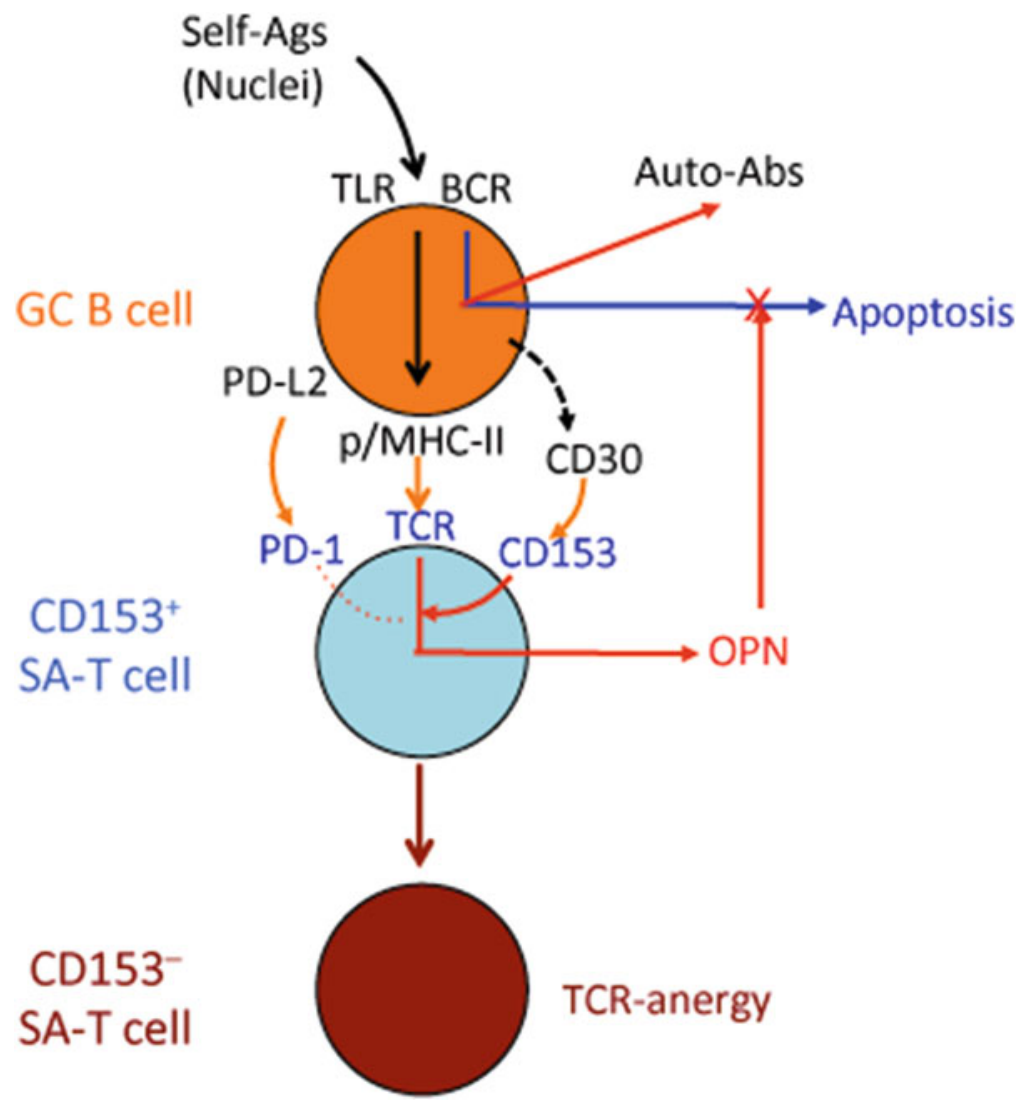

Fig. 6 CD153+ SA-T cells specifically respond to own GC-B cells and produce abundant OPN, which prevent the B cells from BCR-induced apoptosis. The TCR-signaling pathway is enhanced via CD153 co-stimulatory activity but is unaffected by negative PD-1 signaling. The CD153+ SA-T cells may eventually be rendered TCR anergic via a tolerance checkpoint and become CD153- cells

\section{Conclusion and Perspectives}

Among a number of changes in immune function with age is an increasing risk for autoimmunity [1]. We previously proposed that the increase of SA-T cells chronically with age and acutely with a leukemic condition might be related to the decrease of $\mathrm{T}$ cell genesis due to physiological thymic involution and leukemia-associated T-lymphocytopenia, respectively [6]. In a lymphopenic condition, the peripheral $\mathrm{CD}^{+} \mathrm{T}$ cells undergo homeostatic proliferation, which depends on homeostatic cytokines and tonic TCR-signal via MHC II-bearing self-peptides [5]. As such, the sustained $\mathrm{CD}^{+} \mathrm{T}$ cell homeostatic proliferation may lead to an increased risk for autoimmunity [11]. It was reported that $\mathrm{CD}^{+} \mathrm{T}$ cell homeostatic proliferation 
underlies the development of autoimmune diabetes in female NOD mice [12]. Lymphopenia is one of the characteristic features in lupus disease, and therefore we propose that $\mathrm{CD}^{+} \mathrm{T}$ cell homeostatic proliferation underlies the robust and premature development of SA-T cells. A recent report suggests that homeostatically proliferating PD $-1^{+} \mathrm{CD}^{+} \mathrm{T}$ cells may show different energy metabolism from $\mathrm{CD} 4^{+}$ T cells driven by antigens in immune response [13]. Homeostatic proliferation is not associated with the transition to the effector phase as in immune response, and the possibility that the persistence of such a proliferative state with particular energy metabolism leads to a higher propensity for the progression of cellular senescence would be intriguing (Fig. 7). In conclusion, our current results indicate that SA-T cells play an important part in lupus pathogenesis and may provide a novel therapeutic clue for controlling human SLE.

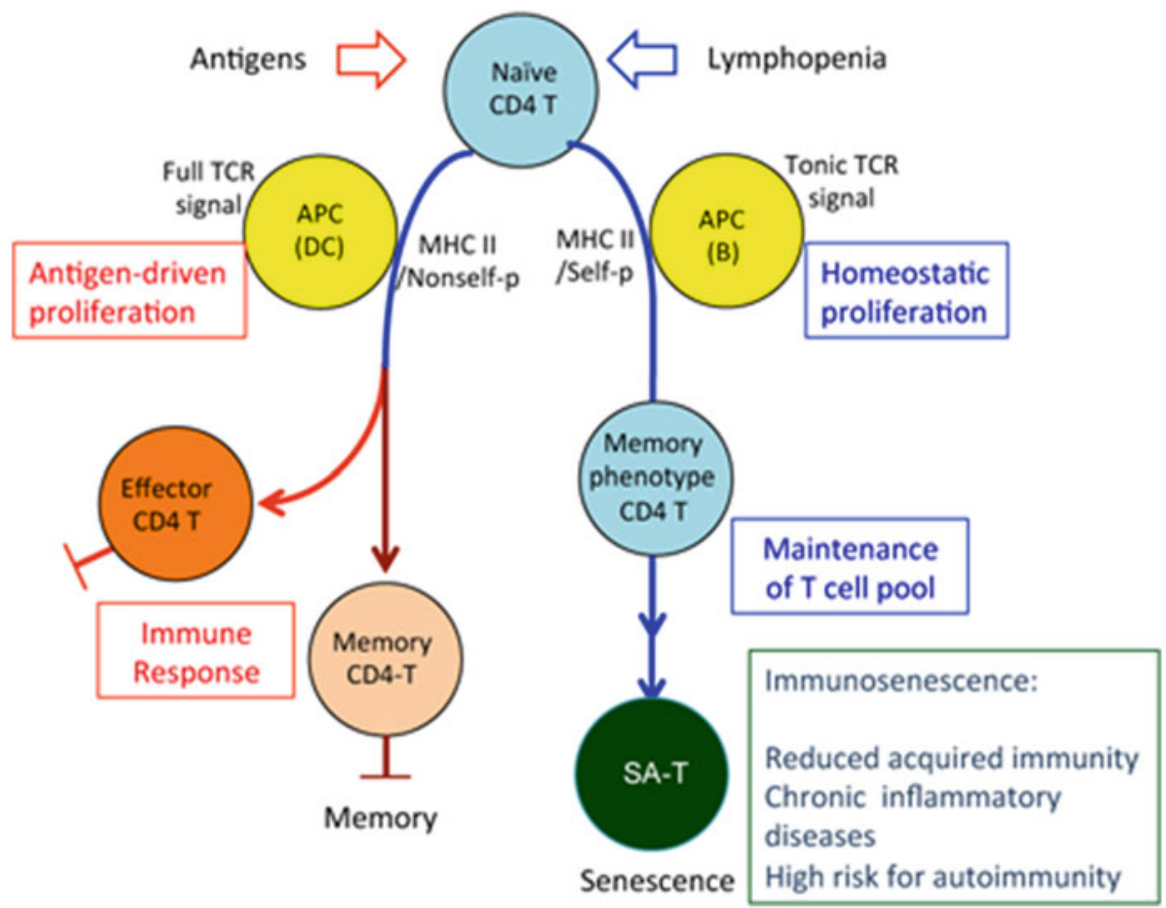

Fig. 7 Normal naïve $\mathrm{CD}^{+} \mathrm{T}$ cells are activated in response to the specific foreign antigens presented by professional antigen-presenting cells (APCs), such as DCs, and show a robust clonal expansion, followed by maturation to the effector cells or quiescent memory cells. In lymphopenic conditions, however, naïve $\mathrm{CD} 4^{+} \mathrm{T}$ cells proliferate polyclonally, called homeostatic proliferation, which depends on the APCs such as B cells presenting self-peptides and homeostatic cytokines, to maintain the peripheral $\mathrm{T}$ cell pool. The sustained homeostatic proliferation may result in an increased risk for autoimmunity as well as T cell senescence to become SA-T cells. The (CD153 $)$ SA-T cells are quite stable and metabolically active, producing abundant inflammatory factors such as OPN and chemokines, and become inflammatory foci in tissues. In the lupus-prone genetic background such as BWF1 with a defective B cell self-tolerance checkpoint, the SA-T cells are involved in auto-reactive $\mathrm{GC}$ reactions and promote overt autoantibody production 
Open Access This chapter is distributed under the terms of the Creative Commons Attribution Noncommercial License, which permits any noncommercial use, distribution, and reproduction in any medium, provided the original author(s) and source are credited.

\section{References}

1. Goronzy JJ, Weyand CM (2012) Immune aging and autoimmunity. Cell Mol Life Sci 69(10):1615-1623

2. Campisi J, d'Adda d, Fagagna F (2007) Cellular senescence: when bad things happen to good cells. Nat Rev Mol Cell Biol 8(9):729-740

3. Freund A, Orjalo AV, Desprez PY, Campisi J (2010) Inflammatory networks during cellular senescence: causes and consequences. Trends Mol Med 16(5):238-246

4. Chinn IK et al (2012) Changes in primary lymphoid organs with aging. Semin Immunol 24:309-320

5. Sprent J, Surh CD (2011) Normal T cell homeostasis: the conversion of naive cells into memory-phenotype cells. Nat Immunol 12(6):478-484

6. Shimatani K, Nakashima Y, Hattori M, Hamazaki Y, Minato N (2009) PD-1+ memory phenotype CD4+ T cells expressing C/EBPalpha underlie $\mathrm{T}$ cell immunodepression in senescence and leukemia. Proc Natl Acad Sci U S A 106(37):15807-15812

7. Vinuesa CG, Sanz I, Cook MC (2009) Dysregulation of germinal centres in autoimmune disease. Nat Rev Immunol 9(12):845-857

8. Grammer AC et al (2003) Abnormal germinal center reactions in systemic lupus erythematosus demonstrated by blockade of CD154-CD40 interactions. J Clin Invest 112(10):1506-1520

9. Kim WY, Sharpless NE (2006) The regulation of INK4/ARF in cancer and aging. Cell 127(2):265-275

10. Krishnamurthy $\mathbf{J}$ et al (2004) Ink4a/Arf expression is a biomarker of aging. J Clin Invest 114(9):1299-1307

11. Baccala R, Theofilopoulos AN (2005) The new paradigm of T-cell homeostatic proliferationinduced autoimmunity. Trends Immunol 26(1):5-8

12. King C, Ilic A, Koelsch K, Sarvetnick N (2004) Homeostatic expansion of T cells during immune insufficiency generates autoimmunity. Cell 117(2):265-277

13. Chang $\mathrm{CH}$ et al (2013) Posttranscriptional control of $\mathrm{T}$ cell effector function by aerobic glycolysis. Cell 153(6):1239-1251 
Part II

Translational Research for Innovative Medicine 


\title{
IL-6: A New Era for the Treatment of Autoimmune Inflammatory Diseases
}

\author{
Tadamitsu Kishimoto, Sujin Kang, and Toshio Tanaka
}

\begin{abstract}
A series of studies have revealed that IL-6 has pleiotropic activity in various cells and that its deregulated expression is responsible for the development of multiple autoimmune inflammatory diseases. A humanized antibody against the IL-6 receptor, tocilizumab, has exhibited outstanding therapeutic efficacy against rheumatoid arthritis, juvenile idiopathic arthritis, Castleman's disease, and other autoimmune inflammatory diseases, leading to its clinical use for treatment of several diseases. These findings indicate that overproduction of IL-6 is responsible for the pathogenesis of autoimmune inflammatory diseases, in which an imbalance between Th17 cells and regulatory $\mathrm{T}$ cells and autoantibodies play central roles. Recent studies have suggested that IL-6 blockade therapy can rectify these underlying immunological abnormalities in autoimmunity. However, the causes of deregulated IL-6 production in these diseases remain unknown. A novel IL-6-regulating molecule, Arid5a, specifically stabilizes IL-6 mRNA and sustains its overproduction; thus, Arid5a plays an important role in promoting inflammation and autoimmune diseases. Indeed, in Arid5a-knockout mice, experimental autoimmune encephalomyelitis does not develop, and lipopolysaccharide stimulation does not induce elevated expression of IL-6. Arid5a can counteract the destabilizing effect of a regulatory RNase, Regnase-1, on IL-6 mRNA; Regnase-1 knockout mice spontaneously develop various fatal autoimmune diseases. Further analyses of these RNA-binding proteins as well as other regulatory molecules for IL-6 expression are expected to elucidate the molecular mechanisms underlying deregulated synthesis of IL-6, and facilitate investigations of the pathogenesis of specific diseases.
\end{abstract}

Keywords IL-6 $\bullet$ Tocilizumab $\bullet$ Autoimmunity $\bullet$ Inflammation $\bullet$ Arid5a $\bullet$ Regnase-1

\footnotetext{
T. Kishimoto $(\bowtie)$

Laboratory of Immune Regulation, World Premier International Immunology

Frontier Research Center, Osaka University, 8F IFReC Building, 3-1 Yamada-oka,

Suita City, Osaka 565-0871, Japan

e-mail: kishimoto@ifrec.osaka-u.ac.jp

S. Kang • T. Tanaka

Department of Clinical Application of Biologics, Osaka University Graduate School of

Medicine, Osaka University, 2-2 Yamada-oka, Suita City, Osaka 565-0871, Japan

Department of Immunopathology, World Premier International Immunology Frontier

Research Center, Osaka University, 3-1 Yamada-oka, Suita City, Osaka 565-0871, Japan

(C) The Author(s) 2015

K. Nakao et al. (eds.), Innovative Medicine, DOI 10.1007/978-4-431-55651-0_11
} 


\section{Introduction}

Interleukin 6 (IL-6), a prototypical cytokine with redundant and pleiotropic activities, contributes to host defense against infections and tissue injuries by inducing the acute-phase response and activating immune responses and hematopoiesis. However, excessive or continuous IL-6 production plays a pathological role in an acute severe life-threatening complication, the so-called cytokine storm, and in various chronic autoimmune inflammatory diseases.

A humanized anti-IL-6 receptor (IL-6R) monoclonal antibody (Ab), tocilizumab, was developed on the basis of the idea that IL-6 blockade represents a novel therapeutic strategy for such diseases. Worldwide, clinical trials have proven that tocilizumab is highly efficacious for the treatment of intractable autoimmune inflammatory diseases, including rheumatoid arthritis (RA), systemic and polyarticular juvenile idiopathic arthritis (JIA), and Castleman's disease, leading to its approval for the treatment of these diseases. Moreover, numerous reports regarding off-label use of tocilizumab strongly suggest that an IL-6 blockade strategy is a promising therapeutic approach for treatment of other diseases. In this chapter, we will highlight the pathological role of IL-6 in autoimmune inflammatory diseases, offer perspectives on the future of IL-6 blockade therapy, and discuss the mechanisms potentially responsible for deregulated IL-6 overexpression.

\section{Pleiotropic Function of IL-6}

The gene encoding B cell stimulatory factor 2 (BSF-2), which induces the differentiation of activated B cells into Ab-producing cells, was successfully cloned in 1986 [1]. Later, BSF-2 was found to be identical to hepatocyte-stimulating factor, hybridoma growth factor, and interferon (IFN)- $\beta 2$, and the molecule was subsequently renamed IL-6 [2]. Human IL-6 consists of 212 amino acids with a 28-amino acid signal peptide and a core protein of about $20 \mathrm{kDa}$. Natural IL-6 is $21-26 \mathrm{kDa}$ with glycosylation, although the glycosyl moiety does not affect its biological activity.

\section{Effect of IL-6 in Immune Response}

IL-6, originally identified as BSF-2, promotes the differentiation of activated B cells into immunoglobulin (Ig)-producing plasma cells. IL-6 also acts as a growth factor for myeloma cells, and some myeloma cells spontaneously produce IL-6 [3]. Moreover, IL-6 promotes the survival of the plasmablasts, cells that secrete immunoglobulin or pathological autoantibodies, e.g., anti-aquaporin 4 (AQP4) in patients with neuromyelitis optica (NMO) [4]. Thus, continuous overexpression of IL-6 results in autoantibody production as well as hypergammaglobulinemia. 
IL-6 also affects T cells. IL- 6 is one of the factors that determine how naïve CD4 ${ }^{+}$ T cells differentiate into particular effector T cell subsets. IL-6 in combination with transforming growth factor (TGF)- $\beta$ preferentially induces differentiation into Th17 cells, whereas IL-6 inhibits TGF $\beta$-induced regulatory T cell (Treg) development. The resultant predominance of Th17 cells over Treg cells may be responsible for the breakdown of immunological tolerance, and may therefore be pathologically involved in the development of autoimmune inflammatory diseases [5]. Indeed, in several autoimmune disease models, IL-6 blockade at the priming step suppresses the development of the imbalance in antigen-specific effector T cell subsets, and thus development of the autoimmune diseases themselves, irrespective of the antigens used for immunization [6-8]. IL-6 also promotes T follicular helper cell differentiation as well as production of IL-21, which also promotes immunoglobulin synthesis [9].

\section{Effect of IL-6 in Acute-Phase Response}

IL-6 is a major regulator of the initiation of acute-phase responses. IL-6 induces hepatocytes to produce acute-phase proteins such as C-reactive protein (CRP), serum amyloid A (SAA), fibrinogen, hepcidin, and $\alpha 1$-antichymotrypsin, whereas it inhibits the production of fibronectin, albumin, and transferrin [10]. CRP and SAA are biological markers of inflammation occurring somewhere in the body. Administration of tocilizumab completely normalizes the serum levels of these proteins, indicating that their synthesis depends primarily on IL-6 [11, 12]. Increased levels of acute-phase proteins signal a state of emergency and contribute to host defense, whereas continuous expression of high levels of SAA or hepcidin leads to complications of chronic inflammatory diseases, such as amyloid A amyloidosis or anemia, respectively $[12,13]$.

\section{Other Effects of IL-6}

IL-6 also acts on other cells [2, 14]. IL-6 produced by bone-marrow stromal cells stimulates synovial fibroblasts to express the receptor activator of the nuclear factor kappa beta (NF- $\mathrm{kB}$ ) ligand (RANKL), which is indispensable for the differentiation and activation of osteoclasts. Osteoclast activation leads to bone resorption and osteoporosis [15]. IL-6 also promotes production of vascular endothelial growth factor by fibroblasts, leading to angiogenesis and increased vascular permeability, which are pathological features of inflammatory lesions observed in synovial tissues of RA and in other conditions [16]. IL-6 promotes the production of collagen in dermal fibroblasts and their differentiation into myofibroblasts, which are predominantly responsible for skin fibrosis in patients with systemic sclerosis (SSc) [17]. Moreover, IL-6 can interact with vascular endothelial cells, the endocrine system including the hypothalamic-pituitary-adrenal axis, neuropsychological systems, and other systems. 


\section{IL-6 Signaling Systems}

The IL-6 signaling cascade is triggered by binding of IL-6 to its specific receptor, IL-6R [18]. The receptor constitutes the IL-6-binding chain, which exists in two different forms, $80 \mathrm{kDa}$ transmembrane IL-6R and 50-55 kDa soluble IL-6R (sIL6R) [18, 19], and the $130 \mathrm{kDa}$ gp130 constitutes the signal-transducing chain [20]. The expression of transmembrane IL-6R is limited to cells such as hepatocytes and leukocytes, whereas sIL-6R is present in blood and tissue fluid. After IL-6 binding to either transmembrane IL-6R or sIL-6R, the resultant complex induces homodimerization of gp130 [21] and triggers a downstream signal cascade. The pleiotropic effects of IL-6 are due to the broad range of gp130 expression on various cells [22]. IL-6R is the specific receptor for IL-6, whereas the signal-transducing chain gp 130 is shared by other members of the IL- 6 family of cytokines, including leukemia inhibitory factor, oncostatin M, ciliary neurotrophic factor, IL-11, cardiotrophin 1, cardiotrophin-like cytokine, IL-27, and IL-35. The findings that all IL-6 family members use the same molecule (gp130) for signal transduction resolved a longstanding mystery regarding why these cytokines exhibit functional redundancy [18, $22,23]$.

Activation of gp130 leads in turn to activation of two downstream signaling cascades, the Janus kinase (JAK)-signal transducer and activator of transcription 3 (STAT3) pathway and the JAK-SH2-domain-containing protein tyrosine phosphatase-2-mitogen-activated protein kinase pathway. Various IL-6-responsive genes are induced by activation of the transcription factor STAT3, which also controls the expression of suppressor of cytokine signaling 1 (SOCS1) and SOCS3. In this context, SOCS1 binds to tyrosine-phosphorylated JAK [24], and SOCS3 binds to tyrosine-phosphorylated gp130 [25], thereby terminating IL-6 signaling via a negative-feedback loop.

\section{Regulatory Mechanisms of IL-6 Synthesis}

In the serum of healthy individuals, the IL-6 level is less than $5 \mathrm{pg} / \mathrm{ml}$; however, the IL-6 concentration increases dramatically during emergent events such as infections and tissue injuries. At sites of infections, neutrophils, monocytes, and macrophages promptly produce IL-6 following through recognition of components of exogenous pathogens via pathogen-associated molecular pattern receptors [26]. In cases of non-infectious inflammation caused by burns or trauma, IL-6 is produced by these immune cells through the stimulation of damage-associated molecular patterns, which are released from damaged or dying cells. Besides immune-competent cells, other types of cells, including endothelial cells, mesenchymal cells, fibroblasts, and cancer cells, are also able to synthesize IL-6 [14]. 
When the emergent stimuli are completely removed from the host, the signal transduction cascade by IL-6-mediated activation is terminated through negative regulatory systems such as ligand-induced internalization, degradation of gp130, and recruitment of SOCS [24, 25], leading to normalization of the CRP level in serum. Accordingly, synthesis of IL-6 is tightly regulated at the transcriptional and post-transcriptional level.

\section{Transcriptional and Post-transcriptional Regulation of IL-6}

Several factors regulate the fate of IL-6 mRNA at either the transcriptional or posttranscriptional stage. The promoter region of the human IL- 6 gene includes binding sites for cis-regulatory elements such as activator protein 1 , interferon regulatory factor 1, a CCAAT enhancer binding protein $\beta$ (C/EBP $\beta$, also termed NF-IL6), specificity protein 1 , and NF- $\kappa B$ [27, 28]. Stimulation of Toll-like receptor (TLR) with bacterial or viral pathogen components, or pro-inflammatory cytokines such as IL-1, IL-6, and TNF- $\alpha$, can activate cis-regulatory elements, leading to IL-6 synthesis, whereas several microRNAs (miRs) directly or indirectly down-regulate IL-6 transcription. For instance, miR-155 interacts with the $3^{\prime}$-untranslated regions (UTR) of $\mathrm{C} / \mathrm{EBP} \beta$ to suppress $\mathrm{C} / \mathrm{EBP} \beta$ expression, whereas miR146a/b targets IL-1 receptor-associated kinase 1 to indirectly inhibit IL-6 transcription.

In general, the mechanisms of post-transcriptional regulation of cytokine expression target either the 5'-UTR of mRNAs to modulate initiation of translation or the $3^{\prime}$-UTR of mRNAs to modulate their stability [29, 30]. Several recently identified RNA-binding proteins and miRs regulate the stability of IL-6 mRNA [27, 28]. For instance, miR-26a binds to the 3'-UTR of IL-6 mRNA in hepatocellular carcinoma, suppressing the expression of STAT3 target genes. In addition, open reading frame 57 of Kaposi sarcoma-associated herpes virus competes with miR-608a for binding to the IL-6 mRNA, thereby stabilizing it.

One of the RNA-binding proteins that influence IL-6 expression, regulatory RNase-1 (Regnase-1, also known as Zc3h12a), interacts with the 3'-UTR of IL-6 mRNA, leading to its destabilization at steady state [31]. Regnase-1-deficient mice spontaneously develop autoimmune diseases with splenomegaly and lymphadenopathy. On the other hand, stimulation of IL-1R/TLR causes phosphorylation of

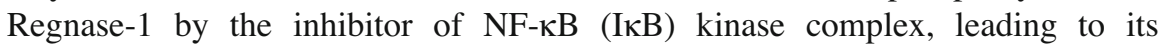
ubiquitination and degradation. The degradation of Regnase-1 is required for expression of IL-6 mRNA; however, re-expression of Regnase-1, which is induced by NF- $\kappa \mathrm{B}$ activation, prevents excessive IL-6 production [32].

We recently identified an RNA-binding protein, AT-rich interactive domaincontaining protein 5a (Arid5a), which interacts with the $3^{\prime}$-UTR and selectively stabilizes the IL-6 mRNA but not TNF- $\alpha$ or IL-12 mRNA [33]. Expression of Arid5a is dramatically upregulated in T cells under Th17-skewing conditions and in 
macrophages stimulated by lipopolysaccharide (LPS), IL-1 $\beta$, and IL-6. Thus, there exists a positive-feedback loop between IL-6 and Arid5a. In untreated RA patients, expression of Arid5a is elevated in $\mathrm{CD}^{+} \mathrm{T}$ cells in the peripheral blood, whereas tocilizumab treatment decreases Arid5a expression [34]. Furthermore, Arid5adeficient mice exhibit reduced serum IL-6 levels upon LPS challenge, and fail to develop experimental autoimmune encephalomyelitis. Arid5a counteracts the destabilizing function of Regnase-1 on IL-6 mRNA, suggesting not only that the balance between Arid5a and Regnase-1 is an important determinant of the stability of IL-6 mRNA, but also that the predominance of Arid5a expression over Regnase-1 promotes inflammatory processes and development of autoimmune diseases [27, 35] (Fig. 1).

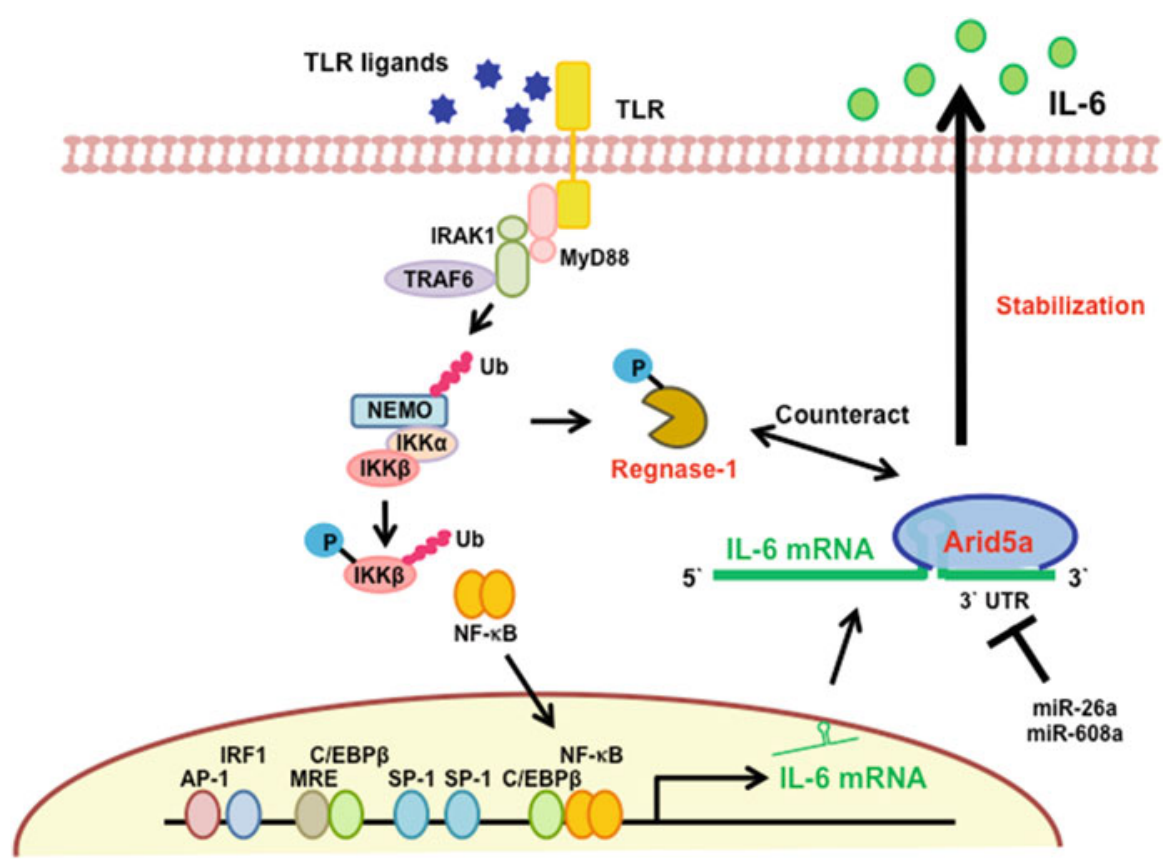

Fig. 1 Regulatory mechanism of IL-6 production. In the innate immune response, pathogenassociated molecular patterns are recognized by pathogen recognition receptors, leading to expression of pro-inflammatory cytokines. For example, Toll-like receptor (TLR)-4 recognizes lipopolysaccharide (LPS), which is a component of the outer membrane of Gram-negative bacteria; TLR4 activates the nuclear factor of kappa beta $(N F-\kappa B)$ signaling pathway and promotes the transcription of IL-6 mRNA. Regnase- 1 promotes IL-6 mRNA degradation, whereas Arid5a inhibits the destabilizing effect of Regnase- 1 . The balance between Arid5a and Regnase- 1 is important for the regulation of IL-6 mRNA. IRAK1 IL-1 receptor-associated kinase 1, MyD88 myeloid differentiation primary response $88, T R A F 6$ tumor necrosis factor receptor-associated factor $6, U b$ ubiquitination, $N E M O \mathrm{NF}-\kappa \mathrm{B}$ essential modulator, $I K K$ inhibitor of NF- $\kappa \mathrm{B}$ kinase, $A P-1$ activator protein 1, IRF1 interferon regulatory factor 1, MRE multiple response element, $C / E B P \beta$ CCAAT enhancer binding protein $\beta, S P-1$ specificity protein $1, U T R$ untranslated region, miR microRNA 


\section{Pathological Role of IL-6 in Autoimmune Inflammatory Diseases}

As described elsewhere, the immediate and transient expression of IL-6 contributes to host defense against infections and tissue injuries. However, deregulated excessive IL-6 synthesis during this protective process or persistent IL-6 production leads to the development of a severe acute life-threatening complication, the so-called cytokine storm or chronic autoimmune inflammatory diseases, respectively. The association of IL-6 with disease development was first demonstrated in a case of benign heart tumor, cardiac myxoma. The cultured fluid from the myxoma tissue of a patient, who had presented with fever, polyarthritis, an elevated CRP level, anemia, and hypergammaglobulinemia with positivity for anti-nuclear factor, was found to contain a large quantity of IL-6 [36]. Subsequently, excessive IL-6 expression was also detected in the synovial fluids of RA, swollen lymph nodes of Castleman's disease, myeloma cells [3], and peripheral blood cells or infiltrating cells in tissues involved in various other autoimmune inflammatory diseases [14, 37]. The extent of the elevation of the serum IL-6 level depends on the particular disease and its disease severity, but in cytokine storms such as those associated with septic shock, the concentration can reach as high as the microgram per milliliter level [38].

\section{IL-6-Targeting Strategies: A New Era for the Treatment of Autoimmune Inflammatory Diseases}

In view of the pathological role of IL-6 in various autoimmune inflammatory diseases, IL-6 targeting was evaluated as a novel therapeutic strategy against these diseases [37]. Tocilizumab, a humanized anti-IL-6R monoclonal Ab of the IgG1 class, was generated by grafting the complementarity-determining regions of a mouse anti-human IL-6R Ab onto human IgG1 [39]. This Ab can block IL-6mediated signal transduction by inhibiting IL-6 binding to both transmembrane IL-6R and sIL-6R. Numerous worldwide clinical trials verified the outstanding efficacy and tolerable safety of tocilizumab, leading to its current approval for the treatment of RA in more than 130 countries; systemic JIA (sJIA) in Japan, India, the USA, and the EU; and polyarticular JIA and Castleman's disease in Japan and India $[27,40,41]$.

\section{Efficacy of Tocilizumab in Rheumatoid Arthritis}

RA is an autoimmune disease characterized by systemic and joint inflammation, which causes joint destruction, dramatically reduces activity of daily living (ADL) and quality of life (QOL), and shortens lifespan [42]. Clinical trials demonstrated 
that tocilizumab effectively suppresses disease activity and protects against progression of joint destruction, as well as ameliorating the effect of RA on ADL and QOL [43]. Although other biologics are currently used to treat RA, including TNF inhibitors, an inhibitor of $\mathrm{T}$ cell stimulation, and a B cell depleting agent, tocilizumab appears to be the most powerful with regard to suppression of disease activity. Tocilizumab is the only biologic proven to be more efficacious as a monotherapy than the anchor disease-modifying antirheumatic drug, methotrexate (MTX). TNF inhibitors require the concomitant use of MTX to achieve their maximal effect, but tocilizumab monotherapy exhibited no clinically relevant inferiority to tocilizumab combination therapy with MTX [44]. Moreover, direct comparison of monotherapy with tocilizumab and adalimumab, a fully human anti-TNF- $\alpha$ Ab, demonstrated that tocilizumab was superior to adalimumab, as determined by various indices of disease activity [45]. On the basis of these findings, in the newest RA management recommendation by the European League Against Rheumatism, tocilizumab was positioned as the first-line biologic, especially in patients for whom biological monotherapy must be initiated [46]. Furthermore, because of the suppressive effects of tocilizumab in the synthesis of acute-phase proteins such as SAA and hepcidin, it is appropriate to select tocilizumab for cases of RA complicated by amyloid A amyloidosis or anemia associated with chronic disorders [43].

\section{Efficacy of Tocilizumab in Systemic Juvenile Idiopathic Arthritis}

sJIA is a subtype of chronic childhood arthritis that leads to joint destruction, functional disability, and growth impairment, accompanied by systemic inflammation [47]. IL-6 is markedly elevated in the blood of sJIA patients, and the IL-6 level is well correlated with disease activity. In a phase III trial of Japanese 56 patients with sJIA, $91 \%, 86 \%$, and $68 \%$ of the patients exhibited American College of Rheumatology (ACR) Pedi responses of $30 \%, 50 \%$, and $70 \%$, respectively, at week 6 [48]. Subsequently, in a global phase III trial, 112 children with active sJIA randomly received placebo or tocilizumab. At week 12, the primary endpoint (absence of fever + improvement of $30 \%$ or greater in at least three of the six variables in the ACR core set for JIA) was met in significantly more patients in the tocilizumab-treated group than in the placebo group (85\% vs. $24 \%$ ) [49]. At week $52,82 \%$ and $59 \%$ of the patients who received tocilizumab attained improvements of $70 \%$ and $90 \%$, respectively. The striking responsiveness of sJIA to tocilizumab has led to widespread recognition that we have started a new era in the treatment of this disease, long considered to be one of the most intractable juvenile diseases [50]. 


\section{Efficacy of Tocilizumab in Castleman's Disease}

Castleman's disease is a lymphoproliferative disease with benign hyperplastic lymph nodes characterized by follicular hyperplasia and capillary proliferation accompanied by endothelial hyperplasia [51]. Deregulated IL-6 expression in transgenic mice produces a syndrome resembling Castleman's disease [52], whereas IL-6 blockade inhibits inflammatory manifestations in these animals [53]. IL-6 is highly expressed in germinal center B cells of hyperplastic lymph nodes of patients with Castleman's disease, and surgical removal of a solitary involved lymph node leads to clinical improvement and reduced serum IL-6 concentrations [54]. In two open-label clinical trials of tocilizumab for Castleman's disease, the drug exerted a significant ameliorative effect with regard to clinical symptoms and laboratory findings $[55,56]$.

\section{Efficacy of Tocilizumab in Other Autoimmune Inflammatory Diseases}

Case reports, series, and pilot studies of the off-label applications of tocilizumab have produced favorable outcomes, indicating that tocilizumab may be used for the treatment of multiple conditions including autoimmune diseases, chronic inflammatory diseases, and autoinflammatory diseases [27, 40, 41]. The results accumulated so far strongly suggest that IL-6 blockade represents an innovative therapeutic strategy for SSc, large-vessel vasculitis, amyloid A amyloidosis, NMO, adult-onset Still's disease (AOSD), and cytokine release syndrome (CRS).

$\mathrm{SSc}$ is a connective tissue disorder characterized by tissue fibrosis, vasculopathy, and immune abnormalities [57]. Several lines of evidence suggest that IL-6 is a potential therapeutic target cytokine in SSc. First, IL-6 is expressed at high levels in patient serum and involved skin, and the serum level is well correlated with the severity of skin symptoms. Second, IL-6 promotes collagen production and expression of $\alpha$-smooth muscle actin by dermal fibroblasts, as well as endothelial cell activation and apoptosis in endothelial cell-neutrophil co-cultures. Moreover, in models of SSc induced by bleomycin, IL-6 blockade by administration of antiIL-6R Ab suppresses myofibroblast activation, resulting in reduced dermal fibrosis [58]. We observed the beneficial effects of tocilizumab for two patients with SSc [59]. Histological analyses revealed thinning of collagen fiber bundles and reduction of activated myofibroblasts in the dermis after a 6-month treatment, suggesting that IL-6 blockade strategy is a promising approach for the treatment of SSc.

The pathological role of IL-6 has been well documented in large-vessel vasculitis, e.g., giant cell arteritis (GCA) and Takayasu arteritis (TA) [60]. Serum concentrations of IL-6 are elevated at the onset and during clinical relapse of GCA and TA, 
and tissue-infiltrating cells produce major quantities of IL-6 and IFN- $\gamma$ in patients with both diseases. Tocilizumab treatment has resulted in rapid and prominent beneficial effects in patients with GCA and TA who had been refractory to conventional treatment regimens [40, 41]. Moreover, tocilizumab monotherapy induced disease remission in five patients with GCA. These results strongly suggest that IL-6 inhibition represents a promising therapeutic strategy for large-vessel vasculitis.

A serious complication of chronic inflammatory diseases is amyloid A amyloidosis, in which amyloid fibril deposition causes progressive deterioration in various organs [12]. Sustained high concentrations of SAA, an amyloid fibril precursor protein, correlate with progression of renal amyloid diseases. Tocilizumab is the most powerful known inhibitor of SAA production. Case studies of amyloid A amyloidosis complicated by RA, JIA, vasculitis syndrome, Behcet's disease, and latent tuberculosis have revealed the striking clinical effect of tocilizumab on gastrointestinal symptoms and renal function [12, 40, 41].

$\mathrm{NMO}$ is a chronic inflammatory disorder predominantly affecting the spinal cord and optic nerves. In this disease, autoantibodies directed against the astrocyte waterchannel protein, AQP4, play a pathologic role [61]. Several studies have reported a significant increase of IL-6 in the cerebrospinal fluid of patients with NMO. Moreover, the population of plasmablasts with the $\mathrm{CD} 19^{\text {int }} \mathrm{CD} 27^{\text {high }} \mathrm{CD} 38^{\text {high }} \mathrm{CD} 180^{\text {negative }}$ phenotype is elevated in the peripheral blood of NMO patients, and anti-AQP4 Abs are produced by these plasmablasts [4]. IL-6 increases the survival of plasmablasts, as well as AQP4 Ab secretion, whereas tocilizumab reduces the survival of these cells. Clinical improvement and reduction of serum levels of anti-AQP4 Abs have been reported in several NMO patients who were refractory to conventional treatment regimens $[62,63]$.

AOSD, a chronic inflammatory disease characterized by four cardinal symptoms (spiking fever, evanescent maculopapular rash, arthritis, and leukocytosis) pathologically resembles sJIA and is considered to be an adult-onset type of sJIA [64]. As expected, numerous case studies and pilot studies have shown that tocilizumab treatment improved clinical symptoms and signs in AOSD patients who had been refractory to conventional treatment $[40,41]$. These favorable results also indicate that, just as for sJIA, tocilizumab may become a first-line biologic for the treatment of AOSD.

CRS is a potentially fatal immune reaction, which is sometimes induced by hyperactivation of $\mathrm{T}$ cells after $\mathrm{T}$ cell-engaging therapies [65]. Serum levels of IL-6, IL-10, and IFN- $\gamma$ are significantly elevated in patients with CRS. Two recent reports showed that CRS - which occurred in two patients with acute lymphoblastic leukemia (ALL) treated with chimeric antigen receptor-modified $\mathrm{T}$ cells with specificity for CD19 (CTL019 cells) and in one ALL patient treated with blinatumomab, a CD19/CD3-bispecific Ab that engages the $\mathrm{T}$ cell receptor-could be abrogated by a single injection of tocilizumab $[66,67]$. These findings indicate that IL-6 inhibition represents an innovative therapeutic approach for T cell-mediated CRS or other complications with hypercytokinemia, such as systemic inflammatory response syndrome, septic shock, macrophage activation syndrome, and hemophagocytic syndrome. 
The beneficial effects of tocilizumab strongly suggest that it will be widely applicable for the treatment of chronic autoimmune inflammatory diseases and acute systemic inflammatory complications. However, further clinical evaluations are essential in order to determine additional indications for tocilizumab therapy. A list of ongoing clinical trials of tocilizumab registered at ClinicalTrials.gov is provided in Table 1.

Table 1 Ongoing clinical trials of tocilizumab registered at ClinicalTrials.gov

\begin{tabular}{|c|c|c|}
\hline Targeted disease & Status & Identifier \\
\hline Relapsing polychondritis & Phase II & NCT01041248 \\
\hline Diabetes mellitus (type 2), obesity & Phase II & NCT01073826 \\
\hline Graves' ophthalmopathy & Phase III & NCT01297699 \\
\hline \multirow[t]{2}{*}{ Cardiovascular disease in RA (vs. etanercept) } & Phase IV & NCT01331837 \\
\hline & Phase IV & NCT01752335 \\
\hline \multirow[t]{2}{*}{ Polymyalgia rheumatica } & Phase II & NCT01396317 \\
\hline & Phase II & NCT01713842 \\
\hline \multirow[t]{3}{*}{ Giant cell arteritis } & Phase II & NCT01450137 \\
\hline & Phase III & NCT01791153 \\
\hline & Phase II & NCT01910038 \\
\hline Takayasu arteritis & Phase III & NCT02101333 \\
\hline \multirow[t]{2}{*}{ Acute GVHD } & Phase I/II & NCT01475162 \\
\hline & Phase II & NCT01757197 \\
\hline Chronic GVHD & Phase II & NCT02174263 \\
\hline Non-ST-elevation myocardial infarction & Phase II & NCT01491074 \\
\hline Systemic sclerosis & Phase II/III & NCT01532869 \\
\hline Transplant rates awaiting kidney transplantation & Phase I/II & NCT01594424 \\
\hline Renal graft inflammation & Phase II & NCT02108600 \\
\hline sJIA-associated uveitis & Phase I/II & NCT01603355 \\
\hline Non-infectious uveitis & Phase I/II & NCT01717170 \\
\hline Recurrent ovarian cancer & Phase I/II & NCT01637532 \\
\hline Behcet's syndrome & Phase II & NCT01693653 \\
\hline \multirow[t]{2}{*}{ Schizophrenia } & Phase I & NCT01696929 \\
\hline & Phase IV & NCT02034474 \\
\hline Erdheim-Chester disease & Phase II & NCT01727206 \\
\hline Primary Sjogren's syndrome & Phase II/III & NCT01782235 \\
\hline Fibrous dysplasia of bone & Phase II & NCT01791842 \\
\hline Hemophagocytic lymphohistiocytosis & Phase II & NCT02007239 \\
\hline Polymyositis/dermatomyositis & Phase II & NCT02043548 \\
\hline HIV infection & Phase I/II & NCT02049437 \\
\hline
\end{tabular}

$R A$ rheumatoid arthritis, GVHD graft-versus-host disease, sJIA systemic juvenile idiopathic arthritis, HIV human immunodeficiency virus 


\section{Mechanisms Through Which Tocilizumab Is Efficacious in Various Autoimmune Inflammatory Diseases}

The efficacy of tocilizumab in amyloid A amyloidosis and anemia associated with chronic disorders can be explained by its suppression of the responsible proteins, SAA and hepcidin, respectively, but the mechanisms through which tocilizumab is efficacious in various phenotypically different autoimmune inflammatory diseases remain incompletely understood [68]. As observed in NMO, tocilizumab treatment could decrease autoantibody (anti-AQP4) production by acting on plasmablasts [4, 62]. IL-21 promotes plasma cell differentiation and preferentially induces IgG4 production [9]. One study demonstrated that tocilizumab treatment causes a selective reduction in IL-21 production of memory/activated T cells, leading to a reduction in the serum levels of IgG4-specific anti-citrullinated peptide Ab in RA patients [69]. Several recent reports demonstrated that tocilizumab therapy led to an increase in the number of Treg cells in peripheral blood $\mathrm{CD}^{+} \mathrm{T}$ cell fractions in RA patients [70-72] (Fig. 2). These effects may account for the correction of immunological abnormalities. In addition, the treatment reportedly resulted in improvement in various other biomarkers related to inflammation, bone and cartilage metabolism, angiogenesis,

a

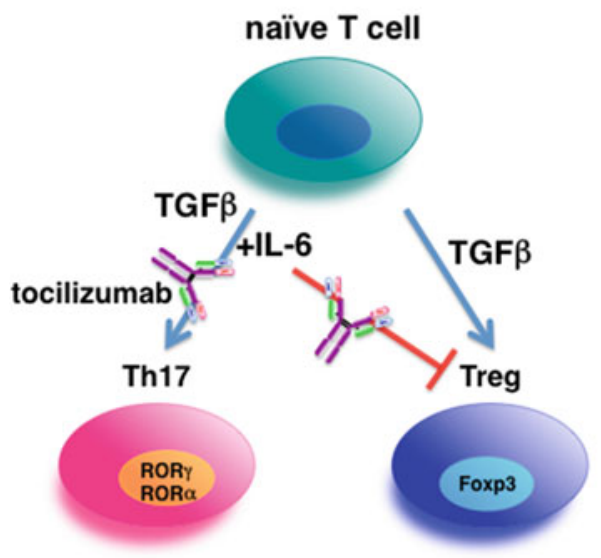

b

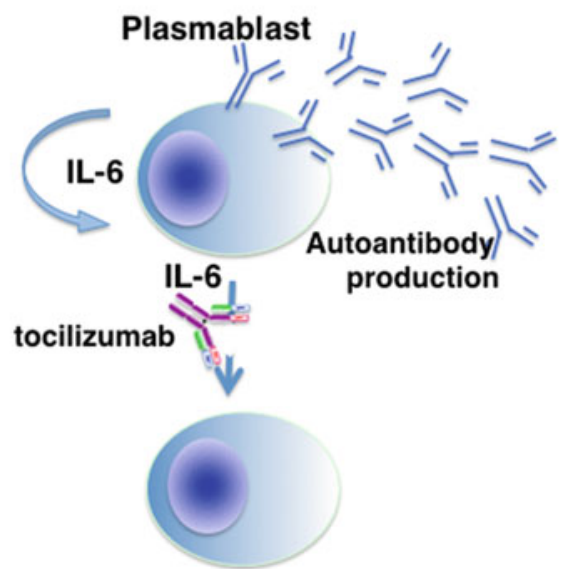

Fig. 2 IL-6-targeted therapy can rectify immunological abnormalities. Although the pathogenesis of autoimmune inflammatory diseases is not fully understood, an imbalance between effector $\mathrm{CD} 4{ }^{+} \mathrm{T}$ cell subsets $(\mathrm{Th} 17 \pm \mathrm{Th} 1 / \mathrm{Treg} \uparrow)$ and autoantibodies play central roles in their development. (a) Overexpression of IL-6 may lead to an imbalance in effector T cell subsets (Th17/Treg $\uparrow$ ), whereas tocilizumab therapy can rectify the imbalance. (b) IL-6, acting in an autocrine or paracrine manner, increases the survival of plasmablasts that produce autoantibodies, whereas tocilizumab treatment decreases the survival of these cells, leading to the suppression of autoantibody synthesis. TGF $\beta$ transforming growth factor $\beta$, Treg regulatory $\mathrm{T}$ cells, ROR retinoid-related orphan receptor, Foxp3 forkhead box p3 
glucose metabolism, fibrosis, etc. These findings indicate that the clinical effects of tocilizumab are mediated by several mechanisms. Another possible explanation for the prominent effect of tocilizumab is that the primary cause of successfully treated diseases is deregulated synthesis of IL-6, but that phenotypic differences arise because of differences in the cell populations that produce IL-6. Thus, to facilitate investigations of the pathogenesis of specific diseases, it will be necessary to elucidate the mechanisms responsible for excessive or continuous IL-6 production.

\section{Concluding Remarks}

Tocilizumab was developed on the basis of a comprehensive view of the IL-6-mediated signaling system, the pathological significance of IL-6 in various diseases, and progress in bioengineering techniques. Tocilizumab is now positioned as a first-line biologic for the treatment of moderate to severe RA and sJIA, and is the only drug approved for Castleman's disease. This great success has resulted in a paradigm shift in the treatment of these diseases, and has accelerated the development of other various IL-6 inhibitors [73]. Within the next decade, we anticipate that IL-6 inhibitors will be widely used for the treatment of various diseases currently considered to be intractable; as a result, some of these diseases will no longer be refractory to therapy.

Although fundamental research on the IL-6-mediated signaling pathway was able to solve the long-standing mystery regarding why cytokines exhibit redundant and pleiotropic activity, another mystery persists regarding why IL-6 is excessively or continuously expressed in various diseases. Accurate and detailed analyses of the proteins such as Arid5a and Regnase-1 and miRs that regulate IL-6 synthesis will be helpful in solving this mystery. Clarification of the mechanism(s) involved will inspire the identification of more specific target molecules and investigations into the pathogenesis of specific diseases.

Conflict of Interest T. Kishimoto holds a patent for tocilizumab and has received royalties for Actemra. T.Tanaka has received a grant and payment for lectures including service on speaker's bureaus from Chugai Pharmaceutical Co.,Ltd. S. Kang declares no conflict of interest. The Department of Clinical Application of Biologics of Osaka University Graduate School of Medicine is an endowment department, supported with an unrestricted grant from Chugai Pharmaceutical Co.,Ltd.

Open Access This chapter is distributed under the terms of the Creative Commons Attribution Noncommercial License, which permits any noncommercial use, distribution, and reproduction in any medium, provided the original author(s) and source are credited.

\section{References}

1. Hirano T, Yasukawa K, Harada $\mathrm{H}$ et al (1986) Complementary DNA for a novel human interleukin (BSF-2) that induces B lymphocytes to produce immunoglobulin. Nature 324:73-76

2. Kishimoto T (1989) The biology of interleukin-6. Blood 74:1-10 
3. Kawano M, Hirano T, Matsuda T et al (1988) Autocrine generation and requirement of BSF-2/ IL-6 for human multiple myelomas. Nature 332:83-85

4. Chihara N, Aranami T, Sato $\mathrm{W}$ et al (2011) Interleukin 6 signaling promotes anti-aquaporin 4 autoantibody production from plasmablasts in neuromyelitis optica. Proc Natl Acad Sci U S A 108:3701-3706

5. Kimura A, Kishimoto T (2010) IL-6: regulator of Treg/Th17 balance. Eur J Immunol 40:1830-1835

6. Fujimoto M, Serada S, Mihara M et al (2008) Interleukin-6 blockade suppresses autoimmune arthritis in mice by the inhibition of inflammatory Th17 responses. Arthritis Rheum 58:3710-3719

7. Serada S, Fujimoto M, Mihara M et al (2008) IL-6 blockade inhibits the induction of myelin antigen-specific Th17 cells and Th1 cells in experimental autoimmune encephalomyelitis. Proc Natl Acad Sci U S A 105:9041-9046

8. Haruta H, Ohguro N, Fujimoto M et al (2011) Blockade of interleukin-6 signaling suppresses not only th17 but also interphotoreceptor retinoid binding protein-specific Th1 by promoting regulatory $\mathrm{T}$ cells in experimental autoimmune uveoretinitis. Invest Ophthalmol Vis Sci 52:3264-3271

9. Ma CS, Deenick EK, Batten M et al (2012) The origins, function, and regulation of T follicular helper cells. J Exp Med 209:1241-1253

10. Heinrich PC, Castell JV, Andus T (1990) Interleukin-6 and the acute phase response. Biochem J 265:621-636

11. Nishimoto N, Terao K, Mima $T$ et al (2008) Mechanisms and pathologic significances in increase in serum interleukin-6 (IL-6) and soluble IL-6 receptor after administration of an antiIL-6 receptor antibody, tocilizumab, in patients with rheumatoid arthritis and Castleman disease. Blood 112:3959-3964

12. Tanaka T, Hagihara K, Hishitani Y et al (2011) Tocilizumab for the treatment of AA amyloidosis. In: Isil Adadan G (ed) Amyloidosis - an insight to disease of systems and novel therapies. INTECH Open Access Publisher, Rijeka, pp 155-170

13. Nemeth E, Rivera S, Gabayan V et al (2004) IL-6 mediates hypoferremia of inflammation by inducing the synthesis of the iron regulatory hormone hepcidin. J Clin Invest 113:1271-1276

14. Akira S, Taga T, Kishimoto T (1993) Interleukin-6 in biology and medicine. Adv Immunol 54:1-78

15. Hashizume M, Hayakawa N, Mihara M (2008) IL-6 trans-signalling directly induces RANKL on fibroblast-like synovial cells and is involved in RANKL induction by TNF-alpha and IL-17. Rheumatology (Oxford) 47:1635-1640

16. Nakahara H, Song J, Sugimoto M et al (2003) Anti-interleukin-6 receptor antibody therapy reduces vascular endothelial growth factor production in rheumatoid arthritis. Arthritis Rheum 48:1521-1529

17. Duncan MR, Berman B (1991) Stimulation of collagen and glycosaminoglycan production in cultured human adult dermal fibroblasts by recombinant human interleukin 6. J Invest Dermatol 97:686-692

18. Kishimoto T, Akira S, Taga T (1992) Interleukin-6 and its receptor: a paradigm for cytokines. Science 258:593-597

19. Yamasaki K, Taga T, Hirata $Y$ et al (1988) Cloning and expression of the human interleukin-6 (BSF-2/IFN beta 2) receptor. Science 241:825-828

20. Hibi M, Murakami M, Saito M et al (1990) Molecular cloning and expression of an IL-6 signal transducer, gp130. Cell 63:1149-1157

21. Murakami M, Hibi M, Nakagawa N et al (1993) IL-6-induced homodimerization of gp130 and associated activation of a tyrosine kinase. Science 260:1808-1810

22. Taga T, Kishimoto T (1997) Gp130 and the interleukin-6 family of cytokines. Annu Rev Immunol 15:797-819

23. Kishimoto T, Taga T, Akira S (1994) Cytokine signal transduction. Cell 76:253-262 
24. Naka T, Narazaki M, Hirata M et al (1997) Structure and function of a new STAT-induced STAT inhibitor. Nature 387:924-929

25. Schmitz J, Weissenbach M, Haan S et al (2000) SOCS3 exerts its inhibitory function on interleukin-6 signal transduction through the SHP2 recruitment sites of gp130. J Biol Chem 275:12848-12856

26. Bianchi ME (2007) DAMPs, PAMPs and alarmins: all we need to know about danger. J Leukoc Biol 81:1-5

27. Kang S, Tanaka T, Kishimoto T (2015) Therapeutic uses of anti-interleukin-6 receptor antibody. Int Immuno 127:21-29

28. Wolf J, Rose-John S, Garbers C (2014) Interleukin-6 and its receptors: a highly regulated and dynamic system. Cytokine 70:11-20

29. Anderson P (2008) Post-transcriptional control of cytokine production. Nat Immunol 9:353-359

30. Chen CY, Shyu AB (1995) AU-rich elements: characterization and importance in mRNA degradation. Trends Biochem Sci 20:465-470

31. Matsushita K, Takeuchi O, Standley DM et al (2009) Zc3h12a is an RNase essential for controlling immune responses by regulating mRNA decay. Nature 458:1185-1190

32. Iwasaki H, Takeuchi O, Teraguchi S et al (2011) The IkappaB kinase complex regulates the stability of cytokine-encoding mRNA induced by TLR-IL-1R by controlling degradation of Regnase-1. Nat Immunol 12:1167-1175

33. Masuda K, Ripley B, Nishimura R et al (2013) Arid5a controls IL-6 mRNA stability, which contributes to elevation of IL-6 level in vivo. Proc Natl Acad Sci U S A 110:9409-9414

34. Saito Y, Kagami SI, Sanayama Y et al (2014) At-rich-interactive domain-containing protein 5A functions as a negative regulator of retinoic acid receptor-related orphan nuclear receptor $\gamma \mathrm{t}$-induced Th17 cell differentiation. Arthritis Rheum 66:1185-1194

35. Tanaka T, Kishimoto T (2014) The biology and medical implications of interleukin-6. Cancer Immunol Res 2:288-294

36. Hirano T, Taga T, Yasukawa K et al (1987) Human B-cell differentiation factor defined by an anti-peptide antibody and its possible role in autoantibody production. Proc Natl Acad Sci U S A 84:228-231

37. Kishimoto T (2005) Interleukin-6: from basic science to medicine-40 years in immunology. Annu Rev Immunol 23:1-21

38. Waage A, Brandtzaeq P, Halstensen A et al (1989) The complex pattern of cytokines in serum from patients with meningococcal septic shock. Association between interleukin 6, interleukin 1, and fatal outcome. J Exp Med 169:333-338

39. Sato K, Tsuchiya M, Saldanha J et al (1993) Reshaping a human antibody to inhibit the interleukin 6-dependent tumor cell growth. Cancer Res 3:851-856

40. Tanaka T, Narazaki M, Kishimoto T (2012) Therapeutic targeting of the interleukin-6 receptor. Annu Rev Pharmacol Toxicol 52:199-219

41. Tanaka T, Narazaki M, Ogata A, Kishimoto T (2014) A new era for the treatment of inflammatory autoimmune diseases by interleukin-6 blockade strategy. Semin Immunol 26:88-96

42. Mclnnes IB, Schett G (2011) The pathogenesis of rheumatoid arthritis. N Engl J Med 365:2205-2219

43. Tanaka T, Hishitani Y, Ogata A (2014) Monoclonal antibodies in rheumatoid arthritis: comparative effectiveness of tocilizumab with tumor necrosis factor inhibitors. Biologics 8:141-153

44. Dougados M, Kissel K, Sheeran T et al (2013) Adding tocilizumab or switching to tocilizumab monotherapy in methotrexate inadequate responders: 24-week symptomatic and structural results of a 2-year randomised controlled strategy trial in rheumatoid arthritis (ACT-RAY). Ann Rheum Dis 72:43-50

45. Gabay C, Emery P, van Vollenhoven R et al (2013) Tocilizumab monotherapy versus adalimumab monotherapy for treatment of rheumatoid arthritis (ADACTA): a randomised, doubleblind, controlled phase 4 trial. Lancet 381:1541-1550 
46. Smolen JS, Landewe R, Breedveld FC et al (2014) EULAR recommendations for the management of rheumatoid arthritis with synthetic and biological disease-modifying antirheumatic drugs: 2013 update. Ann Rheum Dis 73:510-515

47. Yokota S, Tanaka T, Kishimoto T (2012) Efficacy, safety and tolerability of tocilizumab in patients with systemic juvenile idiopathic arthritis. Ther Adv Musculoskelet Dis 4:387-397

48. Yokota S, Imagawa T, Mori M et al (2008) Efficacy and safety of tocilizumab in patients with systemic-onset juvenile idiopathic arthritis: a randomised, double-blind, placebo-controlled, withdrawal phase III trial. Lancet 371:998-1006

49. De Benedetti F, Brunner HI, Ruperto N et al (2012) Randomized trial of tocilizumab in systemic juvenile idiopathic arthritis. N Engl J Med 367:2385-2395

50. Sandborg C, Mellins ED (2012) A new era in the treatment of systemic juvenile idiopathic arthritis. N Engl J Med 367:2439-2440

51. Schulte KM, Talat N (2010) Castleman's disease - a two compartment model of HHV8 infection. Nat Rev Clin Oncol 7:533-543

52. Brandt SJ, Bodine DM, Dunbar CE et al (1990) Dysregulated interleukin 6 expression produces a syndrome resembling Castleman's disease in mice. J Clin Invest 86:592-599

53. Katsume A, Saito H, Yamada Y et al (2002) Anti-interleukin 6 (IL-6) receptor antibody suppresses Castleman's disease like symptoms emerged in IL-6 transgenic mice. Cytokine 20:304-311

54. Yoshizaki K, Matsuda T, Nishimoto N et al (1989) Pathogenic significance of interleukin-6 (IL-6/BSF-2) in Castleman's disease. Blood 74:1360-1367

55. Nishimoto N, Sasai M, Shima Y et al (2000) Improvement in Castleman's disease by humanized anti-interleukin-6 receptor antibody therapy. Blood 95:56-61

56. Nishimoto N, Kanakura Y, Aozasa K et al (2005) Humanized anti-interleukin-6 receptor antibody treatment of multicentric Castleman disease. Blood 106:2627-2632

57. Gabrielli A, Avvedimento EV, Krieg T (2009) Scleroderma. N Engl J Med 360:1989-2003

58. Kitaba S, Murota H, Terao M et al (2012) Blockade of interleukin-6 receptor alleviates disease in mouse model of scleroderma. Am J Pathol 180:165-176

59. Shima Y, Kuwahara Y, Murota $H$ et al (2010) The skin of patients with systemic sclerosis softened during the treatment with anti-IL-6 receptor antibody tocilizumab. Rheumatology (Oxford) 49:2408-2412

60. Unizony S, Stone JH, Stone JR (2013) New treatment strategies in large-vessel vasculitis. Curr Opin Rheumatol 25:3-9

61. Sahraian MA, Radue EW, Minagar A (2013) Neuromyelitis optica: clinical manifestations and neuroimaging features. Neurol Clin 31:139-152

62. Araki M, Aranami T, Matsuoka T et al (2013) Clinical improvement in a patient with neuromyelitis optica following therapy with the anti-IL-6 receptor monoclonal antibody tocilizumab. Mod Rheumatol 23:827-831

63. Araki M, Matsuoka T, Miyamoto K et al (2014) Efficacy of the anti-IL-6 receptor antibody tocilizumab in neuromyelitis optica: a pilot study. Neurology 82:1302-1306

64. Gerfaud-Valentin M, Jamilloux Y, Iwaz J et al (2014) Adult-onset Still's disease. Autoimmun Rev 13:708-722

65. Lee DW, Gardner R, Porter DL et al (2014) Current concepts in the diagnosis and management of cytokine release syndrome. Blood 124:188-195

66. Grupp SA, Kalos M, Barrett D et al (2013) Chimeric antigen receptor-modified T cells for acute lymphoid leukemia. N Engl J Med 368:1509-1518

67. Teachey DT, Rheingold SR, Maude SL et al (2013) Cytokine release syndrome after blinatumomab treatment related to abnormal macrophage activation and ameliorated with cytokinedirected therapy. Blood 121:5154-5157

68. Tanaka T (2013) Can IL-6 blockade rectify imbalance between Tregs and Th17 cells? Immunotherapy 5:695-697

69. Carbone G, Wilson A, Diehl SA et al (2013) Interleukin-6 receptor blockade selectively reduces IL-21 production by CD4 T cells and IgG4 autoantibodies in rheumatoid arthritis. Int J Biol Sci 9:279-288 
70. Samson M, Audia S, Janikashvili N et al (2012) Brief report: inhibition of interleukin-6 function corrects Th17/Treg cell imbalance in patients with rheumatoid arthritis. Arthritis Rheum 64:2499-2503

71. Pesce B, Soto L, Sabugo F et al (2013) Effect of interleukin-6 receptor blockade on the balance between regulatory $\mathrm{T}$ cells and T helper type 17 cells in rheumatoid arthritis patients. Clin Exp Immunol 171:237-242

72. Thiolat A, Semerano L, Pers YM et al (2014) Interleukin-6 receptor blockade enhances CD39+ regulatory $\mathrm{T}$ cell development in rheumatoid arthritis and in experimental arthritis. Arthritis Rheum 66:273-283

73. Jones SA, Scheller J, Rose-John S (2011) Therapeutic strategies for the clinical blockade of IL-6/gp130 signaling. J Clin Invest 21:3375-3383 


\title{
Pathogenesis of Non-alcoholic Steatohepatitis and Its Potential Therapeutic Strategies
}

\author{
Yoshihiro Ogawa, Takayoshi Suganami, Michiko Itoh, and Miyako Tanaka
}

\begin{abstract}
Non-alcoholic steatohepatitis (NASH) is closely associated with progression to liver cirrhosis and hepatocellular carcinoma. We reported that melanocortin 4 receptor-deficient mice (MC4R-KO mice), when fed a high-fat diet, provide a novel rodent model of NASH. Recently, we have identified a unique histological feature termed "hepatic crown-like structures" (hCLS) in the livers of MC4R-KO mice and NASH patients. In hCLS, CD11c-positive macrophages aggregate to surround hepatocytes with large lipid droplets, similar to the "crownlike structure (CLS)" described in obese adipose tissue. Interestingly, we have recently reported that macrophage-induced C-type lectin (Mincle) is involved in CLS formation and fibrogenic gene expression in obese adipose tissue, suggesting the pathophysiologic role of CLS in obesity-induced adipose tissue fibrosis. Collectively, our data provide evidence that hCLS serves as an origin of hepatic inflammation and fibrosis during the progression from simple steatosis to NASH and thus helps in elucidation of the pathogenesis of NASH, pursuit of specific biomarkers, and evaluation of potential therapeutic strategies.
\end{abstract}

Keywords Adipose tissue $\bullet$ Crown-like structure $\bullet$ Fibrosis $\bullet$ Inflammation $\bullet$ Liver - Macrophage-inducible C-type lectin (Mincle) - Metabolic syndrome • Nonalcoholic steatohepatitis (NASH) • Parenchymal cells $\bullet$ Stromal cells

\footnotetext{
Y. Ogawa $(\varangle)$

Department of Molecular Endocrinology and Metabolism, Graduate School of Medical and Dental Sciences, Tokyo Medical and Dental University, Tokyo 113-8510, Japan

Japan Agency for Medical Research and Development, CREST, Tokyo 100-0004, Japan e-mail: ogawa.mem@tmd.ac.jp

T. Suganami

Department of Organ Network and Metabolism, Graduate School of Medical and Dental Sciences, Tokyo Medical and Dental University, Tokyo 113-8510, Japan

Japan Science and Technology Agency, PRESTO, Tokyo, Japan

M. Itoh • M. Tanaka

Department of Organ Network and Metabolism, Graduate School of Medical and Dental Sciences, Tokyo Medical and Dental University, Tokyo 113-8510, Japan

K. Nakao et al. (eds.), Innovative Medicine, DOI 10.1007/978-4-431-55651-0_12
} 


\section{Introduction}

The metabolic syndrome is defined as a combination of visceral fat obesity, impaired glucose metabolism, atherogenic dyslipidemia, and blood pressure elevation, all of which are independent risk factors for atherosclerotic diseases. The molecular basis for the clustering of such independent risks of atherosclerosis is not currently unclear, with visceral fat obesity considered most important. There is considerable evidence that lifestyle diseases are a tissue-remodeling disease. Indeed, obese adipose tissue is characterized by adipocyte hypertrophy, increased angiogenesis and infiltration of immune cells such as macrophages and lymphocytes, tissue fibrosis, and increased production of proinflammatory adipokines [1]. The functional and morphological changes observed in obese adipose tissue are very similar to those described in vascular remodeling during the process of atherosclerosis. We have called the dynamic changes in obese adipose tissue "adipose tissue remodeling" and have been interested in the molecular basis underlying adipose tissue remodeling and its pathophysiological implication in metabolic diseases.

\section{Interaction Between Adipocytes and Macrophages in Obese Adipose Tissue}

We and others previously reported that during the progression from visceral fat obesity to a variety of lifestyle diseases, hypertrophied adipocytes can secrete a number of cytokines and chemokines such as monocyte chemoattractant protein-1 (MCP-1) to stimulate the recruitment of C-C chemokine receptor type 2 (CCR2)positive monocytes into obese adipose tissue as proinflammatory M1 macrophages. Once infiltrated, macrophages may be activated in response to saturated fatty acids released from hypertrophied adipocytes and produce a large amount of proinflammatory cytokines such as tumor necrosis factor- $\alpha$ (TNF- $\alpha$ ), which, in turn, augments the inflammatory response and adipocyte lipolysis to increase the release of fatty acids in hypertrophied adipocytes. These observations led us to speculate that there is an intimate crosstalk between parenchymal cells, adipocytes, stromal cells, and macrophages, which establishes a vicious cycle that augments obesity-induced adipose tissue inflammation [2, 3]. Our data also suggest that saturated fatty acids serve as a naturally occurring ligand for Toll-like receptor 4 (TLR4) complex, a well-known pathogen sensor expressed in infiltrating macrophages [3].

As the site of crosstalk between adipocytes and macrophages, there is a unique histological feature termed a "crown-like structure" (CLS) in obese adipose tissue [4], which is composed of dead parenchymal adipocytes surrounded by macrophages.

In CLS, macrophages are considered to scavenge the residual lipid droplets of dead or dying adipocytes. It is likely that the adipocyte-macrophage interaction can stimulate adipocyte lipolysis and thus increase the release of free fatty acids, particularly saturated fatty acids, from obese adipose tissue, which may be accumulated 
in non-adipose tissue as ectopic fat, thereby inducing a variety of metabolic effects called lipotoxicity [5].

\section{Development of a Novel Rodent Model of NASH}

Non-alcoholic fatty liver disease (NAFLD) is defined as increased accumulation of lipids in the liver without a history of excess alcohol consumption. It is considered the hepatic manifestation of the metabolic syndrome. Non-alcoholic steatohepatitis (NASH), the most aggressive subset of NAFLD, is closely associated with the development of liver cirrhosis and hepatocellular carcinoma. The pathogenesis of NASH, the well-known "two-hit hypothesis", has been proposed for many years; however, the detailed mechanism of disease progression from simple steatosis to NASH is currently unclear, partly because there are no appropriate animal models that reflect the liver condition of human NASH and partly because liver biopsy is required for its definite diagnosis.

Melanocortin-4 receptor (MC4R) is a seven-transmembrane $\mathrm{G}$ protein-coupled receptor that is expressed in the hypothalamic nuclei implicated in the regulation of food intake and body weight. Its pathogenic mutations are the most common known monogenic cause of human obesity. We recently reported that MC4R-deficient (MC4R-KO) mice develop a liver condition similar to human NASH when fed a high-fat diet (HFD) or a Western diet (WD), which is associated with obesity, insulin resistance, and dyslipidemia [6]. Notably, they exhibited enhanced adipose tissue inflammation, which may contribute to excessive lipid accumulation and enhanced fibrosis in the liver. Moreover, they developed well-differentiated HCC for a prolonged time. Our data suggest that MC4R-KO mice would provide a novel rodent model of NASH with which to investigate the sequence of events that encompass diet-induced obesity, hepatic steatosis, liver fibrosis, and HCC. Histologically, they showed hepatocyte ballooning degeneration, increased infiltration of inflammatory cells such as macrophages and lymphocytes, and pericellular fibrosis, all of which are hallmarks of human NASH. Immunohistochemical staining with $\alpha$-smooth muscle action $(\alpha \mathrm{SMA})$ revealed that hepatic stellate cells are activated in the liver from MC4R-KO mice even before the onset of fibrosis.

\section{Identification of Hepatic Crown-Like Structure (hCLS)}

During the course of obesity, MC4R-KO mice developed a number of CLS in the adipose tissue. We found bizarre microstructures in livers from MC4R-KO mice, which are very similar to CLS in obese adipose tissue [7]. We have called them "hepatic crown-like structures" (hCLS), where macrophages aggregate to surround hepatocytes with large lipid droplets. Although there was no significant difference in the number of F4/80-positive macrophages between the genotypes, the number of 
hCLS was increased in MC4R-KO mice relative to wild-type mice, suggesting that the distribution of macrophages was altered in the livers from MC4R-KO mice. Importantly, there was a significant correlation between the number of hCLS and the extent of liver fibrosis. These observations, taken together, suggest that hCLS rather than macrophages themselves are associated with the development of liver fibrosis during the progression from simple steatosis to NASH.

To further explore the functional significance of hCLS in the development of liver fibrosis, we depleted macrophages in livers from MC4R-KO mice by the clodronate liposome method. In MC4R-KO mice, macrophages scattered in the liver were effectively depleted, although those in hCLS appeared to be intact. Accordingly, treatment with clodronate liposome significantly reduced the F4/80-positive areas in the liver from MC4R-KO mice. However, there was no significant change in the numbers of hCLS and $\alpha$ SMA-positive cells after the treatment. In this setting, treatment with clodronate liposome resulted in a significant reduction of expression of inflammatory and fibrogenic genes in the liver from wild-type mice. By contrast, in MC4R-KO mice, there was no significant change in the gene expression after the treatment. These observations suggest that hCLS macrophages are resistant to the treatment and that hCLS is functionally associated with hepatic inflammation and fibrosis in NASH liver. Immunofluorescence and electron microscopy analyses revealed that hCLS is composed of CD11c-positive macrophages and dead parenchymal hepatocytes with large lipid droplets, and is spatially associated with $\alpha$ SMA-positive activated stellate cells and collagen deposition, which support the notion that hCLS is involved in the development of hepatic inflammation and fibrosis.

We also found that hCLS occurs in patients with NASH or even in those with simple steatosis in proportion to the extent of liver fibrosis. Importantly, there was no such increase in hCLS in patients with chronic viral hepatitis, suggesting that hCLS is rather specific to metabolic liver diseases.

Figure 1 illustrates the role of hCLS in the pathogenesis of NASH. Increased flux of free fatty acids from visceral adipose tissue to the liver via the portal vein as a result of adipose tissue inflammation can cause simple steatosis. Parenchymal hepatocytes that are being overloaded with lipid and are thus dead or dying are surrounded by macrophages to form hCLS in the fatty liver. In the NASH liver, macrophages can interact with and engulf dead hepatocytes within hCLS, thereby activating fibrogenic cells to stimulate hepatic fibrosis as an adaptive repair response to tissue injury. It is, therefore, likely that hCLS serves as an origin of hepatic inflammation and fibrosis during the progression from simple steatosis to NASH [7].

\section{Macrophage-Induced C-Type Lectin (Mincle)}

Macrophage-induced C-type lectin (Mincle) is a type II transmembrane $\mathrm{Ca}^{+}$dependent carbohydrate binding lectin. It is expressed abundantly in macrophages, where it is induced in response to LPS. Recent studies show that Mincle is a pathogen sensor that recognizes the mycobacterial glycolipid trehalose dimycolate 


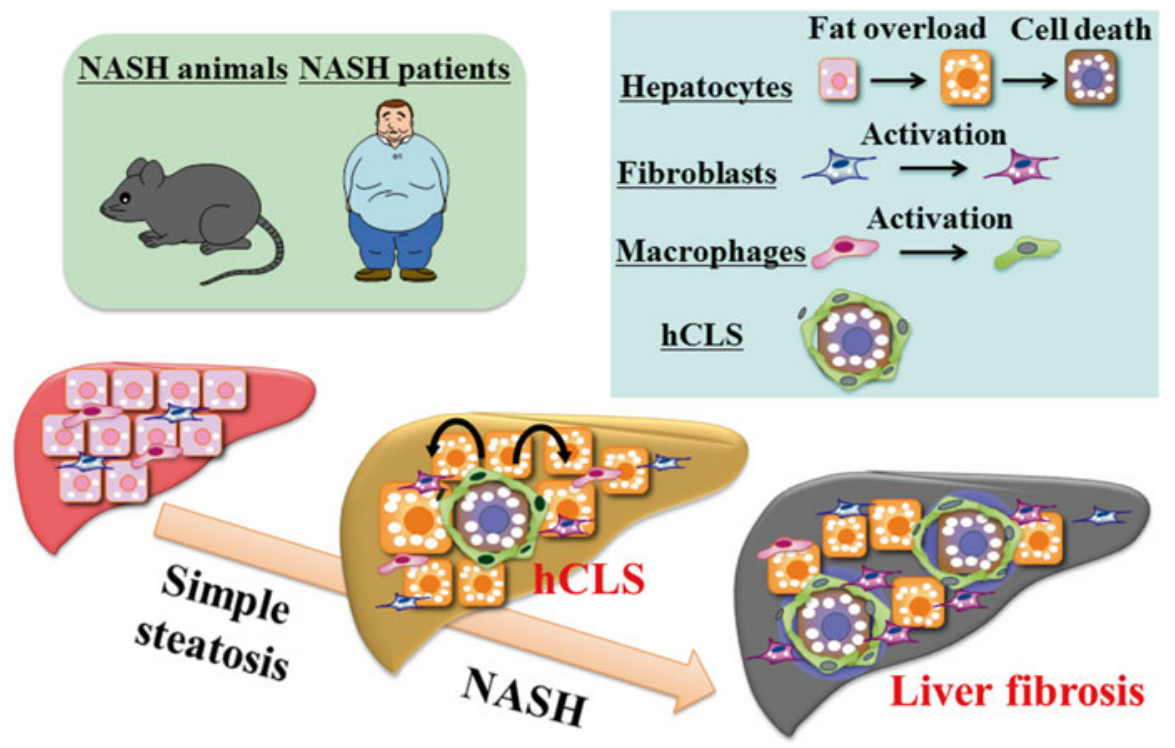

Fig. 1 Possible role of hCLS during the progression from normal healthy liver and simple steatosis to NASH

(TDM) [8]. More interestingly, it can also recognize an endogenous ligand that is released from dead cells as a danger signal [9]. We previously reported that during the interaction between adipocytes and macrophages, saturated fatty acids can induce Mincle expression in macrophages through the activation of the TLR4/ nuclear factor $\kappa \mathrm{B}(\mathrm{NF}-\kappa \mathrm{B})$ pathway [10].

Mincle mRNA expression is induced in obese adipose tissue, which occurs mostly in the stromal vascular fraction. FACS analysis revealed that Mincle is induced in the infiltrating macrophages in obese adipose tissue; a substantial fraction of the macrophages is Mincle-positive. By in situ hybridization analysis, we found that Mincle mRNA expression is restricted to CLS macrophages in obese adipose tissue. We also found that Mincle expression is induced in the adipose tissue of obese humans [10]. There is a significantly positive correlation between Mincle mRNA levels and BMI, suggesting the role of Mincle in the pathogenesis of obesity. These observations suggest that Mincle plays a role in adipose tissue remodeling.

\section{Role of Mincle in Adipose Tissue Remodeling and Ectopic Fat Accumulation}

To explore the role of Mincle in adipose tissue remodeling in vivo, we examined the metabolic phenotype of Mincle-KO mice during the HFD feeding. There was no apparent difference in body weight between the genotypes throughout the 
experimental period. At 8 weeks of HFD feeding, when macrophages were not massively infiltrated, there was no significant difference in adipose tissue and liver weight between the genotypes. Notably, at 16 weeks of HFD feeding, when macrophages were massively infiltrated, Mincle-KO mice showed increased adipose tissue weight with a reciprocal reduction of liver weight. Histological analysis of the adipose tissue revealed that adipocytes seemed to be enlarged in Mincle-KO mice in response to the HFD feeding. Although there was no significant difference in F4/80positive macrophages between the genotypes, the number of CLS was significantly reduced in Mincle-KO mice. Since the number of hCLS is more positively correlated with the extent of liver fibrosis than that of macrophages in MC4R-KO mice [7], we examined adipose tissue fibrosis by Masson trichrome staining. There was extensive interstitial fibrosis in the epididymal fat depot from wild-type mice, which was markedly attenuated in Mincle-KO mice together with a significant reduction of collagen deposition. Interestingly, there was no significant difference in collagen deposition in the subcutaneous fat depot between the genotypes; this may be partly because Mincle expression is not markedly increased in obese subcutaneous fat depots, where macrophage infiltration was not so remarkable. Thus, Mincle-KO mice exhibited increased adipose tissue weight with increased adipocyte cell size with significant reduction of CLS and reduced adipose tissue fibrosis.

We next examined the liver phenotypes of Mincle-KO mice and found that hepatic steatosis is markedly attenuated in Mincle-KO mice. Accordingly, liver TG content and serum ALT concentrations were significantly reduced in Mincle-KO mice. We also examined glucose metabolism and insulin sensitivity by glucose and insulin tolerance tests and confirmed improved glucose metabolism and insulin sensitivity in Mincle-KO mice.

During the paracrine interaction between adipocytes and macrophages, saturated fatty acids, which are released via the macrophage-induced adipocyte lipolysis, are able to induce Mincle in the infiltrating CLS macrophages through the TLR4/NF- $\kappa B$ pathway $[10,11]$. Mincle may recognize as-yet-unidentified endogenous ligands released from dead adipocytes as a danger signal, thereby stimulating adipose tissue fibrosis. In Mincle-KO mice, where adipose tissue fibrosis is attenuated, adipocytes can be hypertrophied enough to store lipid, which may reduce ectopic fat accumulation in the liver. Accordingly, our data suggest that Mincle plays a critical role in the formation of CLS and adipose tissue fibrosis during the course of obesity, which may reduce lipid-storage capacity in adipose tissue and enhance ectopic lipid accumulation [11].

\section{Summary}

Our data suggest that CLS and hCLS provide the unique microenvironment where dead or dying parenchymal and stromal cells crosstalk in close proximity in vivo (Fig. 2). In NASH liver, parenchymal hepatocytes, when they are overloaded with lipids and thus dead or dying, report their dysfunctional state to the adjacent stromal 


\section{hCLS}
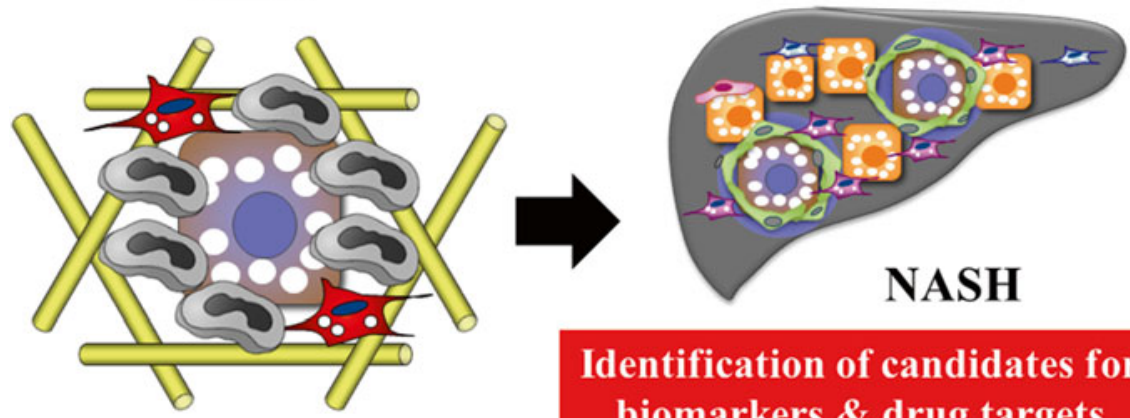

Identification of candidates for biomarkers \& drug targets

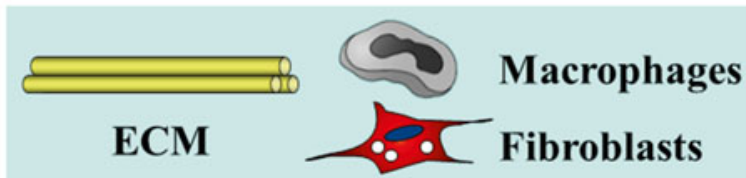

Fig. 2 Detailed analysis of hCLS helps identify candidate molecules to be used as biomarkers and/or drug targets specific for NASH

cells such as macrophages and fibroblasts via multiple danger signals or dying messages. Given that Mincle, when induced in macrophages in CLS, plays a critical role in the development of adipose fibrosis, it is tempting to speculate that Mincle is also involved in the progression of hepatic fibrosis. Detailed analysis of hCLS may provide a clue to discover candidate molecules to be used as biomarkers and/or drug targets specific for NASH.

Acknowledgments We thank the members of the Ogawa laboratory for discussions. Part of this study has been supported by the Uehara Memorial Foundation.

Open Access This chapter is distributed under the terms of the Creative Commons Attribution Noncommercial License, which permits any noncommercial use, distribution, and reproduction in any medium, provided the original author(s) and source are credited.

\section{References}

1. Suganami T, Ogawa Y (2010) Adipose tissue macrophages: their role in adipose tissue remodeling. J Leukoc Biol 88:33-39

2. Suganami T, Nishida J, Ogawa Y (2005) A paracrine loop between adipocytes and macrophages aggravates inflammatory changes: role of free fatty acids and tumor necrosis factor $\alpha$. Arterioscler Thromb Vasc Biol 25:2062-2068

3. Suganami T, Tanimoto-Koyama K, Nishida J, Itoh M, Yuan X, Mizuarai S, Kotani H, Yamaoka S, Miyake K, Aoe S, Kamei Y, Ogawa Y (2007) Role of the Toll-like receptor 4/NF-кB pathway 
in saturated fatty acid-induced inflammatory changes in the interaction between adipocytes and macrophages. Arterioscler Thromb Vasc Biol 27:84-91

4. Cinti S, Mitchell G, Barbatelli G, Murano I, Ceresi E, Faloia E, Wang S, Fortier M, Greenberg AS, Obin MS (2005) Adipocyte death defines macrophage localization and function in adipose tissue of obese mice and humans. J Lipid Res 46:2347-2355

5. Suganami T, Tanaka M, Ogawa Y (2012) Adipose tissue inflammation and ectopic lipid accumulation. Endocr J 59:849-857

6. Itoh M, Suganami T, Nakagawa N, Tanaka M, Yamamoto Y, Kamei Y, Terai S, Sakaida I, Ogawa Y (2011) Melanocortin-4 receptor-deficient mice as a novel mouse model of nonalcoholic steatohepatitis. Am J Pathol 179:2454-2463

7. Itoh M, Kato H, Suganami T, Konuma K, Marumoto Y, Terai S, Sakugawa H, Kanai S, Hamaguchi M, Fukaishi T, Akiyoshi K, Komohara Y, Takeya M, Sakaida I, Ogawa Y (2013) Hepatic crown-like structure: a unique histological feature in non-alcoholic steatohepatitis in mice and humans. PLoS One 8:e82163

8. Ishikawa E, Ishikawa T, Morita YS, Toyonaga K, Yamada H, Takeuchi O, Kinoshita T, Akira S, Yoshikai Y, Yamasaki S (2009) Direct recognition of the mycobacterial glycolipid, trehalose dimycolate, by C-type lectin Mincle. J Exp Med 206:2879-2888

9. Yamasaki S, Ishikawa E, Sakuma M, Hara H, Ogata K, Saito T (2008) Mincle is an ITAMcoupled activating receptor that senses damaged cells. Nat Immunol 9:1179-1188

10. Ichioka M, Suganami T, Tsuda N, Shirakawa I, Hirata Y, Satoh-Asahara N, Shimoda Y, Tanaka M, Kim-Saijo M, Miyamoto Y, Kamei Y, Sata M, Ogawa Y (2011) Increased expression of macrophage-inducible C-type lectin in adipose tissue of obese mice and humans. Diabetes 60:819-826

11. Tanaka M, Ikeda K, Suganami T, Komiya C, Ochi K, Shirakawa I, Hamaguchi M, Nishimura S, Manabe I, Matsuda T, Kimura K, Inoue H, Inagaki Y, Aoe S, Yamasaki S, Ogawa Y (2014) Macrophage-inducible C-type lectin underlies obesity-induced adipose tissue fibrosis. Nat Commun 5:e4982 


\title{
Multifaceted Translational Approach to Major Mental Illness
}

\begin{abstract}
Akira Sawa
Abstract This is a short summary of my presentation in the Uehara Memorial Foundation Symposium 2014. Classification of mental illness in current diagnostic systems [such as the Diagnostic and Statistical Manual of Mental Disorders (DSM)] is built on clinical reliability and utility, but not on etiological validity. Thus, the DSM has not provided an ideal classification of mental disorders. To overcome the dilemma, the National Institute of Mental Health (NIMH) recently proposed the Research Domain Criteria (RDoC) framework. This new framework emphasizes a dimensional approach on the basis of neurocircuitry mechanism. There were three aims in my presentation. First, I summarized the concept of research strategies on the basis of dimensional approach, with a link to the trajectory from early development to adolescence in the disease pathology. Second, I introduced an example of a research infrastructure that could support translational and clinical study for mental illness. Third, I showed two studies that were utilizing the infrastructure.
\end{abstract}

Keywords Mental disorders - Diagnostic and statistical manual of mental disorders (DSM) • Research domain criteria (RDoC) • Dimensional approach • Developmental trajectory $\bullet$ Translational research

\section{Introduction}

The Uehara Memorial Foundation Symposium 2014 was held on June 15, 16, and 17 in Tokyo, under the theme of Innovative Medicine-Basic Research and Development. The role of my presentation was to provide information on the frontline of clinical and translational research in psychiatry to the audience.

Although psychiatry is obviously a domain of modern medicine, studying brain disorders, the classic problem of the brain-mind discontinuity has slowed down scientific progress in psychiatry. Consequently, many different disciplines have

\footnotetext{
A. Sawa, M.D. ( $₫)$

Johns Hopkins Schizophrenia Center, Departments of Psychiatry, Mental Health, and Neuroscience, Institute of Human Genetics, Program in Cellular and Molecular Medicine, Johns Hopkins University Schools of Medicine and Public Health, Baltimore, MD, USA e-mail: asawa1@jhmi.edu
} 
competed with each other in psychiatry in the past century. Such factionalism was also a major obstacle to the development of psychiatry as a key domain of modern medicine. Finally, as a major collaborative effort among psychiatrists, a comprehensive diagnostic manual that standardizes the classification of psychiatric disorders was issued as the Diagnostic and Statistical Manual of Mental Disorders (DSM) third edition in 1980. The DSM has been useful in standardizing clinical practice in psychiatry with high reliability. However, the DSM has been formulated under ignorance of (or minimal attention to) the etiological and biological validity in its disease classification. Thus, this nature of the DSM has been a major deficit in building research on the basis of disease classification defined by this manual.

To overcome this dilemma, the National Institute of Mental Health (NIMH) included in its new strategic plan the following aim: "develop, for research purposes, new ways of classifying mental disorders based on dimensions of observable behavior and neurobiological measures" [1]. According to the Cuthbert article, "the implementation of this aim was named the Research Domain Criteria project or RDoC." The RDoC is formulated in a matrix: it has multiple dimensions that cut across traditional diagnostic categories, such as attention and perception. Meanwhile, biological correlates for each dimension are considered at multiple levels, such as the molecular, cellular, circuitry, and behavioral levels.

There is another important viewpoint to develop research for mental illness. This is the viewpoint of the developmental trajectory. Many mental disorders, such as schizophrenia and psychotic disorders, mood disorders, and substance use disorders, appear in young adulthood, and epidemiological and clinical studies have indicated that major risks for such disorders occur in developmental stages. Thus, it is very important to study the mechanisms of how disease-associated risk factors at earlier ages are accumulated and result in the onset of the disease in the developmental trajectory. The NIMH has also stated that dimensional approaches such as the RDoC may be used in conjunction with attention to neurodevelopment and disease-associated environmental factors.

Thus, the aim of my presentation in the symposium was to introduce these two elements essential to research for mental disorders (e.g., a dimensional approach and attention to the neurodevelopmental trajectory in disease pathology), and then to present a representative infrastructure of translational and clinical research on the based on these two elements.

\section{Major Mental Illness Caused by a Combination of Multiple Genetic Risk Factors and Environmental Stressors in the Pathological Trajectory}

The majority of mental disorders are caused by a combination of multiple genetic risk factors and environmental stressors (Fig. 1). The combination is not random but is likely to result in certain numbers of common biological pathways. It is possible that these pathways underlie clinical manifestations, which are organized in a 
Many genes

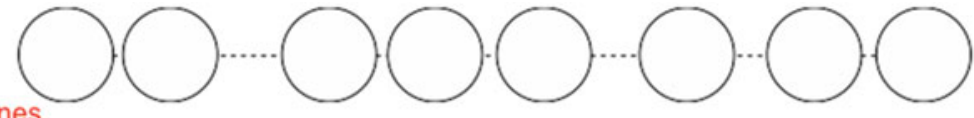
Environmental factors
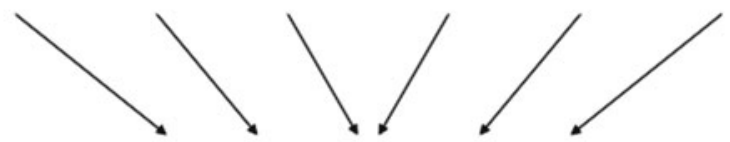

Pathways
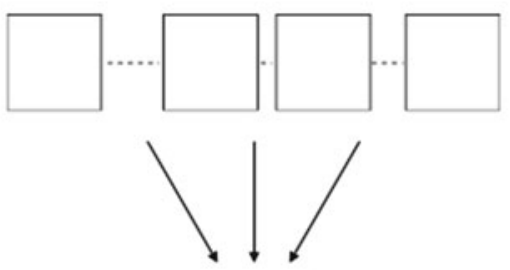

\section{Symptoms}

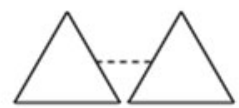

Fig. 1 From etiologies to clinical manifestations: the majority of mental disorders are caused by a combination of multiple genetic risk factors and environmental stressors

dimensional way. If we take schizophrenia (SZ) and related psychotic conditions, the risk factors and stressors are likely to contribute to the pathology in early development and adolescence (Fig. 2). Nonetheless, the onset of the disease is in late adolescence and young adulthood. Thus, it is crucial to study the disease mechanisms in the developmental trajectory. In many medical diseases, the significance of early detection and early intervention has been highlighted. Accordingly, in the case of SZ, studying the transition from risk stages to the onset (the prodromal stage) is regarded as one of the most important research topics at present. Given that current medications for psychosis target only symptomatic aspects and are limited in their efficacy, many investigators optimistically expect that the study of the prodromal stage may identify novel therapeutic targets that are associated with the disease progression and overcome the current limitations (Fig. 2). In regard to the mechanisms associated with the disease progression in the prodromal stage, it is possible that aberrant activation of stress-related cascades underlie disturbed postnatal brain maturation, which results in brain dysfunction and manifestation of mental symptoms (Fig. 3) [2, 3].

\section{Research Infrastructure That Allows a Multifaceted Translational Approach to Major Mental Illness}

After the introduction of the dimensional approach in the developmental trajectory, particularly in the context of SZ and psychotic conditions, I presented an example of a research infrastructure that supports translational and clinical study for mental 


\section{Classic target}

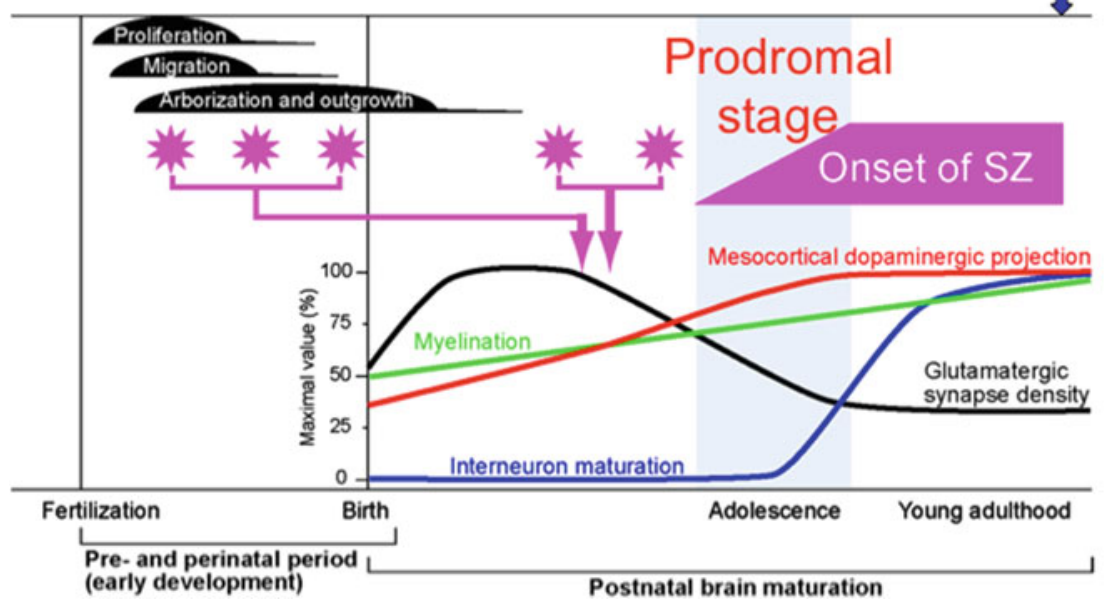

Fig. 2 Developmental trajectory of schizophrenia and related psychotic conditions. Although the onset of SZ is in young adulthood, epidemiological studies have suggested that many environmental risk insults for SZ occur during early development and adolescence (asterisks). Biological studies have also provided evidence that roles for genetic risk factors are associated with neurodevelopment (Adopted and modified from Jaaro-Peled et al. [2])

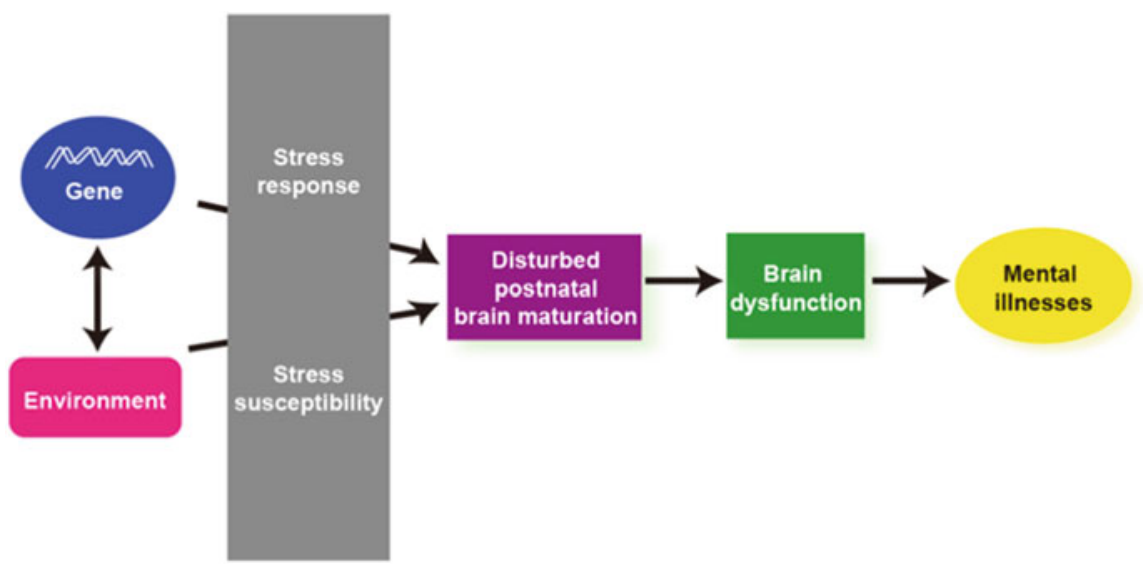

Fig. 3 Gene environmental interactions that underlie disturbed postnatal brain maturation, brain dysfunction, and mental disorders. Genetic and environmental factors for SZ are likely to converge and affect proper cortical maturation in adolescence, which may underlie the pathology of SZ 


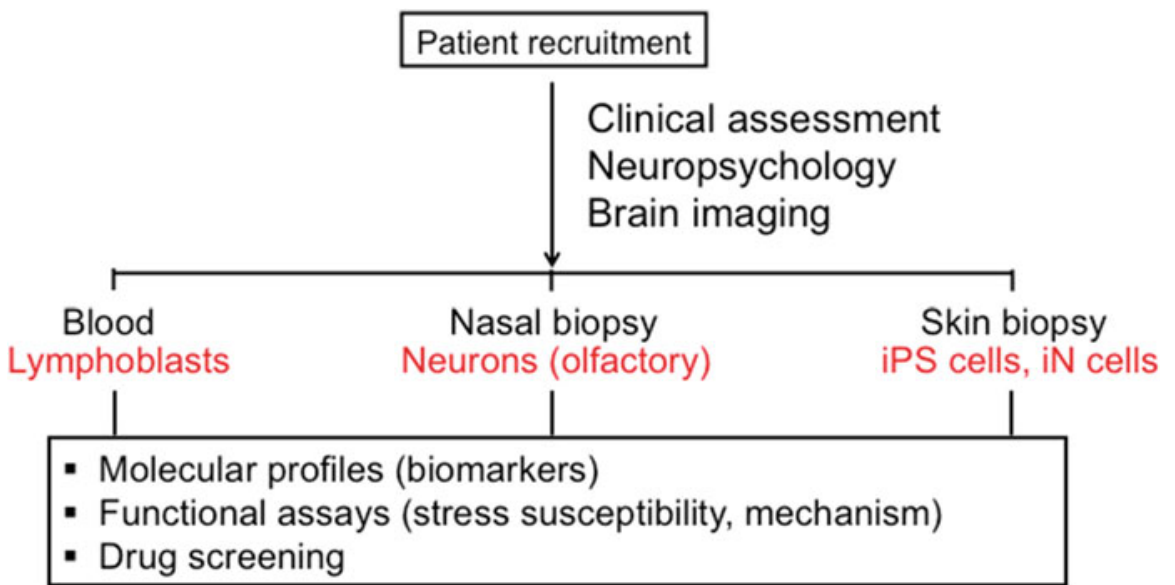

Fig. 4 An example of research infrastructure that supports translational and clinical study for mental disorders. In this infrastructure, whenever study subjects (patients and matched controls) are enrolled, they are assessed clinically and neuropsychologically. They also participate in brain imaging studies as well as tissue biopsies. Taken together, according to the ethical guideline, investigators can build multifaceted translational study on this research platform

disorders (Fig. 4). In this infrastructure, whenever study subjects (patients and matched controls) are enrolled, they are assessed clinically and neuropsychologically. They also participate in brain imaging studies, including positron emission tomography (PET) and magnetic resonance imaging (MRI), which are essential to address brain circuitry disturbance directly in vivo. In parallel, the study participants donate their tissues in multiple ways, including blood draw, skin biopsy, and nasal biopsy. We can obtain an olfactory epithelium (OE) sample easily by nasal biopsy. OE includes neuroprogenitor cells, immature neurons, and mature neurons: thus, by studying OE-derived cells and tissues, we can examine molecular and cellular signatures relevant to neuronal cells (Fig. 5) [4]. The information from the cells and tissues can be correlated with the brain imaging characteristics and clinical manifestations from the same subjects. As described above, RDoC-oriented studies are expected to study biological correlates and clinical manifestations at multiple levels, such as the molecular, cellular, circuitry, and behavioral levels. Thus, this infrastructure can meet such expectations. The disease trajectory may be addressed by designing a longitudinal study in this infrastructure.

\section{Two Representative Studies that Use the Research Infrastructure}

We previously reported that a specific phosphorylation of DISC1 (serine residue at the 710th amino acid of mouse DISC1) has a crucial role in the switch of cell fate from neuroprogenitor proliferation to postmitotic differentiation [5]. DISC1 has 

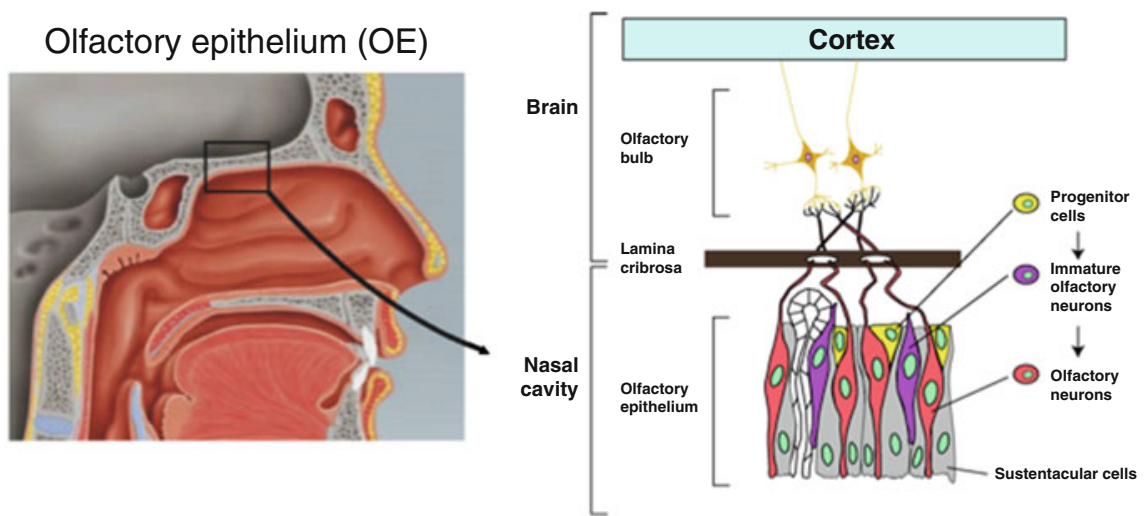

Fig. 5 Olfactory biopsy as a effective approach to obtain biospecimens with neuronal molecular signature. An olfactory epithelium (OE) sample, which is easily obtained by nasal biopsy, includes neuroprogenitor cells, immature neurons, and mature neurons; thus, by studying OE-derived cells and tissues, we can examine molecular and cellular signatures relevant to neuronal cells (Adopted from Sawa and Cascella [4])
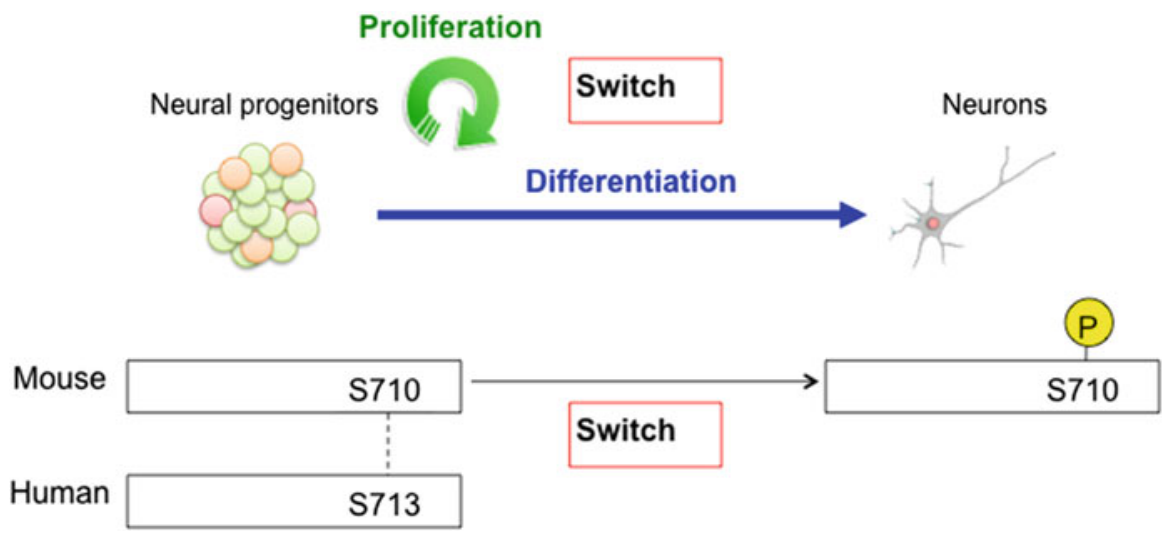

Fig. 6 A specific phosphorylation of DISC1 controls cell fate from neuroprogenitor proliferation to postmitotic neuronal differentiation: an entry point for translational study of mental illness. DISC1 has been involved as a biological hub protein for multiple pathways associated with mental illnesses. Phosphorylation of DISC1 at one specific amino acid residue is known to be crucial for neurodevelopment in mice. However, it remains elusive how this phosphorylation (serine residue at 713th amino acid of human DISC1) underlies cellular, circuitry, and behavioral manifestations associated with mental disorders. The infrastructure introduced in Fig. 4, including the utilization of olfactory cells (see Fig. 5) for study of the phosphorylation, allows us to address this question

been involved as a biological hub protein for multiple pathways associated with mental illnesses [6]. It remains elusive how this phosphorylation (serine residue at the 713th amino acid of human DISC1) underlies cellular, circuitry, and behavioral manifestations associated with mental disorders (Fig. 6). Thus, I presented our 
preliminary data that studied the phosphorylation in olfactory cells from patients and controls, in correlation with their brain imaging characteristics and clinical traits.

In addition, I also presented studies in which the molecular signature of the cerebrospinal fluid was examined from patients in the prodromal stage and with the first episode of psychosis, in comparison with matched controls. In the first set of publications from the study, we observed robust changes in the molecules associated with immune and inflammatory responses, as well as oxidative stress [7, 8]. I also introduced a perspective of how we can study such molecular information in correlation with brain imaging characteristics and clinical manifestations.

Acknowledgments I thank the Uehara Memorial Foundation and the meeting organizers. I also thank Ms. Yukiko Lema for organizing the manuscript. I am supported by the NIH, NARSAD, Stanley Foundation, RUSK Foundation, and Maryland Stem Cell Research Foundation.

Open Access This chapter is distributed under the terms of the Creative Commons Attribution Noncommercial License, which permits any noncommercial use, distribution, and reproduction in any medium, provided the original author(s) and source are credited.

\section{References}

1. Cuthbert BN (2014) The RDoC framework: facilitating transition from ICD/DSM to dimensional approaches that integrate neuroscience and psychopathology. World Psychiatr 13(1):2835. doi:10.1002/wps. 20087

2. Jaaro-Peled H, Hayashi-Takagi A, Seshadri S, Kamiya A, Brandon NJ, Sawa A (2009) Neurodevelopmental mechanisms of schizophrenia: understanding disturbed postnatal brain maturation through neuregulin-1-ErbB4 and DISC1. Trends Neurosci 32(9):485-495. doi:10.1016/j.tins.2009.05.007

3. Sawa A, Seidman LJ (2014) Is prophylactic psychiatry around the corner? Combating adolescent oxidative stress for adult psychosis and schizophrenia. Neuron 83(5):991-993. doi:10.1016/j.neuron.2014.08.028

4. Sawa A, Cascella NG (2009) Peripheral olfactory system for clinical and basic psychiatry: a promising entry point to the mystery of brain mechanism and biomarker identification in schizophrenia. Am J Psychiatry 166(2):137-139. doi:10.1176/appi.ajp.2008.08111702

5. Ishizuka K, Kamiya A, Oh EC, Kanki H, Seshadri S, Robinson JF, Murdoch H, Dunlop AJ, Kubo K, Furukori K, Huang B, Zeledon M, Hayashi-Takagi A, Okano H, Nakajima K, Houslay MD, Katsanis N, Sawa A (2011) DISC1-dependent switch from progenitor proliferation to migration in the developing cortex. Nature 473(7345):92-96. doi:10.1038/nature09859

6. Brandon NJ, Sawa A (2011) Linking neurodevelopmental and synaptic theories of mental illness through DISC1. Nat Rev Neurosci 12(12):707-722. doi:10.1038/nrn3120

7. Coughlin JM, Ishizuka K, Kano SI, Edwards JA, Seifuddin FT, Shimano MA, Daley EL, Zandi PP, Leweke FM, Cascella NG, Pomper MG, Yolken RH, Sawa A (2012) Marked reduction of soluble superoxide dismutase-1 (SOD1) in cerebrospinal fluid of patients with recent-onset schizophrenia. Mol Psychiatry 18:10-11. doi:10.1038/mp.2012.6

8. Hayes LN, Severance EG, Leek JT, Gressitt KL, Rohleder C, Coughlin JM, Leweke FM, Yolken RH, Sawa A (2014) Inflammatory molecular signature associated with infectious agents in psychosis. Schizophr Bull 40(5):963-972. doi:10.1093/schbul/sbu052 


\title{
Translational Research of Leptin in Lipodystrophy and Its Related Diseases
}

\author{
Ken Ebihara and Kazuwa Nakao
}

\begin{abstract}
Leptin, an adipocyte-derived hormone, plays crucial roles in the regulation of energy expenditure and food intake. Through analyses of leptin transgenic mice, we have demonstrated that leptin has pleiotropic effects such as regulation of insulin sensitivity and lipid metabolism. Lipodystrophy is a disease characterized by a lack of adipose tissue, which leads to metabolic disorders including insulin resistant diabetes, hypertriglyceridemia, and fatty liver. We demonstrated that leptin deficiency plays an important role in the pathogenesis of metabolic disorders in lipodystrophy. We also demonstrated the efficacy of leptin replacement therapy in lipodystrophy. Leptin improves insulin sensitivity at least partly by cancellation of lipotoxicity in the liver and skeletal muscle. It is also possible that leptin improves insulin secretion by cancellation of lipotoxicity in pancreatic beta cells. Using animal models, we demonstrated that leptin activates hepatic AMP-activated protein kinase (AMPK), and hepatic AMPK activation is involved in the therapeutic effects of leptin. To elucidate the pathogenic mechanism of hyperphagia in lipodystrophy, we measured food-related neural activity by fMRI and investigated subjective feelings of appetite. We found insufficiency of postprandial suppression of food-related neural activity and formation of satiety feelings in patients with lipodystrophy, which might be largely due to leptin deficiency. In March 2013, marketing and manufacturing approval was granted for metreleptin for the treatment of lipodystrophy in Japan on the basis of the results of our investigator-initiated trial. This is the first global approval of leptin formulation. Leptin has potential as a drug for the treatment of more common metabolic diseases including diabetes, hyperlipidemia, and fatty liver.
\end{abstract}

\footnotetext{
K. Ebihara $(\square)$

Institute for Advancement of Clinical and Translational Science,

Kyoto University Hospital, Kyoto, Japan

Division of Endocrinology and Metabolism, Jichi Medical University, Shimotuke, Japan e-mail: kebihara@jichi.ac.jp

K. Nakao, M.D., Ph.D.

Professor, Medical Innovation Center, Kyoto University Graduate

School of Medicine, Kyoto, Japan

(C) The Author(s) 2015

K. Nakao et al. (eds.), Innovative Medicine, DOI 10.1007/978-4-431-55651-0_14
} 
Keywords Leptin $\bullet$ Lipodystrophy $\bullet$ Metreleptin $\bullet$ Lipotoxicity $\bullet$ AMPK (5'-AMPactivated protein kinase) $\bullet$ fMRI (functional magnetic resonance image) $\bullet$ Reward system

\section{Introduction}

Leptin, an adipocyte-derived hormone originally identified from hereditary obese mice (ob/ob mice) [1], plays crucial physiologic roles in the regulation of energy expenditure and food intake [2-6]. In obese animals and subjects, plasma leptin concentrations are increased in proportion to the degree of adiposity [7-9], indicating that leptin is a satiety signal communicating the size of adipose stores to the brain [10-12] and that leptin resistance is related to obesity [7, 13-15]. Leptin deficiency in human subjects is associated with morbid obesity with insulin resistance, indicating the physiological role of leptin in both animal models and humans [16, 17]. Leptin is implicated in a number of manifestations seen in obese animal models [11, 18-21]. In this chapter, we introduce our past basic and clinical studies for clinical application in lipodystrophy and its related diseases.

\section{Transgenic Mice Overexpressing Leptin}

To explore the clinical implications of leptin in vivo, we generated leptin transgenic (LepTg) mice displaying elevated plasma leptin concentrations comparable to those seen in obese subjects [22]. A fusion gene comprising the human SAP promoter upstream of the mouse leptin cDNA coding sequences was designed to target hormone expression to the liver [23, 24]. Overexpression of leptin in the liver resulted in the complete disappearance of both white adipose tissues in mice [22]. Such a phenotype did not occur when transgene expression was targeted to adipose tissue, the endogenous site of leptin production, using adipocyte-specific promoters [25]. The hyperleptinemia seen in these LepTg mice provides a unique experimental system in which the long-term effects of leptin are investigated in vivo [18-22]. LepTg mice exhibit augmented glucose metabolism and increased insulin sensitivity of both skeletal muscle and liver [22], supporting the concept that leptin acts as an antidiabetic hormone in vivo [26-28]. These studies suggest the potential usefulness of leptin treatment of diabetes and obesity.

\section{Crossbreeding Experiment of LepTg Mice with A-ZIPTg Mice}

Lipodystrophy is a disease characterized by a lack of adipose tissue. It can be developed by a genetic abnormality, immune disorder, viral infection, or drugs. Irrespective of the etiology, loss of adipose tissue leads to severe insulin-resistant 
diabetes, hypertriglyceridemia, and fatty liver [29]. The precise mechanism by which this paucity of fat results in these metabolic disorders remains to be elucidated. Plasma leptin concentrations are markedly reduced in patients with lipodystrophy and in rodent models of lipodystrophy [30-33]. Given leptin's antidiabetic action, leptin deficiency may play a role in the pathogenesis of metabolic disorders in lipodystrophy; thus, leptin may be a drug for patients with lipodystrophy.

A mouse model of generalized lipodystrophy (A-ZIPTg mice) was generated by expressing in adipose tissue a protein that inactivates basic zipper transcription factors [32]. To assess the pathophysiological role and therapeutic potential of leptin in metabolic disorders associated with lipodystrophy, we crossed LepTg mice and A-ZIPTg mice to produce doubly transgenic (LepTg/A-ZIPTg) mice virtually lacking adipose tissue and expressing approximately tenfold higher levels of leptin than normal controls [34]. LepTg/A-ZIPTg mice were hypophagic in comparison with A-ZIPTg mice and exhibited decreased hepatic steatosis. Glucose and insulin tolerance tests displayed increased insulin sensitivity and normal glucose tolerance in LepTg/A-ZIPTg mice, which was comparable to LepTg mice. Pair-feeding experiments demonstrated that the effects of leptin were not solely due to decreased food intake. These results demonstrate that leptin can improve insulin resistance and diabetic manifestations in a mouse model of severe systemic lipodystrophy, indicating that leptin is therapeutically useful in the treatment of lipoatrophic diabetes [34].

\section{Leptin Replacement Therapy in Japanese Patients with Lipodystrophy}

Four-month leptin replacement therapy has been reported to improve glucose and lipid metabolism in lipodystrophy patients in the USA [35]. To elucidate the efficacy, safety, and mechanisms underlying leptin replacement therapy in Asian patients with lipodystrophy, we treated seven Japanese patients, two acquired and five congenital types, with a physiological replacement dose of leptin [36, 37]. Leptin replacement therapy dramatically improved fasting glucose levels (mean $\pm \mathrm{SE}$, $172 \pm 20$ to $120 \pm 12 \mathrm{mg} / \mathrm{dl}, \mathrm{P}<0.05$ ) and triglyceride levels (mean $\pm \mathrm{SE}, 700 \pm 272$ to $260 \pm 98 \mathrm{mg} / \mathrm{dl}, \mathrm{P}<0.05$ ) within 1 week. By 2 months, six of seven patients were able to discontinue all antidiabetic drugs, including insulin.

To investigate the underlying mechanism of metabolic improvement by leptin, we evaluated insulin sensitivity using a hyperinsulinemic euglycemic glucose clamp study in human patients (Fig. 1). The glucose infusion rate as an index of insulin sensitivity was distinctly low at baseline but was improved month by month after the initiation of leptin therapy. We also evaluated the glucose tolerance and the ability of insulin secretion with the oral glucose tolerance test (Fig. 2). After 2 months of leptin therapy, the glucose level was dramatically improved and, at the same time, the ability of insulin secretion was also clearly improved. In lipodystrophy, triglyceride accumulates excessively in the cells of non-adipose tissues including the liver and 


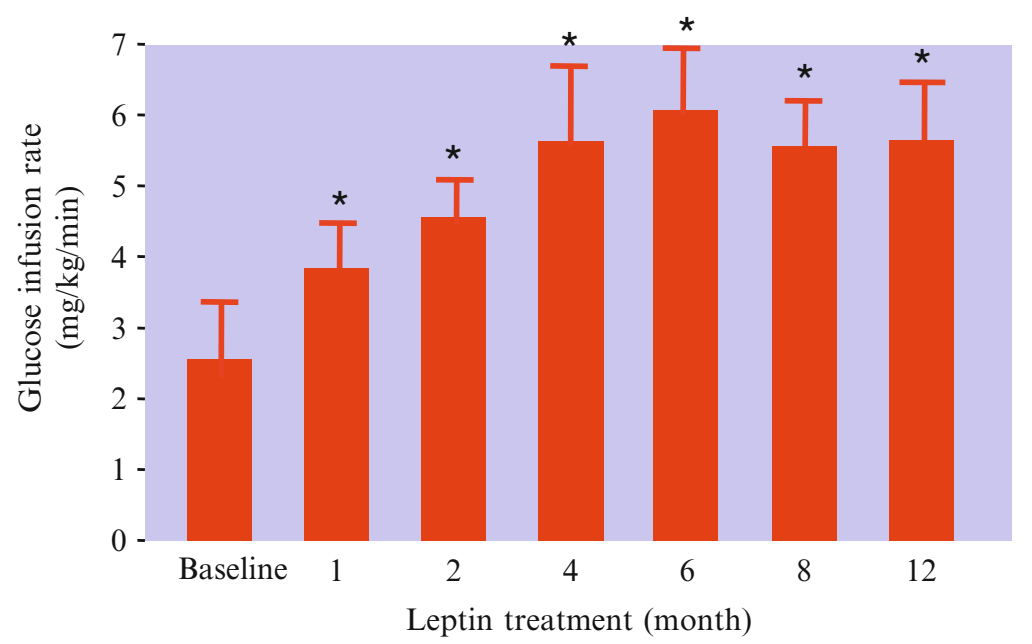

Fig. 1 Glucose infusion rate in a hyperinsulinemic euglycemic glucose clamp study in 10 patients with generalized lipodystrophy. Insulin sensitivity was distinctly low at baseline but improved month by month after the initiation of leptin therapy. ${ }^{*} \mathrm{p}<0.05$ vs baseline
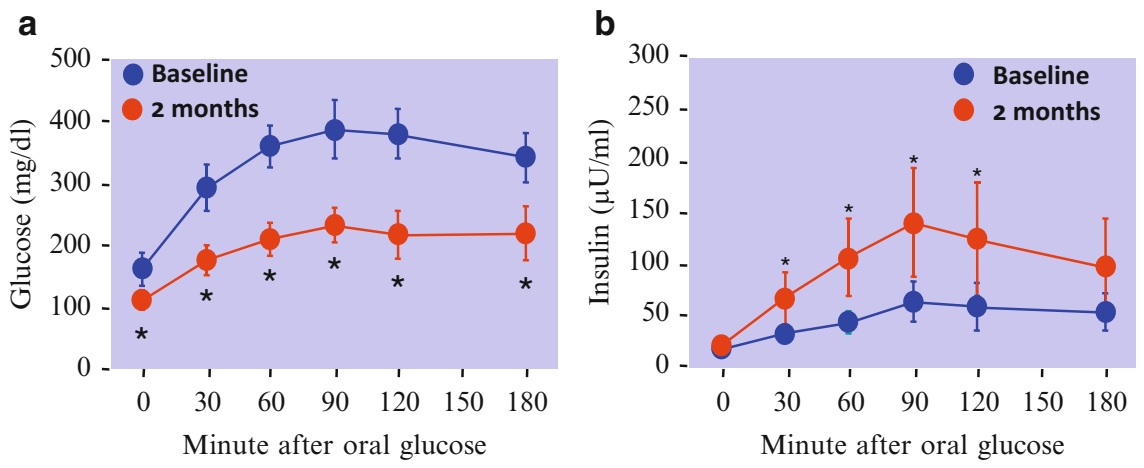

Fig. $275 \mathrm{~g}$ glucose tolerance test in 10 patients with generalized lipodystrophy at baseline and 2 months after the initiation of leptin replacement therapy. (a) Plasma glucose. (b) Insulin levels before and after an oral glucose load. Leptin replacement therapy improved glucose tolerance and insulin secretion. ${ }^{*} \mathrm{p}<0.05$ vs baseline

skeletal muscle [38]. In the liver, the amount of triglyceride accumulation is known to be correlated with the severity of insulin resistance [39]. Triglyceride accumulation in skeletal muscle also leads to insulin resistance [39]. In pancreatic beta cells, triglyceride accumulation is known to impair insulin secretion [39]. Cellular dysfunction caused by ectopic fat deposition has been referred as "lipotoxicity" [40]. To investigate the cancellation of lipotoxicity as a potential mechanism by which 
a

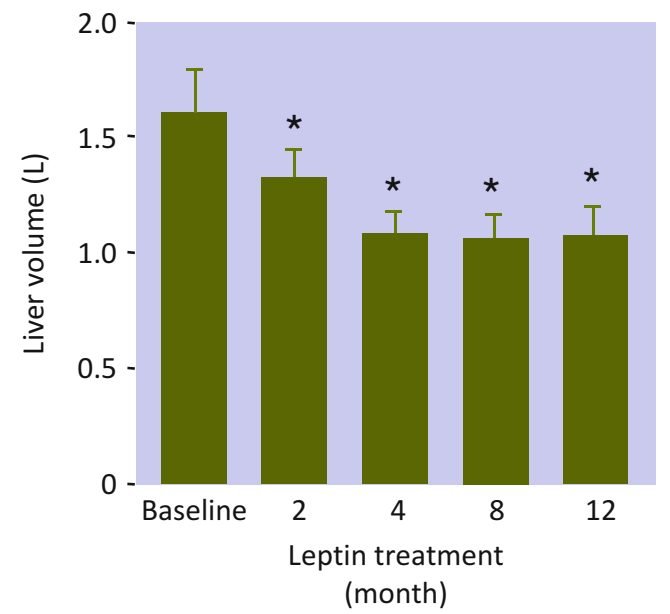

b

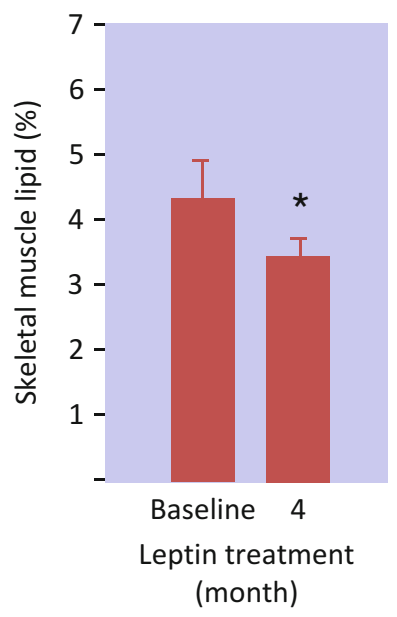

Fig. 3 Ectopic fat deposition in insulin-target tissues. (a) Liver volume calculated by CT in 10 patients with generalized lipodystrophy before and after the initiation of leptin replacement therapy. (b) Skeletal muscle lipid content estimated by MRI before and 4 months after the initiation of leptin replacement therapy. $* \mathrm{p}<0.05$ vs baseline

leptin improves insulin sensitivity and insulin secretion, we evaluated the tissue lipid content in the liver and skeletal muscle in patients with lipodystrophy (Fig. 3). Liver volume as an index of fatty liver was calculated by computed tomography (CT). Leptin therapy effectively decreased liver volume. Skeletal muscle lipid content was estimated by magnetic resonance imaging (MRI). Skeletal muscle lipid content was also significantly decreased after 4 months of leptin therapy. These results suggest that leptin improves insulin sensitivity at least partly by cancellation of lipotoxicity in the liver and skeletal muscle. Although we did not evaluate lipid content in pancreatic beta cells in this study, it is also possible that leptin improves insulin secretion by cancellation of lipotoxicity in beta cells.

We also evaluated subjective feelings of appetite with a $100 \mathrm{~mm}$ visual analog scale [41] in patients before and after the initiation of leptin therapy. On this assessment, participants were instructed to rate how hungry they were by marking on a scale before and after each meal. A higher score indicated a greater extent of hunger. As shown in Fig. 4, the self-reported hunger scores before meals were not different between before and after the initiation of leptin therapy in most patients. In contrast, after meals, the score was effectively suppressed after leptin treatment in most patients. These results indicate that leptin reinforces the formation of satiety feelings after a meal. 

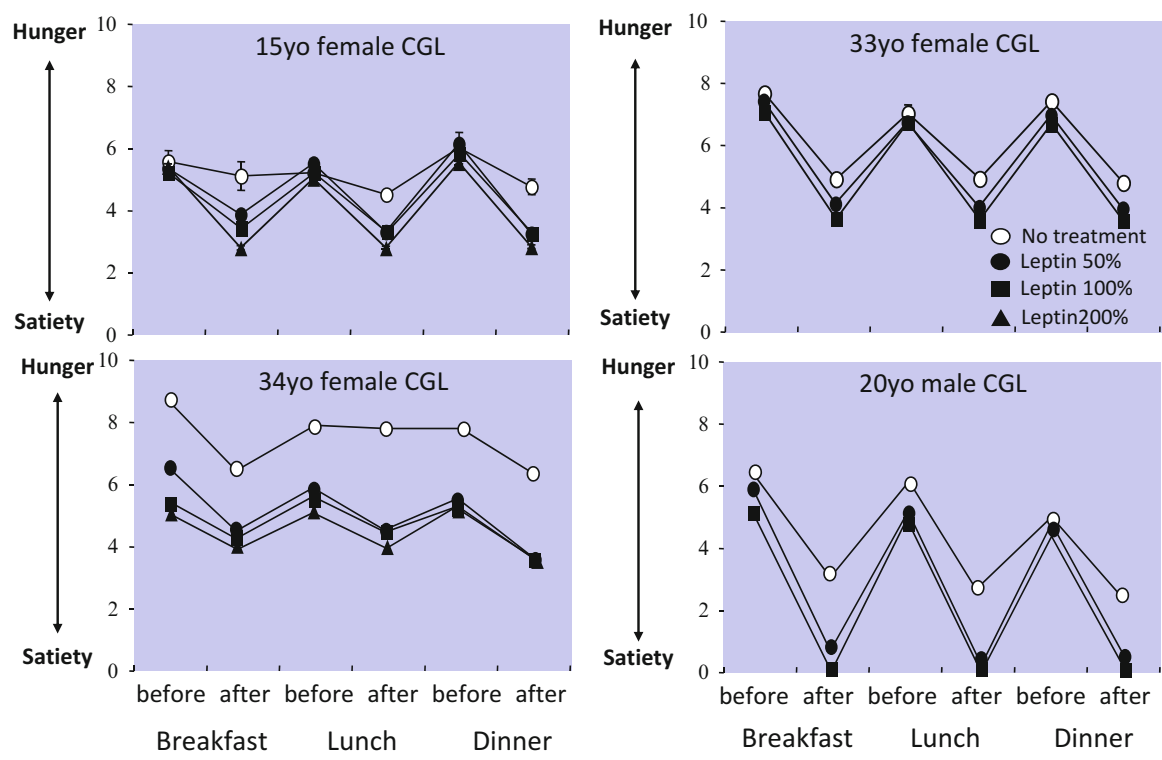

Fig. 4 Subjective appetite feelings evaluated with a $100 \mathrm{~mm}$ visual analog scale in representative patients with generalized lipodystrophy with or without leptin treatment. Patients were treated at $50 \%, 100 \%$, and $200 \%$ of the replacement dose. CGL congenital generalized lipodystrophy

\section{Significance of Hepatic AMPK in the Metabolic Action of Leptin}

Leptin effectively improves insulin sensitivity accompanied by dramatic reduction of fat content in the liver and skeletal muscle in patients with lipodystrophy [35, 37, 38]. Using rodent models, it was demonstrated that leptin activates $5^{\prime}$-AMP-activated protein kinase (AMPK) in the skeletal muscle through both central and direct pathways [42]. AMPK is a heterotrimeric enzyme that is conserved from yeast to humans and functions as a "fuel gauge" to monitor the status of cellular energy. AMPK potently stimulates fatty acid oxidation by inhibiting the activity of acetylCoA carboxylase. Thus, AMPK activation by leptin is a plausible mechanism by which leptin reduces ectopic fat in the skeletal muscle. In addition to the skeletal muscle, recent studies have shown the physiological significance of AMPK in the liver [43, 44]. However, the effect of leptin on hepatic AMPK activity remained to be determined. The role of AMPK in the pathogenesis of metabolic abnormalities in lipodystrophy also remained unclear. We investigated the effect of leptin on hepatic AMPK activities and the pathophysiological role of AMPK in A-ZIPTg mice, a mouse model of generalized lipodystrophy [45].

We demonstrated that leptin activates hepatic AMPK through the central nervous system and alpha-adrenergic sympathetic nerves. AMPK activities were decreased in the fatty liver of A-ZIP/F-1 mice, and leptin administration increased AMPK 
activities in the liver as well as in skeletal muscle with a significant reduction in triglyceride content. Activation of hepatic AMPK with A769662 also led to a decrease in hepatic triglyceride content and blood glucose levels in A-ZIP/F-1 mice. These results indicate that downregulation of hepatic AMPK activities plays a pathophysiological role in the metabolic disturbances of lipodystrophy, and that hepatic AMPK activation is involved in the therapeutic effects of leptin.

\section{fMRI Analysis of Food-Related Brain Activity in Patients with Lipodystrophy}

Lipodystropic patients also exhibit eating disorders, which makes diet therapy difficult [46]. Leptin replacement therapy was shown to suppress appetite in lipodystrophic patients $[46,47]$. However, there is no report on the comparison of eating behaviors between healthy subjects and patients with lipodystrophy. Therefore, the pathophysiological role of leptin in eating disorders in patients with lipodystrophy remains unclear. From experimental studies in human and animals, it has long been established that leptin suppresses energy intake mainly by acting on the hypothalamus [11, 48]. However, there is little information about how the neural networks including the hypothalamus are influenced by leptin signals. Recently the advent of functional neuroimaging techniques such as functional magnetic resonance imaging (fMRI) has been providing novel insights into homeostatic and hedonic aspects of human eating behavior. fMRI measurements of food-related neural activity in congenital leptin-deficient patients were reported [49]. These analyses revealed that leptin treatment modulates neural activity in reward and food-related areas such as the ventral striatum and orbitofrontal cortex.

To reveal the pathogenic mechanism of eating disorders in lipodystrophic patients, we measured food-related neural activity by fMRI scans and investigated subjective feelings of appetite under both fasting and postprandial conditions in patients and age- and sex-matched healthy subjects [51]. In addition, we performed the same sequential analyses in the same patients with leptin replacement therapy [51]. Although there was little difference in the enhancement of neural activity by food stimuli between patients and controls under fasting, postprandial suppression of neural activity was insufficient in many regions of interest including the amygdala, insula, nucleus accumbens, caudate, putamen, and globus pallidus in patients compared with controls. Leptin treatment effectively suppressed postprandial neural activity in many of these regions of interest, whereas it showed little effect under fasting in patients. Consistent with these results, postprandial formation of satiety feelings was insufficient in patients compared with controls, which was effectively reinforced by leptin treatment. These results demonstrate the insufficiency of postprandial suppression of food-related neural activity and formation of satiety feelings in patients with lipodystrophy, which might be largely due to leptin deficiency. This study also demonstrated that leptin has little involvement in the regulation of neural activity and eating behavior under fasting, whereas leptin plays a significant role in 
these regulations under postprandial conditions. We found that leptin suppressed neural activity in regions involved in the reward system such as the amygdala, hippocampus, NcA, and caudate. Further study is needed to elucidate the role of the reward system on appetite regulation by leptin.

\section{Conclusions}

Metreleptin is an analog of human leptin originally developed by Amgen and has been used for treatment in patients with lipodystrophy also in Japan. In July 2012, Shionogi originally filed a New Drug Application (NDA), which was based on the results of our investigator-initiated trial conducted by the Kyoto University Graduate School of Medicine with assistance from the Translational Research Center (the current Institute for Advancement of Clinical and Translational Science) at Kyoto University Hospital. In March 2013, marketing and manufacturing approval was granted by the Japanese Ministry of Health, Labour and Welfare for subcutaneous metreleptin for the treatment of lipodystrophy. This is the first global approval of leptin formulation [51]. In patients with lipodystrophy, leptin improved insulin resistance, hypertriglyceridemia, and fatty liver. We have demonstrated the therapeutic usefulness of leptin in insulin-deficient diabetes, non-obese type 2 diabetes, type 2 diabetes with mild obesity, hypertriglyceridemia, and non-alcoholic fatty liver [45, 52-56]. Leptin has potential as a drug for the treatment of more common metabolic diseases including diabetes, hyperlipidemia, and fatty liver.

Open Access This chapter is distributed under the terms of the Creative Commons Attribution Noncommercial License, which permits any noncommercial use, distribution, and reproduction in any medium, provided the original author(s) and source are credited.

\section{References}

1. Zhang Y, Proenca R, Maffei M, Barone M, Leopold L, Friedman JM (1994) Positional cloning of the mouse obese gene and its human homologue. Nature 372:425-432

2. Friedman JM, Halaas JL (1998) Leptin and the regulation of body weight in mammals. Nature 395:763-770

3. Pelleymounter MA, Cullen MJ, Baker MB, Hecht R, Winters D, Boone T, Collins F (1995) Effects of the obese gene product on body weight regulation in ob/ob mice. Science 269:540-543

4. Halaas JL, Gajiwala KS, Maffei M, Cohen SL, Chait BT, Rabinowitz D, Lallone RL, Burley SK, Friedman JM (1995) Weight-reducing effects of the plasma protein encoded by the obese gene. Science 269:543-546

5. Campfield LA, Smith FJ, Guisez Y, Devos R, Burn P (1995) Recombinant mouse OB protein: evidence for a peripheral signal linking adiposity and central neural networks. Science 269:546-549

6. Satoh N, Ogawa Y, Katsuura G, Numata Y, Tsuji T, Hayase M, Ebihara K, Masuzaki H, Hosoda $\mathrm{K}$, Yoshimasa Y et al (1999) Sympathetic activation of leptin via the ventromedial hypothalamus: leptin-induced increase in catecholamine secretion. Diabetes 48:1787-1793 
7. Frederich RC, Hamann A, Anderson S, Lollmann B, Lowell BB, Flier JS (1995) Leptin levels reflect body lipid content in mice: evidence for diet-induced resistance to leptin action. Nat Med 1:1311-1314

8. Maffei M, Halaas J, Ravussin E, Pratley RE, Lee GH, Zhang Y, Fei H, Kim S, Lallone R, Ranganathan S et al (1995) Leptin levels in human and rodent: measurement of plasma leptin and ob RNA in obese and weight-reduced subjects. Nat Med 1:1155-1161

9. Considine RV, Sinha MK, Heiman ML, Kriauciunas A, Stephens TW, Nyce MR, Ohannesian JP, Marco CC, McKee LJ, Bauer TL et al (1996) Serum immunoreactive-leptin concentrations in normal-weight and obese humans. N Engl J Med 334:292-295

10. Isse N, Ogawa Y, Tamura N, Masuzaki H, Mori K, Okazaki T, Satoh N, Shigemoto M, Yoshimasa Y, Nishi S et al (1995) Structural organization and chromosomal assignment of the human obese gene. J Biol Chem 270:27728-27733

11. Satoh N, Ogawa Y, Katsuura G, Tsuji T, Masuzaki H, Hiraoka J, Okazaki T, Tamaki M, Hayase M, Yoshimasa Y et al (1997) Pathophysiological significance of the obese gene product, leptin, in ventromedial hypothalamus (VMH)-lesioned rats: evidence for loss of its satiety effect in VMH-lesioned rats. Endocrinology 138:947-954

12. Imagawa K, Numata Y, Katsuura G, Sakaguchi I, Morita A, Kikuoka S, Matumoto Y, Tsuji T, Tamaki M, Sasakura K et al (1998) Structure-function studies of human leptin. J Biol Chem 273:35245-35249

13. Caro JF, Kolaczynski JW, Nyce MR, Ohannesian JP, Opentanova I, Goldman WH, Lynn RB, Zhang PL, Sinha MK, Considine RV (1996) Decreased cerebrospinal-fluid/serum leptin ratio in obesity: a possible mechanism for leptin resistance. Lancet 348:159-161

14. Halaas JL, Boozer C, Blair-West J, Fidahusein N, Denton DA, Friedman JM (1997) Physiological response to long-term peripheral and central leptin infusion in lean and obese mice. Proc Natl Acad Sci U S A 94:8878-8883

15. Tanaka T, Masuzaki H, Yasue S, Ebihara K, Shiuchi T, Ishii T, Arai N, Hirata M, Yamamoto H, Hayashi $\mathrm{T}$ et al (2007) Central melanocortin signaling restores skeletal muscle AMP-activated protein kinase phosphorylation in mice fed a high-fat diet. Cell Metab $5: 395-402$

16. Montague CT, Farooqi IS, Whitehead JP, Soos MA, Rau H, Wareham NJ, Sewter CP, Digby JE, Mohammed SN, Hurst JA et al (1997) Congenital leptin deficiency is associated with severe early-onset obesity in humans. Nature 387:903-908

17. Strobel A, Issad T, Camoin L, Ozata M, Strosberg AD (1998) A leptin missense mutation associated with hypogonadism and morbid obesity. Nat Genet 18:213-215

18. Yura S, Ogawa Y, Sagawa N, Masuzaki H, Itoh H, Ebihara K, Aizawa-Abe M, Fujii S, Nakao K (2000) Accelerated puberty and late-onset hypothalamic hypogonadism in female transgenic skinny mice overexpressing leptin. J Clin Invest 105:749-755

19. Aizawa-Abe M, Ogawa Y, Masuzaki H, Ebihara K, Satoh N, Iwai H, Matsuoka N, Hayashi T, Hosoda K, Inoue G et al (2000) Pathophysiological role of leptin in obesity-related hypertension. J Clin Invest 105:1243-1252

20. Elefteriou F, Takeda S, Ebihara K, Magre J, Patano N, Kim CA, Ogawa Y, Liu X, Ware SM, Craigen WJ et al (2004) Serum leptin level is a regulator of bone mass. Proc Natl Acad Sci U S A 101:3258-3263

21. Suganami T, Mukoyama M, Mori K, Yokoi H, Koshikawa M, Sawai K, Hidaka S, Ebihara K, Tanaka T, Sugawara A et al (2005) Prevention and reversal of renal injury by leptin in a new mouse model of diabetic nephropathy. FASEB J 19:127-129

22. Ogawa Y, Masuzaki H, Hosoda K, Aizawa-Abe M, Suga J, Suda M, Ebihara K, Iwai H, Matsuoka N, Satoh N et al (1999) Increased glucose metabolism and insulin sensitivity in transgenic skinny mice overexpressing leptin. Diabetes 48:1822-1829

23. Ogawa Y, Itoh H, Tamura N, Suga S, Yoshimasa T, Uehira M, Matsuda S, Shiono S, Nishimoto H, Nakao K (1994) Molecular cloning of the complementary DNA and gene that encode mouse brain natriuretic peptide and generation of transgenic mice that overexpress the brain natriuretic peptide gene. J Clin Invest 93:1911-1921 
24. Masuzaki H, Ogawa Y, Isse N, Satoh N, Okazaki T, Shigemoto M, Mori K, Tamura N, Hosoda K, Yoshimasa Y et al (1995) Human obese gene expression. Adipocyte-specific expression and regional differences in the adipose tissue. Diabetes 44:855-858

25. Ioffe E, Moon B, Connolly E, Friedman JM (1998) Abnormal regulation of the leptin gene in the pathogenesis of obesity. Proc Natl Acad Sci U S A 95:11852-11857

26. Kamohara S, Burcelin R, Halaas JL, Friedman JM, Charron MJ (1997) Acute stimulation of glucose metabolism in mice by leptin treatment. Nature 389:374-377

27. Liu L, Karkanias GB, Morales JC, Hawkins M, Barzilai N, Wang J, Rossetti L (1998) Intracerebroventricular leptin regulates hepatic but not peripheral glucose fluxes. J Biol Chem 273:31160-31167

28. Cusin I, Zakrzewska KE, Boss O, Muzzin P, Giacobino JP, Ricquier D, Jeanrenaud B, Rohner-Jeanrenaud F (1998) Chronic central leptin infusion enhances insulin-stimulated glucose metabolism and favors the expression of uncoupling proteins. Diabetes 47:1014-1019

29. Goldstein BJ (1994) Syndrome of extreme insulin resistance. In: Kahn CR, Weir GC (eds) Joslin's diabetes mellitus. Lea \& Febiger, Philadelphia

30. Andreelli F, Hanaire-Broutin H, Laville M, Tauber JP, Riou JP, Thivolet C (2000) Normal reproductive function in leptindeficient patients with lipoatrophic diabetes. J Clin Endocrinol Metab 85:715-719

31. Pardini VC, Victoria IM, Rocha SM, Andrade DG, Rocha AM, Pieroni FB, Milagres G, Purisch S, Velho G (1998) Leptin levels, beta-cell function, and insulin sensitivity in families with congenital and acquired generalized lipoatrophic diabetes. J Clin Endocrinol Metab 83:503-508

32. Moitra J, Mason MM, Olive M, Krylov D, Gavrilova O, Marcus-Samuels B, Feigenbaum L, Lee E, Aoyama T, Eckhaus M et al (1998) Life without white fat: a transgenic mouse. Genes Dev 12:3168-3181

33. Shimomura I, Hammer RE, Richardson JA, Ikemoto S, Bashmakov Y, Goldstein JL, Brown MS (1998) Insulin resistance and diabetes mellitus in transgenic mice expressing nuclear SREBP-1c in adipose tissue: model for congenital generalized lipodystrophy. Genes Dev 12:3182-3194

34. Ebihara K, Ogawa Y, Masuzaki H, Shintani M, Miyanaga F, Aizawa-Abe M, Hayashi T, Hosoda K, Inoue G, Yoshimasa Y et al (2001) Transgenic overexpression of leptin rescues insulin resistance and diabetes in a mouse model of lipoatrophic diabetes. Diabetes 50:1440-1448

35. Oral EA, Simha V, Ruiz E, Andewelt A, Premkumar A, Snell P, Wagner AJ, DePaoli AM, Reitman ML, Taylor SI et al (2002) Leptin-replacement therapy for lipodystrophy. N Engl J Med 346:570-578

36. Ebihara K, Masuzaki H, Nakao K (2004) Long-term leptinreplacement therapy for lipoatrophic diabetes. N Engl J Med 351:615-616

37. Ebihara K, Kusakabe T, Hirata M, Masuzaki H, Miyanaga F, Kobayashi N, Tanaka T, Chusho H, Miyazawa T, Hayashi T et al (2007) Efficacy and safety of leptin-replacement therapy and possible mechanisms of leptin actions in patients with generalized lipodystrophy. J Clin Endocrinol Metab 92:532-541

38. Petersen KF, Oral EA, Dufour S, Befroy D, Ariyan C, Yu C, Cline GW, DePaoli AM, Taylor SI, Gorden P et al (2002) Leptin reverses insulin resistance and hepatic steatosis in patients with severe lipodystrophy. J Clin Invest 109:1345-1350

39. Shulman GI (2000) Cellular mechanisms of insulin resistance. J Clin Invest 106:171-176

40. Unger RH (1995) Lipotoxicity in the pathogenesis of obesity-dependent NIDDM. Genetic and clinical implications. Diabetes 44:863-870

41. Chaput JP, Gilbert JA, Gregersen NT, Pedersen SD, Sjödin AM (2010) Comparison of 150-mm versus 100-mm visual analogue scales in free living adult subjects. Appetite 49:691-695

42. Minokoshi Y, Kim YB, Peroni OD, Fryer LG, Müller C, Carling D, Kahn BB (2002) Leptin stimulates fatty-acid oxidation by activating AMP-activated protein kinase. Nature 415:339-343 
43. Assifi MM, Suchankova G, Constant S, Prentki M, Saha AK, Ruderman NB (2005) AMP-activated protein kinase and coordination of hepatic fatty acid metabolism of starved/ carbohydrate-refed rats. Am J Physiol Endocrinol Metab 289:E794-E800

44. Shaw RJ, Lamia KA, Vasquez D, Koo SH, Bardeesy N, Depinho RA, Montminy M, Cantley LC (2005) The kinase LKB1 mediates glucose homeostasis in liver and therapeutic effects of metformin. Science 310:1642-1646

45. Miyamoto L, Ebihara K, Kusakabe T, Aotani D, Yamamoto-Kataoka S, Sakai T, Aizawa-Abe M, Yamamoto Y, Fujikura J, Hayashi T et al (2012) Leptin activates hepatic 5'-AMP-activated protein kinase through sympathetic nervous system and $\alpha 1$-adrenergic receptor: a potential mechanism for improvement of fatty liver in lipodystrophy by leptin. J Biol Chem 287:40441-40447

46. McDuffie JR, Riggs PA, Calis KA, Freedman RJ, Oral EA, DePaoli AM, Yanovski JA (2004) Effects of exogenous leptin on satiety and satiation in patients with lipodystrophy and leptin insufficiency. J Clin Endocrinol Metab 89:4258-4263

47. Moran SA, Patten N, Young JR, Cochran E, Sebring N, Reynolds J, Premkumar A, Depaoli AM, Skarulis MC, Oral EA et al (2004) Changes in body composition in patients with severe lipodystrophy after leptin replacement therapy. Metabolism 53:513-519

48. Schwartz MW, Seeley RJ, Campfield LA, Burn P, Baskin DG (1996) Identification of targets of leptin action in rat hypothalamus. J Clin Invest 98:1101-1106

49. Farooqi IS, Bullmore E, Keogh J, Gillard J, O'Rahilly S, Fletcher PC (2007) Leptin regulates striatal regions and human eating behavior. Science 317:1355

50. Aotani D, Ebihara K, Sawamoto N, Kusakabe T, Aizawa-Abe M, Kataoka S, Sakai T, Iogawa H, Ebihara C, Fujikura $\mathbf{J}$ et al (2012) Functional magnetic resonance imaging analysis of food-related brain activity in patients with lipodystrophy undergoing leptin replacement therapy. J Clin Endocrinol Metab 97:3663-3671

51. Chou K, Perry CM (2013) Metreleptin: first global approval. Drugs 73:989-997

52. Miyanaga F, Ogawa Y, Ebihara K, Hidaka S, Tanaka T, Hayashi S, Masuzaki H, Nakao K (2003) Leptin as an adjunct of insulin therapy in insulin-deficient diabetes. Diabetologia 4610:1329-1337

53. Naito M, Fujikura J, Ebihara K, Miyanaga F, Yokoi H, Kusakabe T, Yamamoto Y, Son C, Mukoyama M, Hosoda K et al (2011) Therapeutic impact of leptin on diabetes, diabetic complications, and longevity in insulin-deficient diabetic mice. Diabetes 60:2265-2273

54. Kusakabe T, Tanioka H, Ebihara K, Hirata M, Miyamoto L, Miyanaga F, Hige H, Aotani D, Fujisawa T, Masuzaki H et al (2009) Beneficial effects of leptin on glycaemic and lipid control in a mouse model of type 2 diabetes with increased adiposity induced by streptozotocin and a high-fat diet. Diabetologia 52:675-683

55. Matsuoka N, Ogawa Y, Masuzaki H, Ebihara K, Aizawa-Abe M, Satoh N, Ishikawa E, Fujisawa Y, Kosaki A, Yamada K et al (2001) Decreased triglyceride-rich lipoproteins in transgenic skinny mice overexpressing leptin. Am J Physiol Endocrinol Metab 280:E334-E339

56. Yamamoto-Kataoka S, Ebihara K, Aizawa-Abe M, Nishio M, Kusakabe T, Yamamoto Y, Aotani D, Sakai T, Zhao M, Ebihara C et al (2014) Leptin improves fatty liver independently of insulin sensitization and appetite suppression in hepatocyte-specific Pten-deficient mice with insulin hypersensitivity. Horm Metab Res (in press) 


\title{
Translational Research of the Activation of the C-Type Natriuretic Peptide (CNP)- Guanylyl Cyclase-B Pathway for Skeletal Dysplasia
}

\author{
Akihiro Yasoda and Kazuwa Nakao
}

\begin{abstract}
The natriuretic peptide family consists of three endogenous ligands: atrial natriuretic peptide (ANP), brain natriuretic peptide (BNP), and C-type natriuretic peptide (CNP). They exert their biological actions through two subtypes of particulate guanylyl cyclase (GC): GC-A for ANP and BNP, and GC-B for CNP. Among the natriuretic peptide family members, ANP and BNP are cardiac hormones that are produced and released from the atrium and ventricle of the heart, respectively, and play important roles in the regulation of the cardiovascular system. On the other hand, although CNP and its receptor, GC-B, exist ubiquitously in the body, we elucidated that the CNP/GC-B system is a crucial stimulator of endochondral bone growth, using CNP or GC-B knockout or transgenic mice. We planned to utilize the activation of the CNP/GC-B pathway as a novel therapeutic strategy for skeletal dysplasia, which consists of multiple skeletal diseases including those with impaired bone growth. We tried to investigate this effect on impaired skeletal growth in a mouse model of achondroplasia, the most common form of skeletal dysplasias, and successfully recovered the skeletal phenotype by using transgenic technology or by administration of synthetic CNP. In the future, the activation of the CNP/ GC-B system may constitute a novel therapeutic strategy for the treatment of skeletal dysplasias.
\end{abstract}

Keywords Natriuretic peptide family $\bullet$ C-type natriuretic peptide (CNP) $\bullet$ Guanylyl cyclase-B (GC-B) • Endochondral bone growth • Transgenic mouse • Knockout mouse $\bullet$ Skeletal dysplasia $\bullet$ Achondroplasia

\footnotetext{
A. Yasoda, M.D., Ph.D. ( $\square)$

Diabetes, Endocrinology and Nutrition, Kyoto University Graduate School of Medicine,

Kyoto, Japan

e-mail: yasoda@kuhp.kyoto-u.ac.jp

K. Nakao, M.D., Ph.D.

Professor, Medical Innovation Center, Kyoto University Graduate

School of Medicine, Kyoto, Japan

(C) The Author(s) 2015

K. Nakao et al. (eds.), Innovative Medicine, DOI 10.1007/978-4-431-55651-0_15
} 


\section{Introduction}

The natriuretic peptide family consists of three endogenous ligands: atrial natriuretic peptide (ANP), brain natriuretic peptide (BNP), and C-type natriuretic peptide (CNP) [1]. They exert their biological actions through two subtypes of particulate guanylyl cyclase (GC): GC-A for ANP and BNP, and GC-B for CNP [2]. Among the natriuretic peptide family members, ANP and BNP are cardiac hormones that are produced and released from the atrium and ventricle of the heart, respectively, and play important roles in the regulation of the cardiovascular system [3-7]. CNP, a third member of the natriuretic peptide family, was first purified from porcine brain [8]. Therefore, CNP was thought to be the primary natriuretic peptide in the brain, but has been proved to exist ubiquitously in the body, e.g., in the blood vessels, gonads, adrenal gland, etc. [9-15] (Fig. 1). Furthermore, analysis of the $\mathrm{CNP} / \mathrm{GC}-\mathrm{B}$ system in genetically engineered mice revealed that CNP and GC-B play a pivotal role in the regulation of endochondral bone growth.

\section{The CNP/GC-B System}

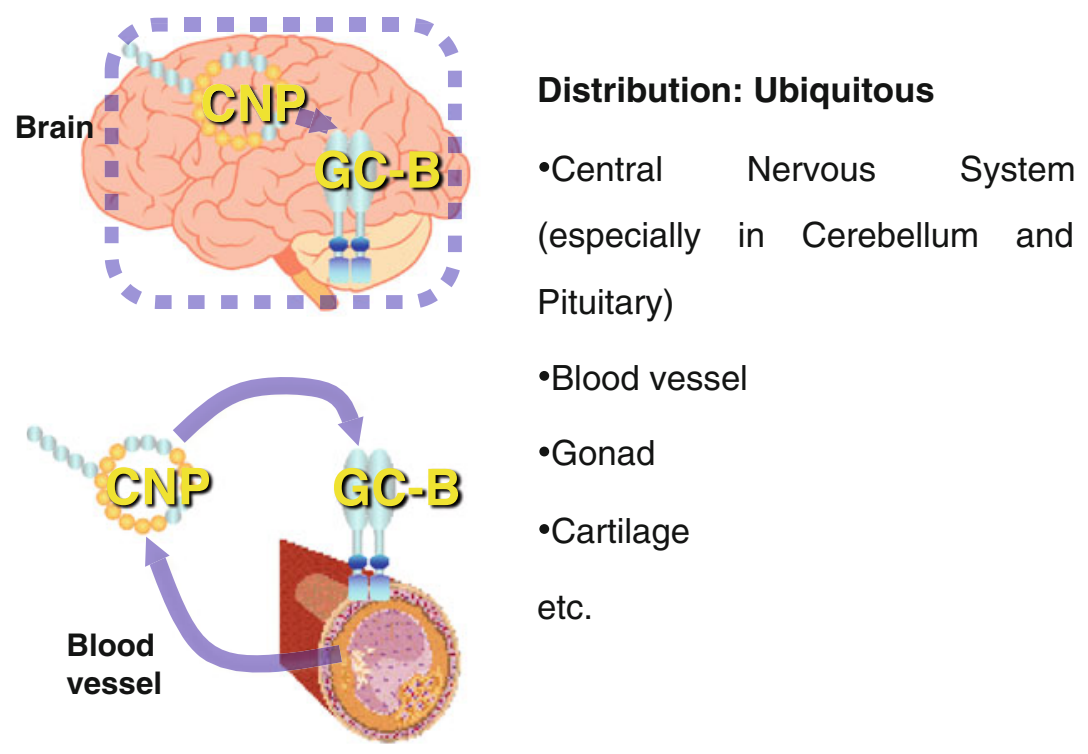

Fig. 1 Schematic representation of the distribution of the CNP/GC-B system. CNP and its receptor, GC-B, exist ubiquitously in the body 


\section{Skeletal Phenotypes of the CNP/GC-B System in Genetically Engineered Mice}

Targeted disruption of the CNP gene (Nppc) causes prominent short stature due to impaired bone growth in mice [16] (Fig. 2). Mammalian bones are formed through two different mechanisms, endochondral ossification and membranous ossification. Most mammalian bones are formed through endochondral ossification, in which the growth of bone is dependent on the growth of cartilaginous anlage as the scaffold of bone; later, it is replaced by calcified bone [17]. In the process of endochondral ossification, chondrocytes in the growth plate undergo proliferation, hypertrophy, and cell death, and finally are replaced by osteoblasts. The short-stature phenotype of CNP knockout (CNP-KO) mice results from impaired bone growth through endochondral ossification. Histological analysis of the growth plate of CNP-KO mice revealed that every chondrocyte layer of the growth plate is narrower in CNP-KO mice than in wild-type mice. Furthermore, mice depleted with the GC-B gene (Npr2) exhibit the same short-stature phenotype observed in CNP-KO mice, demonstrating that the $\mathrm{CNP} / \mathrm{GC}-\mathrm{B}$ system is a physiologically important stimulator of endochondral bone growth [18].

Fig. 2 Gross phenotype (upper panel) and growth curve (lower panel) of the CNP-KO mouse
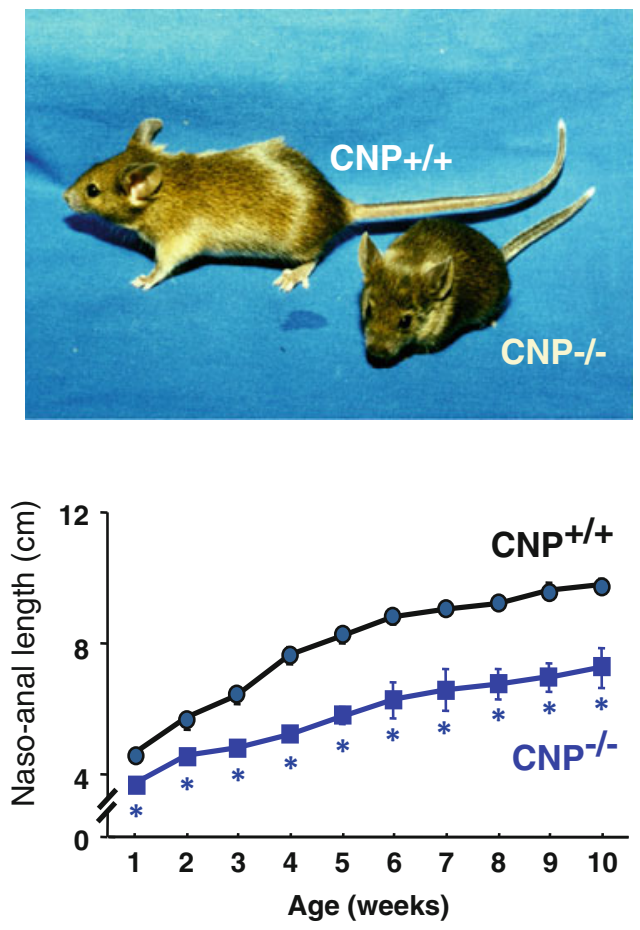

$* \mathrm{p}<0.01$ vs $\mathrm{CNP}^{+/+}$ 
Fig. 3 Cartilage-specific CNP transgenic mouse.

(a) Gross appearance of female cartilage-specific CNP transgenic (col2-CNP-Tg) mouse and its wild-type litter mate at the age of 10 weeks. (b) Immunohistochemical staining for type $\mathrm{X}$ collagen of a histological section of the tibial growth plates
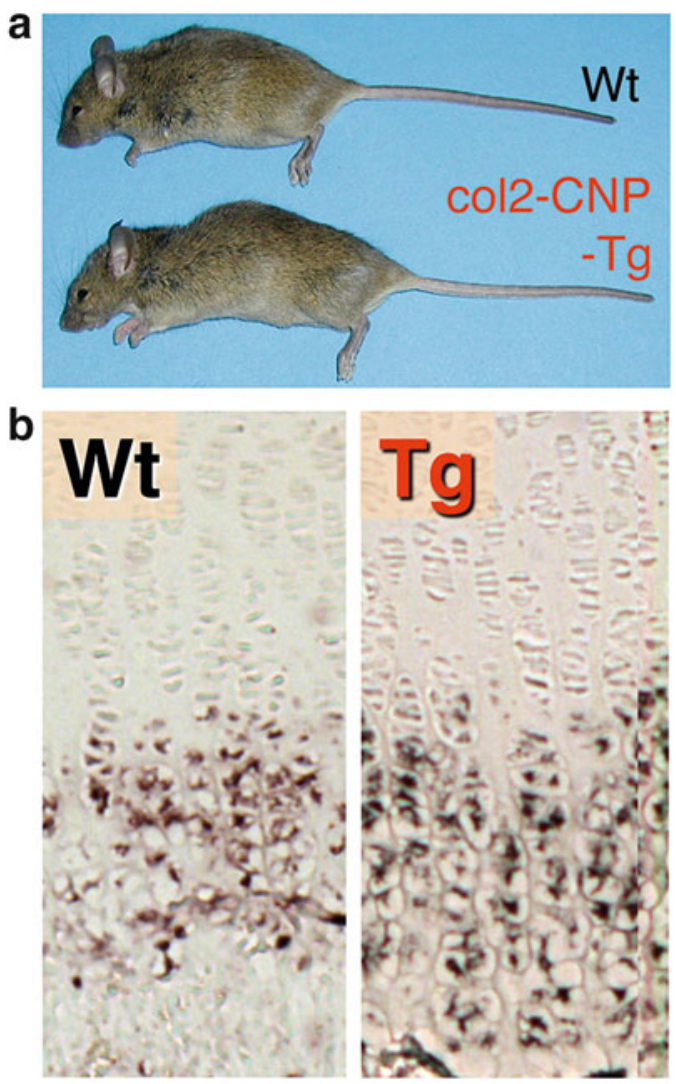

In contrast, cartilage-specific CNP transgenic mice under the control of type II collagen promoter (col2-CNP-Tg mice) exhibit prominent overgrowth of bones formed through endochondral ossification [19] (Fig. 3a). In contrast to CNP- or GC-B-KO mice, every chondrocyte layer, especially hypertrophic chondrocyte layer, of the growth plate of col2-CNP-Tg mice is wider than that of wild-type mice (Fig. 3b). Collectively, the CNP/GC-B system is a physiological stimulator of endochondral bone growth and can potently stimulate bone growth as a local regulator.

\section{Importance of CNP/GC-B Signaling in Endochondral Bone Growth in Humans: Lessons from Rare Congenital Skeletal Disorders}

Skeletal dysplasias are human genetic disorders of the skeleton, whose feature is impairment of bones. In 2004, Bartels et al., reported that acromesomelic dysplasia, type Maroteaux, one form of skeletal dysplasia with a severely impaired bone 
growth phenotype, is caused by a loss-of-function mutation in the human GC-B gene [20]. In fact, the skeletal phenotype observed in this disorder was just the same as that of GC-B-KO mice, and here for the first time, the CNP/GC-B signaling was proved to be a physiological and potent stimulator of endochondral bone growth not only in mice but also in humans. Later, in 2012, Miura et al., reported a Japanese case of skeletal overgrowth phenotype and showed that it was caused by a constitutively activated mutation in the GC-B gene [21]. They also reported a Korean case of skeletal overgrowth caused by a constitutive active mutation in the GC-B gene [22]. Thus, the notion that the CNP/GC-B system is a potent stimulator of endochondral bone in human is firmly established so far.

\section{Translational Research into Activation of CNP/GC-B Signaling for Skeletal Dysplasias}

Following these results, we planned to start translational research into activation of the CNP/GC-B system for disorders with impaired bone growth in skeletal dysplasias. Achondroplasia is the most common form of skeletal dysplasias, with a birth prevalence of $\sim 1: 10,000$. Patients suffering from achondroplasia exhibit short stature (final height: $\sim 130 \mathrm{~cm}$ in males and $\sim 120 \mathrm{~cm}$ in females) and a short-limbed dwarf phenotype owing to impaired endochondral bone growth, and it had been shown to be caused by a constitutive active mutation in the FGF receptor 3 (FGFR3) gene, followed by a decrease in the proliferation of chondrocytes in the growth plate $[23,24]$. Current therapies for achondroplasia consist of distraction osteogenesis, an orthopedic procedure, and administration of growth hormone [25]. Although distraction osteogenesis gives better results, it lays a burden on patients and limits their quality of life. Growth hormone has a minimal effect. Therefore, we started translational research into activation of the CNP/GC-B system for this disease. We obtained transgenic mice with targeted overexpression of activated FGFR3 (G380R mutation in humans) in cartilage, using type II collagen promoter as a mouse model of achondroplasia (Ach mice) [26]. As shown in Fig. 4, Ach mice show short stature and an impaired bone growth phenotype, and mimic the skeletal phenotype of achondroplastic patients. We achieved targeted overexpression of CNP in the growth plate of Ach mice by crossing them with col2-CNP-Tg mice. The short stature observed in Ach mice, owing to their impaired bone growth, was recovered in the double transgenic Ach/col2-CNP-Tg mice and grew almost comparable to that of wild-type mice (Fig. 4). As was previously reported, the length of the Ach mice was significantly shorter at least at the third week after birth, and more than $10 \%$ shorter after 6 weeks of age, than that of their wild-type litter mates. In Ach/col2-CNP-Tg mice, the decrease in the length of Ach mice was recovered, and the length became comparable to that of their wild-type litter mates. A soft x-ray picture revealed that the shortening of Ach bones was almost completely recovered in double transgenic bones. But the width of the cranium, which is made through membranous 


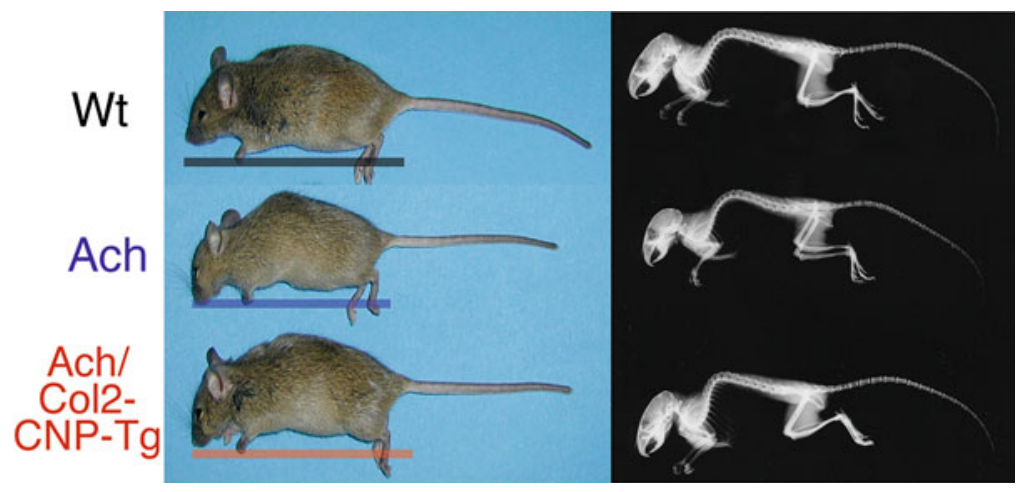

Fig. 4 Skeletal rescue of achondroplastic model mouse by targeted overexpression of CNP in cartilage. The gross appearance (left) and soft x-ray picture (right) of litter mates of the mice at the age of 3 months are depicted

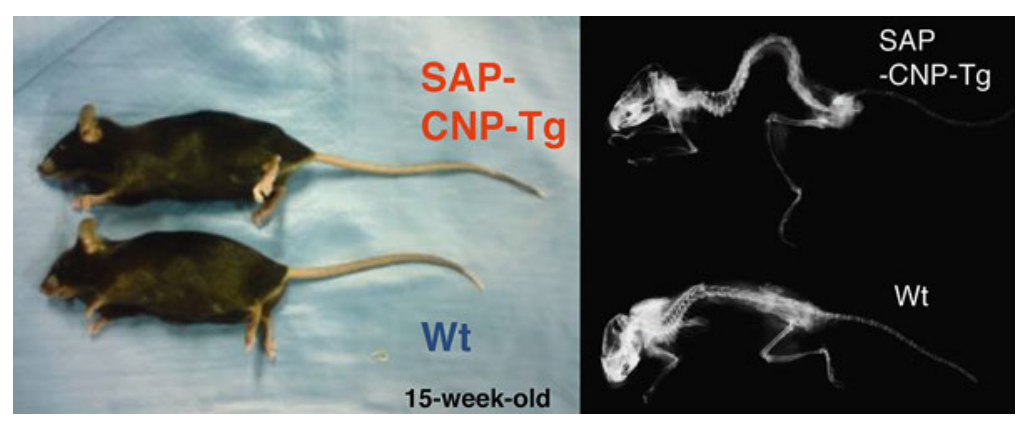

Fig. 5 Generation of CNP transgenic mice with increased circulating CNP under the control of human serum amyloid P component (SAP) promoter. Gross appearance (left) and soft x-ray picture (right) of litter mates of the mice at the age of 15 weeks are depicted

ossification, was not changed. Histological examination of the tibial growth plate showed that the width of the growth plate of Ach mice was shorter than that of wildtype mice, but was restored in the growth plate of the double transgenic mice [19].

Next, in order to test the effect of elevated plasma concentrations of CNP on endochondral bone growth, we generated transgenic mice with an elevated plasma concentration of CNP, using human serum amyloid $\mathrm{P}$ component promoter, which enables targeted overexpression of CNP in the liver (SAP-CNP-Tg mice) (Fig. 5). The resultant SAP-CNP-Tg mice, which have about two times the plasma CNP concentration of wild-type mice, exhibited skeletal overgrowth just like col2-CNPTg mice [27]. Then we mated Ach mice with the SAP-CNP-Tg mice and tried to rescue the impaired endochondral bone growth of Ach mice by increased circulating CNP. We could successfully rescue the short-stature phenotype of Ach mice in the double transgenic Ach/SAP-CNP-Tg mice (Fig. 6a). After 6 weeks of age, the short stature observed in Ach mice was completely rescued in Ach/SAP-CNP-Tg mice. 
a

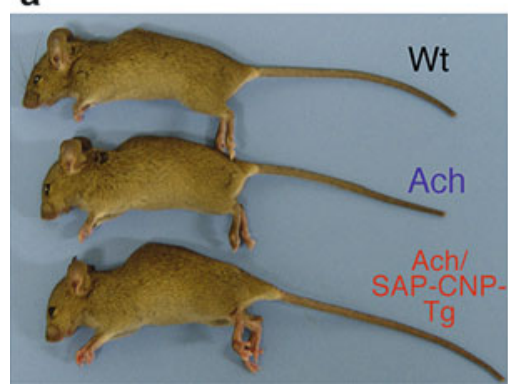

b

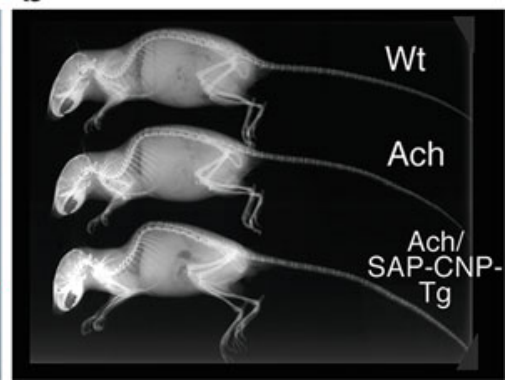

Fig. 6 Skeletal rescue of achondroplastic model mouse by increased circulating CNP. Gross appearance (left) and soft $\mathrm{x}$-ray picture (right) of litter mates of the mice at the age of 3 months are depicted

Skeletal analysis by a soft $x$-ray picture also exhibited that the impaired bone growth of Ach mice was rescued by increased circulating CNP (Fig. 6b). The lengths of the humerus, radius, ulna, femur, and tibia of the double transgenic mice were rather longer than those of wild-type mice. Histological examination revealed that the shortened growth plate of Ach mice was rescued in Ach/SAP-CNP-Tg mice and became rather elongated in comparison with the wild-type mice [28].

Finally, we tried to administrate CNP to Ach mice. Ach mice were about $10 \%$ shorter than wild-type mice at the beginning of administration at the age of 3 weeks. Administration of CNP at the dose of $1 \mu \mathrm{g} / \mathrm{kg} / \mathrm{min}$ stimulated the growth of Ach mice, and their length was almost comparable to that of wild-type mice at the end of the 4-week administration period (Fig. 7a). A soft x-ray picture revealed that the impaired skeletal growth observed in Ach mice was recovered by administration of CNP. Administration of $0.1 \mu \mathrm{g} / \mathrm{kg} / \mathrm{min}$ of CNP considerably rescued the shortness of Ach bones, and $1 \mu \mathrm{g} / \mathrm{kg} / \mathrm{min}$ elongated them to an extent rather longer than those of vehicle-treated wild-type mice. Histological examination revealed that the narrowed growth plate of Ach mice was recovered and became comparable to that of wildtype mice through administration of CNP at the dose of $1 \mu \mathrm{g} / \mathrm{kg} / \mathrm{min}$ (Fig. 7b) [28].

\section{Conclusions and Future Prospects}

We have elucidated that CNP/GC-B signaling is a physiological stimulator of endochondral bone growth and exhibited that the activation of this pathway strongly stimulates longitudinal bone growth in mice model. Furthermore, clinical reports showed that decreased signaling of this pathway causes impaired endochondral bone growth (acromesomelic dysplasia, type Maroteaux), and increased signaling causes skeletal overgrowth phenotype in humans. We started translational research into CNP/GC-B therapy for skeletal dysplasias using a mouse model of achondroplasia, and successfully rescued their impaired skeletal growth phenotype. 
Fig. 7 Skeletal rescue of achondroplastic model mouse by continuous intravenous injection of CNP at the dose of $1 \mu \mathrm{g} / \mathrm{kg} / \mathrm{min}$. Gross appearance (a) and histological pictures of the vertebral growth plates (b, safranin-O staining) of the mice at the end of the 4-week treatment

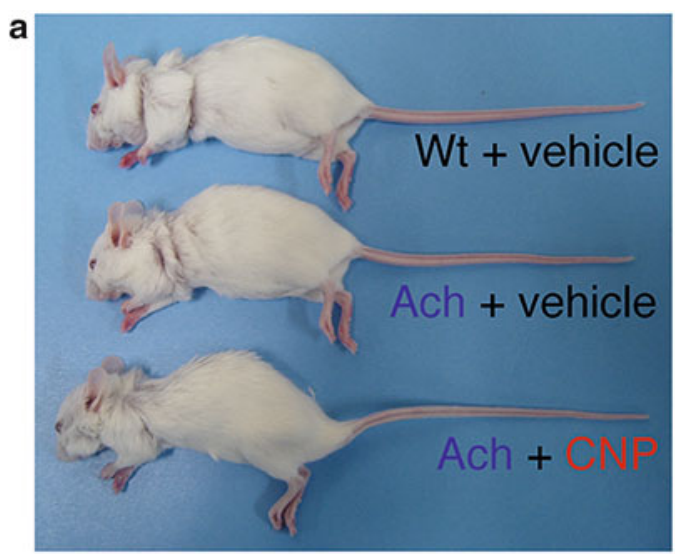

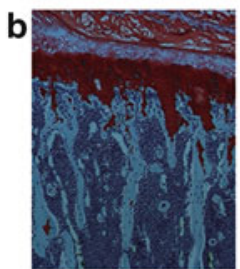

Wt

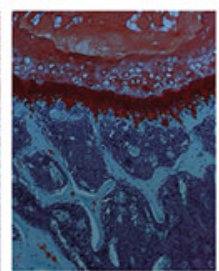

Ach

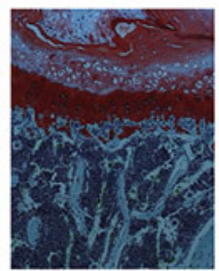

Ach

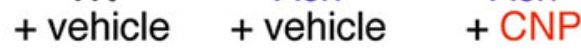

Nevertheless, there remain several problems to be solved to make CNP/GC-B therapy a reality for skeletal dysplasias. In the preclinical study described here, we had to administrate CNP intravenously, not subcutaneously, because CNP is easily degraded by subcutaneous endopeptidase. First, therefore, in the event that we use CNP, we must develop a way of administering CNP. Second, in relevance to this point, modification of the clearance system of CNP may be another approach for activating the CNP/GC-B system [29].

Acknowledgments We thank Dr. D. M. Ornitz for the Ach mice used in this work.

Open Access This chapter is distributed under the terms of the Creative Commons Attribution Noncommercial License, which permits any noncommercial use, distribution, and reproduction in any medium, provided the original author(s) and source are credited.

\section{References}

1. Nakao K, Ogawa Y, Suga S, Imura H (1992) Molecular biology and biochemistry of the natriuretic peptide system. I: natriuretic peptides. J Hypertens 10:907-912

2. Nakao K, Ogawa Y, Suga S, Imura H (1992) Molecular biology and biochemistry of the natriuretic peptide system. II: natriuretic peptide receptors. J Hypertens 10:1111-1114 
3. Mukoyama M, Nakao K, Hosoda K et al (1991) Brain natriuretic peptide as a novel cardiac hormone in humans. Evidence for an exquisite dual natriuretic peptide system, atrial natriuretic peptide and brain natriuretic peptide. J Clin Invest 87:1402-1412

4. Mukoyama M, Nakao K, Saito Y et al (1990) Increased human brain natriuretic peptide in congestive heart failure. N Engl J Med 323:757-758

5. Mukoyama M, Nakao K, Saito Y et al (1990) Human brain natriuretic peptide, a novel cardiac hormone. Lancet 335:801-802

6. Saito Y, Nakao K, Arai H et al (1989) Augmented expression of atrial natriuretic polypeptide gene in ventricle of human failing heart. J Clin Invest 83:298-305

7. Saito Y, Nakao K, Nishimura K et al (1987) Clinical application of atrial natriuretic polypeptide in patients with congestive heart failure: beneficial effects on left ventricular function. Circulation 76:115-124

8. Sudoh T, Minamino N, Kangawa K, Matsuo H (1990) C-type natriuretic peptide (CNP): a new member of natriuretic peptide family identified in porcine brain. Biochem Biophys Res Commun 168:863-870

9. Suga S, Nakao K, Itoh H et al (1992) Endothelial production of C-type natriuretic peptide and its marked augmentation by transforming growth factor-beta. Possible existence of "vascular natriuretic peptide system". J Clin Invest 90:1145-1149

10. Komatsu Y, Nakao K, Itoh H, Suga S, Ogawa Y, Imura H (1992) Vascular natriuretic peptide. Lancet 340:622

11. Suga S, Itoh H, Komatsu Y et al (1993) Cytokine-induced C-type natriuretic peptide (CNP) secretion from vascular endothelial cells-evidence for CNP as a novel autocrine/paracrine regulator from endothelial cells. Endocrinology 133:3038-3041

12. Komatsu Y, Itoh H, Suga S et al (1996) Regulation of endothelial production of C-type natriuretic peptide in coculture with vascular smooth muscle cells. Role of the vascular natriuretic peptide system in vascular growth inhibition. Circ Res 78:606-614

13. Kawai M, Naruse M, Yoshimoto T et al (1996) C-type natriuretic peptide as a possible local modulator of aldosterone secretion in bovine adrenal zona glomerulosa. Endocrinology 137:42-46

14. Zhang M, Su YQ, Sugiura K, Xia G, Eppig JJ (2010) Granulosa cell ligand NPPC and its receptor NPR2 maintain meiotic arrest in mouse oocytes. Science 330:366-369

15. Kiyosu C, Tsuji T, Yamada K, Kajita S, Kunieda T (2012) NPPC/NPR2 signaling is essential for oocyte meiotic arrest and cumulus oophorus formation during follicular development in the mouse ovary. Reproduction 144:187-193

16. Chusho H, Tamura N, Ogawa Y et al (2001) Dwarfism and early death in mice lacking C-type natriuretic peptide. Proc Natl Acad Sci U S A 98:4016-4021

17. Kronenberg HM (2003) Developmental regulation of the growth plate. Nature 423:332-336

18. Tamura N, Doolittle LK, Hammer RE, Shelton JM, Richardson JA, Garbers DL (2004) Critical roles of the guanylyl cyclase B receptor in endochondral ossification and development of female reproductive organs. Proc Natl Acad Sci U S A 101:17300-17305

19. Yasoda A, Komatsu Y, Chusho $\mathrm{H}$ et al (2004) Overexpression of CNP in chondrocytes rescues achondroplasia through a MAPK-dependent pathway. Nat Med 10:80-86

20. Bartels CF, Bukulmez H, Padayatti P et al (2004) Mutations in the transmembrane natriuretic peptide receptor NPR-B impair skeletal growth and cause acromesomelic dysplasia, type Maroteaux. Am J Hum Genet 75:27-34

21. Miura K, Namba N, Fujiwara M et al (2012) An overgrowth disorder associated with excessive production of cGMP due to a gain-of-function mutation of the natriuretic peptide receptor 2 gene. PLoS One 7:e42180

22. Miura K, Kim OH, Lee HR et al (2014) Overgrowth syndrome associated with a gain-offunction mutation of the natriuretic peptide receptor 2 (NPR2) gene. Am J Med Genet A 164A:156-163

23. Shiang R, Thompson LM, Zhu YZ et al (1994) Mutations in the transmembrane domain of FGFR 3 cause the most common genetic form of dwarfism, achondroplasia. Cell 78:335-342 
24. Rousseau F, Bonaventure J, Legeai-Mallet L et al (1994) Mutations in the gene encoding fibroblast growth factor receptor-3 in achondroplasia. Nature 371:252-254

25. Cattaneo R, Villa A, Catagni M, Tentori L (1988) Limb lengthening in achondroplasia by Ilizarov's method. Int Orthop 12:173-179

26. Naski MC, Colvin JS, Coffin JD, Ornitz DM (1998) Repression of hedgehog signaling and BMP4 expression in growth plate cartilage by fibroblast growth factor receptor 3. Development 125:4977-4988

27. Kake T, Kitamura H, Adachi Y et al (2009) Chronically elevated plasma C-type natriuretic peptide level stimulates skeletal growth in transgenic mice. Am J Physiol Endocrinol Metab 297:E1339-E1348

28. Yasoda A, Kitamura H, Fujii T et al (2009) Systemic administration of C-type natriuretic peptide as a novel therapeutic strategy for skeletal dysplasias. Endocrinology 150:3138-3144

29. Matsukawa N, Grzesik WJ, Takahashi N et al (1999) The natriuretic peptide clearance receptor locally modulates the physiological effects of the natriuretic peptide system. Proc Natl Acad Sci U S A 96:7403-7408 


\title{
Clarity and Challenges in Tissue Fibrosis
}

\author{
Scott L. Friedman
}

\begin{abstract}
The tremendous progress in understanding the mechanisms of tissue fibrosis has led to realistic hopes for effective antifibrotic therapies in a range of diseases, including hepatic fibrosis, idiopathic pulmonary fibrosis, and renal fibrosis, as well as fibrotic disorders of muscle, heart, skin and bone marrow. Common mechanisms across these different tissues have unearthed targets that may be relevant to many organs. Best understood are pathways leading to hepatic fibrosis, which also predispose to hepatocellular carcinoma. Hepatic stellate cells are the principal fibrogenic cells in the liver following their activation into myofibroblasts, and their detailed characterization has unearthed many targets for therapy. Increasingly, investigators now rely on genetic mouse models to define contributions of specific molecules, in hopes of antagonizing these molecules as therapeutic targets. Both genomic and molecular approaches are unveiling new patterns of gene expression and molecules. A robust framework for antifibrotic drug discovery has been developed, and many agents are in clinical trials. With iterative evaluation of drug candidates in both animal models and humans, accelerated progress in bringing these drugs to patients is anticipated.
\end{abstract}

Keywords Hepatic fibrosis • Stellate cells • Antifibrotic • Cirrhosis • Pulmonary fibrosis $\bullet$ Autophagy $\bullet$ Fibrogenesis $\bullet$ Macrophages

\section{Introduction}

The topic of tissue fibrosis has become increasingly important in clinical medicine with the realization that up to $45 \%$ of all deaths in the industrialized world are due to fibrotic diseases of various organs [1]. Great strides have been made in understanding the cellular basis, molecular mechanisms, and both tissue specific and generalized features of fibrosis across organs.

Generally, fibrosis mechanisms are similar across different tissues, including the heart, lung, liver, pancreas, kidney, bone marrow, CNS, and skin, among others [2].

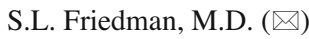

Division of Liver Diseases, Icahn School of Medicine at Mount Sinai,

1425 Madison Ave., Room 11-70C, New York, NY, Box 1123 10029-6574, USA

e-mail: Scott.Friedman@mssm.edu 
In each case, common events include injury to epithelial tissues that leads to activation of resident mesenchymal cells, or myofibroblasts. While the mechanisms of fibrogenesis are similar, the likelihood of regeneration and regression varies widely across organs. Also highly variable is the underlying regenerative capacity of each organ, with the liver being most regenerative and the lung showing the least capacity for tissue regeneration. Ongoing epithelial injury typically tips the balance toward progressive fibrosis rather than regression, yet the factors underlying this critical balance are not well understood.

\section{Liver Fibrosis}

\section{Mechanisms}

In the liver, fibrosis is a common pathway among many different etiologies, including viral hepatitis B and C, nonalcoholic steatohepatitis, and alcoholic liver disease, as well as inherited metabolic disorders, drug-induced liver injury, immune disorders, and neonatal cholestatic syndromes [3]. As noted above, the liver is relatively unique in the long duration of fibrosis, such that, typically, chronic injury must persist for decades before advanced fibrosis, also known as cirrhosis, develops. Once cirrhosis is present, however, the liver harbors a dramatically increased risk of hepatocellular carcinoma (HCC), which currently has the fastest rising tumor incidence in the world [4]. Moreover, HCC is the second leading cause of cancer mortality worldwide, yet curative therapies are rarely available unless aggressive screening methods are used to detect small tumors that are resectable or amenable to local tissue ablation.

The mechanisms of fibrosis in the liver have been greatly clarified thanks to the development decades ago of methods to isolate and grow the primary fibrogenic cells in the liver, the hepatic stellate cells. The cell type is a resident pericyte, which has the unusual feature of storing large amounts of vitamin A, or retinoids, in perinuclear cytoplasmic droplets [5]. Thus, this cell type is the primary storage depot for retinoids in the body. Following injury, hepatic stellate cells undergo a very characteristic activation, or trans-differentiation, yielding a highly fibrogenic and contractile cell. During this process, they release retinoids and acquire more prominent contractile filaments, as well as a whole range of new features that collectively can be considered a "conspiracy to make a scar". This activation includes upregulation of energetic receptors, increased pro-inflammatory and profibrogenic cytokines, and increased signals that promote cell survival.

More recently, additional subtle features of stellate cell activation and liver fibrosis have been uncovered. Prominent among these is an increasingly nuanced appreciation for inflammatory cell subset composition that changes from normal to injured liver, including different types of NK cells, NKT cells, and other lymphocyte subsets, as well as alterations in macrophage composition [6, 7]. Additionally, there has been increasing appreciation for the contribution of adipokines to fibrosis 
progression and regression [8, 9]. In general, adiponectin is considered antiinflammatory and antifibrotic, whereas leptin is pro-inflammatory and fibrogenic. Additional adipokines are under study, and their relevance to human disease has been heightened by the growing epidemic of nonalcoholic fatty liver disease, [10] which promises to dwarf the public health impact of viral hepatitis in the USA and Europe, and possibly Asia [11].

Another important development has been the discovery that activated hepatic stellate cells can revert to an inactivated phenotype when liver injury resolves [1214]. Previously, apoptosis had been the major mechanism invoked to account for loss of activated stellate cells when fibrosis regresses [15]. However, now that reversion to an inactivated state has been validated in animal models, this indicates that there is a reservoir of cells that can both activate and then de-activate. Moreover, the activated cells are distinct from truly quiescent stellate cells in their capacity to re-activate more briskly.

\section{Role of Autophagy}

Our studies have recently focused on a vital intracellular pathway known as autophagy [16] and its contribution to hepatic stellate cell activation. The work was spurred by a seminal observation reported by Czaja and colleagues in 2009 [17], demonstrating that hepatocytes utilize autophagy to provide intracellular energy when stressed, through hydrolysis of intracellular lipids. This raised the interesting prospect that stellate cells, which are "professional" lipid-storing cells, might also exploit autophagy to metabolize intracellular lipids. We addressed this prospect by inhibiting autophagy either chemically or genetically in hepatic stellate cells to assess the impact on stellate cell activation [18]. Through a variety of methods, our data clearly indicated that autophagy of stellate cells is required to provide shortterm but vital intracellular energy as the cell undergoes the highly energy intensive process of activation. Moreover, the block in stellate cell activation conferred by autophagy inhibition could be overcome if the cells were provided exogenous energy in the form of oleic acid, a monounsaturated omega- 9 fatty acid.

Of broader significance, our work demonstrated that autophagy is equally vital to fibrogenesis in mesenchymal cells from other tissues as well. Specifically, embryonic fibroblasts from mice genetically lacking Atg5, a key autophagy regulatory protein, have reduced expression of collagen I, beta-PDGF receptor, alpha smooth muscle actin, and matrix metalloproteinase-2 [18]. Similarly, blocking autophagy in mouse mesangial cells and human pulmonary fibroblasts yielded similar antagonism of fibrogenic properties.

Taken together, these findings implicate autophagy in a new context in which the pathway is essential for energy production in cellular fibrosis. While a simplistic interpretation would be that autophagy blockade could lead to decreased fibrosis, in reality autophagy is an essential pathway for the homeostasis of epithelial cells, and many animal models demonstrate that blocking autophagy in these cells is 
deleterious to organ function. Therefore, any therapeutic exploitation of autophagy inhibition would need to be highly targeted only to fibrogenic cells and not affect surrounding epithelia or other cell types.

\section{Genetic Models of Liver Fibrosis}

In more recent studies, we have begun using genetic models to explore other features of stellate cell activation and biology. We recently developed a mouse model in which stellate cells can be selectively ablated [19]. The model utilizes transgenic expression of the herpes simplex thymidylate kinase gene, which, when expressed in proliferating cells, renders them susceptible to killing by the antiviral drug ganciclovir. To perform these studies, induction of liver injury was required in order to provoke stellate cell proliferation so they would be susceptible to killing. As expected, depletion of stellate cells led to reduced fibrosis and diminished expression of stellate cell activation markers, both following carbon tetrachloride or duct ligation models of injury and fibrosis. We also analyzed inflammatory gene expression and specifically detected increased hepatic expression of interleukin-10 and interferon-gamma following depletion. These results reinforce the central role of stellate cells in liver fibrosis, but also highlight its contribution to modifying the inflammatory milieu in liver injury.

\section{Reversibility of Hepatic Fibrosis}

Among the most exciting discoveries has been the recognition that hepatic fibrosis and even cirrhosis are reversible. While animal studies supported this conclusion for many years, there is now strong human evidence for fibrosis regression. The evidence is clearest in patients who have either complete suppression of hepatitis $\mathrm{B}$ or cured hepatitis $C[20,21]$. In each circumstance, removing active viral infection leads to substantial fibrosis regression and restoration of normal architecture, even in a majority of cirrhotic patients.

The mechanisms underlying fibrosis reversibility represent a fertile avenue for uncovering targets whose manipulation could accelerate fibrosis regression in human disease, even when the primary etiology is not controlled-for example, nonalcoholic steatohepatitis. In the liver, increasing evidence implicates subsets of hepatic 'LY6C-lo' macrophages as harboring a fibrolytic profile that contributes to matrix degradation when liver injury subsides [22]. Therapeutic efforts to exploit this finding include cytokine therapies that could amplify subpopulations of fibrolytic macrophages, or even ex vivo cell differentiation using genetic techniques, followed by re-infusion of fibrolytic cells. There are, however, other pathways of 
matrix degradation, whose quantitative contribution to fibrosis regression in vivo are not completely clarified. For example, dendritic cells secrete MMP-9 [23], whereas neutrophils secrete MMP-8. Further clarification of the mechanisms underlying fibrosis regression are likely to emerge with continued use of more refined genetic mouse models, combined with the ability to analyze in detail the features of fibrosis regression in human liver disease [24]. The latter prospect is especially attractive with the availability of direct-acting antivirals for hepatitis $\mathrm{C}$, which promise to cure disease in greater than $90 \%$ of patients with fewer side effects then previous interferon-based therapies [25, 26]. Thus, with more patients who have advanced fibrosis achieving an HCV cure, it will be highly informative to analyze liver tissues in order to characterize those features that contribute to fibrosis regression in human disease.

\section{Genomics of Fibrosis}

With the era of "big data" upon us, we are now using genomic methods to evaluate large data sets in hopes of uncovering unique and important antifibrotic drug targets. Several databases exist in which gene expression patterns in response to known drugs can be interrogated for different cell types, including mesenchymal cells [27]. Using this approach, we seek to identify stellate cell-specific genes whose antagonism could yield an antifibrotic effect. Antagonism could be achieved using either neutralizing antibodies, siRNAs, or small molecules. The regulatory paths for approval for such agents are yet to be clarified, but there is intense activity in the drug development world, in partnership with the FDA, to accelerate the development of clinical trial designs that can shorten drug testing and bring effective therapies to patients with fibrotic diseases more quickly.

From a clinical perspective, there are important lessons about development and testing of antifibrotic drugs acquired from years of testing agents for idiopathic pulmonary fibrosis $[2,28,29]$. The contrast to hepatic fibrosis is interesting, in that pulmonary fibrosis is a catastrophic illness with a median survival of 3 years, in contrast to the slow progress of hepatic fibrosis. In part, this poor prognosis has driven more aggressive efforts to test and approve antifibrotic drugs faster. Partly as a result of this more extensive trial experience, pulmonary fibrosis trials are now underpinned by well-validated functional tests, whose improvement is acceptable to regulatory agencies as an indication of drug efficacy. Moreover, methods have been developed to sample bronchoalveolar lavage macrophages as biosensors of drug efficacy and some specific trials have sought to antagonize TGF-beta activation. Studies of liver fibrosis will benefit from lessons learned in the pulmonary field, and drugs proven effective in the latter group may be equally valuable to patients with chronic liver disease who are at risk for cirrhosis. 


\section{Framework for Antifibrotic Therapies}

A framework for organizing our understanding of potential antifibrotic therapy delivery is well established. The first principle is to determine if a particular pathway or target is a "core" or "regulatory" one [30]. Core pathways are typically common to two or more tissues and species, are essential for fibrosis, and are presumed to have an earlier evolutionary role. In contrast, a regulatory pathway might be one that is more tissue specific and therefore less likely to elicit off-target effects following its antagonism in a clinical setting. A recent example of an ideal core pathway is alphaV integrin, based on studies by Henderson et al. [31], which demonstrated that this pathway is critical to driving fibrosis in several tissues. Moreover, development of small-molecule antagonists to this integrin are well underway and represent a drug development path that has already been well established for other disease indications [32].

The point of attack for fibrosis therapies and liver include: (1) reducing the primary disease - for example, antivirals or abstinence from alcohol, among others; (2) reducing tissue injury and fostering epithelial repair through the use of epithelial protectants, anti-inflammatory agents, or modulation of inflammatory cell subsets, as described above; (3) blocking myofibroblast proliferation, angiogenesis, or contractility, using specific cytokine receptor antagonists; (4) promoting apoptosis of activated myofibroblasts; (5) stimulating metalloproteinase activity, either through induction of these enzymes or through antagonism of their natural inhibitors specifically, tissue inhibitors of metalloproteinases. A detailed description of the agents in these different categories is beyond the scope of this review, but the reader is referred to several articles [33-35].

\section{Summary}

There are four major conclusions of this work:

1. Tissue fibrosis mechanisms are sufficiently clarified to expect progress in developing and testing antifibrotic drugs.

2. While most mechanisms of fibrosis are shared across organs, each site or tissue presents unique challenges to drug development and clinical trial design.

3. Clinical trial development will require robust, validated endpoints that correlate with clinical outcomes.

4. We are reaching a "tipping point" of interest and emerging clinical trials that will establish proof of principal for antifibrotic drugs.

Open Access This chapter is distributed under the terms of the Creative Commons Attribution Noncommercial License, which permits any noncommercial use, distribution, and reproduction in any medium, provided the original author(s) and source are credited. 


\section{References}

1. Wynn TA (2008) Cellular and molecular mechanisms of fibrosis. J Pathol 214(2):199-210

2. Friedman SL, Sheppard D, Duffield JS, Violette S (2013) Therapy for fibrotic diseases: nearing the starting line. Sci Transl Med 5(167):167sr161. doi:10.1126/scitranslmed.3004700

3. Kocabayoglu P, Friedman SL (2013) Cellular basis of hepatic fibrosis and its role in inflammation and cancer. Front Biosci (Sch Edit) 5:217-230. doi:S368 [pii]

4. Villanueva A, Hernandez-Gea V, Llovet JM (2012) Medical therapies for hepatocellular carcinoma: a critical view of the evidence. Nat Rev Gastroenterol Hepatol 10(1):34-42. doi:nrgastro.2012.199 [pii] 10.1038/nrgastro.2012.199

5. Friedman SL (2008) Hepatic stellate cells - protean, multifunctional, and enigmatic cells of the liver. Physiol Rev 88(1):125-172

6. Gao B, Radaeva S (2013) Natural killer and natural killer T cells in liver fibrosis. Biochim Biophys Acta 1832(7):1061-1069. doi:10.1016/j.bbadis.2012.09.008

7. Iredale JP, Thompson A, Henderson NC (2013) Extracellular matrix degradation in liver fibrosis: biochemistry and regulation. Biochim Biophys Acta 1832(7):876-883. doi:10.1016/j. bbadis.2012.11.002

8. Handy JA, Fu PP, Kumar P, Mells JE, Sharma S, Saxena NK, Anania FA (2011) Adiponectin inhibits leptin signalling via multiple mechanisms to exert protective effects against hepatic fibrosis. Biochem J 440(3):385-395. doi:10.1042/BJ20102148

9. Marra F, Bertolani C (2009) Adipokines in liver diseases. Hepatology 50(3):957-969. doi:10.1002/hep. 23046

10. Wree A, Broderick L, Canbay A, Hoffman HM, Feldstein AE (2013) From NAFLD to NASH to cirrhosis-new insights into disease mechanisms. Nat Rev Gastroenterol Hepatol 10:627636. doi:nrgastro.2013.149 [pii] 10.1038/nrgastro.2013.149

11. Loomba R, Sanyal AJ (2013) The global NAFLD epidemic. Nat Rev Gastroenterol Hepatol 10(11):686-690. doi:10.1038/nrgastro.2013.171

12. Kisseleva T, Cong M, Paik Y, Scholten D, Jiang C, Benner C, Iwaisako K, Moore-Morris T, Scott B, Tsukamoto H, Evans SM, Dillmann W, Glass CK, Brenner DA (2012) Myofibroblasts revert to an inactive phenotype during regression of liver fibrosis. Proc Natl Acad Sci U S A 109:9448-9453. doi:1201840109 [pii] 10.1073/pnas.1201840109

13. Troeger JS, Mederacke I, Gwak GY, Dapito DH, Mu X, Hsu CC, Pradere JP, Friedman RA, Schwabe RF (2012) Deactivation of hepatic stellate cells during liver fibrosis resolution in mice. Gastroenterology 143(4):1073-1083 e1022. doi:S0016-5085(12)00925-0 [pii] 10.1053/j.gastro.2012.06.036

14. Friedman SL (2012) Fibrogenic cell reversion underlies fibrosis regression in liver. Proc Natl Acad Sci U S A 109(24):9230-9231. doi:10.1073/pnas.1206645109

15. Iredale JP, Benyon RC, Pickering J, McCullen M, Northrop M, Pawley S, Hovell C, Arthur MJ (1998) Mechanisms of spontaneous resolution of rat liver fibrosis. Hepatic stellate cell apoptosis and reduced hepatic expression of metalloproteinase inhibitors. J Clin Invest 102(3):538-549

16. Choi AM, Ryter SW, Levine B (2013) Autophagy in human health and disease. N Engl J Med 368(7):651-662. doi:10.1056/NEJMra1205406

17. Singh R, Kaushik S, Wang Y, Xiang Y, Novak I, Komatsu M, Tanaka K, Cuervo AM, Czaja MJ (2009) Autophagy regulates lipid metabolism. Nature 458(7242):1131-1135. doi:nature07976 [pii] 10.1038/nature07976

18. Hernandez-Gea V, Ghiassi-Nejad Z, Rozenfeld R, Gordon R, Fiel MI, Yue Z, Czaja MJ, Friedman SL (2012) Autophagy releases lipid that promotes fibrogenesis by activated hepatic stellate cells in mice and in human tissues. Gastroenterology 142(4):938-946. doi:S00165085(12)00012-1 [pii] 10.1053/j.gastro.2011.12.044

19. Puche JE, Lee YA, Jiao J, Aloman C, Fiel MI, Munoz U, Kraus T, Lee T, Yee HF Jr, Friedman SL (2013) A novel murine model to deplete hepatic stellate cells uncovers their role in amplifying liver damage in mice. Hepatology 57(1):339-350. doi:10.1002/hep.26053 
20. Marcellin P, Gane E, Buti M, Afdhal N, Sievert W, Jacobson IM, Washington MK, Germanidis G, Flaherty JF, Schall RA, Bornstein JD, Kitrinos KM, Subramanian GM, McHutchison JG, Heathcote EJ (2013) Regression of cirrhosis during treatment with tenofovir disoproxil fumarate for chronic hepatitis B: a 5-year open-label follow-up study. Lancet 381(9865):468_ 475. doi:10.1016/S0140-6736(12)61425-1

21. D’Ambrosio R, Aghemo A, Rumi MG, Ronchi G, Donato MF, Paradis V, Colombo M, Bedossa P (2012) A morphometric and immunohistochemical study to assess the benefit of a sustained virological response in hepatitis $\mathrm{C}$ virus patients with cirrhosis. Hepatology 56(2):532-543. doi:10.1002/hep.25606

22. Ramachandran P, Pellicoro A, Vernon MA, Boulter L, Aucott RL, Ali A, Hartland SN, Snowdon VK, Cappon A, Gordon-Walker TT, Williams MJ, Dunbar DR, Manning JR, van Rooijen N, Fallowfield JA, Forbes SJ, Iredale JP (2012) Differential Ly-6C expression identifies the recruited macrophage phenotype, which orchestrates the regression of murine liver fibrosis. Proc Natl Acad Sci U S A 109(46):E3186-E3195. doi:1119964109 [pii] 10.1073/ pnas.1119964109

23. Jiao J, Sastre D, Fiel MI, Lee UE, Ghiassi-Nejad Z, Ginhoux F, Vivier E, Friedman SL, Merad M, Aloman C (2012) Dendritic cell regulation of carbon tetrachloride-induced murine liver fibrosis regression. Hepatology 55(1):244-255. doi:10.1002/hep.24621

24. Snowdon VK, Fallowfield JA (2011) Models and mechanisms of fibrosis resolution. Alcohol Clin Exp Res 35(5):794-799. doi:10.1111/j.1530-0277.2010.01400.x

25. Liang TJ, Ghany MG (2014) Therapy of hepatitis C-back to the future. N Engl J Med 370(21):2043-2047. doi:10.1056/NEJMe1403619

26. Pawlotsky JM (2014) New hepatitis C virus (HCV) drugs and the hope for a cure: concepts in anti-HCV drug development. Semin Liver Dis 34(1):22-29. doi:10.1055/s-0034-1371007

27. Dudley JT, Sirota M, Shenoy M, Pai RK, Roedder S, Chiang AP, Morgan AA, Sarwal MM, Pasricha PJ, Butte AJ (2011) Computational repositioning of the anticonvulsant topiramate for inflammatory bowel disease. Sci Transl Med 3(96):96ra76. doi:10.1126/scitranslmed.3002648

28. O'Connell OJ, Kennedy MP, Henry MT (2011) Idiopathic pulmonary fibrosis: treatment update. Adv Ther 28(11):986-999. doi:10.1007/s12325-011-0066-5

29. Wynn TA (2011) Integrating mechanisms of pulmonary fibrosis. J Exp Med 208(7):1339_ 1350. doi:jem.20110551 [pii] 10.1084/jem.20110551

30. Mehal WZ, Iredale J, Friedman SL (2011) Scraping fibrosis: expressway to the core of fibrosis. Nat Med 17(5):552-553. doi:nm0511-552 [pii] 10.1038/nm0511-552

31. Henderson NC, Arnold TD, Katamura Y, Giacomini MM, Rodriguez JD, McCarty JH, Pellicoro A, Raschperger E, Betsholtz C, Ruminski PG, Griggs DW, Prinsen MJ, Maher JJ, Iredale JP, Lacy-Hulbert A, Adams RH, Sheppard D (2013) Targeting of alphav integrin identifies a core molecular pathway that regulates fibrosis in several organs. Nat Med 19(12):1617-1624. doi:10.1038/nm.3282

32. Zhu J, Choi WS, McCoy JG, Negri A, Zhu J, Naini S, Li J, Shen M, Huang W, Bougie D, Rasmussen M, Aster R, Thomas CJ, Filizola M, Springer TA, Coller BS (2012) Structureguided design of a high-affinity platelet integrin alphaIIbbeta3 receptor antagonist that disrupts $\operatorname{Mg}(2)(+)$ binding to the MIDAS. Sci Transl Med 4(125):125ra132. doi:10.1126/ scitranslmed.3003576

33. Hellerbrand C (2014) Molecular targets for antifibrotic therapy in liver disease: using magic bullets for crossfire rather than a one-sided shotgun attack. Gut 63(7):1039-1041. doi:10.1136/ gutjnl-2013-305908

34. Schuppan D, Kim YO (2013) Evolving therapies for liver fibrosis. J Clin Invest 123(5):18871901. doi:10.1172/JCI66028

35. Cohen-Naftaly M, Friedman SL (2011) Current status of novel antifibrotic therapies in patients with chronic liver disease. Ther Adv Gastroenterol 4(6):391-417. doi:10.1177/17562 83X11413002 10.1177_1756283X11413002 [pii] 


\title{
TRP Channels: Their Function and Potentiality as Drug Targets
}

\author{
Motohiro Nishida, Koichiro Kuwahara, Daisuke Kozai, \\ Reiko Sakaguchi, and Yasuo Mori
}

\begin{abstract}
The transient receptor potential (TRP) proteins are a family of ion channels that act as cellular sensors as well as signal integrators. Several members of the TRP family are sensitive to changes in cellular redox status. Among them, TRPA1 is remarkably susceptible to various oxidants and is known to mediate neuropathic pain and respiratory, vascular, and gastrointestinal functions, making TRPA1 an attractive therapeutic target. However, a method to achieve selective modulation of TRPA1 by small molecules has not yet been established. Most recently, we found that a novel $N$-nitrosamine compound activates TRPA1 by $S$-nitrosylation (the addition of a nitric oxide (NO) group to cysteine thiol) and does
\end{abstract}

\footnotetext{
M. Nishida

Division of Cardiocirculatory Signaling, Okazaki Institute for Integrative Bioscience, National Institute for Physiological Sciences, National Institutes of Natural Sciences, Myodaiji-cho, Okazaki, Aichi 444-8787, Japan

K. Kuwahara

Department of Cardiovascular Medicine, Graduate School of Medicine, Kyoto University, Sakyo-ku, Kyoto 606-8507, Japan

D. Kozai

Department of Synthetic Chemistry and Biological Chemistry, Graduate School of Engineering, Kyoto University, Katsura Campus, Nishikyo-ku, Kyoto 615-8510, Japan

R. Sakaguchi

Department of Synthetic Chemistry and Biological Chemistry, Graduate School of Engineering, Kyoto University, Katsura Campus, Nishikyo-ku, Kyoto 615-8510, Japan

World Premier International Research Initiative-Institute for Integrated Cell-Material Sciences, Kyoto University, Katsura Campus, Nishikyo-ku, Kyoto 615-8510, Japan

Y. Mori, Ph.D. ( $\square)$

Department of Technology and Ecology, Hall of Global Environmental Studies, Kyoto University, Katsura Campus, Nishikyo-ku, Kyoto 615-8510, Japan

World Premier International Research Initiative-Institute for Integrated Cell-Material Sciences, Kyoto University, Katsura Campus, Nishikyo-ku, Kyoto 615-8510, Japan e-mail: mori@sbchem.kyoto-u.ac.jp
} 
so with significant selectivity over other NO-sensitive TRP channels. It is proposed that this subtype selectivity is conferred through synergistic effects of electrophilic cysteine transnitrosylation and molecular recognition of the non-electrophilic moiety on the $N$-nitrosamine. On the other hand, TRPCs are typical receptor-activated $\mathrm{Ca}^{2+}$-permeable cation channels, which sense messenger molecules generated downstream of phospholipase activation. Previously, activation of TRPC3 and TRPC6 by diacylglycerol has been reported to play important roles in the pathogenesis of cardiac hypertrophy. Also, a pyrazole compound, Pyr3, which selectively inhibits TRPC3, suppresses cardiac hypertrophy in animal models in vitro and in vivo. We have most recently found that Pyr3 and related compounds are effective in suppressing cardiac fibrosis and ischemia responsible for cardiac remodeling as well. Thus, in this chapter, we describe the molecular pharmacology of TRP modulators and discuss their modulatory mechanisms and pharmacological actions.

Keywords Electrophile $\cdot$ Nitric oxide $\cdot$ Non-electrophilic compound $\bullet$ Oxidative stress $\bullet$ Transnitrosylation $\bullet$ TRP channel $\bullet$ TRPA1

\section{Introduction}

In 1989, the transient receptor potential (TRP) protein was first identified as being encoded by the trp gene of Drosophila [1]. The TRP protein superfamily consists of a diverse group of calcium ion $\left(\mathrm{Ca}^{2+}\right)$-permeable non-selective cation channels, and is found in most living organisms [2-4]. Mammalian TRP channels are currently divided into TRPC (canonical), TRPV (vanilloid), TRPM (melastatin), TRPP (polycystic kidney disease), TRPML (mucolipin) and TRPA (ankyrin) subfamilies. TRP channels have a tetrameric subunit stoichiometry, and each subunit contains cytoplasmic N- and C-terminal regions, six transmembrane (TM) domains and a pore-forming region between TM5 and TM6. TRP channels are sensitive to a variety of stimuli, including receptor stimulation, temperature, plant-derived compounds, environmental irritants, osmotic pressure, mechanical stress, $\mathrm{pH}$, and voltage from the extracellular and intracellular milieu, and are involved in diverse physiological and pathological processes [2, 5-16].

Certain TRP channels respond well to mediators of oxidative stress, such as reactive oxygen species (ROS), reactive nitrogen species (RNS), and other electrophiles [17-20]. Canonically known as damaging molecules causing cellular dysfunction, ROS and RNS are increasingly recognized as cell-signaling molecules [21, 22]. The first identified ROS-sensitive TRP channel, TRPM2, is activated by hydrogen peroxide $\left(\mathrm{H}_{2} \mathrm{O}_{2}\right)$ and mediates several cellular responses, including cell death and chemokine production [23-26]. TRPM7, which can be modulated by both ROS and RNS, is an essential mediator of anoxic cell death [27, 28]. Some members of the TRPC and TRPV subfamily, including TRPC5 and TRPV1, are activated by $\mathrm{H}_{2} \mathrm{O}_{2}$, nitric oxide (NO), and reactive disulfides [29]. In addition, TRPA1 is remarkably 
activated by various oxidants, including ROS, RNS, reactive disulfides, and other electrophiles [30-33].

Among TRP subfamilies, TRPCs are typical receptor-activated $\mathrm{Ca}^{2+}$-permeable cation channels, which sense messenger molecules generated downstream of phospholipase activation. Previously, Kuwahara [34] and Nishida [35] separately showed that TRPC3 and TRPC6 activated by DAG play important roles in the pathogenesis of cardiac hypertrophy. Also, Mori developed a pyrazole compound, Pyr3, which selectively inhibits TRPC3 and suppresses cardiac hypertrophy in animal models in vitro and in vivo [36]. In our recent progress in studying the pharmacological action of Pyr3 and related compounds, their modulators have turned out to be highly effective in suppressing cardiac fibrosis and ischemia responsible for cardiac remodeling. Thus, TRPC3 and TRPC6 are emerging as critical targets in development of drugs relevant to therapies for heart failure.

\section{Modulation of Trpa1 Channel Activity}

\section{Oxidation Sensitivity of the Trpal Channel}

TRPA1 responds to various oxidative stress mediators and environmental electrophiles (Table 1). Cysteine residues within a protein are direct targets for the oxidant signal reaction $[74,75]$. TRPA1 is not an exception in this characteristics. Its activation by oxidants is proposed to be mediated via oxidative modification of the free sulfhydryl group of cysteine residues, as described for the activation of TRPC5 and TRPV1 [29, 76].

For TRPA1, the oxidation sites have been identified (Fig. 1). Simultaneous mutation of three cysteine residues within the cytoplasmic N-terminus of human TRPA1 (Cys621, Cys641, and Cys665) decreases TRPA1 channel activation by several exogenous cysteine-modifying electrophiles, such as isothiocyanates (e.g. AITC), $\alpha, \beta$-unsaturated aldehyde compounds (e.g. acrolein, $N$-methylmaleimide, and cinnamaldehyde), allicin from garlic, and diallyl disulfide [30, 37, 38]. Lys710 is also suggested to be involved in the activation of TRPA1 by AITC. Three cysteine residues in mouse TRPA1 (Cys415, Cys422, and Cys622, conserved in the human homolog as Cys414, Cys421, and Cys621) were independently identified as the target sites for AITC and cinnamaldehyde [31]. Intracellular $\mathrm{Zn}^{2+}$ also activates human TRPA1 by interacting with Cys641 and C-terminal Cys1021/His983 [78]. Systematic evaluation of TRP channels was performed using a series of reactive disulfides, such as bis(5-nitro-2-pyridyl) disulfide and diallyl disulfide [33]. These compounds possess a different electron acceptor (oxidation) capacity (manifested as redox potential), and these studies revealed that only TRPA1 responds to the inert electrophile diallyl disulfide among TRP channels. Thus, TRPA1 can sense inert oxidant $\mathrm{O}_{2}$, and $\mathrm{O}_{2}$ activation of TRPA1 is by oxidation of Cys633 and/or Cys856, located intracellularly within, respectively, the $\mathrm{N}$-terminal region and the putative linker region between TM4 and TM5 [33]. In addition, TRPA1 cysteine residues 
Table 1 TRPA1 modulators

\begin{tabular}{|c|c|c|}
\hline Class of compound & Compound & Reference \\
\hline \multirow[t]{23}{*}{ Activators (electrophilic) } & Allyl isothiocyanate (AITC) & {$[30]$} \\
\hline & Acrolein & {$[30]$} \\
\hline & $\mathrm{N}$-methylmaleimide & {$[30]$} \\
\hline & Cinnamaldehyde & {$[30]$} \\
\hline & Bis(5-nitro-2-pyridyl) disulfide & {$[33]$} \\
\hline & Allicin & {$[37,38]$} \\
\hline & Diallyl disulfide & {$[30]$} \\
\hline & 2-Chloroacetophenone & {$[17,39]$} \\
\hline & Methylvinylketone & {$[40]$} \\
\hline & Umbellulone & [41] \\
\hline & Ligustilide & {$[42]$} \\
\hline & Hydroxy- $\alpha$-sanshool $(\alpha-\mathrm{SOH})$ & [43] \\
\hline & 6-Shogaol & [43] \\
\hline & Etodolac & {$[44]$} \\
\hline & Glibenclamide & {$[45]$} \\
\hline & Auranofin & {$[46]$} \\
\hline & 4-Hydroxy-2-nonenal & {$[47]$} \\
\hline & 4-Hydroxyhexenal & {$[48]$} \\
\hline & 4-Oxo-2-nonenal & {$[48]$} \\
\hline & Nitrooleic acid & [49] \\
\hline & $15 \mathrm{~d}-\mathrm{PGJ} \mathrm{J}_{2}$ & [48] \\
\hline & Methylglyoxal & {$[50,51]$} \\
\hline & Oleocanthal & {$[52]$} \\
\hline \multirow[t]{15}{*}{ Activators (non-electrophilic) } & Icilin & {$[30]$} \\
\hline & 2-Aminoethyl diphenylborinate & {$[31]$} \\
\hline & Carvacrol & {$[53]$} \\
\hline & Flufenamic acid & {$[54]$} \\
\hline & Isoflurane & {$[55]$} \\
\hline & Farnesyl thiosalicylic acid & {$[56]$} \\
\hline & NPPB & {$[57]$} \\
\hline & Thymol & {$[53]$} \\
\hline & 2,6-Diisopropylphenol (propofol) & {$[53]$} \\
\hline & Docosahexaenoic acid (DHA) & {$[58]$} \\
\hline & Arachidonic acid & [59] \\
\hline & 6-Paradol & {$[43,60]$} \\
\hline & 6-Gingerol & {$[43,60]$} \\
\hline & Capsiate & {$[61]$} \\
\hline & 1,4-Cineol & {$[62]$} \\
\hline \multirow[t]{5}{*}{ Inhibitors } & Isovelleral & {$[40]$} \\
\hline & HC-030031 & {$[63]$} \\
\hline & Chembridge-5861528 & {$[64]$} \\
\hline & AP-18 & {$[49]$} \\
\hline & A-967079 & {$[65]$} \\
\hline
\end{tabular}


Table 1 (continued)

\begin{tabular}{|c|c|c|}
\hline Class of compound & Compound & Reference \\
\hline & AZ868 & {$[66]$} \\
\hline & ADM_09 & [67] \\
\hline & Camphor & [68] \\
\hline & 1,8-Cineol & {$[62]$} \\
\hline \multirow[t]{5}{*}{ Bimodal modulators } & Borneol & [69] \\
\hline & AMG5445 (inhibits human/activates rat) & [70] \\
\hline & $\begin{array}{l}\text { Menthol (activates human/concentration } \\
\text { dependently activates or inhibits } \\
\text { mouse/no effect on Drosophila) }\end{array}$ & [71] \\
\hline & Caffeine (inhibits human/activates mouse) & {$[72]$} \\
\hline & CMP1 (inhibits human/activates rat) & [73] \\
\hline
\end{tabular}

Fig. 1 Predicted structural features of TRPA1 with putative position of critical residues involved in human TRPA1 modulation by compounds. The TRPA1 subunit, which has six transmembrane domains (TM), a pore-forming region between TM5 and TM6, and many ankyrin repeats (indicated by ovals) in the cytoplasmic $\mathrm{N}$-terminal region [77], assembles into tetramers to form a cation channel. Collectively, indicated residues (indicated by filled circles) are reported to be important for TRPA1 activation or inhibition by several compounds [30-33, $69,71,73,78-80]$
TRPA1

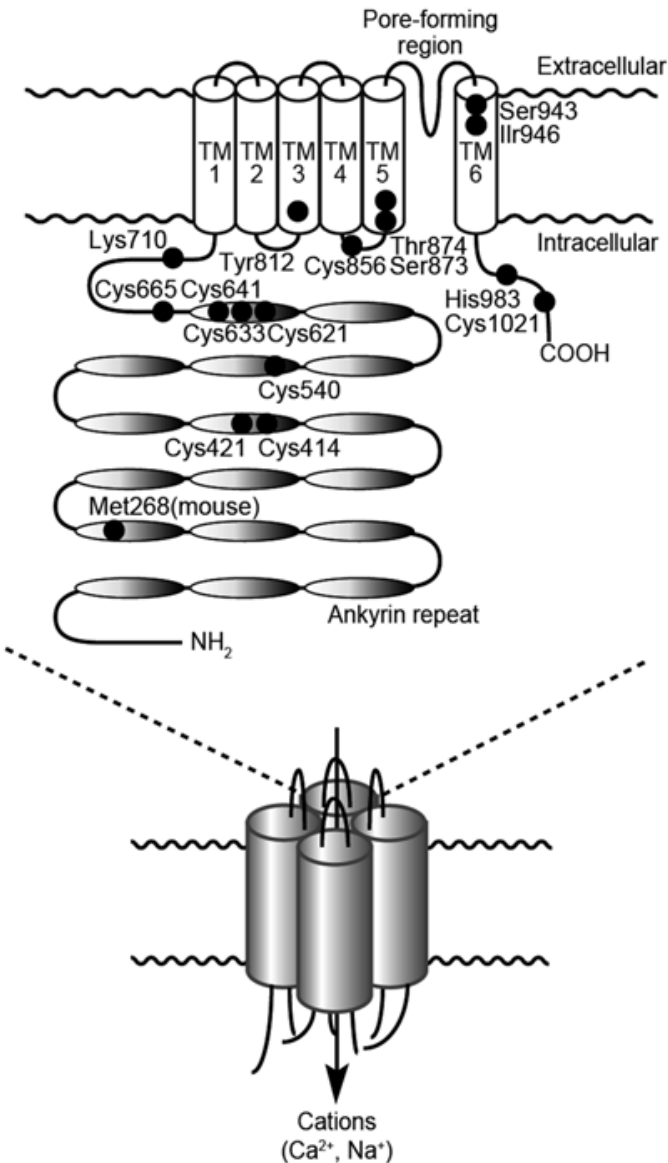


seem also to be critical for TRPA1 activation by other exogenous compounds, including irritants (tear gases, such as 2-chloroacetophenone [39, 81], and $\alpha, \beta$ unsaturated carbonyl-containing compounds, such as methylvinylketone [40, 82]), some plant constituents (umbellulone [41], ligustilide [42], hydroxy- $\alpha$-sanshool $(\alpha-\mathrm{SOH})$ and 6-shogaol [43]), and others (the cyclooxygenase-2 inhibitor etodolac [44], the anti-diabetic drug glibenclamide [45], the gold-containing disease-modifying anti-rheumatic drug auranofin [46], and CMP1 (4-methyl- $N$-[2,2,2-trichloro-1(4-nitro-phenylsulfanyl)-ethyl]-benzamide) [73]). Therefore, TRPA1 is unarguably a receptor for exogenous oxidative/electrophilic compounds.

TRPA1 is also modified via oxidative cysteine modification by endogenous oxidants and electrophiles. TRPA1 is activated by $\mathrm{H}_{2} \mathrm{O}_{2}$ [17, 32, 48, 83], hypochlorite [17], ozone [84], and the ROS generated by ultraviolet light [85]. In addition to ROS, TRPA1 is also activated by RNS such as NO [32, 83, 86] and peroxynitrite [83]. Functional characterization of site-directed mutants of TRPA1 collectively demonstrates that specific cytoplasmic N-terminal cysteine residues and a lysine residue (Cys421, Cys621, Cys641, Cys665, and Lys710 in human TRPA1) are the primary targets of ROS and RNS [17, 32, 86].

In addition to ROS and RNS, lipid peroxidation products such as 4-hydroxy-2nonenal, 4-hydroxyhexenal, 4-oxo-2-nonenal, nitrooleic acid, and 15-deoxy- $\Delta^{12,14}$ prostaglandin $\mathrm{J}_{2}\left(15 \mathrm{~d}-\mathrm{PGJ}_{2}\right)$ activate TRPA1 channels through oxidative modification of the cysteine residues [32, 47-49, 56, 63, 87]. Labeling experiments using biotinylated $15 \mathrm{~d}-\mathrm{PGJ}_{2}$ demonstrated that Cys621 mediates the binding of $15 \mathrm{~d}-\mathrm{PGJ}_{2}$ to human TRPA1 [32]. Another electrophilic dicarbonyl compound, methylglyoxal (MG), which is believed to be associated with the development of diabetic neuropathy, also activates TRPA1 by hemithioacetal formation [50, 51]. Taken together, we can surmise that endogenous electrophilic products activate TRPA 1 channels by cysteine oxidation.

\section{Modulation of Trpa1 by Other Activators and Inhibitors}

Various non-electrophilic activators and inhibitors have also been demonstrated to modulate TRPA1 (Table 1). For example, icilin, 2-aminoethyl diphenylborinate, and carvacrol are compounds with no obvious reactivity toward cysteine residues and activate TRPA1 in a way that is not disrupted by cysteine mutations [30, 31, 81]. TRPA1 is also activated by non-reactive compounds including non-steroidal antiinflammatory drugs, such as flufenamic acid [54]; general anesthetics, such as isoflurane [55]; and farnesyl thiosalicylic acid (FTS) [56]. The chloride channel blocker NPPB (5-nitro-2-(3-phenylpropylamino)benzoic acid) activates TRPA1, and a structure-activity relationship study using a group of NPPB analogs indicates that its phenylalkane, carboxylic, and nitro groups are critical for its activation of TRPA1 [57]. NPPB and FTS are suggested to have similar molecular mechanisms of action at TRPA1. Thymol, 2,6-diisopropylphenol (propofol), and related simple alkyl phenols also activate TRPA1 [53]. TRPA1 is also activated by polyunsaturated fatty acids, which should contain at least three double bonds and 18 carbon atoms, such 
as docosahexaenoic acid (DHA) [58], and by arachidonic acid and its derivatives [59]. 6-Paradol and 6-gingerol activate TRPA1, whereas the non-TRPA1 agonist capsaicin does not, suggesting that a phenol core of these compounds is not sufficient to confer TRPA1 activation [43, 60]. Moreover, capsiate, a non-pungent capsaicin analog, also activates TRPA1 through a mechanism distinct from cysteine and histidine modification [61]. Therefore, TRPA1 activation by non-reactive compounds is dependent on their chemical structures rather than cysteine oxidation.

Inhibitors such as the synthetic compounds HC-030031, Chembridge-5861528 (a derivative of HC-030031), AP-18, A-967079 (a derivative of AP-18), AMG5445, and AZ868 have been developed for TRPA1 [64, 66, 70, 88-93]. Another, ADM_09, is an antagonist of TRPA1 with a putative dual-binding mode of action, which involves the synergic combination of $\mathrm{Ca}^{2+}$-mediated binding of the carnosine group and disulfide formation by its lipoic acid group [67]. Camphor and 1,8-cineol are naturally occurring inhibitors of human TRPA1, but 1,4-cineol is an activator [62]. Borneol is a more effective natural inhibitor than camphor and 1,8-cineol, and the hydroxyl group of borneol is suggested to contribute to its inhibitory action [69].

Several compounds have species-specific modulatory effects on TRPA1. AMG5445 inhibits human TRPA1, but activates rat TRPA1 [70]. The pharmacological profile of the human and rhesus monkey TRPA1 is relatively distinct from mouse and rat TRPA1 [94]. Importantly, findings of species-specific effects have helped to identify the critical region that determines TRPA1 modulation (Fig. 1). Menthol is known to be a bimodal modulator of mouse TRPA1, whereas it does not inhibit human TRPA1, and Drosophila TRPA1 is insensitive to menthol [71]. Chimera and mutagenesis studies indicate that specific residues within TM5 (notably Ser876 and Thr877 of mouse TRPA1, corresponding to Ser873 and Thr874 of human TRPA1) are critical for menthol responsiveness. Furthermore, the region from TM5 to TM6 in mouse and human TRPA1 is the critical domain determining the inhibitory effects of menthol. The same two residues (Ser and Thr within TM5) are also critical for the sensitivity of TRPA1 to AMG5445, AP-18, and A-967079 [65, 71]. DHA sensitivity is limited to human and mouse TRPA1; Drosophila TRPA1 does not respond to DHA [58]. Neither the cytoplasmic N-terminal region nor TM5 of TRPA1 is directly involved in DHA sensing.

Caffeine, which is not a reactive chemical reagent, activates mouse TRPA1, but suppresses human TRPA1 [72]. A mutation of Met268 in the N-terminal cytoplasmic region of mouse TRPA1 to the human form (Pro) changes caffeine action from activation to suppression [79]. An electrophilic compound, CMP1, a structural ana$\log$ of AMG5445, inhibits human TRPA1 and activates rat TRPA1 via modification of human Cys621 and rat Cys622, respectively [73, 95]. The specific mutations Ala946Ser and Met949Ile in the upper portion of the TM6 region of rat TRPA1 change the effect of CMP1 from activatory to inhibitory. Therefore, these studies demonstrate that specific regions and residues within TRPA1 determine the TRPA1 modulatory activity of non-electrophilic compounds, and that the key domains/ residues vary between compounds. Furthermore, while direct physical interaction of non-electrophilic compounds with TRPA1 is likely to be critical for modulation, it is unclear whether or not these critical sites are involved in binding. 
There have been other studies regarding chemical structures important in molecular recognition of activators by TRPA1. Isovelleral, a fungal natural product, which contains an $\alpha, \beta$-unsaturated aldehyde moiety, activates TRPA1 independently of cysteine oxidation [40]. A major compound in extra-virgin olive oil, oleocanthal (OC), is an electrophile that does not require cysteine residues to activate TRPA1 [52]. A structure-activity relationship study using synthetic OC analogs indicated that OC requires both aldehyde groups to activate TRPA1. The mouse Cys622Ser TRPA1 mutant is still sensitive to umbellulone, albeit less so than wild-type TRPA1 [41]. Zhong et al. suggest that umbellulone is a mechanistically hybrid activator, apparently combining covalent interaction at a reactive cysteine with noncovalent interaction with a second site on TRPA1 [41]. Thus, chemical structure recognition by TRPA1, a clearly distinct mechanism from cysteine oxidation, is supposed to be important even for TRPA1 activation by some specific electrophiles.

\section{Subtype-Selective S-Nitrosylation by a Novel Nitrosamine}

Protein $S$-nitrosylation, the covalent attachment of an NO moiety to the sulfur atom of cysteine residues to form $S$-nitrosothiol, regulates various protein functions to mediate NO bioactivity [96]. Receptor-activated (TRPC5, TRPC1, and TRPC4) and thermosensor (TRPV1, TRPV3, TRPV4, and TRPA1) TRP channels are activated by exogenous NO-releasing donors through $S$-nitrosylation [29, 32], but with very limited TRP subtype selectivity. Recently, this problem was partly solved with our finding that the 7-azabenzobicyclo[2.2.1] heptane (ABBH) $N$-nitrosamine selectively activates TRPA1 through transnitrosylation [80].

Although protein $S$-nitrosylation is widely accepted, questions regarding target selectivity of $S$-nitrosylation signaling are incompletely understood [97]. NO is produced in vivo by only three NO synthase (NOS) isoforms [98], and NO is reactive and diffusible within cells. Binding of NOS to targets or their adaptors has been demonstrated at select sites of nitrosylation reactions, but there are many $S$-nitrosylated proteins $(>1,000)[96,99,100]$. Recent studies have identified that protein-protein transnitrosylation, the transfer of the NO group from one protein to another in the absence of apparent NO release, is a potentially important targeting pathway [99, 101, 102]. Transnitrosylation is exemplified by transnitrosylation of X-linked inhibitor of apoptosis by SNO-caspase-3 in apoptotic cell death [103-106]. Here, a binding interaction between the two proteins is also required for transnitrosylation, because a binding-deficient mutant of one protein abrogates this protein-protein transnitrosylation $[105,106]$.

In our effort to develop transnitrosylation-based subtype-selective activators of TRP channels, it was necessary that we first identify a synthetic NO donor that has only transnitrosylative reactivity. However, $\operatorname{SNAP}(S$-nitroso- $N$-acetyl-DL-penicillamine) and NOR3 $(( \pm)-(E)$-4-ethyl-2-[(E)-hydroxyimino]-5-nitro-3-hexenamide) are NO-releasing donors. $S$-Nitrosoglutathione is known to be a biological transnitrosylating agent, but also actively releases NO [107, 108]. In contrast, the ABBH 


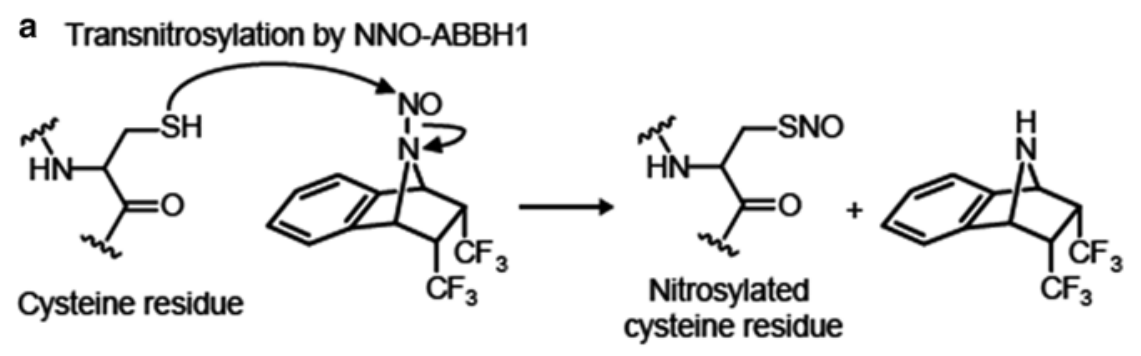

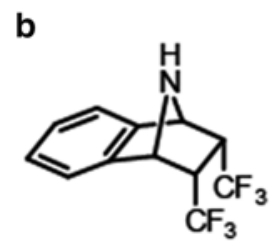

$\mathrm{NH}-\mathrm{ABBH}$

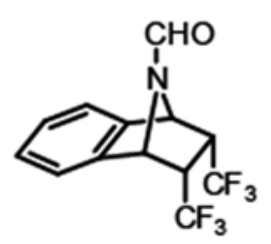

NCHO-ABBH

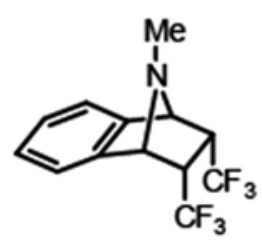

NMe-ABBH

Fig. 2 Selective S-nitrosylation of human TRPA1 by a novel N-nitrosamine. (a) Chemical mechanism underlying the transnitrosylating action of NNO-ABBH1 on protein thiol group. (b) Chemical structures of non-electrophilic analogs of NNO-ABBH1

$N$-nitrosamines constitute a new class of $\mathrm{NO}$ donors that, at physiological $\mathrm{pH}$ and temperature, transnitrosylate thiols to generate $S$-nitrosothiols without releasing NO [109-111]. Surprisingly, our intracellular $\mathrm{Ca}^{2+}$-imaging measurements have demonstrated that $N$-nitroso-2-exo,3-exo-ditrifluoromethyl-7-azabenzobicyclo[2.2.1] heptane (NNO-ABBH1) induces robust $\mathrm{Ca}^{2+}$ influx via recombinant human TRPA1 channels, but not via other SNAP-activated TRP channels, suggesting that NNOABBH1 selectively $S$-nitrosylates TRPA1 [80] (Fig. 2). SNAP $S$-nitrosylates both TRPA1 and TRPV1, but NNO-ABBH1 $S$-nitrosylates only TRPA1. Importantly, TRPA1 activation by NNO-ABBH1 is suppressed by specific cysteine mutations but not by NO scavenging, indicating that transnitrosylation underlies the activation of TRPA1 by NNO-ABBH1. This is supported by a positive correlation of N-NO bond reactivity and TRPA1-activating potency in a congeneric series of ABBH $N$-nitrosamines. Cys540, Cys641, and Cys665 of human TRPA1 are involved in its modification by NNO-ABBH1. Because Cys641 and Cys665 are also required for responsiveness to SNAP [32, 86], Cys540 may be a unique target for NNO-ABBH1.

Several non-electrophilic analogs of NNO-ABBH1 $-\mathrm{N}-\mathrm{H}$ (NH-ABBH), $N$-formyl (NCHO-ABBH), and $N$-methyl (NMe-ABBH) (Fig. 2)—also activated TRPA1 but less potently than NNO-ABBH1. They also did not cause $S$-nitrosylation of TRPA1, and their activity was not affected by cysteine mutation of TRPA1. Interestingly, the NMe-ABBH sensitivity of TRPA1 was significantly enhanced by SNAP at a subthreshold concentration $(10 \mu \mathrm{M})$, supporting the idea that TRPA1 activation by these non-electrophilic analogs may be subject to positive synergistic 
interactions between nitrosylation and molecular recognition. Thus, NNO-ABBH1 may be a hybrid activator. It is reported that a non-electrophilic TRPA1 activator flufenamic acid synergistically potentiates the activation of TRPA1 by AITC [54]. Also, umbellulone has been proposed to activate TRPA1 by combining covalent interaction at a reactive cysteine with noncovalent interaction with a second site on TRPA1 [41]. In contrast to TRPA1, TRPC5 and TRPV1 failed to respond to NMeABBH. Molecular recognition of chemical groups other than NO may explain the subtype-selective activation of TRPA1 by these compounds.

Despite evidence of synergistic effects between cysteine trans-nitrosylation and molecular recognition of the non-electrophilic moiety, it remains unclear how the transnitrosylation site and the non-electrophilic molecular recognition site converge in TRPA1. Also, it is unknown whether NNO-ABBH1 and other non-electrophilic analogs have bimodal and/or species-specific effects on TRPA1. Further detailed studies into TRPA1 modulation by ABBH $N$-nitrosamines will provide a basis for developing new drugs selectively targeting $S$-nitrosylation of TRPA1. In addition, these studies will be expanded toward the development of selective transnitrosylating modulators of other proteins.

\section{TRPC Channels as Therapeutic Targets for Heart Failure}

\section{Structure and Function of TRPC Channels}

Seven mammalian homologs (TRPC1-C7) have so far been identified and expressed in the heart. While TRPC4 and TRPC5 share about $65 \%$ amino acid homology in their group, TRPC3, TRPC6, and TRPC7 display the greatest homology covering $\sim 75 \%$ of the amino acid sequence [112]. TRPC1, TRPC3, and TRPC6 have been identified to play a role in cardiovascular diseases, especially pathological cardiac hypertrophy and heart failure.

The mammalian TRPC proteins include three to four ankyrin repeats and coiledcoil domain in the cytoplasmic $\mathrm{N}$-terminal sequence that are essential for tetrameric channel assembly, and six putative transmembrane domains, and amino acid sequence identity ( $\geq 30 \%$ ) over the N-terminal $\sim 750-900$ amino acids in the internal C-terminus, which includes the TRP box motif with the conserved EWKFAR residues and another coiled-coil motif. The higher-order structure of TRPC3 channels was recently solved using single particle cryo-electron microscopy [113]. The ice structure is lace-like and very open, with a very large overall volume. The TRPC channels appear to form assemblies of homotetramers or heterotetramers at least within given structural subfamilies; i.e., TRPC1/4/5 or TRPC3/6/7 [114]. TRPC1, TRPC4, and TRPC5 channels are activated by inositol-1,4,5-trisphosphate $\left(\mathrm{IP}_{3}\right)$-dependent mechanisms, while TRPC3, TRPC6, and TRPC7 are directly activated by diacylglycerol (DAG) independently of the store depletion-induced mechanism [115]. Meanwhile, it is reported that the direct interaction of TRPC3 
with $\mathrm{IP}_{3} \mathrm{R}$ or ryanodine receptor $(\mathrm{RyR})$ is required for TRPC3 activation $[116,117]$ and the DAG-induced activation of native TRPC7 in DT40 B lymphocytes [118]. The N-termini and C-termini serve as the sites for protein scaffolding, including $\mathrm{IP}_{3} \mathrm{R}$, RyR, caveolin [119], phospholipase C (PLC) $\gamma[120,121]$, protein kinase C $\beta$ [122], and $\mathrm{Na}^{+} / \mathrm{H}^{+}$exchanger regulatory factor (NHERF) 1 [123]. These interactions are often found to regulate subcellular localization of the respective TRPC proteins. For example, interaction of the ankyrin domain in TRPC6 with the ring finger protein (RNF24) is essential for retention of TRPC6 in the Golgi apparatus [124], and association of TRPC3 at the N-terminus with vesicle-associated membrane protein (VAMP) 2 is required for vesicular trafficking of TRPC3 [125].

TRPC1 is considered unique because no other family member shares highsequence homology. TRPC1 first emerged as a candidate subunit of SOCs [126-129]. Recently, our study has implicated the critical involvement of TRPC1 in coordination with elementary $\mathrm{Ca}^{2+}$ signaling events that promote functional coupling between the ER and plasma membrane in receptor-induced $\mathrm{Ca}^{2+}$ signaling [130]. Thus, TRPC1 may not only function as a $\mathrm{Ca}^{2+}$-permeable channel-forming subunit, but also as an accessory protein to form the $\mathrm{Ca}^{2+}$ signaling complex.

The activation mechanism of TRPC channels is not only linked to PLC activation by receptor stimulation, but also linked to physical stimulations such as mechanical stretch, hypoxia, and oxidative stress [131]. TRPC1 and TRPC6 have been suggested to be a component of the tarantula toxin-sensitive mechanosensitive cation channels $[132,133]$. For example, the excessive mechanical stress-induced muscle contractility in myocytes with Duchenne muscular dystrophy was blunted by inhibition or deletion of TRPC6 [134]. On the other hand, intracellular lipid mediators, such as DAG and 12-hydroxy-eicosatetraenoic acid (12-HETE), reportedly mediate TRPC6 channel activation induced by oxidative stress [135] and mechanical stretch [136]. Thus, TRPC6 protein signaling complex, including TRPC1 and TRPC3, may function as both mechanosensitive and mechanoactivated cation channels in the cardiovascular system.

\section{Role of TRPC Channels in Pathological Cardiac Remodeling}

Cardiovascular disease is a leading cause of morbidity and mortality, accounting for more than a quarter of all deaths worldwide. Especially, heart failure is a final stage of all cardiovascular diseases, and the 5-year survival rate after diagnosis is less than $50 \%$ [137]. Several drugs that modulate neurohumoral activation, such as $\beta$-adrenergic receptor antagonists, angiotensin-converting enzyme (ACE) inhibitors, angiotensin (Ang) type 1 receptor (AT1R) antagonists, and mineral corticoid receptor antagonists, have been introduced for the treatment of heart failure [138]. Treatment with these drugs has been shown to improve the prognosis in patients with heart failure with reduced systolic function, but the mortality for heart failure still remains unacceptably high. Thus, additional approaches are greatly required as the prevalence of cardiovascular diseases continues to rise and exact a huge societal cost. 
Induction of pathologic remodeling (i.e., structural and morphological changes of organs) and organ dysfunction is a common prominent feature of these disorders that is mediated by excessive and sustained neurohumoral and mechanical stimulation. In the cardiovascular system, the TRPC family has been particularly found to play a role in cardiovascular diseases. As heart failure is developed through hypertrophy [139], many studies have investigated the mechanisms of hypertrophy [140]. TRPC1, TRPC3, and TRPC6 are often upregulated in several rodent models of cardiac hypertrophy, and their inhibition ameliorates the associated cardiovascular dysfunction [141]. In human heart failure, upregulation of TRPC5 and TRPC6 was observed [34, 142]. Although the subtype of upregulated TRPC channels at hypertrophy may differ between mice and humans, these findings suggest that the expression of TRPC channels is increased on hypertrophy, and upregulated TRPC may activate local $\mathrm{Ca}^{2+}$ signaling essential for the progression of pathological cardiac remodeling and failure.

TRPC expression is regulated by the $\mathrm{Ca}^{2+}$-dependent protein phosphatase, calcineurin, and its downstream target, nuclear factor of activated T cells (NFAT) $[143,144]$. Increases in the frequency or amplitude of $\mathrm{Ca}^{2+}$ transients evoked by $\mathrm{Ca}^{2+}$ influx-induced $\mathrm{Ca}^{2+}$ release in excitable cardiomyocytes is thought to encode signals for induction of hypertrophic gene expression [145, 146]. Activation of TRPC channels induces local $\mathrm{Ca}^{2+}$ signaling through an increase in the frequency of $\mathrm{Ca}^{2+}$ transient via $\mathrm{Na}^{+}$influx-dependent membrane depolarization and/or direct $\mathrm{Ca}^{2+}$ influx, which leads to NFAT transcriptional activation through calcineurindependent dephosphorylation and nuclear translocation of NFAT. TRPC3-mediated $\mathrm{Ca}^{2+}$ influx has been shown to regulate hypertrophic gene expression without affecting cardiac beating and cell size [147]. As the promoter region of the TRPC6 gene contains NFAT binding sites, activation of plasma membrane TRPC channels may serve as a positive-feedback mechanism to amplify TRPC-mediated $\mathrm{Ca}^{2+}$ signaling in the heart [34]. In addition, NFAT transcriptional activation requires association of co-factor(s) with NFAT, and TRPC6 upregulation is also mediated by stressactivated protein kinases (c-Jun $\mathrm{NH}_{2}$-terminal kinase and p38 mitogen-activated protein kinase) upon receptor stimulation in cardiac fibroblasts [148, 149]. Thus, multiple transcriptional pathways including the calcineurin-NFAT pathway have been linked to maladaptive cardiovascular remodeling via TRPC upregulation.

Mice with cardiomyocyte-specific overexpression of TRPC 3 and TRPC6 show heightened sensitivity to mechanical stress and increased expression of a sensitive marker for pathological hypertrophy $[34,150]$. In contrast, pressure overloadinduced cardiac hypertrophy is suppressed by double deletions of TRPC $3 / 6$ genes in C57BL6/J background mice, although single deletion of TRPC3 and TRPC6 genes never suppresses cardiac hypertrophy [151]. As TRPC3 and TRPC6 form heteromultimer channels and regulate agonist- and mechanical stretch-induced hypertrophic growth of rat neonatal cardiomyocytes [35] and mice lacking TRPC6 were reported to have mRNA upregulation for TRPC3 [152], TRPC3 and TRPC6 proteins may compensatively work with each other. TRPC6 is abundantly expressed 
in cardiac fibroblasts, and fibroblasts lacking the TRPC6 gene were refractory to transdifferentiation [149]. TRPC6 gene-deleted mice show impaired dermal and cardiac wound healing after injury, suggesting an obligate function for TRPC6 and calcineurin in promoting myofibroblast differentiation.

TRPC1 is also thought to play a pathologic role in the heart, with increased expression observed in rodent hearts with cardiomyocyte hypertrophy. Ohba et al. first reported the potential involvement of TRPC1 channels in pressure overloadinduced hypertrophy [153]. Among TRPC1, TRPC3, TRPC5, and TRPC6, TRPC1 expression was increased in abdominal aortic-banded rats. Endothelin-1 (ET-1) stimulation resulted in the increased expression of brain natriuretic protein (BNP), atrial natriuretic factor (ANF), and TRPC1 as well as an increased cell surface area in neonatal myocytes. ET-1 stimulation also increased $\mathrm{Ca}^{2+}$ entry possibly through TRPC channels. Knockdown of TRPC1 with siRNA prevented Gq-coupled receptor-stimulated hypertrophic responses. Mice with global TRPC1 gene deletion show less cardiac hypertrophy and left ventricular dysfunction in response to pressure overload or neurohormonal stimulation in comparison with wild-type 129Sv background mice [154]. Although it is still unclear whether TRPC1 gene deletion never suppresses physiological (adaptive) hypertrophy in vivo, TRPC1 might be also a therapeutic target for heart failure.

\section{Negative Feedback Mechanism in TRPC Channels}

Phosphorylation of TRPC $3 / 6$ proteins by protein kinase $\mathrm{C}(\mathrm{PKC})$, protein kinase A (PKA), and protein kinase $\mathrm{G}$ (PKG) has been widely accepted as a major posttranslational modification that negatively regulates TRPC channel activity. PKG can directly phosphorylate human TRPC3 at Thr-11 and Ser-263, and human TRPC6 at Thr-70 and Ser-322. PKG is activated by NO, atrial natriuretic peptide (ANP), or inhibition of phosphodiesterase (PDE)-5, each of which negatively regulates pathological cardiac hypertrophy. The physiological importance of PKG-dependent negative regulation of TRPC6 channel activity by NO was originally identified as a mechanism of endothelium-dependent vasodilation [155]. As PKA and PKG recognize a similar substrate sequence, PKA-dependent phosphorylation of rodent TRPC6 at Thr-69 was also revealed to serve as an endothelium-independent vasodilation [156]. Increased PKG activity attenuates $\mathrm{Ca}^{2+} /$ calcineurin-dependent cardiomyocyte hypertrophy induced by receptor stimulation and mechanical stretch, and mutation of the PKG phosphorylation site on TRPC6 canceled this inhibitory effect [157]. In contrast, decreased cGMP/PKG signaling by deletion of the guanylate cyclase (GC)-A gene was associated with development of spontaneous cardiac hypertrophy through TRPC $3 / 6$ channel activation [158]. Actually, this hypertrophy was attenuated by treatment with Pyr2, an inhibitor of all TRPC channels. 


\section{Suppression of Pathological Cardiac Hypertrophy by TRPC3/6 Inhibition}

Several reagents that inhibit TRPC3/6 channel activity have been shown to suppress cardiac hypertrophy in vivo and in vitro. For example, $\alpha 1$ adrenergic receptorstimulated hypertrophic responses were blocked by 2-aminoethoxydiphenylborane (2-APB) and N-\{4-[3,5-bis(trifluoromethyl)-1H-pyrazol- 1yl]phenyl $\}$-4-methyl1,2,3-thiadiazole-5-carboxamide (BTP2; also called Pyr2), but not by verapamil, a voltage-dependent L-type $\mathrm{Ca}^{2+}$ channel blocker [142]. Indirect inhibition of TRPC3/6 channel activities by PDE-5 inhibitors [159] and ANP [158] can also suppresses pathological hypertrophy through phosphorylation of TRPC6 at Thr69. Mori developed a pyrazole compound, Pyr3, which selectively inhibits TRPC3 channel activity with an $\mathrm{IC}_{50}$ value of $0.7 \mu \mathrm{M}$ [36]. Interestingly, Pyr3 showed more potent inhibitory effects on mechanical stretch-induced NFAT activation and hypertrophic growth of rat neonatal cardiomyocytes, suggesting that Pyr3 is more selective for native TRPC 3/6 heteromultimer channels in the heart. Indeed, left ventricular dilation and dysfunction induced by pressure overload [36] or genetic deletion of muscle LIM protein [160] were actually reduced by a low concentration $(0.1 \mathrm{mg} / \mathrm{kg} /$ day) of Pyr3 treatment. Moreover, Pyr3 treatment also suppressed oxidative stress and cardiac fibrosis in mouse hearts with dilated cardiomyopathy, and mechanical stretch-induced production of ROS in rat cardiomyocytes. Two recently identified selective TRPC3/6 inhibitors, GSK2332255B and GSK2833503A (IC (I0 $_{3}, 3-21 \mathrm{nM}$ against TRPC3 and TRPC6), also inhibited ET-1-induced hypertrophic responses in adult cardiac myocytes [151]. These findings strongly suggest that TRPC3 and TRPC6 are emerging as critical targets in the development of drugs relevant to therapies for pathological cardiac remodeling and chronic heart failure.

\section{Conclusion}

Because TRPA1 mediates neuropathic pain, vascular dilation, and other functions, it has the potential to be an excellent drug target. Therefore, it is important to understand the mechanisms of both activation and inhibition of TRPA1 by small molecules. Recent studies have revealed that TRPA1 modulation by electrophiles is through cysteine oxidation, and that molecular recognition of chemical structures is a key determinant of TRPA1 modulation not only by non-electrophilic compounds, but also by some specific electrophiles. A novel ABBH $N$-nitrosamine induces selective $S$-nitrosylation of TRPA1 probably through synergistic processes of cysteine oxidation and molecular recognition of the non-electrophilic moiety. However, molecular bases of TRPA1 modulation by non-electrophilic compounds are very poorly understood. Further studies are required to delineate the entire mechanism. Similarly, further research is needed to define in detail the molecular mechanisms by which chemical ligands induce the activation of other TRP channels, such as 
TRPV1 and TRPM8 [10, 161]. This might support our understanding of TRPA1 mechanisms. TRPA 1 channel activity is also modulated by $\mathrm{Ca}^{2+}$, receptor stimulation, $\mathrm{pH}$, osmotic pressure, and temperature [60, 162-168], so a better understanding of the complexities of its modulation is critical to the development of novel TRPA1-specific drugs. It will also improve our appreciation of the physiological and pathological functions of TRPA1.

In terms of TRPCs, a growing body of evidence has suggested that direct or indirect inhibition of TRPC $3 / 6$ channel activity improves pathological cardiac remodeling and heart failure in mice, although the molecular mechanisms underlying regulation of transition of the heart from adaptation to maladaptation by TRPC $3 / 6$ channels are still uncovered. A pyrazole-derivative compound is also reported to inhibit SOCs as well as TRPC3 [169], but our findings strongly suggest that a pyrazole-derivative compound (especially Pyr3) will become a promising seed for the treatment of chronic heart failure.

Open Access This chapter is distributed under the terms of the Creative Commons Attribution Noncommercial License, which permits any noncommercial use, distribution, and reproduction in any medium, provided the original author(s) and source are credited.

\section{References}

1. Montell C, Rubin GM (1989) Molecular characterization of the Drosophila trp locus: a putative integral membrane protein required for phototransduction. Neuron 2(4):1313-1323

2. Clapham DE (2003) TRP channels as cellular sensors. Nature 426(6966):517-524. doi:10.1038/nature02196

3. Montell C (2005) The TRP superfamily of cation channels. Sci STKE 2005(272):re3. doi:10.1126/stke.2722005re3

4. Venkatachalam K, Montell C (2007) TRP channels. Annu Rev Biochem 76:387-417. doi:10.1146/annurev.biochem.75.103004.142819

5. Patapoutian A, Peier AM, Story GM, Viswanath V (2003) ThermoTRP channels and beyond: mechanisms of temperature sensation. Nat Rev Neurosci 4(7):529-539. doi:10.1038/nrn1141

6. Clapham DE, Julius D, Montell C, Schultz G (2005) International Union of Pharmacology. XLIX Nomenclature and structure-function relationships of transient receptor potential channels. Pharmacol Rev 57(4):427-450. doi:10.1124/pr.57.4.6

7. Voets T, Talavera K, Owsianik G, Nilius B (2005) Sensing with TRP channels. Nat Chem Biol 1(2):85-92. doi:10.1038/nchembio0705-85

8. Christensen AP, Corey DP (2007) TRP channels in mechanosensation: direct or indirect activation? Nat Rev Neurosci 8(7):510-521. doi:10.1038/nrn2149

9. Nilius B, Owsianik G, Voets T, Peters JA (2007) Transient receptor potential cation channels in disease. Physiol Rev 87(1):165-217. doi:10.1152/physrev.00021.2006

10. Vriens J, Nilius B, Vennekens R (2008) Herbal compounds and toxins modulating TRP channels. Curr Neuropharmacol 6(1):79-96. doi:10.2174/157015908783769644

11. Wu LJ, Sweet TB, Clapham DE (2010) International Union of Basic and Clinical Pharmacology. LXXVI Current progress in the mammalian TRP ion channel family. Pharmacol Rev 62(3):381-404. doi:10.1124/pr.110.002725

12. Numata T, Kiyonaka S, Kato K, Takahashi N, Mori Y (2011) Activation of TRP channels in mammalian systems. In: Zhu MX (ed) TRP channels. CRC Press, Boca Raton 
13. Santoni G, Farfariello V (2011) TRP channels and cancer: new targets for diagnosis and chemotherapy. Endocr Metab Immune Disord Drug Targets 11(1):54-67

14. Gees M, Owsianik G, Nilius B, Voets T (2012) TRP channels. Compr Physiol 2(1):563-608. doi:10.1002/cphy.c110026

15. Julius D (2013) TRP channels and pain. Annu Rev Cell Dev Biol 29:355-384. doi:10.1146/ annurev-cellbio-101011-155833

16. Sousa-Valente J, Andreou AP, Urban L, Nagy I (2014) Transient receptor potential ion channels in primary sensory neurons as targets for novel analgesics. $\mathrm{Br} \mathrm{J}$ Pharmacol 171(10):2508-2527. doi:10.1111/bph.12532

17. Bessac BF, Jordt SE (2008) Breathtaking TRP channels: TRPA1 and TRPV1 in airway chemosensation and reflex control. Physiology 23:360-370. doi:10.1152/physiol.00026.2008

18. Miller BA, Zhang W (2011) TRP channels as mediators of oxidative stress. Adv Exp Med Biol 704:531-544. doi:10.1007/978-94-007-0265-3_29

19. Kozai D, Ogawa N, Mori Y (2014) Redox regulation of transient receptor potential channels. Antioxid Redox Signal 21(6):971-986. doi:10.1089/ars.2013.5616

20. Simon F, Varela D, Cabello-Verrugio C (2013) Oxidative stress-modulated TRPM ion channels in cell dysfunction and pathological conditions in humans. Cell Signal 25(7):1614-1624. doi:10.1016/j.cellsig.2013.03.023

21. Droge W (2002) Free radicals in the physiological control of cell function. Physiol Rev 82(1):47-95. doi:10.1152/physrev.00018.2001

22. Brieger K, Schiavone S, Miller FJ Jr, Krause KH (2012) Reactive oxygen species: from health to disease. Swiss Med Wkly 142:w13659. doi:10.4414/smw.2012.13659

23. Hara Y, Wakamori M, Ishii M, Maeno E, Nishida M, Yoshida T, Yamada H, Shimizu S, Mori E, Kudoh J, Shimizu N, Kurose H, Okada Y, Imoto K, Mori Y (2002) LTRPC2 Ca2 +permeable channel activated by changes in redox status confers susceptibility to cell death. Mol Cell 9(1):163-173

24. Yamamoto S, Shimizu S, Kiyonaka S, Takahashi N, Wajima T, Hara Y, Negoro T, Hiroi T, Kiuchi Y, Okada T, Kaneko S, Lange I, Fleig A, Penner R, Nishi M, Takeshima H, Mori Y (2008) TRPM2-mediated Ca2 + influx induces chemokine production in monocytes that aggravates inflammatory neutrophil infiltration. Nat Med 14(7):738-747. doi:10.1038/ nm1758

25. Naziroglu M (2011) TRPM2 cation channels, oxidative stress and neurological diseases: where are we now? Neurochem Res 36(3):355-366. doi:10.1007/s11064-010-0347-4

26. Knowles H, Li Y, Perraud AL (2013) The TRPM2 ion channel, an oxidative stress and metabolic sensor regulating innate immunity and inflammation. Immunol Res 55(1-3):241-248. doi:10.1007/s12026-012-8373-8

27. Aarts M, Iihara K, Wei WL, Xiong ZG, Arundine M, Cerwinski W, MacDonald JF, Tymianski M (2003) A key role for TRPM7 channels in anoxic neuronal death. Cell 115(7):863-877

28. McNulty S, Fonfria E (2005) The role of TRPM channels in cell death. Pflugers Arch: Eur J Physiol 451(1):235-242. doi:10.1007/s00424-005-1440-4

29. Yoshida T, Inoue R, Morii T, Takahashi N, Yamamoto S, Hara Y, Tominaga M, Shimizu S, Sato Y, Mori Y (2006) Nitric oxide activates TRP channels by cysteine S-nitrosylation. Nat Chem Biol 2(11):596-607. doi:10.1038/nchembio821

30. Hinman A, Chuang HH, Bautista DM, Julius D (2006) TRP channel activation by reversible covalent modification. Proc Natl Acad Sci U S A 103(51):19564-19568. doi:10.1073/ pnas.0609598103

31. Macpherson LJ, Dubin AE, Evans MJ, Marr F, Schultz PG, Cravatt BF, Patapoutian A (2007) Noxious compounds activate TRPA1 ion channels through covalent modification of cysteines. Nature 445(7127):541-545. doi:10.1038/nature05544

32. Takahashi N, Mizuno Y, Kozai D, Yamamoto S, Kiyonaka S, Shibata T, Uchida K, Mori Y (2008) Molecular characterization of TRPA1 channel activation by cysteine-reactive inflammatory mediators. Channels 2(4):287-298 
33. Takahashi N, Kuwaki T, Kiyonaka S, Numata T, Kozai D, Mizuno Y, Yamamoto S, Naito S, Knevels E, Carmeliet P, Oga T, Kaneko S, Suga S, Nokami T, Yoshida J, Mori Y (2011) TRPA1 underlies a sensing mechanism for O2. Nat Chem Biol 7(10):701-711. doi:10.1038/ nchembio. 640

34. Kuwahara K, Wang Y, McAnally J, Richardson JA, Bassel-Duby R, Hill JA, Olson EN (2006) TRPC6 fulfills a calcineurin signaling circuit during pathologic cardiac remodeling. J Clin Invest 116(12):3114-3126. doi:10.1172/JCI27702

35. Onohara N, Nishida M, Inoue R, Kobayashi H, Sumimoto H, Sato Y, Mori Y, Nagao T, Kurose H (2006) TRPC3 and TRPC6 are essential for angiotensin II-induced cardiac hypertrophy. EMBO J 25(22):5305-5316. doi:10.1038/sj.emboj.7601417

36. Kiyonaka S, Kato K, Nishida M, Mio K, Numaga T, Sawaguchi Y, Yoshida T, Wakamori M, Mori E, Numata T, Ishii M, Takemoto H, Ojida A, Watanabe K, Uemura A, Kurose H, Morii T, Kobayashi T, Sato Y, Sato C, Hamachi I, Mori Y (2009) Selective and direct inhibition of TRPC3 channels underlies biological activities of a pyrazole compound. Proc Natl Acad Sci U S A 106(13):5400-5405. doi:10.1073/pnas.0808793106

37. Bautista DM, Movahed P, Hinman A, Axelsson HE, Sterner O, Hogestatt ED, Julius D, Jordt SE, Zygmunt PM (2005) Pungent products from garlic activate the sensory ion channel TRPA1. Proc Natl Acad Sci U S A 102(34):12248-12252. doi:10.1073/pnas.0505356102

38. Macpherson LJ, Geierstanger BH, Viswanath V, Bandell M, Eid SR, Hwang S, Patapoutian A (2005) The pungency of garlic: activation of TRPA1 and TRPV1 in response to allicin. Curr Biol: CB 15(10):929-934. doi:10.1016/j.cub.2005.04.018

39. Brone B, Peeters PJ, Marrannes R, Mercken M, Nuydens R, Meert T, Gijsen HJ (2008) Tear gasses CN, CR, and CS are potent activators of the human TRPA1 receptor. Toxicol Appl Pharmacol 231(2):150-156. doi:10.1016/j.taap.2008.04.005

40. Escalera J, von Hehn CA, Bessac BF, Sivula M, Jordt SE (2008) TRPA1 mediates the noxious effects of natural sesquiterpene deterrents. J Biol Chem 283(35):24136-24144. doi:10.1074/ jbc.M710280200

41. Zhong J, Minassi A, Prenen J, Taglialatela-Scafati O, Appendino G, Nilius B (2011) Umbellulone modulates TRP channels. Pflugers Arch: Eur J Physiol 462(6):861-870. doi:10.1007/s00424-011-1043-1

42. Zhong J, Pollastro F, Prenen J, Zhu Z, Appendino G, Nilius B (2011) Ligustilide: a novel TRPA1 modulator. Pflugers Arch: Eur J Physiol 462(6):841-849. doi:10.1007/s00424-011-1021-7

43. Riera CE, Menozzi-Smarrito C, Affolter M, Michlig S, Munari C, Robert F, Vogel H, Simon SA, le Coutre J (2009) Compounds from Sichuan and Melegueta peppers activate, covalently and non-covalently, TRPA1 and TRPV1 channels. Br J Pharmacol 157(8):1398-1409. doi:10.1111/j.1476-5381.2009.00307.x

44. Wang S, Dai Y, Kogure Y, Yamamoto S, Zhang W, Noguchi K (2013) Etodolac activates and desensitizes transient receptor potential ankyrin 1. J Neurosci Res 91(12):1591-1598. doi:10.1002/jnr.23274

45. Babes A, Fischer MJ, Filipovic M, Engel MA, Flonta ML, Reeh PW (2013) The anti-diabetic drug glibenclamide is an agonist of the transient receptor potential Ankyrin 1 (TRPA1) ion channel. Eur J Pharmacol 704(1-3):15-22. doi:10.1016/j.ejphar.2013.02.018

46. Hatano N, Suzuki H, Muraki Y, Muraki K (2013) Stimulation of human TRPA1 channels by clinical concentrations of the antirheumatic drug auranofin. Am J Physiol Cell Physiol 304(4):C354-C361. doi:10.1152/ajpcell.00096.2012

47. Trevisani M, Siemens J, Materazzi S, Bautista DM, Nassini R, Campi B, Imamachi N, Andre E, Patacchini R, Cottrell GS, Gatti R, Basbaum AI, Bunnett NW, Julius D, Geppetti P (2007) 4-Hydroxynonenal, an endogenous aldehyde, causes pain and neurogenic inflammation through activation of the irritant receptor TRPA1. Proc Natl Acad Sci U S A 104(33):13519-13524. doi:10.1073/pnas.0705923104

48. Andersson DA, Gentry C, Moss S, Bevan S (2008) Transient receptor potential A1 is a sensory receptor for multiple products of oxidative stress. J Neurosci: Off J Soc Neurosci 28(10):2485-2494. doi:10.1523/JNEUROSCI.5369-07.2008 
49. Taylor-Clark TE, Ghatta S, Bettner W, Undem BJ (2009) Nitrooleic acid, an endogenous product of nitrative stress, activates nociceptive sensory nerves via the direct activation of TRPA1. Mol Pharmacol 75(4):820-829. doi:10.1124/mol.108.054445

50. Eberhardt MJ, Filipovic MR, Leffler A, de la Roche J, Kistner K, Fischer MJ, Fleming T, Zimmermann K, Ivanovic-Burmazovic I, Nawroth PP, Bierhaus A, Reeh PW, Sauer SK (2012) Methylglyoxal activates nociceptors through transient receptor potential channel A1 (TRPA1): a possible mechanism of metabolic neuropathies. J Biol Chem 287(34):28291-28306. doi:10.1074/jbc.M111.328674

51. Andersson DA, Gentry C, Light E, Vastani N, Vallortigara J, Bierhaus A, Fleming T, Bevan S (2013) Methylglyoxal evokes pain by stimulating TRPA1. PLoS One 8(10):e77986. doi:10.1371/journal.pone.0077986

52. Peyrot des Gachons C, Uchida K, Bryant B, Shima A, Sperry JB, Dankulich-Nagrudny L, Tominaga M, Smith AB 3rd, Beauchamp GK, Breslin PA (2011) Unusual pungency from extra-virgin olive oil is attributable to restricted spatial expression of the receptor of oleocanthal. J Neurosci: Off J Soc Neurosci 31(3):999-1009. doi:10.1523/JNEUROSCI.1374-10.2011

53. Lee SP, Buber MT, Yang Q, Cerne R, Cortes RY, Sprous DG, Bryant RW (2008) Thymol and related alkyl phenols activate the hTRPA1 channel. Br J Pharmacol 153(8):1739-1749. doi:10.1038/bjp.2008.85

54. Hu H, Tian J, Zhu Y, Wang C, Xiao R, Herz JM, Wood JD, Zhu MX (2010) Activation of TRPA1 channels by fenamate nonsteroidal anti-inflammatory drugs. Pflugers Arch: Eur J Physiol 459(4):579-592. doi:10.1007/s00424-009-0749-9

55. Matta JA, Cornett PM, Miyares RL, Abe K, Sahibzada N, Ahern GP (2008) General anesthetics activate a nociceptive ion channel to enhance pain and inflammation. Proc Natl Acad Sci U S A 105(25):8784-8789. doi:10.1073/pnas.0711038105

56. Maher M, Ao H, Banke T, Nasser N, Wu NT, Breitenbucher JG, Chaplan SR, Wickenden AD (2008) Activation of TRPA1 by farnesyl thiosalicylic acid. Mol Pharmacol 73(4):1225-1234. doi: $10.1124 / \mathrm{mol} .107 .042663$

57. Liu K, Samuel M, Ho M, Harrison RK, Paslay JW (2010) NPPB structure-specifically activates TRPA1 channels. Biochem Pharmacol 80(1):113-121. doi:10.1016/j.bcp.2010.03.005

58. Motter AL, Ahern GP (2012) TRPA1 is a polyunsaturated fatty acid sensor in mammals. PLoS One 7(6):e38439. doi:10.1371/journal.pone.0038439

59. Redmond WJ, Gu L, Camo M, McIntyre P, Connor M (2014) Ligand determinants of fatty acid activation of the pronociceptive ion channel TRPA1. PeerJ 2:e248. doi:10.7717/peerj.248

60. Bandell M, Story GM, Hwang SW, Viswanath V, Eid SR, Petrus MJ, Earley TJ, Patapoutian A (2004) Noxious cold ion channel TRPA1 is activated by pungent compounds and bradykinin. Neuron 41(6):849-857

61. Shintaku K, Uchida K, Suzuki Y, Zhou Y, Fushiki T, Watanabe T, Yazawa S, Tominaga M (2012) Activation of transient receptor potential A1 by a non-pungent capsaicin-like compound, capsiate. Br J Pharmacol 165(5):1476-1486. doi:10.1111/j.1476-5381.2011.01634.x

62. Takaishi M, Fujita F, Uchida K, Yamamoto S, Sawada Shimizu M, Hatai Uotsu C, Shimizu M, Tominaga M (2012) 1,8-cineole, a TRPM8 agonist, is a novel natural antagonist of human TRPA1. Mol Pain 8:86. doi:10.1186/1744-8069-8-86

63. Taylor-Clark TE, Undem BJ, Macglashan DW Jr, Ghatta S, Carr MJ, McAlexander MA (2008) Prostaglandin-induced activation of nociceptive neurons via direct interaction with transient receptor potential A1 (TRPA1). Mol Pharmacol 73(2):274-281. doi:10.1124/ mol.107.040832

64. Wei H, Chapman H, Saarnilehto M, Kuokkanen K, Koivisto A, Pertovaara A (2010) Roles of cutaneous versus spinal TRPA1 channels in mechanical hypersensitivity in the diabetic or mustard oil-treated non-diabetic rat. Neuropharmacology 58(3):578-584. doi:10.1016/j. neuropharm.2009.12.001

65. Nakatsuka K, Gupta R, Saito S, Banzawa N, Takahashi K, Tominaga M, Ohta T (2013) Identification of molecular determinants for a potent mammalian TRPA1 antagonist by utilizing species differences. J Mol Neurosci: MN 51(3):754-762. doi:10.1007/s12031-013-0060-2 
66. Vallin KS, Sterky KJ, Nyman E, Bernstrom J, From R, Linde C, Minidis AB, Nolting A, Narhi K, Santangelo EM, Sehgelmeble FW, Sohn D, Strindlund J, Weigelt D (2012) N-1Alkyl-2-oxo-2-aryl amides as novel antagonists of the TRPA1 receptor. Bioorg Med Chem Lett 22(17):5485-5492. doi:10.1016/j.bmcl.2012.07.032

67. Nativi C, Gualdani R, Dragoni E, Di Cesare ML, Sostegni S, Norcini M, Gabrielli G, la Marca G, Richichi B, Francesconi O, Moncelli MR, Ghelardini C, Roelens S (2013) A TRPA1 antagonist reverts oxaliplatin-induced neuropathic pain. Sci Rep 3:2005. doi:10.1038/ srep02005

68. Xu H, Blair NT, Clapham DE (2005) Camphor activates and strongly desensitizes the transient receptor potential vanilloid subtype 1 channel in a vanilloid-independent mechanism. $\mathrm{J}$ Neurosci: Off J Soc Neurosci 25(39):8924-8937. doi:10.1523/JNEUROSCI.2574-05.2005

69. Takaishi M, Uchida K, Fujita F, Tominaga M (2014) Inhibitory effects of monoterpenes on human TRPA1 and the structural basis of their activity. J Physiol Sci: JPS 64(1):47-57. doi:10.1007/s12576-013-0289-0

70. Klionsky L, Tamir R, Gao B, Wang W, Immke DC, Nishimura N, Gavva NR (2007) Speciesspecific pharmacology of Trichloro(sulfanyl)ethyl benzamides as transient receptor potential ankyrin 1 (TRPA1) antagonists. Mol Pain 3:39. doi:10.1186/1744-8069-3-39

71. Xiao B, Dubin AE, Bursulaya B, Viswanath V, Jegla TJ, Patapoutian A (2008) Identification of transmembrane domain 5 as a critical molecular determinant of menthol sensitivity in mammalian TRPA1 channels. J Neurosci: Off J Soc Neurosci 28(39):9640-9651. doi:10.1523/ JNEUROSCI.2772-08.2008

72. Nagatomo K, Kubo Y (2008) Caffeine activates mouse TRPA1 channels but suppresses human TRPA1 channels. Proc Natl Acad Sci U S A 105(45):17373-17378. doi:10.1073/ pnas.0809769105

73. Chen J, Zhang XF, Kort ME, Huth JR, Sun C, Miesbauer LJ, Cassar SC, Neelands T, Scott VE, Moreland RB, Reilly RM, Hajduk PJ, Kym PR, Hutchins CW, Faltynek CR (2008) Molecular determinants of species-specific activation or blockade of TRPA1 channels. J Neurosci: Off J Soc Neurosci 28(19):5063-5071. doi:10.1523/JNEUROSCI.0047-08.2008

74. Satoh T, Lipton SA (2007) Redox regulation of neuronal survival mediated by electrophilic compounds. Trends Neurosci 30(1):37-45. doi:10.1016/j.tins.2006.11.004

75. Bindoli A, Rigobello MP (2013) Principles in redox signaling: from chemistry to functional significance. Antioxid Redox Signal 18(13):1557-1593. doi:10.1089/ars.2012.4655

76. Salazar H, Llorente I, Jara-Oseguera A, Garcia-Villegas R, Munari M, Gordon SE, Islas LD, Rosenbaum T (2008) A single N-terminal cysteine in TRPV1 determines activation by pungent compounds from onion and garlic. Nat Neurosci 11(3):255-261. doi:10.1038/nn2056

77. Gaudet R (2008) A primer on ankyrin repeat function in TRP channels and beyond. Mol Biosyst 4(5):372-379

78. Hu H, Bandell M, Petrus MJ, Zhu MX, Patapoutian A (2009) Zinc activates damage-sensing TRPA1 ion channels. Nat Chem Biol 5(3):183-190. doi:10.1038/nchembio.146

79. Nagatomo K, Ishii H, Yamamoto T, Nakajo K, Kubo Y (2010) The Met268Pro mutation of mouse TRPA1 changes the effect of caffeine from activation to suppression. Biophys $\mathrm{J}$ 99(11):3609-3618. doi:10.1016/j.bpj.2010.10.014

80. Kozai D, Kabasawa Y, Ebert M, Kiyonaka S, Firman OY, Numata T, Takahashi N, Mori Y, Ohwada T (2014) Transnitrosylation directs TRPA1 selectivity in N-nitrosamine activators. Mol Pharmacol 85(1):175-185. doi:10.1124/mol.113.088864

81. Bessac BF, Sivula M, von Hehn CA, Escalera J, Cohn L, Jordt SE (2008) TRPA1 is a major oxidant sensor in murine airway sensory neurons. J Clin Invest 118(5):1899-1910. doi:10.1172/JCI34192

82. Sadofsky LR, Boa AN, Maher SA, Birrell MA, Belvisi MG, Morice AH (2011) TRPA1 is activated by direct addition of cysteine residues to the N-hydroxysuccinyl esters of acrylic and cinnamic acids. Pharmacol Res: Off J Ital Pharmacol Soc 63(1):30-36. doi:10.1016/j. phrs.2010.11.004 
83. Sawada Y, Hosokawa H, Matsumura K, Kobayashi S (2008) Activation of transient receptor potential ankyrin 1 by hydrogen peroxide. Eur J Neurosci 27(5):1131-1142. doi:10.1111/j.1460-9568.2008.06093.x

84. Taylor-Clark TE, Undem BJ (2010) Ozone activates airway nerves via the selective stimulation of TRPA1 ion channels. J Physiol 588(Pt 3):423-433. doi:10.1113/jphysiol.2009.183301

85. Hill K, Schaefer M (2009) Ultraviolet light and photosensitising agents activate TRPA1 via generation of oxidative stress. Cell Calcium 45(2):155-164. doi:10.1016/j.ceca.2008.08.001

86. Miyamoto T, Dubin AE, Petrus MJ, Patapoutian A (2009) TRPV1 and TRPA1 mediate peripheral nitric oxide-induced nociception in mice. PLoS One 4(10):e7596. doi:10.1371/ journal.pone. 0007596

87. Taylor-Clark TE, McAlexander MA, Nassenstein C, Sheardown SA, Wilson S, Thornton J, Carr MJ, Undem BJ (2008) Relative contributions of TRPA1 and TRPV1 channels in the activation of vagal bronchopulmonary $\mathrm{C}$-fibres by the endogenous autacoid 4-oxononenal. $\mathrm{J}$ Physiol 586(14):3447-3459. doi:10.1113/jphysiol.2008.153585

88. McNamara CR, Mandel-Brehm J, Bautista DM, Siemens J, Deranian KL, Zhao M, Hayward NJ, Chong JA, Julius D, Moran MM, Fanger CM (2007) TRPA1 mediates formalin-induced pain. Proc Natl Acad Sci U S A 104(33):13525-13530. doi:10.1073/pnas.0705924104

89. Petrus M, Peier AM, Bandell M, Hwang SW, Huynh T, Olney N, Jegla T, Patapoutian A (2007) A role of TRPA1 in mechanical hyperalgesia is revealed by pharmacological inhibition. Mol Pain 3:40. doi:10.1186/1744-8069-3-40

90. Strassmaier T, Bakthavatchalam R (2011) Transient receptor potential A1 modulators. Curr Top Med Chem 11(17):2227-2236

91. Andrade EL, Meotti FC, Calixto JB (2012) TRPA1 antagonists as potential analgesic drugs. Pharmacol Ther 133(2):189-204. doi:10.1016/j.pharmthera.2011.10.008

92. Brederson JD, Kym PR, Szallasi A (2013) Targeting TRP channels for pain relief. Eur J Pharmacol 716(1-3):61-76. doi:10.1016/j.ejphar.2013.03.003

93. Klement G, Eisele L, Malinowsky D, Nolting A, Svensson M, Terp G, Weigelt D, Dabrowski M (2013) Characterization of a ligand binding site in the human transient receptor potential ankyrin 1 pore. Biophys J 104(4):798-806. doi:10.1016/j.bpj.2013.01.008

94. Bianchi BR, Zhang XF, Reilly RM, Kym PR, Yao BB, Chen J (2012) Species comparison and pharmacological characterization of human, monkey, rat, and mouse TRPA1 channels. J Pharmacol Exp Ther 341(2):360-368. doi:10.1124/jpet.111.189902

95. Chen J, Kym PR (2009) TRPA1: the species difference. J Gen Physiol 133(6):623-625. doi:10.1085/jgp.200910246

96. Hess DT, Matsumoto A, Kim SO, Marshall HE, Stamler JS (2005) Protein S-nitrosylation: purview and parameters. Nat Rev Mol Cell Biol 6(2):150-166. doi:10.1038/nrm1569

97. Smith BC, Marletta MA (2012) Mechanisms of S-nitrosothiol formation and selectivity in nitric oxide signaling. Curr Opin Chem Biol 16(5-6):498-506. doi:10.1016/j. cbpa.2012.10.016

98. Alderton WK, Cooper CE, Knowles RG (2001) Nitric oxide synthases: structure, function and inhibition. Biochem J 357(Pt 3):593-615

99. Stamler JS, Hess DT (2010) Nascent nitrosylases. Nat Cell Biol 12(11):1024-1026. doi:10.1038/ncb1110-1024

100. Seth D, Stamler JS (2011) The SNO-proteome: causation and classifications. Curr Opin Chem Biol 15(1):129-136. doi:10.1016/j.cbpa.2010.10.012

101. Anand P, Stamler JS (2012) Enzymatic mechanisms regulating protein S-nitrosylation: implications in health and disease. J Mol Med 90(3):233-244. doi:10.1007/s00109-012-0878-z

102. Nakamura T, Lipton SA (2013) Emerging role of protein-protein transnitrosylation in cell signaling pathways. Antioxid Redox Signal 18(3):239-249. doi:10.1089/ars.2012.4703

103. Pawloski JR, Hess DT, Stamler JS (2001) Export by red blood cells of nitric oxide bioactivity. Nature 409(6820):622-626. doi:10.1038/35054560

104. Mitchell DA, Marletta MA (2005) Thioredoxin catalyzes the S-nitrosation of the caspase-3 active site cysteine. Nat Chem Biol 1(3):154-158. doi:10.1038/nchembio720 
105. Kornberg MD, Sen N, Hara MR, Juluri KR, Nguyen JV, Snowman AM, Law L, Hester LD, Snyder SH (2010) GAPDH mediates nitrosylation of nuclear proteins. Nat Cell Biol 12(11):1094-1100. doi:10.1038/ncb2114

106. Nakamura T, Wang L, Wong CC, Scott FL, Eckelman BP, Han X, Tzitzilonis C, Meng F, Gu Z, Holland EA, Clemente AT, Okamoto S, Salvesen GS, Riek R, Yates JR 3rd, Lipton SA (2010) Transnitrosylation of XIAP regulates caspase-dependent neuronal cell death. Mol Cell 39(2):184-195. doi:10.1016/j.molcel.2010.07.002

107. Foster MW, McMahon TJ, Stamler JS (2003) S-nitrosylation in health and disease. Trends Mol Med 9(4):160-168

108. Makita N, Kabasawa Y, Otani Y, Firman SJ, Hashimoto M, Nakaya M, Nishihara H, Nangaku M, Kurose H, Ohwada T, Iiri T (2013) Attenuated desensitization of beta-adrenergic receptor by water-soluble N-nitrosamines that induce S-nitrosylation without NO release. Circ Res 112(2):327-334. doi:10.1161/CIRCRESAHA.112.277665

109. Ohwada T, Miura M, Tanaka H, Sakamoto S, Yamaguchi K, Ikeda H, Inagaki S (2001) Structural features of aliphatic N-nitrosamines of 7-azabicyclo[2.2.1]heptanes that facilitate N-NO bond cleavage. J Am Chem Soc 123(42):10164-10172

110. Yanagimoto T, Toyota T, Matsuki N, Makino Y, Uchiyama S, Ohwada T (2007) Transnitrosation of thiols from aliphatic N-nitrosamines: S-nitrosation and indirect generation of nitric oxide. J Am Chem Soc 129(4):736-737. doi:10.1021/ja0658259

111. Ohwada T, Ishikawa S, Mine Y, Inami K, Yanagimoto T, Karaki F, Kabasawa Y, Otani Y, Mochizuki M (2011) 7-azabicyclo[2.2.1]heptane as a structural motif to block mutagenicity of nitrosamines. Bioorg Med Chem 19(8):2726-2741. doi:10.1016/j.bmc.2011.02.049

112. Vazquez G, Wedel BJ, Aziz O, Trebak M, Putney JW Jr (2004) The mammalian TRPC cation channels. Biochim Biophys Acta 1742(1-3):21-36. doi:10.1016/j.bbamcr.2004.08.015

113. Mio K, Ogura T, Kiyonaka S, Hiroaki Y, Tanimura Y, Fujiyoshi Y, Mori Y, Sato C (2007) The TRPC3 channel has a large internal chamber surrounded by signal sensing antennas. J Mol Biol 367(2):373-383. doi:10.1016/j.jmb.2006.12.043

114. Hofmann T, Schaefer M, Schultz G, Gudermann T (2002) Subunit composition of mammalian transient receptor potential channels in living cells. Proc Natl Acad Sci U S A 99(11):7461-7466. doi:10.1073/pnas.102596199

115. Hofmann T, Obukhov AG, Schaefer M, Harteneck C, Gudermann T, Schultz G (1999) Direct activation of human TRPC6 and TRPC3 channels by diacylglycerol. Nature 397(6716):259263. doi:10.1038/16711

116. Kiselyov K, Xu X, Mozhayeva G, Kuo T, Pessah I, Mignery G, Zhu X, Birnbaumer L, Muallem S (1998) Functional interaction between InsP3 receptors and store-operated Htrp3 channels. Nature 396(6710):478-482. doi:10.1038/24890

117. Kiselyov KI, Shin DM, Wang Y, Pessah IN, Allen PD, Muallem S (2000) Gating of storeoperated channels by conformational coupling to ryanodine receptors. Mol Cell 6(2):421-431

118. Vazquez G, Bird GS, Mori Y, Putney JW Jr (2006) Native TRPC7 channel activation by an inositol trisphosphate receptor-dependent mechanism. J Biol Chem 281(35):25250-25258. doi:10.1074/jbc.M604994200

119. Brazer SC, Singh BB, Liu X, Swaim W, Ambudkar IS (2003) Caveolin-1 contributes to assembly of store-operated $\mathrm{Ca} 2+$ influx channels by regulating plasma membrane localization of TRPC1. J Biol Chem 278(29):27208-27215. doi:10.1074/jbc.M301118200

120. Patterson RL, van Rossum DB, Ford DL, Hurt KJ, Bae SS, Suh PG, Kurosaki T, Snyder SH, Gill DL (2002) Phospholipase C-gamma is required for agonist-induced Ca2+ entry. Cell 111(4):529-541

121. Nishida M, Sugimoto K, Hara Y, Mori E, Morii T, Kurosaki T, Mori Y (2003) Amplification of receptor signalling by $\mathrm{Ca} 2+$ entry-mediated translocation and activation of PLCgamma2 in B lymphocytes. EMBO J 22(18):4677-4688. doi:10.1093/emboj/cdg457

122. Numaga T, Nishida M, Kiyonaka S, Kato K, Katano M, Mori E, Kurosaki T, Inoue R, Hikida M, Putney JW Jr, Mori Y (2010) Ca2+ influx and protein scaffolding via TRPC3 sustain PKCbeta and ERK activation in B cells. J Cell Sci 123(Pt 6):927-938. doi:10.1242/jcs.061051 
123. Mery L, Strauss B, Dufour JF, Krause KH, Hoth M (2002) The PDZ-interacting domain of TRPC4 controls its localization and surface expression in HEK293 cells. J Cell Sci 115(Pt 17):3497-3508

124. Lussier MP, Lepage PK, Bousquet SM, Boulay G (2008) RNF24, a new TRPC interacting protein, causes the intracellular retention of TRPC. Cell Calcium 43(5):432-443. doi:10.1016/j.ceca.2007.07.009

125. Singh BB, Lockwich TP, Bandyopadhyay BC, Liu X, Bollimuntha S, Brazer SC, Combs C, Das S, Leenders AG, Sheng ZH, Knepper MA, Ambudkar SV, Ambudkar IS (2004) VAMP2-dependent exocytosis regulates plasma membrane insertion of TRPC3 channels and contributes to agonist-stimulated Ca2+ influx. Mol Cell 15(4):635-646. doi:10.1016/j. molcel.2004.07.010

126. Wes PD, Chevesich J, Jeromin A, Rosenberg C, Stetten G, Montell C (1995) TRPC1, a human homolog of a Drosophila store-operated channel. Proc Natl Acad Sci U S A 92(21):9652-9656

127. Zitt C, Zobel A, Obukhov AG, Harteneck C, Kalkbrenner F, Luckhoff A, Schultz G (1996) Cloning and functional expression of a human $\mathrm{Ca} 2+-$ permeable cation channel activated by calcium store depletion. Neuron 16(6):1189-1196

128. Zhu X, Jiang M, Peyton M, Boulay G, Hurst R, Stefani E, Birnbaumer L (1996) trp, a novel mammalian gene family essential for agonist-activated capacitative $\mathrm{Ca} 2+$ entry. Cell 85(5):661-671

129. Liu X, Cheng KT, Bandyopadhyay BC, Pani B, Dietrich A, Paria BC, Swaim WD, Beech D, Yildrim E, Singh BB, Birnbaumer L, Ambudkar IS (2007) Attenuation of store-operated $\mathrm{Ca} 2+$ current impairs salivary gland fluid secretion in TRPC1(-I-) mice. Proc Natl Acad Sci U S A 104(44):17542-17547. doi:10.1073/pnas.0701254104

130. Mori Y, Wakamori M, Miyakawa T, Hermosura M, Hara Y, Nishida M, Hirose K, Mizushima A, Kurosaki M, Mori E, Gotoh K, Okada T, Fleig A, Penner R, Iino M, Kurosaki T (2002) Transient receptor potential 1 regulates capacitative $\mathrm{Ca}(2+)$ entry and $\mathrm{Ca}(2+)$ release from endoplasmic reticulum in B lymphocytes. J Exp Med 195(6):673-681

131. Poteser M, Graziani A, Rosker C, Eder P, Derler I, Kahr H, Zhu MX, Romanin C, Groschner K (2006) TRPC3 and TRPC4 associate to form a redox-sensitive cation channel. Evidence for expression of native TRPC3-TRPC4 heteromeric channels in endothelial cells. J Biol Chem 281(19):13588-13595. doi:10.1074/jbc.M512205200

132. Maroto R, Raso A, Wood TG, Kurosky A, Martinac B, Hamill OP (2005) TRPC1 forms the stretch-activated cation channel in vertebrate cells. Nat Cell Biol 7(2):179-185. doi:10.1038/ ncb1218

133. Spassova MA, Hewavitharana T, Xu W, Soboloff J, Gill DL (2006) A common mechanism underlies stretch activation and receptor activation of TRPC6 channels. Proc Natl Acad Sci U S A 103(44):16586-16591. doi:10.1073/pnas.0606894103

134. Seo K, Rainer PP, Lee DI, Hao S, Bedja D, Birnbaumer L, Cingolani OH, Kass DA (2014) Hyperactive adverse mechanical stress responses in dystrophic heart are coupled to transient receptor potential canonical 6 and blocked by cGMP-protein kinase G modulation. Circ Res 114(5):823-832. doi:10.1161/CIRCRESAHA.114.302614

135. Weissmann N, Sydykov A, Kalwa H, Storch U, Fuchs B, Mederos y Schnitzler M, Brandes RP, Grimminger F, Meissner M, Freichel M, Offermanns S, Veit F, Pak O, Krause KH, Schermuly RT, Brewer AC, Schmidt HH, Seeger W, Shah AM, Gudermann T, Ghofrani HA, Dietrich A (2012) Activation of TRPC6 channels is essential for lung ischaemia-reperfusion induced oedema in mice. Nat Commun 3:649. doi:10.1038/ncomms1660

136. Inoue R, Jensen LJ, Jian Z, Shi J, Hai L, Lurie AI, Henriksen FH, Salomonsson M, Morita H, Kawarabayashi Y, Mori M, Mori Y, Ito Y (2009) Synergistic activation of vascular TRPC6 channel by receptor and mechanical stimulation via phospholipase C/diacylglycerol and phospholipase A2/omega-hydroxylase/20-HETE pathways. Circ Res 104(12):1399-1409. doi:10.1161/CIRCRESAHA.108.193227

137. Levy D, Kenchaiah S, Larson MG, Benjamin EJ, Kupka MJ, Ho KK, Murabito JM, Vasan RS (2002) Long-term trends in the incidence of and survival with heart failure. N Engl J Med 347(18):1397-1402. doi:10.1056/NEJMoa020265 
138. Klein L, O'Connor CM, Gattis WA, Zampino M, de Luca L, Vitarelli A, Fedele F, Gheorghiade M (2003) Pharmacologic therapy for patients with chronic heart failure and reduced systolic function: review of trials and practical considerations. Am J Cardiol 91(9A):18F-40F

139. Levy D, Garrison RJ, Savage DD, Kannel WB, Castelli WP (1990) Prognostic implications of echocardiographically determined left ventricular mass in the Framingham Heart Study. N Engl J Med 322(22):1561-1566. doi:10.1056/NEJM199005313222203

140. Frey N, Olson EN (2003) Cardiac hypertrophy: the good, the bad, and the ugly. Annu Rev Physiol 65:45-79. doi:10.1146/annurev.physiol.65.092101.142243

141. Eder P, Molkentin JD (2011) TRPC channels as effectors of cardiac hypertrophy. Circ Res 108(2):265-272. doi:10.1161/CIRCRESAHA.110.225888

142. Bush EW, Hood DB, Papst PJ, Chapo JA, Minobe W, Bristow MR, Olson EN, McKinsey TA (2006) Canonical transient receptor potential channels promote cardiomyocyte hypertrophy through activation of calcineurin signaling. J Biol Chem 281(44):33487-33496. doi:10.1074/ jbc.M605536200

143. Wilkins BJ, Dai YS, Bueno OF, Parsons SA, Xu J, Plank DM, Jones F, Kimball TR, Molkentin JD (2004) Calcineurin/NFAT coupling participates in pathological, but not physiological, cardiac hypertrophy. Circ Res 94(1):110-118. doi:10.1161/01.RES.0000109415.17511.18

144. Heineke J, Molkentin JD (2006) Regulation of cardiac hypertrophy by intracellular signalling pathways. Nat Rev Mol Cell Biol 7(8):589-600. doi:10.1038/nrm1983

145. Roderick HL, Bootman MD (2007) Pacemaking, arrhythmias, inotropy and hypertrophy: the many possible facets of IP3 signalling in cardiac myocytes. J Physiol 581(Pt 3):883-884. doi:10.1113/jphysiol.2007.133819

146. Colella M, Grisan F, Robert V, Turner JD, Thomas AP, Pozzan T (2008) Ca2+ oscillation frequency decoding in cardiac cell hypertrophy: role of calcineurin/NFAT as $\mathrm{Ca} 2+$ signal integrators. Proc Natl Acad Sci U S A 105(8):2859-2864. doi:10.1073/pnas.0712316105

147. Brenner JS, Dolmetsch RE (2007) TrpC3 regulates hypertrophy-associated gene expression without affecting myocyte beating or cell size. PLoS One 2(8):e802. doi:10.1371/journal. pone.0000802

148. Nishida M, Onohara N, Sato Y, Suda R, Ogushi M, Tanabe S, Inoue R, Mori Y, Kurose H (2007) Galpha12/13-mediated up-regulation of TRPC6 negatively regulates endothelin-1induced cardiac myofibroblast formation and collagen synthesis through nuclear factor of activated $\mathrm{T}$ cells activation. J Biol Chem 282(32):23117-23128. doi:10.1074/jbc. M611780200

149. Davis J, Burr AR, Davis GF, Birnbaumer L, Molkentin JD (2012) A TRPC6-dependent pathway for myofibroblast transdifferentiation and wound healing in vivo. Dev Cell 23(4):705-715. doi:10.1016/j.devcel.2012.08.017

150. Nakayama H, Wilkin BJ, Bodi I, Molkentin JD (2006) Calcineurin-dependent cardiomyopathy is activated by TRPC in the adult mouse heart. FASEB J: Off Publ Fed Am Soc Exp Biol 20(10):1660-1670. doi:10.1096/fj.05-5560com

151. Seo K, Rainer PP, Shalkey Hahn V, Lee DI, Jo SH, Andersen A, Liu T, Xu X, Willette RN, Lepore JJ, Marino JP Jr, Birnbaumer L, Schnackenberg CG, Kass DA (2014) Combined TRPC3 and TRPC6 blockade by selective small-molecule or genetic deletion inhibits pathological cardiac hypertrophy. Proc Natl Acad Sci U S A 111(4):1551-1556. doi:10.1073/ pnas.1308963111

152. Dietrich A, Mederos YSM, Gollasch M, Gross V, Storch U, Dubrovska G, Obst M, Yildirim E, Salanova B, Kalwa H, Essin K, Pinkenburg O, Luft FC, Gudermann T, Birnbaumer L (2005) Increased vascular smooth muscle contractility in TRPC6-/- mice. Mol Cell Biol 25(16):6980-6989. doi:10.1128/MCB.25.16.6980-6989.2005

153. Ohba T, Watanabe H, Murakami M, Takahashi Y, Iino K, Kuromitsu S, Mori Y, Ono K, Iijima T, Ito H (2007) Upregulation of TRPC1 in the development of cardiac hypertrophy. J Mol Cell Cardiol 42(3):498-507

154. Seth M, Zhang ZS, Mao L, Graham V, Burch J, Stiber J, Tsiokas L, Winn M, Abramowitz J, Rockman HA, Birnbaumer L, Rosenberg P (2009) TRPC1 channels are critical for hypertrophic signaling in the heart. Circ Res 105(10):1023-1030. doi:10.1161/CIRCRESAHA.109.206581 
155. Takahashi S, Lin H, Geshi N, Mori Y, Kawarabayashi Y, Takami N, Mori MX, Honda A, Inoue R (2008) Nitric oxide-cGMP-protein kinase G pathway negatively regulates vascular transient receptor potential channel TRPC6. J Physiol 586(Pt 17):4209-4223. doi:10.1113/ jphysiol.2008.156083

156. Nishioka K, Nishida M, Ariyoshi M, Jian Z, Saiki S, Hirano M, Nakaya M, Sato Y, Kita S, Iwamoto T, Hirano K, Inoue R, Kurose H (2011) Cilostazol suppresses angiotensin II-induced vasoconstriction via protein kinase A-mediated phosphorylation of the transient receptor potential canonical 6 channel. Arterioscler Thromb Vasc Biol 31(10):2278-2286. doi:10.1161/ ATVBAHA.110.221010

157. Koitabashi N, Aiba T, Hesketh GG, Rowell J, Zhang M, Takimoto E, Tomaselli GF, Kass DA (2010) Cyclic GMP/PKG-dependent inhibition of TRPC6 channel activity and expression negatively regulates cardiomyocyte NFAT activation Novel mechanism of cardiac stress modulation by PDE5 inhibition. J Mol Cell Cardiol 48(4):713-724. doi:10.1016/j. yjmcc.2009.11.015

158. Kinoshita H, Kuwahara K, Nishida M, Jian Z, Rong X, Kiyonaka S, Kuwabara Y, Kurose H, Inoue R, Mori Y, Li Y, Nakagawa Y, Usami S, Fujiwara M, Yamada Y, Minami T, Ueshima K, Nakao K (2010) Inhibition of TRPC6 channel activity contributes to the antihypertrophic effects of natriuretic peptides-guanylyl cyclase-A signaling in the heart. Circ Res 106(12):1849-1860. doi:10.1161/CIRCRESAHA.109.208314

159. Nishida M, Watanabe K, Sato Y, Nakaya M, Kitajima N, Ide T, Inoue R, Kurose H (2010) Phosphorylation of TRPC6 channels at Thr69 is required for anti-hypertrophic effects of phosphodiesterase 5 inhibition. J Biol Chem 285(17):13244-13253. doi:10.1074/jbc. M109.074104

160. Kitajima N, Watanabe K, Morimoto S, Sato Y, Kiyonaka S, Hoshijima M, Ikeda Y, Nakaya M, Ide T, Mori Y, Kurose H, Nishida M. (2011) TRPC3-mediated Ca2+ influx contributes to Rac1-mediated production of reactive oxygen species in MLP-deficient mouse hearts. Biochem Biophys Res Commun 409(1):108-113

161. Steinberg X, Lespay-Rebolledo C, Brauchi S (2014) A structural view of ligand-dependent activation in thermoTRP channels. Front Physiol 5:171. doi:10.3389/fphys.2014.00171

162. Doerner JF, Gisselmann G, Hatt H, Wetzel CH (2007) Transient receptor potential channel A1 is directly gated by calcium ions. J Biol Chem 282(18):13180-13189. doi:10.1074/jbc. M607849200

163. Fujita F, Uchida K, Moriyama T, Shima A, Shibasaki K, Inada H, Sokabe T, Tominaga M (2008) Intracellular alkalization causes pain sensation through activation of TRPA1 in mice. J Clin Invest 118(12):4049-4057

164. Zhang XF, Chen J, Faltynek CR, Moreland RB, Neelands TR (2008) Transient receptor potential A1 mediates an osmotically activated ion channel. Eur J Neurosci 27(3):605-611. doi:10.1111/j.1460-9568.2008.06030.x

165. Caspani O, Heppenstall PA (2009) TRPA1 and cold transduction: an unresolved issue? J Gen Physiol 133(3):245-249. doi:10.1085/jgp.200810136

166. Nilius B, Prenen J, Owsianik G (2011) Irritating channels: the case of TRPA1. J Physiol 589(Pt 7):1543-1549. doi:10.1113/jphysiol.2010.200717

167. Nilius B, Appendino G, Owsianik G (2012) The transient receptor potential channel TRPA1: from gene to pathophysiology. Pflugers Arch: Eur J Physiol 464(5):425-458. doi:10.1007/ s00424-012-1158-z

168. Bautista DM, Pellegrino M, Tsunozaki M (2013) TRPA1: a gatekeeper for inflammation. Annu Rev Physiol 75:181-200

169. Schleifer H, Doleschal B, Lichtenegger M, Oppenrieder R, Derler I, Frischauf I, Glasnov TN, Kappe CO, Romanin C, Groschner K (2012) Novel pyrazole compounds for pharmacological discrimination between receptor-operated and store-operated $\mathrm{Ca}(2+)$ entry pathways. $\mathrm{Br} \mathrm{J}$ Pharmacol 167(8):1712-1722 


\title{
Autophagic Cell Death and Cancer Chemotherapeutics
}

\author{
Shigeomi Shimizu
}

\begin{abstract}
Autophagy usually functions in cell-protective events. However, it may also be utilized as a cell-suicide mechanism, which is known as "autophagic cell death." Autophagic cell death is frequently induced in cells that lack apoptotic machinery, such as p53-deficient cancer cells. Therefore, small compounds that activate autophagic cell death are good candidates for anticancer chemotherapeutics to combat p53-deficient cancers. This chapter focuses on recent advances in autophagy/ autophagic cell death and their relationship with tumorigenesis.
\end{abstract}

Keywords Autophagy • Autophagic cell death • p53 • Apoptosis • Ulk1 • Atg5

\section{Apoptosis and Other Types of Cell Death}

Apoptosis is a form of programmed cell death (PCD) and its molecular basis is well understood. Mammalian cells possess two apoptotic signaling pathways, which are known as the intrinsic and extrinsic pathways. In the intrinsic pathway, mitochondria play a crucial role by increasing membrane permeability to release several apoptogenic molecules (e.g., cytochrome $c$, Smac, and Omi) into the cytoplasm. After its release, cytochrome $c$ associates with Apaf-1, which activates the caspase cascade to execute apoptotic cell death. Smac and Omi accelerate caspase activation by inhibiting IAP family proteins that function as endogenous caspase inhibitors. The Bcl-2 family proteins are well-characterized regulators of apoptosis, which directly modulate mitochondrial membrane permeability [1]. This family of proteins contains anti-apoptotic members, such as Bcl-2 and $\mathrm{Bcl}-\mathrm{x}_{\mathrm{L}}$, and pro-apoptotic members, including multidomain Bax and Bak, as well as numerous $\mathrm{BH}$-only proteins. Multidomain pro-apoptotic Bax and Bak are functionally redundant and play a direct role in increasing mitochondrial membrane permeability, thereby leading to the release of apoptogenic proteins. $\mathrm{BH} 3$-only proteins function as death transducers by activating Bax/Bak or inactivating anti-apoptotic Bcl-2 family members [1].

\footnotetext{
S. Shimizu $(\bowtie)$

Department of Pathological Cell Biology, Medical Research Institute, Tokyo Medical and Dental University, 1-5-45 Yushima, Bunkyo-ku, Tokyo 113-8510, Japan

e-mail: shimizu.pcb@mri.tmd.ac.jp 
Until 10 years ago, the evidence indicated that apoptosis is the primary mediator of physiological and pathological cell death. However, a detailed analysis of Bax/ Bak double-knockout mice with a totally inhibited intrinsic apoptotic pathway showed that PCD mainly proceeds normally in organisms that lack apoptosis [2, 3]. These findings encouraged researchers to identify other pathways of PCD, i.e., nonapoptotic cell death. Several types of non-apoptotic cell death have been elucidated, such as programmed necrosis [4] and autophagic cell death [5]. Previously, necrosis was considered to be a form of accidental cell death due to physicochemical stressors but recent evidence has demonstrated the existence of regulated (or programmed) necrosis. Regulated necrosis is a genetically controlled form of cell death, which is characterized morphologically by cytoplasmic granulation and cellular swelling. Necroptosis is one of the best-characterized types of programmed necrosis, which is activated by several kinases, including RIP1, RIP3, and MLKL, whereas it is inhibited by the small compound necrostatin-1 [6]. Autophagic cell death is another type of non-apoptotic cell death that occurs via autophagy activation. Autophagy has long been considered a cell-protective mechanism but recent evidence indicates that the hyperactivation of autophagy is sometimes utilized as a cell-suicide mechanism. This chapter summarizes the current knowledge of autophagy and autophagic cell death.

\section{Autophagy}

Autophagy is a catabolic process that digests cellular contents within lysosomes. Autophagy is a low-level constitutive function, which is accelerated by a variety of cellular stressors such as nutrient starvation, DNA damage, and organelle damage. Autophagy is a protective mechanism that facilitates the degradation of superfluous or damaged cellular constituents, although hyperactivation of autophagy can lead to cell death.

In this multistep process, autophagy substrates, such as cytoplasm and damaged organelles, are sequestered inside isolation membranes, which eventually mature into double-membrane structures called autophagosomes. Autophagosomes subsequently fuse with lysosomes to form autolysosomes where the sequestered components are digested (Fig. 1) [7]. The molecular basis of autophagy was elucidated in autophagy-defective mutant yeasts [8]. The subsequent identification of vertebrate homologs greatly expanded our understanding of the molecular mechanisms of autophagy.

Autophagy is driven by over 30 proteins (Atgs), which are well conserved from yeasts to mammals [7]. Atg1 [also called Unc51-like kinase 1 (Ulk1)] is a serine/ threonine kinase that is essential for the initiation of autophagy [9]. Autophagy is also regulated by phosphatidylinositol 3-kinase (PI3K) type III, which is a component of a multi-protein complex that includes Atg6 (Beclin1). PI3K promotes invagination of the membrane at domains rich in phosphatidylinositol-3-phosphate, which are called omegasomes, thereby initiating the generation of the isolation membrane [10]. The subsequent expansion and closure of isolation membranes are 


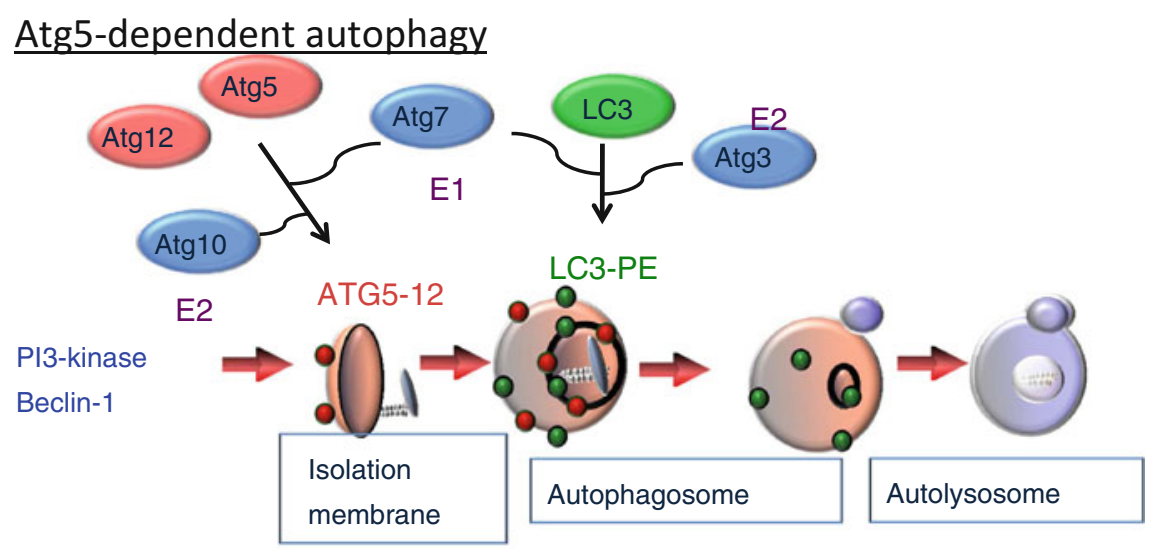

\section{Atg5-independent autophagy}

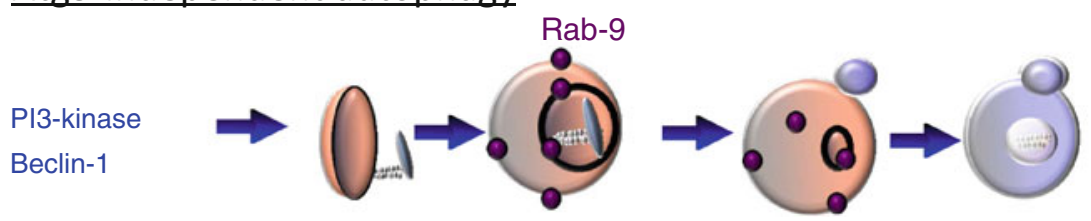

Fig. 1 Hypothetical model of macroautophagy. There are at least two modes of macroautophagy, i.e., conventional and alternative macroautophagy. Conventional macroautophagy depends on Atg5 and Atg7, is associated with LC3 modification, and may originate from ER-mitochondria contact membrane. In contrast, alternative macroautophagy occurs independent of Atg 5 or Atg7 expression and LC3 modification. The generation of autophagic vacuoles in this type of macroautophagy may originate from Golgi membranes and late endosomes (LE) in a Rab9-dependent manner. Although both of these processes lead to bulk degradation of damaged proteins or organelles by generating autolysosomes, they seem to be activated by different stimuli in different cell types and have different physiological roles

mediated by two ubiquitin-like conjugation pathways: the Atg5-Atg12 pathway and the MAP-LC3 pathway [7]. In cells that lack these ubiquitin-like conjugation systems, such as Atg5- and Atg7-deficient cells, autophagosome formation is largely disrupted, which indicates the necessity for Atg5 and Atg7 in autophagy. Ubiquitin-like conjugation of phosphatidylethanolamine to LC3 facilitates the translocation of LC3 from the cytosol to the sites of origin of the autophagic membrane. This form of translocation is recognized as a reliable marker of autophagy.

\section{Alternative Macroautophagy}

Although Atg5 has long been considered as an essential molecule for autophagy, we recently identified an Atg5-independent type of autophagy, which is induced when cells are severely stressed, such as in the event of DNA damage. The morphology of 
Atg5-independent autophagic structures is indistinguishable from that of those formed during Atg5-dependent autophagy, i.e., isolation membranes, autophagosomes, and autolysosomes [11]. Thus, we designated this form of Atg5-independent autophagy as "alternative macroautophagy" (Fig. 1). This alternative macroautophagy is driven by Ulk1 and PI3K complexes during the initiation steps. These molecules also function during the initiation of conventional autophagy. However, this pathway is not mediated by other components such as Atg9 and proteins in the ubiquitin-like protein conjugation system (Atg5, Atg7, and LC3), which extend conventional autophagic membranes. Alternative macroautophagy requires the extension of autophagic membranes, thus several unidentified molecules may mediate this function.

Alternative macroautophagy is not an atypical form of autophagy because it occurs in a wide variety of cells, including embryonic fibroblasts and thymocytes, as well as in several tissues such as the heart, brain, and liver [11]. Erythrocyte maturation is a representative example of a physiological alternative macroautophagy. Erythrocytes undergo enucleation and clearance of mitochondria during terminal differentiation, and the possible involvement of autophagy in the latter process was proposed on the basis of a morphological analysis. However, the maturation process is normal in Atg5-deficient erythrocytes. In agreement, an ultrastructural analysis showed that mitochondria are also engulfed and digested by autophagic structures in both the wild-type and Atg5-deficient reticulocytes. These results indicate that conventional macroautophagy does not eliminate mitochondria from erythrocytes. In contrast, mitochondrial clearance is significantly reduced in Ulk1-deficient reticulocytes where alternative macroautophagy is absent. These data suggest that Ulk1-mediated alternative macroautophagy plays a pivotal role in the elimination of mitochondria from erythrocytes $[12,13]$. Ongoing research should provide a more detailed picture of the physiological and pathological relevance of alternative macroautophagy in the near future.

\section{Autophagic Cell Death}

Autophagy is activated by most cellular stressors, thus numerous autophagycontaining cells are often observed in regions where cell death occurs (Fig. 2). In most cases, autophagy has a cell protection function against cellular stressors, but autophagy is utilized as a cell-suicide mechanism in some cases [5]. Certain modes of cell death that are accompanied by protective autophagy are not referred to as "autophagic cell death." In particular, the term "autophagic cell death" should be used when cell death is performed via autophagy activation. This can be demonstrated by the suppression of cell death by autophagy inhibitors (e.g., 3-methyl adenine and wortmannin) or by the genetic ablation of autophagy (e.g., knockout or siRNA silencing of essential autophagy genes). If autophagy inhibition does not prevent cell death, this process should not be referred to as autophagic cell death. 


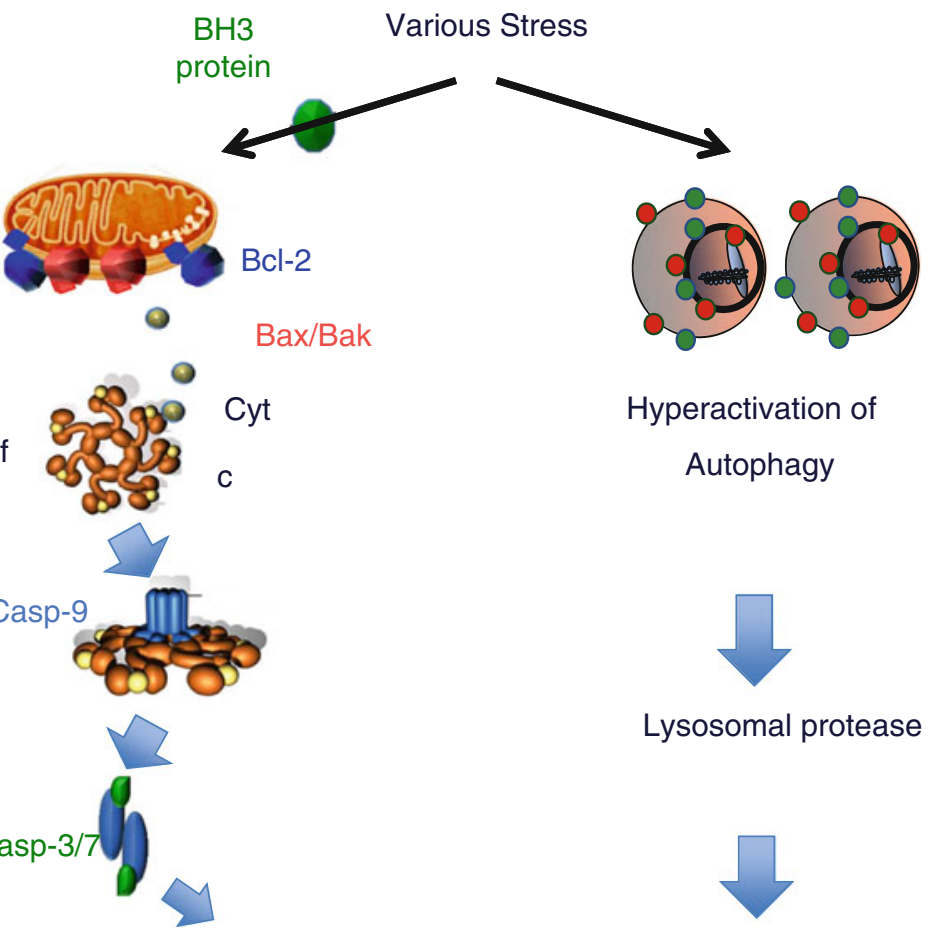

Apoptosis

Autophagic Cell Death

Fig. 2 Molecular mechanism of apoptosis and autophagic cell death. An increase in the permeability of the outer mitochondrial membrane is crucial for apoptosis to occur and is regulated by multidomain pro-apoptotic members of the Bcl-2 family (Bax and Bak), resulting in the release of cytochrome $\mathrm{c}$ into the cytoplasm. Then, cytochrome $\mathrm{c}$ associates with Apaf-1, which activates the caspase cascade to execute apoptotic cell death. Apoptosis-associated mitochondrial membrane permeability is primarily controlled by Bcl-2 family members. When apoptosis is blocked, various apoptotic stimuli activate autophagy, resulting in the induction of autophagic cell death

Autophagic cell death occurs in several distinct settings. First, caspase-independent, autophagy-dependent PCD occurs during mammalian embryogenesis. Second, autophagy may induce developmental cell death during regression of the salivary glands in Drosophila [14]. Third, autophagy-mediated cell death is induced in apoptosis-resistant cells, particularly in the absence of the pro-apoptotic proteins Bax and Bak [5]. The latter situation should provide an excellent experimental system for investigating autophagic cell death because the process can be observed at the cellular level.

Bax/Bak-deficient cells do not undergo apoptosis after exposure to a variety of apoptotic stimuli, although these cells still die in numerous autophagic structures (Fig. 2). This type of cell death is inhibited by autophagy inhibitors or by silencing autophagy genes such as Atg5 and Atg6. Thus, Atg5-dependent autophagy 
is required for the death of Bax/Bak-deficient cells after exposure to apoptotic stimuli [5]. Although we discovered Atg5-independent alternative autophagy, it is not involved in the autophagic cell death of Bax/Bak-deficient cells. However, this does not necessarily indicate that alternative autophagy is irrelevant for autophagic cell death. In other situations, alternative autophagy may potentially induce autophagic cell death.

\section{Cancer and Autophagic Cell Death}

It has been suggested that autophagic cell death may participate in physiological and pathological events. A large body of evidence indicates that inhibition of apoptosis is critical for tumorigenesis, but the elimination of cancer cells may also be mediated by autophagic cell death, and there is evidence that decreased autophagic activity is related to tumorigenesis. For example, the levels of Beclin-1 (Atg6) are lower in some types of cancers of the ovary, breast, and prostate because of monoallelic mutations [15]. Furthermore, mice that are heterozygous for the beclin-1 gene are cancer prone $[15,16]$, which strongly suggests that inhibition of Beclin-1 expression contributes to the pathogenesis of cancer. Moreover, Atg5, LC3, and Fip200 are associated with myeloma, glioblastoma, and breast cancer, respectively $[17,18]$, thereby suggesting that the failure of cells to undergo autophagy leads to tumor progression.

Several mechanisms may explain how tumorigenesis is mediated by the failure of cells to undergo autophagy: (1) accumulation of p62, a substrate of autophagy, leads to NF-kB activation [19]; (2)accumulation of p62 stabilizes Nrf2, which makes tumor cells resistant to hypoxic stress [20]; (3) retention of damaged organelles, including mitochondria, increases the level of active oxygen species and increases the mutation rate; and (4) defective elimination of cancer cells due to the loss of autophagic cell death [21]. These mechanisms may be cell- and stimulus-type specific; however, we believe that it is reasonable to conclude that the failure of autophagic cell death is one of the most crucial mechanisms involved in tumorigenesis because autophagic cell death occurs in normal cells (e.g., fibroblasts or thymocytes) but not in most cancer cells. Furthermore, in some cancer cells, the magnitude of JNK activation, which is a crucial factor for autophagic cell death, is significantly lower compared with that in normal cells after exposure to apoptotic stimuli [21]. In these cancer cells, the JNK activity level may not reach the threshold level required to induce autophagic cell death. This conclusion is supported by evidence that the enforced expression of activated JNK in cancer cells induces autophagic cell death. Taken together, it is likely that insufficient activation of JNK followed by the failure of autophagic cell death may induce uncontrolled growth of cells, which ultimately acquire the malignant phenotype. 


\section{Autophagic Cell Death and Cancer Chemotherapeutics}

Many molecularly targeted anticancer agents have been developed on the basis of apoptosis. However, autophagic cell death is also involved in the mechanisms of carcinogenesis. Therefore, we speculate that cancer may be cured by inducing autophagic death in cancer cells. This type of treatment would be effective for cancers that do not respond to existing anticancer agents (i.e., agents with apoptosisbased mechanisms of action). In addition, synergistic effects with existing anticancer agents are expected. Thus, we are now trying to develop molecularly targeted anticancer agents based on the induction of autophagic cell death. To meet this objective, we have established a high-throughput assay system that can monitor excessive autophagy and cell death-inducing activity. Using this assay, we screened a low molecular weight compound library to identify chemicals with autophagic cell death-inducing activities and successfully identified 24 candidate compounds. In particular, four of these compounds exhibited strong anticancer activities. At present, we are optimizing these compounds for development as drugs based on pharmacokinetic investigations and structure-activity relationships. In the near future, we aim to develop anticancer agents that will be effective against cancers that are resistant to the anticancer agents used in current clinical practice.

Open Access This chapter is distributed under the terms of the Creative Commons Attribution Noncommercial License, which permits any noncommercial use, distribution, and reproduction in any medium, provided the original author(s) and source are credited.

\section{References}

1. Tsujimoto Y (2003) Cell death regulation by the Bcl-2 protein family in the mitochondria. J Cell Physiol 195:158-167

2. Lindsten T, Ross AJ, King A et al (2000) The combined functions of proapoptotic Bcl-2 family members bak and bax are essential for normal development of multiple tissues. Mol Cell 6:1389-1399

3. Wei MC, Zong WX, Cheng EH et al (2001) Proapoptotic BAX and BAK: a requisite gateway to mitochondrial dysfunction and death. Science 292:727-730

4. Degterev A, Huang Z, Boyce M et al (2005) Chemical inhibitor of nonapoptotic cell death with therapeutic potential for ischemic brain injury. Nat Chem Biol 1:112-119

5. Shimizu S, Kanaseki T, Mizushima N et al (2004) A role of Bcl-2 family of proteins in nonapoptotic programmed cell death dependent on autophagy genes. Nat Cell Biol 6:1221-1228

6. Vandenabeele P, Galluzzi L, Vanden Berghe T et al (2010) Molecular mechanisms of necroptosis: an ordered cellular explosion. Nat Rev Mol Cell Biol 11:700-714

7. Mizushima N, Yoshimori T, Ohsumi Y (2011) The role of Atg proteins in autophagosome formation. Annu Rev Cell Dev Biol 27:107-132

8. Nakatogawa H, Suzuki K, Kamada Y et al (2009) Dynamics and diversity in autophagy mechanisms: lessons from yeast. Nat Rev Mol Cell Biol 10:458-467

9. Kabeya Y, Kamada Y, Baba M et al (2005) Atg17 functions in cooperation with Atg1 and Atg13 in yeast autophagy. Mol Biol Cell 16:2544-2553 
10. Axe EL, Walker SA, Manifava M et al (2008) Autophagosome formation from membrane compartments enriched in phosphatidylinositol 3-phosphate and dynamically connected to the endoplasmic reticulum. J Cell Biol 182:685-701

11. Nishida Y, Arakawa S, Fujitani K et al (2009) Discovery of Atg5/Atg7-independent alternative macroautophagy. Nature 461:654-658

12. Kundu M, Lindsten T, Yang CY et al (2008) Ulk1 plays a critical role in the autophagic clearance of mitochondria and ribosomes during reticulocyte maturation. Blood 112:1493-1502

13. Honda S, Arakawa S, Nishida Y et al (2014) Ulk1-mediated Atg5-independent macroautophagy mediates elimination of mitochondria from embryonic reticulocytes. Nat Commun 5. Article number:4004

14. Baehrecke EH (2003) Autophagic programmed cell death in Drosophila. Cell Death Differ 9:940-945

15. Qu X, Yu J, Bhagat G et al (2003) Promotion of tumorigenesis by heterozygous disruption of the beclin 1 autophagy gene. J Clin Invest 112:1809-1820

16. Yue Z, Jin S, Yang C et al (2003) Beclin 1, an autophagy gene essential for early embryonic development, is a haploinsufficient tumor suppressor. Proc Natl Acad Sci U S A 100:15077-15082

17. Iqbal J, Kucuk C, deLeeuw RJ et al (2009) Genomic analyses reveal global functional alterations that promote tumor growth and novel tumor suppressor genes in natural killer-cell malignancies. Leukemia 23:1139-1151

18. Huang X, Bai HM, Chen L et al (2010) Reduced expression of LC3B-II and Beclin 1 in glioblastoma multiforme indicates a down-regulated autophagic capacity that relates to the progression of astrocytic tumors. J Clin Neurosci 17:1515-1519

19. Mathew R, Karp CM, Beaudoin B et al (2009) Autophagy suppresses tumorigenesis through elimination of p62. Cell 137:1062-1075

20. Takamura A, Komatsu M, Hara T et al (2011) Autophagy-deficient mice develop multiple liver tumors. Genes Dev 25:795-800

21. Shimizu S, Konishi A, Nishida Y et al (2010) Involvement of JNK in the regulation of autophagic cell death. Oncogene 29:2070-2082 


\title{
Adrenomedullin as a Potential Therapeutic Agent for Refractory Ulcerative Colitis
}

\author{
Kazuo Kitamura, Shinya Ashizuka, Haruhiko Inatsu, and Toshihiro Kita
}

\begin{abstract}
Adrenomedullin (AM) was originally isolated from human pheochromocytoma as a biologically active peptide with potent vasodilating action, but it also has a wide range of physiological properties including cardiovascular protection, neovascularization, and the ability to suppress apoptosis. It is constitutively produced by various tissues including the gastrointestinal tract. AM production and secretion can be induced by pro-inflammatory cytokines such as tumor necrosis factor- $\alpha$ and interleukin-1, as well as by lipopolysaccharide. Conversely, AM causes the downregulation of inflammatory cytokines in cultured cells and downregulates inflammatory processes in various models of colitis, including those induced by acetic acid and by dextran sulfate sodium. AM works by exerting anti-inflammatory and antibacterial effects and by stimulating mucosal regeneration and supporting maintenance of the colonic epithelial barrier. The present findings suggest that AM could serve as a novel agent for treating refractory ulcerative colitis.
\end{abstract}

Keywords Endogenous peptide - Inflammatory bowel disease - Autoimmune response $\bullet$ Cytokines $\bullet$ Translational research $\bullet$ Hypotensive peptide

\section{Abbreviations}

$\begin{array}{ll}\text { AM } & \text { Adrenomedullin } \\ \text { AZA } & \text { Azathioprine } \\ \text { CD } & \text { Crohn's disease } \\ \text { CGRP } & \text { Calcitonin gene related peptide } \\ \text { CRLR } & \text { Calcitonin receptor-like receptor } \\ \text { DSS } & \text { Dextran sulfate sodium } \\ \text { HIF } & \text { Hypoxia-induced factor } \\ \text { IBD } & \text { Inflammatory bowel disease }\end{array}$

K. Kitamura, M.D., Ph.D. $(\bowtie) \bullet$ S. Ashizuka • H. Inatsu • T. Kita Department of Internal Medicine, Circulatory and Body Fluid Regulation, Faculty of Medicine, University of Miyazaki, 5200 Kihara, Kiyotake, Miyazaki City, Miyazaki 889-1692, Japan

e-mail: kazuokit@med.miyazaki-u.ac.jp 


$\begin{array}{ll}\text { IELs } & \text { Intraepithelial T lymphocytes } \\ \text { IFN } & \text { Interferon } \\ \text { IL } & \text { Interleukin } \\ \text { iNOS } & \text { Inducible nitric oxide synthase } \\ \text { IR } & \text { Immunoreactive } \\ \text { LPS } & \text { Lipopolysaccharide } \\ \text { PAMP } & \text { Proadrenomedullin N-terminal } 20 \text { peptide } \\ \text { PSL } & \text { Prednisolone } \\ \text { RAMP } & \text { Receptor activity-modifying protein } \\ \text { STAT } & \text { Signal transducer and activator of transcription } \\ \text { TCR } & \text { T-cell receptor } \\ \text { TGF } & \text { Transforming growth factor } \\ \text { TNF } & \text { Tumor necrosis factor } \\ \text { UC } & \text { Ulcerative colitis } \\ \text { UCDAI } & \text { Ulcerative Colitis Disease Activity Index } \\ \text { VSMCs } & \text { Vascular smooth muscle cells }\end{array}$

\section{Introduction}

Inflammatory bowel disease (IBD) is a refractory ailment, probably involving an autoimmune response to one or more as yet unknown triggers in the intestinal tract. The etiology of IBD is unknown, but it is thought to involve genetic, immunological, and environmental factors. The two major types of IBD are ulcerative colitis (UC) and Crohn's disease (CD). An estimated 1.4 million persons are afflicted with these diseases in the USA $[1,2]$ and the estimated number of patients with IBD in Japan has recently increased to over 100,000 . Therefore, the number of patients with severe and refractory UC has also increased, and consequently, new and effective drugs for refractory UC are highly desirable.

Adrenomedullin (AM) was originally isolated from a human pheochromocytoma as a potent vasodilatory peptide that could elevate levels of platelet cAMP [3]. In addition to hypotension and other properties, AM regulates cellular growth and differentiation, stimulates angiogenesis, and modulates hormone secretion. AM also appears to inhibit inflammation and support tissue homeostasis by suppressing the synthesis of pro-inflammatory cytokines and promoting wound healing [4]. In fact, AM ameliorates several types of colitis induced in animal models, and because it is an endogenous biologically active peptide, it should be a safe and effective drug for the treatment of IBD.

This chapter presents general information about AM, its physiological and pathophysiological roles within gastrointestinal organs, and its therapeutic effects in animal models with colitis and in patients with refractory UC. 


\section{Basic Characteristics of AM}

AM is a biologically active peptide that was initially isolated from a human pheochromocytoma arising from the adrenal medulla [3]. The human AM gene is located at the distal end of the short arm of chromosome 11 (p15.1-3) [5]. The AM protein expressed in humans is composed of 52 amino acids (Fig. 1) and it belongs to the calcitonin peptide superfamily, which also includes calcitonin gene-related peptide (CGRP), amylin, and adrenomedullin-2/intermedin [6]. Functional AM or CGRP receptors comprise calcitonin receptor-like receptor (CRLR) and a receptor activity-modifying protein (RAMP) [7]. Three types of RAMP are expressed in mammalian tissue, and CRLR can function as either a CGRP receptor or an AM receptor, depending on the co-expressed RAMP subtype (Fig. 1).

The precursor of human AM (human preproAM) comprises 185 amino acids and includes the AM sequence [8]. In addition to AM, proadrenomedullin (proAM) contains a novel 20-residue peptide called proadrenomedullin N-terminal 20 peptide (PAMP), which is also processed from the AM precursor (Fig. 1), but PAMP is not discussed here.

$\mathrm{AM}$ is essentially ubiquitous in human tissues. Immunoreactive AM has been identified in cardiovascular, renal, respiratory, gastrointestinal, reproductive,

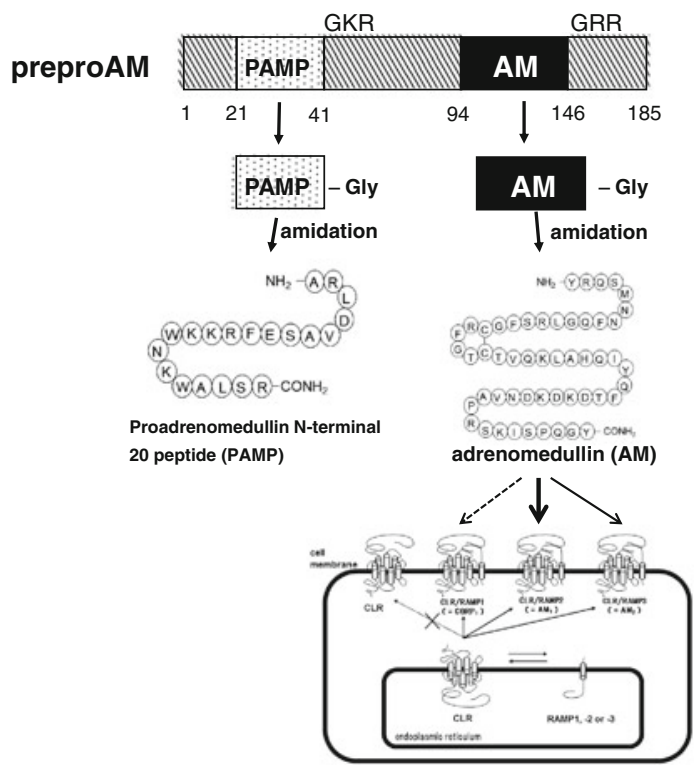

Fig. 1 Processing and biosynthesis of AM and PAMP from preproAM and relationships between $\mathrm{AM}$ and its receptor. When processed from preproAM, AM-Gly, an intermediate form (iAM), is produced and converted by an amidation enzyme to mature AM (mAM) with an amide structure at the C-terminal [8]. The mature form of PAMP is thought to be produced via a similar process. CRLR serves as a CGRP receptor when co-expressed with RAMP1, and as an AM type 1 or 2 (AM1 or AM2) receptor when co-expressed with either RAMP2 or RAMP3, respectively [7] 
neurological, endocrine, and immune tissues [9]. AM is produced in stromal as well as parenchymal cells, and numerous factors can influence its synthesis. For example, cardiovascular hormones such as angiotensin II and endothelin increase AM production in vascular smooth muscle cells (VSMCs). The production of AM is also regulated by physical factors such as shear stress, ventricular wall stress, and hypoxia, as well as by inflammatory cytokines such as tumor necrosis factor (TNF)- $\alpha$ and interleukin (IL)-1 [11]. Lipopolysaccharide (LPS) also stimulates the synthesis and secretion of AM in endothelial cells, VSMCs, and various other cell types [10].

AM has generally been characterized as a cardiovascular hormone. Consistent with such a classification, AM is an essential cardiovascular peptide involved in regulating the circulation and controlling body fluids. In addition, AM reportedly exerts angiogenic, lymphangiogenic, antioxidant, tissue protective and reparative, and anti-inflammatory effects. These multiple biological activities of AM have been described in two recent reviews $[4,11]$.

\section{Pathophysiological Roles of AM in Inflammation}

Plasma AM concentrations are elevated in patients with hypertension, congestive heart failure, myocardial infarction, or renal diseases, and during the acute phase of stroke [12-14]. Two molecular forms of AM circulate in the blood of humans and rats; one is a mature form of AM with an amidated C-terminal (AM-NH2), and the other is an intermediate form with a non-amidated C-terminal glycine (AM-Gly). Plasma and tissue AM concentrations have been measured in several studies, but most of them detected immunoreactive AM as total AM comprising AM-NH2 and AM-Gly $[12,13,15]$. Figure 2 shows a comparison of plasma mature and total AM concentrations in several diseases. Both mature and total AM levels in plasma are increased in arthritis and inflammatory bowel diseases in addition to hypertension and renal diseases.
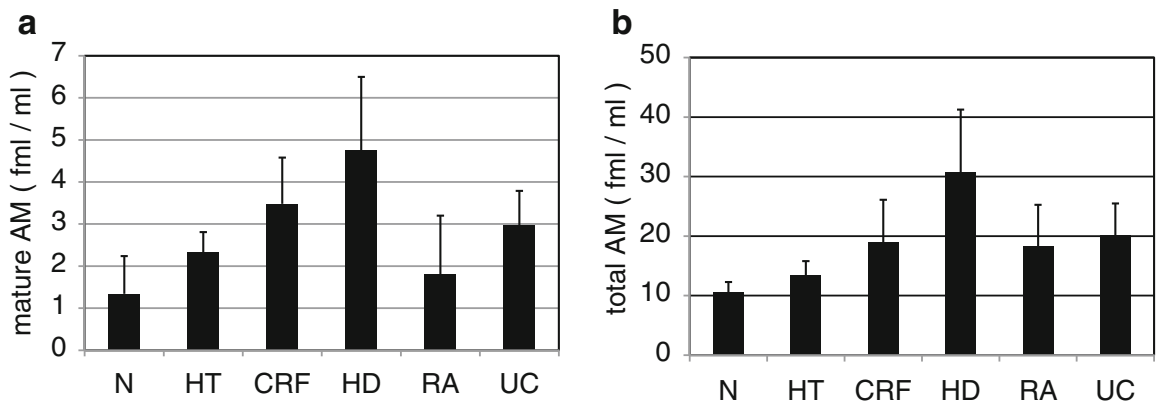

Fig. 2 Comparison of plasma concentrations of mature (a) and total(b) AM in healthy volunteers and patients with hypertension, renal failure, and inflammatory disorders (Modified and redrawn from Ohta et al. [12, 15]) 
Given its biological activity as a circulation control factor with potent hypotensive and natriuretic activity, elevated AM in cardiovascular disease might inhibit disease progression. Plasma AM concentrations are also increased in patients with septic shock [16, 17], and we also found high levels of plasma AM in patients with UC (Fig. 2). Pro-inflammatory cytokines such as TNF- $\alpha$ and IL-1, and LPS can induce AM synthesis. Conversely, AM causes the downregulation of inflammatory cytokines in Swiss 3 T3 fibroblasts [18] and rat alveolar macrophages [19]. In addition, AM possesses immunomodulatory activities that downregulate inflammatory processes in various models, including those of arthritis [20] and pancreatitis [21]. These findings suggest that beyond having vasorelaxant activity, AM might negatively regulate the inflammatory response. Consistent with this notion, AM secretion and plasma levels are both increased in models of endotoxic shock [22]. Furthermore, heterozygous AM knockout mice are significantly more sensitive to endotoxic shock than wild-type mice [23], whereas transgenic mice overexpressing AM are resistant to endotoxic shock [24]. Taken together, these findings suggest that AM is an anti-inflammatory peptide that exerts protective effects in the context of endotoxic shock and inflammatory disorders.

$\mathrm{AM}$ is also widely distributed in the gastrointestinal tract, and is expressed at high levels in the stomach and colon. Immunohistochemical analysis has revealed AM-immunoreactive (IR) cells in the pyloric glands, where they also stain positively for chromogranin A and gastrin [25]. AM-IR cells are also found in the mucosal and glandular epithelia of the digestive tract, as well as in the endocrine and neuroendocrine systems [9]. Tissues surrounding gastric ulcers stain intensely for AM [26], and $\mathrm{AM}$ expression in these regions tends to increase during the healing and scarring stages of gastric ulcers, suggesting that AM is involved in gastric mucosal healing. Indeed, AM has significant ulcer-healing activities in animal models of gastric ulcers $[27,28]$. The mechanism through which AM stimulates ulcer healing is presumed to involve the preservation of gastric blood flow, the inhibition of gastric acid secretion, the stimulation of angiogenesis, and the proliferation of mucosal epithelial cells. On the other hand, AM expression is increased in human colon cancer cells [29, 30]. Thus, AM may contribute to the pathogenesis of colon cancer through its ability to stimulate angiogenesis and cell proliferation.

\section{Preclinical Pharmacological Effect of AM in Experimental Model of Colitis}

As summarized above, AM apparently exerts anti-inflammatory effects by inhibiting the expression of TNF- $\alpha$ and IL- $1 \beta$, and it protects against experimentally induced ulcers of the gastric mucosa. AM immunoreactivity has been detected throughout the gastrointestinal tract, with concentrations being comparably high in the stomach and colon [25]. Although little is understood about the physiological and pathophysiological functions of AM in the gastrointestinal tract, these findings suggest that endogenously expressed AM in the colon could play an important 

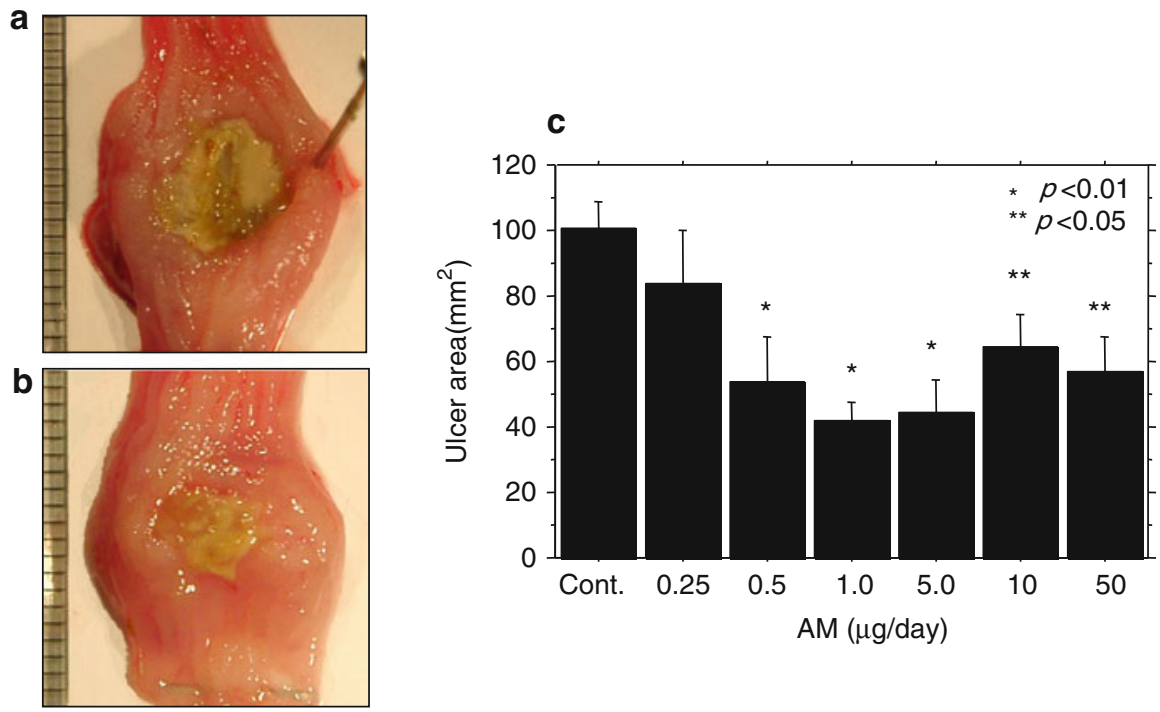

Fig. 3 Macroscopic and histological appearance of colonic ulcers induced by subserosal injection of acetic acid. Rats were treated with saline (a) or $\mathrm{AM}(\mathbf{b})$ for 5 days. Rats given AM had milder ulceration and less edema. Effects of AM $(0.25-50.0 \mu \mathrm{g} /$ day $)$ on acetic acid-induced colonic ulcers at 5 days after induction (c) ( $n=5$ per group). AM dose-dependently reduced the ulcer area, but this effect was diminished at higher doses (Modified and redrawn from Ashizuka et al. [31])

protective role against inflammatory bowel diseases. To test this hypothesis, we assessed the effect of synthetic AM on acetic acid-induced colitis and cytokine production [31]. Colitis was induced using the method of Kojima et al. [32], after which AM or saline was administered anally once a day for 3-10 days. The synthetic AM significantly reduced the size and severity of the ulcerative lesions and also reduced edema (Fig. 3a, b). Microscopically, less severe ulceration, reduced edema, and milder infiltration of the lamina propria by inflammatory cells were found in samples of colonic mucosa from rats treated with AM. Furthermore, IL-6 levels in the affected tissues were significantly lower in AM-treated rats than in control rats. On the other hand, AM did not significantly affect levels of interferon- $\gamma$ (IFN- $\gamma$ ). The ability of AM to reduce areas of ulceration was dose dependent, with the beneficial effect of AM waning somewhat at higher doses (Fig. 3c).

We further evaluated the effects of AM and its mechanism of action in dextran sulfate sodium (DSS)-induced colitis, a popular experimental model of UC, focusing on epithelial barrier function $[33,34]$. Control mice given DSS developed profound and sustained weight loss with symptoms of diarrhea and hematochezia (a major symptom of colitis) whereas body weight was maintained and clinical symptoms were suppressed in mice treated with AM. Histological analysis of tissue samples collected from control mice on day 10 after DSS administration revealed remarkable thickening of the colonic wall with pronounced crypt abscesses, 
endothelial erosions, and destroyed epithelial integrity. In contrast, the mice treated with AM exhibited far less severe histological manifestations, although the colon wall had become mildly thickened. Intestinal intraepithelial T lymphocytes (IELs) are essential for maintaining epithelial function and preventing inflammatory responses, and we found that IELs isolated from the large intestine of AM-treated mice consistently produced less IFN- $\gamma$, TNF- $\alpha$, and IL- 6 than those from control mice, and that production of transforming growth factor- $\beta$ (TGF- $\beta$ ) was induced. The induction of DSS colitis leads to changes in IEL profiles, with a relative decrease in the numbers of T cell receptor (TCR) $\gamma \delta+$ cells that express TGF- $\beta$ and might suppress inflammation by acting as regulatory $\mathrm{T}$ cells. Treating mice with AM prevents the TCR $\gamma \delta$ IEL population from becoming reduced, which would in turn inhibit the production of inflammatory cytokines and increase the production of TGF- $\beta$, thus suppressing signal transducer and activator of transcription (STAT)-3 and STAT-1 activation in epithelial cells. The mRNA expression of junctional molecules that contribute to the structure and function of tight junctions is obviously reduced in DSS colitis. However, such a reduction is much smaller in AM-treated mice, which might contribute to the suppression of the disease trajectory.

AM also exerts potent activity against various bacteria $[35,36]$ - for example, Escherichia coli-and the fact that the AM distribution within the mucosa is very similar to that of the defensin family of proteins [36] suggests that AM may also help defend against infection. In addition, the gastrointestinal tracts of AM-treated mice contain significantly fewer bacterial anaerobes than those of control mice [33]. The numbers of facultative anaerobes reportedly correlate with IBD activity in humans [37] and thus AM may protect against disruption of the mucosal epithelium through the suppression and translocation of anaerobes in the intestinal mucosa.

Reports from other laboratories also suggest that AM exerts beneficial effects against experimental models of colitis. For example, AM suppresses the inflammatory response and mediates the partial regeneration of mucosal immune tolerance in a model of TNBS-induced colitis, which reflects severe IBD [38]. In addition, $\mathrm{AM}$ is an efficient counter-regulatory agent that protects and improves the microcirculation that becomes adversely affected by cyclooxygenase (COX)-2 and inducible nitric oxide synthase (iNOS)/NO abnormalities during inflammation $[39,40]$. Hayashi et al. recently reported that AM ameliorates DSS-induced colitis, possibly via suppression of the systemic and local production of cytokines, such as TNF- $\alpha$ and IL-6, which accelerate ulcer healing and colonic mucosal regeneration [41]. AM also appears to reduce inflammatory indices and histological inflammation in DSS-induced murine colitis, and these beneficial effects are associated with mucosal protection through the fine tuning of hypoxia-induced factor (HIF) activity [42].

Given its effectiveness in these experimental models of colitis, we suggest that AM has potential as a novel agent for the treatment of IBD, and that it works by exerting anti-inflammatory and antibacterial effects and by stimulating mucosal regeneration and helping to maintain the colonic epithelial barrier. 


\section{Exploratory Clinical Study of AM as a Therapeutic Agent for Refractory Ulcerative Colitis}

AM confers benefits upon patients with cardiovascular diseases such as myocardial infarction, congestive heart failure, hypertension, pulmonary hypertension, and limb ischemia. The first clinical pilot study of intravenous AM in patients with acute myocardial infarction [43] found that an infusion of $12.5-25 \mathrm{ng} / \mathrm{kg} / \mathrm{min}$ of AM for $12 \mathrm{~h}$ did not significantly affect the hemodynamic parameters of the patients, but significantly improved the wall motion index in infarct areas at 3 months postinfarction, compared with baseline. Intravenously infused AM thus appears to exert potentially protective cardiovascular effects without severe adverse effects, which allows it to serve as a possible adjunct to percutaneous coronary intervention.

We found that AM infusions cause similar and steady decreases in blood pressure (Fig. 4a) and several markers of arteriosclerosis (such as pulse wave velocity) in patients with essential hypertension and primary aldosteronism (PA) [44, 45]. The infused AM suppressed aldosterone release to values within the normal range in the PA group, but had limited effect in the control group and did not alter adrenocorticotropic hormone-cortisol system in both groups (Fig. 4b). An infusion of AM $(15 \mathrm{ng} / \mathrm{kg} / \mathrm{min})$ induced an increase of circulating C-reactive protein (CRP) in all participants, which suggests that AM can inhibit or stimulate inflammation, depending upon the milieu. Consistent with this notion, AM downregulates TNF- $\alpha$, an important mediator of cytokine production, in macrophages and Swiss 3 T3 cells [18, 19], but stimulates IL-6 production in NR8383 and Swiss 3 T3 cells [46, 47]. Furthermore, bell-shaped dose-response curves (Fig. 3c) suggest that the beneficial effect of AM on ulcerative area is dose dependent, and that this effect tends to wane at higher doses. This means that an AM dose of $15 \mathrm{ng} / \mathrm{kg} / \mathrm{min}$ is too high for treating

a

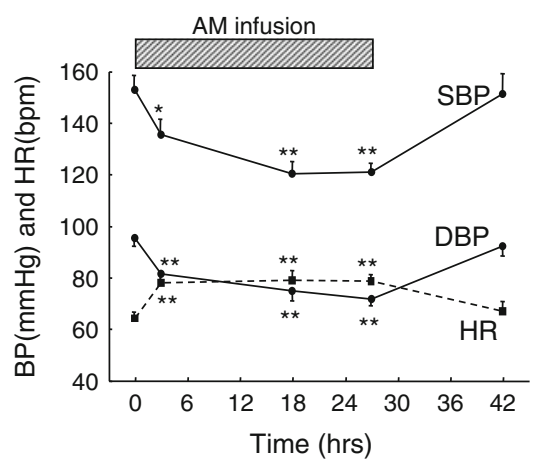

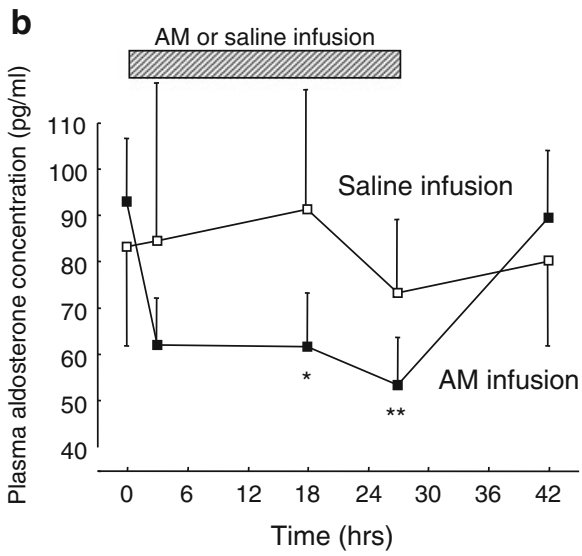

Fig. 4 Changes in blood pressure (a) and plasma aldosterone levels (b) during infusion of AM or vehicle in patients with essential hypertension and primary aldosteronism (Modified and redrawn from Ashizuka et al. [48]) 
IBD. Therefore, we clinically treated UC by administering AM at a dose of $9 \mathrm{ng} / \mathrm{kg} /$ min, which does not evoke severe hypotension or stimulate CRP production. Below, we describe the first patient with UC whom we treated using AM. The excellent results indicated that AM can effectively treat refractory UC [48, 34].

A 68-year-old woman who was undergoing treatment for diabetes presented with a 3-year history of refractory UC. During previous flare-ups, clinical remission was achieved using a high-dose steroid infusion and leukocytapheresis, but insufficient mucosal repair required a regimen of continuous prednisolone (PSL) with azathioprine (AZA). However, the patient's condition worsened, with symptoms of severe abdominal pain and bloody stools. Colonoscopy revealed deep ulcerations and erosions throughout the large intestine (Fig. 5a). Higher doses of PSL in combination with leukocytapheresis failed to induce remission (Ulcerative Colitis Disease Activity Index (UCDAI) score:7). After ischemic heart disease, cerebrovascular disease, and malignancy were ruled out, AM (9 ng/ $/ \mathrm{kg} / \mathrm{min})$ was intravenously administered for 12 days ( $8 \mathrm{~h} /$ day), which alleviated the abdominal pain and bloody stool after a few days. No adverse effects occurred except for a slight decline in blood pressure. Colonoscopy 2 weeks later revealed significant mucosal regeneration (Fig. 5b), which gastroenterologists had never encountered before, and the patient's UCDAI score had declined to 2. Colonoscopy 3 months later revealed that

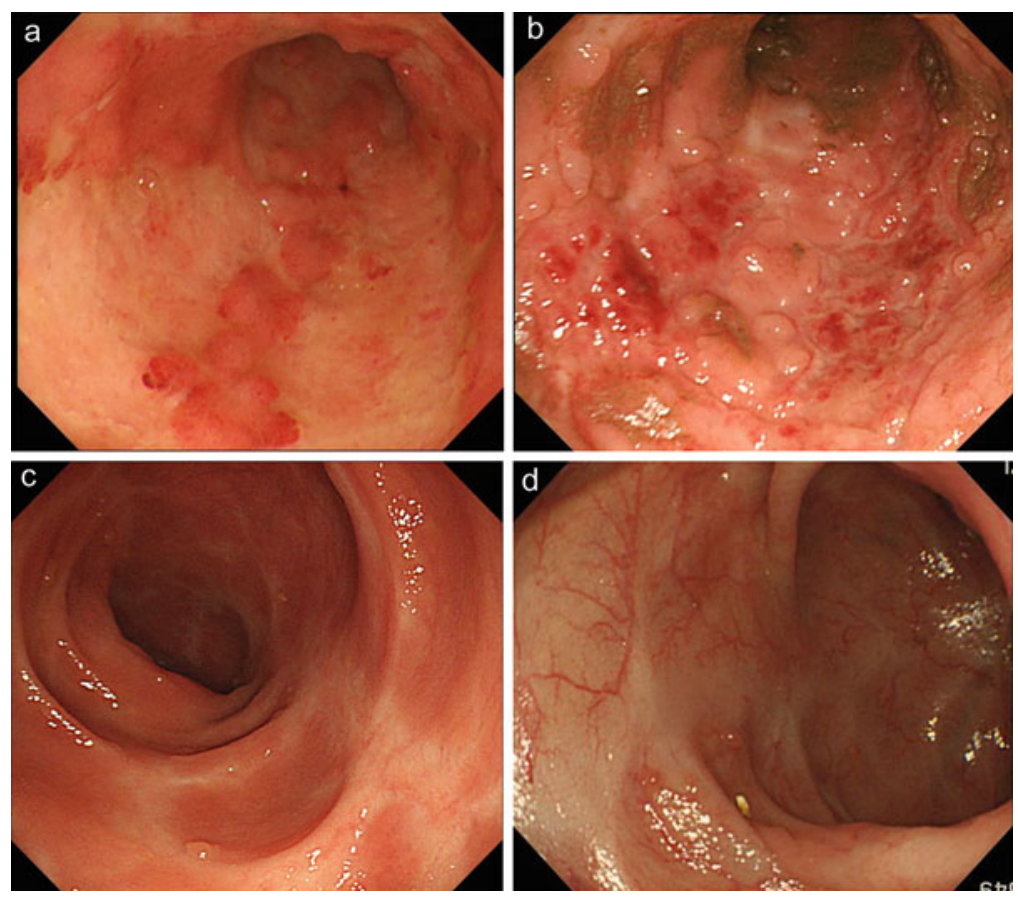

Fig. 5 Colonoscopy findings: (a) before; (b) 2 weeks after; (c) 3 months after; and (d) 12 months after treatment with AM (Modified from Ashizuka et al. [48]) 
all of the colonic lesions had healed with scarring and the patient's UCDAI score had reached 0, which prompted withdrawal of the PSL (Fig. 5a). Thus, AM appears to be effective against refractory UC in animal models of colitis and for patients with refractory UC.

Table 1 summarizes the comparison of AM therapy and existing methods currently used to treat IBD that all act through the induction and maintenance of remission [2, 34]. These include aminosalicylates (5-ASA), steroids (prednisolone), immune modifiers (azathioprine, 6-MP and methotrexate), and biologics (inflix-

Table 1 Comparison of AM therapy and methods currently used to treat IBD

\begin{tabular}{|c|c|c|c|}
\hline Agent & Mechanism & Merit & Demerit \\
\hline $\begin{array}{l}\text { Anti-inflammatory } \\
\text { agent }\end{array}$ & $\begin{array}{l}\text { Inhibition of LTB4 } \\
\text { production }\end{array}$ & \multirow[t]{2}{*}{ Safety } & \multirow[t]{2}{*}{$\begin{array}{l}\text { Insufficient for severe } \\
\text { UC }\end{array}$} \\
\hline SASP/5-ASA & $\begin{array}{l}\text { Removal of reactive } \\
\text { oxygen }\end{array}$ & & \\
\hline Hormonal agent & $\begin{array}{l}\text { Inhibition of inflammatory } \\
\text { cytokines }\end{array}$ & \multirow[t]{3}{*}{$\begin{array}{l}\text { Potent anti- } \\
\text { inflammatory } \\
\text { effect }\end{array}$} & $\begin{array}{l}\text { Cumulative toxicity } \\
\text { (metabolic } \\
\text { abnormality) }\end{array}$ \\
\hline \multirow[t]{2}{*}{ Predonisolone } & $\begin{array}{l}\text { Inhibition of cell } \\
\text { proliferation and } \\
\text { differentiation }\end{array}$ & & \multirow[t]{2}{*}{$\begin{array}{l}\text { Susceptibility to } \\
\text { infection }\end{array}$} \\
\hline & $\begin{array}{l}\text { Inhibition of cell } \\
\text { infiltration }\end{array}$ & & \\
\hline Immunosuppressor & $\begin{array}{l}\text { Inhibition of DNA } \\
\text { synthesis }\end{array}$ & \multirow[t]{3}{*}{$\begin{array}{l}\text { Maintenance of } \\
\text { remission }\end{array}$} & Myelosuppression \\
\hline \multirow[t]{2}{*}{ Azathioprine/6MP } & \multirow[t]{2}{*}{ Immunosuppressor } & & $\begin{array}{l}\text { Susceptibility to } \\
\text { infection }\end{array}$ \\
\hline & & & Malignant lymphoma? \\
\hline Immunosuppressor & \multirow[t]{3}{*}{ Inhibition of IL-2 } & \multirow{3}{*}{$\begin{array}{l}\text { Potent anti- } \\
\text { inflammatory } \\
\text { effect }\end{array}$} & $\begin{array}{l}\text { Monitoring the } \\
\text { concentration in blood }\end{array}$ \\
\hline \multirow[t]{2}{*}{$\begin{array}{l}\text { Cyclosporine, } \\
\text { Tacrolims }\end{array}$} & & & $\begin{array}{l}\text { Susceptibility to } \\
\text { infection }\end{array}$ \\
\hline & & & $\begin{array}{l}\text { Finger tremor, Renal } \\
\text { damage }\end{array}$ \\
\hline \multirow[t]{2}{*}{ Anti-cytokine agent } & \multirow[t]{3}{*}{ Anti-TNF- $\alpha$} & \multirow{3}{*}{$\begin{array}{l}\text { Potent anti- } \\
\text { inflammatory } \\
\text { effect }\end{array}$} & Tachyphylaxis, Allergy \\
\hline & & & $\begin{array}{l}\text { Susceptibility to } \\
\text { infection (tuberculosis) }\end{array}$ \\
\hline $\begin{array}{l}\text { Infliximab, } \\
\text { Adalimumab }\end{array}$ & & & Malignant lymphoma? \\
\hline $\begin{array}{l}\text { Blood component } \\
\text { removal }\end{array}$ & \multirow[t]{2}{*}{$\begin{array}{l}\text { Removal of activated } \\
\text { leukocyte }\end{array}$} & \multirow[t]{2}{*}{ Safety } & $\begin{array}{l}\text { Delayed effect } \\
(\sim 2 \text { weeks })\end{array}$ \\
\hline LCAP/GMA & & & Troublesome \\
\hline Endogenous peptide & Mucosal healing & Safety & \multirow[t]{3}{*}{ Hypotensive effect } \\
\hline \multirow[t]{2}{*}{ Adrenomedullin } & $\begin{array}{l}\text { Inhibition of inflammatory } \\
\text { cytokines }\end{array}$ & \multirow[t]{2}{*}{ New mechanism } & \\
\hline & $\begin{array}{l}\text { Angiogenesis, } \\
\text { Amelioration of ischemia }\end{array}$ & & \\
\hline
\end{tabular}


imab). However, the results obtained with these medications are not completely satisfactory in about one quarter of UC patients, as their effects are either insufficient or lead to complications. Surgical treatment (colectomy) may be considered for such patients.

In addition to conventional steroid therapy, immune modifiers or biologics are commonly used to treat steroid-resistant or steroid-dependent refractory UC. Although these therapies can be effective, potentially serious infections such as fungal diseases and tuberculosis are a concern, especially in patients with diabetes mellitus. In addition, immune modifiers can cause malignant lymphoma in young patients. Therefore, new and effective drugs that lack these adverse side effects are highly desirable. We found that a continuous intravenous infusion of AM ameliorated the symptoms of intractable UC without adverse side effects. Moreover, endoscopic evaluation after AM therapy showed remarkable mucosal regeneration and healing with neovascularization at previously ulcerative or erosive lesions. These results suggest that AM is a potentially useful therapeutic agent with a novel mechanism of action that involves anti-inflammatory effects with mucosal and vascular regeneration.

\section{Conclusion}

Here, we have provided general information about AM, its physiological and pathophysiological roles in the gastrointestinal tract, and translational research demonstrating the safety and value of AM for treating refractory UC. AM is a multifunctional cardiovascular hormone that exerts curative effects on cardiocirculatory dynamics. Thus, AM has been investigated mainly in the context of cardiovascular disease. However, AM can also protect organs and regenerate tissues, and it exerts anti-inflammatory and angiogenic effects while stimulating epithelial and mucosal cell proliferation. This suggests that AM could help to treat various diseases in addition to cardiovascular disease. With respect to its use in the treatment of refractory $\mathrm{UC}$, AM has no antigenicity because it is an endogenous peptide. Moreover, its mode of action differs from those of existing immunomodulators such as steroids, immune modifiers, and biologics. Additional study is required to confirm whether or not AM could treat refractory UC.

Acknowledgments This study was supported in part by the 8th Specified Research Grant from the Uehara Memorial Foundation; Grants-in-Aid for Scientific Research from the Ministry of Education, Culture, Sports, Science and Technology of Japan; and Health and Labor Science Research Grants for Translational Research from the Ministry of Health, Labor and Welfare, Japan.

Open Access This chapter is distributed under the terms of the Creative Commons Attribution Noncommercial License, which permits any noncommercial use, distribution, and reproduction in any medium, provided the original author(s) and source are credited. 


\section{References}

1. Latella G, Papi C (2012) Crucial steps in the natural history of inflammatory bowel disease. World J Gastroenterol: WJG 18(29):3790-3799. doi:10.3748/wjg.v18.i29.3790

2. Engel MA, Khalil M, Neurath MF (2012) Highlights in inflammatory bowel disease-from bench to bedside. Clin Chem Lab Med: CCLM/FESCC 50(7):1229-1235

3. Kitamura K, Kangawa K, Kawamoto M, Ichiki Y, Nakamura S, Matsuo H, Eto T (1993) Adrenomedullin: a novel hypotensive peptide isolated from human pheochromocytoma. Biochem Biophys Res Commun 192(2):553-560. doi:10.1006/bbrc.1993.1451

4. Cheung BM, Tang F (2012) Adrenomedullin: exciting new horizons. Recent Pat Endocr Metab Immune Drug Discovery 6(1):4-17

5. Ishimitsu T, Kojima M, Kangawa K, Hino J, Matsuoka H, Kitamura K, Eto T, Matsuo H (1994) Genomic structure of human adrenomedullin gene. Biochem Biophys Res Commun 203(1):631-639. doi:10.1006/bbrc.1994.2229

6. Hong Y, Hay DL, Quirion R, Poyner DR (2012) The pharmacology of adrenomedullin 2/intermedin. Br J Pharmacol 166(1):110-120. doi:10.1111/j.1476-5381.2011.01530.x

7. McLatchie LM, Fraser NJ, Main MJ, Wise A, Brown J, Thompson N, Solari R, Lee MG, Foord SM (1998) RAMPs regulate the transport and ligand specificity of the calcitonin-receptor-like receptor. Nature 393(6683):333-339. doi:10.1038/30666

8. Kitamura K, Sakata J, Kangawa K, Kojima M, Matsuo H, Eto T (1993) Cloning and characterization of cDNA encoding a precursor for human adrenomedullin. Biochem Biophys Res Commun 194(2):720-725. doi:10.1006/bbrc.1993.1881

9. Asada Y, Hara S, Marutsuka K, Kitamura K, Tsuji T, Sakata J, Sato Y, Kisanuki A, Eto T, Sumiyoshi A (1999) Novel distribution of adrenomedullin-immunoreactive cells in human tissues. Histochem Cell Biol 112(3):185-191

10. Minamino N, Kikumoto K, Isumi Y (2002) Regulation of adrenomedullin expression and release. Microsc Res Tech 57(1):28-39. doi:10.1002/jemt.10048

11. Kuwasako K, Kitamura K, Nagata S, Hikosaka T, Takei Y, Kato J (2011) Shared and separate functions of the RAMP-based adrenomedullin receptors. Peptides 32(7):1540-1550. doi:10.1016/j.peptides.2011.05.022

12. Ohta H, Tsuji T, Asai S, Tanizaki S, Sasakura K, Teraoka H, Kitamura K, Kangawa K (1999) A simple immunoradiometric assay for measuring the entire molecules of adrenomedullin in human plasma. Clin Chim Acta; Int J Clin Chem 287(1-2):131-143

13. Hirayama N, Kitamura K, Imamura T, Kato J, Koiwaya Y, Tsuji T, Kangawa K, Eto T (1999) Molecular forms of circulating adrenomedullin in patients with congestive heart failure. J Endocrinol 160(2):297-303

14. Kato J, Kobayashi K, Etoh T, Tanaka M, Kitamura K, Imamura T, Koiwaya Y, Kangawa K, Eto T (1996) Plasma adrenomedullin concentration in patients with heart failure. J Clin Endocrinol Metab 81(1):180-183. doi:10.1210/jcem.81.1.8550749

15. Ohta H, Tsuji T, Asai S, Sasakura K, Teraoka H, Kitamura K, Kangawa K (1999) One-step direct assay for mature-type adrenomedullin with monoclonal antibodies. Clin Chem 45(2):244-251

16. Nishio K, Akai Y, Murao Y, Doi N, Ueda S, Tabuse H, Miyamoto S, Dohi K, Minamino N, Shoji H, Kitamura K, Kangawa K, Matsuo H (1997) Increased plasma concentrations of adrenomedullin correlate with relaxation of vascular tone in patients with septic shock. Crit Care Med 25(6):953-957

17. Ueda S, Nishio K, Minamino N, Kubo A, Akai Y, Kangawa K, Matsuo H, Fujimura Y, Yoshioka A, Masui K, Doi N, Murao Y, Miyamoto S (1999) Increased plasma levels of adrenomedullin in patients with systemic inflammatory response syndrome. Am J Respir Crit Care Med 160(1):132-136. doi:10.1164/ajrccm.160.1.9810006

18. Isumi Y, Kubo A, Katafuchi T, Kangawa K, Minamino N (1999) Adrenomedullin suppresses interleukin-1beta-induced tumor necrosis factor-alpha production in Swiss 3 T3 cells. FEBS Lett 463(1-2):110-114 
19. Kamoi H, Kanazawa H, Hirata K, Kurihara N, Yano Y, Otani S (1995) Adrenomedullin inhibits the secretion of cytokine-induced neutrophil chemoattractant, a member of the interleukin-8 family, from rat alveolar macrophages. Biochem Biophys Res Commun 211(3):1031-1035. doi:10.1006/bbrc.1995.1914

20. Gonzalez-Rey E, Chorny A, O'Valle F, Delgado M (2007) Adrenomedullin protects from experimental arthritis by down-regulating inflammation and Th1 response and inducing regulatory T cells. Am J Pathol 170(1):263-271. doi:10.2353/ajpath.2007.060596

21. Onur OE, Guneysel O, Akoglu H, Denizbasi A, Onur E (2007) Adrenomedullin reduces the severity of cerulein-induced acute pancreatitis. Peptides 28(11):2179-2183. doi:10.1016/j. peptides.2007.08.028

22. Matsui E, Kitamura K, Yoshida M, Kato J, Asada Y, Sumiyoshi A, Eto T (2001) Biosynthesis and secretion of adrenomedullin and proadrenomedullin $\mathrm{N}$-terminal 20 peptide in a rat model of endotoxin shock. Hypertens Res: Off J Jpn Soc Hypertens 24(5):543-549

23. Saito R, Shimosawa T, Ogihara T, Maruyama N, Fujita T, Okamura N, Nakahara K (2012) Function of adrenomedullin in inflammatory response of liver against LPS-induced endotoxemia. APMIS: Acta Pathol Microbiol Immunol Scand 120(9):706-711. doi:10.1111/j.1600-0463.2012.02892.x

24. Shindo T, Kurihara H, Maemura K, Kurihara Y, Kuwaki T, Izumida T, Minamino N, Ju KH, Morita H, Oh-hashi Y, Kumada M, Kangawa K, Nagai R, Yazaki Y (2000) Hypotension and resistance to lipopolysaccharide-induced shock in transgenic mice overexpressing adrenomedullin in their vasculature. Circulation 101(19):2309-2316

25. Washimine H, Asada Y, Kitamura K, Ichiki Y, Hara S, Yamamoto Y, Kangawa K, Sumiyoshi A, Eto T (1995) Immunohistochemical identification of adrenomedullin in human, rat, and porcine tissue. Histochem Cell Biol 103(4):251-254

26. Hashimoto H, Akimoto M, Maeda A, Shigemoto M, Yamashita K, Yokoyama I (2000) Changes in vasoactive substances during gastric ulcer healing. J Cardiovasc Pharmacol 36(5 Suppl 1):S278-S281

27. Cantarella G, Martinez G, Cutuli VM, Loreto C, D'Alcamo M, Prato A, Amico-Roxas M, Bernardini R, Clementi G (2005) Adrenomedullin modulates COX-2 and HGF expression in reserpine-injuried gastric mucosa in the rat. Eur J Pharmacol 518(2-3):221-226. doi:10.1016/j. ejphar.2005.06.001

28. Hirsch AB, McCuen RW, Arimura A, Schubert ML (2003) Adrenomedullin stimulates somatostatin and thus inhibits histamine and acid secretion in the fundus of the stomach. Regul Pept 110(3):189-195

29. Hikosaka T, Tsuruda T, Nagata S, Kuwasako K, Tsuchiya K, Hoshiko S, Inatsu H, Chijiiwa K, Kitamura K (2011) Adrenomedullin production is increased in colorectal adenocarcinomas; its relation to matrix metalloproteinase-9. Peptides 32(9):1825-1831. doi:10.1016/j.peptides.2011.07.012

30. Kim JY, Park WD, Lee S, Park JH (2012) Adrenomedullin is involved in the progression of colonic adenocarcinoma. Mol Med Rep 6(5):1030-1034. doi:10.3892/mmr.2012.1045

31. Ashizuka S, Ishikawa N, Kato J, Yamaga J, Inatsu H, Eto T, Kitamura K (2005) Effect of adrenomedullin administration on acetic acid-induced colitis in rats. Peptides 26(12):2610-2615. doi:10.1016/j.peptides.2005.05.007

32. Kojima R, Hamamoto S, Moriwaki M, Iwadate K, Ohwaki T (2001) [The new experimental ulcerative colitis model in rats induced by subserosal injection of acetic acid]. Nihon Yakurigaku Zasshi Folia Pharmacol Jpn 118(2):123-130

33. Ashizuka S, Inagaki-Ohara K, Kuwasako K, Kato J, Inatsu H, Kitamura K (2009) Adrenomedullin treatment reduces intestinal inflammation and maintains epithelial barrier function in mice administered dextran sulphate sodium. Microbiol Immunol 53(10):573-581. doi:10.1111/j.1348-0421.2009.00159.x

34. Ashizuka S, Inatsu H, Inagaki-Ohara K, Kita T, Kitamura K (2013) Adrenomedullin as a potential therapeutic agent for inflammatory bowel disease. Curr Protein Pept Sci 14(4):246-255

35. Marutsuka K, Nawa Y, Asada Y, Hara S, Kitamura K, Eto T, Sumiyoshi A (2001) Adrenomedullin and proadrenomudullin N-terminal 20 peptide (PAMP) are present in human colonic epithelia and exert an antimicrobial effect. Exp Physiol 86(5):543-545 
36. Allaker RP, Grosvenor PW, McAnerney DC, Sheehan BE, Srikanta BH, Pell K, Kapas S (2006) Mechanisms of adrenomedullin antimicrobial action. Peptides 27(4):661-666. doi:10.1016/j.peptides.2005.09.003

37. Cummings JH, Macfarlane GT, Macfarlane S (2003) Intestinal bacteria and ulcerative colitis. Curr Issues Intest Microbiol 4(1):9-20

38. Gonzalez-Rey E, Fernandez-Martin A, Chorny A, Delgado M (2006) Therapeutic effect of urocortin and adrenomedullin in a murine model of Crohn's disease. Gut 55(6):824-832. doi:10.1136/gut.2005.084525

39. Talero E, Alvarez de Sotomayor M, Sanchez-Fidalgo S, Motilva V (2011) Vascular contribution of adrenomedullin to microcirculatory improvement in experimental colitis. Eur J Pharmacol 670(2-3):601-607. doi:10.1016/j.ejphar.2011.09.032

40. Talero E, Sanchez-Fidalgo S, de la Lastra CA, Illanes M, Calvo JR, Motilva V (2008) Acute and chronic responses associated with adrenomedullin administration in experimental colitis. Peptides 29(11):2001-2012. doi:10.1016/j.peptides.2008.07.013

41. Hayashi Y, Narumi K, Tsuji S, Tsubokawa T, Nakaya MA, Wakayama T, Zuka M, Ohshima T, Yamagishi M, Okada T (2011) Impact of adrenomedullin on dextran sulfate sodium-induced inflammatory colitis in mice: insights from in vitro and in vivo experimental studies. Int $\mathrm{J}$ Colorectal Dis 26(11):1453-1462. doi:10.1007/s00384-011-1254-0

42. MacManus CF, Campbell EL, Keely S, Burgess A, Kominsky DJ, Colgan SP (2011) Antiinflammatory actions of adrenomedullin through fine tuning of HIF stabilization. FASEB J: Off Publ Fed Am Soc Exp Biol 25(6):1856-1864. doi:10.1096/fj.10-170316

43. Kataoka Y, Miyazaki S, Yasuda S, Nagaya N, Noguchi T, Yamada N, Morii I, Kawamura A, Doi K, Miyatake K, Tomoike H, Kangawa K (2010) The first clinical pilot study of intravenous adrenomedullin administration in patients with acute myocardial infarction. J Cardiovasc Pharmacol 56(4):413-419. doi:10.1097/FJC.0b013e3181f15b45

44. Kita T, Suzuki Y, Kitamura K (2010) Hemodynamic and hormonal effects of exogenous adrenomedullin administration in humans and relationship to insulin resistance. Hypertens Res: Off J Jpn Soc Hypertens 33(4):314-319. doi:10.1038/hr.2009.236

45. Kita T, Tokashiki M, Kitamura K (2010) Aldosterone antisecretagogue and antihypertensive actions of adrenomedullin in patients with primary aldosteronism. Hypertens Res: Off J Jpn Soc Hypertens 33(4):374-379. doi:10.1038/hr.2010.8

46. Isumi Y, Minamino N, Kubo A, Nishimoto N, Yoshizaki K, Yoshioka M, Kangawa K, Matsuo H (1998) Adrenomedullin stimulates interleukin-6 production in Swiss 3 T3 cells. Biochem Biophys Res Commun 244(2):325-331. doi:10.1006/bbrc.1998.8261

47. Wong LY, Cheung BM, Li YY, Tang F (2005) Adrenomedullin is both proinflammatory and antiinflammatory: its effects on gene expression and secretion of cytokines and macrophage migration inhibitory factor in NR8383 macrophage cell line. Endocrinology 146(3):13211327. doi:10.1210/en.2004-1080

48. Ashizuka S, Kita T, Inatsu H, Kitamura K (2013) Adrenomedullin: a novel therapy for intractable ulcerative colitis. Inflamm Bowel Dis 19(2):E26-E27. doi:10.1002/ibd.22891 


\title{
RNA Activation
}

\author{
X.Y. Zhao, J. Voutila, Nagy A. Habib, and Vikash Reebye
}

\begin{abstract}
The ability to manipulate gene expression is invaluable for understanding the molecular pathogenesis of disease as well as for developing novel therapeutics. RNA interference provides a robust platform for the knockdown of a specific gene at the post-transcriptional level, but activation of specific genes traditionally has been limited to ligand-mediated activation of signal transduction pathways or introduction of exogenous transgenes from expression vectors. Recent work has shown that small RNA molecules targeted to the promoter region of a gene can activate gene expression. This phenomenon, called RNA activation, provides a tool for specific activation of endogenous genes, and introduces a new role for noncoding RNAs in the regulation of gene expression. These small RNAs are typically 21-nucleotide duplexes, and have been shown to activate a wide variety of genes in many cell types and across species. The application of this technology will prove invaluable for basic research through gain-of-function studies and potentially targeted gene activation for disease intervention. This chapter will cover what is currently defined on the mechanism of RNA activation, and will explore the possible application of this technology for novel therapeutics.
\end{abstract}

Keywords RNA activation $\bullet$ Non-coding RNA $\bullet$ Small RNA $\bullet$ Gene regulation • Regenerative medicine $\bullet$ RNA therapeutics

\section{Introduction}

Noncoding RNA (ncRNA) gained popularity as tools for loss-of-function studies by their ability to cause target transcript degradation through small inhibitory RNAs (siRNAs). The full compendium of noncoding RNAs now includes miRNA, PIWI-interacting RNA (piRNA), and long noncoding RNA (lncRNA) to carry out

X.Y. Zhao $\bullet$ N.A. Habib $(\varangle) \bullet$ V. Reebye $(\square)$

Department of Surgery and Cancer, Imperial College London, Hammersmith Hospital,

Du Cane Road, London W12 0NN, UK

e-mail: nagy.habib@imperial.ac.uk; v.reebye@imperial.ac.uk

J. Voutila

MiNA Therapeutics Limited, 96 Kensington High Street, London W8 4SG, UK

(C) The Author(s) 2015

K. Nakao et al. (eds.), Innovative Medicine, DOI 10.1007/978-4-431-55651-0_20 
crucial roles such as transcriptional, post-transcriptional, and chromatin-modifying regulators. Although ncRNAs were predominantly seen as inhibitors of gene expression, we now recognize that these small RNA strands have much more complex functions including strong transcriptional upregulation; concepts which ultimately may have implications in the etiology of cancer $[1,2]$.

Small ncRNAs are classified into three main categories, all of which modulate gene expression by targeting transcripts within the cytoplasm. These include microRNAs (miRNAs), which inhibit protein translation by base-pairing with imperfect complementarity to target sequences within mRNA molecules; PIWIinteracting RNAs (piRNAs), which are involved in gene silencing by targeting transposons in animal germ cells; and small interfering RNAs (siRNAs) or RNA interference (RNAi), which target homologous mRNA sequences to degrade transcripts, leading to post-transcriptional gene silencing [3-5]. A new addition can be made to this category, in the form of activating RNAs, the discovery of which was made serendipitously [6]. While attempting to silence E-cadherin gene transcription, using small double-stranded RNAs (dsRNAs) in human cells, Li et al. [7] observed that several promoter-targeted dsRNA molecules were able to activate gene and protein expression. A similar event was also confirmed independently by Janowski et al. [8] who demonstrated that expression of the progesterone receptor (PR) could be activated by specific dsRNAs interacting upstream of the transcription start site (TSS) within the promoter region of mammalian cells and even beyond the 3' terminus region of the PR mRNA [9]. This phenomenon of activating RNA has now adopted numerous nomenclatures across the scientific community, including RNA activation (RNAa), small activating RNAs or short-activating RNAs (saRNAs, which will be used for this chapter), and antigene RNAs (agRNAs). To date, the number of characterized saRNA targets, which include E-cadherin, PR, p21, KLF4, and p53 amongst others, demonstrate that this non-integrative approach to selectively activate target genes will undoubtedly make an invaluable contribution to gain-of-function studies in translational medicine. The future of RNA therapy will entail the ability to synthesise saRNA duplexes for desired gene targets and the loading of these therapeutic agents into appropriate carrier particles for biodistribution to target organs. This chapter will provide an overview on saRNAs and their potential introduction into therapeutic applications.

\section{Molecular Mechanism}

\section{saRNA-Associated Proteins}

\section{Argonaute Proteins}

Despite entering a decade since the first report of activating RNA was made to the scientific community, a clear and complete mechanistic understanding of how these molecules work is still being sought after. The accumulation of work over this time 
has highlighted important components that demonstrate how saRNA molecules might function. These include the orchestration of numerous RNA-binding enzymes, epigenetic changes, and recruitment of the transcriptional activation machinery. The most accepted mechanism of action currently includes the association of saRNAs with the Argonaute (Ago) protein family. Mammalian cells contain four variants of Ago. These are Ago1, Ago2, Ago3, and Ago4, and are classed as catalytic proteins which exert important biological roles in embryonic development and cell differentiation. The Ago proteins are particularly well characterized for their contribution in gene-silencing pathways guided by small RNA molecules [10]. Human Ago1, Ago3, and Ago4 are clustered on chromosome 1, whereas Ago2 is located on chromosome 8 . Although the clustering might not be indicative of independent function, Ago2 demonstrates a more prominent role in siRNA-mediated gene silencing through its ability to cleave mRNA strands [11]. During the canonical RNA interference process, siRNAs are recruited by Ago2 for the formation of RNA-induced silencing complex (RISC). Now it appears that Ago2 is also implicated in molecular events that orchestrate RNA-dependent gene activation. Its exact role has yet to be fully defined; however, it is believed that Ago2 cleaves out the passenger strand from saRNA duplexes, after which the Ago2/saRNA guide strand translocates to the nucleus $[7,12,13]$. The Ago2/saRNA complex is then believed to recognize complementary sequences on either the promoter or enhancer regions of target genes or even on nascent promoter RNA $[14,15]$. The guide strand also recruits chromatinmodifying enzymes to the cis-regulatory elements of target genes to modulate epigenetic changes and allow transcriptional activation of target genes. This multicomplex protein module regulates and stabilizes histone modification processes, which, as a result, leads to a longer duration of gene regulation when saRNA effects are compared with the more transient dynamics of siRNA [10, 16].

\section{Heterogeneous Nuclear RNA-Binding Proteins}

The saRNA guide strand mediates the interaction of functional proteins that form part of the ribonucleoprotein complex. Historically, the role of heterogeneous nuclear proteins (hnRNPs) has been known to be crucial in regulating gene transcription and other processes such as DNA and RNA strand stability and subcellular shuttling of mRNA [17-19]. HnRNPs are now known to be intricately implicated in modulating saRNA activity as demonstrated by saRNA-induced recruitment of hnRNP-K. Recently, Jia et al. discovered that hnRNPs (A1, A2/B1, and C1/C2) were involved in promoter-associated saRNA. HnRNPA2/B1, in particular, was crucially important for saRNA-induced activation of target genes in both the in vitro and in vivo settings [20-22]. Whether additional proteins are also involved is still unknown; however, defining the role of each component will undoubtedly bring us closer to understanding the nuclear mechanism of saRNA-dependent gene activation. 


\section{Histone Modification Enzymes}

The kinetics of saRNA appear to be distinct from classical RNAi. While siRNAinduced post-transcriptional gene silencing can be observed within hours after transfection and lasts for up to 7 days; the dynamics of saRNA-induced gene activation, on the other hand, reveal a typical delay of up to $48 \mathrm{~h}$ following transfection into mammalian cells [12, 16, 23, 24]. For example, Place et al. [16] noticed that MOF or E2F1 silencing by siRNA began to emerge $6 \mathrm{~h}$ following transfection, with levels subsiding by $24 \mathrm{~h}$. In contrast, E-cadherin and p 21 activation by saRNA was observed at $48 \mathrm{~h}$, with levels almost maximally increasing by $72 \mathrm{~h}$. In some cell lines, saRNA-induced gene upregulation is seen to be preserved for almost 2 weeks. These discoveries, accompanied by the observation of Janowski's team, suggest that the delayed response of saRNAs can be attributed to the more complex orchestration of events that need to occur for targeted gene activation.

saRNA initially requires shuttling into the nucleus to its intended target. The interaction of Ago2 with saRNA, as mentioned above, and subsequent cleavage of the guide strand are believed to be the key initiation processes. Once the Ago2/ saRNA guide complex accesses the gene target site together with their association with hnRNPs, a series of histone modifications is thought to occur. This observation originates from studies where agents that block histone modification appeared to suppress the effects of saRNAs, while agents that decrease acetylation of histone (H3K4), H3K14, and H3K9 appeared to upregulate saRNA-targeted upregulation of the PR gene and E-cadherin [25-29]. DNA-bound Ago2/saRNA guide might then regulate changes in DNA methylation. Since the prolonged duration of saRNAinduced gene activation occurs for up to 7 days, this suggests that major regulatory modifications ensue. This presents an attractive feature of saRNA for therapeutic use $[16,25]$.

\section{Target Site Selection of saRNA}

The recognition that RNA duplexes strongly activate transcription of target genes has unfortunately made very slow progress toward exploitation for therapeutic use. The main hurdle on this front has been the lack of a mechanistic understanding of how saRNAs induce targeted transcriptional activation, and whether the same process occurs systematically across all variants of activating RNAs or whether it refers to RNAa, agRNA, or saRNA. On the basis of existing concepts, activating RNA appears to involve components of the transcriptional and epigenetic machinery to exert their effects on target genes. However, the genomic target site of saRNA still holds subjective observations, which are sometimes contradictory. saRNAs generally demonstrate strand pattern preference, and this is guided by just one strand (either the sense or the antisense strand) [10]. The interaction of the single 'guide' strand with Ago2 is thought to be drawn toward either complementary sense strand or, as seen with the PR and low-density lipoprotein receptor (LDLR), this can 
be with the antisense transcript where the saRNA-Ago complex interacts with heterochromatic protein 1- $\gamma$ (HP1 $\gamma$ ) and RNA polymerase II (RNAPII) [30-33].

Some have suggested that saRNAs target sequences downstream from the $3^{\prime}$ untranslated region $\left(3^{\prime}\right.$-UTR) of the intended target gene. Others have suggested that saRNAs demonstrate optimal function when they overlap the TSS of the intended target gene or are within a 200-1,200 bp region upstream of the TSS [9, 25, 34].

To further add to the complicated mechanism of how saRNAs recognize their targets, the efficacy of its activity is also dependent on the selection method of the guide strand and the systematic understanding of how, in conjunction with its interaction with Ago2, it can mediate specificity. This is an important feature, as the effects of RNA activation changes significantly in response to subtle modifications in the sequence of the activating RNA. Moreover, the specific sequence of the saRNA is also thought to strongly influence its subcellular localization, as this is clearly seen with miRNAs where their distribution in the cytoplasm or nucleus is affected on the basis of the sequence they contain. Again, these features are yet to be fully characterized with saRNA duplexes [35, 36].

Since Ago2 recruitment of saRNA initiates a strong transcriptional activation effect in mammalian cells, it is thought that target genes must be accessible to the Ago2/saRNA complex. Therefore, the effectiveness of the saRNA would be dependent on epigenetic alterations. [7]. This concept, again, is subjective, as there is evidence to show that transcription factors that are able to drive pluripotency can be induced by saRNAs in human mesenchymal stem cells [37]. The Ago2 processing unit has also created some dispute, where it is thought that Ago2-induced degradation of the strand does not emerge on the noncoding transcript of the target gene. Others have suggested that Ago2-induced antisense cleavage is directly influenced by the saRNA sequence [38-40]. This also remains to be defined. Despite these mechanistic oversights, the existence of iterative approaches to design and select saRNA duplexes for any desired target gene, and the established potential of their use in medical research, provide certainty that there will be strong drive to decipher the mechanism of action of saRNAs. During this time many promising targets for gain-of-function therapies will be at the ready.

\section{Application of saRNA}

\section{Stem Cell Differentiation}

RNA activation presents itself as a powerful biological tool to selectively enhance the transcriptional expression of target genes. Recently, it has been demonstrated that once the process of target gene site selection is optimized, synthetic doublestranded saRNA can be synthesized and used appropriately as a workflow to achieve a variety of desired biological effects. For regenerative medicine, the focus on inducible pluripotent stem cells or transdifferentiation of adult hematopoietic stem 
cells toward surrogate organ cell phenotypes could be greatly impacted by the use of saRNAs. Driving CD34 ${ }^{+}$hematopoietic stem cells toward an insulin-expressing phenotype is currently an attractive 'autologous therapy' for patients with type 1 diabetes. While numerous methods, which include changes in the growth factor and cytokine microenvironment of stem cells, have paved the way to achieving this, the introduction of saRNA targeting the islet- $\beta$ cell master transcription factor (MAFA) has bypassed many hurdles. saRNAs targeting MAFA have significantly reduced the duration of transdifferentiation and greatly enhanced maturation of adult hematopoietic $\mathrm{CD}_{34}{ }^{+}$stem cells toward an insulin-secreting phenotype that responds to changes in the glucose gradient [41]. Although this strategy may not completely eliminate the demand for pharmacological insulin replacement, it might offer a baseline to reduce the risk of non-compliance in many patient groups with diabetes who are entirely dependent on insulin replacement therapy.

The ability to modulate pluripotent reprogramming factors, such as Kruppel-like factor 4 (KFL4) and c-Myc, by synthetic saRNA is likely to accelerate stem cell research [37]. Combined expression of OCT3/4, SOX2, KLF4, and c-Myc already allows reprogramming of adult fibroblasts into induced pluripotent stem cells for a myriad of applications [42]. Achieving this with synthetic saRNA that can be used safely across different platforms will undoubtedly improve the scope for using this technology as a clinically safe and efficient alternative to reprogram genes for regenerative medicine.

\section{Clinical Therapy (Regenerative Medicine)}

Over the past decade, a number of clinical trials have been initiated to evaluate the safety and efficacy of a variety of innovative RNA-based therapeutic strategies. Although most of these systems inhibit gene expression, block protein function, or induce potent immune responses of cytotoxic $\mathrm{T}$ lymphocytes to control tumor growth, RNA therapeutics are now increasingly being validated toward gain-offunction studies. saRNA that enhances transcription of $\mathrm{C} / \mathrm{EBP} \alpha$, a member of the $\mathrm{C} / \mathrm{EBP}$ family of transcription factors, in hepatocellular carcinoma (HCC-HepG2) cells was shown to have a powerful anti-proliferation role in hepatocytes by differentially regulating tumor suppressors [24]. It was demonstrated that peripheral injection of the same saRNA formulated with a clinically relevant synthetic carrier molecule, such as PAMAM dendrimers, into an animal model of liver failure and hepatocellular carcinoma drastically improved both liver function and tumor burden $[24,43]$. While the current of list of saRNA targets continue to grow, the prospect of gain-of-function therapy with tumor suppressor genes relevant to liver, prostate, breast, and bladder cancer gives great promise for oncology [44-47]. SaRNA-induced activation of p21 and Wilm's tumor 1 gene (WT1) has been shown to inhibit cell proliferation as well as accelerating apoptosis in HCC (HepG2) and suppression of cell invasion in breast cancer cells (MCF7) [48-51]. It has also been shown that saRNA-induced p21 activation causes cellular senescence to repress or 
terminate androgen-independent prostate cancer cell (PC3) proliferation [52-54]. Additionally, the most challenging issue of innate or acquire resistance to chemotherapy can now potentially be addressed, since it has been demonstrated that saRNA-based intervention targeting p21 enhances the chemosensitivity of cisplatin in A549 non-small-cell lung carcinoma [55]. With new formulations of clinically efficient carrier molecules on the horizon for organ-specific biodistribution, this alone offers a promising and optimistic future for cancer survival rates.

\section{Concluding Remarks}

It should be noted that the reports and reviews cited in this chapter have only come to light over the past 5 years. Just as it took over two decades for siRNAs to be fully appreciated and integrated as a powerful tool for loss-of-function studies in clinical research, the future of saRNA is undoubtedly promising. Its potential to regulate gene expression endogenously in living cells - and, as a result, its ability to selectively modulate signaling networks for known diseases or for stem cell reprogramming will likely attract the attention of the pharmaceutical industry [56]. This alone sets the momentum for more mechanistic work to prevail in deciphering the complicated but truly fascinating concept of gene activation via man-made RNA duplexes. saRNA-based therapeutics will hopefully become integrated into 'personalized' medicine for more precise patient care.

Open Access This chapter is distributed under the terms of the Creative Commons Attribution Noncommercial License, which permits any noncommercial use, distribution, and reproduction in any medium, provided the original author(s) and source are credited.

\section{References}

1. Nagano T, Mitchell JA, Sanz LA et al (2008) The air noncoding RNA epigenetically silences transcription by targeting G9a to chromatin. Science 322:1717-1720

2. Wang KC, Chang HY (2011) Molecular mechanisms of long noncoding RNAs. Mol Cell 43:904-914

3. Carthew RW, Sontheimer EJ (2009) Origins and mechanisms of miRNAs and siRNAs. Cell 136:642-655

4. Castel SE, Martienssen RA (2013) RNA interference in the nucleus: roles for small RNAs in transcription, epigenetics and beyond. Nat Rev Genet 14:100-112

5. Thomson T, Lin H (2009) The biogenesis and function of PIWI proteins and piRNAs: progress and prospect. Annu Rev Cell Dev Biol 25:355-376

6. Pushparaj PN, Aarthi JJ, Kumar SD et al (2008) RNAi and RNAa-the yin and yang of RNAome. Bioinformation 2:235-237

7. Li L-C, Okino ST, Zhao H et al (2006) Small dsRNAs induce transcriptional activation in human cells. Proc Natl Acad Sci U S A 103:17337-17342

8. Janowski BA, Younger ST, Hardy DB et al (2007) Activating gene expression in mammalian cells with promoter-targeted duplex RNAs. Nat Chem Biol 3:166-173 
9. Yue X, Schwartz JC, Chu Y et al (2010) Transcriptional regulation by small RNAs at sequences downstream from 3' gene termini. Nat Chem Biol 6:621-629

10. Portnoy V, Huang V, Place R et al (2011) Small RNA and transcriptional upregulation. Wiley Interdiscip Rev RNA 2:748-760

11. Liu J, Carmell MA, Rivas FV et al (2004) Argonaute2 is the catalytic engine of mammalian RNAi. Science 305:1437-1441

12. Jiao AL, Slack FJ (2014) RNA-mediated gene activation. Epigenetics 9:27-36

13. Matranga C, Tomari Y, Shin C et al (2005) Passenger-strand cleavage facilitates assembly of siRNA into Ago2-containing RNAi enzyme complexes. Cell 123:607-620

14. Rand TA, Petersen S, Du F et al (2005) Argonaute2 cleaves the anti-guide strand of siRNA during RISC activation. Cell 123:621-629

15. Wang KC, Chang HY (2011) Molecular mechanisms of long noncoding RNAs. Mol Cell 43:904-914

16. Place RF, Noonan EJ, Foldes-Papp Z et al (2010) Defining features and exploring chemical modifications to manipulate RNAa activity. Curr Pharm Biotechnol 11:518-526

17. He Y, Smith R (2009) Nuclear functions of heterogeneous nuclear ribonucleoproteins A/B. Cell Mol Life Sci 66:1239-1256

18. Krecic AM, Swanson MS (1999) hnRNP complexes: composition, structure, and function. Curr Opin Cell Biol 11:363-371

19. Glisovic T, Bachorik JL, Yong J et al (2008) RNA-binding proteins and post-transcriptional gene regulation. FEBS Lett 582:1977-1986

20. Schwartz JC, Younger ST, Nguyen NB et al (2008) Antisense transcripts are targets for activating small RNAs. Nat Struct Mol Biol 15:842-848

21. Tsai MC, Manor O, Wan Y et al (2010) Long noncoding RNA as modular scaffold of histone modification complexes. Science 329:689-693

22. Hu J, Chen Z, Xia D et al (2012) Promoter associated small double-stranded RNA interacts with heterogeneous nuclear ribonucleoprotein A2/B1 to induce transcriptional activation. Biochem J 447:407-416

23. Dykxhoorn DM, Novina CD, Sharp PA (2003) Killing the messenger: short RNAs that silence gene expression. Nat Rev Mol Cell Biol 4:457-467

24. Reebye V, Saetrom P, Mintz P et al (2014) A novel RNA oligonucleotide improves liver function and inhibits liver carcinogenesis in vivo. Hepatology 59:216-227

25. Janowski BA, Younger ST, Hardy DB et al (2007) Activating gene expression in mammalian cells with promoter-targeted duplex RNAs. Nat Chem Biol 3:166-173

26. Roh TY, Cuddapah S, Cui K et al (2006) The genomic landscape of histone modifications in human T cells. Proc Natl Acad Sci U S A 103:15782-15787

27. Strahl BD, Ohba R, Cook RG et al (1999) Methylation of histone H3 at lysine 4 is highly conserved and correlates with transcriptionally active nuclei in Tetrahymena. Proc Natl Acad Sci U S A 96:14967-14972

28. Santos-Rosa H, Schneider R, Bannister AJ et al (2002) Active genes are trimethylated at K4 of histone H3. Nature 419:407-411

29. Shi Y, Sawada J, Suiet G et al (2003) Coordinated histone modifications mediated by a CtBP co-repressor complex. Nature 422:735-738

30. Matsui M, Sakurai F, Elbashir S et al (2010) Activation of LDL receptor expression by small RNAs complementary to a noncoding transcript that overlaps the LDLR promoter. Chem Biol 17:1344-1355

31. Guang S, Bochner AF, Burkhart KB et al (2010) Small regulatory RNAs inhibit RNA polymerase II during the elongation phase of transcription. Nature 465:1097-1101

32. Buhler M, Verdel A, Moazed D (2006) Tethering RITS to a nascent transcript initiates RNAi- and heterochromatin-dependent gene silencing. Cell 125:873-886

33. Song JJ, Liu J, Tolia NH et al (2003) The crystal structure of the Argonaute2 PAZ domain reveals an RNA binding motif in RNAi effector complexes. Nat Struct Biol 10:1026-1032

34. Huang V, Qin Y, Wang J et al (2010) RNAa is conserved in mammalian cells. PLoS One $5: \mathrm{e} 8848$ 
35. Liao JY, Ma LM, Guo YH et al (2010) Deep sequencing of human nuclear and cytoplasmic small RNAs reveals an unexpectedly complex subcellular distribution of miRNAs and tRNA 3' trailers. PLoS One 5:e10563

36. Hwang HW, Wentzel EA, Mendell JT (2007) A hexanucleotide element directs microRNA nuclear import. Science 315:97-100

37. Voutila J, Sætrom P, Mintz P et al (2012) Gene expression profile changes after short-activating RNA-mediated induction of endogenous pluripotency factors in human mesenchymal stem cells. Mol Ther Nucleic Acids 1:e35

38. Modarresi F, Faghihi MA, Lopez-Toledano MA et al (2012) Inhibition of natural antisense transcripts in vivo results in gene-specific transcriptional upregulation. Nat Biotechnol 30:453-459

39. Schwartz JC, Younger ST, Nguyen N-B et al (2008) Antisense transcripts are targets for activating small RNAs. Nat Struct Mol Biol 15:842-848

40. Matsui M, Chu Y, Zhang H et al (2013) Promoter RNA links transcriptional regulation of inflammatory pathway genes. Nucleic Acids Res 41:1-24

41. Reebye V, Sætrom P, Mintz PJ et al (2013) A short-activating RNA oligonucleotide targeting the islet $\beta$-cell transcriptional factor MafA in CD34 (+) cells. Mol Ther Nucleic Acids 2:e97

42. Nakatake Y, Fukui N, Iwamatsu Y et al (2006) Klf4 cooperates with Oct3/4 and Sox 2 to activate the Lefty 1 core promoter in embryonic stem cells. Mol Cell Biol 26:7772-7782

43. Shcharbin D, Shakhbazau A, Bryszewska M (2013) Poly (amidoamine) dendrimer complexes as a platform for gene delivery. Expert Opin Drug Deliv 10:1687-1698

44. Kosaka M, Kang MR, Yang G et al (2012) Targeted p21WAF1/CIP1 activation by RNAa inhibits hepatocellular carcinoma cells. Nucleic Acid Ther 22:335-343

45. Place RF, Wang J, Noonan EJ et al (2012) Formulation of small activating RNA into lipidoid nanoparticles inhibits xenograft prostate tumor growth by inducing p21 expression. Mol Ther Nucleic Acids 1:e15

46. Junxia W, Ping G, Yuan H et al (2010) Double strand RNA-guided endogeneous E-cadherin up-regulation induces the apoptosis and inhibits proliferation of breast carcinoma cells in vitro and in vivo. Cancer Sci 101:1790-1796

47. Kang MR, Yang G, Place RF et al (2012) Intravesical delivery of small activating RNA formulated into lipid nanoparticles inhibits orthotopic bladder tumor growth. Cancer Res 72:5069-5079

48. Qin Q, Lin YW, Zheng XY et al (2012) RNAa-mediated overexpression of WT1 induces apoptosis in HepG2 cells. World J Surg Oncol 10:11

49. Kosaka M, Kang MR, Yang G et al (2012) Targeted p21WAF1/CIP1 activation by RNAa inhibits hepatocellular carcinoma cells. Nucleic Acid Ther 22:335-343

50. Junxia W, Ping G, Yuan H et al (2010) Double strand RNA-guided endogeneous E-cadherin up-regulation induces the apoptosis and inhibits proliferation of breast carcinoma cells in vitro and in vivo. Cancer Sci 101:1790-1796

51. Zhao F, Pan S, Gu Y et al (2014) Small activating RNA restores the activity of the tumor suppressor HIC-1 on breast cancer. PLoS One 9:e86486

52. Place RF, Wang J, Noonan EJ et al (2012) Formulation of small activating RNA into lipidoid nanoparticles inhibits xenograft prostate tumor growth by inducing p21 expression. Mol Ther Nucleic Acids 1:e15

53. Phalke S, Mzoughi S, Bezzi M et al (2012) p53-Independent regulation of p21Waf1/Cip1 expression and senescence by PRMT6. Nucleic Acids Res 40:9534-9542

54. Hu Z, Zhang D, Hao J et al (2014) Induction of DNA damage and p21-dependent senescence by Riccardin $\mathrm{D}$ is a novel mechanism contributing to its growth suppression in prostate cancer cells in vitro and in vivo. Cancer Chemother Pharmacol 73:397-407

55. Wei J, Zhao J, Long M et al (2010) p21WAF1/CIP1 gene transcriptional activation exerts cell growth inhibition and enhances chemosensitivity to cisplatin in lung carcinoma cell. BMC Cancer 10:632

56. Donner A (2014) It is an RNA world. SciBX 7:28 
Part III

New Technology for Innovative Medicine 


\title{
Proceedings of the Uehara Memorial Foundation. Innovative Medicine: Basic Research and Development. Cardiac Reprogramming for Heart Repair
}

\author{
Masaki Ieda
}

\begin{abstract}
Heart disease remains the leading cause of death worldwide. Terminally differentiated cardiomyocytes do not possess regenerative capacity, and heart disease is irreversible. Stem cell-derived cardiomyocytes are an attractive cell source for heart regeneration, but the risk of tumor formation due to contamination of stem cells, the complicated process of cell transplantation, and poor survival of the transplanted cells may be challenges for this approach. The discovery of reprogramming of fibroblasts into induced pluripotent stem cells (iPSCs) by the Yamanaka factors, Oct4, Sox2, Klf4, and c-Myc, inspired a new strategy to generate desired cell types from fibroblasts. It has been demonstrated that a diverse range of cell types, such as pancreatic $\beta$ cells, blood cells, neurons, chondrocytes, and hepatocytes, can be directly generated from fibroblasts, using lineage-specific transcription factors. We first reported that functional cardiomyocytes can be generated from mouse fibroblasts using cardiac-specific transcription factors, Gata4, Mef2c, and Tbx5 (GMT) in vitro. Our subsequent work revealed that GMT can also convert resident cardiac fibroblasts into cardiomyocyte-like cells in infarcted mouse hearts. We also demonstrated that Gata4, Mef2c, Tbx5, Myocd, and Mesp1 (GMTMM) can convert human fibroblasts into cardiomyocyte-like cells, and that addition of miR133 to GMT or GMTMM promoted cardiac reprogramming in mouse and human fibroblasts. Intriguingly, miR-133 directly suppressed Snai1, a master gene of epithelial-to-mesenchymal transition, which in turn repressed fibroblast signatures and promoted cardiac reprogramming. Here, I review the recent studies in cardiac reprogramming and discuss the perspectives and challenges of this innovative technology toward regenerative therapy.
\end{abstract}

\footnotetext{
M. Ieda, M.D., Ph. D. (ه)

Department of Clinical and Molecular Cardiovascular Research, Keio University School of Medicine, 35 Shinanomachi, Shinjuku-ku, Tokyo 160-8582, Japan

Department of Cardiology, Keio University School of Medicine, 35 Shinanomachi, Shinjuku-ku, Tokyo 160-8582, Japan
}

JST, CREST, 35 Shinanomachi, Shinjuku-ku, Tokyo 160-8582, Japan

e-mail: mieda@z8.keio.jp

K. Nakao et al. (eds.), Innovative Medicine, DOI 10.1007/978-4-431-55651-0_21 
Keywords Heart $\bullet$ Regeneration $\bullet$ Induced cardiomyocytes $\bullet$ Direct reprogramming $\bullet$ Transcription factors $\bullet$ miRNA

\section{Introduction}

Cardiovascular disease remains a leading cause of death worldwide for which current therapeutic regimens remain limited. As terminally differentiated cardiomyocytes have little regenerative capacity following injury, the demand for cardiac regenerative therapy is high. Following myocardial injury, endogenous cardiac fibroblasts, which account for more than half of the cells in the heart, proliferate and synthesize extracellular matrix, leading to fibrosis and heart failure. The large population of cardiac fibroblasts might be a potential source of cardiomyocytes for regenerative applications, if they could be reprogrammed into functional cardiomyocytes in situ.

The discovery of induced pluripotent stem cells (iPSCs) by Drs. Takahashi and Yamanaka provided a new strategy, which is direct generation of specific cell types from fibroblasts, using a combination of lineage-specific factors without mediating through a stem cell state. Recent studies demonstrated that direct reprogramming yields a diverse range of medically relevant cell types, including pancreatic $\beta$ cells, neurons, chondrocytes, and hepatocytes from fibroblasts [1-7]. Recently, we and others reported that cardiac and skin fibroblasts could be directly reprogrammed into cardiomyocyte-like cells by several combinations of cardiac-specific factors [8-12]. We discovered that a combination of three cardiac-specific transcription factors, Gata4, Mef $2 c$, and Tbx5, directly induced cardiomyocyte-like cells from mouse fibroblasts in vitro [8]. Subsequent studies revealed that direct gene transfer of the cardiac reprogramming factors in vivo could convert resident cardiac fibroblasts into cardiomyocytes in infarcted hearts, and improved cardiac function after myocardial infarction (MI) [12-14]. We and others also reported that human fibroblasts could be reprogrammed into cardiomyocyte-like cells by addition of cardiac transcription factors and miRNAs to GMT [15-17]. More recently, we found that cardiac-enriched miRNA, miR-133a (miR-133), promoted cardiac reprogramming in mouse embryonic fibroblasts (MEFs), adult mouse cardiac fibroblasts, and human cardiac fibroblasts (HCFs) in combination with GMT or GMTMM transduction. Mechanistically, miR-133 suppressed the fibroblast program by directly repressing Snai1, a master regulator of epithelial-to-mesenchymal transition (EMT), and thereby promoted cardiac reprogramming. This chapter will discuss recent studies of direct cardiac reprogramming using defined factors and provide a future perspective of this new technology. 


\section{Cell Fate Conversion by Transcription Factors}

John Gurdon and colleagues demonstrated that the nucleus of a differentiated frog cell, when transferred into an enucleated egg, could be converted to the totipotent zygote and give rise to a whole new frog [18]. Davis et al. found that $M y o D$, a master gene of skeletal muscle, converted fibroblasts into myoblasts [19-21]. Despite the race to identify single transcription factors that could guide cell fate similarly to MyoD for other lineages, including cardiomyocytes, the MyoD paradigm appeared to be an exception rather than the rule. Takahashi and Yamanaka achieved a breakthrough in this field by overexpressing four embryonic stem cell (ESC)-specific transcription factors in fibroblasts and generating pluripotent stem cells, iPS cells. Using retroviral vectors, they expressed 24 candidate genes and selected for reprogramming by expression of Fbx15, a gene specifically expressed in pluripotent stem cells. The combination of 24 factors induced formation of colonies with characteristic ESC morphology, and successive selection led to the minimally required core set of four genes, comprising Oct4, Sox2, Klf4, and c-Myc [22]. Many laboratories have since improved iPSC generation techniques to show that iPSCs share all features of ESCs, including expression of pluripotency markers, epigenetic marks, and the ability to generate chimeric mice.

The generation of iPSCs sparked a new idea, which is converting one cell type directly into another, using a combination of lineage-specific factors instead of single master genes. For example, Vierbuchen et al. converted mouse fibroblasts into functional neurons in vitro, using the neuronal lineage-specific transcription factors Ascll, Brn2, and Myt1l [4]. Zhou et al. provided the first evidence of cellular reprogramming in vivo using defined factors [5]. They showed that gene transfer of three transcription factors, $N g n 3, P d x 1$, and Mafa [23], efficiently reprogrammed pancreatic exocrine cells into functional $\beta$ cells in the mouse. The newly generated $\beta$ cells in vivo were indistinguishable from endogenous islet $\beta$ cells in terms of their structure and gene expression.

\section{Direct Reprogramming of Mouse Fibroblasts into Cardiomyocytes in Vitro}

In our study, we used cardiac fibroblasts (CFs) as a cell source for cardiac reprogramming. CFs are found throughout cardiac tissues along with cardiomyocytes, accounting for more than half of the cells in the heart, and may be a potential cell source for cardiac repair. To determine the candidate factors of cardiac reprogramming, we identified the genes that are specifically expressed in embryonic cardiomyocytes. We developed a novel cell purification system in which embryonic cardiomyocytes and CFs can be purified using FACS [24]. Using this system, we selected 14 factors as candidates for cardiac reprogramming, which are specifically expressed in cardiomyocytes and are critical for heart development. 
We next developed a screening system in which the induction of mature cardiomyocytes from fibroblasts could be analyzed quantitatively by FACS. We generated $\alpha$ myosin heavy chain $(\alpha M H C)$ promoter-driven EGFP-IRES-Puromycin transgenic mice $(\alpha \mathrm{MHC}-\mathrm{GFP})$, in which only mature cardiomyocytes expressed green fluorescent protein (GFP) $[8,25,26]$. Transduction of all 14 factors into fibroblasts induced $1.7 \%$ of $\mathrm{GFP}^{+}$cells, and serial removal of individual factors demonstrated that a combination of three factors (Gata4, Mef2c, and Tbx5) were sufficient for efficient $\mathrm{GFP}^{+}$cell induction (around $15 \%$ ) from CFs. The three cardiac reprogramming factors, Gata4, Mef2c, and Tbx5, are core cardiac transcription factors during early heart development [27-29] and are known to interact with each other, coactivate cardiac gene expression, and promote cardiomyocyte differentiation [30-32]. We designated these $\mathrm{GFP}^{+}$cardiomyocyte-like cells as induced cardiomyocytes (iCMs). The iCMs expressed several cardiac marker proteins, such as sarcomeric $\alpha$-actinin, cardiac troponin T, and atrial natriuretic factor (Fig. 1). They also had well-defined sarcomeric structures similar to neonatal cardiomyocytes. The global gene expression profile of $\mathrm{GFP}^{+} \mathrm{iCMs}$ is not identical but similar to neonatal cardiomyocytes, and different from original CFs. We also found that functionally important cardiac genes, such as ion channel and sarcomere genes, were upregulated more in 4-week iCMs than in 2-week iCMs, suggesting that cardiac differentiation occurred over several weeks. The chromatin state of iCMs was also similar to cardiomyocytes but different from fibroblasts in histone modifications and DNA methylation patterns [8]. These results suggest that iCMs are cardiomyocyte-like cells in gene expression and epigenetic states. We also demonstrated that iCMs possessed functional properties characteristic of cardiomyocytes. The iCMs exhibited intracellular $\mathrm{Ca}^{2+}$ transient and action potentials after 2-4 weeks of culture. In addition, CF-derived iCMs contracted spontaneously. To determine if the iCMs could arise from cells in other organs, we transduced the three factors into mouse tail-tip fibroblasts (TTFs). TTF-derived iCMs expressed cardiac markers and

a

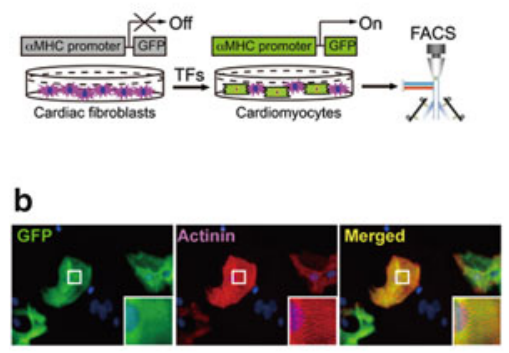

C

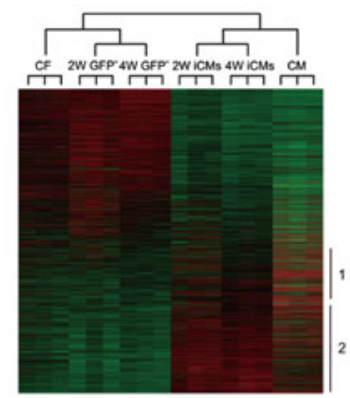

d

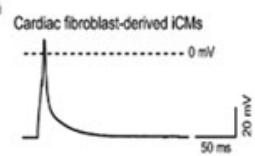

Adut mouse ventricular cardicmyocyies

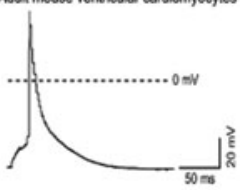

Fig. 1 Cardiac reprogramming in mouse fibroblasts. (a) Screening system for cardiac reprogramming factors. The $\alpha$-MHC-GFP turns on, if reprogramming succeeds. (b) Immunohistochemistry for iCMs. Mouse fibroblasts were converted into cardiomyocyte-like cells (iCMs) by transduction of Gata4, Mef2c, and Tbx5. The iCMs expressed cardiac proteins and had striated muscle structures. (c) Microarray analyses for fibroblasts, iCMs, and cardiomyocytes. (d)Action potential recordings from iCMs and cardiomyocytes (Cited from Ieda et al. [8]) 
spontaneous intracellular $\mathrm{Ca}^{2+}$ transients, while the percentage of cardiac troponin $\mathrm{T}^{+}$cells was $50 \%$ less than that of CF-derived iCMs. The TTF-derived iCMs had some functional characteristics of cardiomyocytes, such as spontaneous intracellular $\mathrm{Ca}^{2+}$ transients, but we did not observe cellular contraction, suggesting that TTFs are more resistant to cardiac reprogramming by GMT. These findings exclude the possibility that iCMs arise from contamination of cardiomyocytes or cardiac progenitors in the fibroblast population [33, 34].

While direct conversion of somatic cells to $\beta$ cells and neurons was reported, the "route" of cell fate change was not clear. There are two possibilities for the reprogramming of fibroblasts into differentiated cardiomyocytes. One is direct conversion of fibroblasts into differentiated cardiomyocytes, and the other is that fibroblasts first revert to a cardiac progenitor/stem cell state before further cardiac differentiation. We were able to genetically test these two possibilities by using mice expressing Isl1Cre-yellow fluorescent protein (YFP) and Mesp1-Cre-YFP obtained by crossing Isl1-Cre or Mesp1-Cre mice with R26R-EYFP mice [35]. Isl1 and Mesp1 are transiently expressed in the early cardiac progenitor cells before further cardiac differentiation $[36,37]$. We found that iCMs induced from Isl1-Cre-YFP and Mesp1-Cre-YFP fibroblasts did not express YFP, suggesting that the fibroblasts directly reprogrammed into differentiated cardiomyocytes without passing through a cardiac progenitor cell state. We next investigated whether GMT-transduced CFs can be transplanted in vivo and be reprogrammed into cardiomyocytes within the heart. CFs infected with GMT expressed $\alpha$-MHC GFP and sarcomeric markers, and converted into cardiomyocytelike cells in the mouse hearts after 2 weeks of cell transplantation.

Following our initial report, other groups also reported generation of cardiomyocyte-like cells from mouse fibroblasts based on the same factors and microRNAs. Song et al. reported that adding Hand2 to GMT converted adult CFs and TTFs into functional cardiomyocyte-like cells more efficiently than GMT alone [12]. Protze et al. found that the combination of Mef2c, myocardin, and Tbx5 upregulated a broad spectrum of cardiac genes and induced functional cardiomyocytes from MEFs and CFs [11]. Jayawardena et al. reported that a combination of muscle-specific microRNAs, mir-1, 133, 208, and 499, can convert CFs into functional cardiomyocyte-like cells [10]. Thus, several combinations of cardiogenic factors can induce cardiac reprogramming, which is similar to the experience in the iPSC field. Although the induction of fully matured cardiac cells remains low in culture, and further refinements are needed, these results suggest that fibroblasts transduced with cardiac reprogramming factors ex vivo can be delivered to damaged myocardium for regenerative purposes.

\section{Cardiac Reprogramming in Vivo by Gene Transfer of Defined Factors}

On the basis of the findings of direct cardiac reprogramming in vitro, we investigated whether gene transfer of GMT into mouse injured hearts could similarly induce new cardiomyocyte generation [14]. Transduction of GMT retroviruses 
converted adult CFs from infarcted hearts into iCMs in vitro. Injection of GMT retroviruses into $\alpha \mathrm{MHC}-\mathrm{GFP}$ transgenic mouse hearts induced the expression of $\alpha \mathrm{MHC}-\mathrm{GFP}$, a reporter of cardiomyocytes, in $3 \%$ of virus-infected fibroblasts. A mixture of GMT injection into the immunosuppressed mouse hearts induced cardiac protein expression in retrovirus-infected cells within 2 weeks, with a conversion rate of approximately $1 \%$, although few cells showed striated muscle structures. Next, to transduce GMT more efficiently in vivo, we developed a new polycistronic retrovirus vector expressing GMT separated by $2 \mathrm{~A}$ "self-cleaving" peptides (3F2A). In vivo gene transfer of $3 \mathrm{~F} 2 \mathrm{~A}$ into infarcted hearts resulted in generation of $\mathrm{iCMs}$ in fibrotic tissues, which expressed sarcomeric $\alpha$-actinin, cardiac troponin $\mathrm{T}$, and several cardiac-specific genes, and had clear cross-striations. These results suggest that $3 \mathrm{~F} 2 \mathrm{~A}-\mathrm{iCMs}$ are more mature cardiomyocytes, and that the polycistronic vector can be used for cellular reprogramming in vivo.

In parallel with our study, two other groups also published reports on in vivo cardiac reprogramming in infarcted mouse hearts. Qian et al. found that fibroblasts in infarcted hearts were converted into cardiomyocyte-like cells by GMT retroviral gene transfer [13]. Song et al. reported that adding Hand2 to GMT converted endogenous CFs into functional cardiomyocyte-like cells more efficiently than GMT alone in vivo [12]. Both studies demonstrated that the in vivo iCMs had wellorganized sarcomeric structures and exhibited functional characteristics of adult ventricular cardiomyocytes, including cellular contraction, electrophysiological properties, and functional coupling to other cardiac cells. They also demonstrated that retroviral gene transfer of reprogramming factors into infarcted hearts significantly improved cardiac function and reduced fibrosis 2 and 3 months after myocardial infarction. Although all three studies, including ours, demonstrated in vivo cardiac reprogramming, the approaches used to address this issue differed. The other two groups used mainly fibroblast-lineage tracing mice to demonstrate cardiac conversion from CFs, while we co-transduced GMT with marker genes (GFP or DsRed) to demonstrate cardiac induction in the infected fibroblasts, using $\alpha \mathrm{MHC}-\mathrm{GFP}$ transgenic and nude mice. Although it is not clear how many newly generated iCMs remained in the injured hearts in the longer-term follow-up, and to what extent the iCMs contributed to the improvement in cardiac function, these findings might inform new regenerative strategies for repairing injured hearts. Further work in larger animals and more efficient gene delivery system in vivo are needed for future research.

\section{Gata4/Mef2c/Tbx5/Myocd/Mesp1 Reprogram Human Fibroblasts into Cardiomyocyte-Like Cells}

We next analyzed whether human fibroblasts could be directly converted to iCMs by defined factors [17]. We found that GMT was not sufficient for cardiac induction in HCFs. Thus, we screened additional factors that enhance reprogramming, and 
found that addition of Mesp1 and Myocd to GMT upregulated a broader spectrum of cardiac genes more efficiently than GMT alone. The HCFs and human dermal fibroblasts transduced with GMT, Mesp1, and Myocd (GMTMM) changed the cell morphology to a rod-like or polygonal shape, expressed a broad range of cardiac genes and concomitantly suppressed fibroblast genes, and exhibited spontaneous $\mathrm{Ca}^{2+}$ oscillations. Moreover, the cells matured to exhibit action potentials and contract synchronously in coculture with murine cardiomyocytes. The EdU assay revealed that the human iCMs did not pass through a mitotic stem cell state and were directly generated from fibroblasts.

Human cardiac reprogramming was also reported by other groups. Nam et al. reported that a combination of Gata4, Hand2, Tbx5, Myocd, miR-1, and miR-133 induced $13 \%$ of adult HCFs to express cardiac troponin T protein and that a small subset of the iCMs exhibited spontaneous contractility after 11 weeks in culture [15]. Islas et al. reported that transient overexpression of Ets2 and Mesp1 could reprogram human dermal fibroblasts into cardiac progenitor-like cells [16]. The induced cardiac progenitor-like cells differentiated into immature cardiomyocytes and exhibited calcium activities. These findings may represent an important initial step toward potential therapeutic applications of the direct reprogramming approach in clinical situations. However, human cardiac reprogramming process is slower and less efficient than mouse reprogramming, much like the induction of human iPSCs compared with mouse iPSCs. Future studies are needed to thoroughly optimize conditions for human cardiomyocyte generation and maturation and to characterize the properties of human iCMs.

\section{MiR-133 Promotes Cardiac Reprogramming by Repressing Snai1 and Silencing the Fibroblast Profile}

As discussed, induction of functional cardiomyocytes in vitro was inefficient and molecular mechanisms of direct reprogramming remained undefined. In contrast, the in vivo iCMs were more fully reprogrammed than their counterparts in vitro, suggesting the presence of undefined factors that may enhance reprogramming. Identification of such potent reprogramming factors could provide new insights into the mechanisms of cardiac reprogramming. We selected four cardiac miRNAs as candidate factors, which are specifically expressed in cardiomyocytes and skeletal muscles and are important for heart development and physiology. FACS analyses using $\alpha$ MHC-GFP mice revealed that these miRNAs alone did not reprogram MEFs into iCMs, but addition of miR-133 to GMT strongly enhanced cardiac induction compared with other miRNAs. The time courses of reprogramming between GMT and GMT/miR-133 revealed that the GMT/miR-133-iCMs showed sarcomeric structures after 7 days of infection, which normally takes 2 weeks with GMT alone. Moreover, cell contraction started from 10 days after GMT/miR-133 transduction, which generally took 4 weeks, and the number of beating cells increased over time, 
with sevenfold more contractile cells achieved compared with GMT alone. The global gene expression profiles of iCMs induced with GMT or GMT/miR-133 revealed that among 23,474 probes, 46 cardiac-enriched genes were upregulated and 129 fibroblast-related genes were downregulated by GMT/miR-133 induction. Molecularly, we found that Snai1, a basic helix-loop-helix transcription factor and a master regulator of EMT, was a direct target of miR-133 and had two conserved miR-133-binding sites within the 3' UTR. Snail knockdown suppressed fibroblast genes, upregulated cardiac genes, and induced more contracting iCMs in combination with GMT transduction, recapitulating the effects of miR-133 overexpression. In contrast, overexpression of Snail in GMT/miR-133-transduced cells activated the fibroblast signature and inhibited generation of beating iCMs. Moreover, miR133-mediated Snai1 repression was also critical for cardiac reprogramming in adult mice and HCFs in combination with GMT or GMTMM. These results are consistent with the cell fate conversion, in which the target cell program is progressively activated and the starting-cell profile is concomitantly suppressed during reprogramming. Although further work in identifying more efficient protocols and understanding molecular mechanisms of cardiac reprogramming are needed, these reports demonstrate that the cardiac reprogramming strategy might be a potential approach for heart regeneration in the future [38].

\section{Perspectives and Challenges of Cardiac Reprogramming for Clinical Applications}

As discussed above, the cardiac reprogramming field has extensively progressed and may change regenerative medicine in the future. The directly induced cardiac cells appear to quickly exit the cell cycle following the lineage conversion, and the utility of iCMs in vitro might be limited. Alternatively, direct induction of cardiac progenitor cells, as shown in neural stem/progenitor cell reprogramming, may be a good approach to solve this issue [39]. In contrast, introduction of cardiac reprogramming factors directly into the damaged heart may convert the endogenous cardiac fibroblast population, which represents $>50 \%$ of cardiac cells, into new functional cardiomyocytes in situ, and may improve cardiac function. This in vivo reprogramming approach may have several advantages compared with cell transplantation-based regeneration (Fig. 2). First, the process is simple and short; second, avoiding the reprogramming of pluripotent cells before cardiac differentiation would greatly lower the risk of tumor formation; and third, direct injection of defined factors obviates the need for cell transplantation, for which long-term cardiac cell survival remains a challenge.

Although direct cardiac induction using defined factors has been demonstrated by several laboratories, the reprogramming efficiency remains low and many pitfalls remain that may lead to reprogramming failure [40, 41]. Future studies will be needed to thoroughly optimize the protocol for iCM generation, and to characterize 


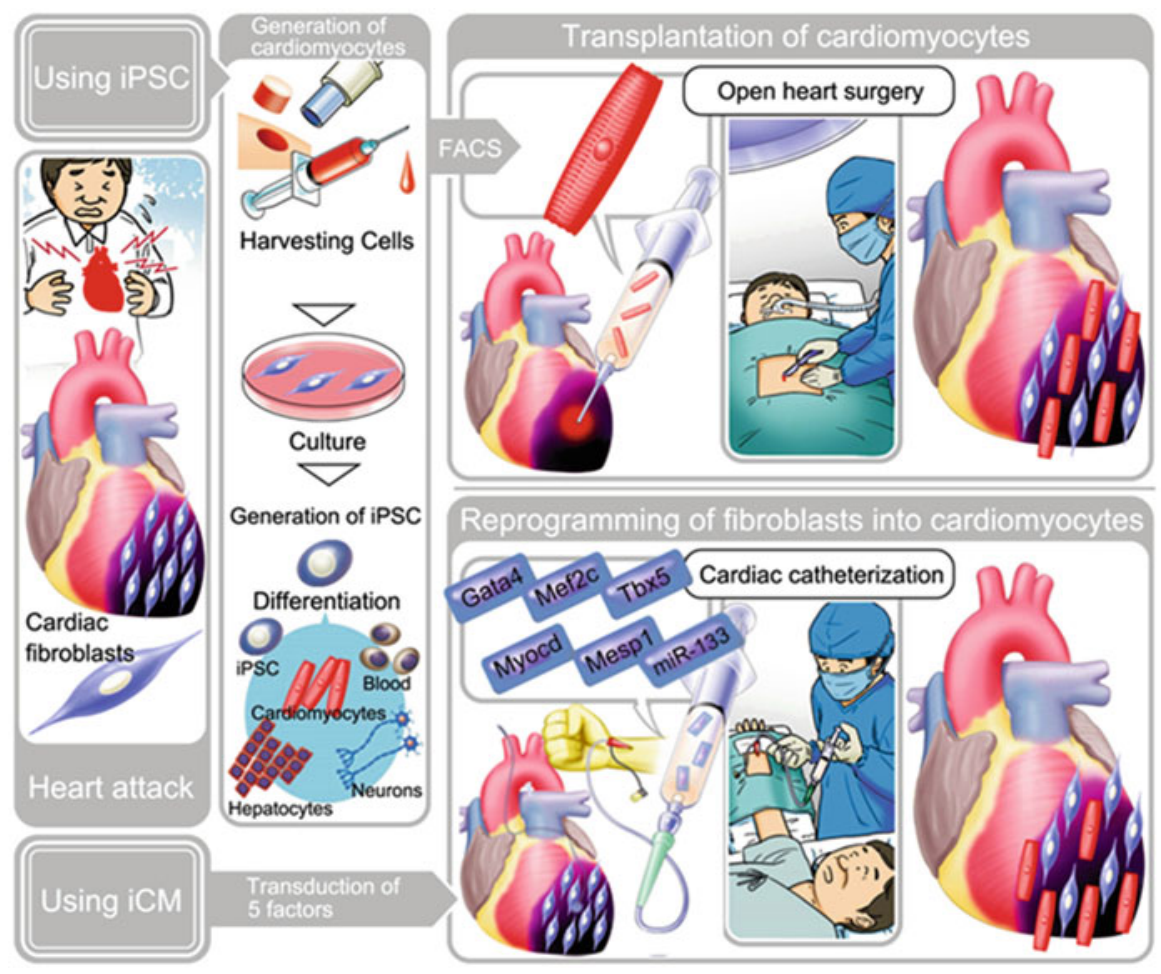

Fig. 2 Future heart regenerative therapies. The upper panel indicates the cell transplantation strategy using iPSC-derived cardiomyocytes to repair the damaged heart. The lower panel indicates the direct cardiac reprogramming strategy, which converts endogenous cardiac fibroblasts into iCMs in situ using defined factors

the properties of iCMs. Given that secreted proteins, electrical and mechanical stimulation, and cell-to-cell contact might promote cardiac differentiation and reprogramming in our experience, the future of this new technology seems to be bright and promising. Highly standardized protocols that make the process more efficient and more easily transferable between different laboratories should be developed in the future to push this field forward.

\section{Conclusions}

Cellular reprogramming has long been recognized as a possibility, although the impact of cell type conversion by defined factors was most prominently exemplified only recently by the discovery of iPSCs. This landmark finding fundamentally altered approaches to regenerative medicine, and provided a broad strategy to induce desired cell types by introducing lineage-specific factors. Detailed analyses 
of the properties of generated cells and understanding of the molecular mechanisms of reprogramming might be necessary to advance this nascent technology for future clinical applications.

Acknowledgments The author is grateful to the members of the Ieda Lab and many other labs for their critical work and discussions. The author apologizes to other authors whose work has not been cited, because of space limitations. The author was supported by a research grant from The Uehara Memorial Foundation for this paper.

\section{Disclosures None.}

Open Access This chapter is distributed under the terms of the Creative Commons Attribution Noncommercial License, which permits any noncommercial use, distribution, and reproduction in any medium, provided the original author(s) and source are credited.

\section{References}

1. Han DW, Tapia N, Hermann A, Hemmer K, Hoing S, Arauzo-Bravo MJ, Zaehres H, Wu G, Frank S, Moritz S, Greber B, Yang JH, Lee HT, Schwamborn JC, Storch A, Scholer HR (2012) Direct reprogramming of fibroblasts into neural stem cells by defined factors. Cell Stem Cell 10:465-472

2. Huang P, He Z, Ji S, Sun H, Xiang D, Liu C, Hu Y, Wang X, Hui L (2011) Induction of functional hepatocyte-like cells from mouse fibroblasts by defined factors. Nature 475:386-389

3. Marro S, Pang ZP, Yang N, Tsai MC, Qu K, Chang HY, Sudhof TC, Wernig M (2011) Direct lineage conversion of terminally differentiated hepatocytes to functional neurons. Cell Stem Cell 9:374-382

4. Vierbuchen T, Ostermeier A, Pang ZP, Kokubu Y, Sudhof TC, Wernig M (2010) Direct conversion of fibroblasts to functional neurons by defined factors. Nature 463:1035-1041

5. Zhou Q, Brown J, Kanarek A, Rajagopal J, Melton DA (2008) In vivo reprogramming of adult pancreatic exocrine cells to beta-cells. Nature 455:627-632

6. Hiramatsu K, Sasagawa S, Outani H, Nakagawa K, Yoshikawa H, Tsumaki N (2011) Generation of hyaline cartilaginous tissue from mouse adult dermal fibroblast culture by defined factors. J Clin Invest 121:640-657

7. Sekiya S, Suzuki A (2011) Direct conversion of mouse fibroblasts to hepatocyte-like cells by defined factors. Nature 475:390-393

8. Ieda M, Fu JD, Delgado-Olguin P, Vedantham V, Hayashi Y, Bruneau BG, Srivastava D (2010) Direct reprogramming of fibroblasts into functional cardiomyocytes by defined factors. Cell $142: 375-386$

9. Efe JA, Hilcove S, Kim J, Zhou H, Ouyang K, Wang G, Chen J, Ding S (2011) Conversion of mouse fibroblasts into cardiomyocytes using a direct reprogramming strategy. Nat Cell Biol $13: 215-222$

10. Jayawardena TM, Egemnazarov B, Finch EA, Zhang L, Payne JA, Pandya K, Zhang Z, Rosenberg P, Mirotsou M, Dzau VJ (2012) MicroRNA-mediated in vitro and in vivo direct reprogramming of cardiac fibroblasts to cardiomyocytes. Circ Res 110:1465-1473

11. Protze S, Khattak S, Poulet C, Lindemann D, Tanaka EM, Ravens U (2012) A new approach to transcription factor screening for reprogramming of fibroblasts to cardiomyocyte-like cells. J Mol Cell Cardiol 53:323-332

12. Song K, Nam YJ, Luo X, Qi X, Tan W, Huang GN, Acharya A, Smith CL, Tallquist MD, Neilson EG, Hill JA, Bassel-Duby R, Olson EN (2012) Heart repair by reprogramming nonmyocytes with cardiac transcription factors. Nature 485:599-604 
13. Qian L, Huang Y, Spencer CI, Foley A, Vedantham V, Liu L, Conway SJ, Fu JD, Srivastava D (2012) In vivo reprogramming of murine cardiac fibroblasts into induced cardiomyocytes. Nature 485:593-598

14. Inagawa K, Miyamoto K, Yamakawa H, Muraoka N, Sadahiro T, Umei T, Wada R, Katsumata Y, Kaneda R, Nakade K, Kurihara C, Obata Y, Miyake K, Fukuda K, Ieda M (2012) Induction of cardiomyocyte-like cells in infarct hearts by gene transfer of Gata4, Mef2c, and Tbx5. Circ Res 111:1147-1156

15. Nam YJ, Song K, Luo X, Daniel E, Lambeth K, West K, Hill JA, Dimaio JM, Baker LA, Bassel-Duby R, Olson EN (2013) Reprogramming of human fibroblasts toward a cardiac fate. Proc Natl Acad Sci U S A 110:5588-5593

16. Islas JF, Liu Y, Weng KC, Robertson MJ, Zhang S, Prejusa A, Harger J, Tikhomirova D, Chopra M, Iyer D, Mercola M, Oshima RG, Willerson JT, Potaman VN, Schwartz RJ (2012) Transcription factors ETS2 and MESP1 transdifferentiate human dermal fibroblasts into cardiac progenitors. Proc Natl Acad Sci U S A 109:13016-13021

17. Wada R, Muraoka N, Inagawa K, Yamakawa H, Miyamoto K, Sadahiro T, Umei T, Kaneda R, Suzuki T, Kamiya K, Tohyama S, Yuasa S, Kokaji K, Aeba R, Yozu R, Yamagishi H, Kitamura T, Fukuda K, Ieda M (2013) Induction of human cardiomyocyte-like cells from fibroblasts by defined factors. Proc Natl Acad Sci U S A 110:12667-12672

18. Gurdon JB, Elsdale TR, Fischberg M (1958) Sexually mature individuals of Xenopus laevis from the transplantation of single somatic nuclei. Nature 182:64-65

19. Berkes CA, Tapscott SJ (2005) MyoD and the transcriptional control of myogenesis. Semin Cell Dev Biol 16:585-595

20. Tapscott SJ (2005) The circuitry of a master switch: Myod and the regulation of skeletal muscle gene transcription. Development 132:2685-2695

21. Davis RL, Weintraub H, Lassar AB (1987) Expression of a single transfected cDNA converts fibroblasts to myoblasts. Cell 51:987-1000

22. Takahashi K, Yamanaka S (2006) Induction of pluripotent stem cells from mouse embryonic and adult fibroblast cultures by defined factors. Cell 126:663-676

23. Zhou Q, Law AC, Rajagopal J, Anderson WJ, Gray PA, Melton DA (2007) A multipotent progenitor domain guides pancreatic organogenesis. Dev Cell 13:103-114

24. Ieda M, Tsuchihashi T, Ivey KN, Ross RS, Hong TT, Shaw RM, Srivastava D (2009) Cardiac fibroblasts regulate myocardial proliferation through betal integrin signaling. Dev Cell $16: 233-244$

25. Ieda M, Kanazawa H, Kimura K, Hattori F, Ieda Y, Taniguchi M, Lee JK, Matsumura K, Tomita Y, Miyoshi S, Shimoda K, Makino S, Sano M, Kodama I, Ogawa S, Fukuda K (2007) Sema3a maintains normal heart rhythm through sympathetic innervation patterning. Nat Med 13:604-612

26. Gulick J, Subramaniam A, Neumann J, Robbins J (1991) Isolation and characterization of the mouse cardiac myosin heavy chain genes. J Biol Chem 266:9180-9185

27. Zhao R, Watt AJ, Battle MA, Li J, Bondow BJ, Duncan SA (2008) Loss of both GATA4 and GATA6 blocks cardiac myocyte differentiation and results in acardia in mice. Dev Biol 317:614-619

28. Srivastava D (2006) Making or breaking the heart: from lineage determination to morphogenesis. Cell 126:1037-1048

29. Olson EN (2006) Gene regulatory networks in the evolution and development of the heart. Science 313:1922-1927

30. Ghosh TK, Song FF, Packham EA, Buxton S, Robinson TE, Ronksley J, Self T, Bonser AJ, Brook JD (2009) Physical interaction between TBX5 and MEF2C is required for early heart development. Mol Cell Biol 29:2205-2218

31. Garg V, Kathiriya IS, Barnes R, Schluterman MK, King IN, Butler CA, Rothrock CR, Eapen RS, Hirayama-Yamada K, Joo K, Matsuoka R, Cohen JC, Srivastava D (2003) GATA4 mutations cause human congenital heart defects and reveal an interaction with TBX5. Nature 424:443-447 
32. Morin S, Charron F, Robitaille L, Nemer M (2000) GATA-dependent recruitment of MEF2 proteins to target promoters. Embo J 19:2046-2055

33. Andersen DC, Andersen P, Schneider M, Jensen HB, Sheikh SP (2009) Murine "cardiospheres" are not a source of stem cells with cardiomyogenic potential. Stem Cells 27:1571-1581

34. Davis DR, Zhang Y, Smith RR, Cheng K, Terrovitis J, Malliaras K, Li TS, White A, Makkar R, Marban E (2009) Validation of the cardiosphere method to culture cardiac progenitor cells from myocardial tissue. PLoS One 4:e7195

35. Srinivas S, Watanabe T, Lin CS, William CM, Tanabe Y, Jessell TM, Costantini F (2001) Cre reporter strains produced by targeted insertion of EYFP and ECFP into the ROSA26 locus. BMC Dev Biol 1:4

36. Bondue A, Lapouge G, Paulissen C, Semeraro C, Iacovino M, Kyba M, Blanpain C (2008) Mesp1 acts as a master regulator of multipotent cardiovascular progenitor specification. Cell Stem Cell 3:69-84

37. Laugwitz KL, Moretti A, Lam J, Gruber P, Chen Y, Woodard S, Lin LZ, Cai CL, Lu MM, Reth M, Platoshyn O, Yuan JX, Evans S, Chien KR (2005) Postnatal is11+ cardioblasts enter fully differentiated cardiomyocyte lineages. Nature 433:647-653

38. Nam YJ, Song K, Olson EN (2013) Heart repair by cardiac reprogramming. Nat Med 19:413-415

39. Lujan E, Chanda S, Ahlenius H, Sudhof TC, Wernig M (2012) Direct conversion of mouse fibroblasts to self-renewing, tripotent neural precursor cells. Proc Natl Acad Sci U S A 109:2527-2532

40. Chen JX, Krane M, Deutsch MA, Wang L, Rav-Acha M, Gregoire S, Engels MC, Rajarajan K, Karra R, Abel ED, Wu JC, Milan D, Wu SM (2012) Inefficient reprogramming of fibroblasts into cardiomyocytes using gata4, mef2c, and tbx5. Circ Res 111:50-55

41. Srivastava D, Ieda M (2012) Critical factors for cardiac reprogramming. Circ Res 111:5-8 


\title{
Development of a New In Vivo Optical Probe for Biological Diagnosis and Therapy
}

\author{
Michitaka Ozaki, Takeaki Ozawa, and Yuma Yamada
}

\begin{abstract}
Real-time imaging of the specific markers of lesions in the living body will provide valuable information in various physiopathological situations. Clinically, real-time imaging will definitely aid accurate diagnosis and rational therapy, especially in the surgical field.

In this chapter, we describe some unique optical probes for "biological imaging" and our recent challenge in undertaking development of a new type of in vivo probe. The reduction-oxidation-sensitive green fluorescent protein (roGFP) and bioluminescent luciferase probe for caspase-3 activity have been useful for understanding of the dynamic changes of liver redox states and apoptotic cell death. To overcome the difficulty of imaging in deeper lesions by optical probes, we newly developed a far-red bioluminescent probe. Lastly, we have undertaken the challenge to develop an innovative optical probe that switches "on" only when the probe recognizes a target molecule to reduce non-specific signals in vivo. The project of developing this unique probe is still underway.

Regarding a carrying system of the probe into cells in vivo, we have developed a liposome with cell-penetrating octa-arginine peptides (R8) and a pH-sensitive fusogenic peptide (GALA), which delivers the functional proteins into cells efficiently and rapidly in vivo.

We believe that these optical probes will provide a new avenue toward new diagnosis and therapy to come in the future.
\end{abstract}

Keywords Optical probe $\bullet$ In vivo imaging • Fluorescence • Bioluminescence • Oxidative stress $\bullet$ Apoptosis $\bullet$ Liver $\bullet$ Liposome

\footnotetext{
M. Ozaki, M.D., Ph.D. (四)

Laboratory of Molecular and Functional Bio-Imaging (LMFBI), Faculty of Health Sciences, Graduate School of Health Sciences, Hokkaido University,

N-12, W-5, Kita-ku, Sapporo, Hokkaido 060-0812, Japan

e-mail: ozaki-m@med.hokudai.ac.jp

T. Ozawa

Department of Chemistry, School of Science, The University of Tokyo,

7-3-1 Hongo, Bunkyo-ku, Tokyo 113-0033, Japan

Y. Yamada

Laboratory for Molecular Design of Pharmaceutics, Faculty of Pharmaceutical Sciences,

Hokkaido University, N-12, W-5, Kita-ku, Sapporo, Hokkaido 060-0812, Japan
}

K. Nakao et al. (eds.), Innovative Medicine, DOI 10.1007/978-4-431-55651-0_22 


\section{Abbreviations}

$\begin{array}{ll}\text { AdpcFluc } & \text { Adenovirus vector encoding pcFluc-DEVD } \\ \text { Dlk1 } & \text { Human Delta-like protein } \\ \text { AP-1 } & \text { Activator protein 1 } \\ \text { ATP } & \text { Adenosine triphosphate } \\ \text { BG } & \text { Benzylguanine } \\ \text { BRET } & \text { Bioluminescence resonance energy transfer } \\ \text { CLuc } & \text { Cypridina luciferase } \\ \text { DEVD } & \text { Asp-Glu-Val-Asp (a substrate sequence of caspase-3) } \\ \text { FBP } & \text { Far-red bioluminescent protein } \\ \text { FLuc } & \text { Firefly luciferase } \\ \text { FlucC-SNAP } & \text { C-terminal fragment of luciferase conjugated with SNAP } \\ \text { FlucN-SNAP } & \text { N-terminal fragment of luciferase conjugated with SNAP } \\ \text { H/R } & \text { Hypoxia and reoxygenation } \\ \text { I/R } & \text { Ischemia and reperfusion } \\ \text { MAPK } & \text { Mitogen-activated Protein Kinase } \\ \text { MEND } & \text { Multi-functional envelope-type nano device } \\ \text { NF-kB } & \text { Nuclear factor-kappa B } \\ \text { OS } & \text { Oxidative stress } \\ \text { pcFluc-DEVD } & \text { Cyclic luciferase reflecting caspase-3 activity } \\ \text { PET } & \text { Positron emission tomography } \\ \text { roGFP } & \text { Reduction-oxidation sensitive green fluorescent protein } \\ \text { ROS } & \text { Reactive oxygen species } \\ \text { R8 } & \text { Octa-arginine peptide } \\ \text { SNAP } & \text { A } 182 \text { resides }\end{array}$

SNAP A 182 residues polypeptide that can be fused to any protein of interest

\section{Introduction}

Real-time monitoring of molecular markers of lesions in the living body will provide valuable information on various physiopathological phenomena. This will aid understanding of lesions qualitatively as well as quantitatively. Clinically, this will lead to more accurate diagnosis and rational therapy. Especially in the surgical field, this kind of technology will enable us to "see" the biological characteristics (and potential pathological/progressive behavior) of the lesions during operation as well as its location, size, and expansion, which will definitely help the surgeon select the best surgical procedure for the patient.

The conventional imaging technologies commonly used in clinical practice include CT, PET-CT, and MRI. These technologies provide useful and geographical information about the location, size, shape, and expansion (including distant metastasis), and aid accurate clinical diagnosis and pre-operative staging. However, they 
Table 1 Comparison between conventional imaging technology and optical imaging

\begin{tabular}{l|l|l}
\hline Type of imaging & Advantages & Disadvantages \\
\hline $\begin{array}{l}\text { Conventional } \\
\text { imaging } \\
\text { technology (CT/ }\end{array}$ & $\begin{array}{l}\text { (1) Can image exact size of lesions } \\
\text { macroscopically }\end{array}$ & $\begin{array}{l}\text { (1) Does not provide information } \\
\text { on biological features of lesions }\end{array}$ \\
\cline { 2 - 3 } & (2) Can image whole body & $\begin{array}{l}\text { (2) Does not show exact locations } \\
\text { during operation }\end{array}$ \\
\cline { 2 - 3 } & $\begin{array}{l}\text { (3) Enables preoperative diagnosis } \\
\text { and clinical staging }\end{array}$ & $\begin{array}{l}\text { (3) High cost and space } \\
\text { occupying }\end{array}$ \\
\hline $\begin{array}{l}\text { Optical imaging } \\
\text { (fluorescence/ } \\
\text { bioluminescence) }\end{array}$ & $\begin{array}{l}\text { (1) Can image molecules/cells of } \\
\text { interest; enables biological imaging } \\
\text { of lesions }\end{array}$ & $\begin{array}{l}\text { (1) Not good at examining deep } \\
\text { lesions }\end{array}$ \\
\cline { 2 - 3 } & $\begin{array}{l}\text { (2) Low cost and space saving; } \\
\text { enables intra-operative real-time } \\
\text { imaging }\end{array}$ & $\begin{array}{l}\text { (2) Needs fluorescent/ } \\
\text { bioluminescent reagents for } \\
\text { imaging }\end{array}$ \\
\cline { 2 - 3 } & \begin{tabular}{l} 
(3) Relative estimation \\
\hline
\end{tabular}
\end{tabular}

do not show the biological characteristics of the lesion, nor its exact location during the operation. In addition, they require a lot of space and much expense for installation.

On the other hand, optical imaging by fluorescence or bioluminescence will provide biological information with sophisticated probes at low cost and in a small space. Disadvantages of optical imaging are that it requires a customized probe (fluorescence or bioluminescence) and has difficulty in imaging deeper lesions. In addition, signals from optical probes are relative, not absolute (Table 1).

\section{An Optical Probe for In Vivo Imaging of Caspase-3 Activity}

Many optical probes have been developed to visualize protease activities for biological in vivo imaging [1-7]. They have been used to analyze the dynamic changes in the cell and organ stresses and injuries, including hypoxia and reoxygenation $(\mathrm{H} / \mathrm{R})$ and ischemia and reperfusion (I/R) of cells and organs, respectively. $\mathrm{I} / \mathrm{R}$-induced injury is an important concern in various clinical situations, including surgical resection of organs, transplantation of organs and cells, and myocardial/ cerebral/intestinal infarction. In these clinical situations, prolonged ischemia followed by reperfusion results in extended organ injury caused by apoptosis, necrosis, and organ failure [8]. Although the mechanisms of I/R-induced injury are complex, post-ischemic apoptotic and/or necrotic injury play a pivotal role in subsequent organ failure $[9,10]$. Therefore, noninvasive monitoring of cell and organ injury (e.g., caspase activity) in vivo is definitely beneficial and is able to provide important clues for treatment.

We previously developed a novel probe of cyclic luciferase reflecting caspase-3 activity (pcFluc-DEVD) [2]. Two fragments of DnaE inteins are fused with neighboring $\mathrm{N}$-terminal and $\mathrm{C}$-terminal ends of firefly luciferase, which are connected 
with DEVD (Asp-Glu-Val-Asp [a substrate sequence of caspase-3]). After translation into a single polypeptide, the $\mathrm{N}$-terminal and $\mathrm{C}$-terminal ends of the luciferase are ligated by protein splicing, producing a closed circular polypeptide chain. The cyclic luciferase structure is distorted and therefore almost loses its bioluminescent activity. When caspase- 3 is activated in cells, the DEVD chain is cleaved, which restores firefly luciferase (Fluc) luminescent activity by returning to the original structure (Fig. 1) [11].

This cyclic luciferase was first applied for quantitative measurement of caspase-3 activity in living cells. HeLa cells transfected with this probe were stimulated with staurosporine, an inducer of apoptosis. This bioluminescent probe based on cyclic luciferase allowed precise and quantitative measurements of caspase- 3 activity in living cells. Moreover, the response of this cyclic luciferase to caspase- 3 activation was rapid and paralleled biochemically assessed apoptosis.

This imaging technology by cyclic luciferase could be applied for in vivo realtime imaging of caspase- 3 activity in living mice. We successfully imaged the caspase-3 activity in post-ischemic liver using our caspase-3 probe (Fig. 2). By transfecting the adenovirus vector encoding pcFluc-DEVD (AdpcFluc), we investigated whether or not this probe could monitor caspase-3 dependent apoptosis in the mouse liver during I/R (Fig. 2) [11]. We confirmed the efficacy of the probe in detecting apoptotic liver injury in a mouse model, which showed the potency for future clinical application.

\section{bioluminescent probe for Caspase-3 activity -distorted circular luciferase senses caspase-3 activity -}

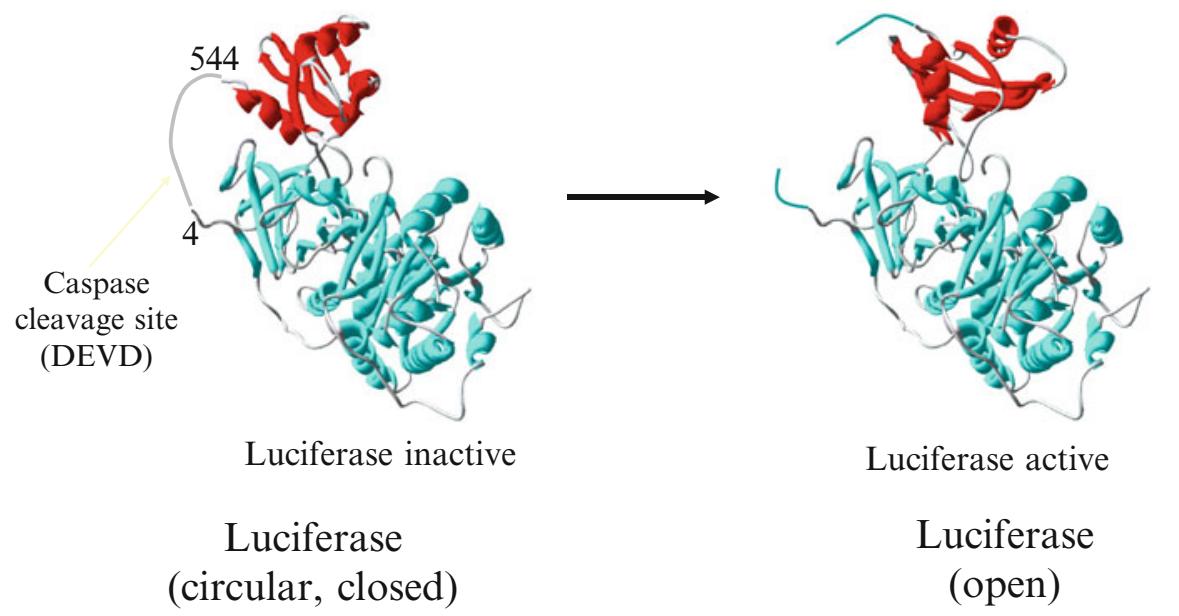

Fig. 1 Bioluminescent probe for caspase-3 activity. The principle for monitoring activity of caspase-3 is based on cyclic firefly luciferase (Modified from Ozaki et al. [11], with permission) 


\section{In vivo imaging of caspase-3 activity by the optic probe}
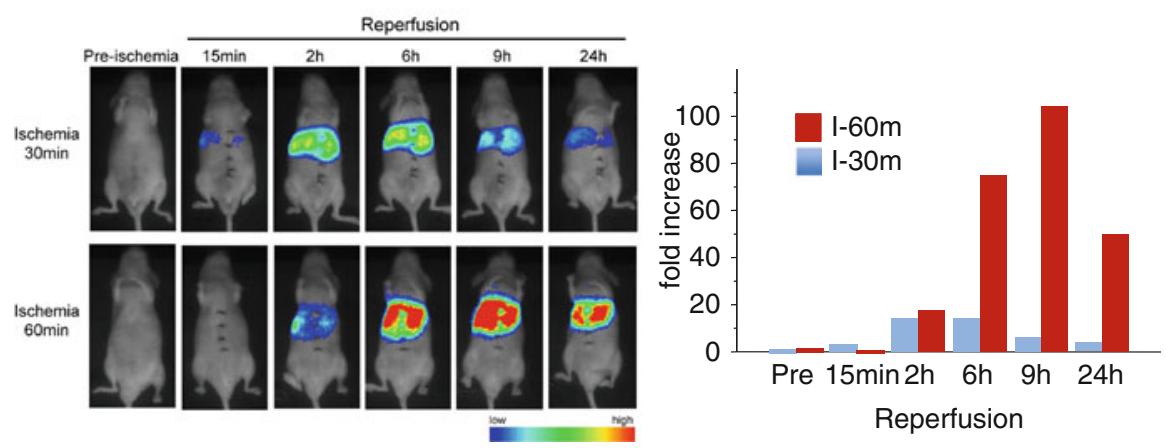

Fig. 2 In vivo imaging of caspase-3 activity by the optical probe. An ischemic insult to the liver induced caspase-3 activation (Modified from Ozaki et al. [11], with permission)

\section{An Optical Probe for In Vivo Imaging of the Hepatic Redox State}

Reactive oxygen species (ROS) generated during I/R of organs certainly play a pivotal role in I/R-induced organ injury and failure [12]. Hepatic I/R results in massive cell death (a) through apoptosis and necrosis mediated by excessive generation of ROS in hepatocytes early after I/R [13]; and (b) through necrosis caused by neutrophilic infiltration in the later phase [14]. According to the numerous studies on liver $\mathrm{I} / \mathrm{R}$ in mice, organ injury in the early post-I/R period is definitely mediated by generation of ROS in hepatocytes $[15,16]$. Excessive ROS directly exert deleterious effects on cells and tissues through lipid peroxidation, protein degradation, and DNA damage. In addition, redox-sensitive molecules, such as nuclear factor-kappa $\mathrm{B}(\mathrm{NF}-\kappa \mathrm{B})$, activator protein 1 (AP-1), and some mitogen-activated protein kinases (MAPKs), are potentially activated under these oxidative conditions and play pivotal roles in I/R-induced injury [17]. Therefore, monitoring cellular redox states by visualizing cellular ROS in post-I/R tissue has been one of the possible strategies for forecasting I/R-induced injury and failure [18]. These considerations suggest that the post-I/R redox state may be a good prognostic indicator of liver injury and function after liver surgery and transplantation, implying that in vivo monitoring may provide more diagnostic and therapeutic options in clinical liver surgery and transplantation.

For in vivo assays of tissue oxidative stress (OS), tissues are usually homogenized and subsequently assayed, either with redox-sensing electrodes or by measuring the ratios of the reduced and oxidized forms of glutathione and ascorbate. Recently, the redox states of tissues have also become assessable by using the dyes 5- (and 6-) carboxy-2,7-dichlorodihydrofluorescein diacetate [19] and dihydrofluorescein diace- 


\section{In vivo imaging of I/R-induced oxidative stress of mouse liver}

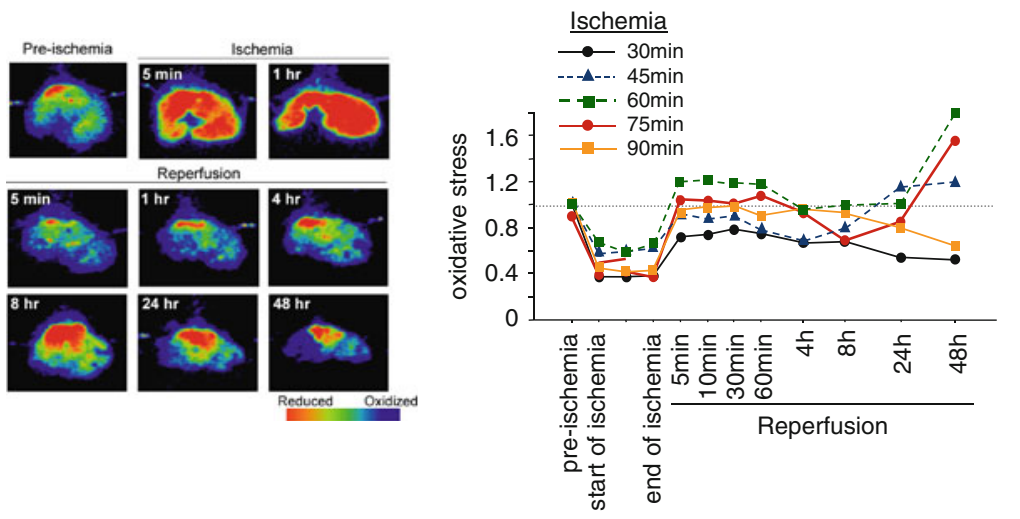

Fig. 3 In vivo imaging of I/R-induced oxidative stress of the mouse liver. The roGFP probe showed dynamic changes in liver redox states. The left panel depicts representative images of the dynamic changes after $60 \mathrm{~min}$ of ischemia, followed by reperfusion (oxidation, green to blue; reduction, orange to red) (Modified from Haga et al. [10], with permission)

tate [20]. Although such approaches allow evaluation of the sum of the oxidized and reduced species present, and thereby estimation of the overall redox state of the tissue, these techniques do not allow measurement of redox potentials in real time. More importantly, homogenization of tissues precludes the possibility of monitoring dynamic changes in OS in the same animal and organ.

A newly developed reduction-oxidation-sensitive green fluorescent protein (roGFP) probe (GFP with mutations of C48S, S147C, Q204C, and S65T) [1] could be applied for in vivo imaging. Use of the roGFP probe enables evaluation of cell and organ redox states non-invasively and continuously. Adenovirally transduced roGFP in the mouse liver was shown to act as a good indicator of pre- and postischemic liver redox states (Fig. 3) [10]. Through use of this roGFP probe, postoperative liver injury may be predicted by real-time monitoring of liver redox states.

\section{Optical Imaging of Deeper Lesions in the Living Body}

An increasing number of antibodies have been applied for targeting antigens on the surface of cancer cells for clinical diagnosis and therapy, on the basis of the fact that some specific antigens expressed on the cancer cell surface reflect malignant behaviors including invasion, metastasis, and neo-vascularization [21-23]. Realtime visualization of tumor-specific antigens in the living body will enable timely understanding of cancer expansion and its biological behaviors, and prediction of the patient's prognosis in the near future. 
Fluorescence and bioluminescence imaging technologies have played important roles in molecular imaging in cells and small animals [24-26]. Photon detection is easy to use, cost saving, and space saving, compared with radioisotope imaging. Basically, bioluminescence imaging is achieved with a luciferin-luciferase reaction in the presence of sufficient amounts of molecular oxygen and adenosine triphosphate (ATP). However, most bioluminescence spectra are observed in the visible region, which overlaps with the absorption spectra of hemoglobin and tissue constituents, therefore attenuating the bioluminescence intensity in living animals.

Recently, a self-illuminating quantum dot probe was developed to improve the light penetration based on bioluminescence resonance energy transfer (BRET) between the bioluminescence of Renilla luciferase and quantum dots [27]. The multivalent conjugation of Renilla luciferase to single dots allowed for highly efficient BRET between luciferase and quantum dots. However, the large size of the conjugate caused problems in metabolism and localization in vivo [28]. Several far-red fluorescent protein variants showing maximal emission at around $650 \mathrm{~nm}$ have been developed for in vivo imaging [29], but are not well characterized as energy acceptors for BRET systems. On the other hand, the organic dye indocyanine and its derivatives have molecular weights of less than 1,200 Da; they produce far-red fluorescence and have been widely used for in vivo imaging [30].

Luciferase conjugated to such organic dyes is expected to create new possibilities for in vivo application. Cypridina luciferase (CLuc) catalyzes the oxidation of Cypridina luciferin to yield light emission peaking at $460 \mathrm{~nm}$ [31].

The luciferase genes from both Cypridina hilgendorfii and C. noctiluca have been cloned [32], the latter of which we used. This self-secretory CLuc of $62 \mathrm{kDa}$ has some unique properties as a bioluminescent enzyme [33]. The secreted protein contains 17 disulfide bond pairs and is highly stable under physiological conditions. Its turnover rate (1400 luciferin molecules per minute) is the highest among known luciferases [34]. We have expressed recombinant CLuc in yeast and applied it to ELISA. We conjugated a far-red fluorescent indocyanine derivative to biotinylated CLuc via glycol chains and named this far-red bioluminescent protein (FBP).

A monoclonal antibody against human Delta-like protein (anti-Dlk1), one of the embryonic antigens expressed on the surface of many cancer cells, including hepatocellular carcinoma, was then produced. Using anti-Dlk1 monoclonal antibody linked to FBP via biotin-avidin interaction, we achieved bioluminescence imaging of cancer cells in vivo in mice (Fig. 4).

However, very unfortunately, there were some non-specific signals from the tissues that did not express Dlk-1, though the tumor implanted into the liver itself was imaged clearly by this probe. This may have been because excessive and unbound antibodies circulating in the blood or captured in tissues emitted signals when the substrate was injected. In order to apply this probe to clinical application, nonspecific signals must be reduced as much as possible. So, we have launched the following new project. 
In vivo imaging by a new optic probe for deeper lesions in mouse
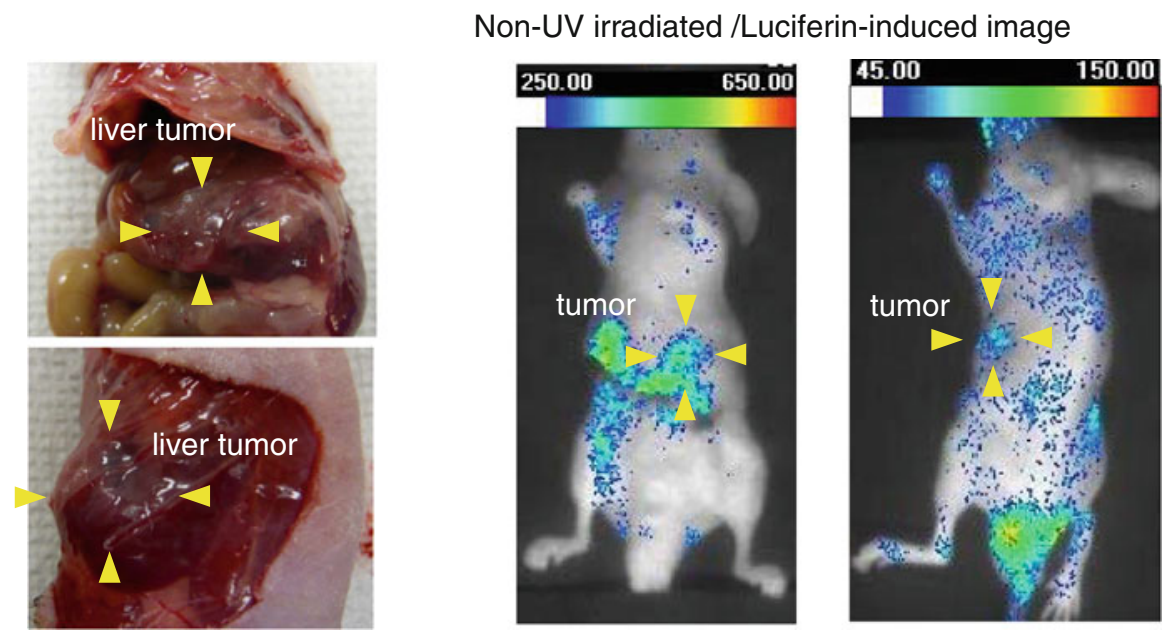

Image was obtained $24 \mathrm{hr}$ after Luciferin adm (iv)

(400mg/200ml i.v.)

Fig. 4 In vivo imaging by a new optical probe for deeper lesions in the mouse. A liver tumor of the mouse was imaged, but some non-specific signals were observed, probably because of excessive and unbound antibodies

\section{Development of an In Vivo Optical Probe for Antigen-Specific Imaging Inside/Outside Cells}

For accurate biological diagnosis and rational therapy in clinical practice, we need to know the biological features of the lesion as well as its size, shape, and extent. For this purpose, we plan to develop a new optical probe, which will image the target molecules only after binding to the antigen. This does not allow unbound antibody to emit light signals, and therefore it will lead to the reduction of nonspecific signals in vivo. Additionally, the probe should be applied to targets inside as well as outside cells in vivo, because there are lots of diagnostic and therapeutic targets inside as well as outside cells. In order to create a new type of in vivo optical probe, the following two technologies have to be developed (Fig. 5).

(A) Development of an Optical Probe Activated Only When it Binds to the Target Molecule

In order to develop a probe activated after binding to the target antigen in vivo, we have designed a new type of luciferase-antibody conjugated probe. We planned to prepare split firefly luciferases, the $\mathrm{N}$-terminal and C-terminal fragments of luciferase conjugated with SNAP (a 182-residue polypeptide that can be fused to any 


\section{Strategy for Ag-specific imagingin vivo}

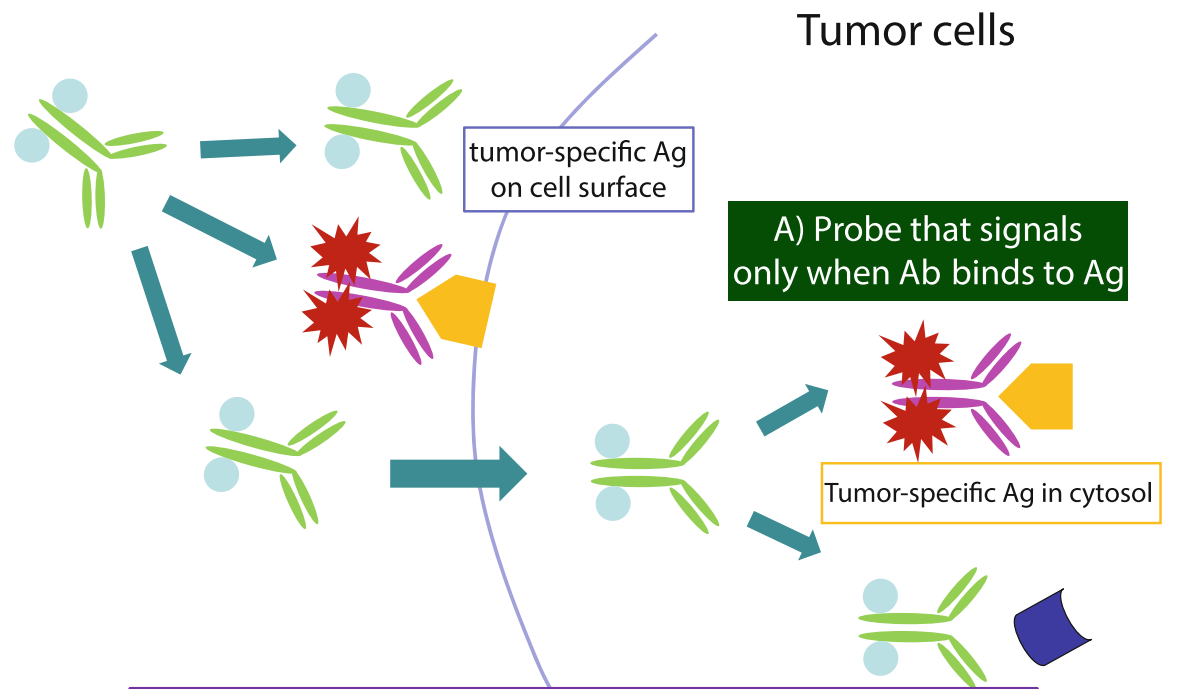

B) Safe and stable delivery of a probe into cell/cytosol

Fig. 5 Strategy for antigen-specific imaging in vivo

protein of interest) - FlucN-SNAP and FlucC-SNAP, respectively - and to prepare a polyclonal antibody covalently connected with benzylguanine (Ab-BG). When the antibodies with FlucN and FlucC recognize and bind to the close epitopes of the same antigen, split luciferase fragments will be reconstituted and activated (Fig. 6). Theoretically, the probe should enable us to see the target molecule in tissues and cells only when it exists.

We plan first to express FlucN-SNAP and FlucC-SNAP proteins by Escherichia coli, and then to bind benzylguanine (BG) with polyclonal Ab. Lastly, we will conjugate these proteins (Fig. 7).

To date, we have succeeded in obtaining sufficient amounts of FlucN-SNAP and FlucC-SNAP proteins by the E. coli expression system, and we have confirmed the good binding capacity of FlucN-SNAP and FlucC-SNAP proteins to BG. So, we have bound $\mathrm{BG}$ with polyclonal $\mathrm{Ab}$, and performed conjugation with Fluc-SNAPs. We are now examining the probe function in our in vitro system.

(B) Development of a Safe and Stable System for Delivery of Optical Probes into Cells

In order to develop a new system to deliver sufficient amounts of optical probe safely into cells in vivo, we are now developing a new liposome-based delivery system.

There seem to be two important processes for developing an effective and rapid delivery system (Fig. 8). First, the carrier including probes in it should be 


\section{Concept for antigen-dependent optic probe}

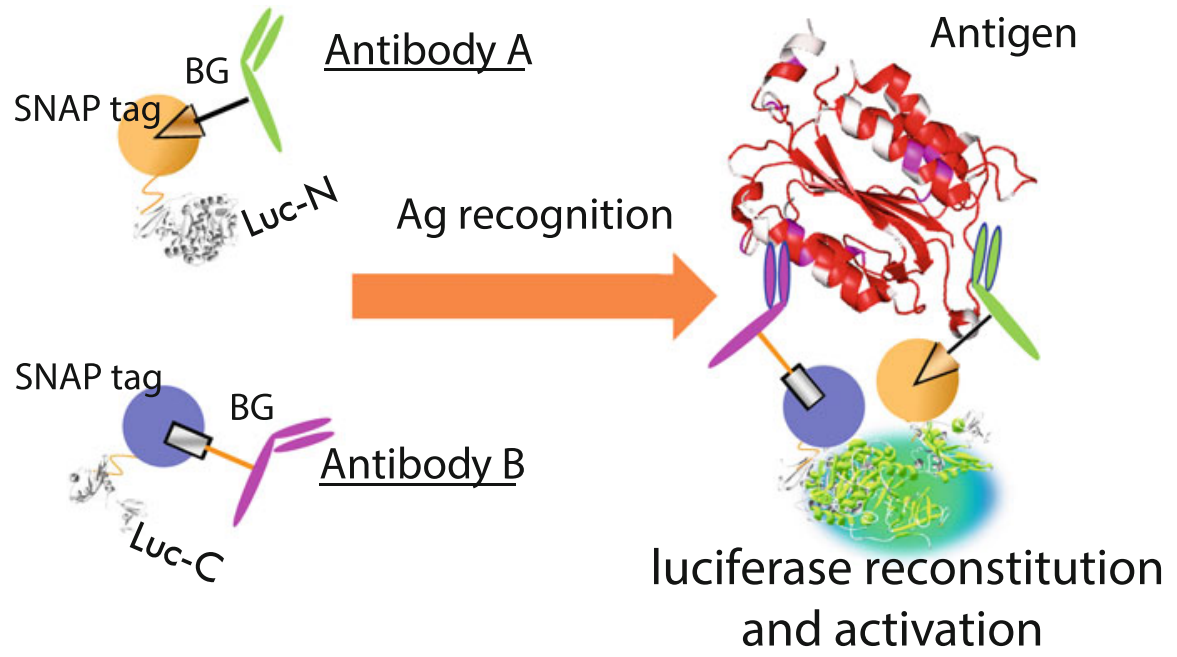

Fig. 6 Concept for an antigen-dependent optical probe. When the probes with C-/N-terminal fragments of luciferase recognize the antigen, luciferase is reconstructed and activated

\section{Procedures of making a probe}

\section{(1) Expression of FlucN-SNAP \& FlucC-SNAP}

\begin{tabular}{|c|c|c|}
\hline FlucN & SNAP & $6 \mathrm{His}$ \\
\hline FlucC & SNAP & $6 \mathrm{His}$ \\
\hline
\end{tabular}

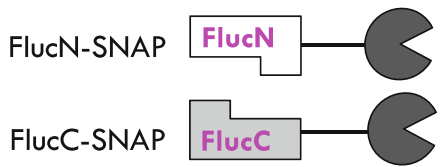

(2) Binding BG (Ligand) to polyclonal $\mathbf{A b}$

$\checkmark$ :Benzylguanine group

SNAP-tagged
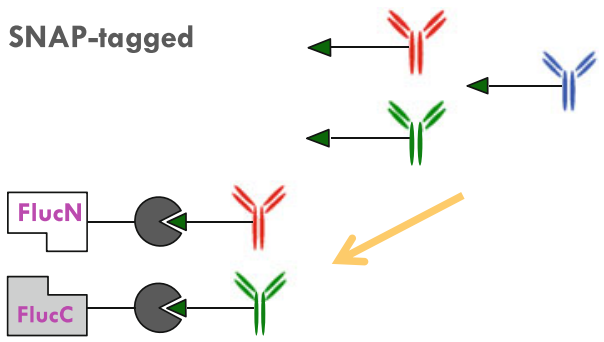

(3) Conjugation of Fluc fragments and antibodies by SNAP-BG binding

Fig. 7 Procedure for making a probe: (1) expression of FlucN-SNAP and FlucC-SNAP; (2) binding BG (ligand) to polyclonal Ab; (3) conjugation of Fluc fragments and antibodies by SNAP-BG binding 


\section{Strategy for cellular delivery of the probe}

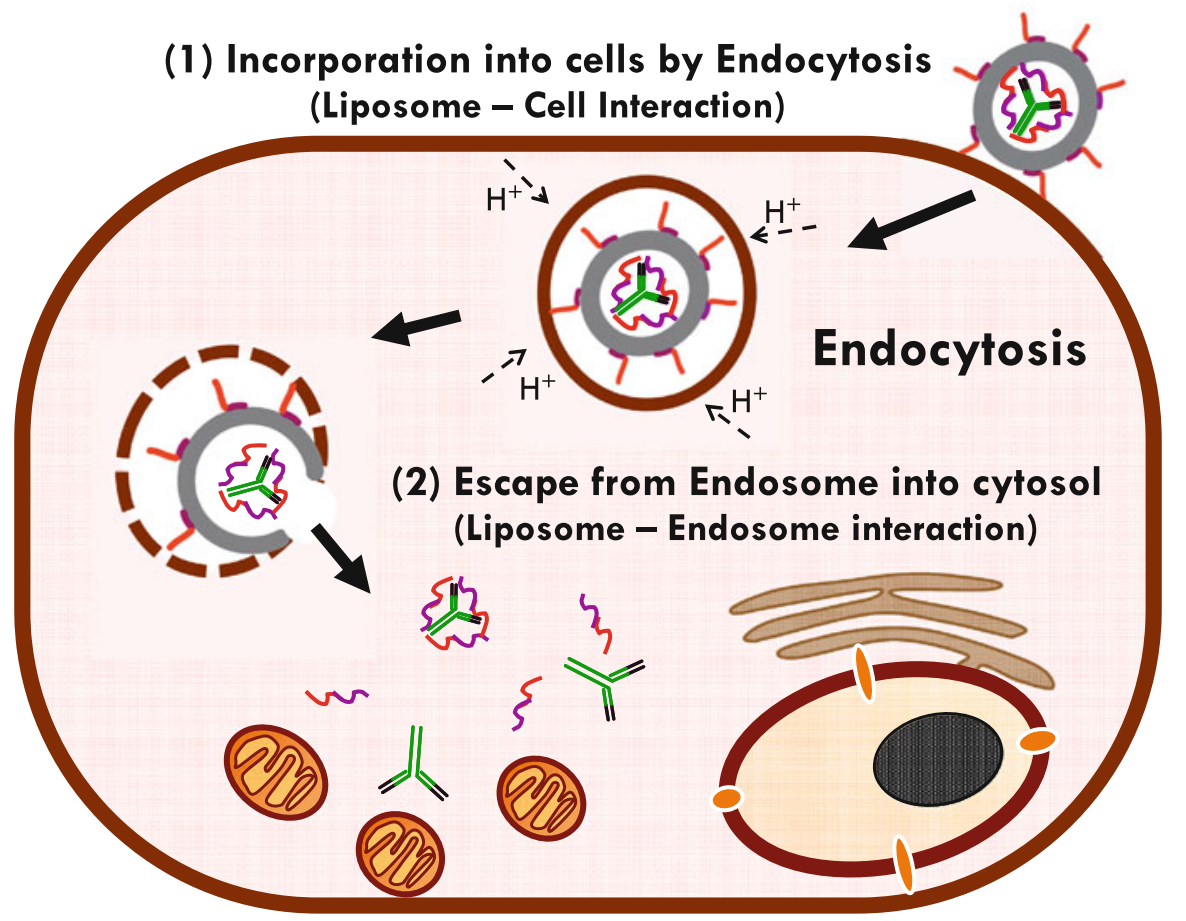

Fig. 8 Two important processes for efficient cellular delivery of probes: (1)incorporation into cells by endocytosis (a liposome-cell interaction); (2) escape from the endosome into the cytosol (a liposome-endosome interaction)

incorporated into cells sufficiently and rapidly. Second, the incorporated probe should be released from the lysosome into the cytosol. These processes should not be harmful to the cells and the probes.

We have already made a prototype liposome-based carrier for efficient drug delivery in vivo. We further added a cell-permeable octa-arginine peptide (R8) to the liposome, expecting better incorporation into cells (Fig. 9). In the in vitro experiments, the R8 liposome successfully delivered the "antibody" into the cells much better than the liposome without R8. The R8 liposome, however, failed to escape the "antibody" from the endosome into the cytosol.

Next, we applied GALA to make the probe release rapidly and efficiently from the endosome into the cytosol (Fig. 10). GALA is a 30-amino-acid peptide with a glutamic acid-alanine-leucine-alanine repeat that contains a histidine and tryptophan residue. GALA changes its conformation from a random coil to an amphipathic alpha-helix when $\mathrm{pH}$ values are between 7.0 and 5.0. At neutral $\mathrm{pH}$, GALA is water soluble, while at acidic $\mathrm{pH}$, GALA binds to bilayer membranes. So it is expected to promote rapid fusion of the liposome with the endosomal membrane in the cell. The fusogenic liposome was modified with a cell-penetrating R8 and also GALA. 
Multi-functional Envelope-type Nano Device (MEND) : R8-Liposome

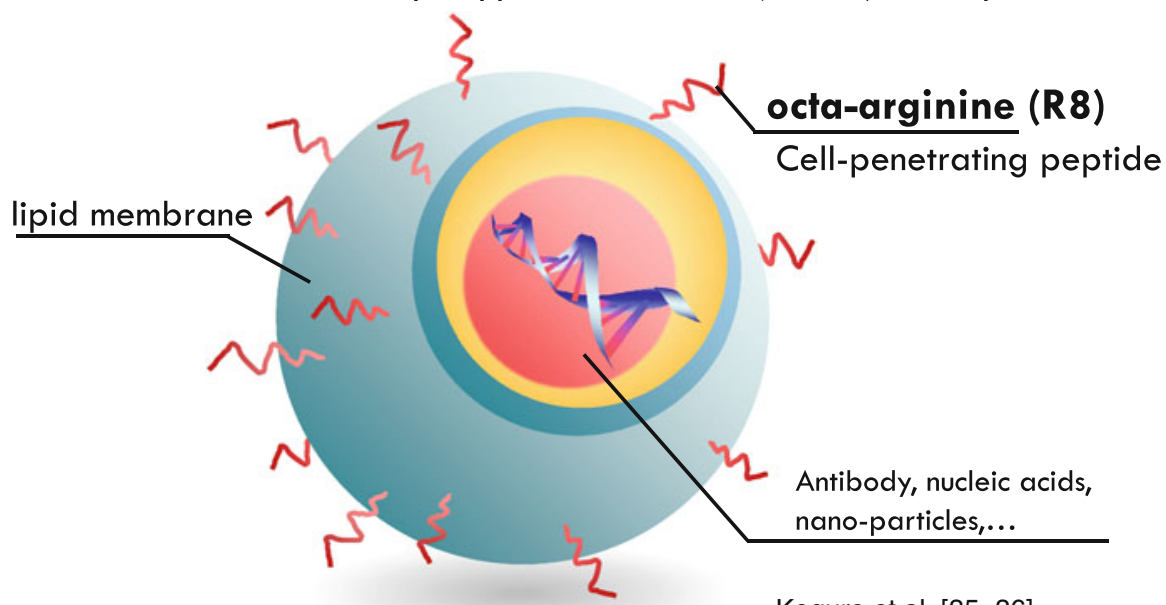

Kogure et al. [35, 36]

Nakamura et al. [37]

Fig. 9 Concept of a multifunctional envelope-type nano device (MEND). Octa-arginine (R8) is bound to the liposome surface (R8 liposome)

\section{GALA: $\mathrm{pH}$ sensitive fusogenic peptide}

\section{GALA: WEAALAEALAEALAEHLAEALAEALEALAA}
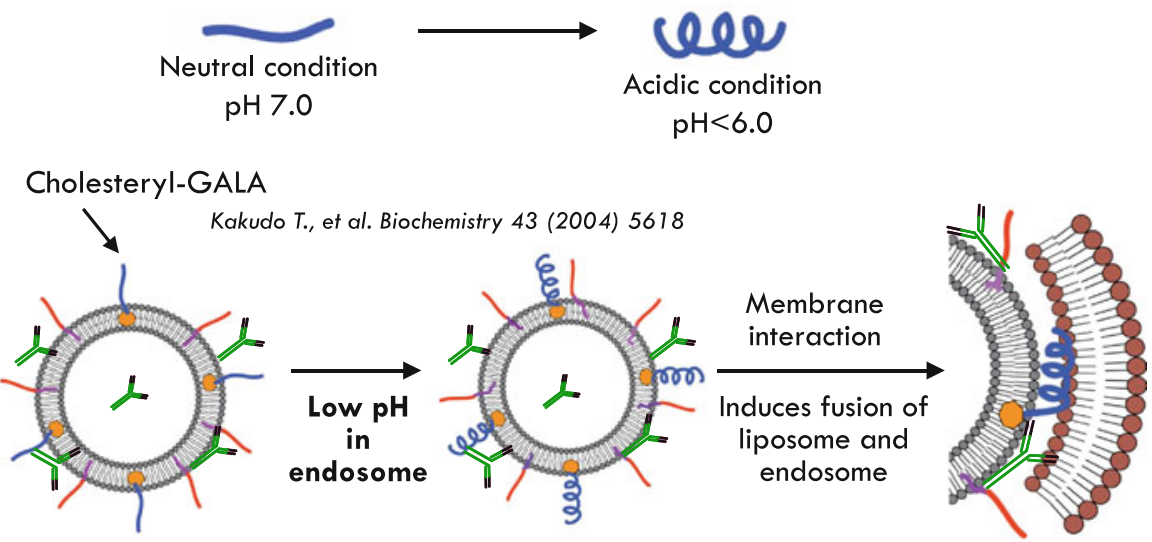

Fig. 10 The GALA pH-sensitive fusogenic peptide changes its conformation depending on $\mathrm{pH}$ values and is expected to promote lipid-membrane fusion 
The presence of R8 enhanced the cellular uptake of antibodies, while the mounting of GALA reduced the endosomal entrapment, resulting in release of the antibody into the cytosol within $30 \mathrm{~min}$ in in vitro experiments. Moreover, compared with the commercially available reagents for protein delivery, the R8/GALAmodified liposome was superior in terms of both cellular uptake and transport into the cytosol. The specific antibody delivered by the R8/GALA liposome was detected by its epitope, which indicated that the R8/GALA liposome basically did not deteriorate the protein function.

\section{Conclusion}

In this chapter, we have described possible application of an optical probe for "biological imaging" and our recent challenge in undertaking development of a new type of in vivo probe.

In the mouse liver I/R model, the fluorescent probe, roGFP, and bioluminescent luciferase probe, pcFluc-DEVD, were useful in understanding the dynamic changes of liver redox states and apoptotic cell death. We could understand the pathological significance of OS and apoptosis in I/R-induced liver injury. Because of the difficulty of imaging of deeper lesions by optical probes, we developed a unique probe loaded on an antibody. This probe successfully imaged a target molecule in deeper regions in mice, but also revealed another problem - an in vivo non-specific signal from excessive and unbound antibodies. Lastly, we undertook the challenge to develop an innovative optical probe which switches "on" only when the probe recognizes a target molecule. The project of developing this unique probe is still underway but seems very promising at this time.

Regarding a carrying system of the probe to target molecules in vivo, we developed a liposome bound with a cell-penetrating R8 and GALA. The R8/GALA liposome delivered the "antibody" to the cells and also the cytosol efficiently and rapidly in vivo.

In the future, we plan to prove the efficacy of the new probe carried by the R8/ GALA liposome in in vivo experiments.

Open Access This chapter is distributed under the terms of the Creative Commons Attribution Noncommercial License, which permits any noncommercial use, distribution, and reproduction in any medium, provided the original author(s) and source are credited.

\section{References}

1. Dooley CT, Dore TM, Hanson GT et al (2004) Imaging dynamic redox changes in mammalian cells with green fluorescent protein indicators. J Biol Chem 279:22284-22293

2. Kanno A, Yamanaka Y, Hirano H et al (2007) Cyclic luciferase for real-time sensing of caspase-3 activities in living mammals. Angew Chem Int Ed 46:7595-7599

3. Zhu L, Xie J, Swierczewska M et al (2011) Real-time video imaging of protease expression in vivo. Theranostics 1:18-27 
4. Lexman B, Hall DE, Bhojani MS et al (2002) Noninvasive real-time imaging of apoptosis. Proc Natl Acad Sci U S A 99:16551-16555

5. Ray P, De A, Patel M et al (2008) Monitoring caspase-3 activation with a multimodality imaging sensor in living subjects. Clin Cancer Res 14:5801-5809

6. Yhee JY, Kim SA, Koo H et al (2012) Optical imaging of cancer-related proteases using nearinfrared fluorescence matrix metalloproteinase-sensitive and cathepsin B-sensitive probes. Theranostics 2:e0017

7. Kim GB, Kim Y-P (2012) Analysis of protease activity using quantum dots. Theranostics 2:e004

8. Ozaki M, Deshpande SS, Angkeow P et al (2000) Inhibition of the Rac1 GTPase protects against nonlethal ischemia/reperfusion-induced necrosis and apoptosis in vivo. FASEB $J$ $14: 418-429$

9. Lucchesi BR (1993) Complement activation, neutrophils, and oxygen radicals in reperfusion injury. Stroke 24:I41-I47

10. Haga S, Remington S, Morita N et al (2009) Hepatic ischemia induced immediate oxidative stress after reperfusion and determined the severity of the reperfusion-induced damage. Antioxid Redox Signal 11(10):2563-2572

11. Ozaki M, Haga S, Ozawa T (2012) In vivo monitoring of liver damage by caspase-3 probe. Theranostics 2(2):207-214

12. Robin E, Guzy RD, Loor G et al (2007) Oxidant stress during simulated ischemia primes cardiomyocytes for cell death during reperfusion. J Biol Chem 282:19133-19143

13. Tsung A, Kaizu T, Nakao A et al (2005) Ethyl pyruvate ameliorates liver ischemia-reperfusion injury by decreasing hepatic necrosis and apoptosis. Transplantation 79:196-204

14. Montalvo-Jave EE, Escalante-Tattersfield T, Ortega-Salgado JA et al (2008) Factors in the pathophysiology of the liver ischemia-reperfusion injury. J Surg Res 147:153-159

15. Haga S, Terui K, Fukai M et al (2008) Preventing hypoxia/reoxygenation damage to hepatocytes by p66(shc) ablation: up-regulation of anti-oxidant and anti-apoptotic proteins. J Hepatol 48:422-432

16. Terui K, Enosawa S, Haga $S$ et al (2004) Stat3 confers resistance against hypoxia/ reoxygenation-induced oxidative injury in hepatocytes through upregulation of Mn-SOD. J Hepatol 41:957-965

17. Thannickal VJ, Fanburg BL (2000) Reactive oxygen species in cell signaling. Am J Physiol Lung Cell Mol Physiol 279:L1005-L1028

18. Guo L, Haga S, Enosawa $S$ et al (2004) Improved hepatic regeneration with reduced injury by redox factor-1 in a rat small-sized liver transplant model. Am J Transplant 4:879-887

19. Jiang J, Kini V, Belikova $\mathrm{N}$ et al (2004) Cytochrome $\mathrm{c}$ release is required for phosphatidylserine peroxidation during Fas-triggered apoptosis in lung epithelial A549 cells. Lipids 39:1133-1142

20. Jones MA, Raymond MJ, Yang Z et al (2007) NADPH oxidase-dependent reactive oxygen species formation required for root hair growth depends on ROP GTPase. J Exp Bot $58: 126-127$

21. Adams R, Meade A, Wasan H et al (2008) Cetuximab therapy in first-line metastatic colorectal cancer and intermittent palliative chemotherapy: review of the COIN trial. Expert Rev Anticancer Ther 8:1237-1245

22. Boltze J, Förschler A, Nitzsche B et al (2008) Permanent middle cerebral artery occlusion in sheep: a novel large animal model of focal cerebral ischemia. J Cereb Blood Flow Metab 28:1951-1964

23. Ilowite NT (2008) Update on biologics in juvenile idiopathic arthritis. Curr Opin Rheumatol 20:613-618

24. Contag PR, Olomu IN, Stevenson DK et al (1998) Bioluminescent indicators in living mammals. Nat Med 4:245-247

25. Hoffman RM, Yang M (2005) Dual-color, whole-body imaging in mice. Nat Biotechnol 23:790 
26. Ntziachristos V, Ripoll J, Wang LV et al (2005) Looking and listening to light: the evolution of whole-body photonic imaging. Nat Biotechnol 23:313-320

27. So MK, Xu C, Loening AM et al (2006) Self-illuminating quantum dot conjugates for in vivo imaging. Nat Biotechnol 24:339-343

28. Frangioni JV (2006) Self-illuminating quantum dots light the way. Nat Biotechnol $24: 326-328$

29. Shcherbo D, Merzlyak EM, Chepurnykh TV et al (2007) Bright far-red fluorescent protein for whole-body imaging. Nat Methods 4:741-746

30. Frangioni JV (2003) In vivo near-infrared fluorescence imaging. Curr Opin Chem Biol 7:626-634

31. Shimomura O, Johnson FH (1971) Mechanism of the luminescent oxidation of Cypridina luciferin. Biochem Biophys Res Commun 44:340-346

32. Thompson EM, Nagata S, Tsuji FI (1989) Cloning and expression of cDNA for the luciferase from the marine ostracod Vargula hilgendorfii. Proc Natl Acad Sci U S A 86:6567-6571

33. Shimomura O, Johnson FH, Saiga Y (1961) Purification and properties of Cypridina luciferase. J Cell Comp Physiol 58:113-123

34. Shimomura O, Johnson FH, Masugi T (1969) Cypridina bioluminescence: light-emitting oxyluciferin-luciferase complex. Science 164:1299-1300 


\title{
Introduction of Mesenchymal Stem Cells for Liver Surgery (Hepatectomy and Transplantation)
}

\author{
Shinji Uemoto, Yasuhiro Fujimoto, Takumi Teratani, Hiroyuki Kanazawa, \\ Junji Iwasaki, Zhao Xiangdong, Yuki Masano, Shintaro Yagi, Koichiro Hata, \\ and Eiji Kobayashi
}

\begin{abstract}
In liver transplantation, prolonged ischemia and/or a relatively small graft (living, split, reduced) are the risk factors for liver dysfunction. Novel measures to enhance liver function with a smaller graft can be a clue for safe partial or living-donor liver transplantation or safe hepatectomy for malignant disease. The therapeutic potential and immunomodulatory effects of mesenchymal stem cells (MSCs) have been reported. In this chapter, recent finding on the positive effect of MSCs for liver transplantation and hepatectomy are discussed.

Our rat experiment revealed that introduction of MSCs provides trophic support to the I/R-injured liver by inhibiting hepatocellular apoptosis and by stimulating regeneration, which is shown with the pig model as well. In the rat liver transplantation model, portal transfusion of the MSCs ameliorates the injury of the liver graft after prolonged cold preservation and transplantation. Those findings together suggest a potential advantage with partial or living-donor liver transplantation. The most severe complication with cell therapy is embolus formation due to cell aggre-
\end{abstract}

\footnotetext{
S. Uemoto, M.D., Ph.D. ( $\square)$

Professor, Division of HBP Surgery and Transplantation,

Department of Surgery, Kyoto University Graduate School of Medicine,

54 Kawara-cho, Shogoin, Sakyo, Kyoto 606-8507, Japan

e-mail: uemoto@kuhp.kyoto-u.ac.jp

Y. Fujimoto, M.D., Ph.D.

Division of HBP Surgery and Transplantation, Department of Surgery, Kyoto University Graduate School of Medicine, 54 Kawara-cho, Shogoin, Sakyo, Kyoto 606-8507, Japan

Division of HBP Surgery, Department of Surgery, Shizuoka City Shizuoka Hospital, 10-93

Ote-machi, Aoi-ku, Shizuoka City, Shizuoka 420-8630, Japan

T. Teratani, Ph.D. • E. Kobayashi, M.D., Ph.D.

Division of Development Advanced Therapy, Center for Development of Advanced Medical Technology, Jichi Medical University,

3311-1, Yakushiji, Shimotsuke, Tochigi 329-0498, Japan

H. Kanazawa, M.D., Ph.D. • J. Iwasaki, M.D., Ph.D. • Z. Xiangdong, M.D., Ph.D.

Y. Masano, M.D. • S. Yagi, M.D., Ph.D. • K. Hata, M.D., Ph.D.

Division of HBP Surgery and Transplantation, Department of Surgery, Kyoto University

Graduate School of Medicine, 54 Kawara-cho, Shogoin, Sakyo, Kyoto 606-8507, Japan
}

K. Nakao et al. (eds.), Innovative Medicine, DOI 10.1007/978-4-431-55651-0_23 
gation. However, with modification of the solution, we can keep cells in a suspended form for several hours, which secures safe administration of MSCs.

Keywords Liver transplantation - Mesenchymal stem cell • Hepatectomy • Ischemia $\bullet$ Reperfusion $\bullet$ Liver failure $\bullet$ Living donor $\bullet$ Partial liver transplantation

\section{Clinical Liver Transplantation: Deceased Donor vs. Living Donor}

\section{Statistics}

Liver transplantation has been successfully used as a treatment for end-stage liver disease, though there has been discussion regarding where the liver graft is obtained, i.e., from a deceased donor or a living donor. In the USA, the majority of liver transplantation is done with deceased donors, and the number of living-donor liver transplantations (LDLTs) is limited. The reason why LDLT is not popular in the USA is due to the death of a donor in 2002. Since 2001, the number of patients who have undergone LDLT has declined. On the other hand, in Japan, most liver transplantation is done with LDLT. Though the organ transplantation law took effect in 1997, the number of deceased donor liver transplantations (DDLTs) has been minimal; this is partly because the law itself is very strict in comparison with those of Western countries. Even since modification of the law in 2010, the annual number of DDLTs has been limited to below 50. The ratio of the numbers of LDLTs to DDLTs is $10: 1$ [1].

\section{Operations and the Post-transplant Course}

In DDLT, the whole liver is used, and it has a high reserve capacity (Fig. 1). In other words, the liver volume is sufficient for the recipient throughout the postoperative course from the immediate post-transplant period. That is true with LDLT as well; therefore, a larger graft is preferable in terms of the recipient's postoperative course. However, harvesting a larger graft from a living donor may cause complications, and there have been some reports of donor deaths. Currently, we primarily use smaller grafts such as left lobe grafts, in our program, though small-for-size syndrome (SFSS) occasionally follows in recipients, which can be lethal. Since LDLT is a type of partial liver transplantation (PLTx), the possibility of SFSS is unavoidable. Functional impairment conditions such as prolonged cholestasis, ascites, coagulopathy, and encephalopathy are considered to result from SFSS that occurs after LDLT [2]. They strongly affect patient survival and clinical costs for the whole treatment. Therefore, novel measures to enhance liver function with smaller grafts may be the key to safe PLTx or LDLT. 


\section{Postoperative course (human): Whole vs. Partial LTX}
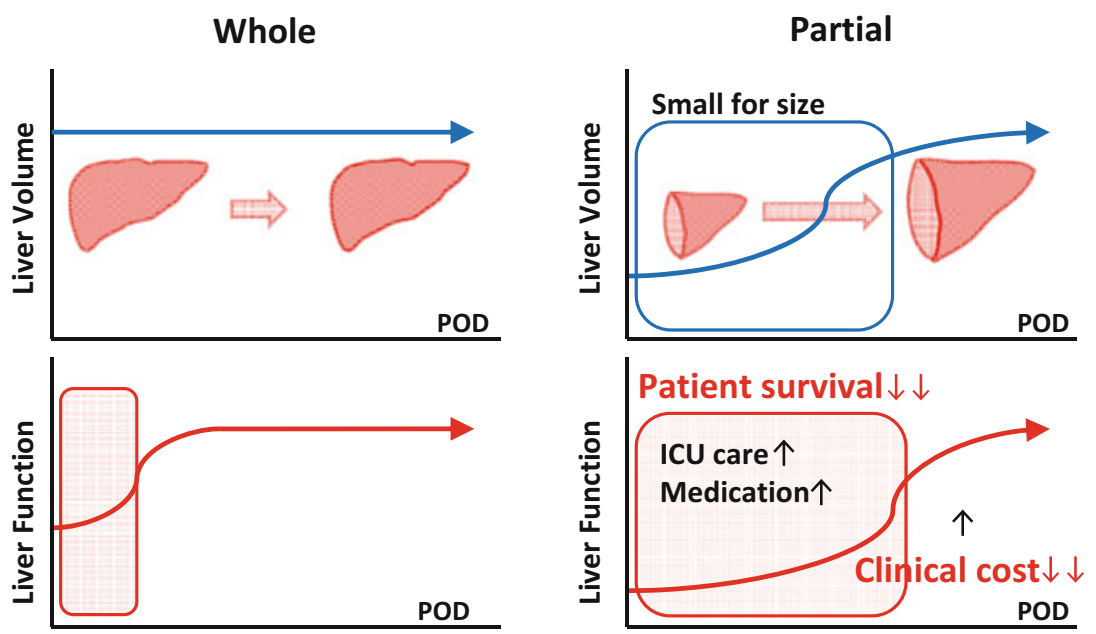

Fig. 1 Postoperative course of whole vs. partial liver transplantation, showing the difference between DDLT and LDLT after the operation. There is no change in size with DDLT, whereas graft enlargement is observed with LDLT. Functional recovery follows volume recovery; therefore, there should be some critical period with LDLT after operation

\section{Mesenchymal Stem Cells}

\section{Characteristics}

Mesenchymal stem cells (MSCs), present in the human body in large amounts, can be candidates for stem cell-based therapy for liver diseases. Recently, the therapeutic potential and immunomodulatory effect of MSCs have been revealed. Adipose tissue-derived mesenchymal stem cells (AT-MSCs), because of their high accessibility with minimal invasiveness, are especially attractive in the context of future clinical applications. MSCs can differentiate into multiple types of lineages, such as chondrogenic, osteogenic, and adipogenic, depending on the microenvironment around them. Importantly, human MSCs represent an advantageous cell type for allogeneic transplantation, as they are immunoprivileged with low human leukocyte antigen (HLA) I and no HLA II expression, thereby reducing the risk of allogeneic transplant rejection and preventing graft-versus-host disease (GVHD). According to Banas et al., adipose tissue-derived (AT) MSCs can improve liver functions, which we have verified by changes in the levels of biochemical parameters. Ammonia, uric acid, glutamic-pyruvic transaminase, and glutamic-oxaloacetic transaminase concentrations returned to a nearly normal level after AT-MSC transplantation. It was shown that AT-MSCs secrete interleukin 1 receptor (IL-1R), IL-6, 
IL-8, granulocyte colony-stimulating factor (G-CSF), granulocyte-macrophage colony-stimulating factor (GM-CSF), monocyte chemotactic protein 1, nerve growth factor, and hepatocyte growth factor, suggesting that AT-MSCs account for broad therapeutic efficacy in animal models of liver diseases and in the clinical settings of liver disease treatment [3].

\section{Isolation Techniques}

AT-MSCs can be derived from subcutaneous adipose tissue. Adipose tissue was minced with scissors and scalpels into less than $3 \mathrm{~mm}$ pieces and, after gentle shaking with an equal volume of $\operatorname{PBS}(-)$, the mixture was separated into two phases. The upper phase (containing stem cells, adipocytes, and blood), after washing with phosphate-buffered saline (PBS) (-), was enzymatically dissociated with $0.075 \%$ collagenase (type I)/PBS(-) for $1 \mathrm{~h}$ at $37^{\circ} \mathrm{C}$ with gentle shaking. The dissociated tissue was then mixed with an equal volume of DMEM, supplemented with $10 \%$ fetal bovine serum (FBS) and incubated for $10 \mathrm{~min}$ at room temperature. The solution was then separated into two phases. The lower phase was centrifuged at $1500 \mathrm{rpm}$ for $5 \mathrm{~min}$ at $20^{\circ} \mathrm{C}$. The cellular pellet was resuspended in $160 \mathrm{mM} \mathrm{NH}_{4} \mathrm{Cl}$ to eliminate erythrocytes and passed through a $40 \mu \mathrm{m}$ mesh filter into a new tube. The cells were resuspended in an equal volume of DMEM/10 \% FBS and then centrifuged. Isolation resulted in obtaining $7.7 \times 10^{6}$ of adherent cells for a primary culture from $5 \mathrm{~g}$ of adipose tissue (approximately $1.0 \times 10^{5}$ to $4.6 \times 10^{6} / 1 \mathrm{~g}$ ) after $7-10$ days of culture. The cells were suspended in DMEM/10 \% FBS plated in a concentration of $1-5 \times 10^{6}$ cells $/ 75 \mathrm{~cm}^{2}$. The cells with $70-80 \%$ confluence were harvested with $0.25 \%$ trypsin EDTA and then re-plated at $1.0 \times 10^{5}$ cells $/ 60 \mathrm{~mm}$ dish and used for analysis [4].

\section{Mesenchymal Stem Cells for Rat Hepatectomy}

\section{Rat Hepatectomy Model}

Our rat model provided the ideal animal model to research I/R injury after major hepatectomy by the Pringle maneuver or liver transplantation with small-for-size grafts (Fig. 2) [5]. Intestinal congestion was avoided during the application of hepatic ischemia by bypassing portal flow through nonischemic lobes (the right and caudate lobes). Moreover, the left lateral and the left portion of the medial lobes, as well as the nonischemic lobes, were excised at the onset of reflow to mimic the clinical condition of liver transplantation with small-for-size grafts or major hepatectomy by the Pringle maneuver in order to research postischemic liver functions. 


\section{Hepatectomy (70\%) with I/R injury model (rat)}

Lewis-rats, male

Warm ischemic time :40 min

Hepatectomy $70 \%$

MSC: Lewis-Rat, BM-MSC (passage 6 - 8)

MSC group $(n=6): 1 \times 10^{6}$ cells/body

Control group $(n=6)$ :PBS

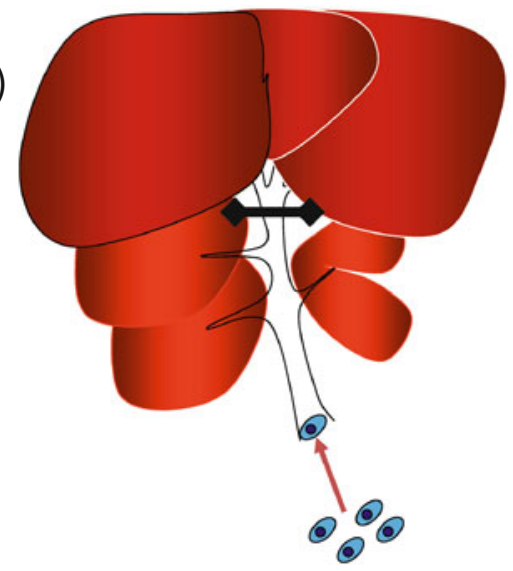

Fig. 2 Hepatectomy (70 \%) with the I/R injury model in the rat.

After 40 min of warm ischemia, $70 \%$ hepatectomy was performed, followed by injection of MSCs via the portal vein

\section{Outcome After MSC Injection}

Male Lewis rats were separated into two groups: an MSC group given MSCs after reperfusion as a treatment, and a control group given phosphate-buffered saline after reperfusion as a placebo. The results of liver function tests, pathologic changes in the liver, and the remnant liver regeneration rate were assessed. The fate of transplanted MSCs in the luciferase-expressing rats was examined by in vivo luminescent imaging. The MSC group showed peak luciferase activity of transplanted MSCs in the remnant liver $24 \mathrm{~h}$ after reperfusion, after which luciferase activity gradually declined. The elevation of serum alanine transaminase levels was significantly reduced by the MSC injection. Histopathological findings showed that the vacuolar change was lesser in the MSC group than in the control group. In addition, a significantly lower percentage of TUNEL-positive cells was observed in the MSC group than in the controls. The remnant liver regeneration rate was accelerated in the MSC group. The above results suggest that MSC transplantation provides trophic support to the I/R-injured liver by inhibiting hepatocellular apoptosis and by stimulating regeneration. 


\section{Mesenchymal Stem Cells for Pig Ischemia/Reperfusion Injury}

\section{Pig Ischemia Reperfusion Model}

Following rat hepatectomy experiments, a preclinical pig model was introduced (Fig. 3). In our model, long warm ischemia of up to $90 \mathrm{~min}$ is simulated for Pringle's procedure during hepatectomy or ischemia/reperfusion injury during liver transplantation. Inflow occlusion, such as Pringle's occlusion for a long period, causes mesenteric congestion, which affects the outcome and can sometimes be lethal. Therefore, a portacaval shunt between the splenic vein and the internal jugular vein was placed during inflow occlusion. The clamp was place at the hepatoduodenal ligament for $90 \mathrm{~min}$ with intensive monitoring for vital signs. After the declamp, MSCs $\left(1.0 \times 10^{7} / \mathrm{kg}\right)$ were injected in the MSC group. An initial problem with the MSC injection was probable hepatic embolism. Injection of the MSCs just after collection from the plates led to severe aggregation of cells, followed by embolus formation. Accordingly, we modified our protocol by immersing the MSCs in an ET-Kyoto solution so that the cells would not aggregate. Thereafter,

\section{Warm ischemia and reperfusion model (pig)}
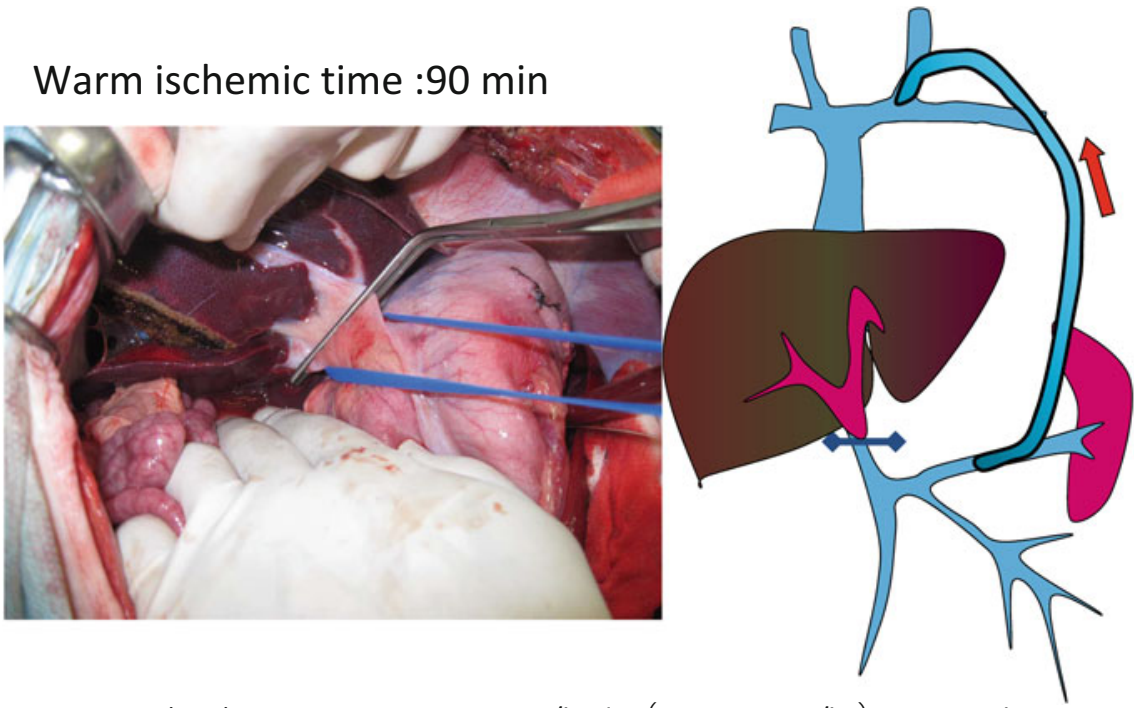

MSC group $(n=3)$ : AT-MSC $2.3 \pm 0.2 \times 10^{8} /$ body $\left(1.0 \pm 0.1 \times 10^{7} / \mathrm{kg}\right)$ via portal vein Control group $(n=3)$ : saline

Fig. 3 Warm ischemia and reperfusion model in the pig.

After 90 min of warm ischemia using Pringle's procedure, MSCs were injected via the portal vein. During Pringle's procedure, a portacaval shunt was placed in order to avoid mesenteric congestion 
no lethal complication was encountered. After the procedure, the pig was monitored for survival and daily laboratory examinations including AST, ALT, LDH, and portal venous pressure.

\section{Outcome After MSC Injection (Fig. 4)}

All pigs in the MSC group ( $n=5)$ and control group $(n=5)$ survived during an observation period for 7 days. In the control group, AST, ALT, and LDH peaked several hours after the operation and returned to normal in 3 days. However, this increase in laboratory parameters was not observed in the MSC group. In terms of adverse effects, portal venous pressure (PVP) was kept stable without an increase throughout the course, in both the MSC group and the control group. Therefore, the modified method for cell preparation is effective to avoid cell aggregation and leads to safe application of this method in the clinical setting. As we see from the laboratory results, the protective effect of MSCs is suggested in prolonged warm ischemia during surgery. This may lead to wider indications in liver surgery and transplantation.

\section{Liver enzymes and PVP: I/R model (pig)}
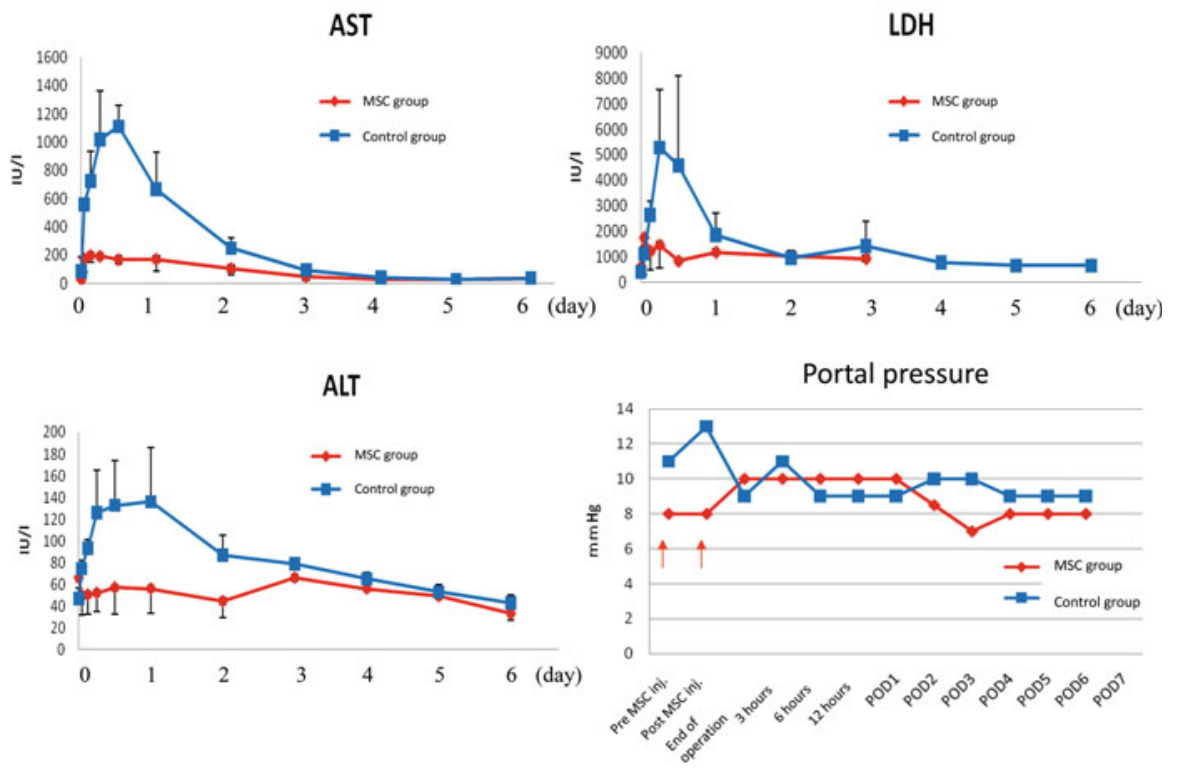

Fig. 4 Liver enzymes and PVP in the I/R model in the pig.

In the pig warm ischemia model, AST, ALT, and LDH were well controlled by MSC administration. In spite of the MSC administration, portal pressure was maintained without a significant increase 


\section{Mesenchymal Stem Cells for Rat Whole Liver Transplantation with a Long Preservation Period}

\section{Rat Liver Transplantation Model}

In the previous section, we showed that MSCs provide a protective effect against hepatic ischemia- reperfusion injury and stimulate liver regeneration in rat hepatectomy. We further evaluated the effect of MSCs on liver transplantation after prolonged cold preservation by using a rat liver transplantation model. Adipose tissue-derived MSCs from luciferase-transgenic Lewis (Luc-Tg LEW) rats were cultured and preserved in ET-Kyoto solution before use. Male Lewis rats were used as transplantation donors and recipients. The rats were randomly assigned to two groups: an MSC group and a control group. The liver grafts were retrieved and preserved in UW solution at $4{ }^{\circ} \mathrm{C}$ for $24 \mathrm{~h}$. After preservation, $1 \times 10^{5}$ of MSCs in $0.2 \mathrm{ml}$ of ET-Kyoto solution were transfused via the portal vein of the graft in the MSC group, and ET-Kyoto solution only was transfused in the control group. Then the liver graft was orthotopically transplanted into a recipient rat by the cuff technique of Kamada and Calne, with minor modification. Both the superior and inferior hepatic vena cava were anastomosed by a running suture. The portal vein was connected by a polyethylene cuff and the bile duct by a stent. The hepatic artery was reconstructed by the sleeve method. The survival of the recipient rats was evaluated for 7 days after transplantation.

\section{Outcome After MSCs Injection}

The anhepatic times were $15.5 \pm 0.7 \mathrm{~min}$ in the MSC group and $15.4 \pm 1.3 \mathrm{~min}$ in the control group. The inferior vena cava clamping times were $27.6 \pm 1.2 \mathrm{~min}$ and $27.1 \pm 1.7 \mathrm{~min}$, respectively. Both parameters did not differ significantly between the groups. Liver enzymes were better in the MSC group. In the control group, prominent necrosis and ballooning of cells in the adjacent area were observed, whereas the findings were almost normal in the MSC group (Fig. 5). In the MSC group, DLK/OV6 double positive cells were observed, whereas positive cells were minimal in the control group. These data support the positive effect of MSCs in ameliorating ischemia/reperfusion injury. The regenerative effect of MSCs was shown as well. The 7-day survival was significantly better in the MSC group than in the control group (7/8 vs. 3/8; Fisher's exact test, $p<0.05$ ) (Fig. 6). Portal transfusion of the MSCs ameliorated the injury of the liver graft after prolonged cold preservation and transplantation. 


\section{Histology: LTx with long preservation model (rat)}

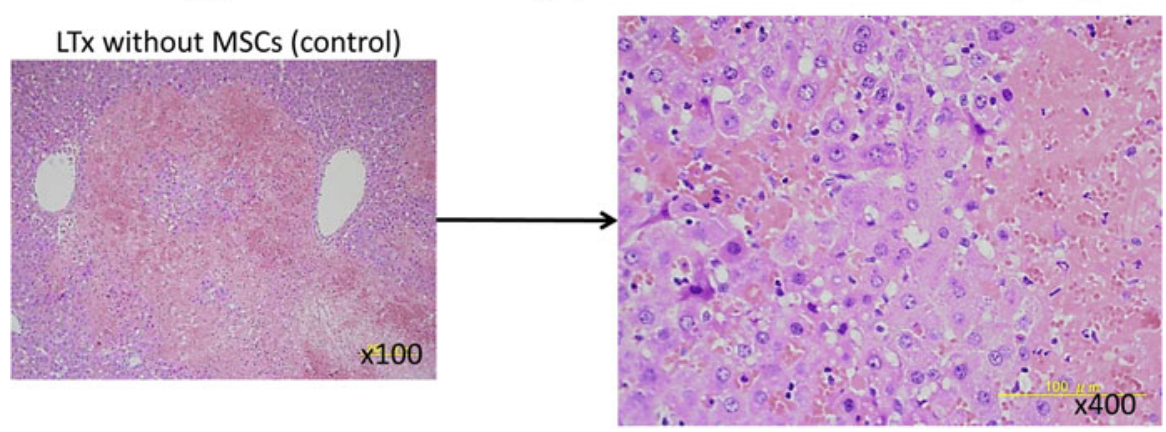

LTx with MSCs
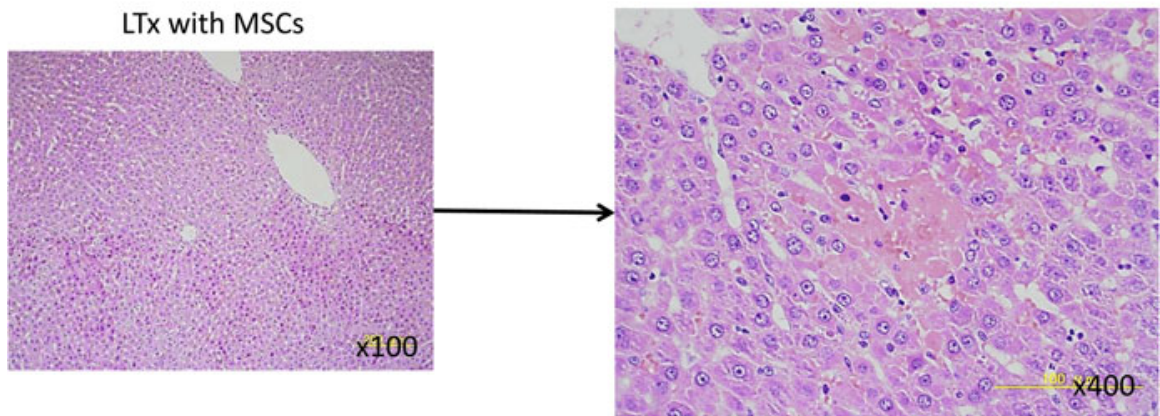

Fig. 5 Histology in liver transplantation using the long-preservation model in the rat.

An almost normal structure with minimal necrosis was maintained in the MSC group, whereas significant necrosis and hepatocyte ballooning in the adjacent area were observed in the control group

\section{Experimental Models for Clinical Application}

\section{Reduced Liver Transplantation (Rat) as a Model for Living- Donor Liver Transplantation}

During the donor operation, the right lobe (RL) of the liver was selected as the graft for the $20 \%$ PLTx group, while the superior right lobe (SRL) was selected for the $15 \%$ PLTx group, on the basis of the rat liver anatomy. Liver grafts were preserved in ice-cold HTK solution before implantation for $180 \mathrm{~min}$. The SHVC was sewn with an 8-0 Prolene continuous suture, and the portal vein was anastomosed using a cuff technique. After confirming reflow, the infrahepatic vena cava (IHVC) was anastomosed using an 8-0 nylon continuous suture. The hepatic artery was connected using a sleeve technique, and the bile duct was reconstructed over an 


\section{Survival after liver transplantation (rat)}

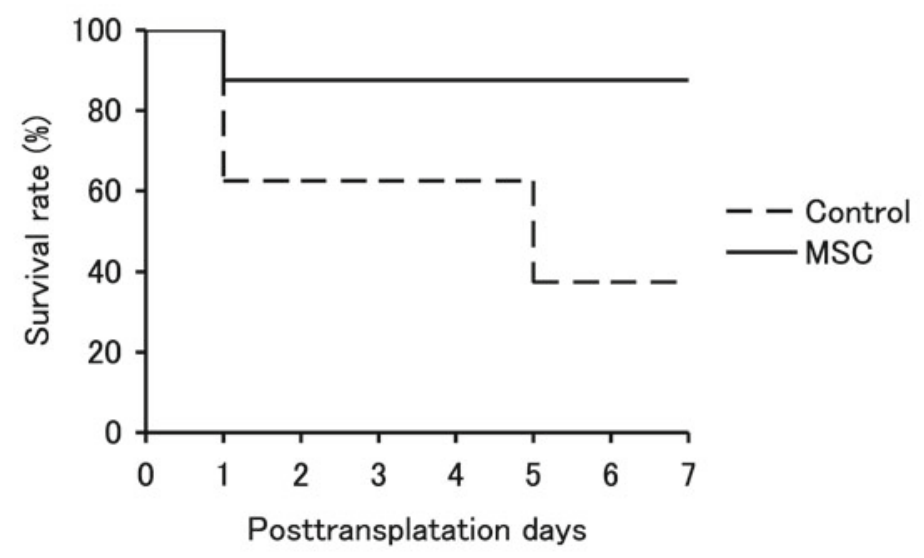

$7 / 8$ vs. $3 / 8$, MSC vs. control; $p<0.05$

Fig. 6 Survival after liver transplantation in the rat.

After 24-h preservation, only $38 \%$ (3/8) achieved long-term survival. In contrast, with administration of MSCs, $88 \%(7 / 8)$ survived at 7 days after transplantation

intraluminal stent. With this model, 7-day survival was $100 \%$ with the $20 \%$ graft and $17 \%$ with the $15 \%$ graft. Experiments assessing the efficacy of MSCs are under evaluation.

\section{Partial Liver Transplantation (Pig) as a Model for Living-Donor Liver Transplantation}

For this preclinical study, the Clawn miniature pig was used for the partial liver transplantation model [6]. The porcine liver consists of four lobes. The right lateral lobe (RLL) accounts for $30 \%$ of the whole liver. The structure of the porta hepatis was carefully dissected and cut in order to resect the lobes other than the RLL. Seventy percent of the liver was resected in the donor, not on the back table, to confirm hemostasis. Then the graft was preserved in the preservation solution for 3-24 h, followed by implantation into the recipient. In the recipient, prior to total hepatectomy, a portacaval shunt was placed to avoid mesenteric congestion. The inferior vena cava (suprahepatic and infrahepatic), portal vein, hepatic artery, and bile duct were sewn (Fig. 7). 


\section{Recipient Operation with reduced graft (pig)}

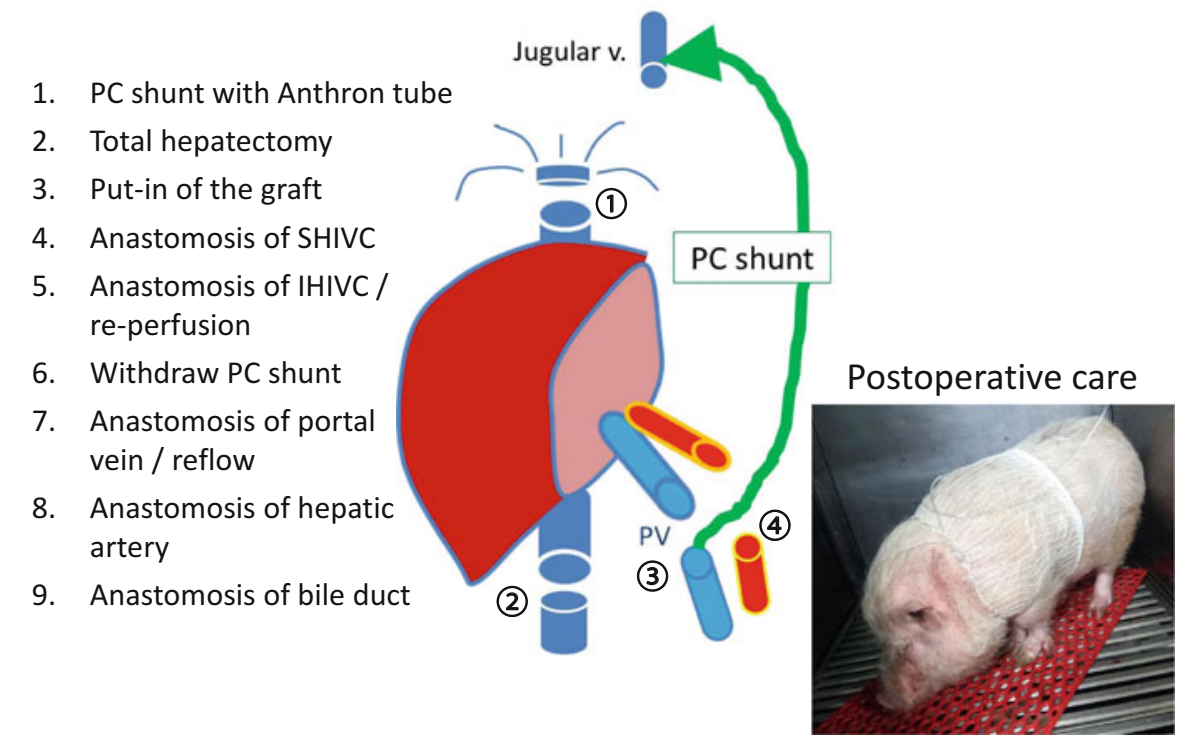

Fig. 7 Recipient operation with a reduced graft in the pig.

In order to avoid mesenteric congestion, a portacaval shunt was placed prior to total hepatectomy. Following total hepatectomy, implantation of the graft was done with multiple anastomoses

\section{Future Perspectives}

\section{Source for MSCs}

MSC have been isolated from almost every type of tissue stroma, such as the bone marrow, adipose tissue, umbilical cord blood, placenta, kidney, liver, heart, and spleen. There are many reports on the positive immunomodulatory effect or regenerative effect of MSCs of bone marrow origin. However, retrieval of bone marrow from healthy volunteers or patients seems invasive. On the other hand, Banas et al. reported a stronger effect of adipose tissue-derived MSCs compared with BM-MSCs. Moreover, subcutaneous adipose tissue can be safely retrieved under local anesthesia or during a major operation. We have been focusing on utilizing MSCs from adipose tissue. The remaining problem is from whom and when adipose tissue can be retrieved. Potential donors are the patient him- or herself, the donor of the organ in transplantation, or a third party. One of the advantages of MSCs compared with other cell sources is that MSCs express less HLA, leading to less rejection. Moreover, the regenerative or immunomodulatory effects are expected only in the short term, not in the long term. For use in cancer patients, we propose use of the patient's own MSCs without immunosuppression. On the other hand, cells from all 
three sources can be utilized in transplant recipients under immunosuppression. For an elective cancer operation, the patient's own MSCs can be retrieved and cultured for several weeks. However, stocked cells from a third party can be used for organ transplantation performed on an emergency basis [7, 8].

\section{Complications}

The most severe complication we have encountered is embolus formation due to cell aggregation. However, with the modification using ET-Kyoto solution, we can keep cells in a suspended form for several hours, which secures safe administration of the MSCs. ET-Kyoto solution is commercially available, and its safety is established. Potential transformation of MSCs into malignant cells can be problematic; however, the lifespan of the cells is limited, according to our study, and they can be eliminated within 2 weeks. Mild rejection will help to eliminate cells after a certain period as well.

\section{Conclusion}

Thanks to the support of the Uehara Memorial Foundation, we have been able to show the positive effect of MSCs with liver surgery. Cell damage during ischemia reperfusion injury is ameliorated, and liver regeneration after hepatectomy is enhanced by use of intravenous or intraportal MSCs. These positive effects have been shown both in vitro and in vivo including in a preclinical pig model.

Open Access This chapter is distributed under the terms of the Creative Commons Attribution Noncommercial License, which permits any noncommercial use, distribution, and reproduction in any medium, provided the original author(s) and source are credited.

\section{References}

1. Uemoto S (2010) [Liver transplantation]. Nihon Rinsho 68(12):2277-2280

2. Yagi $S$ et al (2012) Small-for-size syndrome in living donor liver transplantation. Hepatobiliary Pancreat Dis Int 11(6):570-576

3. Banas A et al (2008) IFATS collection: in vivo therapeutic potential of human adipose tissue mesenchymal stem cells after transplantation into mice with liver injury. Stem Cells 26(10):2705-2712

4. Banas A et al (2007) Adipose tissue-derived mesenchymal stem cells as a source of human hepatocytes. Hepatology 46(1):219-228

5. Kanazawa $\mathrm{H}$ et al (2011) Bone marrow-derived mesenchymal stem cells ameliorate hepatic ischemia reperfusion injury in a rat model. PLoS One 6(4):e19195 
6. Hori T et al (2014) How to successfully resect $70 \%$ of the liver in pigs to model an extended hepatectomy with an insufficient remnant or liver transplantation with a small-for-size graft. Surg Today 44(11):2201-2207

7. Dahlke MH et al (2009) Toward MSC in solid organ transplantation: 2008 position paper of the MiSOT Study Group. Transplantation 88(5):614-619

8. Franquesa M et al (2013) Mesenchymal Stem Cells in Solid Organ Transplantation (MiSOT) Fourth Meeting: lessons learned from first clinical trials. Transplantation 96(3):234-238 


\title{
Synaptic and Axonal Plasticity Induction in the Human Cerebral Cortex
}

\author{
Yoshikazu Ugawa
}

\begin{abstract}
This chapter summarizes a newly developed method (quadripulse stimulation (QPS)) to induce neural plasticity in the human brain.

What Is QPS? One stimulation burst consisting of four monophasic pulses is given every $5 \mathrm{~s}$ for $30 \mathrm{~min}$. In total, 360 bursts (1440 pulses) are given in one session. Short-interval QPS potentiates excitability and longer-interval QPS depresses the target area. QPS at intervals of $5 \mathrm{~ms}$ (QPS5) induces long-term potentiation (LTP) most efficiently and QPS50 induces long-term depression (LTD) most effectively in the human primary motor cortex (M1).

After QPS, no changes were found in the threshold, GABAergic function of M1, or acetylcholine function. In contrast, EPSP summation and sharpness of the IO curve are bidirectionally modulated by QPS. These findings indicate that excitatory synaptic efficacy is bidirectionally modulated by QPS. The effect is specific to the activated neurons. These all are consistent with synaptic LTP/LTD.

Dopamine enhanced both LTP of QPS5 and LTD of QPS50. It is again compatible with plasticity induction in animals. These are consistent with the concept that LTD was enhanced by the D1 agonist and LTD by the D1 and D2 agonists together, but the D1 agonist alone or the D2 agonist alone induced no changes in LTD.

In PD patients, even at an early stage, QPS induced neither LTP- nor LTD-like effects in the motor cortex. This lack of plasticity was normalized by L-Dopa intake in parallel with motor symptom improvements.
\end{abstract}

Keywords Synaptic plasticity - Axonal plasticity - Human motor cortex • Transcranial magnetic stimulation (TMS) • Quadripulse stimulation (QPS) • Dopamine $\bullet$ Dopamine agonist $\bullet$ Parkinson's disease $\bullet$ Long-term potentiation (LTP) - Long-term depression (LTD)

\footnotetext{
Y. Ugawa $(\bowtie)$

Department of Neurology, School of Medicine, Fukushima Medical University,

1 Hikarigaoka, Fukushima 960-1295, Japan

e-mail: ugawa-tky@umin.net
} 


\section{Introduction}

Neural plasticity is one main mechanism to support the flexibility of the human brain. It underlies several important human brain functions such as memory (hippocampus), motor learning (cerebellum), motor programming (basal ganglia), and so on. It has also some relation to reward learning, decision making, and addiction. It is well known that plasticity plays some roles also in the recovery of damaged function in some neurological disorders, such as cerebrovascular disease, Parkinson disease, dystonia, and so on. This chapter summarizes a newly developed stimulation method (quadripulse stimulation (QPS)) to induce neural plasticity in the human brain.

\section{What Is QPS? [1]}

There are several stimulation methods to induce plastic changes in the human brain. QPS must be the most powerful and reliable one for human plasticity induction. Figure 1 shows the protocol of QPS, and Fig. 2 demonstrates a set of four stimulators for QPS. After baseline (motor evoked potential) MEP recordings, one session

\section{Quadripulse stimulation (QPS)}

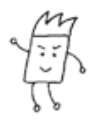

(Pre)

(min)

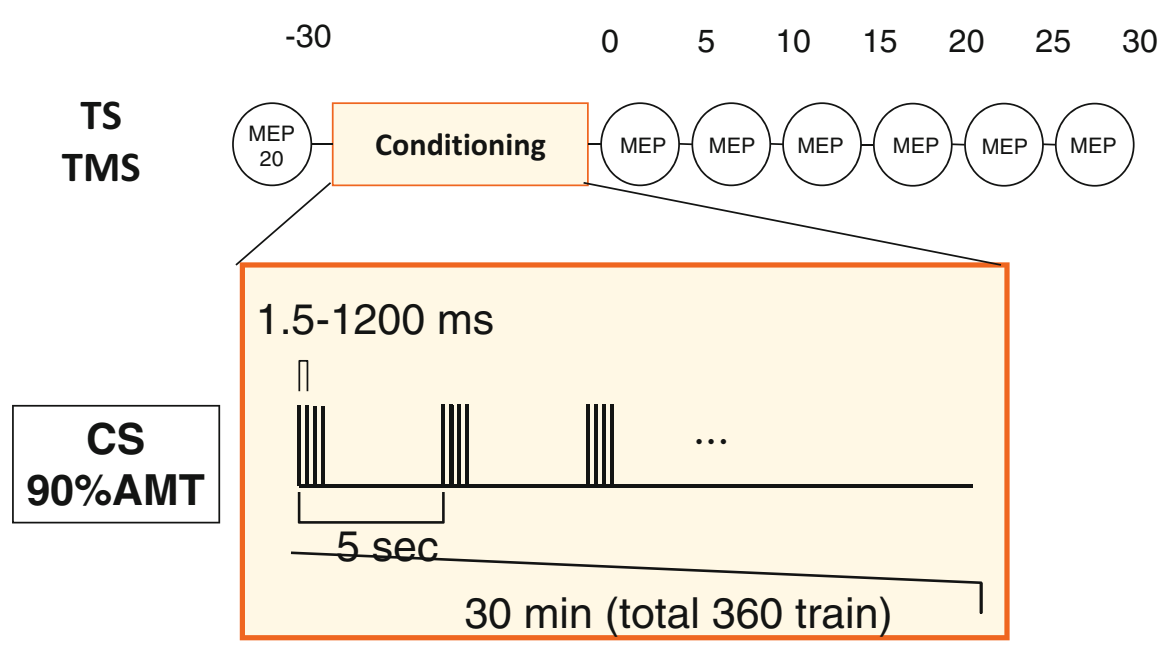

Fig. 1 QPS protocol 


\section{Quadripulse stimulation (QPS)}

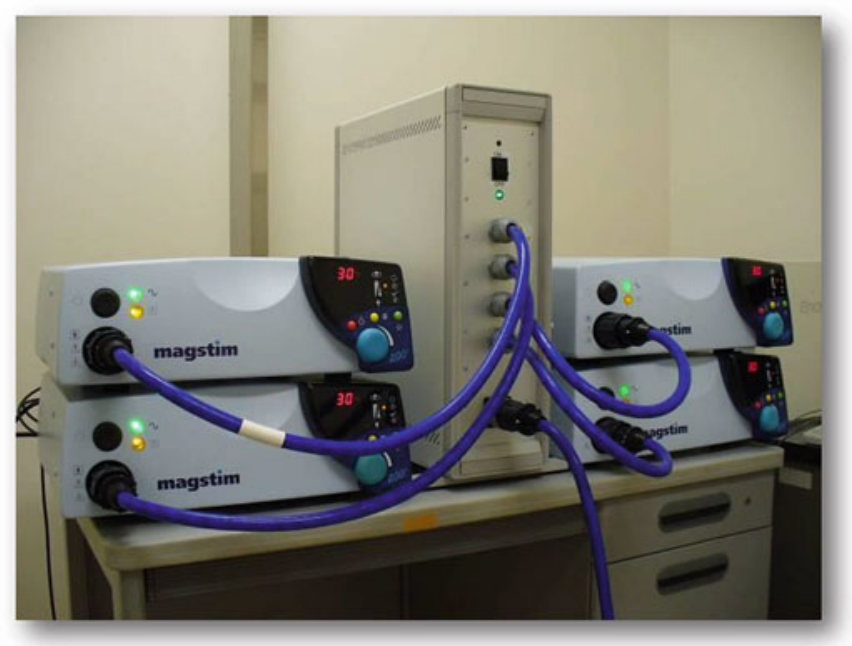

Fig. 2 Transcranial magnetic stimulator set up for QPS. Four monophasic magnetic stimulators are combined by a combine module

of QPS stimulation was performed and MEPs were followed by single-pulse transcranial magnetic stimulation (TMS) with the same intensity used in the baseline recordings (Fig. 1). In QPS, one stimulation burst consisting of four monophasic TMS pulses is given every $5 \mathrm{~s}$ for $30 \mathrm{~min}$. In total, 360 bursts (1,440 pulses) are given in one session. The interval of TMS pulses was changed in different QPS conditioning sessions from 1.5 to $1250 \mathrm{~ms}$. We evaluated the motor cortical excitability using the size of MEPs to TMS pulses at the same intensity.

Figure 3 shows typical MEPs before and after QPS5 (inter-pulse interval (IPI) 5 ms), QPS30, and QPS50. MEPs were enlarged after QPS5, whereas they were decreased in size after QPS30 and PQS50. After QPS, in total, the stimulated cortical area excitability is bidirectionally modulated depending on the interval of magnetic pulses in one burst. Short-interval QPS potentiates excitability, and longer-interval QPS depresses the target area. QPS at an interval of $5 \mathrm{~ms}$ (QPS5) induces long-term potentiation (LTP) most efficiently, and QPS50 induces longterm depression (LTD) most effectively in the human primary motor cortex (M1). Figure 4 shows the relation between the QPS effect and the IPI of TMS pulses, which fits well to the well-known Bienenstock-Cooper-Munro (BCM) curve. Their physiological characteristics, bidirectional modulation, dependence on the interval of pulses, and spatial specificity are all compatible with the plasticity reported previously. We conclude that QPS can induce neuronal plasticity in the human brain. 


\section{Plasticity in humans Quadripulse stimulation}

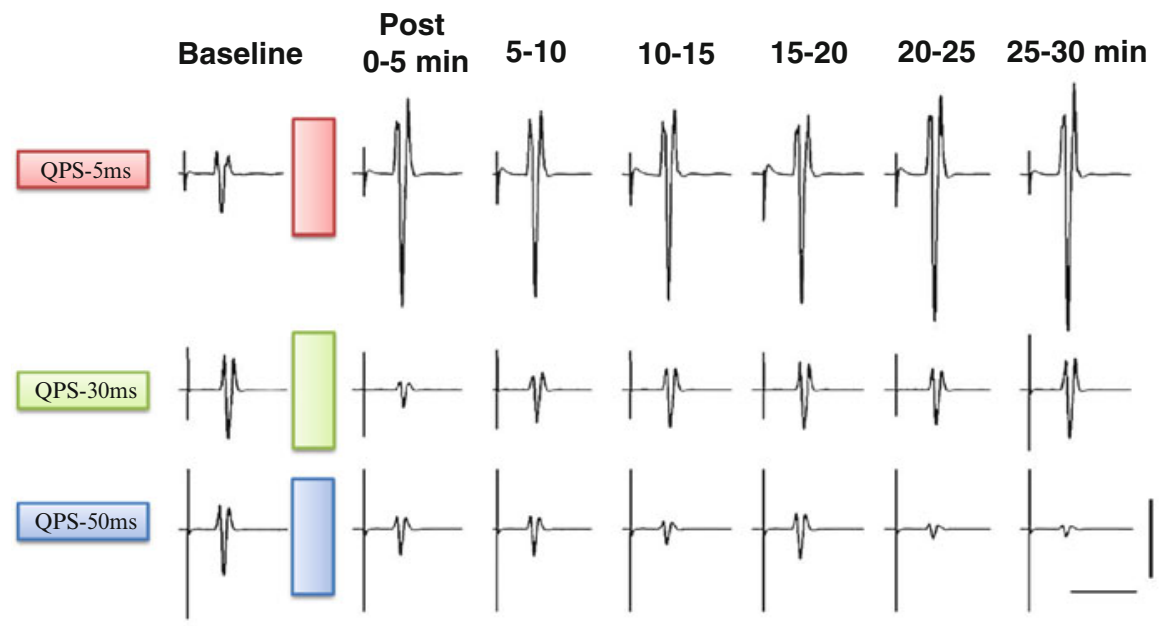

Fig. 3 Typical motor evoked potentials (MEPs) in the QPS experiments. The top row are for QPS5, the middle for QPS30 and the bottom for QPS50. QPS5 prominently enhanced MEPs, and QPS30 or QPS50 depressed MEPs

\section{Quadripulse stimulation (QPS)}

\section{Stimulus-Response function}

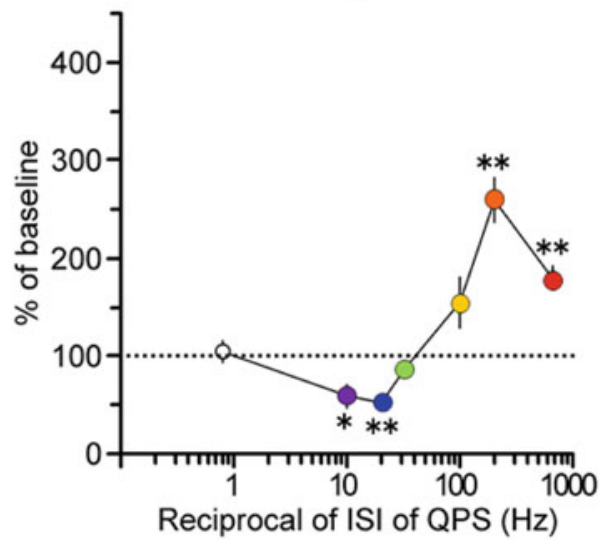

- QPS-1.5ms

- QPS-5ms

O QPS-10ms

○ QPS-30ms

- QPS-50ms

- QPS-100ms

Normalized amplitudes of MEP measured at $\mathbf{3 0}$ min after QPS as a function of the reciprocal of ISI of QPS (QPS frequency).

Fig. 4 Correlation between the main effect and the reciprocal of the inter-pulse interval of QPS. This time course is almost the same the Bienenstock-Cooper-Munro (BCM) curve for synaptic plasticity 


\section{Mechanisms Underlying QPS [1]}

To elucidate the mechanisms underlying QPS, we compared several physiological features between the baseline (before QPS) and after QPS. No changes were found in the threshold (Fig. 5a, d), GABAergic function of M1, or acetylcholine function. In contrast, both intracortical facilitation (ICF) and short-latency cortical facilitation (SICF) were bidirectionally modulated by QPS5 and QPS50 (Fig. 6a, b). Both of them may reflect glutamatergic EPSP summation. The sharpness of the IO curve was also bidirectionally modulated by QPS (Fig. 5c, f). The sharpness of the IO curve should also reflect the excitatory synaptic function of M1. These indicate that excitatory synaptic efficacy is bidirectionally modulated by QPS. Figure 5c, f show the special specificity of the QPS effect to the area conditioned by QPS. These are all consistent with the synaptic LTP/LTD (Fig. 7)

Nakatani-Enomoto et al. [2] supported the safety of QPS by recoding EEGs and measuring serum prolactin levels before and after QPS. Nakamura et al. [3] showed the independency of QPS effects on brain-derived nerve growth factor (BDNF)

\section{MTs, Recruitment curve, Input specificity changes after QPS}
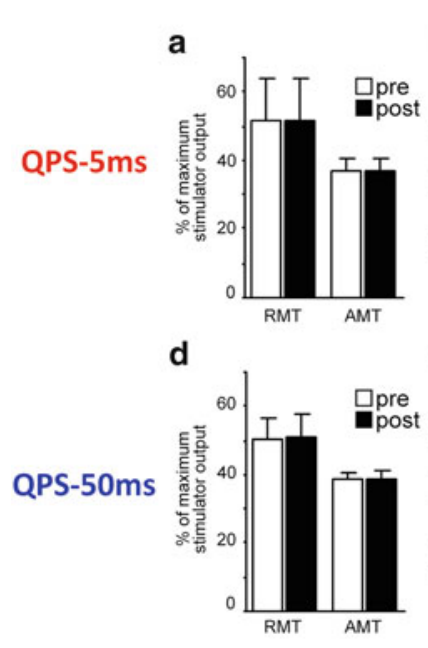

b

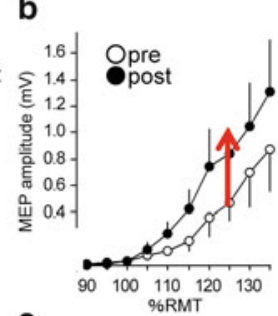

e

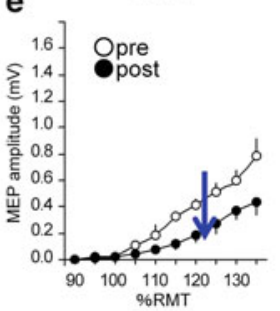

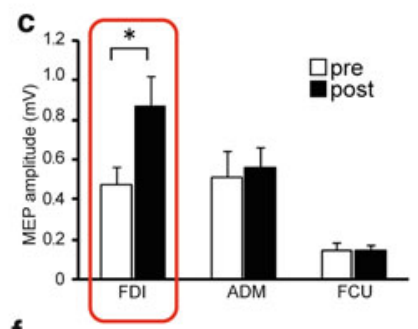

f

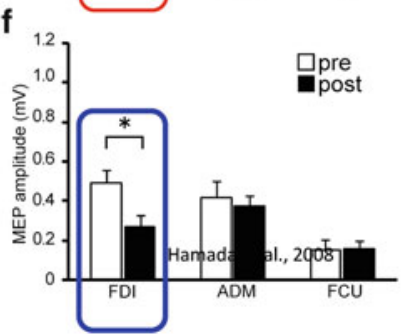

Fig. 5 Physiological characteristics before and after QPS. The threshold is shown in (a) and (d), the input-output curves in (b) and (e), and somatotopic specificity in (c) and (f). They revealed no changes in the threshold, bidirectional modulation of the IO curves, and somatotopic specificity to the target muscle. These are all consistent with bidirectional changes in the excitatory glutamatergic synapses 

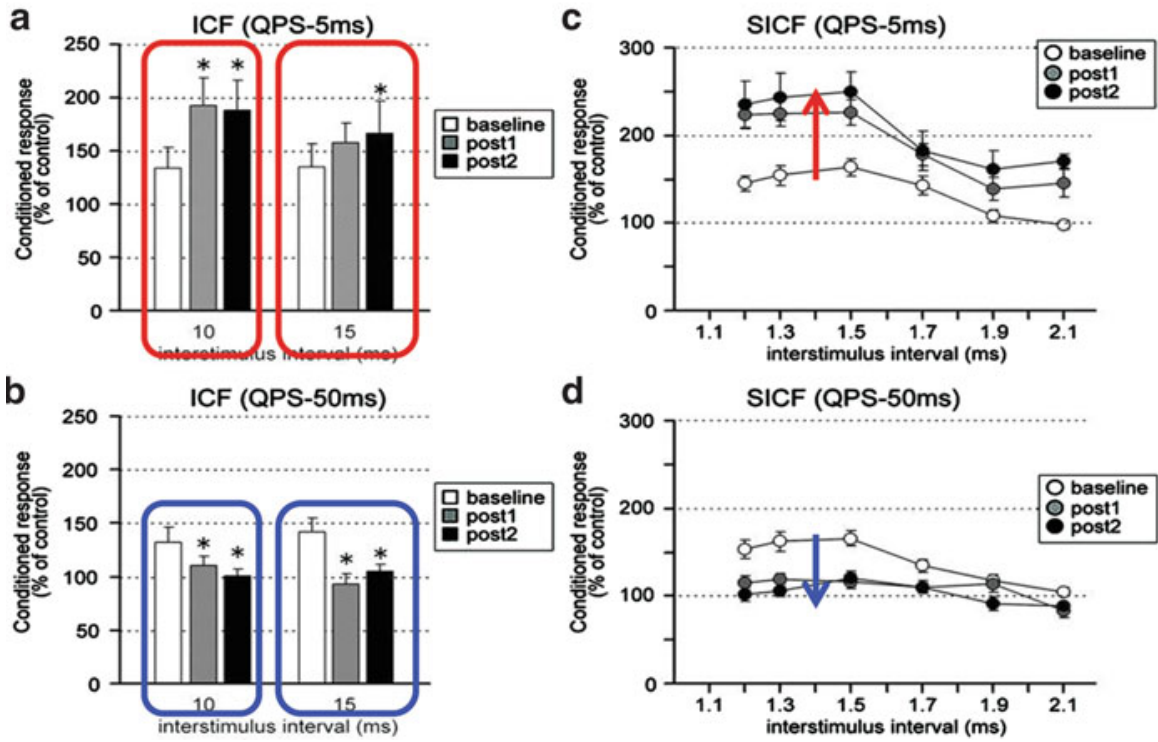

Fig. 6 Intracortical facilitation (ICF) and short-interval intracortical facilitation (SICF) before and after QPS. Both effects were bidirectionally modulated by QPS5 and QPS50. The results are all consistent with bidirectional modulation of glutamatergic synapses

\section{Summary of Results}

- QPS at short intervals

- QPS at long intervals

- MTs

- MEPs at active

- Recruit curve

- Input specificity

- $\mathrm{SICl}$

- ICF, SICF

- SAI, LAI $\rightarrow$ MEP $\uparrow$, long duration (> $75 \mathrm{~min}$ )

$\rightarrow$ MEP $\downarrow$, long duration (>75 $\mathrm{min}$ )

$\rightarrow$ no change

$\rightarrow$ QPS-short, $\uparrow ;$ QPS-long, $\downarrow$

$\rightarrow$ bidirectional modulation

$\rightarrow$ FDI $\downarrow$, ADM no change

$\rightarrow$ no change

$\rightarrow$ bidirectional modulation

$\rightarrow$ no change

Fig. 7 Summary of physiological analyses of the mechanisms underlying QPS effects 
polymorphism on which some plasticity induction methods depend. It is one superior point of QPS compared with other methods, especially when applying these methods to patients, because we do not need to consider BDNF polymorphism in judging the results in the patients.

\section{Dopamine/Dopamine Agonists and Plasticity [5]}

\section{Dopamine/Dopamine Agonists in Normal Subjects [5]}

It is well known that dopamine enhances both LTP and LTD, and the former is one of the D1 effects and the latter is a kind of D2 effect. To study the relation of dopamine or a dopamine agonist with QPS, we compared LTP/LTD effects between the conditions after placebo, L-Dopa, or pramipexole intake in normal volunteers. Figure 8 shows typical MEPs 30 min after QPS5 and QPS50 under the conditions

\section{MEPs before and 30 min after QPS}
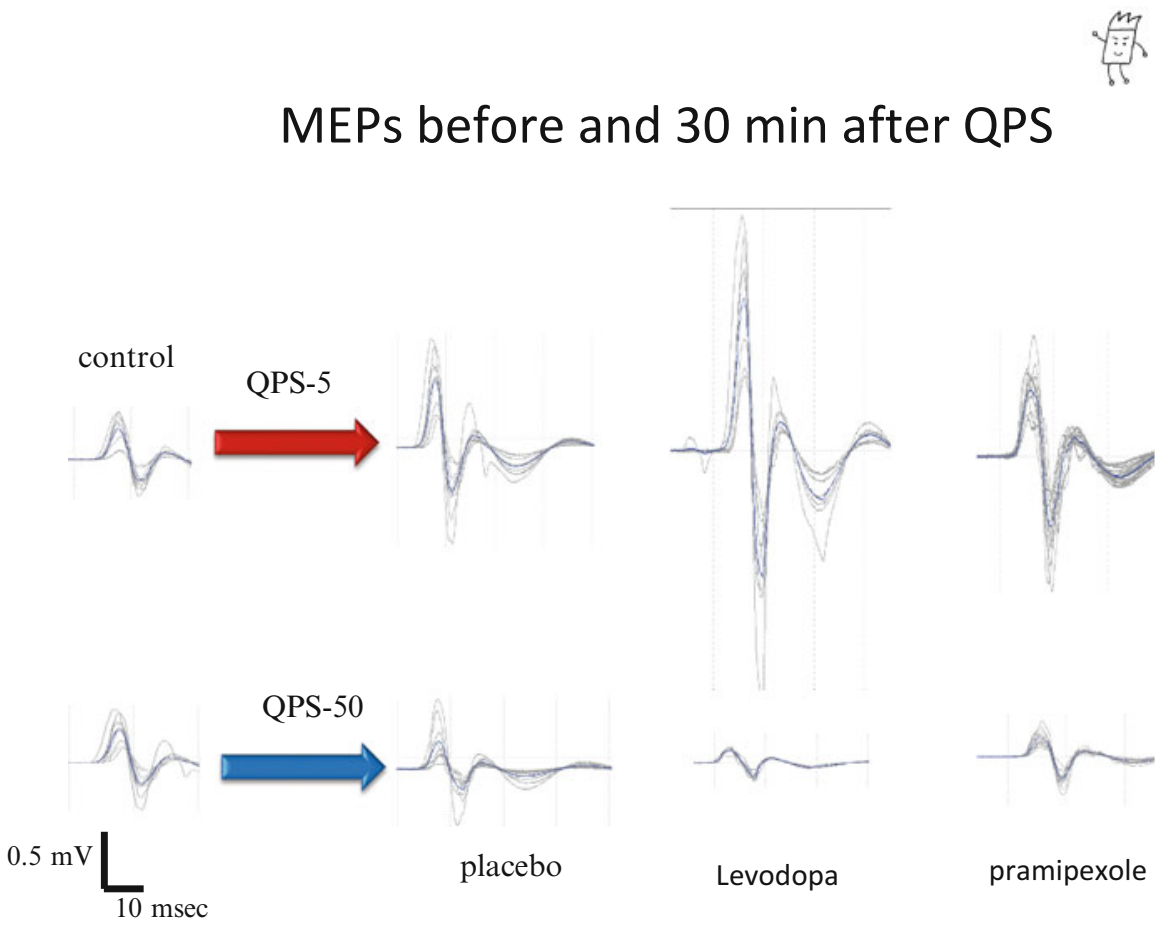

Fig. 8 L-Dopa or pramipexole influence on the QPS. The left column shows the baseline condition, the other three rows show MEPs $30 \mathrm{~min}$ after QPS5 or QPS50 after intake of placebo, L-Dopa, or pramipexole. L-Dopa enhanced both LTP- and LTD-like effects. In contrast, pramipexole did not affect QPS effects 
of placebo, L-Dopa, or pramipexole intake. Under the placebo condition, QPS5 enlarged MEPs and QPS50 made them smaller, as shown above. Under the L-Dopa intake condition, both LTP-like and LTD-like effects were enhanced as compared with the placebo condition. In contrast, neither an LTP-like effect nor an LTD-like effect was modulated by pramipexole. This is again compatible with plasticity induction in animals. Pramipexole, almost purely a D2 agonist, may not enhance LTP because D1 activation strengthens LTP but D2 activation does not. It did not enhance the LTD-like effects, probably because both D1 and D2 coactivation is required for LTD [4].

\section{Dopamine/Dopamine Agonists in PD}

In PD patients, even at an early stage, QPS induced neither LTP nor LTD-like effects in the motor cortex. This lack of plasticity was normalized by L-Dopa intake in parallel with motor symptom improvements.

\section{Meta-plasticity [6]}

Flexibility plays critical roles in brain function, as shown above. However, flexibility or plasticity is sometimes harmful to the brain. Hyperpotentiation of the cerebral cortices may induce epilepsy, and excessive reward-seeking may produce an addiction to drugs or gambling. The brain, therefore, has several mechanisms to maintain the stable state to some extent. In other words, the brain is as flexible as possible, but within a certain safety range. To maintain this safety, the brain has meta-plasticity, which is called "plasticity of plasticity". The meta-plasticity function was studied with some priming stimulation over the primary motor cortex (M1), premotor cortex (PM), or supplementary motor cortex (SMA). Figure 9 shows the experimental protocol of the meta-plasticity experiment. Before the usual QPS experiments, we gave a priming stimulation for a short period $(10 \mathrm{~min})$, which had no influence on MEPs when given alone. Figure 10 shows the MEP time courses with and without priming stimulation (QPS5 for $10 \mathrm{~min}$ ). In all QPS protocols, the QPS5 priming stimulation modulated the main QPS effects in the depressive direction. This is compatible with the meta-plasticity of animal experiments. It indicates that the human motor cortex has a safety-maintaining mechanism, just like animal M1.

\section{Sensory Cortical Plasticity $[7,8]$}

The above studies were all done on M1. We studied the neural plasticity of the sensory cortex in humans, using sensory evoked potentials (SEPs). SEPs were bidirectionally modulated by QPS over M1. QPS5 over M1 potentiated SEPs and QPS50 depressed SEPs, but neither QPS5 nor QPS50 over the sensory cortex (S1) had any 


\section{M1 Priming effects on QPS-induced plasticity}

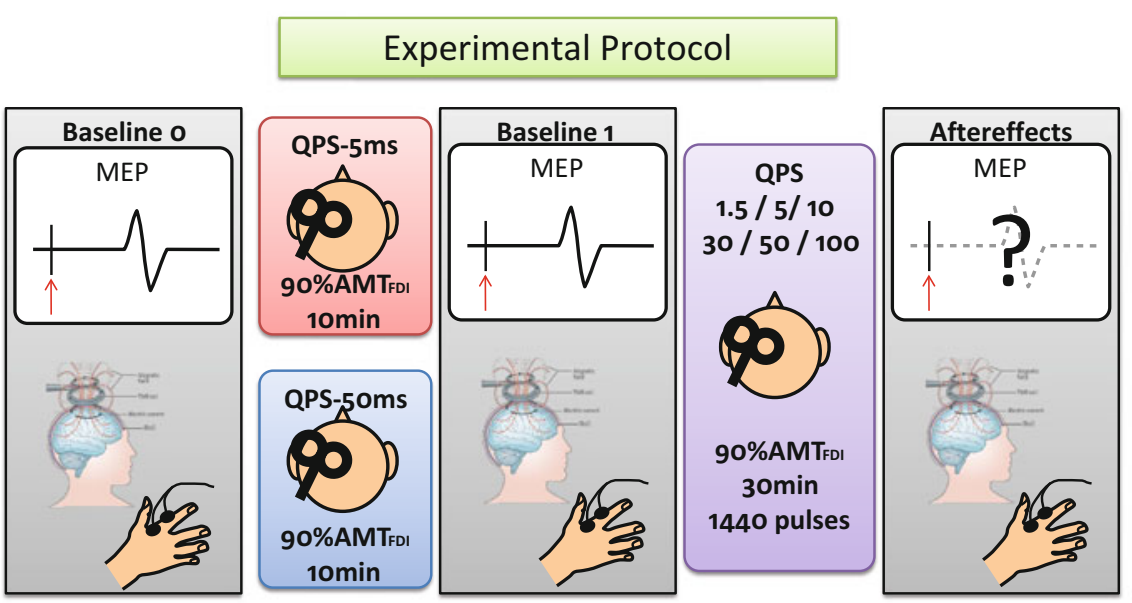

Fig. 9 Protocol for the meta-plasticity experiments

\section{M1 Priming effects on QPS-induced plasticity}
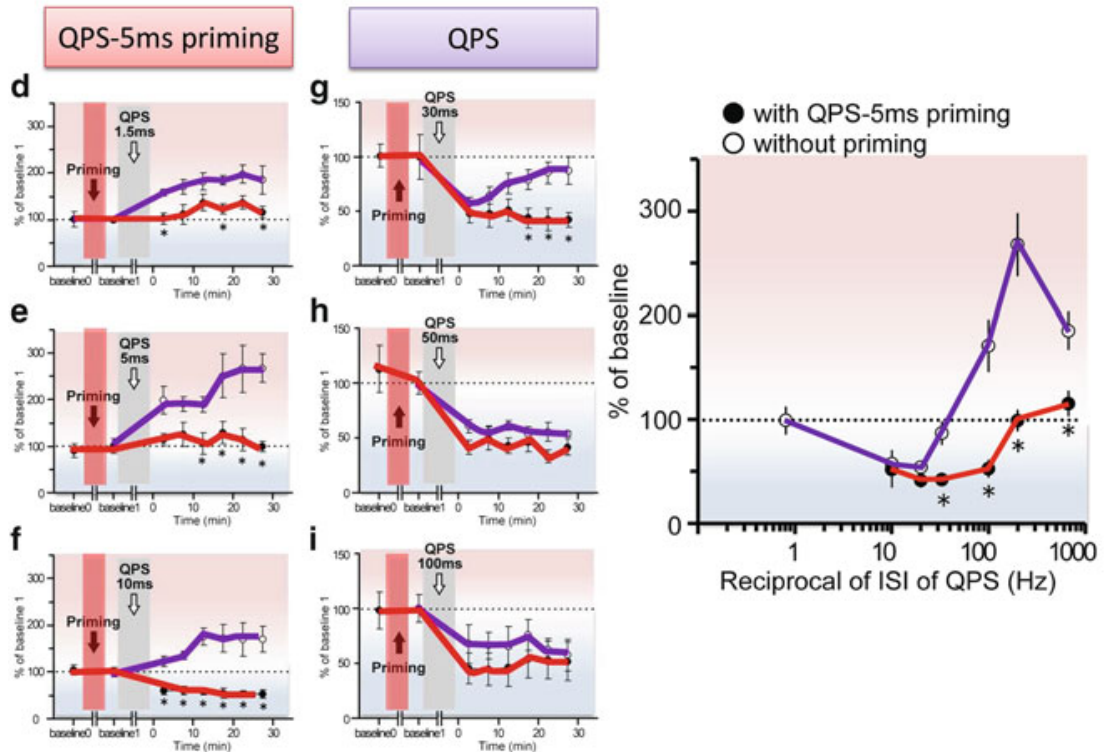

Fig. 10 Time courses of QPS 1.5, 5, 10, 30, 50, and 100 with and without QPS5 priming over M1. All time courses without priming (purple) were depressed by the QPS5 priming stimulation. The facilitatory priming stimulation depressed the main conditioning effects, which is compatible with the meta-plastic influence of the priming stimulation 
influence on SEPs. This indicates that S1 is more influenced by motor cortical excitability changes than S1 excitability itself, through heterotopic synaptic plasticity. This finding may have some relation to sensory gating of motor performance. This directionality of sensory cortical plasticity was broken in myoclonus epilepsy patients. Any QPS protocols induced LTP-like effects in patients with cortical myoclonus but did not induce LTD. The safety-maintaining mechanisms may be broken in these epileptic disorders.

\section{Axonal Plasticity in the Human Motor Cortex}

All of the above works studied synaptic plasticity in the human brain, which is produced by synaptic efficacy changes without any axonal excitability changes (threshold for axonal membrane or the resting membrane potential). In some situations, axonal excitability changes are also induced in association with synaptic plasticity in animal experiments. We studied whether axonal plasticity is also induced in the human motor cortex after QPS over M1 using high-voltage electric stimulation (transcranial electrical stimulation (TES)) of M1. TES activates corticospinal tract axons directly, whereas TMS activates them through at least one synapse in M1 (Fig. 11). We compared the QPS effects on MEPs to TMS and those on MEPs to TES. QPS5 over M1 induced axonal depression in association with synaptic

\section{Site of action in various stimulation methods}

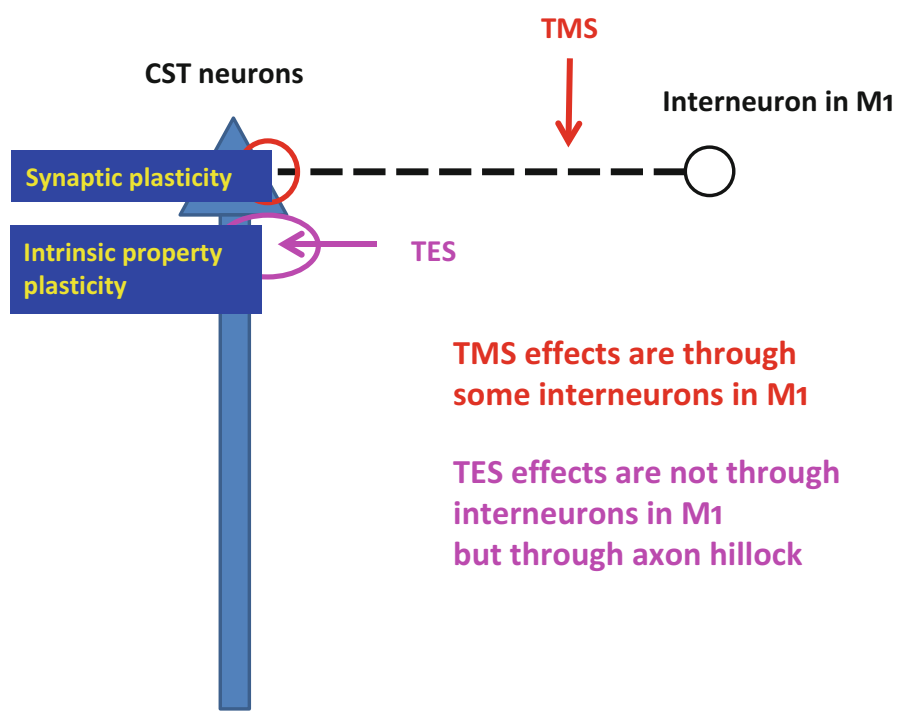

Fig. 11 Supposed activation sites in TMS and TES 


\section{Time course}

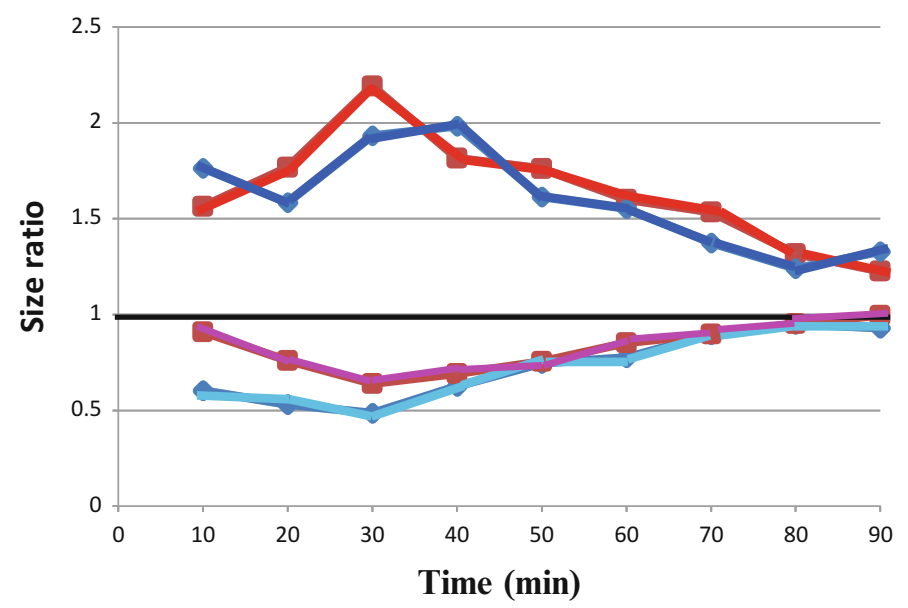

TMS QPS5 TMS QPS50 TES QPS5 -

Fig. 12 Time courses of MEPs to TMS and TES. QPS5 potentiated MEPs to TMS and depressed those to TES. Conversely, QPS50 depressed MEPs to TMS and potentiated those to TES

potentiation, and QPS50 induced axonal potentiation and synaptic depression (Fig. 12). This is the first report of axonal plasticity in the human brain.

\section{Future Projects for QPS}

We would like to finally use QPS for treatment of neurological and psychiatric disorders. Several projects are now ongoing, such as in Parkinson disease, depression, and cerebrovascular diseases. To show clinical efficacy of these methods as treatment, we need to take care of several points: what sham stimulation to use, axonal plasticity effects in addition to synaptic plasticity effects. We are also considering the effects on some distant areas after QPS in treatment applications [9]. I believe QPS will be used as some sort of treatment in the near future.

Open Access This chapter is distributed under the terms of the Creative Commons Attribution Noncommercial License, which permits any noncommercial use, distribution, and reproduction in any medium, provided the original author(s) and source are credited. 


\section{References}

1. Hamada M, Terao Y, Hanajima R et al (2008) Bidirectional long-term motor cortical plasticity and metaplasticity induced by quadripulse transcranial magnetic stimulation. J Physiol 586:3927-3947

2. Nakatani-Enomoto S, Hanajima R, Hamada M et al (2011) Some evidence supporting the safety of quadri-pulse stimulation (QPS). Brain Stimul 4:303-305

3. Nakamura K, Enomoto H, Hanajima R et al (2011) Quadri-pulse stimulation (QPS) induced LTP/LTD was not affected by Val66Met polymorphism in the brain-derived neurotrophic factor (BDNF) gene. Neurosci Lett 487:264-267

4. Calabresi P, Maj R, Mercuri NB, Bernardi G (1992) Coactivation of D1 and D2 dopamine receptors is required for long-term synaptic depression in the striatum. Neurosci Lett 142:95-99

5. Enomoto H, Terao Y, Kadowaki S, Nakamura K, Moriya A, Nakatani-Enomoto S, Kobayashi S, Hanajima R, Ugawa Y (2015) Effects of L-Dopa and pramipexole on plasticity induced by QPS in human motor cortex. J Neural Transm. doi: 10.1007/s00702-015-1374-8

6. Hamada M, Hanajima R, Terao Y et al (2009) Primary motor cortical metaplasticity induced by priming over the supplementary motor area. J Physiol 587:4845-4862

7. Hirose M, Mochizuki H, Tanji Y (2011) On-line effects of quadripulse transcranial magnetic stimulation (QPS) on the contralateral hemisphere studied with somatosensory evoked potentials and near infrared spectroscopy. Exp Brain Res 214(4):577-586

8. Nakatani-Enomoto S, Hanajima R, Hamada M et al (2012) Bidirectional modulation of sensory cortical excitability by quadripulse magnetic stimulation (QPS) in humans. Clin Neurophysiol 123:1415-1421

9. Tsutusmi R, Hanajima R, Terao Y et al (2014) Effects of the motor cortical quadripulse tarancranial magnetic stimulation (QPS) on the contralateral motor cortex and interhemispheric interactions. J Neurophysiol 111:26-35 


\title{
TIM-3 Is a Novel Therapeutic Target for Eradicating Acute Myelogenous Leukemia Stem Cells
}

\author{
Koichi Akashi
}

Abstract Acute myelogenous leukemia (AML) is derived from self-renewing leukemic stem cells (LSCs). We found that T-cell immunoglobulin mucin-3 (TIM-3) is expressed on LSCs in most types of primary AML, except for acute promyelocytic leukemia (M3 by the FAB classification). TIM-3 is not expressed in normal hematopoietic stem cells (HSCs). In a xenogeneic transplantation system, we showed that targeting of TIM-3 by an anti-TIM-3 cytotoxic antibody is sufficient to eradicate human AML LSCs without affecting normal human hematopoiesis. These data strongly suggest that TIM-3 is a promising therapeutic target to cure AML patients.

Keywords Acute myelogenous leukemia $\bullet$ Leukemic stem cell $\bullet$ Cancer stem cell $\bullet$ TIM-3 $\bullet$ Hematopoietic stem cell $\bullet$ Xenotransplantation

\section{Introduction}

In normal hematopoiesis, human hematopoietic stem cells (HSCs) reside within the CD $34^{+} \mathrm{CD} 38^{-}$cell fraction of bone marrow cells. They self-renew and differentiate into mature cells to maintain normal hematopoiesis. Similarly, in acute myelogenous leukemia (AML), leukemic blast cells are derived from a small population called leukemic stem cells (LSCs) or leukemia-initiating cells, which also resides within the $\mathrm{CD} 34^{+} \mathrm{CD} 38^{-}$cell fraction $[1,2]$. LSCs self-renew and give rise to clonogenic leukemic cells, whereas non-LSCs lack the potential to self-renew or maintain leukemia $[1,3,4]$ indicating that AML is hierarchically organized initiating from LSCs.

Conventional chemotherapy currently achieves complete remission in $~ 90 \%$ of AML cases [5, 6]. However, a considerable proportion of AML patients $(\sim 60 \%)$ eventually relapse after intensive chemotherapies. The recurrence of AML in these

K. Akashi ( $\square)$

Department of Medicine and Biosystemic Sciences, Kyushu University Graduate

School of Medicine, 3-1-1 Maidashi, Higashi-Ku, Fukuoka 812-8582, Japan

e-mail: akashi@med.kyushu-u.ac.jp

K. Nakao et al. (eds.), Innovative Medicine, DOI 10.1007/978-4-431-55651-0_25 
patients may be caused by regrowth of surviving LSCs. To selectively kill AML LSCs while sparing normal HSCs, one of the most practical approaches is to target AML LSC-specific surface molecules or molecules required for LSC function. To achieve such specificity, the target molecule should be expressed on LSCs at a high level but not on normal HSCs [7]. The molecule can be expressed in mature blood cells or progenitors, because these cells can anyway be replenished by normal HSCs.

\section{Search for Surface Antigens Specific to AML LSCs}

A number of candidate surface molecules for eradicating AML LSCs have been reported mainly by utilizing cDNA microarray analysis of purified LSCs. Figure 1 shows the results of transcriptome profiling of purified LSCs from AML patients and normal adult HSCs [8]. The molecules strongly expressed in AML LSCs, including CLL-1 [9], CSF1R [10], CD96 [11], and CD99 [12], are specifically expressed in LSCs. CLL-1 is a transmembrane glycoprotein [13]. The proportion of CLL-1-expressing CD34+CD38- AML cells, however, is highly diversified in cases [9]. CD96 is a member of the Ig gene superfamily. CD96 is expressed on activated T cells [14]. The expression level of CD96 protein is also high enough to clearly distinguish AML LSCs from normal HSCs. T-cell immunoglobulin mucin-3 (TIM-3) is expressed in LSCs of most AML types (except for M3) at high levels, but is not expressed in normal HSCs [8]. The expression level of CD25 [15], CD32 [15], CD44 [16], and CD47 [17] in LSCs was only two- to threefold higher at the mRNA level as compared with normal HSCs, and in some AML cases, LSCs did not express these molecules. CD33 and CD123 [18] proteins are expressed at a high level in normal HSCs and myeloid progenitors, including CMPs and GMPs [19], suggesting that targeting these molecules should harm normal hematopoiesis.

It might also be important to understand the function of these therapeutic target molecules in the development of AML. A previous study has shown that anti-CD44 monoclonal antibodies reduced the leukemic burden and blocked secondary engraftment in a NOD-SCID model [16]. This effect on LSCs was mediated in part by the disruption of LSC-niche interactions [16]. Anti-CD47 antibodies can block LSC reconstitution in a NOD-SCID model [17], and this might be due to the activation of phagocytosis by macrophages through inhibition of interaction of CD47 with SIRPA [20].

Recently, we have reported that TIM-3 is expressed on the cell surface of LSCs in most AML types [8, 21]. TIM-3 is not expressed in normal human HSCs [8] (Fig. 1). Furthermore, a recent study has succeeded in prospectively isolating LSCs from residual HSCs within the $\mathrm{CD} 34^{+} \mathrm{CD} 38^{-}$fraction in de novo AML patients by using TIM-3 as a positive LSC marker [12]. Here, we summarize recent progress in studies of TIM-3 and discuss the potential usefulness of TIM-3 for eradicating AML LSCs. TIM-3 has several advantages over other candidate markers. First, TIM-3 protein is not detectable in normal HSCs or in other myelo-erythroid or lymphoid progenitors, although TIM-3 is upregulated in monocyte-lineage committed progenitors. 
a

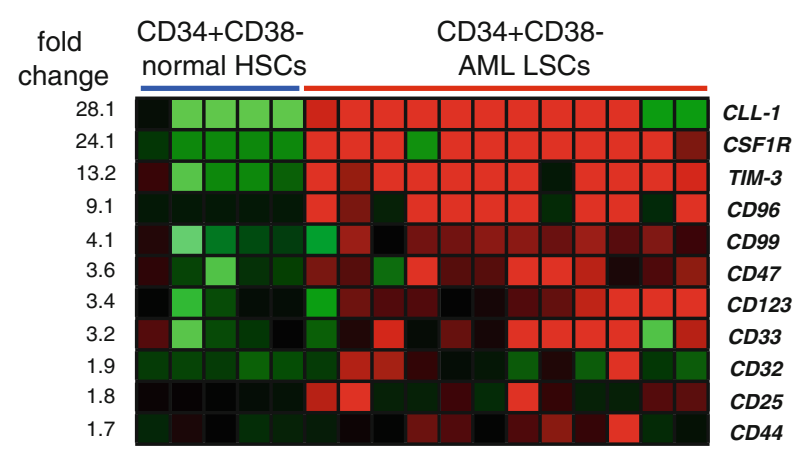

b

Normal
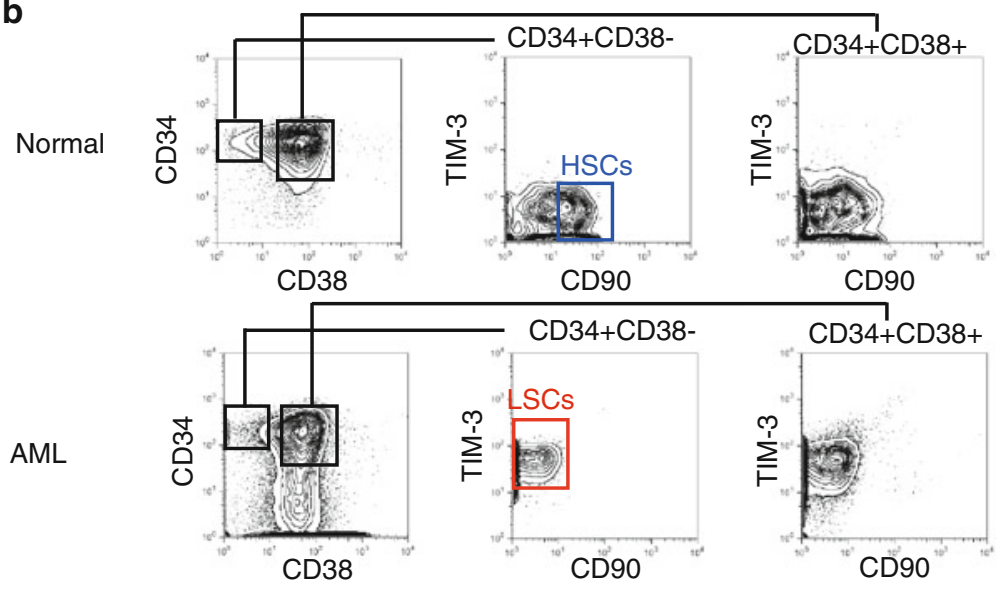

Fig. 1 TIM-3 expression in normal HSCs and AML LSCs. (a) Results of gene expression analysis comparing $\mathrm{CD} 34^{+} \mathrm{CD} 38^{-}$normal HSCs and AML LSCs. Surface molecules highly expressed in LSCs are shown. (b) FACS analysis of TIM-3 protein expression in normal HSCs and AML LSCs. Both $\mathrm{CD} 34^{+} \mathrm{CD} 38^{-} \mathrm{CD} 90^{-}$LSCs and $\mathrm{CD} 34^{+} \mathrm{CD} 38^{+} \mathrm{AML}$ cells express TIM-3, whereas CD $34^{+} \mathrm{CD} 38^{-} \mathrm{CD} 90^{-}$HSCs completely lack TIM-3 expression. TIM-3 expression originates within the $\mathrm{CD} 34^{+} \mathrm{CD} 38^{+}$progenitor fraction in normal human hematopoiesis. A representative FACS analysis is shown here

Second, TIM-3 marks all functional LSCs that can reconstitute human AML in immunodeficient mice in the majority of M0, M1, M2, and M4 AML cases, and its expression level is sufficient to eradicate LSCs by antibody-based treatment.

\section{TIM-3 Expression and Functions in Normal Hematopoiesis}

TIM-3 was originally identified as a surface molecule expressed in interferon (IFN)- $\gamma$-producing CD4 ${ }^{+}$Th1 cells and $\mathrm{CD}^{+} \mathrm{T}$ cytotoxic type 1 (Tc1) cells [22] in murine hematopoiesis. TIM-3, a type 1 cell-surface glycoprotein, has a structure 


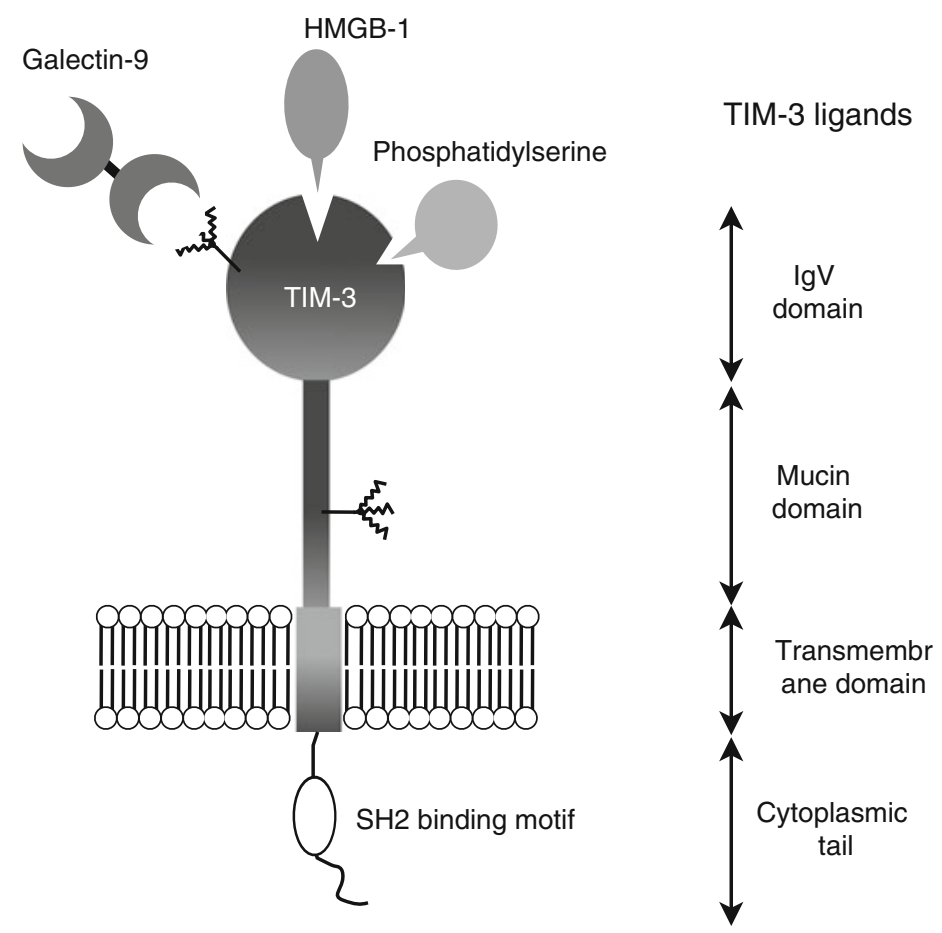

Fig. 2 Structure of TIM-3 molecule and its ligands. TIM-3 is a type 1 cell-surface glycoprotein and has a structure that includes an $\mathrm{N}$-terminal immunoglobulin variable domain followed by a mucin domain, a transmembrane domain, and a cytoplasmic tail with highly conserved six tyrosine residues and a SH2 binding motif. Galectin-9, HMGB1, and PS have been identified as ligands of TIM-3

that includes an $\mathrm{N}$-terminal immunoglobulin variable domain followed by a mucin domain, a transmembrane domain, and a cytoplasmic tail (Fig. 2). In steady-state human hematopoiesis, TIM-3 is expressed in monocytes and in a fraction of NK cells, but not in granulocytes, B cells, or T cells [8]. However, TIM-3 is upregulated in $\mathrm{T}$ cells in response to immune reactions. TIM-3 plays an important role in regulation of Th1-dependent immune responses and immune tolerance [22-24]. Galectin-9, an S-type lectin, has been reported as a TIM-3 ligand in lymphocytes. Galectin-9 has two distinct carbohydrate recognition domains and binds to carbohydrate chains on the IgV domain of TIM-3. TIM-3 has highly conserved six tyrosine residues and a Src homology 2 (SH2) binding motif in its cytoplasmic tail, and stimulation of TIM-3 by galectin- 9 results in increased phosphorylation of tyrosine residues in T cells [25]. Engagement of TIM-3 by galectin-9 induces apoptosis of Th1 cells and inhibits their IFN- $\gamma$ production [26]. These data collectively suggest that TIM-3 is a negative regulator of Th1- and Tc1-driven immune responses.

TIM-3 is also known as a marker of "exhausted" CD8 ${ }^{+} \mathrm{T}$ cells. Exhausted T cells show impaired proliferation and effector function under antigen stimulation. 
One of the major markers for exhausted $\mathrm{T}$ cells is the inhibitory molecule programmed cell death 1 (PD-1), and T cell function is partially restored by blocking the interaction between PD-1 and PD-1 ligand in mice [27]. TIM-3 is also expressed on exhausted $\mathrm{CD}^{+} \mathrm{T}$ cells in patients with chronic viral infections, including human immunodeficiency virus (HIV) [28], hepatitis B virus [29], and hepatitis C virus (HCV) [30]. Blockade of both TIM-3 and PD-1 ligation can significantly restore $\mathrm{T}$ cell proliferation and effector potential, suggesting that both TIM-3 and PD-1 pathways play a major role in $\mathrm{CD} 8^{+} \mathrm{T}$ cell exhaustion [31].

TIM-3 can also modulate the immune reaction pathway to regulate innate immunity. NK cells and some myeloid cells, including monocytes/macrophages, dendritic cells, and mast cells, express TIM-3 in both human and mouse hematopoiesis. In NK cells, TIM-3 is induced on their surface on activation [32,33], but the function of TIM-3 in NK cells remains controversial. It has been reported that TIM-3 is a human NK cell co-receptor to enhance IFN- $\gamma$ production [32], but another report showed that NK cell-mediated cytotoxicity was reduced by cross-linking of TIM-3 [33].

In terms of the myeloid lineage, TIM-3 is expressed in monocytes/macrophages, dendritic cells (DCs), and mast cells [34-37]. In human bone marrow, $\mathrm{CD} 34^{+} \mathrm{CD} 38^{-} \mathrm{CD} 90^{+}$normal HSCs and the majority of $\mathrm{CD} 34^{+} \mathrm{CD} 38^{+}$progenitor cells do not express TIM-3. Within the $\mathrm{CD} 34^{+} \mathrm{CD} 38^{+}$progenitor fraction, human myeloid progenitors can be divided into three subpopulations, such as common myeloid progenitors (CMPs), granulocyte/macrophage progenitors (GMPs), and megakaryocyte/erythrocyte progenitors (MEPs) [38]. TIM-3 is expressed only in a fraction GMPs, but not in CMPs and MEPs. Purified TIM- $3^{+}$GMPs give rise mainly to colony-forming unit-macrophage (CFU-M), whereas TIM- $3^{-}$GMPs can generate various types of myeloid colonies, suggesting that upregulation of TIM-3 occurs in concert with monocyte lineage commitment at the GMP stage in humans [8].

In mature monocytes or dendritic cells, TIM-3 signaling synergizes with that of Toll-like receptors to promote secretion of tumor necrosis factor- $\alpha$ (TNF- $\alpha$ ) inflammatory responses [34]. In addition, TIM-3 on macrophages and DCs recognizes phosphatidylserine (PS) in apoptotic cells through its IgV domain. Binding of PS to TIM-3 does not interfere with that of galectin-9 to TIM-3, as the binding sites of these molecules are located on opposite sides of the $\operatorname{IgV}$ domain. In TIM-3expressing DCs, recognition of PS by TIM-3 induced enhancement of phagocytosis of apoptotic cells and cross-presentation of apoptotic cell-associated antigen to $\mathrm{CD}^{+} \mathrm{T}$ cells [35]. TIM-3 expression and functions in hematopoietic cells are summarized in Fig. 3.

\section{TIM-3 Is a Marker of Malignant Stem Cells in Human AML}

We have identified TIM-3 as an AML LSC-specific surface molecule. We first compared the gene expression profiles of $\mathrm{CD} 34^{+} \mathrm{CD} 38^{-} \mathrm{AML}$ cells and normal HSCs by using cDNA microarray analysis (Fig. 1a). As shown in Fig. 1b, TIM-3 protein is not expressed in $\mathrm{CD} 34^{+} \mathrm{CD} 38^{-} \mathrm{CD} 90^{+}$normal $\mathrm{HSCs}$, but the vast majority 


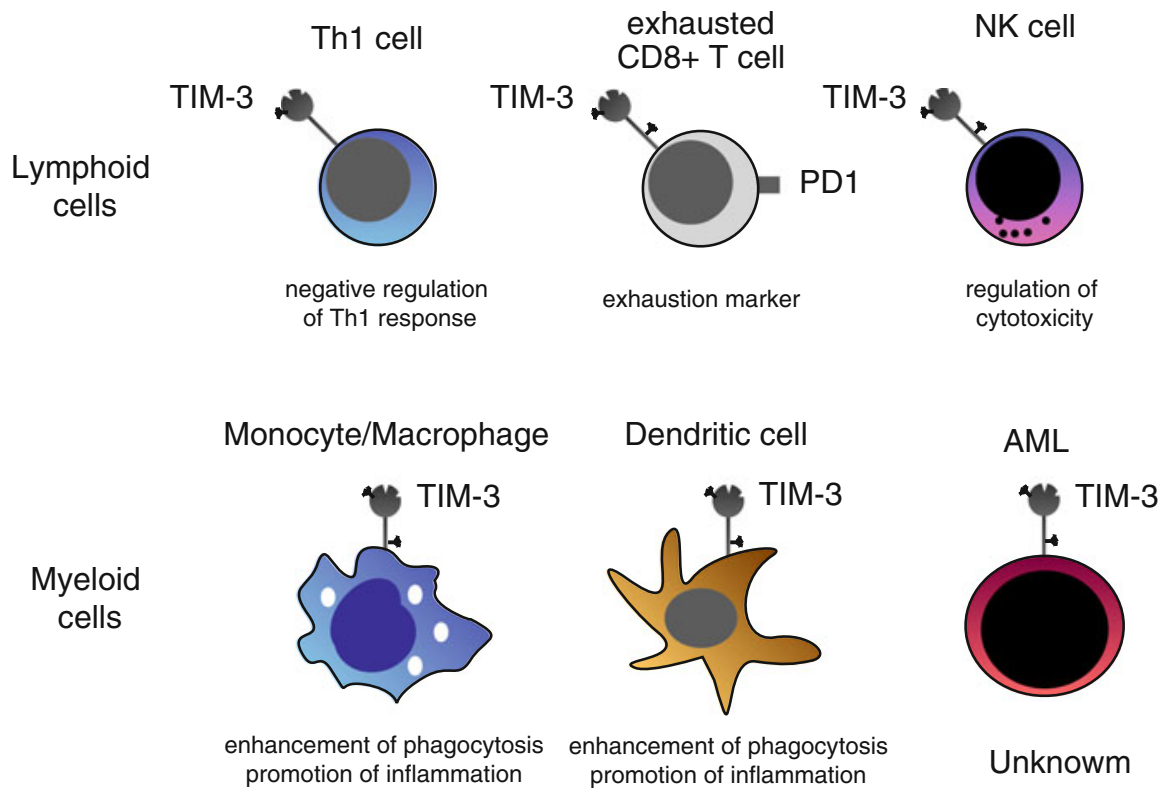

Fig. 3 TIM-3 expression and functions in normal hematopoietic cells. TIM-3 is expressed in Th1 cells, exhausted $\mathrm{CD} 8^{+} \mathrm{T}$ cells, NK cells, monocytes, and dendritic cells in normal hematopoiesis. The functions of TIM-3 differ by cell type and context

of the $\mathrm{CD} 34^{+} \mathrm{CD} 38^{-}$LSCs and the $\mathrm{CD} 34^{+} \mathrm{CD} 38^{+}$cells expressed TIM-3 at a high level in patients with most types of AML except for acute promyelocytic leukemia (M3) $[8,21]$. Another group has also reported that the expression level of TIM-3 is especially high in immature AML cells with core-binding factor translocations or mutations in CEBPA [21].

It is important to note that the TIM- $3^{+}$fraction in AML patients contained all functional LSCs. We separated AML cells into the TIM- $3^{+}$and TIM- $3^{-}$populations and transplanted each population into sublethally irradiated immunodeficient mice, and found that only TIM- $3^{+}$AML cells, but not TIM- $3^{-}$cells, reconstituted human AML in these mice [8]. These data suggest that targeting TIM- $3^{+}$cells is sufficient for eradication of LSCs in AML patients.

\section{Targeting AML-LSCs by Monoclonal Anti-TIM-3 Killing Antibodies in a Xenograft Model}

To utilize TIM-3 to target AML LSCs, it is critical to establish anti-human TIM-3 antibodies that can kill TIM-3-expressing cells in vivo. To achieve successful antibody-based treatment, antibody-dependent cellular cytotoxicity (ADCC) and 
complement-dependent cytotoxicity (CDC) activities are critical to eliminate target cells [39]. Additionally, recent studies have suggested that antibody-dependent cellular phagocytosis (ADCP) could play an important role in killing target cells in vitro [40] and in vivo [41].

An anti-TIM-3 monoclonal antibody (IgG2b) was obtained by immunizing Balb/c mice with L929 cells stably expressing human TIM-3 and soluble TIM-3 protein [8]. In this antibody, the variable portions of the $\mathrm{VH}$ regions of the cloned hybridoma that recognize TIM-3 were grafted onto IgG2a Fc regions, because the IgG2a subclass is most efficient to induce ADCC activity in mice [42, 43]. The clone called ATIK2a was established, and it was effective in killing TIM-3-expressing cell lines by its CDC and ADCC activities [8].

We then tested the effect of ATIK2a on the growth of AML LSCs or normal HSCs in xenograft models. NOD-SCID mice transplanted with $10^{5} \mathrm{CD} 34^{+}$cord blood cells were treated with ATIK2a. These mice were reconstituted with normal hematopoiesis with nearly equal percentages of human cell chimerisms, although human mature monocytes were depleted. In contrast, in mice reconstituted with human AML, ATIK2a exerted profound effects on leukemia development. The mice were transplanted with human AML of M0, M1, and M4 types, and after confirmation of AML development in these mice, ATIK2a was injected six times over 2 weeks. Strikingly, human AML cells disappeared in mice treated with ATIK2a but not in those with control IgG treatment. These data strongly suggest that targeting of AML LSCs by utilizing anti-TIM-3 killing antibodies is a practical approach to cure human AML.

\section{TIM-3 Is a Functional Molecule for AML LSC Maintenance}

Since TIM-3 has a tyrosine residue and $\mathrm{SH} 2$ domain that can activate Src family proteins, we hypothesized that TIM-3 signaling has some function to maintain AML-LSCs. We found that the serum levels of galectin-9, a TIM-3 ligand, were significantly ( $>10$-fold) elevated in AML patients but not in normal individuals on an ELISA assay. Furthermore, TIM- $3^{+}$AML cells had abundant galectin- 9 protein in their cytoplasm, and they secreted galectin- 9 in the sera of mice transplanted with human AML. Mice reconstituted with normal human HSCs or B cell acute lymphoblastic leukemia did not have detectable levels of serum galectin-9. These results collectively suggest that AML cells secreted galectin-9 in an autocrine manner. Furthermore, TIM-3 stimulation by galectin-9 in AML cells in vitro induced significant gene expression changes including NF- $\kappa \mathrm{B}$ target genes (unpublished data). Collectively, it is suggested that AML LSCs had growth and survival advantages through an autocrine stimulation loop of the TIM-3/galectin-9 system. 


\section{Conclusion}

TIM-3 has been shown to play pivotal roles in modulating immune reactions. By transcriptome analysis, we newly identified TIM-3 as a surface molecule specific to AML LSCs but not expressed in normal HSCs. Our in vivo xenogeneic transplantation analysis directly showed that targeting TIM-3 could be an efficient, useful therapeutic approach to eradicate AML LSCs.

Open Access This chapter is distributed under the terms of the Creative Commons Attribution Noncommercial License, which permits any noncommercial use, distribution, and reproduction in any medium, provided the original author(s) and source are credited.

\section{References}

1. Lapidot $\mathrm{T}$ et al (1994) A cell initiating human acute myeloid leukaemia after transplantation into SCID mice. Nature 367(6464):645-648

2. Ishikawa $F$ et al (2007) Chemotherapy-resistant human AML stem cells home to and engraft within the bone-marrow endosteal region. Nat Biotechnol 25(11):1315-1321

3. Bonnet D, Dick JE (1997) Human acute myeloid leukemia is organized as a hierarchy that originates from a primitive hematopoietic cell. Nat Med 3(7):730-737

4. Hope KJ, Jin L, Dick JE (2004) Acute myeloid leukemia originates from a hierarchy of leukemic stem cell classes that differ in self-renewal capacity. Nat Immunol 5(7):738-743

5. Miyawaki S (2012) Clinical studies of acute myeloid leukemia in the Japan Adult Leukemia Study Group. Int J Hematol 96(2):171-177

6. Stein EM, Tallman MS (2012) Remission induction in acute myeloid leukemia. Int J Hematol 96(2):164-170

7. Krause DS, Van Etten RA (2007) Right on target: eradicating leukemic stem cells. Trends Mol Med 13(11):470-481

8. Kikushige $\mathrm{Y}$ et al (2010) TIM-3 is a promising target to selectively kill acute myeloid leukemia stem cells. Cell Stem Cell 7(6):708-717

9. van Rhenen A et al (2007) The novel AML stem cell associated antigen CLL-1 aids in discrimination between normal and leukemic stem cells. Blood 110(7):2659-2666

10. Aikawa $Y$ et al (2010) PU.1-mediated upregulation of CSF1R is crucial for leukemia stem cell potential induced by MOZ-TIF2. Nat Med 16(5):580-585, 1p following 585

11. Hosen $\mathrm{N}$ et al (2007) CD96 is a leukemic stem cell-specific marker in human acute myeloid leukemia. Proc Natl Acad Sci U S A 104(26):11008-11013

12. Jan M et al (2012) Clonal evolution of preleukemic hematopoietic stem cells precedes human acute myeloid leukemia. Sci Transl Med 4(149):149ra118

13. Bakker AB et al (2004) C-type lectin-like molecule-1: a novel myeloid cell surface marker associated with acute myeloid leukemia. Cancer Res 64(22):8443-8450

14. Wang PL et al (1992) Identification and molecular cloning of tactile. A novel human T cell activation antigen that is a member of the Ig gene superfamily. J Immunol 148(8):2600-2608

15. Saito $\mathrm{Y}$ et al (2010) Identification of therapeutic targets for quiescent, chemotherapy-resistant human leukemia stem cells. Sci Transl Med 2(17):17ra9

16. Jin L et al (2006) Targeting of CD44 eradicates human acute myeloid leukemic stem cells. Nat Med 12(10):1167-1174

17. Majeti $\mathrm{R}$ et al (2009) CD47 is an adverse prognostic factor and therapeutic antibody target on human acute myeloid leukemia stem cells. Cell 138(2):286-299

18. Jin L et al (2009) Monoclonal antibody-mediated targeting of CD123, IL-3 receptor alpha chain, eliminates human acute myeloid leukemic stem cells. Cell Stem Cell 5(1):31-42 
19. Taussig DC et al (2005) Hematopoietic stem cells express multiple myeloid markers: implications for the origin and targeted therapy of acute myeloid leukemia. Blood 106(13):4086-4092

20. Takenaka K et al (2007) Polymorphism in Sirpa modulates engraftment of human hematopoietic stem cells. Nat Immunol 8(12):1313-1323

21. Jan M et al (2011) Prospective separation of normal and leukemic stem cells based on differential expression of TIM3, a human acute myeloid leukemia stem cell marker. Proc Natl Acad Sci U S A 108(12):5009-5014

22. Monney L et al (2002) Th1-specific cell surface protein Tim-3 regulates macrophage activation and severity of an autoimmune disease. Nature 415(6871):536-541

23. Sanchez-Fueyo A et al (2003) Tim-3 inhibits T helper type 1-mediated auto- and alloimmune responses and promotes immunological tolerance. Nat Immunol 4(11):1093-1101

24. Sabatos CA et al (2003) Interaction of Tim-3 and Tim-3 ligand regulates T helper type 1 responses and induction of peripheral tolerance. Nat Immunol 4(11):1102-1110

25. van de Weyer PS et al (2006) A highly conserved tyrosine of Tim-3 is phosphorylated upon stimulation by its ligand galectin-9. Biochem Biophys Res Commun 351(2):571-576

26. Zhu C et al (2005) The Tim-3 ligand galectin-9 negatively regulates T helper type 1 immunity. Nat Immunol 6(12):1245-1252

27. Barber DL et al (2006) Restoring function in exhausted CD8 T cells during chronic viral infection. Nature 439(7077):682-687

28. Jones RB et al (2008) Tim-3 expression defines a novel population of dysfunctional T cells with highly elevated frequencies in progressive HIV-1 infection. J Exp Med 205(12):2763-2779

29. Wu W et al (2012) Blockade of Tim-3 signaling restores the virus-specific CD8(+) T-cell response in patients with chronic hepatitis B. Eur J Immunol 42(5):1180-1191

30. Golden-Mason L et al (2009) Negative immune regulator Tim-3 is overexpressed on T cells in hepatitis $\mathrm{C}$ virus infection and its blockade rescues dysfunctional CD4+ and CD8+ T cells. J Virol 83(18):9122-9130

31. Sakuishi K et al (2010) Targeting Tim-3 and PD-1 pathways to reverse T cell exhaustion and restore anti-tumor immunity. J Exp Med 207(10):2187-2194

32. Gleason MK et al (2012) Tim-3 is an inducible human natural killer cell receptor that enhances interferon gamma production in response to galectin-9. Blood 119(13):3064-3072

33. Ndhlovu LC et al (2012) Tim-3 marks human natural killer cell maturation and suppresses cell-mediated cytotoxicity. Blood 119(16):3734-3743

34. Anderson AC et al (2007) Promotion of tissue inflammation by the immune receptor Tim-3 expressed on innate immune cells. Science 318(5853):1141-1143

35. Nakayama $M$ et al (2009) Tim-3 mediates phagocytosis of apoptotic cells and crosspresentation. Blood 113(16):3821-3830

36. Nakae $\mathrm{S}$ et al (2007) TIM-1 and TIM-3 enhancement of Th2 cytokine production by mast cells. Blood 110(7):2565-2568

37. Dekruyff RH et al (2010) T cell/transmembrane, Ig, and mucin-3 allelic variants differentially recognize phosphatidylserine and mediate phagocytosis of apoptotic cells. J Immunol 184(4):1918-1930

38. Manz MG et al (2002) Prospective isolation of human clonogenic common myeloid progenitors. Proc Natl Acad Sci U S A 99(18):11872-11877

39. Nimmerjahn F, Ravetch JV (2007) Antibodies, Fc receptors and cancer. Curr Opin Immunol 19(2):239-245

40. Manches $\mathrm{O}$ et al (2003) In vitro mechanisms of action of rituximab on primary non-Hodgkin lymphomas. Blood 101(3):949-954

41. Oflazoglu E et al (2009) Macrophages and Fc-receptor interactions contribute to the antitumour activities of the anti-CD40 antibody SGN-40. Br J Cancer 100(1):113-117

42. Nimmerjahn F, Ravetch JV (2005) Divergent immunoglobulin g subclass activity through selective Fc receptor binding. Science 310(5753):1510-1512

43. Uchida $\mathrm{J}$ et al (2004) The innate mononuclear phagocyte network depletes B lymphocytes through Fc receptor-dependent mechanisms during anti-CD20 antibody immunotherapy. J Exp Med 199(12):1659-1669 


\title{
TGF- $\beta$ LAP Degradation Products, a Novel Biomarker and Promising Therapeutic Target for Liver Fibrogenesis
}

\author{
Mitsuko Hara, Tomokazu Matsuura, and Soichi Kojima
}

\begin{abstract}
While there are many blood and/or tissue biomarkers as well as algorithms clinically used to assess hepatic fibrosis, a good biomarker and therapeutic target of hepatic fibrogenesis, which reflects prefibrotic changes, has not been established. The most fibrogenic cytokine, transforming growth factor (TGF)- $\beta$, is produced as a latent complex, in which TGF- $\beta$ is trapped by its propeptide. On the surface of activated hepatic stellate cells, plasma kallikrein activates TGF- $\beta$ by cleaving latency-associated protein (LAP) between the $\mathrm{R}^{58}$ and $\mathrm{L}^{59}$ residues, releasing active TGF- $\beta$ from the complex. We made specific antibodies that recognize neo-C-terminal $\left(\mathrm{R}^{58}\right)$ and N-terminal $\left(\mathrm{L}^{59}\right)$ ends of LAP degradation products (LAP-DPs) and found that LAP-DPs may serve as a novel surrogate marker of TGF- $\beta$ activation - namely, generation of active TGF- $\beta$ - and is thus a therapeutic marker for TGF- $\beta$-mediated liver fibrogenesis in patients and can also be used to monitor effects of anti-fibrogenic factors or compounds for discovery of a novel anti-fibrosis drug.
\end{abstract}

Keywords Biomarkers • Hepatic fibrogenesis $\bullet$ TGF- $\beta \bullet$ LAP $\bullet$ Latent TGF- $\beta$ activation $\bullet$ Hepatic stellate cells $\bullet$ Plasma kallikrein $\bullet$ LAP-DP $\bullet$ Drug discovery $\bullet$ Anti-fibrosis drug

\section{Abbreviations}

ECM Extracellular matrix

HSCs Hepatic stellate cells

$\alpha$ SMA $\quad \alpha$ smooth muscle actin

TGF- $\beta 1 \quad$ Transforming growth factor- $\beta 1$

M. Hara • S. Kojima, Ph.D. ( $\varangle)$

Micro-Signaling Regulation Technology Unit, RIKEN Center for Life Science Technologies,

2-1 Hirosawa, Wako, Saitama 351-0918, Japan

e-mail: m-hara@riken.jp; skojima@riken.jp

T. Matsuura

Department of Laboratory Medicine, The Jikei University School of Medicine,

Minato-ku, Tokyo 105-0003, Japan

e-mail: matsuurat@jikei.ac.jp

K. Nakao et al. (eds.), Innovative Medicine, DOI 10.1007/978-4-431-55651-0_26 


$\begin{array}{ll}\text { LAP } & \text { Latency associated protein } \\ \text { SLC } & \text { Small latent complex } \\ \text { LTBP } & \text { Latent TGF- } \beta \text { binding protein } \\ \text { LLC } & \text { Large latent complex } \\ \text { PLN } & \text { Plasmin } \\ \text { PLK } & \text { Plasma kallikrein } \\ \text { LAP-DP } & \text { LAP degradation products } \\ \text { LAP } \beta 1 & \text { TGF- } \beta 1 \text { LAP } \\ \text { BDL } & \text { Bile duct ligation } \\ \text { HBV } & \text { Hepatitis B virus } \\ \text { HCV } & \text { Hepatitis C virus } \\ \text { NASH } & \text { Non-alcoholic steatohepatitis }\end{array}$

\section{Introduction of Liver Fibrogenesis}

Hepatic fibrosis is the excessive accumulation of extracellular matrices (ECM; mainly collagen) in the perisinusoidal space (or space of Disse) in the liver, and an important pathological step developing from chronic hepatitis to liver cirrhosis irrespective of etiologies [1], whereas hepatic fibrogenesis means fibrosis progression or an ongoing reaction producing excessive ECM, sometimes nonsymptomatic, in the liver [2]. While there are many blood and/or tissue biomarkers as well as algorithms clinically used to assess hepatic fibrosis [3-7], the gold standard is still scoring of stained collagen fibers in the biopsy sample [3]. However, biopsy is invasive and risky. Imaging techniques including ultrasound elastography have been developed [3]. In contrast, a good biomarker and therapeutic target of hepatic fibrogenesis, which reflects prefibrotic changes, has not been established [2,3]. Therefore, development of a noninvasive biomarker for hepatic fibrogenesis, which will lead not only to establishment of a novel diagnosis useful to prevent liver fibrosis/ cirrhosis, but also to acceleration of drug discovery and development against liver fibrosis, is in high demand [3].

\section{Activation of Hepatic Stellate Cells}

Hepatic stellate cells (HSCs) play a central role in the pathogenesis of hepatic fibrosis by virtue of their ability to undergo a process termed "activation" [1,2]. During this process, HSCs transform into myofibroblast-like cells accompanying several key phenotypic changes, which collectively increase extracellular matrix accumulation [1-3]. These include (1) cellular proliferation caused by upregulation of mitogenic cytokines and their receptors; (2) morphologic changes with loss of stored 
vitamin A droplets; (3) contractility caused by increased $\alpha$ smooth muscle actin ( $\alpha$ SMA), which may constrict sinusoidal blood flow; and (4) fibrogenesis mainly caused by increased synthesis and release of collagen.

\section{TGF- $\beta$ and Its Activation Reaction}

Among many cytokines and growth factors related to fibrogenesis, the most potentand therefore the most "fibrogenic"-cytokine is the $25 \mathrm{kD}$ homodimeric cytokine, transforming growth factor (TGF)- $\beta$ [8]. The TGF- $\beta$ family is composed of three subtypes (TGF- $\beta 1$, TGF- $\beta 2$, and TGF- $\beta 3$ ), with biological properties that are nearly identical [8]. TGF- $\beta$ is produced as an inactive latent complex, in which active TGF- $\beta$ is trapped by its propeptide, latency-associated protein (LAP), and to exert its biological activities, it must be released from the complex [9]. This reaction is called activation of TGF- $\beta$ (Fig. 1). TGF- $\beta 1$ is produced as a 390 -amino-acid

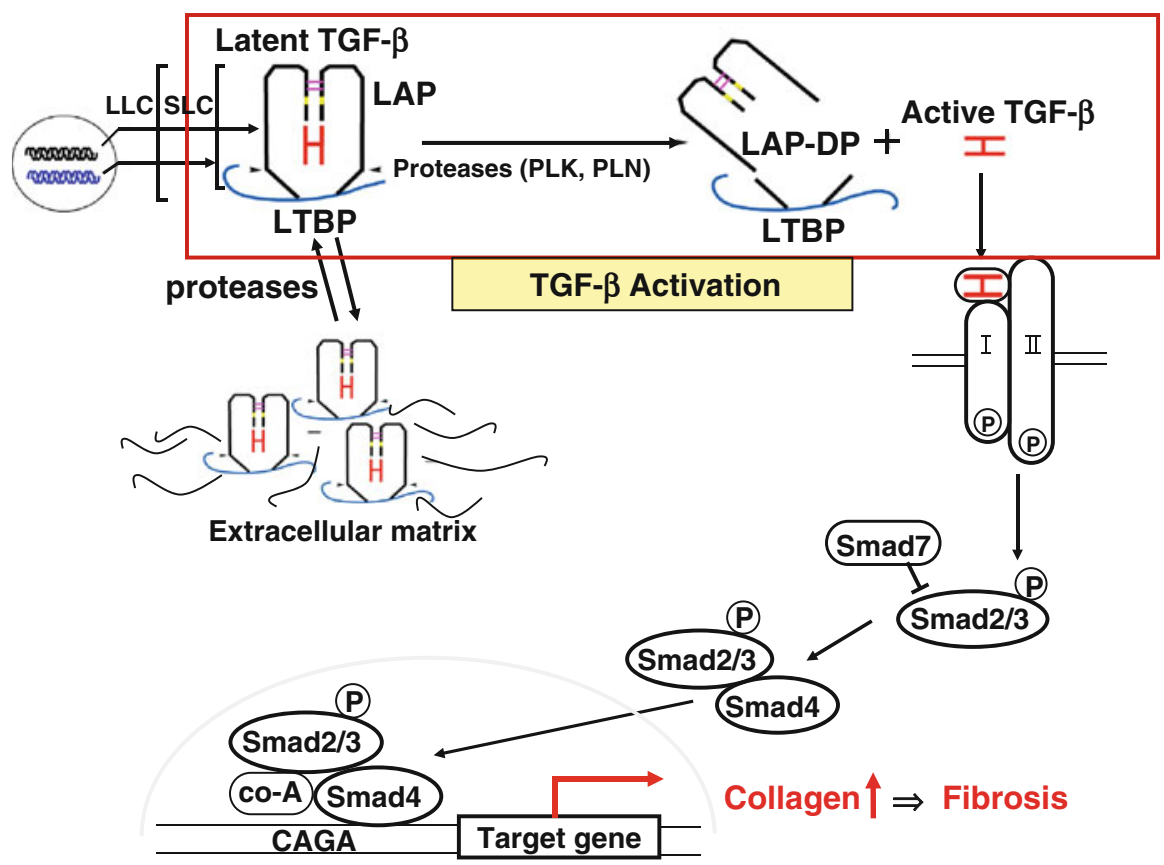

Fig. 1 TGF- $\beta$ activation and signaling pathway. TGF- $\beta$ is produced as a latent complex (LLC) composed of active TGF- $\beta$ trapped by its propeptide LAP (SLC) and a matrix protein LTBP. Upon cleavage of LAP by proteases such as PLK, active TGF- $\beta$ is released from the complex (this reaction is called TGF- $\beta$ activation) and exerts fibrogenic activity (stimulation of collagen synthesis) via binding to its receptors and Smad signaling 
precursor protein consisting of a signal peptide of 29 amino acids, an $\mathrm{N}$-terminal LAP, and a C-terminal region that becomes the active TGF- $\beta 1$ molecule, and each region is dimerized through $\mathrm{S}-\mathrm{S}$ bonds. After processing by cleavage at $\mathrm{R}^{278}-\mathrm{A}^{279}$ by a furin-like protease, the LAP still non-covalently captures the active TGF- $\beta 1$, forming small latent complex (SLC) and preventing active TGF- $\beta 1$ from binding its cognate receptors [9]. The active TGF- $\beta 1$ and the LAP homodimers are $25 \mathrm{kD}$ and $75 \mathrm{kD}$, respectively. SLC is S-S bonded to another gene product, the latent TGF- $\beta$ binding protein (LTBP), via $\mathrm{C}^{33}$ residues, forming the large latent complex (LLC). This complex can be sequestered in the ECM (Fig. 1) [10] because LTBP is a member of an ECM protein family, fibrillin [11].

Activation of latent TGF- $\beta$ is performed through different mechanisms depending on the tissue and cell types and experimental conditions, and several molecules are known to activate TGF- $\beta 1$ in animal models [12-21]. These include integrins [12-15], thrombospondin [16], and proteases, such as matrix metalloproteinases and serine proteases [17-21]. The integrin $\alpha v \beta 6$ binds to and activates latent TGF- $\beta$ and plays a role in regulating pulmonary inflammation and fibrosis as well as biliary fibrosis [12-15]. Thrombospondin 1 is another major activator of latent TGF- $\beta$, especially in the lung and pancreas, by binding to a defined site within LAP and inducing a conformational change in the latent complex [16]. In the normal liver, TGF- $\beta$ is produced and secreted from sinusoidal endothelial cells and Kupffer cells (KCs, resident macrophages in the liver) at low levels. Elevated production of TGF- $\beta$ was seen first in all cell types and then mainly in hepatocytes and HSCs after partial hepatectomy, whereas elevated production of TGF- $\beta$ was seen solely in HSCs after inflammation and fibrosis [22]. TGF- $\beta$ secreted from HPCs is entirely in the latent form, whereas TGF- $\beta$ secreted from HSCs is $50-90 \%$ in the active form [22]. Thus, HSCs are recognized as the major source of active TGF- $\beta$, namely the site of TGF- $\beta$ activation, particularly in the damaged liver [22,23].

We have addressed a potential proteolytic mechanism for latent TGF- $\beta$ activation in HSCs by surface plasmin (PLN) and plasma kallikrein (PLK) during the formation of hepatic fibrosis $[20,21]$. PLN releases latent TGF- $\beta$ from the extracellular matrix and activates it by cleaving LAP from latent TGF- $\beta$ molecules on the HSC surface $[9,20]$. Lyons et al. first reported that PLN digests LAP and activates TGF$\beta 1$ in vitro [24]. Using a protease inhibitor, camostat mesilate, we demonstrated that PLN and PLK are involved in the TGF- $\beta 1$ activation associated with liver fibrosis and impaired liver regeneration in animal models [20,21]. However, it remained to be elucidated whether PLN- and/or PLK-dependent TGF- $\beta 1$ activation also occurs during the pathogenesis of liver fibrosis in patients, as there was no good biomarker reflecting protease-dependent TGF- $\beta 1$ activation reaction. To answer this question, we determined cleavage site within LAP and made specific antibodies that recognize LAP degradation products (LAP-DPs) bearing a neo-amino or carboxyl terminus [25]. 


\section{TGF- $\beta$ LAP-DP Serves as a Surrogate Marker for Its Activation Reaction}

To identify the cleavage sites in LAP during latent TGF- $\beta 1$ activation by PLN and PLK, recombinant human LAP $\beta 1$ was digested with these proteases, the resultant fragments were separated by SDS-polyacrylamide gel electrophoresis (PAGE), and the N-terminal sequence of each LAP-DP was determined using a pulsed liquid protein sequencer Precise 494cLC, which revealed that PLN and PLK primarily cleave LAP $\beta 1$ between the $\mathrm{K}^{56}$ and $\mathrm{L}^{57}$ residues, and the $\mathrm{R}^{58}$ and $\mathrm{L}^{59}$ residues, respectively, during proteolytic activation of latent TGF- $\beta 1$ (Fig. 2) [25].

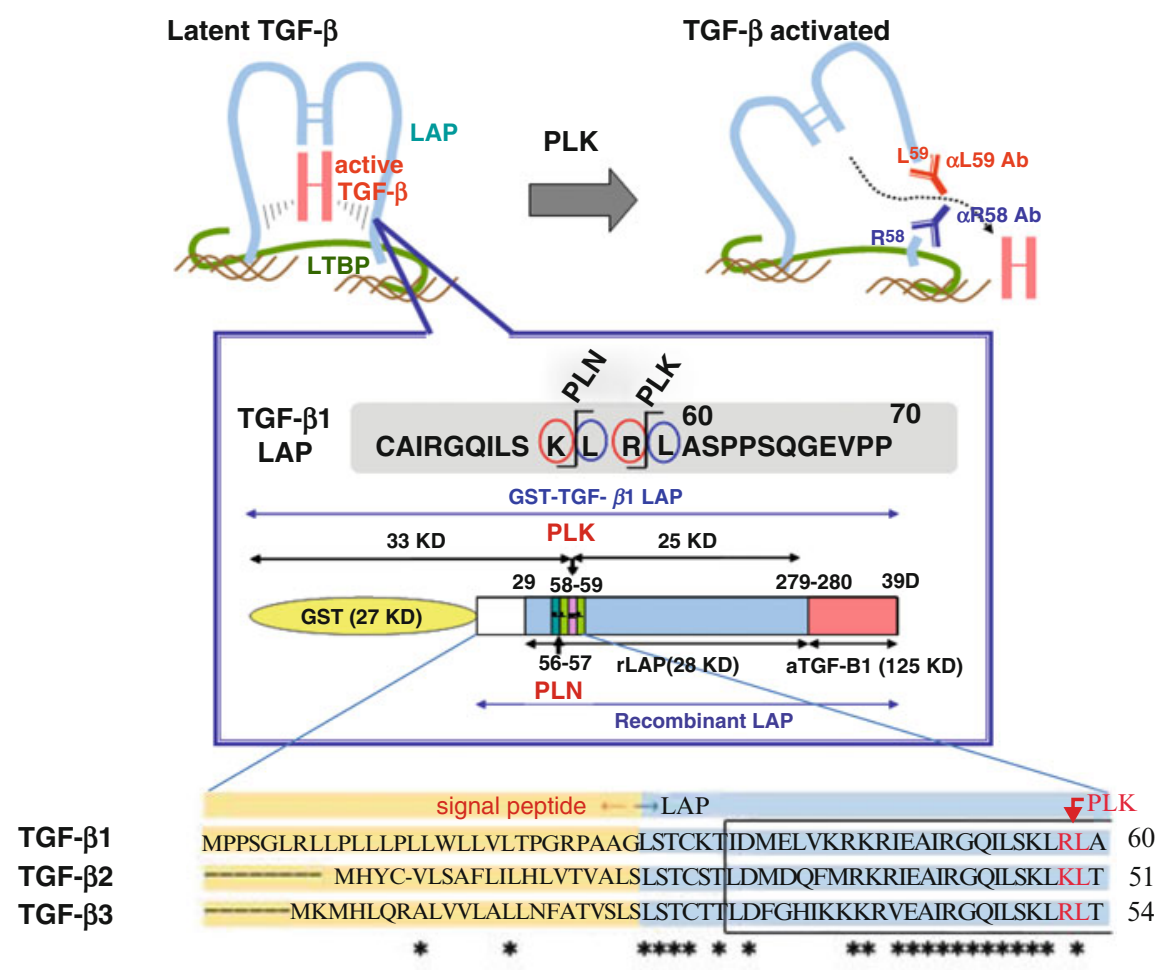

Fig. 2 Cleavage of $\mathrm{K}^{56} \mathrm{LRL}^{59}$ within LAP activates TGF- $\beta$. PLN and PLK cleave LAP between $\mathrm{K}^{56}-\mathrm{L}^{59}$ and $\mathrm{R}^{58}-\mathrm{L}^{59}$ residues, respectively, causing release of active TGF- $\beta 1$ from the latent complex. The amino acid sequences around the PLN and PLK cleavage sites are illustrated. Antibodies that specifically recognize the cutting edges of LAP-DPs were produced. The dark blue " $Y$ " labeled R58 represents antibodies recognizing the C-terminal or N-terminal side LAP-DPs, whereas the red " $Y$ " labeled L59 represents antibodies recognizing the $\mathrm{N}$-terminal or C-terminal side LAP-DPs. A comparison of amino acid sequences from the N-terminus until the PLK cleavage site among three isoforms of TGF- $\beta$ is presented at the bottom 

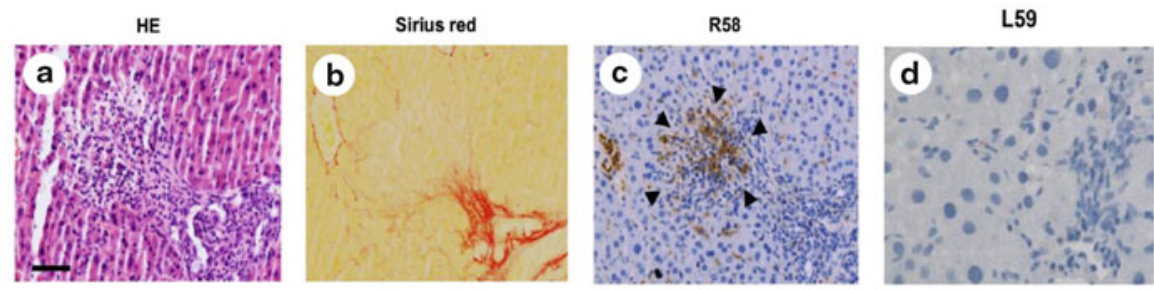

R58

aSMA
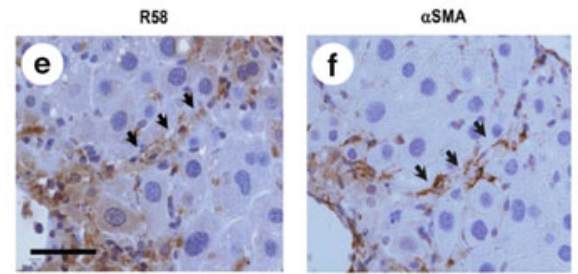

CD31

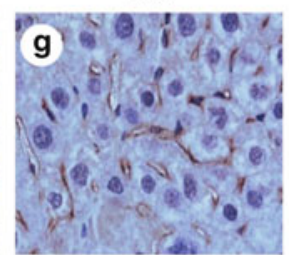

CD68

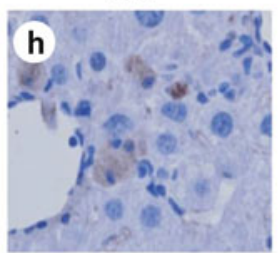

Fig. 3 Emergence of TGF- $\beta$ LAP-DPs in activated HSCs within pre-fibrotic areas in BDL models. Liver sections from BDL-operated mice were stained by HE (a) and Sirius red (b), and immunostained with R58 (c) and L59 (d) antibodies (scale bar=50 $\mu \mathrm{m}$ ), and were immunostained with R58 (e), anti- $\alpha$ SMA (f), anti-CD31 (g), and anti-CD68 (h) antibodies (scale bar=25 $\mu \mathrm{m}$ ). More detailed results are provided elsewhere [25]

To detect PLK-produced LAP-DPs, we made two monoclonal antibodies. One is the R58 antibody detecting N-terminal side LAP-DPs terminating on the $\mathrm{R}^{58}$ residue ( $\mathrm{R}^{58}$ LAP-DPs), and another is the L59 antibody detecting C-terminal side LAPDPs starting from the $\mathrm{L}^{59}$ residue ( $\mathrm{L}^{59} \mathrm{LAP}-\mathrm{DPs}$ ). We established techniques to detect each LAP-DP using these antibodies [25]. The $\mathrm{R}^{58}$ LAP-DPs remaining in tissues or cell surfaces through $\mathrm{S}-\mathrm{S}$ bonded LTBP can be detected mostly in $\alpha$ SMApositive activated stellate cells in liver tissues from both fibrotic animals and patients by immunostaining with the R58 antibody, whereas the ${ }^{59}$ LAP-DPs were not detectable by immunostaining with the L59 antibody [25]. Figure 3 shows the results obtained from bile duct ligation (BDL) mice. These mice often exhibited granulomatous lesions (panel $a$ ), in which fibroblastic cells infiltrated and started ECM production (panel $b$ ). Importantly, the $\mathrm{R}^{58}$ LAP-DPs were detected in granulomatous lesions prior to Sirius red positivity, namely before collagen accumulation (arrowheads in panel c). In contrast, L59 antibody failed to stain the $\mathrm{L}^{59}$ LAP-DPs, although various antigen unmasking procedures were treated (panel $d$ ). We found that the $\mathrm{L}^{59}$ LAP-DPs were released into the blood and could be measured by an ELISA using the L59 antibody (Hara et al., unpublished data). In panels $e-h$, nonparenchymal regions were recognized by antibody R58 (arrowheads in panel $e$ ), and mostly overlapped with $\alpha$ SMA-positive HSCs (arrowheads in panel f), but not with CD31-positive liver sinusoidal endothelial cells (panel g) nor with CD68positive KCs (hepatic macrophages) (panel $h$ ). We further found that the R58 antibody detected TGF- $\beta 1 / 3$ LAP-DPs but not TGF- $\beta 2$ LAP-DPs because of the similarity and difference of the R58 side sequence, respectively (Fig. 2). Finally, we succeeded in detecting $\mathrm{R}^{58}$ LAP-DPs in patients with chronic hepatitis $\mathrm{B}$ and $\mathrm{C}$ virus 
(HBV and $\mathrm{HCV}$, respectively) infection categorized as A1F2 and A2F2, as well as in patients with non-viral hepatitis, such as autoimmune hepatitis and non-alcoholic steatohepatitis (NASH) [25]. A specific cell shape called a "crown-like structure" (CLS) has been referred to as a biomarker for NASH in both an animal model and patients [26]. Recently, we found that $\mathrm{R}^{58}$ LAP-DPs positivity well matched the emergence of CLS [27].

These data suggest the occurrence of a PLK-dependent TGF- $\beta$ activation reaction in patients and indicate that the LAP-DP may be useful as a surrogate marker reflecting PLK-dependent TGF- $\beta 1 / 3$ activation and subsequent fibrogenesis in the fibrotic liver both in animal models and in patients.

\section{Conclusion and Future Subjects}

The most fibrogenic cytokine, TGF- $\beta$, is produced as a latent complex, in which TGF- $\beta$ is trapped by its propeptide, LAP. On the surface of activated HSCs, PLK activates TGF- $\beta$ by cleaving LAP between the $\mathrm{R}^{58}$ and $\mathrm{L}^{59}$ residues, releasing active TGF- $\beta$ from the complex. We made specific antibodies that recognize the neo-Cterminal $\left(\mathrm{R}^{58}\right)$ and $\mathrm{N}$-terminal $\left(\mathrm{L}^{59}\right)$ ends of the LAP-DP, and found that the LAP-DP may serve as a novel surrogate marker of TGF- $\beta$ activation - namely, generation of active TGF- $\beta$ - and is thereby a therapeutic marker for TGF- $\beta$-mediated liver fibrogenesis in patients [25].

Utilizing LAP-DP antibodies, we are developing techniques to visualize the fibrogenic area by positron emission tomography (PET), planning to eliminate activated HSCs with pertussis toxin, and undertaking the challenge to solve the co-crystal structure of LAP and a LAP-DP targeting inhibitor, which binds to the LAP cleavage site, thereby inhibiting TGF- $\beta$ activation and liver fibrosis in HBVinfected chimeric mice (Hara et al., unpublished data). The effectiveness of an inhibitor against the TGF- $\beta$ activation reaction has been reported in the integrinmediated activation of TGF- $\beta[3,15,28]$. LAP-DP is also used to monitor the effects of anti-fibrogenic factors or compounds for discovery of a novel anti-fibrosis drug. For example, we recently found that HCV NS3 protease mimics TGF- $\beta 2$ and enhances liver fibrosis via binding to and activation of the TGF- $\beta$ type I receptor, and that an anti-NS3 antibody raised against the predicted binding sites attenuates liver fibrosis in HCV-infected chimeric mice [29]. In this study, R58 LAP-DP staining nicely showed the anti-fibrogenic potentials of the anti-NS3 antibody.

The technique developed accelerates drug discovery targeting TGF- $\beta$-dependent fibrogenesis in patients suffering from chronic hepatitis.

Acknowledgments This work was supported partly by the Program for Promotion of Fundamental Studies in Health Science of the National Institute of Biomedical Innovation (NIBL) and a grant from the Uehara Memorial Foundation, Japan (to S.K.), Research on the Innovative Development and the Practical Application of New Drugs for Hepatitis B (Principal investigator: Soichi Kojima; H24-B Drug Discovery-Hepatitis-General-003), provided by the Ministry of Health, Labor and Welfare of Japan. 
Open Access This chapter is distributed under the terms of the Creative Commons Attribution Noncommercial License, which permits any noncommercial use, distribution, and reproduction in any medium, provided the original author(s) and source are credited.

\section{References}

1. Bataller R, Brenner DA (2005) Liver fibrosis. J Clin Invest 115:209-218

2. Friedman SL (2008) Mechanism of hepatic fibrogenesis. Gastroenterology 134:1655-1669

3. Schuppan D, Kim YO (2013) Evolving therapies for liver fibrosis. J Clin Invest 123:1887-1901

4. Kuno A, Ikehara Y, Tanaka Y, Ito K, Matsuda A, Sekiya S, Hige S, Sakamoto M, Kage M, Mizokami M, Narimatsu H (2013) A serum "sweet-doughnut" protein facilitates fibrosis evaluation and therapy assessment in patients with viral hepatitis. Sci Rep 3:1065

5. Ikeda K, Izumi N, Tanaka E, Yotsuyanagi H, Takahashi Y, Fukushima J, Kondo F, Fukusato T, Koike K, Hayashi N, Kumada H (2013) Fibrosis score consisting of four serum markers successfully predicts pathological fibrotic stages of chronic hepatitis B. Hepatol Res 43:596-604

6. Fallatah HI (2014) Noninvasive biomarkers of liver fibrosis: an overview. Adv Hepatol. Article ID 357287

7. Kazankov K, Barrera F, Møller HJ, Bibby BM, Vilstrup H, George J, Grønbaek H (2014) Soluble CD163, a macrophage activation marker, is independently associated with fibrosis in patients with chronic viral hepatitis B and C. Hepatology 60:521-530

8. Dooley S, ten Dijke P (2012) TGF- $\beta$ in progression of liver disease. Cell Tissue Res 347:245-256

9. Dabovic B, Rifkin DB (2008) TGF- $\beta$ bioavailability: latency, targeting, and activation. In: Derynck R, Miyazono K (eds) The TGF- $\beta$ family. Cold Spring Harbor Laboratory Press, New York, pp 179-202

10. Breitkof K, Lahme B, Tag CG, Gressner AM (2001) Expression and matrix deposition of latent TGF- $\beta$ binding proteins in normal and fibrotic rat liver and transdifferentiating hepatic stellate cells in culture. Hepatology 33:387-396

11. Zilberberg L, Todorovic V, Dabovic B, Horiguchi M, Couroussé T, Sakai LY, Rifkin DB (2012) Specificity of latent TGF- $\beta$ binding protein (LTBP) incorporation into matrix: role of fibrillins and fibronectin. J Cell Physiol 227:3828-3836

12. Margadant $C$, Sonnenberg A (2010) Integrin-TGF- $\beta$ crosstalk in fibrosis, cancer and wound healing. EMBO Rep 11:97-105

13. Shi M, Zhu J, Wang R, Chen X, Mi L, Walz T, Springer TA (2011) Latent TGF- $\beta$ structure and activation. Nature 474:343-349

14. Henderson NC, Sheppard D (2012) Integrin-mediated regulation of TGF $\beta$ in fibrosis. Biochim Biophys Acta 1832:891-896

15. Patsenker E, Popov Y, Stickel F, Jonczyk A, Goodman SL, Schuppan D (2008) Inhibition of integrin $\alpha v \beta 6$ on cholangiocytes blocks transforming growth factor- $\beta$ activation and retards biliary fibrosis progression. Gastroenterology 135:660-670

16. Ribeiro SM, Poczatek M, Schultz-Cherry S, Villain M, Murphy-Ullrich JE (1999) The activation sequence of thrombospondin-1 interacts with the latency-associated peptide to regulate activation of latent transforming growth factor- $\beta$. J Biol Chem 274:13586-13593

17. Jenkins $G$ (2008) The role of proteases in transforming growth factor- $\beta$ activation. Int $J$ Biochem Cell Biol 40:1068-1078

18. Du X, Shimizu A, Masuda Y, Kuwahara N, Arai T, Kataoka M, Uchiyama M, Kaneko T, Akimoto T, Iino Y, Fukuda Y (2012) Involvement of matrix metalloprotease-2 in the development of renal interstitial fibrosis in mouse obstruction nephropathy. Lab Invest 92:1149-1160 
19. Lyons RM, Gentry LE, Purchio AF, Moses HL (1990) Mechanism of activation of latent recombinant transforming growth factor beta 1 by plasmin. J Cell Biol 110:1361-1367

20. Okuno M, Akita K, Moriwaki H, Kawada N, Ikeda K, Kaneda K, Suzuki Y, Kojima S (2001) Prevention of rat fibrosis by protease inhibitor, Camostat Mesilate, via reduced generation of active TGF- $\beta$. Gastroenterology 120:1784-1800

21. Akita K, Okuno M, Enya M, Imai S, Moriwaki H, Kawada N, Suzuki Y, Kojima S (2002) Impaired liver regeneration in mice by lipopolysaccharide via TNF- $\alpha /$ kallikrein-mediated activation of latent TGF- $\beta$. Gastroenterology 123:352-364

22. Bissell DM, Wang SS, Jarnagin WR, Roll FJ (1995) Cell-specific expression of transforming growth factor- $\beta$ in rat liver. Evidence for autocrine regulation of hepatocyte proliferation. $\mathbf{J}$ Clin Invest 96:447-455

23. Friedman SL (1993) The cellular basis of hepatic fibrosis. N Engl J Med 328:1828-1835

24. Lyons RM, Keski-Oja J, Moses HL (1988) Proteolytic activation of latent transforming growth factor- $\beta$ from fibroblast-conditioned medium. J Cell Biol 106:1659-1665

25. Hara M, Kirita A, Kondo W, Matsuura T, Nagatsuma K, Dohmae N, Ogawa S, Imajoh-Ohmi S, Friedman SL, Rifkin DB, Kojima S (2014) LAP degradation product reflects plasma kallikrein-dependent TGF- $\beta$ activation in patients with hepatic fibrosis. Springerplus 3:221

26. Itoh M, Kato H, Suganami T, Konuma K, Marumoto Y, Terai S, Sakugawa H, Kanai S, Hamaguchi M, Fukaishi T, Aoe S, Akiyoshi K, Komohara Y, Takeya M, Sakaida I, Ogawa Y (2013) Hepatic crown-like structure: a unique histological feature in non-alcoholic steatohepatitis in mice and humans. PLoS One 8, e82163

27. Konuma K, Itoh M, Suganami T, Kanai S, Nakagawa N, Sakai T, Kawano H, Hara M, Kojima S, Izumi Y, Ogawa Y (2015) Eicosapentaenoic acid ameliorates non-alcoholic steatohepatitis in a novel mouse model using Melanocortin-4 receptor-deficient mice. PlosOne 10, e0121528

28. Allison M (2012) Stromedix acquisition signals growing interest in fibrosis. Nat Biotechnol 30:375-376

29. Sakata K, Hara M, Terada T, Watanabe N, Takaya D, Yaguchi S, Matsumoto T, Matsuura T, Shirouzu M, Yokoyama S, Yamaguchi T, Miyazawa K, Aizaki H, Suzuki T, Wakita T, Imoto M, Kojima S (2013) HCV NS3 protease enhances liver fibrosis via binding to and activating TGF- $\beta$ type I receptor. Sci Rep 3:3243 


\title{
Cell-Based Regenerative Therapy for Liver Disease
}

\author{
Kenichi Horisawa and Atsushi Suzuki
}

\begin{abstract}
The liver can regenerate itself in response to acute liver damage. However, chronically induced liver dysfunction interferes with the liver regeneration process and increases the risk of onset of more severe hepatic failure, including hepatic cirrhosis and liver cancer. To develop more efficient therapeutics for chronic liver diseases, cell-based regenerative therapies using functional hepatocyte-like cells derived from pluripotent stem cells are actively under investigation. In addition to such stem cell-based approaches, recent studies have revealed that direct cell-fate conversion from fibroblasts into hepatocyte-like cells can be induced by forced expression of particular sets of transcription factors in fibroblasts. This phenomenon is known as "direct reprogramming" and is expected to be a complementary or alternative technology to the stem cell-based regenerative therapies. In this chapter, we briefly summarize the recent progress and future perspectives of studies on reprogramming technologies, which are directed at the development of cell-based regenerative therapies for liver diseases.
\end{abstract}

Keywords Hepatocyte $\bullet$ Direct reprogramming $\bullet$ iHep cell $\bullet$ iPS cell $\bullet$ Regenerative medicine $\bullet$ Cell transplantation

\footnotetext{
K. Horisawa

Division of Organogenesis and Regeneration, Medical Institute of Bioregulation, Kyushu University, 3-1-1 Maidashi, Higashi-ku, Fukuoka 812-8582, Japan
}
A. Suzuki $(\bowtie)$
Division of Organogenesis and Regeneration, Medical Institute of Bioregulation, Kyushu University, 3-1-1 Maidashi, Higashi-ku, Fukuoka 812-8582, Japan
Core Research for Evolutional Science and Technology (CREST),
Japan Science and Technology Agency, Saitama, Japan
e-mail: suzukicks@bioreg.kyushu-u.ac.jp 


\section{The Liver and Disease}

The liver is the central organ for metabolism, by which it produces energy for life activities and detoxifies various extrinsic and intrinsic harmful substances. In addition, the liver creates endocrine and exocrine materials, such as serum albumin, growth factors, and bile acids. The liver is developed from the foregut endoderm [1] and consists of parenchymal cells, such as hepatocytes, and various non-parenchymal cells, such as biliary epithelial cells, Kupffer cells, pit cells, hepatic stellate cells, mesothelial cells, and sinusoid endothelial cells [2]. Among the diverse kinds of cell populations, the major cell population in the liver is composed of hepatocytes, which play primary physiological roles in this organ.

The liver is one of the few organs capable of regeneration in the body. Since the ancient Greek era, the liver has been known to have a strong intrinsic regenerative ability in vivo. The famous "Prometheus" myth represents the rapid regeneration of this organ [3]. Indeed, deletion of two thirds of the total mass of the liver can be recovered completely in vivo [2]. However, chronic hepatitis generates a highly serious situation for the liver, and often leads to more severe hepatic failure, including hepatic cirrhosis and liver cancer. Thus, various conditions are attributed etiologically to chronic hepatitis. The most common cause of chronic hepatitis is infection by hepatotropic viruses, followed by alcoholic hepatitis, non-alcoholic fatty liver disease, drug-induced hepatitis, and autoimmune hepatitis [4].

\section{Expectations for Cell-Based Regenerative Therapies}

Many types of medical treatments have been applied to patients with chronic hepatitis. Although anti-inflammatory drugs, such as ursodeoxycholic acid, glycyrrhizin, and liver extracts, are widely used to treat all types of chronic hepatitis, such treatments are only symptomatic therapies. For patients with viral hepatitis, antiviral drugs, such as interferon, corticosteroid, ribavirin, lamivudine, and azathioprine, have been specifically employed. However, several side effects associated with these drugs, including fever and drug-resistant strains, appear with high frequency [4].

In the treatment of severe liver failure, organ transplantation is the ultimate solution and has a relatively high postoperative survival rate. However, there are many problems to be solved, including a chronic donor shortage, ethics, and immune rejection [4]. Therefore, cell-based regenerative therapies, which employ in vitroexpanded or newly generated hepatocytes, are expected to be the next-generation therapies.

Although hepatocytes have a high proliferative ability in vivo, this potential disappears immediately upon in vitro culture. Consequently, innovative technologies that can expand, maintain, mature, and create hepatocytes in vitro are required for future cell-based therapies for liver disorders. 


\section{Pluripotent Stem Cell-Based Approaches to Treat Liver Failure}

Among the current technologies aimed toward regenerative therapies for liver diseases, pluripotent stem cell-based approaches could be considered to show the most promise. Pluripotent stem cells can differentiate into any of the cell types responsible for the formation of particular tissues and organs, with the exception of extraembryonic tissues. Embryonic stem (ES) cells have been established as pluripotent stem cells and employed in basic research toward regenerative therapies for liver diseases. Hepatocyte differentiation from murine ES cells is generally induced via methods involving embryoid body (EB) formation [5-8]. Although EB-derived hepatocyte-like cells survive and function in vivo, their low differentiation frequency and associated teratoma formation remain as serious problems [9]. Several studies have tried to purify definitely differentiated hepatocytes or hepatic progenitor cells, using reporter expressions that are regulated by the promoters or enhancers of hepatocyte-specific genes, including albumin and $\alpha$-fetoprotein $(A f p)[10,11]$. Meanwhile, other studies have revealed the molecular mechanisms that regulate hepatocyte differentiation. In particular, employment of a liquidity factor, activin A, was found to efficiently improve both the ratio of hepatocyte differentiation and the quality of parental ES cells [12-14]. Other regulatory molecules required for hepatocyte differentiation have also been discovered [15-17]. By improving the in vitro hepatocyte differentiation of ES cells, many researchers have succeeded in transplantation of mouse/human ES cell-derived hepatocyte-like cells [18-20].

In 2006, Takahashi and Yamanaka [21] reported epoch-making artificial stem cells, termed "induced pluripotent stem" (iPS) cells. These novel stem cells can be expected to overcome the ethical and immunological problems associated with the use of ES cells [21,22]. Soon after the publication of their study on iPS cells, many research groups started to employ these novel stem cells for in vitro hepatocyte differentiation using methods and knowledge accumulated in studies on ES cells [23-26]. Most recently, Takebe et al. [27] reported that vascularized and functional human liver bud-like structures could be formed by human iPS cells in culture and regenerate a part of the liver tissue upon transplantation. This technology has brought iPS cells closer to clinical reality. Nonetheless, iPS cells still have some problems to be solved, including tumorigenesis after transplantation and substantial costs. Thus, more vigorous basic studies are required for clinical-level applications using iPS cell technology.

\section{Direct Reprogramming}

\section{Development of Direct Reprogramming Technologies}

Recently, the strategy of direct cell-fate conversion from one cell type into another cell type, termed "direct reprogramming", has rapidly expanded worldwide and is expected to be a complementary or alternative technology for future cell-based 
regenerative therapies using pluripotent stem cells. This method can generate both mature and progenitor-like cells in specific lineages from other types of cells. Thus, the cells required for treatment of particular diseases could be provided from an alternative source of cells and used as donor cells for transplantation therapy.

The first evidence for direct reprogramming was reported in the 1980s. Davis et al. [28] discovered a single transcription factor, Myod, that could induce fate conversion of fibroblasts into myoblasts. That sensational report encouraged researchers to look for single master transcription factors that could specify and govern the fate of cells in each lineage. However, studies on direct reprogramming subsequently went into decline for a long time, because further master transcription factors were not easy to discover.

In the last decade, the emergence of iPS cells has completely changed the situation for direct reprogramming studies [21, 22]. After the discovery of iPS cells, it was found that sets of transcription factors, rather than single transcription factors, could be successively identified as master regulators capable of inducing fate conversion of cells. At present, various types of cells have been induced from fibroblasts, including neurons [29], cardiomyocytes [30], hepatocytes [31, 32], adipocytes [33], Sertoli cells [34], and chondrocytes [35]. Furthermore, not only fibroblasts but also other types of cells, such as hepatocytes [36], astrocytes [37], Sertoli cells [38], and B cells [39], have been employed as sources of cells for direct reprogramming. These findings imply that direct reprogramming technology will become a universal method for almost all kinds of cells. Although the recent studies on direct reprogramming are summarized in Table 1, studies on direct reprogramming are continuing to increase year by year.

Regarding successful cases of direct reprogramming, three different cell lineages, cardiomyocytes, neurons, and hepatocytes, can be considered to be well-investigated targets for the following reasons: (1)functional failure of these cells is critical for the survival of individuals; (2) a number of patients suffer from diseases associated with malfunction of these cells; and (3) a large number of cells should be prepared in vitro prior to application of these cells for transplantation therapies. Among these three cell types, cardiomyocytes could be considered to be the most widely studied cells in the field of direct reprogramming. Following the publication of the first report by Ieda et al. [30], several groups reported similar methods for cardiomyocyte reprogramming [40-47]. However, the transcription factors used for the induction of cardiomyocyte-like cells were different in each study (Table 1), and the properties of the cells were also inhomogeneous [30, 40-47]. The obtained evidence suggested flexibility in the molecular mechanisms underlying direct reprogramming and a necessity for standardized protocols toward therapeutic applications.

The molecular machinery for the direct induction of neuronal cells in vitro can be considered to be the most deeply investigated example in the field of direct reprogramming. Vierbuchen et al. [29] showed that three transcription factors, Brm2, Ascl1, and Myt11, induced conversion of mouse fibroblasts into neuron-like cells, designated induced neuronal (iN) cells. iN cells had neuron-specific characteristics, including neurite outgrowth, expression of specific neuronal markers, and electrophysiological activities. In addition to the discovery of iN cells, the same group 
Table 1 Recent examples of direct reprogramming studies

\begin{tabular}{|c|c|c|c|c|}
\hline Target cell & Source cell & Factors used & Species & References \\
\hline Neuronal cell & $\begin{array}{l}\text { Embryonic } \\
\text { fibroblast }\end{array}$ & $\begin{array}{l}\text { Brn2, Ascl1, } \\
\text { and Myt11 }\end{array}$ & Mouse & $\begin{array}{l}\text { Vierbuchen } \\
\text { et al. [29] }\end{array}$ \\
\hline $\begin{array}{l}\text { Glutamatergic } \\
\text { neuron }\end{array}$ & Cortical astrocyte & Ngn2 & Mouse & $\begin{array}{l}\text { Berninger et al. } \\
{[56] ; \text { Heinrich }} \\
\text { et al. }[57]\end{array}$ \\
\hline $\begin{array}{l}\text { GABAergic } \\
\text { neuron }\end{array}$ & Cortical astrocyte & $\begin{array}{l}\text { Dlx2, or Dlx2 } \\
\text { and Ascl1 }\end{array}$ & Mouse & $\begin{array}{l}\text { Heinrich et al. } \\
{[57]}\end{array}$ \\
\hline Neuronal cell & Hepatocyte & $\begin{array}{l}\text { Brn2, Ascl1, } \\
\text { and Myt11 }\end{array}$ & Mouse & Marro et al. [36] \\
\hline $\begin{array}{l}\text { Dopaminergic } \\
\text { neuron }\end{array}$ & $\begin{array}{l}\text { Embryonic/skin } \\
\text { fibroblast }\end{array}$ & $\begin{array}{l}\text { Ascl1, Lmx1a, } \\
\text { and Nurr1 }\end{array}$ & $\begin{array}{l}\text { Mouse/ } \\
\text { human }\end{array}$ & $\begin{array}{l}\text { Caiazzo } \\
\text { et al. }[58]\end{array}$ \\
\hline Neuronal cell & $\begin{array}{l}\text { Fetal/postnatal } \\
\text { fibroblast }\end{array}$ & $\begin{array}{l}\text { Brn2, Ascl1, } \\
\text { Myt11, and NeuroD1 }\end{array}$ & Human & Pang et al. [59] \\
\hline Neuronal cell & $\begin{array}{l}\text { Neonatal foreskin/ } \\
\text { adult dermal } \\
\text { fibroblast }\end{array}$ & $\begin{array}{l}\text { Asc11, Brn2, Myt11, } \\
\text { Lmx1a, and Foxa2 }\end{array}$ & Human & $\begin{array}{l}\text { Pfisterer } \\
\text { et al. [60] }\end{array}$ \\
\hline Neuronal cell & Astrocyte & Brn4 & Mouse & Potts et al. [37] \\
\hline Neural stem cell & Sertoli cell & $\begin{array}{l}\text { Pax6, Ngn2, Hes1, } \\
\text { Id1, Ascl1, Brn2, } \\
\text { cMyc, and Klf4 }\end{array}$ & Mouse & Sheng et al. [38] \\
\hline Cardiomyocyte & Cardiac fibroblast & Gata4, Mef2c, and Tbx5 & Mouse & Ieda et al. [30] \\
\hline Cardiomyocyte & Cardiac fibroblast & $\begin{array}{l}\operatorname{miR}-1,133,208 \\
\text { and } 499\end{array}$ & Mouse & $\begin{array}{l}\text { Jayawardena } \\
\text { et al. [40] }\end{array}$ \\
\hline Cardiomyocyte & Cardiac fibroblast & $\begin{array}{l}\text { Gata } 4, \text { Mef2c, } \\
\text { Tbx } 5 \text {, and Hand } 2\end{array}$ & Mouse & Song et al. [41] \\
\hline Cardiomyocyte & Cardiac fibroblast & $\begin{array}{l}\text { Gata } 4, \text { Mef2c, } \\
\text { and Tbx } 5\end{array}$ & Mouse & Chen et al. [42] \\
\hline Cardiomyocyte & $\begin{array}{l}\text { Cardiac/embryonic } \\
\text { fibroblast }\end{array}$ & $\begin{array}{l}\text { Mef2c, Tbx } 5+ \\
\text { Myocd or Gata4 }\end{array}$ & Mouse & Protze et al. [43] \\
\hline Cardiomyocyte & $\begin{array}{l}\text { Embryonic } \\
\text { fibroblast }\end{array}$ & $\begin{array}{l}\text { Gata4, Tbx5, Hand2, } \\
\text { and Myod M3 domain } \\
\text { fused with Mef2c }\end{array}$ & Mouse & Hirai et al. [44] \\
\hline Cardiomyocyte & Dermal fibroblast & $\begin{array}{l}\text { GATA4, MEF2C, TBX5, } \\
\text { MESP1, and MYOCD }\end{array}$ & Human & Wada et al. [45] \\
\hline Cardiomyocyte & Dermal fibroblast & $\begin{array}{l}\text { GATA4, MEF2C, TBX5, } \\
\text { ESRRG, MESP1, } \\
\text { ZFPM2, and MYOCD }\end{array}$ & Human & Fu et al. [46] \\
\hline Cardiomyocyte & $\begin{array}{l}\text { Cardiac/embryonic } \\
\text { fibroblast }\end{array}$ & $\begin{array}{l}\text { Gata } 4, \text { Mef } 2 c, \\
\text { Tbx } 5+\operatorname{miR} 133 \text { or } \\
\text { Mesp } 1 \text { and Myocd }\end{array}$ & $\begin{array}{l}\text { Mouse/ } \\
\text { human }\end{array}$ & $\begin{array}{l}\text { Muraoka } \\
\text { et al. [47] }\end{array}$ \\
\hline Hepatocyte & $\begin{array}{l}\text { Embryonic/dermal } \\
\text { fibroblast }\end{array}$ & Hnf $4 \alpha+$ Foxa 1,2 , or 3 & Mouse & $\begin{array}{l}\text { Sekiya and } \\
\text { Suzuki [31] }\end{array}$ \\
\hline
\end{tabular}


Table 1 (continued)

\begin{tabular}{|c|c|c|c|c|}
\hline Target cell & Source cell & Factors used & Species & References \\
\hline Hepatocyte & Tail-tip fibroblast & $\begin{array}{l}\text { Gata4, Hnf1 } \alpha, \text { Foxa3, } \\
\text { and p19Arf KD }\end{array}$ & Mouse & Huang et al. [32] \\
\hline Hepatocyte & $\begin{array}{l}\text { Fetal limb } \\
\text { fibroblast }\end{array}$ & $\begin{array}{l}\text { FOXA3, HNF1A, } \\
\text { HNF4A, and SV40 Large } \\
\text { T antigen }\end{array}$ & Human & Huang et al. [53] \\
\hline Hepatocyte & $\begin{array}{l}\text { Embryonic } \\
\text { fibroblast }\end{array}$ & $\begin{array}{l}\text { HNF1A, HNF4A, } \\
\text { HNF6, ATF5, } \\
\text { PROX1, CEBP, } \\
\text { MYC, and TP53 KD }\end{array}$ & Human & Du et al. [54] \\
\hline Hepatocyte & Neonatal fibroblast & $\begin{array}{l}\text { HNF1A+FOXA1, } \\
\text { FOXA3, or HNF4A } \\
\text { (mRNA) }\end{array}$ & Human & $\begin{array}{l}\text { Simeonov and } \\
\text { Uppal [52] }\end{array}$ \\
\hline Sertoli cell & $\begin{array}{l}\text { Embryonic } \\
\text { fibroblast }\end{array}$ & $\begin{array}{l}\text { Nr5a1, Wt1, Dmrt1, } \\
\text { Gata4, and Sox9 }\end{array}$ & Mouse & $\begin{array}{l}\text { Buganim } \\
\text { et al. [34] }\end{array}$ \\
\hline Chondrocyte & Dermal fibroblast & $\begin{array}{l}\text { KLF4, MYC, } \\
\text { and SOX9 }\end{array}$ & Human & Tam et al. [35] \\
\hline Erythroid cell & B cell & $\begin{array}{l}\text { Gata1, Scl, } \\
\text { and Cebpa }\end{array}$ & Mouse & $\begin{array}{l}\text { Sadahira } \\
\text { et al. [39] }\end{array}$ \\
\hline $\mathrm{T}$ cell & B cell & Pax5 KO & Mouse & $\begin{array}{l}\text { Cobaleda } \\
\text { et al. [61] }\end{array}$ \\
\hline Monocyte & Skin fibroblast & $\begin{array}{l}\text { Spl1, Cebpa, Mnda, } \\
\text { and Irf8 }\end{array}$ & Mouse & $\begin{array}{l}\text { Suzuki et al. } \\
\text { [62] }\end{array}$ \\
\hline Macrophage & Pre-T cell & Cebpa or Cebpb & Mouse & Laiosa et al. [63] \\
\hline $\begin{array}{l}\text { Macrophage-like } \\
\text { cell }\end{array}$ & $\begin{array}{l}3 \text { T3 cell, } \\
\text { embryonic/skin } \\
\text { fibroblast }\end{array}$ & Pu. $1+$ Cebpa or Cebpb & Mouse & Feng et al. [64] \\
\hline Dendritic cell & Pre-T cell & Pu.1 & Mouse & Laiosa et al. [63] \\
\hline $\begin{array}{l}\text { Multilineage } \\
\text { blood progenitor }\end{array}$ & $\begin{array}{l}\text { Adult/neonatal } \\
\text { dermal fibroblast }\end{array}$ & $\begin{array}{l}\text { OCT4+hematopoietic } \\
\text { cytokine treatment }\end{array}$ & Human & Szabo et al. [65] \\
\hline Megakaryocyte & $\begin{array}{l}3 \text { T3 cell, dermal } \\
\text { fibroblast }\end{array}$ & $\begin{array}{l}\mathrm{Nfe} 2, \mathrm{Mafg} \\
\text { and Mafk }\end{array}$ & $\begin{array}{l}\text { Mouse/ } \\
\text { human }\end{array}$ & Ono et al. [66] \\
\hline $\begin{array}{l}\text { Thymic } \\
\text { epithelial cell }\end{array}$ & $\begin{array}{l}\text { Embryonic } \\
\text { fibroblast }\end{array}$ & Foxn1 & Mouse & $\begin{array}{l}\text { Bredenkamp } \\
\text { et al. [67] }\end{array}$ \\
\hline $\begin{array}{l}\text { Vascular } \\
\text { endothelial cell }\end{array}$ & Amniotic cell & $\begin{array}{l}\text { ETV2, FLI1, ERG1 } \\
\text { with TGFB inhibition }\end{array}$ & Human & $\begin{array}{l}\text { Ginsberg } \\
\text { et al. [68] }\end{array}$ \\
\hline $\mathrm{B}$ cell & $\begin{array}{l}\text { Pancreatic exocrine } \\
\text { cell }\end{array}$ & Ngn3, Pdx1, and Mafa & Mouse & Zhou et al. [69] \\
\hline Brown fat cell & Skin fibroblast & Prdm16 and Cebpb & $\begin{array}{l}\text { Mouse/ } \\
\text { human }\end{array}$ & $\begin{array}{l}\text { Kajimura } \\
\text { et al. [70] }\end{array}$ \\
\hline $\begin{array}{l}\text { Pancreatic } \\
\text { islet cell }\end{array}$ & Hepatocyte & Ngn3 & Mouse & $\begin{array}{l}\text { Desgraz and } \\
\text { Herrera [71] }\end{array}$ \\
\hline
\end{tabular}


revealed that remarkable epigenetic remodeling occurred in iN cells, and that Ascl1 acted as a pioneering factor that could activate closed chromatins during the reprogramming [48]. A better understanding of the molecular mechanisms controlling direct cell-fate conversion could provide great advantages for the development and application of direct reprogramming technology.

\section{Direct Reprogramming of Fibroblasts to Hepatocytes}

As mentioned above, functionally mature hepatocytes are strongly demanded for clinical use. To supply hepatocytes safely and stably without any ethical problems, direct reprogramming of patient-derived non-hepatic cells into hepatocytes appears to be a preferable method. Such newly generated hepatocytes could be technologically and economically useful for future cell-based regenerative therapies for liver diseases. The first studies showing direct conversion of non-hepatic cells into hepatocyte-like cells were independently reported by two groups: one was from our group [31] and the other was from Hui's group [31, 32] (Fig. 1). Although both groups induced conversion of mouse fibroblasts into hepatocyte-like cells,

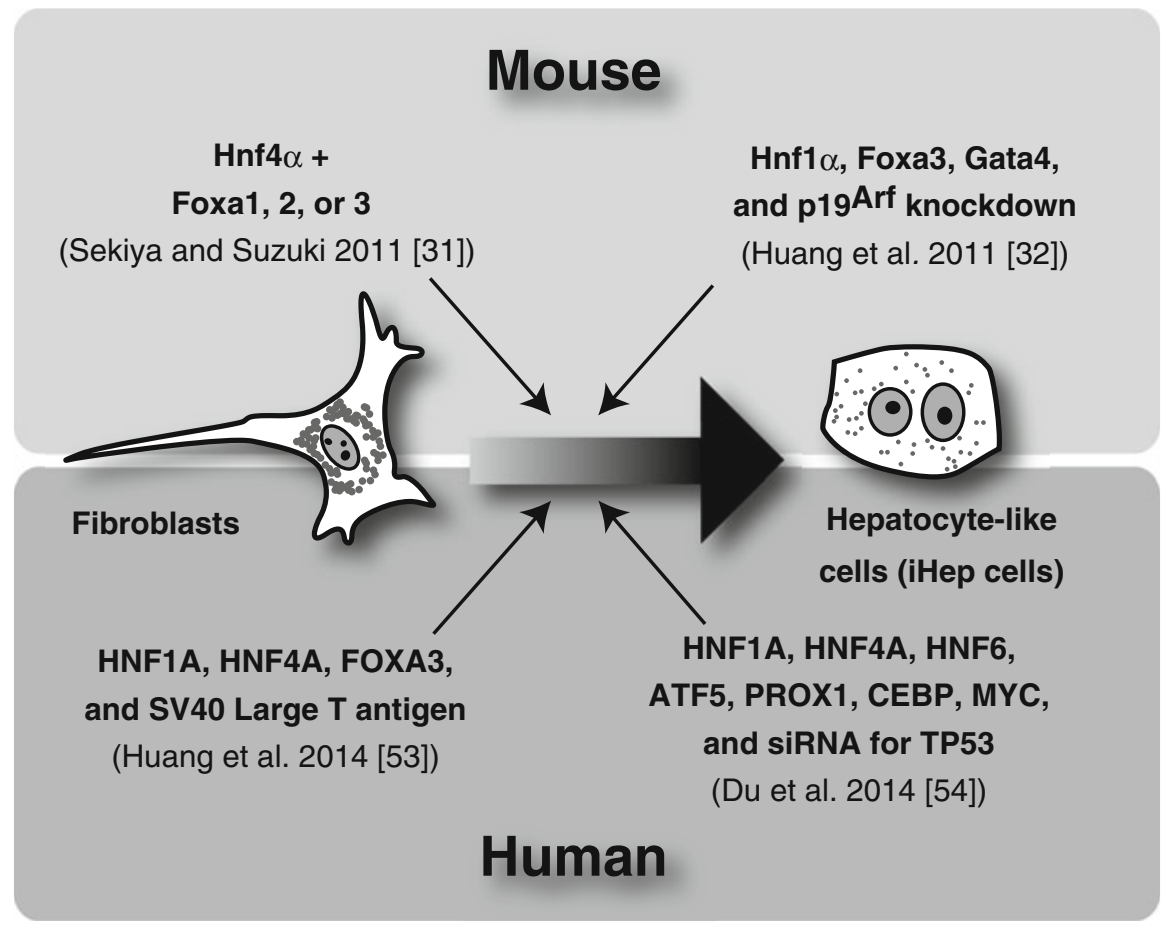

Fig. 1 Direct reprogramming of fibroblasts to iHep cells. iHep cells can be induced by various protocols 
the transcription factors used were different. Our group found that three combinations of two transcription factors, comprising a hepatocyte nuclear receptor, $\mathrm{Hnf} 4 \alpha$ [49], and members of the forkhead domain-containing transcription factor family, Foxa1, Foxa2, and Foxa3 [50], could induce conversion of fibroblasts into hepatocyte-like cells, designated induced hepatocyte-like (iHep) cells [31]. In contrast, Hui's group used a combination of three transcription factors, Gata4, Hnf1 $\alpha$, and Foxa3, with siRNA-based suppression of the expression of a tumor suppressor, p19 ${ }^{\text {Arf }}$ [32]. Although the reprogramming factors were different, iHep cells could be induced in both methods, and their morphological and functional properties were similar $[31,32]$. These findings suggest the presence of lineagespecific core transcriptional networks that can determine the fate of cells.

iHep cells have multiple hepatocyte-specific properties: (1) the morphology and gene expression pattern of iHep cells resemble those of epithelial cells; (2) iHep cells express hepatocyte-specific genes and proteins; (3) iHep cells have functional features of hepatocytes, including glycogen storage, LDL uptake, ammonium metabolism, urea production, cytochrome P450 activity, and drug metabolism; and (4) iHep cells can reconstitute liver tissues, ameliorate hepatic functions of recipient mice, and rescue mice from a deadly hepatic disorder, hereditary tyrosinemia type I, upon transplantation into the liver of a mouse model of the disease. In addition to these features, we recently showed that iHep cells possess the potential to be involved in lipid metabolism, similar to hepatocytes [51]. These findings suggest that iHep cells are useful not only for screening of drugs, but also for the treatment of patients with fatty liver diseases. The morphology and function of iHep cells do not become attenuated during culture or after freeze-preservation, which could be great advantages in the application of iHep cells to cell-based regenerative therapies for liver diseases.

The above-mentioned characteristics suggest that iHep cells could be a potent cell source for future cell transplantation therapies. However, three problems remain to be resolved. First, the level of hepatic function is lower in iHep cells than in primary hepatocytes. Therefore, for use of iHep cells in cell transplantation therapies, the level of hepatic function in iHep cells needs to be improved by inducing the maturation of these cells. Second, viral vectors need to be excluded from the reprogramming procedures, because integration of the virus genomes into the host genomes may induce malignant transformation of cells. Thus, other reprogramming strategies using chemical compounds, growth factors, or non-integrative vectors are expected to be practical. Recently, Simeonov and Uppal [52] demonstrated the potential utility of an mRNA transfection-based technique for the generation of iHep cells. This technology may contribute to safer induction of iHep cells. Third, application of iHep cell technology to human cells is still in the development stage. Recently, two different groups reported data on the induction of human iHep cells $[53,54]$. These studies showed that the technology for iHep cell generation in mouse cells could be reproducible in human cells. However, there are some critical differences between the methods for mouse and human iHep cell induction. In the case of human iHep cells, activation of MYC and SV40 large T antigen and suppression of TP53 are included in the methods for iHep cell generation, which have 
a risk for inducing malignant transformation of these cells (Fig. 1). Thus, it is expected that human iHep cells capable of proliferation without growth boosters, which can avoid the risk of tumorigenesis, should be generated for the application of iHep cells in transplantation therapies.

Conversely, Zhu et al. [55] reported a completely different strategy for induction of hepatocytes in vitro. They initially used OCT4, KLF4, SOX2, and MYC to induce iPS cells. However, they interrupted the conversion process of iPS cells and continued the culture of partially reprogrammed intermediate cells in the presence of various growth factors and chemical compounds that are known inducers of hepatic differentiation. The resultant hepatocyte-like cells showed hepatocytespecific features like iHep cells. Thus, this method could be another alternative to stem cell-based regenerative therapies for liver failures, as well as the technology of iHep cell generation.

\section{Conclusions}

Cell-based regenerative medicine is one of the most promising clinical technologies in the treatment of liver disorders. However, it has remained unclear whether cellbased therapies are actually available in clinical settings without any risk for patients. To reduce the associated risks, the methods for cellular reprogramming and differentiation procedures should be improved in ES cell, iPS cell, and iHep cell technologies. As one of the important approaches, we need to understand the molecular mechanisms underlying the generation of iHep cells and the differentiation of hepatocytes, with a view to updating the technology for direct reprogramming and actualizing the functional maturation of iHep cells for use in cell-based regenerative therapies toward liver diseases. We believe that many current efforts in basic research will open up a new horizon for next-generation therapies using directly reprogrammed cells.

Open Access This chapter is distributed under the terms of the Creative Commons Attribution Noncommercial License, which permits any noncommercial use, distribution, and reproduction in any medium, provided the original author(s) and source are credited.

\section{References}

1. Zaret KS (2002) Regulatory phases of early liver development: paradigms of organogenesis. Nat Rev Genet 3:499-512. doi:10.1038/nrg837

2. Fausto N (2002) Regeneration of liver. eLS. doi:10.1038/npg.els.0001108

3. Taub R (2004) Liver regeneration: from myth to mechanism. Nat Rev Mol Cell Biol 5:836-847. doi: $10.1038 / \mathrm{nrm} 1489$

4. Monga SPS (2011) Molecular pathology of liver diseases. Springer, New York

5. Hamazaki T, Iiboshi Y, Oka M, Papst PJ, Meacham AM, Zon LI, Terada N (2001) Hepatic maturation in differentiating embryonic stem cells in vitro. FEBS Lett 497:15-19. doi:10.1016/S0014-5793(01)02423-1 
6. Chinzei R, Tanaka Y, Shimizu-Saito K, Hara Y, Kakinuma S, Watanabe M, Teramoto K, Arii S, Takase K, Sato C, Terada N, Teraoka H (2002) Embryoid-body cells derived from a mouse embryonic stem cell line show differentiation into functional hepatocytes. Hepatology 36:22-29. doi:10.1053/jhep.2002.34136

7. Yamamoto H, Quinn G, Asari A, Yamanokuchi H, Teratani T, Terada M, Ochiya T (2003) Differentiation of embryonic stem cells into hepatocytes: biological functions and therapeutic application. Hepatology 37:983-993. doi:10.1053/jhep.2003.50202

8. Imamura T, Cui L, Teng R, Johkura K, Okouchi Y, Asanuma K, Ogiwara N, Sasaki K (2004) Embryonic stem cell-derived embryoid bodies in three-dimensional culture system form hepatocyte-like cells in vitro and in vivo. Tissue Eng 10:1716-1724. doi:10.1089/ ten.2004.10.1716

9. Kumashiro Y, Asahina K, Ozeki R, Shimizu-Saito K, Tanaka Y, Kida Y, Inoue K, Kaneko M, Sato T, Teramoto K, Arii S, Teraoka H (2005) Enrichment of hepatocytes differentiated from mouse embryonic stem cells as a transplantable source. Transplantation 79:550-557. doi:10.1097/01.TP.0000153637.44069.C6

10. Heo J, Factor VM, Uren T, Takahama Y, Lee J-S, Major M, Feinstone SM, Thorgeirsson SS (2006) Hepatic precursors derived from murine embryonic stem cells contribute to regeneration of injured liver. Hepatology 44:1478-1486. doi:10.1002/hep.21441

11. Drobinskaya I, Linn T, Saric T, Bretzel RG, Bohlen H, Hescheler J, Kolossov E (2008) Scalable selection of hepatocyte- and hepatocyte precursor-like cells from culture of differentiating transgenically modified murine embryonic stem cells. Stem Cells 26:22452256. doi:10.1634/stemcells.2008-0387

12. Tada S, Era T, Furusawa C, Sakurai H, Nishikawa S, Kinoshita M, Nakao K, Chiba T, Nishikawa S-I (2005) Characterization of mesendoderm: a diverging point of the definitive endoderm and mesoderm in embryonic stem cell differentiation culture. Development 132:4363-4374. doi:10.1242/dev.02005

13. Yasunaga M, Tada S, Torikai-Nishikawa S, Nakano Y, Okada M, Jakt LM, Nishikawa S, Chiba T, Era T, Nishikawa S-I (2005) Induction and monitoring of definitive and visceral endoderm differentiation of mouse ES cells. Nat Biotechnol 23:1542-1550. doi:10.1038/nbt1167

14. D'Amour KA, Agulnick AD, Eliazer S, Kelly OG, Kroon E, Baetge EE (2005) Efficient differentiation of human embryonic stem cells to definitive endoderm. Nat Biotechnol 23:1534-1541. doi:10.1038/nbt1163

15. Gouon-Evans V, Boussemart L, Gadue P, Nierhoff D, Koehler CI, Kubo A, Shafritz DA, Keller G (2006) BMP-4 is required for hepatic specification of mouse embryonic stem cell-derived definitive endoderm. Nat Biotechnol 24:1402-1411. doi:10.1038/nbt1258

16. Kubo A, Kim YH, Irion S, Kasuda S, Takeuchi M, Ohashi K, Iwano M, Dohi Y, Saito Y, Snodgrass R, Keller G (2010) The homeobox gene Hex regulates hepatocyte differentiation from embryonic stem cell-derived endoderm. Hepatology 51:633-641. doi:10.1002/hep.23293

17. Han S, Dziedzic N, Gadue P, Keller GM, Gouon-Evans V (2011) An endothelial cell niche induces hepatic specification through dual repression of Wnt and Notch signaling. Stem Cells 29:217-228. doi:10.1002/stem.576

18. Ishii T, Yasuchika K, Machimoto T, Kamo N, Komori J, Konishi S, Suemori H, Nakatsuji N, Saito M, Kohno K, Uemoto S, Ikai I (2007) Transplantation of embryonic stem cell-derived endodermal cells into mice with induced lethal liver damage. Stem Cells 25:3252-3260. doi:10.1634/stemcells.2007-0199

19. Sharma AD, Cantz T, Vogel A, Schambach A, Haridass D, Iken M, Bleidissel M, Manns MP, Schöler HR, Ott M (2008) Murine embryonic stem cell-derived hepatic progenitor cells engraft in recipient livers with limited capacity of liver tissue formation. Cell Transplant 17:313-323, http://dx.doi.org/10.3727/096368908784153896

20. Basma H, Soto-Gutiérrez A, Yannam GR, Liu L, Ito R, Yamamoto T, Ellis E, Carson SD, Sato S, Chen Y, Muirhead D, Navarro-Alvarez N, Wong RJ, Roy-Chowdhury J, Platt JL, Mercer DF, Miller JD, Strom SC, Kobayashi N, Fox IJ (2009) Differentiation and transplantation of human embryonic stem cell-derived hepatocytes. Gastroenterology 136:990-999. doi:10.1053/j.gastro.2008.10.047 
21. Takahashi K, Yamanaka S (2006) Induction of pluripotent stem cells from mouse embryonic and adult fibroblast cultures by defined factors. Cell 126:663-676. doi:10.1016/j. cell.2006.07.024

22. Takahashi K, Tanabe K, Ohnuki M, Narita M, Ichisaka T, Tomoda K, Yamanaka S (2007) Induction of pluripotent stem cells from adult human fibroblasts by defined factors. Cell 131:861-872. doi:10.1016/j.cell.2007.11.019

23. Song Z, Cai J, Liu Y, Zhao D, Yong J, Duo S, Song X, Guo Y, Zhao Y, Qin H, Yin X, Wu C, Che J, Lu S, Ding M, Deng H (2009) Efficient generation of hepatocyte-like cells from human induced pluripotent stem cells. Cell Res 19:1233-1242. doi:10.1038/cr.2009.107

24. Sullivan GJ, Hay DC, Park I-H, Fletcher J, Hannoun Z, Payne CM, Dalgetty D, Black JR, Ross JA, Samuel K, Wang G, Daley GQ, Lee J-H, Church GM, Forbes SJ, Iredale JP, Wilmut I (2010) Generation of functional human hepatic endoderm from human induced pluripotent stem cells. Hepatology 51:329-335. doi:10.1002/hep.23335

25. Li W, Wang D, Qin J, Liu C, Zhang Q, Zhang X, Yu X, Lahn BT, Mao FF, Xiang AP (2010) Generation of functional hepatocytes from mouse induced pluripotent stem cells. J Cell Physiol 222:492-501. doi:10.1002/jcp.22000

26. Espejel S, Roll GR, McLaughlin KJ, Lee AY, Zhang JY, Laird DJ, Okita K, Yamanaka S, Willenbring H (2010) Induced pluripotent stem cell-derived hepatocytes have the functional and proliferative capabilities needed for liver regeneration in mice. J Clin Invest 120:3120-3126. doi:10.1172/JCI43267

27. Takebe T, Sekine K, Enomura M, Koike H, Kimura M, Ogaeri T, Zhang R-R, Ueno Y, Zheng Y-W, Koike N, Aoyama S, Adachi Y, Taniguchi H (2013) Vascularized and functional human liver from an iPSC-derived organ bud transplant. Nature 499:481-484. doi:10.1038/ nature 12271

28. Davis RL, Weintraub H, Lassar AB (1987) Expression of a single transfected cDNA converts fibroblasts to myoblasts. Cell 51:987-1000. doi:10.1016/0092-8674(87)90585-X

29. Vierbuchen T, Ostermeier A, Pang ZP, Kokubu Y, Südhof TC, Wernig M (2010) Direct conversion of fibroblasts to functional neurons by defined factors. Nature 463:1035-1041. doi: $10.1038 /$ nature 08797

30. Ieda M, Fu J-D, Delgado-Olguin P, Vedantham V, Hayashi Y, Bruneau BG, Srivastava D (2010) Direct reprogramming of fibroblasts into functional cardiomyocytes by defined factors. Cell 142:375-386. doi:10.1016/j.cell.2010.07.002

31. Sekiya S, Suzuki A (2011) Direct conversion of mouse fibroblasts to hepatocyte-like cells by defined factors. Nature 475:390-393. doi:10.1038/nature10263

32. Huang P, He Z, Ji S, Sun H, Xiang D, Liu C, Hu Y, Wang X, Hui L (2011) Induction of functional hepatocyte-like cells from mouse fibroblasts by defined factors. Nature 475:386-389. doi:10.1038/nature10116

33. Zhu J, Pang D, Zhou Y, Tang X, Huang Y, Xie W, Gao F, Lai L, Zhang M, Ouyang H (2012) Direct conversion of porcine embryonic fibroblasts into adipocytes by chemical molecules. Cell Reprogram 14:99-105. doi:10.1089/cell.2011.0074

34. Buganim Y, Itskovich E, Hu Y-C, Cheng AW, Ganz K, Sarkar S, Fu D, Welstead GG, Page DC, Jaenisch R (2012) Direct reprogramming of fibroblasts into embryonic Sertoli-like cells by defined factors. Cell Stem Cell 11:373-386. doi:10.1016/j.stem.2012.07.019

35. Tam WL, DF O, Hiramatsu K, Tsumaki N, Luyten FP, Roberts SJ (2014) Sox9 reprogrammed dermal fibroblasts undergo hypertrophic differentiation in vitro and trigger endochondral ossification in vivo. Cell Reprogram 16:29-39. doi:10.1089/cell.2013.0060

36. Marro S, Pang ZP, Yang N, Tsai M-C, Qu K, Chang HY, Südhof TC, Wernig M (2011) Direct lineage conversion of terminally differentiated hepatocytes to functional neurons. Cell Stem Cell 9:374-382. doi:10.1016/j.stem.2011.09.002

37. Potts MB, Siu JJ, Price JD, Salinas RD, Cho MJ, Ramos AD, Hahn J, Margeta M, Oldham MC, Lim DA (2014) Analysis of Mll1-deficiency Identifies Neurogenic Transcriptional Modules and Brn4 as a Factor for Direct Astrocyte-to-neuron Reprogramming. Neurosurgery. doi:10.1227/NEU.0000000000000452 
38. Sheng C, Zheng Q, Wu J, Xu Z, Wang L, Li W, Zhang H, Zhao X-Y, Liu L, Wang Z, Guo C, Wu H-J, Liu Z, Wang L, He S, Wang X-J, Chen Z, Zhou Q (2012) Direct reprogramming of Sertoli cells into multipotent neural stem cells by defined factors. Cell Res 22:208-218. doi: $10.1038 / \mathrm{cr} .2011 .175$

39. Sadahira K, Fukuchi Y, Kunimono H, Sakurai M, Ikeda Y, Okamoto S, Nakajima H (2012) Direct reprogramming of terminally differentiated B cells into erythroid lineage. FEBS Lett 586:3645-3652. doi:10.1016/j.febslet.2012.08.019

40. Jayawardena TM, Egemnazarov B, Finch EA, Zhang L, Payne JA, Pandya K, Zhang Z, Rosenberg P, Mirotsou M, Dzau VJ (2012) MicroRNA-mediated in vitro and in vivo direct reprogramming of cardiac fibroblasts to cardiomyocytes. Circ Res 110:1465-1473. doi:10.1161/CIRCRESAHA.112.269035

41. Song K, Nam Y-J, Luo X, Qi X, Tan W, Huang GN, Acharya A, Smith CL, Tallquist MD, Neilson EG, Hill JA, Bassel-Duby R, Olson EN (2012) Heart repair by reprogramming nonmyocytes with cardiac transcription factors. Nature 485:599-604. doi:10.1038/nature11139

42. Chen JX, Krane M, Deutsch M-A, Wang L, Rav-Acha M, Gregoire S, Engels MC, Rajarajan K, Karra R, Abel ED, Wu JC, Milan D, Wu SM (2012) Inefficient reprogramming of fibroblasts into cardiomyocytes using Gata4, Mef2c, and Tbx5. Circ Res 111:50-55. doi:10.1161/ CIRCRESAHA.112.270264

43. Protze S, Khattak S, Poulet C, Lindemann D, Tanaka EM, Ravens U (2012) A new approach to transcription factor screening for reprogramming of fibroblasts to cardiomyocyte-like cells. J Mol Cell Cardiol 53:323-332. doi:10.1016/j.yjmcc.2012.04.010

44. Hirai H, Katoku-Kikyo N, Keirstead SA, Kikyo N (2013) Accelerated direct reprogramming of fibroblasts into cardiomyocyte-like cells with the MyoD transactivation domain. Cardiovasc Res 100:105-113. doi:10.1093/cvr/cvt167

45. Wada R, Muraoka N, Inagawa K, Yamakawa H, Miyamoto K, Sadahiro T, Umei T, Kaneda R, Suzuki T, Kamiya K, Tohyama S, Yuasa S, Kokaji K, Aeba R, Yozu R, Yamagishi H, Kitamura T, Fukuda K, Ieda M (2013) Induction of human cardiomyocyte-like cells from fibroblasts by defined factors. Proc Natl Acad Sci U S A 110:12667-12672. doi:10.1073/pnas.1304053110

46. Fu J-D, Stone NR, Liu L, Spencer CI, Qian L, Hayashi Y, Delgado-Olguin P, Ding S, Bruneau BG, Srivastava D (2013) Direct Reprogramming of Human Fibroblasts toward a Cardiomyocyte-like State. Stem Cell Rep 1:235-247. doi:10.1016/j.stemcr.2013.07.005

47. Muraoka N, Yamakawa H, Miyamoto K, Sadahiro T, Umei T, Isomi M, Nakashima H, Akiyama M, Wada R, Inagawa K, Nishiyama T, Kaneda R, Fukuda T, Takeda S, Tohyama S, Hashimoto H, Kawamura Y, Goshima N, Aeba R, Yamagishi H, Fukuda K, Ieda M (2014) MiR-133 promotes cardiac reprogramming by directly repressing Snai1 and silencing fibroblast signatures. EMBO J 33:1565-1581. doi:10.15252/embj.201387605

48. Wapinski OL, Vierbuchen T, Qu K, Lee QY, Chanda S, Fuentes DR, Giresi PG, Ng YH, Marro S, Neff NF, Drechsel D, Martynoga B, Castro DS, Webb AE, Südhof TC, Brunet A, Guillemot F, Chang HY, Wernig M (2013) Hierarchical mechanisms for direct reprogramming of fibroblasts to neurons. Cell 155:621-635. doi:10.1016/j.cell.2013.09.028

49. Hwang-Verslues WW, Sladek FM (2010) HNF4 $\alpha-$ role in drug metabolism and potential drug target? Curr Opin Pharmacol 10:698-705. doi:10.1016/j.coph.2010.08.010

50. Lam EW-F, Brosens JJ, Gomes AR, Koo C-Y (2013) Forkhead box proteins: tuning forks for transcriptional harmony. Nat Rev Cancer 13:482-495. doi:10.1038/nrc3539

51. Miura S, Suzuki A (2014) Acquisition of lipid metabolic capability in hepatocyte-like cells directly induced from mouse fibroblasts. Front Cell Dev Biol 2:43

52. Simeonov KP, Uppal H (2014) Direct reprogramming of human fibroblasts to hepatocyte-like cells by synthetic modified mRNAs. PLoS One 9, e100134. doi:10.1371/journal.pone.0100134

53. Huang P, Zhang L, Gao Y, He Z, Yao D, Wu Z, Cen J, Chen X, Liu C, Hu Y, Lai D, Hu Z, Chen L, Zhang Y, Cheng X, Ma X, Pan G, Wang X, Hui L (2014) Direct reprogramming of human fibroblasts to functional and expandable hepatocytes. Cell Stem Cell 14:370-384. doi:10.1016/j. stem.2014.01.003

54. Du Y, Wang J, Jia J, Song N, Xiang C, Xu J, Hou Z, Su X, Liu B, Jiang T, Zhao D, Sun Y, Shu J, Guo Q, Yin M, Sun D, Lu S, Shi Y, Deng H (2014) Human hepatocytes with drug metabolic function induced from fibroblasts by lineage reprogramming. Cell Stem Cell 14:394-403. doi:10.1016/j.stem.2014.01.008 
55. Zhu S, Rezvani M, Harbell J, Mattis AN, Wolfe AR, Benet LZ, Willenbring H, Ding S (2014) Mouse liver repopulation with hepatocytes generated from human fibroblasts. Nature 508:93-97. doi:10.1038/nature13020

56. Berninger B, Costa MR, Koch U, Schroeder T, Sutor B, Grothe B, Götz M (2007) Functional properties of neurons derived from in vitro reprogrammed postnatal astroglia. J Neurosci 27:8654-8664. doi:10.1523/JNEUROSCI.1615-07.2007

57. Heinrich C, Blum R, Gascón S, Masserdotti G, Tripathi P, Sánchez R, Tiedt S, Schroeder T, Götz M, Berninger B (2010) Directing astroglia from the cerebral cortex into subtype specific functional neurons. PLoS Biol 8, e1000373. doi:10.1371/journal.pbio.1000373

58. Caiazzo M, Dell'Anno MT, Dvoretskova E, Lazarevic D, Taverna S, Leo D, Sotnikova TD, Menegon A, Roncaglia P, Colciago G, Russo G, Carninci P, Pezzoli G, Gainetdinov RR, Gustincich S, Dityatev A, Broccoli V (2011) Direct generation of functional dopaminergic neurons from mouse and human fibroblasts. Nature 476:224-227. doi:10.1038/nature 10284

59. Pang ZP, Yang N, Vierbuchen T, Ostermeier A, Fuentes DR, Yang TQ, Citri A, Sebastiano V, Marro S, Südhof TC, Wernig M (2011) Induction of human neuronal cells by defined transcription factors. Nature 476:220-223. doi:10.1038/nature10202

60. Pfisterer U, Kirkeby A, Torper O, Wood J, Nelander J, Dufour A, Björklund A, Lindvall O, Jakobsson J, Parmar M (2011) Direct conversion of human fibroblasts to dopaminergic neurons. Proc Natl Acad Sci U S A 108:10343-10348. doi:10.1073/pnas.1105135108

61. Cobaleda C, Jochum W, Busslinger M (2007) Conversion of mature B cells into T cells by dedifferentiation to uncommitted progenitors. Nature 449:473-477. doi:10.1038/nature06159

62. Suzuki T, Nakano-Ikegaya M, Yabukami-Okuda H, de Hoon M, Severin J, Saga-Hatano S, Shin JW, Kubosaki A, Simon C, Hasegawa Y, Hayashizaki Y, Suzuki H (2012) Reconstruction of monocyte transcriptional regulatory network accompanies monocytic functions in human fibroblasts. PLoS One 7, e33474. doi:10.1371/journal.pone.0033474

63. Laiosa CV, Stadtfeld M, Xie H, de Andres-Aguayo L, Graf T (2006) Reprogramming of committed $\mathrm{T}$ cell progenitors to macrophages and dendritic cells by C/EBP alpha and PU.1 transcription factors. Immunity 25:731-744. doi:10.1016/j.immuni.2006.09.011

64. Feng R, Desbordes SC, Xie H, Tillo ES, Pixley F, Stanley ER, Graf T (2008) PU.1 and C/EBPalpha/beta convert fibroblasts into macrophage-like cells. Proc Natl Acad Sci U S A 105:6057-6062. doi:10.1073/pnas.0711961105

65. Szabo E, Rampalli S, Risueño RM, Schnerch A, Mitchell R, Fiebig-Comyn A, LevadouxMartin M, Bhatia M (2010) Direct conversion of human fibroblasts to multilineage blood progenitors. Nature 468:521-526. doi:10.1038/nature09591

66. Ono Y, Wang Y, Suzuki H, Okamoto S, Ikeda Y, Murata M, Poncz M, Matsubara Y (2012) Induction of functional platelets from mouse and human fibroblasts by p45NF-E2/Maf. Blood 120:3812-3821. doi:10.1182/blood-2012-02-413617

67. Bredenkamp N, Ulyanchenko S, O’Neill KE, Manley NR, Vaidya HJ, Blackburn CC (2014) An organized and functional thymus generated from FOXN1-reprogrammed fibroblasts. Nat Cell Biol. doi:10.1038/ncb3023

68. Ginsberg M, James D, Ding B-S, Nolan D, Geng F, Butler JM, Schachterle W, Pulijaal VR, Mathew S, Chasen ST, Xiang J, Rosenwaks Z, Shido K, Elemento O, Rabbany SY, Rafii S (2012) Efficient direct reprogramming of mature amniotic cells into endothelial cells by ETS factors and TGF $\beta$ suppression. Cell 151:559-575. doi:10.1016/j.cell.2012.09.032

69. Zhou Q, Brown J, Kanarek A, Rajagopal J, Melton DA (2008) In vivo reprogramming of adult pancreatic exocrine cells to beta-cells. Nature 455:627-632. doi:10.1038/nature07314

70. Kajimura S, Seale P, Kubota K, Lunsford E, Frangioni JV, Gygi SP, Spiegelman BM (2009) Initiation of myoblast to brown fat switch by a PRDM16-C/EBP-beta transcriptional complex. Nature 460:1154-1158. doi:10.1038/nature08262

71. Desgraz R, Herrera PL (2009) Pancreatic neurogenin 3-expressing cells are unipotent islet precursors. Development 136:3567-3574. doi:10.1242/dev.039214 